\title{
FLOODPLAIN INHABITATION A SOCIO-SPATIAL RESILIENCE NETWORK
}

\author{
Rebecca Boyle
}

A thesis submitted in partial fulfilment of the requirements for the degree of

MASTER OF ARCHITECTURE (PROFESSIONAL)

at the

SCHOOL OF ARCHITECTURE

VICTORIA UNIVERSITY OF WELLINGTON

2012 


\section{ACKNOWLEDGEMENTS}

I would like to thank my supervisor Penny for her positivity, guidance and support through the entirety of this research endeavour

$\&$

Maitland City Council for their generosity in meeting with me and granting permission to include the Central Maitland Structure Plan maps.

To Nick for helping me keep life in balance and my thesis in perspective

$\&$

to my friends and family including Mum, Dad, Pat, Soph, Tom, Chris, Ben, Biddy, Bardy, Maureen, Ian, Liz, Kathryn, Fuchsia, Tori and Rach for the distraction, encouragement and nurturing of my passions through the years,

I would like to say,

\section{Thankyou}




\section{PREFACE}

I remember with such clarity that June day, sitting on the verandah of my Granada home in the unrelenting Spanish heat attempting to cool myself down. The radio pop music was droning on in the background; the most recent method of improving my Spanish. When suddenly in amongst indistinguishable words there was a phrase I understood; 'demasiada lluvia en Australia' (too much rain in Australia).

My home town of Newcastle was flooding, along with much of the Hunter Region. My family became isolated from one another and my younger brother found himself at home alone, without electricity, sandbagging the house. Radio became a source of connecting community members.

The most recent devastating widespread flooding within New South Wales and Queensland highlight the continuing issues and safety concerns for the thousands of Australians living on flood prone land. As I sat watching the footage of evacuations, helicopter depictions of townships underwater and isolated residents, I began to contemplate the future of living on flood prone land. One only needs to look at the populated coastline and riverine locations of Australia to realise that humans have and always will seek to reside beside bodies of water. I recognise within myself that throughout my life I too have chosen, like so many before me, to live in coastal and riverine locations, attracted to the recreational, environmental and lifestyle benefits it provides.

Flooding will continue to affect us all either directly or indirectly. There needs to be a way in which the natural inevitability of flooding can be accepted and planned for, rather than continuing to view it as a disastrous event to be feared. Investigations into how vulnerabilities can be reduced and resilience strengthened will benefit the future of thousands of individuals who, despite the risks, will continue to inhabit flood prone land into the future. 
"The catastrophes of the 2010-11 Queensland and Victorian

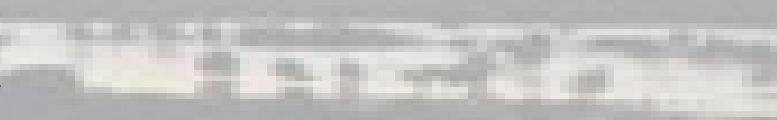
floods the worst and the most costly floods in Australia's history... affirm the necessity to work with the logics of landscape and Fit forces of nature, rather than against them. The harsh conditions of the continent are a mirror of what will occur in different $+\frac{1}{2}$ degrees and variations elsewhere in the world".
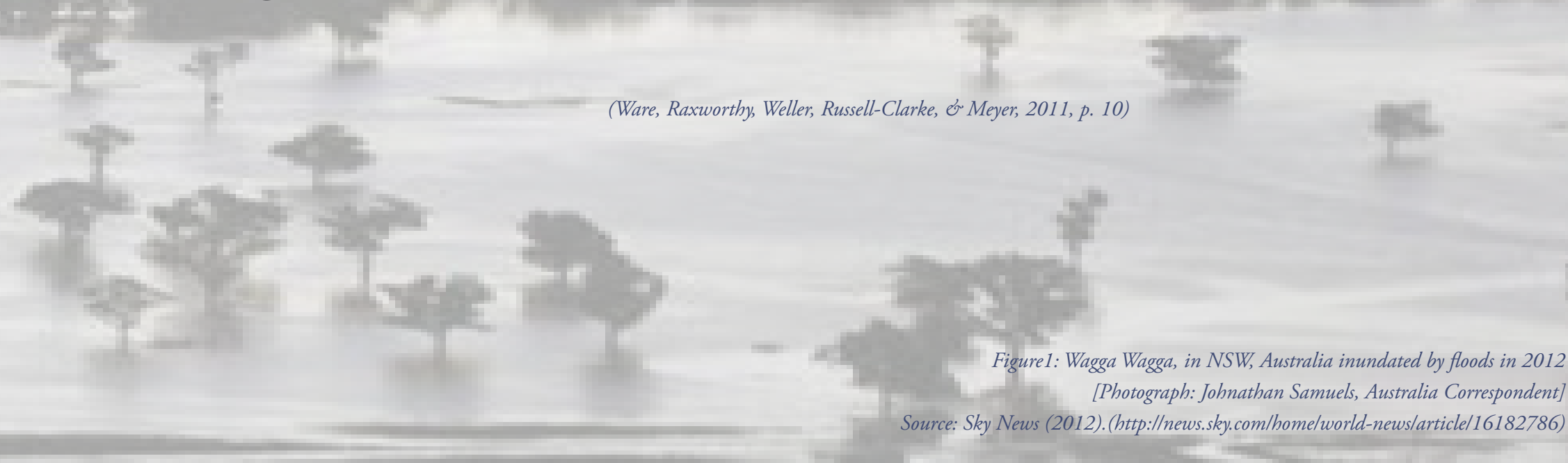


\section{ABSTRACT}

\section{Keywords; Riverine floodplain inhabitation, resilience, Maitland (Australia)}

The inadequacy of current approaches to managing floodplain inhabitation was highlighted in the 2010-11 Queensland, northern New South Wales and Victorian floods; the most costly floods in Australia's history. Despite technological advancements and the prevalence of flood mitigation infrastructure, floods continue to have widespread adverse physical, social, economic, and emotional impacts. This situation is mirrored internationally and is anticipated to worsen as scientists predict an increase in the severity and prevalence of natural disasters such as flooding. In response to this, management of floodplain inhabitation must shift from flood prevention to adaptation.

Adaptation is a key term in ecological resilience, defined as the capacity of a system to adapt and persist in the face of disturbance (Holling 1976). Hendstra et al (2004) suggests that in the context of disaster-resilient cities, resilience can be defined as the "capacity to adapt to stress from hazards and the ability to recover quickly from their impacts" (Henstra, Kovacs, McBean, \& Sweeting, 2004, p. 5). Analysis of ecosystems reveals that interdependence across scales, variety, redundancy, adaptability and feedback are the key resilience principles enabling the system to adapt and maintain stability during flooding.

At present there is a sparsity of literature exploring spatial resilience approaches to improving floodplain inhabitation. Whilst amphibious approaches improve individual resilience, there is a lack of innovative solutions to improve community and city resilience to flooding. Resilience approaches have the potential to reduce safety concerns, financial losses and the emotional stress associated with residing on Australian floodplains. Such approaches acknowledge the interconnected nature of riverine floodplains and their inhabitants. However resilience principles need to be given a physical spatial function within specific social contexts. Architecture provides a platform to test new and retrofit adaptable approaches to promote a more suitable spatial relationship with the river. This thesis will take the theory and literature of resilience and apply it to a site-specific spatial context: Maitland.

Maitland city is built on one of the most flood prone regions in New South Wales (Keys, 1999). Despite the Hunter Valley Flood Mitigation Scheme, which consists of 170 kilometers of levees and flood control structures, flooding continues to occur in and around Maitland. Regardless of these flood risks, Maitland City Council is proposing large scale residential development on the floodplain to encourage population increase. Maitland will be used as the primary case study for investigating the opportunities socio-spatial resilience interventions have for improving the longterm inhabitation of the floodplain. This thesis proposes a multi-scaled approach to examine flood hazard and exposure at the individual, community, city and regional scale. As spatial designers it is imperative that architects play a part in this explorative process. 
Floods are currently perceived as disastrous due to the devastating physical, emotional, cultural and economic impacts they have on inhabitants of the flood prone land. Recent advances in amphibious construction are providing a means to improve individual resilience, but these measures fail to improve community and city resilience to flooding.

RESEARCH QUESTION:

RESEARCH AIMS:

What role can architecture play in strengthening socio-spatial flood resilience at a city and urban scale?

- Establish a new resilience flood management approach

- Explore the role architecture can play in strengthening socio-spatial resilience for future floodplain inhabitation.

- Improve the ability of floodplain communities to remain connected within a functional city structure when impacted upon by flooding.

\section{DECLARATION:}

Note: Unless otherwise indicated north is vertically up the page. Figures within the text without acknowledgement are by the author.

This thesis does not provide a complete overview of flood management, but rather discusses particular aspects that justify resilience being explored as an alternative strategy.

This thesis does not attempt to provide a final solution, but rather a category of innovative possibilities which challenge current perceptions of riverine floodplain inhabitation.

Instead,

this thesis aims to reveal the opportunities a socio-spatial resilience network provide for future floodplain cities, reinforcing the need for further investigation into how practical measures can be implemented to improve the flood resilience of infrastructure and communities of floodplain cities. 


\section{CONTENTS}

TITLE PAGE

ACKNOWLEDGEMENTS

PREFACE

ABSTRACT

PROBLEM, QUESTION, AIMS, DECLARATION

- 1.0 INTRODUCTION •

- 1.1 OVERVIEW OF THE PROBLEM AND ITS CONTEXT

- 1.2 MOTIVATIONS FOR RESEARCH

- 1.3 SCOPE OF RESEARCH

- 1.4 AIM AND OBJECTIVES

- 1.5 THESIS STRUCTURE

- 1.6 DESIGN THROUGH RESEARCH

- 2.1 FLOODS ON FLOODPLAINS

- 2.2 HISTORY OF FLOODPLAIN INHABITATION

- 2.3 TRANSFORMING FLOODPLAIN MANAGEMENT PRACTICES: MANAGING RISK

- 2.4 THE CURRENT HOLISTIC APPROACH

- 2.5 SHORTCOMINGS OF FLOOD MANAGEMENT STANDARDS

- 2.6 A NEW RESILIENCE APPROACH 
3.1 RESILIENCE: A TRANSFORMING THEORY

3.2 KEY PRINCIPLES OF ECOLOGICAL RESILIENCE TO FLOODING

- 3.3 RESILIENCE IN THE CONTEXT OF NATURAL HAZARDS

- 3.4 SOCIO-SPATIAL FLOOD RESILIENCE

- 3.4.1 Society - Regional scale

- 3.4.2 Communities - City scale

- 3.4.3 Neighbourhoods - Urban Scale

- 3.4.4 Individuals and families - Building/lot scale

- 3.5 RESILIENCE FLOODPLAIN MANAGEMENT

- 3.6 CONCLUSION: THE NEED FOR A RESILIENCE FLOOD MANAGEMENT NETWORK

4.1 FLOOD RESILIENCE PRINCIPLES

4.2 SOCIO-SPATIAL SCALES

49

4.3 ADAPTABILITY

4.4 VARIETY AND REDUNDANCY

4.5 FEEDBACK

- 4.6 SUMMARY OF FLOOD RESILIENCE STRATEGIES FOR FLOODPLAIN CITIES

- 4.7 ESTABLISHING A RESILIENCE FLOOD MANAGEMENT MATRIX

- 4.7.1 The resilience flood management matrix

4.7.2 Limitations of the matrix

- 4.8 CONCLUSION 
- 5.1 RESILIENCE STRATEGIES

- 5.2 A CRITERIA TO ANALYSE PRECEDENTS

- 5.3 TONLE SAP LAKE, LOWER MEKONG RIVER BASIN, CAMBODIA

- 5.4 LOWER NINTH WARD, NEW ORLEANS, UNITED STATES OF AMERICA

- 5.5 MAASBOMMEL, WEST MAAS EN WAAL, NETHERLANDS

- 5.6 RESILIENCE FLOOD MANAGEMENT MATRIX: MACRO/MICRO ANALYSIS

- 5.7 KEY FINDINGS FROM PRECEDENT ANALYSIS

- 6.1 CONTEXT OF MAITLAND

6.1.1 Maitland City Council development proposals

- 6.2 RESILIENCE FLOOD MANAGEMENT MATRIX: MAITLAND

- 6.3 SOCIO-SPATIAL FLOOD RESILIENCE ANALYSIS

- 6.3.1 Maitland flood characteristics and flood mitigation strategies

6.3.2 Vulnerability profile of Maitland

- 6.3.3 City scale analysis

6.3.4 Urban scale analysis

- 6.3.5 Residential building and site scale analysis

- 6.4 KEY FINDINGS 
7.0 MAITLAND CASE STUDY : A SOCIO-SPATIAL FLOOD RESILIENCE NETWORK •

7.1 THE AIM OF A SOCIO-SPATIAL RESILIENCE NETWORK

- 7.2 MAITLAND: A NEW RESILIENCE FLOOD MANAGEMENT MATRIX

7.3 DESIGNING A SOCIO-SPATIAL RESILIENCE NETWORK IN MAITLAND

- 7.4 KEY ISSUES OF THE MAITLAND CITY SYSTEM

- 7.5 UNDERSTANDING THE SOCIO-SPATIAL SYSTEM

- 7.6 MAITLAND'S SOCIO- SPATIAL RESILIENCE NETWORK

- 7.7 CURRENT SOCIO-SPATIAL VULNERABILITIES AND OPPORTUNITIES

- 7.7.1 Residents : building and lot scale

- 7.7.2 Neighbourhoods : urban street scale

- 7.7.3 Neighbourhoods : urban levee scale

- 7.7.4 Communities : city services scale

7.8 ARCHITECTURAL FLOOD RESILIENCE PRINCIPLES

- 7.9 EVALUATION OF THE MAITLAND SOCIO-SPATIAL RESILIENCE NETWORK

BIBLIOGRAPHY 
"Sunburnt. Scorched. Saturated. Soggy. The Australian continent is severely affected by extreme weather events. It is fully exposed, cannot hide. It is what it is. And the more man tames, domesticates and (re)designs and (re)engineers it with beautiful parks, waterfront esplanades, suburban allotments and urbanized cores, canalizations and dams, plantations and gardens, the more it is seemingly ravaged again and again by the forces of nature...." 


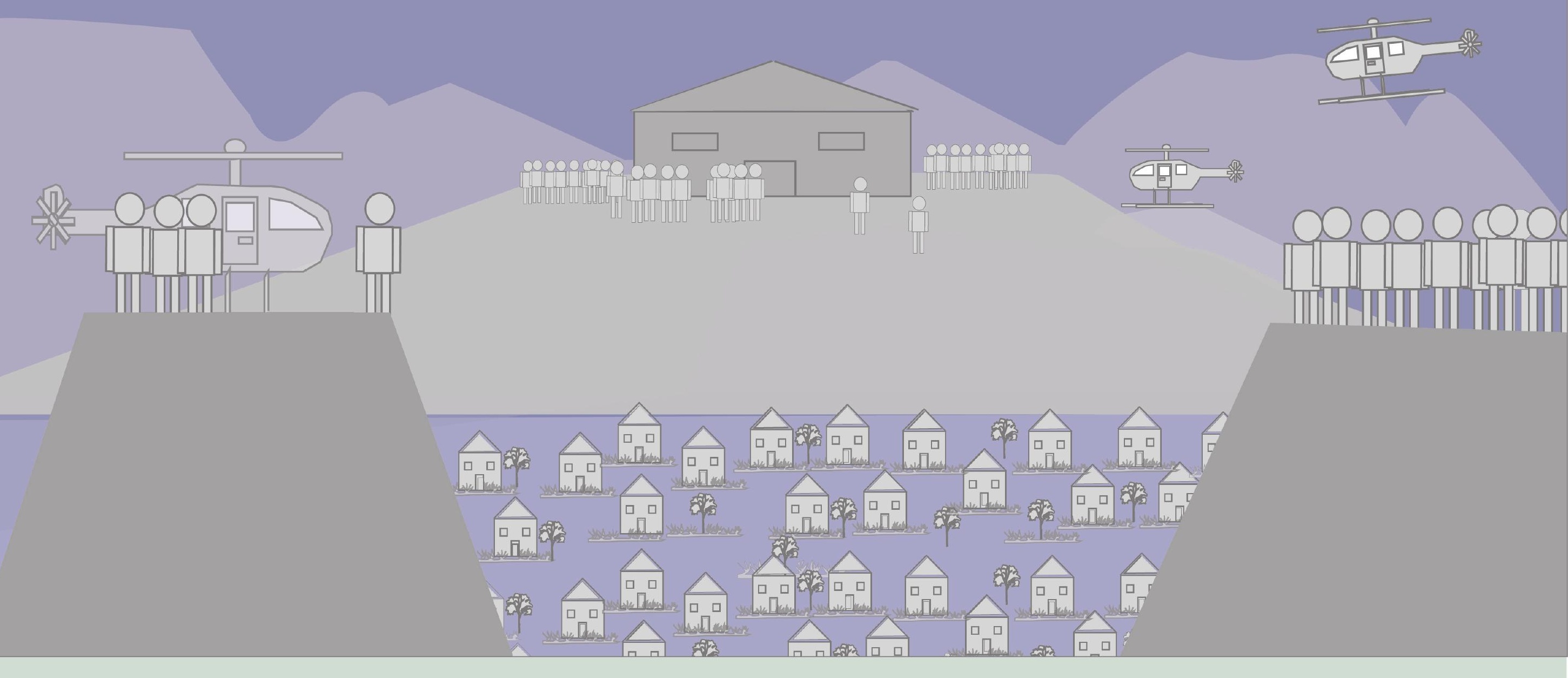

\subsection{INTRODUCTION}

This chapter provides an overview of how architecture engages with the research problem with regard to the need for improved resilience for riverine floodplain inhabitants. The motivations of this thesis and the context of the problem are detailed. The aims and objectives, method and structure of this thesis are also outlined. 
Flooding results in large-scale emotional, physical, financial, cultural and political loss. It is the most costly natural disaster in Australia with an average minimum annual damages bill of $\$ 350$ million (SCARM Report 73, 2000). Extensive flooding across Queensland, Victoria and New South Wales in 2010-2011 directly resulted in six billion dollars worth of damage. This figure does not include the on-going indirect consequences for trade, tourism, agriculture, mining and the manufacturing sectors.

Thousands of Australians are forced to rebuild their lives after floods, within communities now deprived of local infrastructure, basic services and facilities. Current measures often fail to establish a system capable of functioning post flooding. Consequently towns such as Grantham are being relocated to higher ground through Government buy back schemes. Flood standards are being redrafted and there are fresh calls for further investment in flood works. The concern is that this investment in structural flood mitigation work will perpetuate the safety risks associated with increased flood exposure as the community builds more extensively in areas perceived as safe from flooding.

Floods are not predictable, but the magnitude, intensity, spatial distribution and duration of floods can be mitigated and planned for. Mitigation involves a careful balance between structural and non-structural measures, commonly referred to as hard and soft mitigation (Paton \& Johnston, 2006). Bolin \& Standford (1998) argue that if only the physical aspects of a natural disaster are addressed then the hardedged structurally engineered solutions that focus purely on the physical aspects of a natural disaster will fail to address the social aspects of communities' ability to anticipate and respond to flooding. The social and demographic components of communities and the built environment greatly impact on resilience (Mileti, 1999). Whilst it must be recognized that social understanding and awareness reduce risk and improve recovery processes, if the residential, urban and city infrastructure is not adaptable and resilient, it will fail when inundated by floodwaters.
Australian floodplain management has historically been a reactive process focused on preventing flooding through the construction of flood mitigation works. New ideas are now influencing policy and encouraging a more integrated and holistic approach. International explorations of amphibious architecture are transforming the structural preventative method to provide a means to periodically reside on water. Amphibious housing designs provide an opportunity for Australian floodplain inhabitants to achieve greater individual resilience to floods. However, despite the resilient benefits of amphibious approaches, they cannot be viewed as an integrated or complete solution. Catchments, cites, suburbs and houses are an integrated system with the features at each scale impacting upon those at another scale in different time frames. Community as well as individual vulnerability needs to be considered, and integrated with evacuation and recovery strategies to optimally improve resilience. An approach aimed at improving resilience to flood hazards can be best achieved when multiple interventions such as flood adaptive infrastructure, flood risk education and pre-planning for extreme floods are integrated to provide system wide adaptability on different spatial-time scales.

Despite this shift in government management policy, improvements in flood prone housing are beginning to follow a similar structural ad hoc reactive approach. This is seen through construction types, water resistant materials and permanent and temporary flood proofing measures. A lack of mandatory flood prone building codes in Australia means these measures are not standardized. This thesis develops a resilience matrix to improve our understanding of current policy shortcomings and to enhance the local, city and catchment wide planning for flood events. 


\subsection{Motivations for research}

Australia's high annual flood damage costs are an indication that current approaches are failing. The situation is likely to worsen as climate change increases the severity and prevalence of floods, and population growth places more individuals in communities on floodplains. Exploration and implementation of innovative approaches to flood resilience on a city scale are emerging, but research in this field remains limited (Ryan, 2010). There is a need to test resilience approaches in relation to specific spatial components.

Maitland, in the Lower Hunter Region of New South Wales, Australia is situated on a floodplain. The fact that the city is flood prone has historically hampered development and renewal opportunities. This historically significant city is in desperate need of rejuvenation and continues to experience a population decline, whilst the population of the surrounding region grows rapidly. The Maitland City Council has established the Central Maitland Structure Plan (November 2009) with the aim of reviving and reconnecting the city to the river. New residential developments are planned to encourage the floodplain population to increase to the 1954 population size by 2021 . This will inevitably increase the number of people who are put at risk of flooding. Maitland provides the ideal case study to test a new socio-spatial resilience approach.

This thesis will demonstrate the benefits a new resilience approach would have for Maitland, and may be applicable to other cities across Australia. The aim is to increase the robustness and flexibility of the system (individual, community and city) to cope with a variety of flood events.

\subsection{Scope of Research}

Due to the multi-disciplinary nature of the topic this thesis will focus on establishing an architectural and urban design perspective to improve resilience on a series of socio-spatial scales. Whilst principles and theories from the disciplines of ecology, hydrology and policy play a part, this thesis will propose an architectural approach to the issues associated with floodplain inhabitation. This research looks specifically at flooding resulting from rivers. It does not address sea or lake floods. There are limitations on the level of detail and accuracy this thesis achieves in the fields of hydrology, ecology, policy and social-science.

\subsection{Aim and Objectives}

This thesis will use architecture and urban design to:

1. propose spatial interventions to improve the ability of Maitland floodplain inhabitants to anticipate, respond to and recover from floods.

2. design a network of resilience interventions to improve individual, community and city resilience to flooding.

3. propose a model to integrate measures that enable cities, communities and individuals to adapt to the periodic need to live with floods.

The approach recognises the importance of accepting flooding as a natural recurring event rather than something that can be prevented. A multi-scale resilience flood management matrix will be established. This will allow existing flood management strategies to be examined within the context of resilience principles. 
Introduction: This chapter establishes the context of the issues surrounding riverine floodplain habitation and the need for a resilient approach.

Background: The background chapter provides insight into the history of floodplain habitation in the context of flooding perceived as 'disastrous' rather than as naturally recurring mitigated and embraced events. This transformation of floodplain management is discussed with regard to its transformation from historical origins. Examples of this are portrayed through civilisations who recognise the significant values associated with flooding and the need to embrace its characteristics.

Literature Review: Literature on floodplain management is reviewed to highlight the need for a balanced approach between resistance (the prevention of floods) and resilience (achieving temporal stability living with floods). A need for site-specific strategies is revealed based on insight gained from literature on how ecosystems mitigate and maintain stability during floods. Key flood resilience principles are outlined.

Theoretical Framework: A resilience flood management matrix is established. The flood resilience principles are detailed with regards to existing flood management methods and ecological processes. A diagrammatic resilience flood management matrix provides a means to visually engage with the key resilience principles.

Precedent Analysis: Precedent analysis of floodplain habitation in Cambodia, the Netherlands and New Orleans with regard to the flood resilience principles provides insight into the issues associated with inhabiting riverine floodplains. The success and failure of existing measures highlights where interventions have the capacity to improve individual, community and city resilience to flooding.
Conceptual Framework: Combining the social vulnerability characteristics of floodplains with the resilience floodplain management matrix establishes a criteria revealing opportunities for site-specific design interventions.

Case Study: Case study analysis of Maitland, in New South Wales Australia provides a means to test site-specific design interventions. The issues associated with applying the theoretical framework to the site are highlighted. Exploring a variety of interventions at multiple spatial scales demonstrates how a socio-spatial resilience network can improve the long-term inhabitation of riverine floodplains.

Evaluation and Discussion: The conclusions gained from the Maitland case study provide further insight into how a socio-spatial resilience approach improves the flexibility and robustness of a floodplain community and city. The need for further investigation into community connectedness at the city scale and the opportunities a socio-spatial resilience network provides are highlighted. A balance between artificial interventions and natural system adaptations, achieved through applying the resilience flood management matrix, reduces the adverse impact of flooding on floodplains and their inhabitants. 


\subsection{Design through Research}

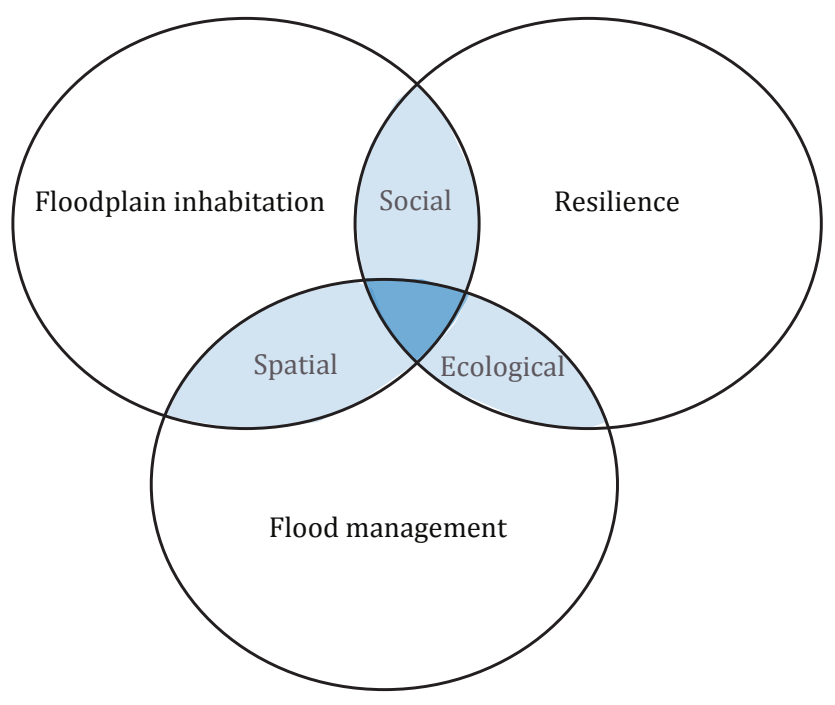

A design through research strategy enables literature review findings and precedent analysis to be explored in a site specific case study. A new approach is established through research into floodplain management and ecological resilience to flooding. Flood resilience strategies will be visually defined and documented. Design allows a method to explore and evaluate ideas from literature and site analysis. A range of possible solutions have been tested and theories applied to gain further insight into the issues facing floodplain communities and to demonstrate the need for a multiscaled approach to improve resilience. Architecture provides a means to spatially test theories arising from ecology, hydrology, engineering, sociology and flood management policy. Design solutions provide a means to analyse possible strategies that could be applied to other Australian riverine floodplains.

Figure 2: The intersection of primary and the subsequent secondary themes of this thesis 
"Beneath the foundations of untold numbers of houses are the floors and foundations of their predecessors; to the archaeologist the land is a palimpsest on which can be read the unfolding of man's settlement history". 

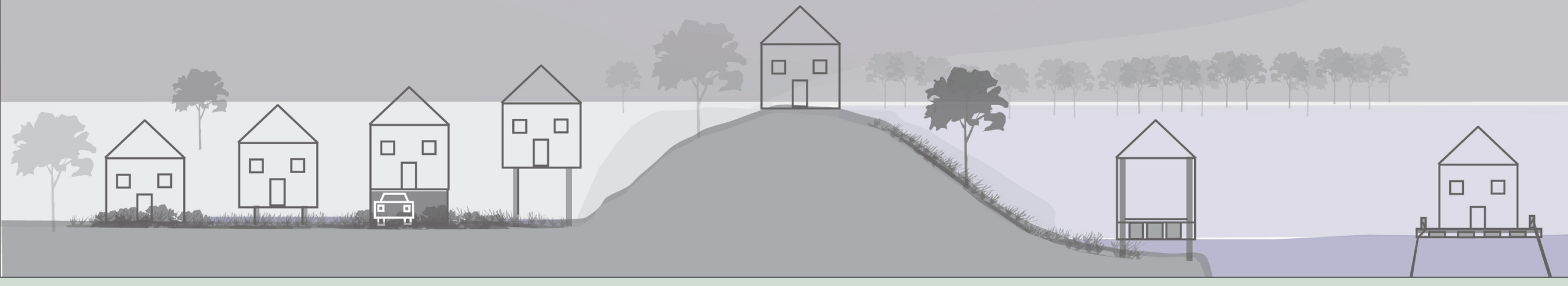

\subsection{BACKGROUND: FLOODPLAIN CONTEXT}

This chapter establishes the riverine floodplain context and discusses current issues associated with inhabiting flood prone land. The historical origins of floodplain management and the transformation of methods are detailed. The need for a new philosophical approach is highlighted. 


\subsection{Floods on floodplains}

This thesis specifically addresses the inundation of floodplains from an overtopping of river banks. The floodplain is the area of land adjacent to a body of water that is prone to periodic flooding. Flooding is defined as; "a general and temporary condition of partial or complete inundation of normally dry land areas from overflow of inland or tidal waters from the unusual and rapid accumulation or runoff of surface waters from any source" (Australian Government, 2011).

Floods are a natural reoccurring hydrological process. The diversity and redundancy of ecosystems in the natural world allows them to adapt to the changed hydrological conditions. In contrast to this, humans inhabiting floodplains are vulnerable to flood hazards due to the damage potential to infrastructure and community. As Gregg \& Houghteon (2006) explain, the spatial and temporal distribution of the event, flood frequency, magnitude, warning, and societal reaction times will determine the impact of the flood hazard.

Flooding along rivers can result from heavy rainfall or as a consequence of levee or dam failure. There are many factors influencing the variability of flood velocity, volume and duration. These include vegetative cover, flood mitigating measures, watershed topography, land use and urban density. Low lying river flooding is characteristically slow in the rate of rise and the floodplain remains inundated for some time. Structural failure of flood engineering works can lead to flash flooding.

The floodplain is regulated with regards to its defined floodway and flood fringe. Development is most commonly prohibited within the floodway as this is perceived to be the natural path of flood waters. This zone characteristically experiences high velocity and high volume water flow. The flood fringe is the area of the floodplain bounding the floodway. It acts as a storage space for the slower moving floodwaters. The topography, vegetation and human development of the catchment influence the floodplain's hydrological processes.

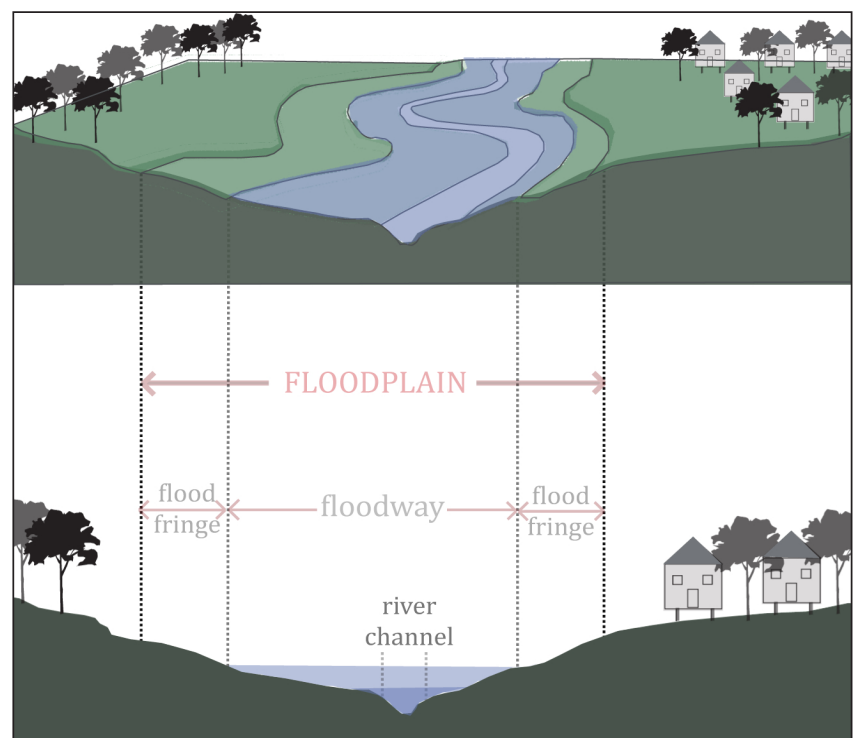

Figure 3: Floodplain, floodway and flood fringe

\subsection{History of floodplain inhabitation}

From prehistoric times human civilisations have chosen to reside near bodies of water to take advantage of the agricultural, industrial, transport, and recreational benefits provided by waterways. "Living on floodplains is second nature to humanity and hence so is cohabiting with the flood risk" (Alexander, 1993, p131). Humans throughout history have adversely affected ecosystems through such practices as hunting, fishing, agricultural land clearing, and urban development. This in turn has altered the structural make up of ecosystems and subsequently influenced the severity and frequency of flooding (Burkett, et al., 2005).

Historically, natural disasters have been perceived as external events requiring management to reduce the impact on unsuspecting and unprepared societies (Ryan, 2010, p44). Communities who choose to reside on floodplains need to recognize the inevitability of floods as a natural event. Humans have a long history of developing techniques to prevent floods. However the perceived safety this creates inevitably leads to increased development and subsequently increased flood risk. 


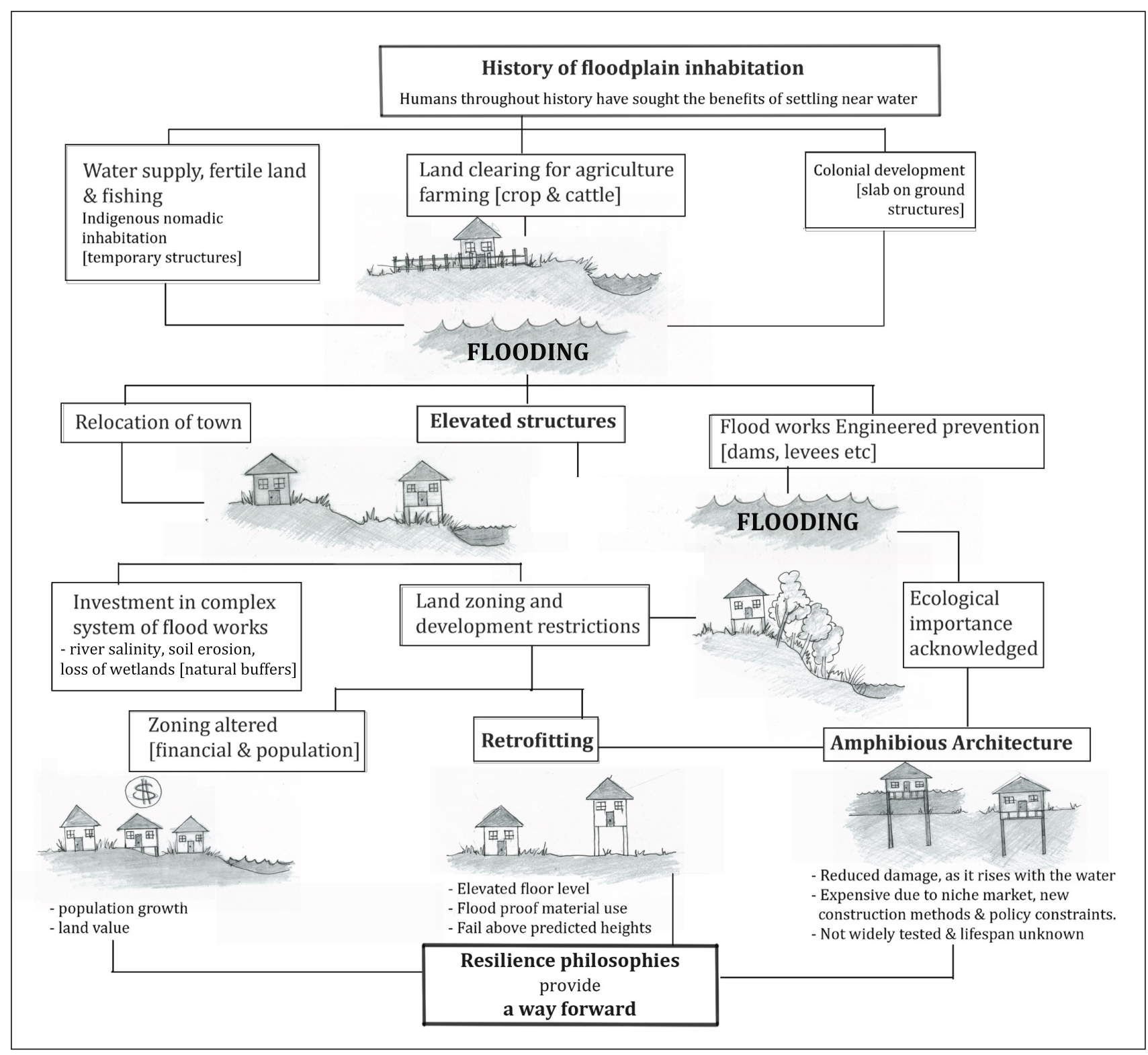

Figure 4: History of floodplain inhabitation 
Pile dwellings, raft structures and ship constructions are the three main construction types utilised through human history for inhabiting floodplains. These design concepts continue to be further developed utilizing improved technology and materials (Flesche \& Burchard, 2005). Stilt houses were the earliest form of flood prone dwellings with archaeological evidence indicating there was a stilt city at Lake Constance in Germany in the Neolithic Period (c. 4000 BC) (Flesche \& Burchard, 2005). Timber construction locally sourced from nearby mangroves enabled the floor structures to be raised above flood levels on stilts (Danby, 1971). Over time the instability of silts in the river mud from tides and seasonal flooding continued to cause issues for statically elevated stilt and pile construction (Oliver, 2003).

Raft structures were a way to avoid this design problem. They were typically constructed from locally sourced reeds to take advantage of floating opportunities, and reduce the impact of fluctuating water levels. The earliest recording of a floating community was the Mesopotamian civilization 5,000 years ago (Flesche $\&$ Burchard, 2005). This technology was then further developed into timber pontoon houses, light weight concrete construction and metal and polystyrene slab construction. Floating villages or villages elevated high on piles or 'stilts' with jetties and pontoons providing connections between them continue to exist today (Oliver, 2003). Pile, raft and ship construction arose from floodplain inhabitants' acceptance of floods as an intrinsic part of their lives
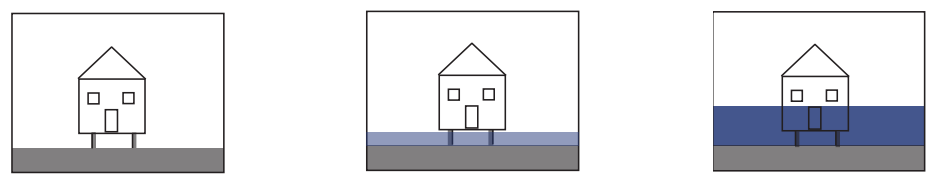

Pile or post construction

Minimal flood proofing measure

- Low flood suitability improved through the use of marine grade timber

- Not suitable for medium flood velocities and depths

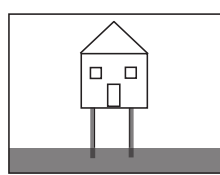

Stilt house construction

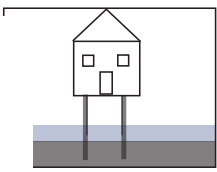

Medium flood proofing measure

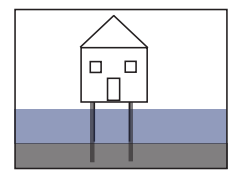

- High flood suitability improved by water resistant material use and stilts extending deep below ground.

- Not suitable for high velocity floods.

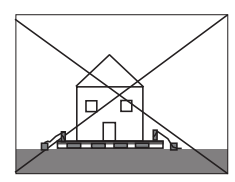

Floating construction

- High suitability to fluctuating water environments improved with marine grade and treated timber use

- Not suitable for high velocity floods with large wave heights
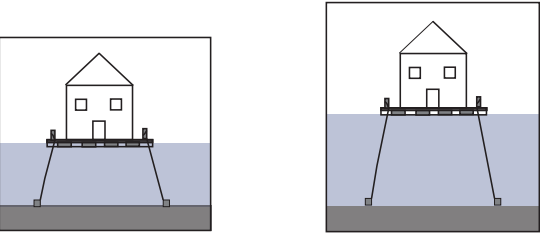

High flood proofing measure

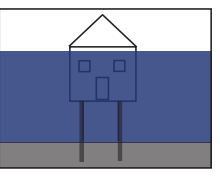

Figure 5: Pile, stilt and raft housing construction 
Historically, floodplain management began as an ad hoc reactive process undertaken by local property owners rather than a central governing body. Such ad hoc arrangements were no longer adequate as populations grew. As a global phenomenon the nineteenth century saw engineering and natural flood mitigation works implemented in flood prone regions in an effort to prevent flooding. According to the Standing Committee on Agriculture and Resource Management (SCARM, 2002) flood management has evolved through four major phases. Australian floodplain management began in the 1960s as a reaction to extensive flooding in New South Wales. In the 1960-70s flood management focused on structural works. Technological methods, such as dams and levees were implemented in an attempt to control nature (Pelling, 2003). The 1970s was characterised by zoning, development controls and land use policies, while the 1980s to 1990 s saw the creation of a governing body for flood emergency management (State Emergency Service (SES) volunteers). From the 1990s the role ecology plays in mitigating floods and the environmental need for periodic flooding were acknowledged. Flood managers began to understand that flood risk eventuates from the interaction between the flood hazard and environmental, built and social vulnerabilities. Flood management now involves holistically analysing the vulnerability of floodplain cities, communities and inhabitants.
Flood management involves structural (flood works, flood proofing) and non-structural (land zoning, land use, emergency evacuation, flood warning and insurance) measures. This means flood management is focused both on resistance (prevention) and resilience (mitigation/distribution of flood risks). Flood management works to mitigate the risks associated with disasters: hazard, vulnerability and exposure (Crichton, 1999). According to the SCARM Report 73; "the primary objective of floodplain management is to reduce the effect of flooding and flood liability on individual owners and occupiers of flood prone property and to reduce private and public losses resulting from floods" (SCARM Report 73, 2000, p.xiv). Flood management utilises historical flood and weather pattern data in an attempt to limit uncertainty, but weather patterns remain variable and unpredictable, and historical data limited. Strategy focus has predominately been at a catchment scale (Zvenbergen, Veerbeek, Gersonius, \& van Herk, 2008). It is now realized that effective flood management is reliant on the involvement of all levels of government, industry and community (The National Flood Risk Advisory Group, 2008). 
Government investment in the construction and maintenance of flood works is vital, but there is ongoing debate about the acceptability and reliability of these flood works. Altered local ecosystems are no longer able to absorb and buffer the floods. Flood engineering structures adversely impact on the natural hydrology of floodplains. On occasion structures such as levees that work to reduce the frequency of flooding by restricting floodwater flow to the river channels can subsequently increase the risk of severe flooding downstream (Folke, Holling, \& Perrings, 1996). The loss of regular inundation on a floodplain also has negative ramifications on the riparian habitat, with ecosystems becoming less efficient, less fertile and less capable of buffering, diffusing, absorbing and storing flood waters (Mileti, 1999).

Nordenson, Seavitt, \& Yarinsk (2010) purport that hard engineering solutions are "costly, unreliable and often ineffective" (p. 14). Ware, Raxworthy, Weller, RussellClarke, \& Meyer (2011) believe that there is sufficient evidence that "feats of hard engineering solutions are not nature-proof (but have) severe consequences for the landscape" (p10). As Klein, Nicholls, \& Thomalla (2004) suggest, preventative flood measures, built to protect nearby communities up to a specific design flood level, are often ignorantly perceived to have removed the flood hazard rather than to have only reduced the occurrence rates. Investment and development subsequently increase behind these structures. The new inhabitants are put at greater risk due to the lack of flood awareness and preparedness. Many of these structures are reaching their design life spans, resulting in greater risks of catastrophic flooding (Mileti, 1999). The growing Australian population exacerbates the problem as urban densification puts greater numbers of people on the floodplain.

Economics plays a significant role in flood management as flood protection standards are determined by accepted economic risk levels. Kelly \& Garvin (2007) calculate a value on the consequences of flooding by: economic damage (national, community, individual), the number of people and properties affected, the resultant harm to individuals, including fatalities, injuries and emotional stress and environmental and ecological damage effecting ecosystem functioning (Kelly \& Garvin, 2007, p. 343).
The SCARM report states that it is neither economically nor practically feasible to provide complete flood protection up to the probable maximum flood (PMF) event. Subsequently a compromise between the communities' accepted risk and affordability is needed (Csiro, 2000). Socio-economic risks involve the number of people anticipated to be inundated, coupled with the recovery capacity of the inhabitants and built infrastructure. There are however changing views on who should be responsible for these flood events: the individual landholder or the government and insurance companies. This was most recently evidenced in the Queensland Flood Enquiry Commission in which specialist organisations and governments were put on trial over the flood disasters.
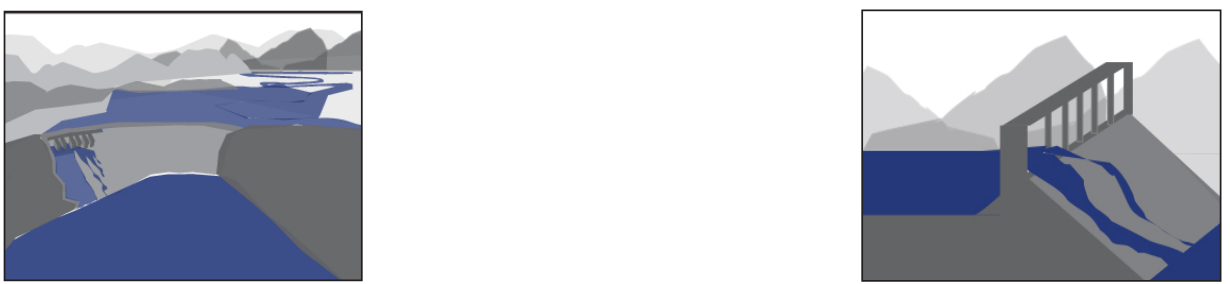

Flood engineering structures such as dams are flood preventative structures

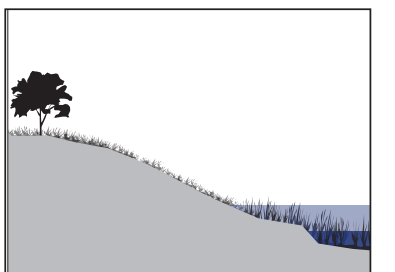

Levees: a combined ecological and structural flood mitigation solution

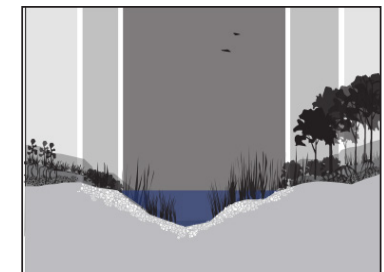

Rejuvenation of riverine ecosystems, forests and wetlands.
Figure 6: Flood engineering structures, levees and rejuvenated ecosystems 


\subsection{The current holistic approach}

A new approach is needed to deal with changing expectations. Bewsher \& Grech (1999) formally recognised the philosophical shift in floodplain management that took place in the 1990s that saw the transition from a preventative engineered focus, to environmentally sympathetic zoning solutions. They recognised the negative impact preventative solutions had had on the functional capacity of the floodplain and natural river systems and altered flood frequencies. Flood prevention strategies led to a false sense of flood security, increased development and reduced flood awareness.

The SCARM report 73 (2000) categorised floodplain management into structural flood mitigation works, land use planning controls, development and building controls, flood emergency measures and flood awareness (pp. 32-36). These categories are indicative of the evolution of flood management. Advancements in computer GIS (Geographical Information System) flood mapping resulted in improved understanding of the specific flood risks experienced within regions. This allowed more appropriate measures to be selected to mitigate the flood hazards Ashley \& Blanksby (2007) similarly recognise that this integrated systems approach ensures all the parts of the complex scheme are adequately addressed. A key issue remains engaging residents in flood prone regions in this discussion.

Flood managers are increasingly aware of the significant role policy, the economy, local ecosystems, the residing society and their culture has on flood risk levels. In response to SCARM (2000) a governing flood emergency body was established to improve strategic evacuation processes and flood education programs undertaken. Australian floodplain ecosystems were recognised as valuable resources and the negative impacts development has on hydrology and ecology became an intrinsic component of decision making (Capon, Parsons, \& Thoms, October 2009). Land use policies were implemented to reduce land clearing rates, soil erosion, high salinity levels, and chemical runoff impacting on water quality, soil quality and ecosystem functioning. Local Governments implemented flood prone land planning guidelines to reduce the risks of being inundated by anticipated floods. This in many cases resulted in a retreat from waterside living.
Education programs attempted to improve societal awareness of the flood hazards to enable improved preparedness, a reduction in exposure levels and an understanding of the significant flood mitigation functions performed by ecosystems, including buffering, absorbing, storing and filtering of flood waters. The process resulted in a rejuvenation of previously neglected wetlands, forests and riverine ecosystems. Despite the recent improvements to flood management, there continue to be major policy deficiencies that contribute to flood vulnerabilities.

\subsection{Shortcomings of flood management standards}

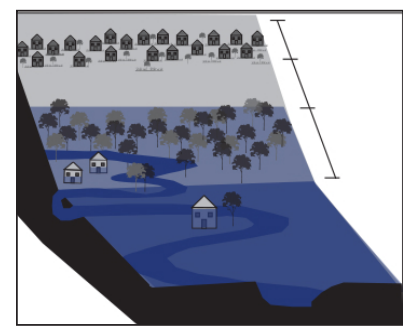

Land use policies and land zoning aim to reduce flood risk

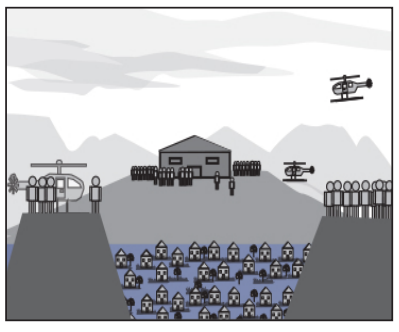

Emergency management focusses on evacuation and recovery from floods
Figure 7: Land use policies and emergency management 
Flood hazard levels at the regional and catchment scale are currently understood in terms of the accumulated technical data and GIS-based flood modeling computer programs. Climate change poses concerns for the validity of information. GISbased flood modeling is also limited as it does not consider the impact of local features. There is a large body of literature that attempts to address the limitations of this situation (Merz, Hall, Disse, \& Schumann, 2010). Ryan (2010) believes "flood risk management strategies must meet present needs while providing an adjustment path for the future" (p. 44) (theory backed in: Ashley R. , Blanksby, Cashman, \& Newman, 2007; Ashley R., Blanksby, Chapman, \& Zhou, 2007; Muller, 2007). Despite widespread scientific acknowledgement of increased severity and prevalence of severe weather events, former land planning and building standards fail to be updated.

The amount of accumulated historical flood data in Australia continues to hamper flood modeling accuracy. Australia has a one in one hundred annual exceedance probability (AEP) policy standard. Flood records in Europe date back $500-1000$ years and thus higher standards are already imposed. For example, in the Netherlands, four standard safety levels of 1:1250,1:2000, 1:4000, and 1:10,000 are allocated to dikes according to the type of likely flooding, population density, and investments within the area (Samules, Klijn, \& Dijkman, 2006). Australian flood management expert Chas Keys believes that the one in one hundred AEP flood standard applied across Australia creates complacency towards flood events within the community and fails to consider site-specific hazard levels relating to population numbers and infrastructure investment.

It must also be noted that the one in one hundred AEP standards applied universally across Australia and much of the world, occurred without sound hydrological reasoning (Smith, 1998). The flood standard is not only confusing in nature, as the standard implies that the flood event takes place only once in one hundred years, but it also results in planning controls being narrowed to a section of the floodplain (Bewsher \& Grech, 1997).

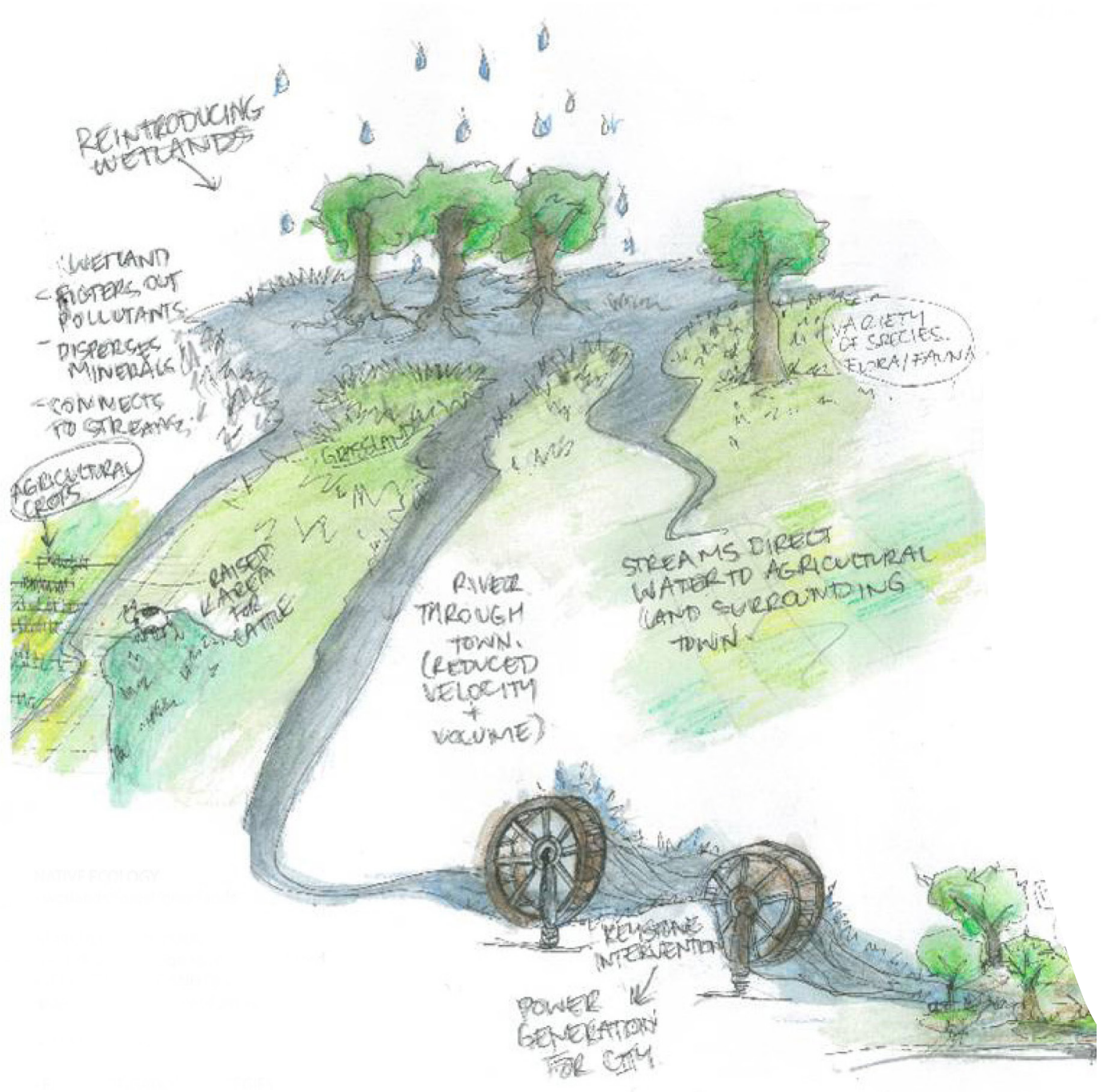

- Native ecology

- Architecture of flood prone housing

- Flood mitigation strategies

- Increasing populations and development proposals 


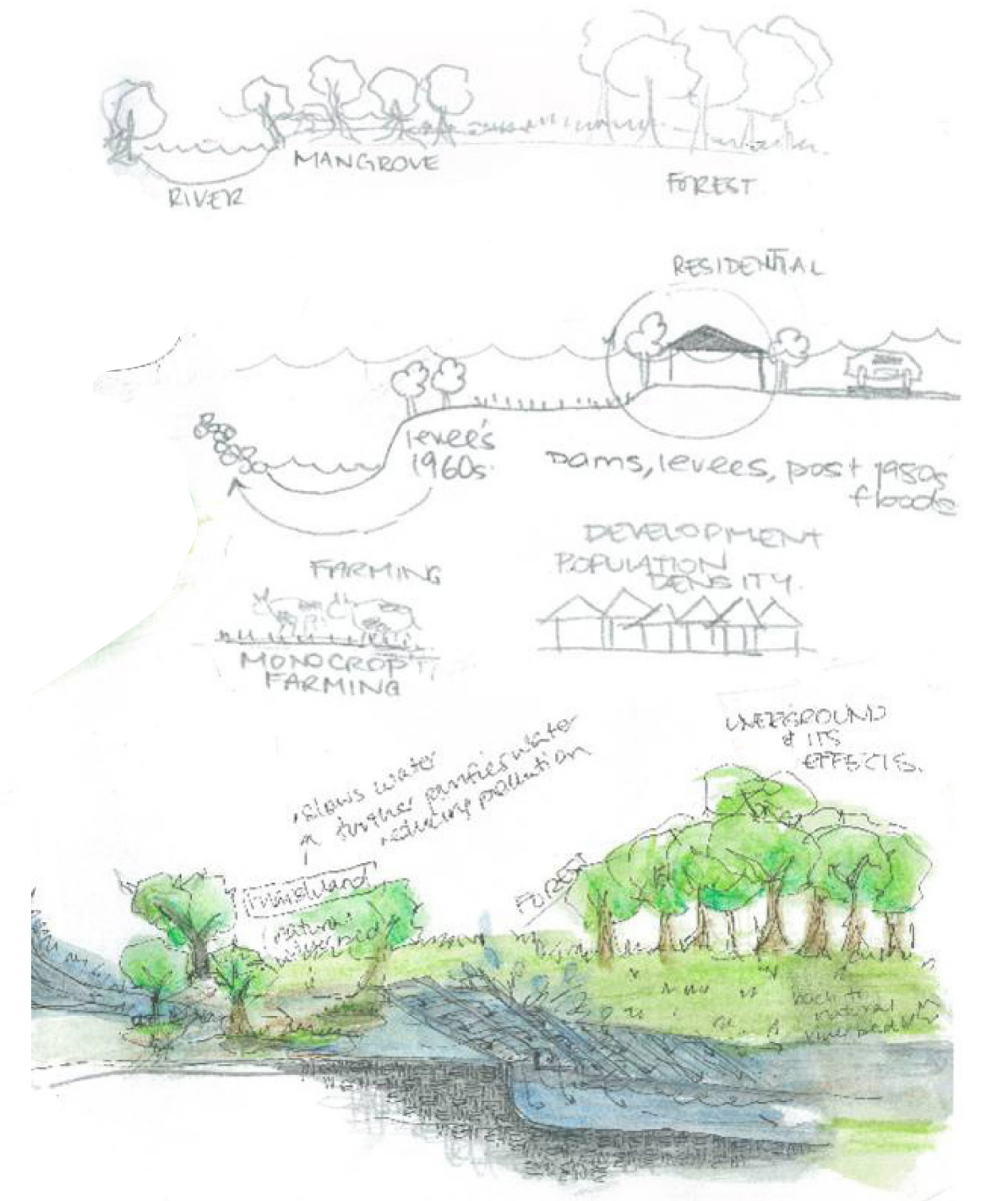

In Australia, councils cross hatch the extent of a one hundred year flood on the Local Environmental Plan (LEP) of Local Development Control Plans. This in many cases has led to the false perception that areas outside the cross hatched area are not flood prone, despite changes in the area's built environment and environmental resilience (Brewsher \& Grech, 1997). Historically, urban expansion has often resulted in land zoning changes where greater numbers of socio-economically disadvantaged people build dwellings and infrastructure on flood prone land. Current population growth trends are also placing increasing pressure on land availability.

Whilst there continues to be a lack of flood building code standards across Australia, local governments have begun imposing flood proofing standards for residential development, including minimum floor standards and water-resistant material use. Dry proofing measures protect a building structure from flood waters up to a depth of one meter. This is the point at which hydrostatic pressure variations between the exterior water levels and the capillary rise through building material elements impact upon structural integrity (Ashley, Garvin, Pasche, Vassilopoulos, $\&$ Zevenbergen, 2007).

Climate change is predicted to increase the frequency and severity of rainfall and severe storm events across Australia. Major flood works, which have formerly provided protection, are likely to become insufficient with greater risk for failure and overtopping. The one in one hundred flood design standards no longer satisfy culturally dictated safety levels. Due to the increased uncertainty and severity of weather events, climate change provides an incentive to plan for the future and anticipate extreme events and trends (Zvenbergen, Veerbeek, Gersonius, \& van Herk, 2008). A new philosophical shift is needed which takes weather changes into account in planning for continued inhabitation of flood prone land.

Figure 8: Exploring ecological impacts of flood management and land use 
2.6 A new resilience approach

Scientists are predicting that weather hazards will increase in severity, frequency and variability in the future. It is therefore imperative that uncertainty is integrated into planning to improve a city's flexibility to adapt to somewhat undefined potential flood hazards.

Resilience promotes a transformation of traditional flood preventative perspectives to an approach aimed at maintaining ecosystem resilience, increasing the adaptive capacity of societies to cope with the uncertainty of floods, and decreasing community vulnerabilities. Flood managers need to recognise that disturbances such as flooding must be "anticipated, accommodated [for] in planning, and even employed in some cases by natural resource managers to maintain ecosystem structure or services" (Burkett, et al., 2005. p 360). Acceptance and understanding of the dynamic risks associated with cities, communities and individuals reduces the relative exposure, subsequent flood impact and recovery periods. Klein R (2003), Huq \& Klein (2003) and Klein, Nicholls, \& Thomalla (2004) have recommended five generic approaches to 'anticipatory adaptation':

1. Increasing the ability of physical infrastructure to withstand the impacts of climate change

2. Increasing the flexibility of potentially vulnerable systems that are managed by humans

3. Enhancing the adaptability of vulnerable natural systems

4. Reversing trends that increase vulnerability

5. Improving public awareness and preparedness

(Klein R. , 2003, p. 36)

Globally, there is a philosophical shift taking place in which resilience is being promoted in flood management. The August 2002 Elbe and Danube floods in Germany triggered this shift across Europe. England produced the Making Space for Water Report (Defra, 2004): a government flood strategy which launched a new approach recognising the importance of planning for flooding, but also the need to live with floods and investigate ways of reducing their impact.
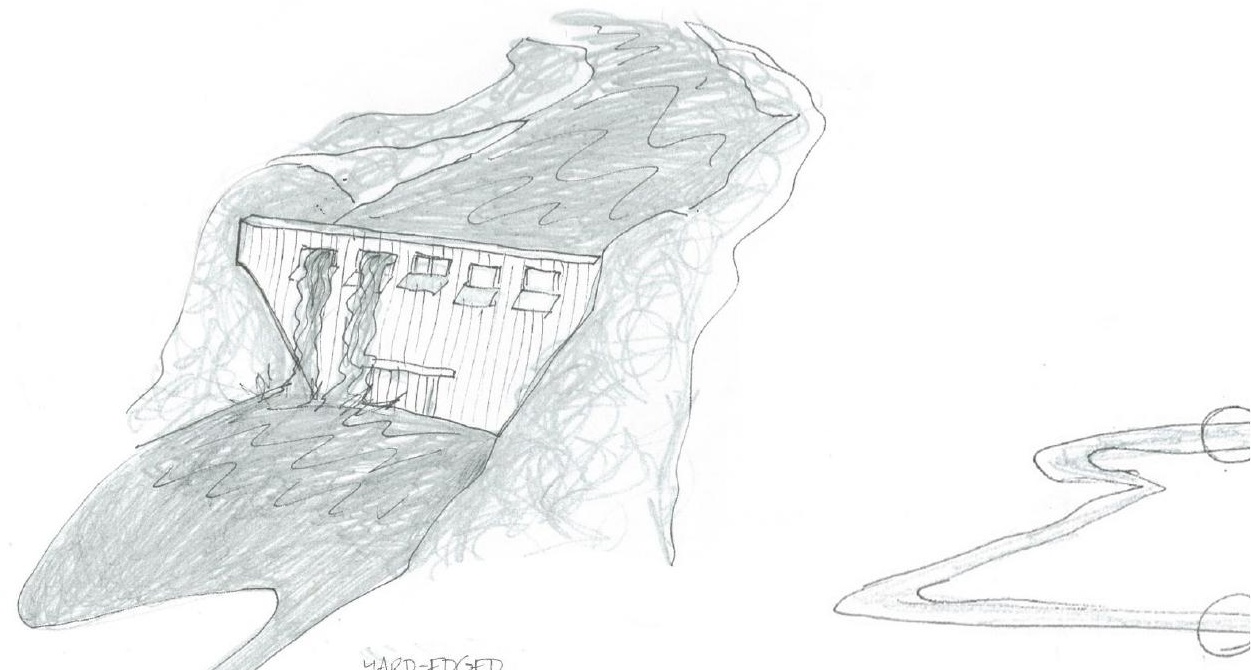

MARD-EDGET

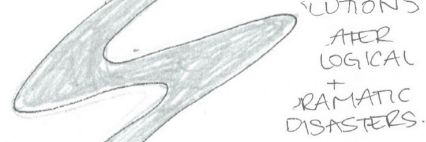

ratamic

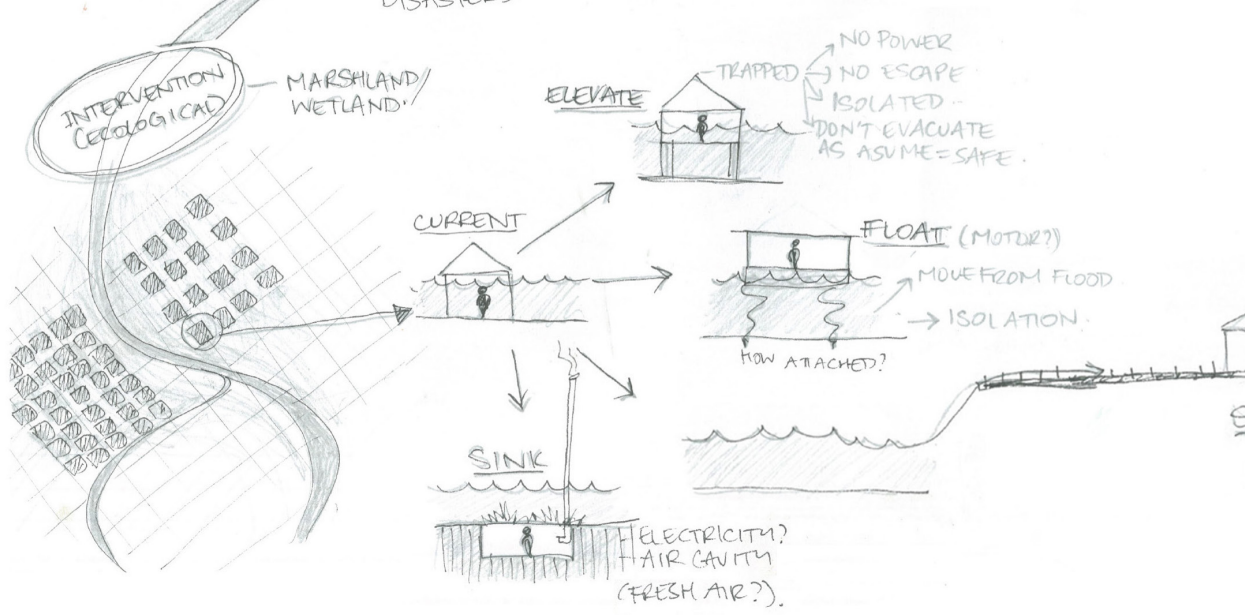

- Issues with flood engineering structures

- Existing housing vulnerabilities

- Explorations into new housing possibilities 
"Our oldest faiths and deepest symbols reflect the primal connection to the natural world, to the living planet that long ago imprinted on the human consciousness a cyclic sense of death and decay, rebirth and renewal". 
Resilience originated in the fields of mathematics and engineering (Bodin \& Wiman, 2004). The concept was initially applied to ecology by Crawford Stanley Holling (1973) as the capacity to maintain stability within multiple domains when impacted upon by disturbances. Resilience determines the persistence of relationships within a system and is a measure of the ability of these systems to absorb changes of state, maintain variables, set parameters, and continue to function when impacted upon by disturbances (Holling 1973, p.17).

The resilience definition provided by Holling prompted a means to describe the non-linear dynamics of ecosystems in their ability to absorb disturbances and continue to function. It also provided the conceptual basis to measure a system's disturbance threshold; the point at which the system is forced to adapt and transform to maintain stability within the changed environment. Holling (1973) defined stability, in contrast to resilience, as the ability of the system to return to a former state of equilibrium after a temporary disturbance.

Ecologists such as Odum (1969) and Tilman (1999) later challenged the theory of stability, established by Holling (1973), believing it would inevitably result in system failure. Resilience became increasingly defined as a dynamic interdependent system capable of evolving continuously in response to external disturbances on different scales (Timmerman, 1981); (Klein, Nicholls, \& Thomalla, 2004). Resilience is thus not only a measure of the system's ability to withstand and recover from disturbances, but also its ability to anticipate and adapt to change (Zvenbergen, Veerbeek, Gersonius, \& van Herk, 2008).

The Resilience Alliance, founded by C.S Holling in 1999 is an international network of social scientists and ecologists who through a socio-ecological resilience lens explore the changeable interactions between humans and nature. The Resilience Alliance endeavours to develop and test adaptive resilience policies relating to climate hazards.

Figure 10: Ecosystem strategies which provide insight for resilience design and construction Source: Authors own table adapted from Watson and Adams (2011),

"Lessons of nature applicable to design and construction", p. 258.
The Resilience Alliance (2002) defines resilience as

1. The amount of disturbance a system can absorb while remaining functionally and structurally stable and

2. The capacity of the system to self-organise and

3. The ability of the system to increase its capacity to learn and adapt.

Resilience has become a multi-disciplinary concept encompassing the complex interaction of natural environments and human societies (Harrison \& Salt, 2006; Gunderson, Allen, \& Holling, 2010). Resilience is influenced by a system's external interdependence and internal flexibility. To understand how ecosystems achieve resilience to natural disturbances such as flooding, there is a need to analyse the underlying system strategies that influence how the system reacts to altered environmental flood conditions.

\section{Strategies used by ecosystems to maintain function and structure during floods}

\begin{tabular}{|l|l|}
\hline Absorption & Watershed planning and design (reservoirs, retention ponds, green roofs) \\
\hline Buffering & Breaks, riparian buffers, rain gardens, shuttering. \\
\hline Core protection & Zoning, decentralisation, self -reliant subsystems \\
\hline Diffusion & Meanders, wetland and coastal zone landscape, open foundations. \\
\hline Rapid response & Smart grid, early warning, emergency responsive systems. \\
\hline Redundant circuits & Green infrastructure, wildlife corridors, and multiple service routes. \\
\hline Storage capacity & Aquifers, wetlands, reservoirs, cisterns. \\
\hline Waste/nutrient recovery & Sustainable storm water design and waste systems. \\
\hline
\end{tabular}


As Poff (2002) explains "river channels and their floodplains are among the most naturally dynamic ecosystems on earth, in large part due to periodic flooding" ( $\mathrm{p} 1497)$. Key resilience principles enable floodplain ecosystems to adapt and maintain stability. The flood characteristics of magnitude, frequency, and duration promote biodiversity, functional redundancy and improve ecosystem productivity. Analysis of ecosystem responses to flooding provides insight into the underlying resilience principles of the system. These principles applied to flood management provide a new approach for achieving flood resilience.

Brian Walker in 'Conserving Biological Diversity Through Ecosystem Resilience' (1995) proposes that species are divided into functional groups, referred to as 'guilds' due to ecological similarity. Ecosystem engineers are responsible for shaping their community. They modify or create habitat through their physical structure or practices (Baskin, 1998). The system drivers, known as keystone species, are responsible for controlling these guilds. A reduction in keystone species will result in a loss of species reliant upon them, although their importance will vary in space and time (Baskin, 1998). A loss in species diversity will have disastrous effects on the ecosystem's flexibility as variety and redundancy ensure species regeneration and reorganisation of the ecosystem following a flood disturbance.

Biodiveristy, functional redundancy, feedback, flexibility and the regenerative ability of species are identified as key principles enabling ecosystems to reorganise to temporal conditions and absorb recurring flood disturbances (Adger, Hughes, Folke, Carpenter, \& Rockstrom, 2005); (Chapin, et al., 2000). These resilience attributes ensure ecosystems continue to deliver vital services during periods of rapid or gradual environmental change. Watson \& Adams (2011) established a table linking key ecosystem principles with their flood management counterpart.

Inter-scale connections, response processes, species diversity and feedback mechanisms are ecosystem characteristics determining the ability of the system to maintain structural and functional stability pre, during and post floods. The underlying flood resilience principles, scale, adaptability, variety and redundancy, and feedback, provide new guiding principles for flood management to work to improve the flood resilience of city systems. However, it must be recognised that societal and spatial factors within the urban environment make achieving resilience to natural hazards far more complex than simply applying the ecosystem resilience principles to flood management. An architectural approach that recognises the need for resilience in communities, in dwellings and in key infrastructure components is needed to enhance this flood management strategy.

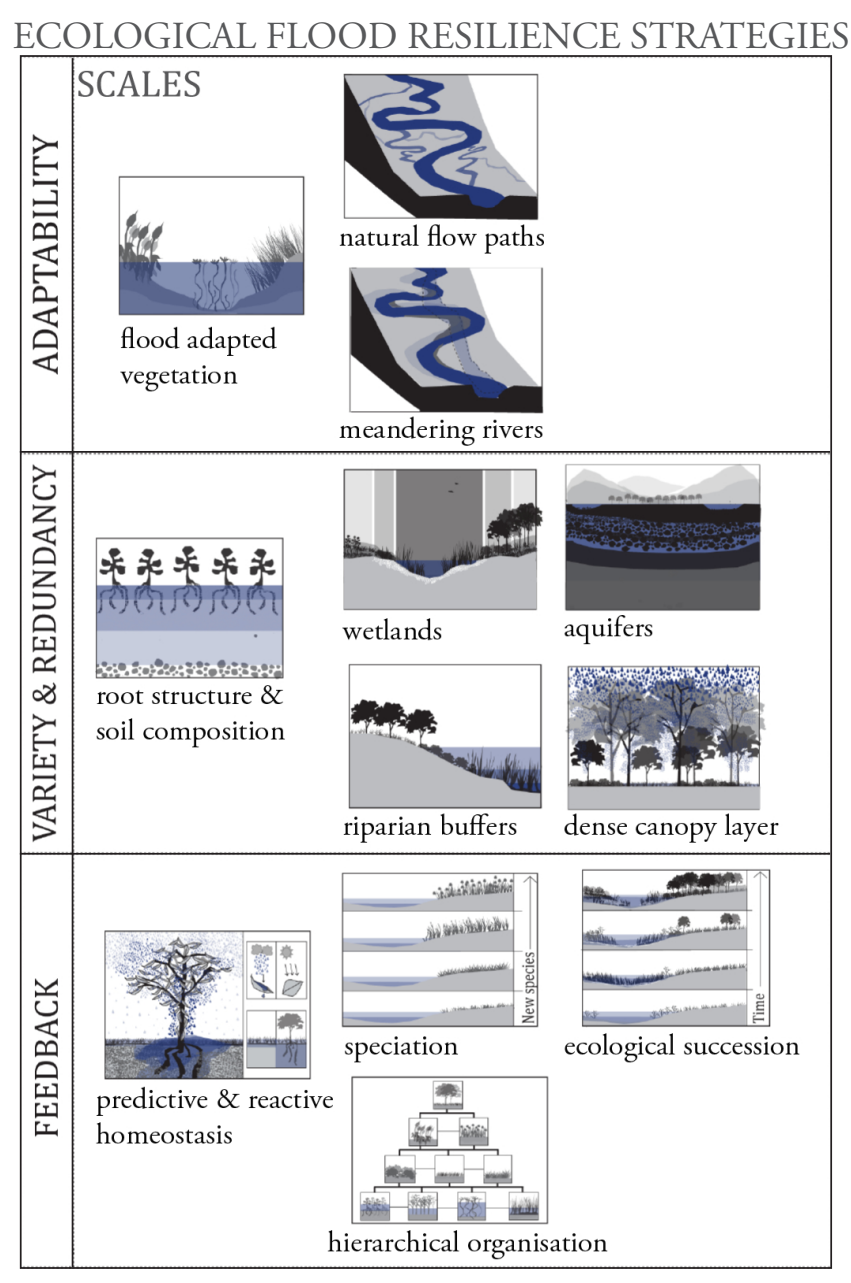

Figure 11: Ecological flood resilience principles 
In the context of human established systems, Kaplan (2005) believes resilience allows for positive valued outcomes to result from flooding if inhabitants embrace and adapt to the conditions. Natural disasters have traditionally been perceived as environmental occurrences that adversely impact on the physical environment of humans (Pelling, 2003). There is an opportunity for society to alter former views and embrace the periodic need to live with water. This will not only benefit floodplain communities but also improve unique riverine ecosystems that reside within the aquatic and terrestrial environments (Poff, 2002).

A floodplain city's ability to adapt to flooding circumstances and the time it takes to recover and return to full functioning capacity can be improved through 'engineering resilience' (Holling 1996; Walker et al 1981); (Plimm 1984; O’Neill et al 1986; Tilman and Downing 1994). There are many varying perspectives on how to engineer resilience. Watson \& Adams (2011) believe resilience design incorporates lessons from nature on how to "protect, restore and enhance" the services of a system when mitigating the impact of flooding (Watson \& Adams, 2011, p257). Mileti (1999) believes human adaptation to natural disasters is a cyclical process involving: "preparedness, response, recovery, and mitigation" (Mileti, 1999, p. 22), while Pelling (2003) posited that vulnerability to natural hazards is based around exposure, resistance and resilience.

Improvements in weather forecasting, GIS computer flood mapping software, and the collection of flood data are successfully working to improve flood prediction. However climate change is expected to lead to greater climate variation and severe weather evens. Ecosystem flood resilience highlights the importance of system redundancy, increasing the variety of flood-adapted functions, increasing a system's strength and flexibility and mitigating flood impacts on a variety of spatial scales. These ecosystem flood resilience principles can be integrated on a variety of social and spatial scales to provide insight into new, innovative long term solutions that embrace the hydrological characteristics of floodplains. For floodplain cities, ecological and social resilience must be analysed on a variety of spatial scales to explore how resilience can be strengthened, and vulnerabilities reduced.

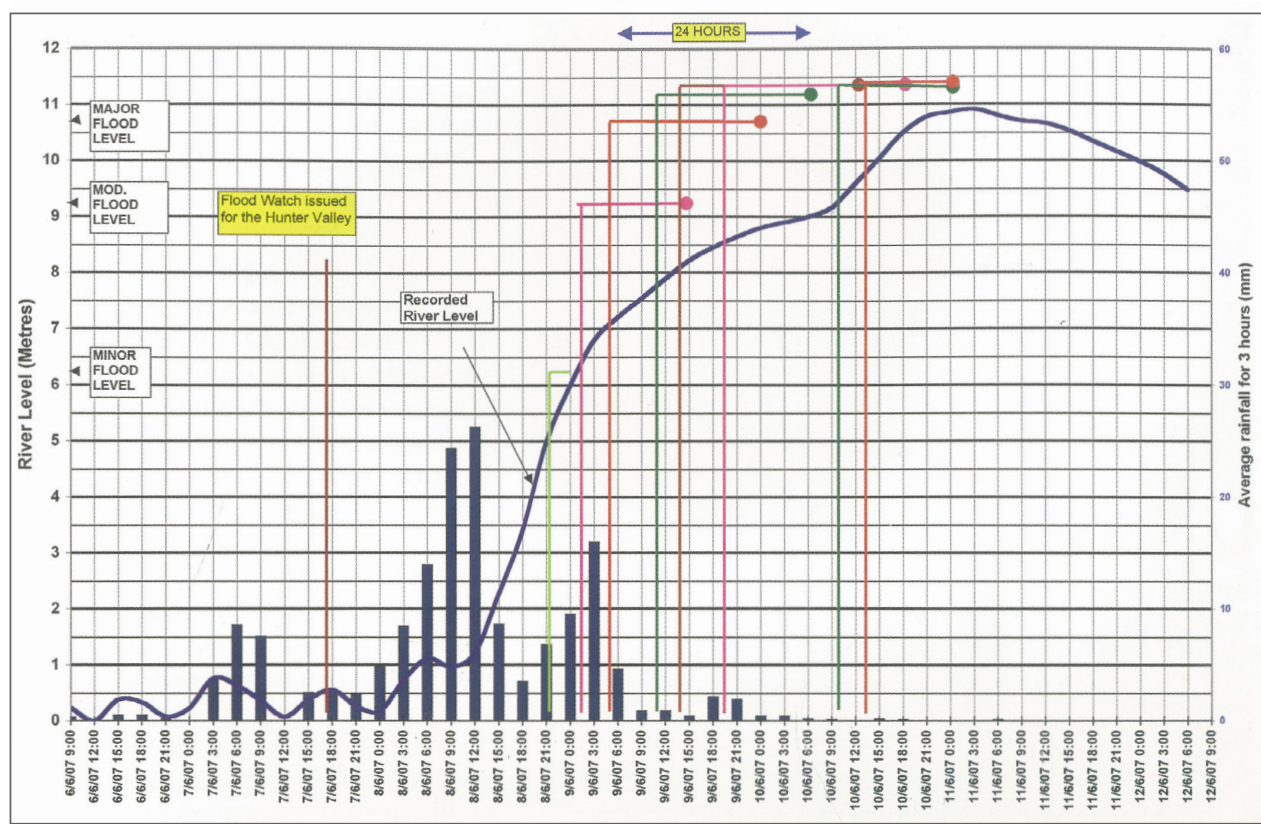

Figure 12: Maitland's Belmore Bridge flood gauge recordings for the June 2007 floods Source: Australian Government Bureau of Meteorology, 2012 


\section{The interacting scales of a catchment system}

Floods impact upon each spatial scale. Resilience at each scale relies on a balance between flood mitigation and the provision of space for water and people.

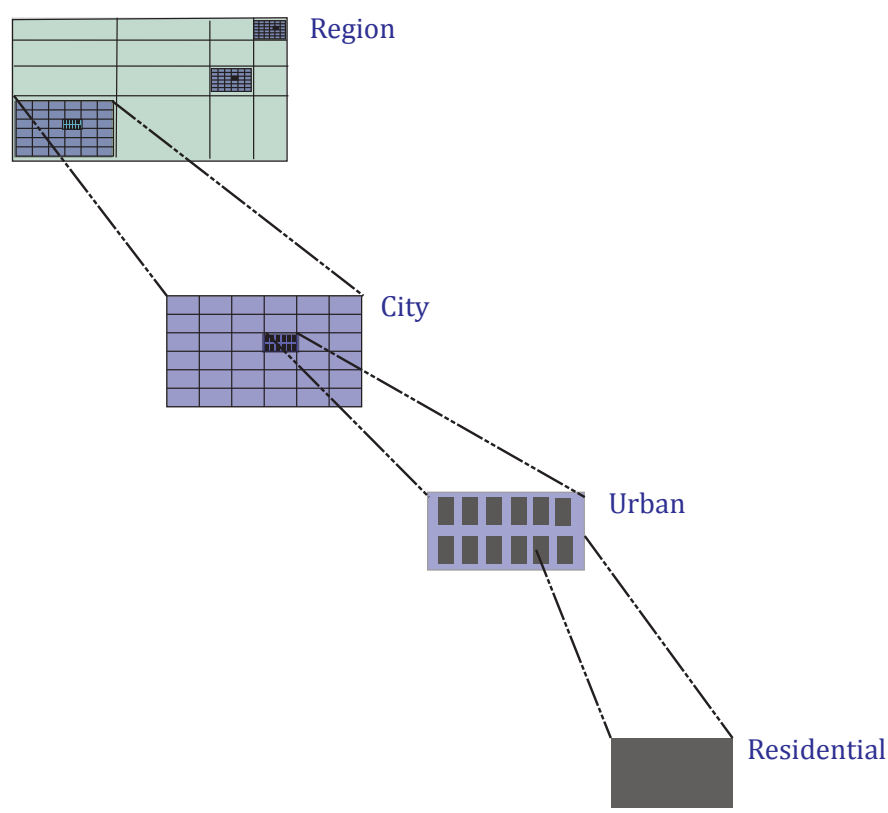

Figure 13: The interacting scales of a catchment system

Flood management currently focusses on a regional catchment scale with flood mapping, and on a residential scale using flood proofing. On a city scale focus is on flood mitigation and preventative structures. There is a failure to address the cities functional and structural resilience to flooding. Similarly there is a failure to address vulnerabilities at the urban scale, with residents becoming isolated.

\subsection{Socio-spatial flood resilience}

It is imperative that there is understanding of the spatial environments within which a floodplain society operates. This relationship is referred to in this thesis as 'sociospatial resilience', which recognises the interconnections of a society, with its urban and ecological spatial realms. The resilience principles relied upon by floodplain ecosystems can be analysed in the context of human habitation and used to help formulate plans for social and structural resilience in a community. "Resilience in risk management of human systems is concerned with the preservation of the day-to-day and other activities of communities and societies within the human species... and the welfare of individuals" (Handmer \& Dovers, 1996; p487). The floodplain city system in this context is defined as the society and ecosystems at risk of inundation from rivers (De Bruijn K. , 2005, p. 1). Systems, such as those of cities, need to be viewed as a series of subsystems interconnected to a broader interacting context.

It must be recognised that vulnerability and resilience effect the adaptive capacity of systems. As Jeff Dayton-Johnson (2004) highlights "adaptive capacity is a function of countries' vulnerability to natural disaster risk and their resilience once such disasters have struck" (Dayton-Johnson, 2004, p. 8). The resilience and vulnerabilities within urban and ecological spatial domains are greatly influenced by systems' autonomous flood adaptive capacity. In ecosystems the non-linear interactions of ecosystems are responsible for generating alternating stable states which enable the system to maintain stability (Gunderson \& Holling, 2002). In cities this is achieved through a variety of strategies acting to mitigate and simultaneously embrace floodwaters, at the regional, city, suburban and building/lot scales. These organised scales act as "sub systems" within the major system (Walker, Holling, Carpenter, \& Kinzig, 2004). An example of ecological measures at these respective scales could be wetlands, green infrastructure, rain gardens and dense vegetation. 
Spatial resilience in the context of natural hazards has been defined by Greiving \& Fleischhauer (2006) as "an area where human beings and their artefacts are threatened by spatially relevant hazards", such as flooding (p110). This definition recognises the urban domain, but fails to acknowledge the significance of ecological resilience. Pelling (2003) notes there is "relatively little theoretical discussion of urban vulnerability or resilience to environmental risk" (p46). The spatial effects of flooding need to be understood and incorporated into social responses to strengthen social resilience.

Social resilience has been compared, contrasted and incorporated with socioecological resilience since the 1970s. A socio-ecological system recognises the cause and effect relationship between ecological and social systems. Ecological systems are made up of organisms interconnected via sub-systems such as the functional processes of organisms. Social systems are similarly made up of organisms, known as humans, which are connected and reliant upon cooperative and interdependent relationships (Anderies, Janssen, \& Ostrom, 2004). Flood hazards affect the social, urban and ecological spatial realms. Whilst socio-ecological resilience recognises the dynamic interconnected nature of societies and ecology, it fails to integrate the urban built environment as an intrinsic component of the environment that must be made resilient. Socio-spatial resilience aims to correct this shortcoming.
Social resilience has become increasingly complex in present day floodplain cities. Social resilience has been defined as "the ability to face internal or external crisis and not only effectively resolve it but also learn from it, be strengthened by it and emerge transformed by it, both individually and as a group" (Brenson-Lazan, 2003). Sapirstein (2006) believes social resilience can be measured by time; "how long would it take for the community to respond to the event, self-organise and incorporate the lessons learned before returning to a [new] normal way of functioning" (Sapirstein, 2006 , p. 3). This differs to social vulnerability, which relates to the damage cost of disturbances, including physical, emotional and economic impact and the number of lives lost. Social resilience is "the ability to face internal or external crisis and not only effectively resolve it but also learn from it, be strengthened by it and emerge transformed by it, both individually and as a group" (Brenson-Lazan, 2003). Like ecological resilience, social resilience is strengthened by redundancy. In modern cities there are a plethora of opportunities to develop relationships and social ties within communities, neighborhoods, workplaces and families.

Dovers and Handmer (1992) distinguish between reactive and proactive aspects of a resilient society as its capacity to anticipate and learn. A reactive approach involves strengthening the system to become resistant to change, whereas a proactive resilience approach accepts the unavoidability of change and establishes a system capable of adapting to new conditions. Social resilience involves a diverse range of individual and collective values including cultural and political values, demographic, economic status and health. Adger (2000) indicates that these secondary social values influence humans' ability to cope with, adapt to and recover from external environmental disturbances such as floods. For social resilience to be successfully achieved it must respond to differing exposure levels experienced by the young, teenagers, middle aged, elderly, sick or disabled peoples. But alongside these demographic groups, the health status, economic status, political policies, educated awareness and cultural values are also proven to greatly impact on social resilience to natural hazards (Smit, et al., 2001). 


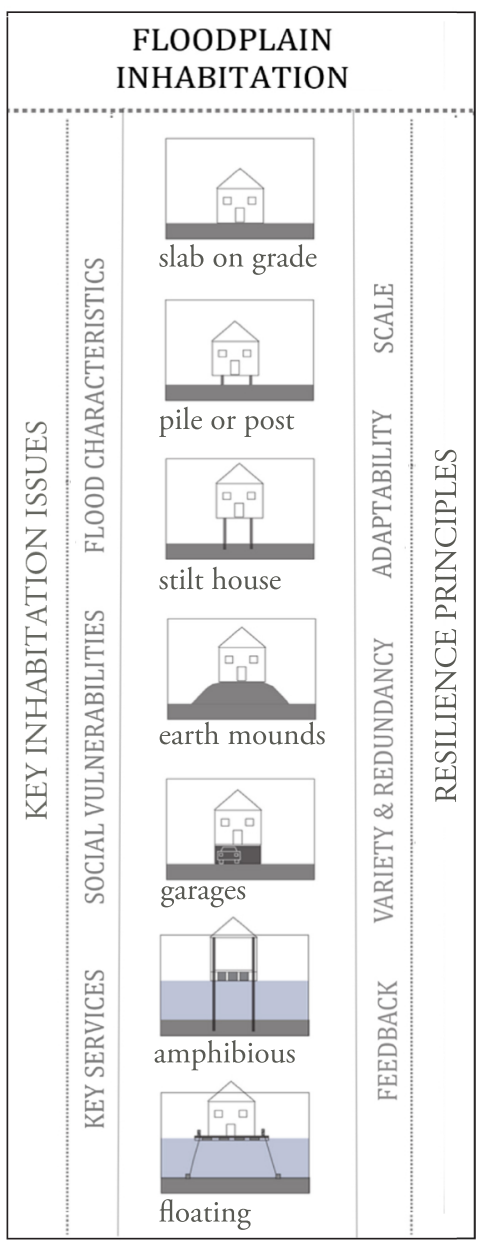

Figure 14: Highlighting the socio-spatial resilience themes and existing methods of living on floodplains.
Modern societies have become increasingly reliant on the delivery of vital services and infrastructure by governing bodies; such as energy, water, waste removal, communication and transportation (Merz, Hall, Disse, \& Schumann, 2010, p515). The centralisation of vital services in modern cities has resulted in a loss of redundancy and thus the systems are at greater risk of failing when impacted upon by disturbances. Burkett et al (2005) identify that non-linear relationships ensure functional redundancy is established at a variety of scales. Redundancy allows the system to respond, adapt and provide feedback to disturbances taking place at regional, city and local scales (Folke, Holling, \& Perrings, 1996). Each scale has its own spatial and temporal attributes (Allen and Starr 1982; O'Neil et al 1986). These scales are intrinsically linked such that a disturbance at one scale influences another. Increasing social resilience relies upon the "strength and scope of the internal connections between the people, organizations and environment that form that society and working to maintain these connections" during and after disastrous events (Sapirstein, 2006, p. 7). Socio-spatial resilience recognises the interconnected nature of the urban and ecological spatial domains with their inhabitants and the multi-scaled impact flood hazards have upon these.

Social values and relationships are connected to respective spatial domains. To comprehend the complex interactions taking place within a city, social resilience and social vulnerability need to be analysed at the regional (society), city (communities), urban scale (neighbourhood) and residential building/lot scale (individuals/families). The physical characteristics of flood hazards such as volume, velocity, duration and frequency vary at different spatial scales. A socio-spatial resilience lens will reveal the intrinsic connections between the flood impacted spatial area and the social vulnerability of floodplain inhabitants. This in turn will highlight the strengths and weaknesses of a city's flood resilience and allow strategies to develop. (Socio-spatial flood resilience strategies are to be further explored in the proceeding chapters.) 
Flood management practices have traditionally focused on the regional catchment scale and accumulated technical data to predict flood vulnerability. Improvements in flood gauges, height indicators and flood mapping have enhanced data accuracy and thus reduced vulnerability (Merz, Hall, Disse, \& Schumann, 2010). GIS computer flood mapping services now allow a means of measuring the success and hydrological impact of these large scale engineering works on the river catchment and its adjoining floodplains. However, as Klein, Nicholls, \& Thomalla (2004) highlight, flood engineering structures "remove rather than reduce the risk, promoting further development in what remain potentially hazardous areas", within a society that becomes increasingly unaware and unprepared for floods (p. 37). The economically disadvantaged move into areas with cheaper land now perceived as "safe" from flood, others continue to live in the area 'made safe' by the engineering structure and the communities risk of a severe flood event is either unaffected or increased. Social vulnerability is not only reliant on social flood awareness and preparedness, but also on an understanding of spatially operating flood strategies. Regional socio-spatial strategies to ameliorate this problem could include land use planning, development guidelines and zoning restrictions, flood management policies and infrastructure development, including the structures that allowed inhabitants to remain during a flood event. These strategies not only have spatial implications, but also have implications on society at large.

\subsubsection{Communities - City scale}

"The very features that make cities feasible and desirable - their architectural structures, population concentrations, places of assembly and interconnected infrastructure systems also put them at high risk to floods" (Godschalk, 2003, p. 136).

Floodplain cities have a rich flood stricken history. However in many cases their respective societies become increasingly unaware or complacent of this history with every flood free year that passes. Although a city's flood vulnerability can be reduced in post flood residential relocation efforts: "once a city has developed and high levels of investment have been made there is a large inertia against relocation" (Klein, Nicholls, \& Thomalla, 2004, p. 37). There is commonly a system of flood mitigating structures implemented to protect floodplain cities from regular flood inundation. These structures are predominately focussed on the perimeter of cities, whilst flood implications upon urban structure such as street networks, are marginalised in priority despite their direct contribution to community connection and individual isolation during floods. The negative ramifications of centralised infrastructure and services are poorly recognised. There is currently a failure to acknowledge the intricate relationship between spatial aspects, such as flood works, and social flood exposure levels, with regards to community connectedness, access to shops and services during flood events, and individual flood awareness, preparedness and response. Resilience at the city scale relies upon "the ability of a community to not only deal with adversity but in doing so to reach a higher level of functioning" (J. Kulig as cited in Pooley, Cohen and O'Connor 2006 p 163).

As an example of a measure to improve socio-spatial resilience on a city scale, a flood evacuation centre that fulfils an everyday community strengthening function, has an alternate energy source, waste and water collection and treatment, and that utilises high insulating flood proof materials and strong construction, will enhance not only the buildings utility in the everyday functioning of the city, but improve the resilience of also the city in the time of flood. Decentralised infrastructure and services, alternate energy generating means (solar, hydro and wind), multiple modes of transport and circulation and green infrastructure housed in a flood resilient structure enhance the socio-spatial resilience of not just individuals, but local city communities. Such measures would not only reduce the adverse impacts on the community during and post flooding, but they would enhance the lives of floodplain inhabitants in the everyday functioning of the city, strengthening the connections between society and ecology.

Timmerman (1981) believes a resilient city is less vulnerable to natural hazards, but as Klein, Nicholls, \& Thomalla (2004) point out there is a lack of "systematic 
and reproducible analysis as to what makes cities resilient and how resilience can be enhanced" (Klein, Nicholls, \& Thomalla, 2004, p. 42). The resilience of cities to flooding requires urban, infrastructure, commercial and residential designs to anticipate and respond to water inundation at varying height levels, velocity rates and duration periods. The decentralisation of infrastructure reduces the chances of a total system collapse and increases the ability of communities to access vital services during floods.

\subsubsection{Neighbourhoods - Urban Scale}

Godschalk et al (1999) call for a "sustainable mitigation policy system whose goal is developing resilient communities, capable of managing extreme events" (Godschalk, Beatley, Berke, Brower, \& Kaiser, 1999, p. 137).

Neighbourhoods in this context refer to the close knit communities who reside locally to one another. Social resilience in this context is spatially defined by the local community groups of a city. A neighbourhood community's socio-spatial resilience is largely determined by the socio-demographics and culture of the community, the community connectedness and the spatial flood resilience of the area in which the community resides. Community vulnerability is significantly influenced by the number of people from at risk groups including those from a low socio-demographic, the young, elderly, sick or disabled. Achieving resilience at the community level is reliant on local access to vital services and local social support structures. When a community has not been directly affected by floods, social flood preparation regarding the implementation of temporary or permanent dry or wet flood measures is reduced.

Neighbourhoods residing in urban floodplain settings are enhanced by established dry retention zones, rain gardens, impermeable surfaces, and vegetative recreational zones positioned in recognition of floodwater flow paths, with sensible placement of urban infrastructure in flood resilient zones. These measures not only work to reduce the likelihood of urban flooding, but also decrease the adverse impact flooding will have in isolating neighbourhood community's from one another and from vital infrastructure and services, thus recognising the intrinsic relationship the social and spatial realms have at the urban scale.

\subsubsection{Individuals and families - Building/lot scale}

There is currently a failure to link urban socio-spatial resilience with individual resilience. Despite the resilience enhancing benefits of residential wet and dry flood proofing measures, residents are inhibited in their ability to achieve social resilience as a result of becoming isolated from community support networks and key services during floods. Spatial resilience is largely determined by the specific location of the residence; in relation to the river, natural flood paths, artificial diversion channels and water retention zones. However social resilience incorporates structural flood proofing measures and amphibious construction, which reduce economic damage, emotional impact and safety concerns, and socio-demographic components, such as being elderly, young, disabled or sick. Flood resilience at this scale has become a priority in order to reduce structural flood damage, but there is currently a failure to sufficiently incorporate social resilience aspects such as the provision of alternate electricity, water sources and transport means. Alex Wilson (2006) refers to this shortcoming as 'passive survivability', which he defines as "the ability of a building to maintain critical life support conditions for its occupants if services such as power, heating, fuel or water are lost for an extended period" (p.20).

A socio-spatial flood resilience approach will encourage cities, communities and individuals to prepare for climate change and nature's characteristic unpredictably (Adger, Hughes, Folke, Carpenter, \& Rockstrom, 2005). It will enable floodplain management policies to transform from traditional preventative aspirations, to policies focused on managing the robustness and flexibility of residential sociospatial systems. This will enable floods to positively shape ongoing development on floodplain cities (Folke C. , 2006). 


\subsection{Resilience floodplain management}

Current flood management practices combine infrastructure, landscape, and social policy in an attempt to improve the ability of societies and their urban environments to withstand floods (Nordenson, Seavitt, \& Yarinsk, 2010, p. 16). They build upon traditional flood management practices which were primarily focused on controlling the natural environment in order to mitigate or eliminate uncertainty. Resilience flood management practices will work towards establishing a symbiotic relationship with the inhabitants of the flood prone land and the reoccurring extreme hydrological conditions of floods and drought. Despite the increasingly recognised advantages resilience thinking provides for managing hazards, there continues to be a lack of integration of theory into policy (Harrison \& Salt, 2006). When applying resilience theory to natural hazards, Mileti (1999) highlights the importance of building local networks and increasing the adaptive capacity of cities.

Watson and Adams (2011) believe the pre-planning and rebuilding processes prior and post a natural hazard foster opportunities for new innovative solutions. Paton and Johnston (2006) similarly perceive floods as catalysts for change; resilience becomes a measure of the ability of communities to adapt to inundation with water. Ryan (2010) highlights that "major flood events in the past century have... significantly increased our understanding and capacity to cope with floods" (Ryan, 2010 , p. 44). Societies who learn from flood experiences and make post-flood adaptations are able to reduce vulnerabilities and improve their adaptive capacity to cope with future flood events.

The adaptive capacity of the city is dependent on the flexibility and robustness of individuals and communities. Educated flood awareness, pre-planning and adaptive infrastructure enable the city to maintain its basic structure and function during and post flood events. The time it takes the city system to return to a stable state is also intrinsic to the resilience concept (Gunderson, Allen, \& Holling, 2010). 
Time plays various functional roles at a variety of spatial scales (Pimm, 1984). Periods of gradual change interact with fast moving processes across a variety of temporal and spatial scales (Bodin and Wiman (2004); Gunderson \& Holling (2002). The time it takes a system to return to a state of equilibrium following a disturbance is now referred to as "engineering resilience" (Holling C. , Engineering resilience versus ecological resilience, 1996).

Flood preparedness involves implementing a variety of time-based and crossscale initiatives in anticipation of floods. These pre-flood initiatives could include warning systems, evacuation processes and emergency supplies. They facilitate effective social response and reduce the city's flood vulnerability (Mileti, 1999, p. 22). Development at present is often seen to exacerbate flood risks, but resilience provides opportunities for innovative future development to become part of the solution. Innovative resilient urban designs coupled with flood mitigation practices establish an environment for pro-active positive renewal to take place alongside investment (Watson \& Adams, 2011).

A network which nurtures a collaborative multi-disciplinary approach to flood management will involve specialists across a variety of fields including hydrologists, ecologists, emergency workers, resource managers, local government planners and state and federal government policy makers (Mileti, 1999, p. 9). A flood-resilience framework would promote both a quantitative and qualitative capacity of the system to absorb and spatially accommodate future varying flood events.

Applying resilience principles to existing flood management strategies will provide greater insight into what a resilience floodplain management framework will involve. Boundaries will need to be established due to the complex multi-scale nature of resilience theory and flood management practices. But it must be recognised that flooding extends beyond a system's defined boundaries. In this way, it encompasses the ecological, economic and socio-cultural domains.
Resilience principles, although lacking in Australian floodplain management have recently been integrated into the Netherlands philosophical approach. Extensive international research has recently focused on shifting former flood preventative philosophies to concepts of resilience. This has arisen from society and academic spheres recognising the innovative potential a resilience approach provides for flood management (De Bruijn, K.M., Klijn, F and Godschalk, M.F).

However despite recent interest in community resilience, few studies have established systematic principles of resilience and practically applied these principles at a city or urban scale (Godschalk D. , Urban hazard mitigation: creating resilient cities, 2003). Zvenbergen, Veerbeek, Gersonius, \& van Herk (2008) also highlight the lack of research investigating a systems approach for defining, measuring, increasing, managing and assessing resilience in urban flood management (p. 83-4); (Carpenter, Walker, Anderies, \& Abel, 2001).

There needs to be greater recognition within the flood management process of the significant influence of social dimensions on vulnerability to hazard. "Society not only determines the vulnerability but also the strategy followed to cope with flood risks. Acceptable levels of risk are [thus] a balance between social and economic values" (De Bruijn K. , 2005, p. 1). The adaptive capacity of individuals, communities and cities to flooding will depend upon the variety of mechanisms in place to ensure basic services and facilities are available during flood events. A sociospatial resilience framework interlinked at a variety of spatial scales and time frames will provide insight for Government policies and interventions traditionally focused at the catchment scale (Ryan, 2010). 


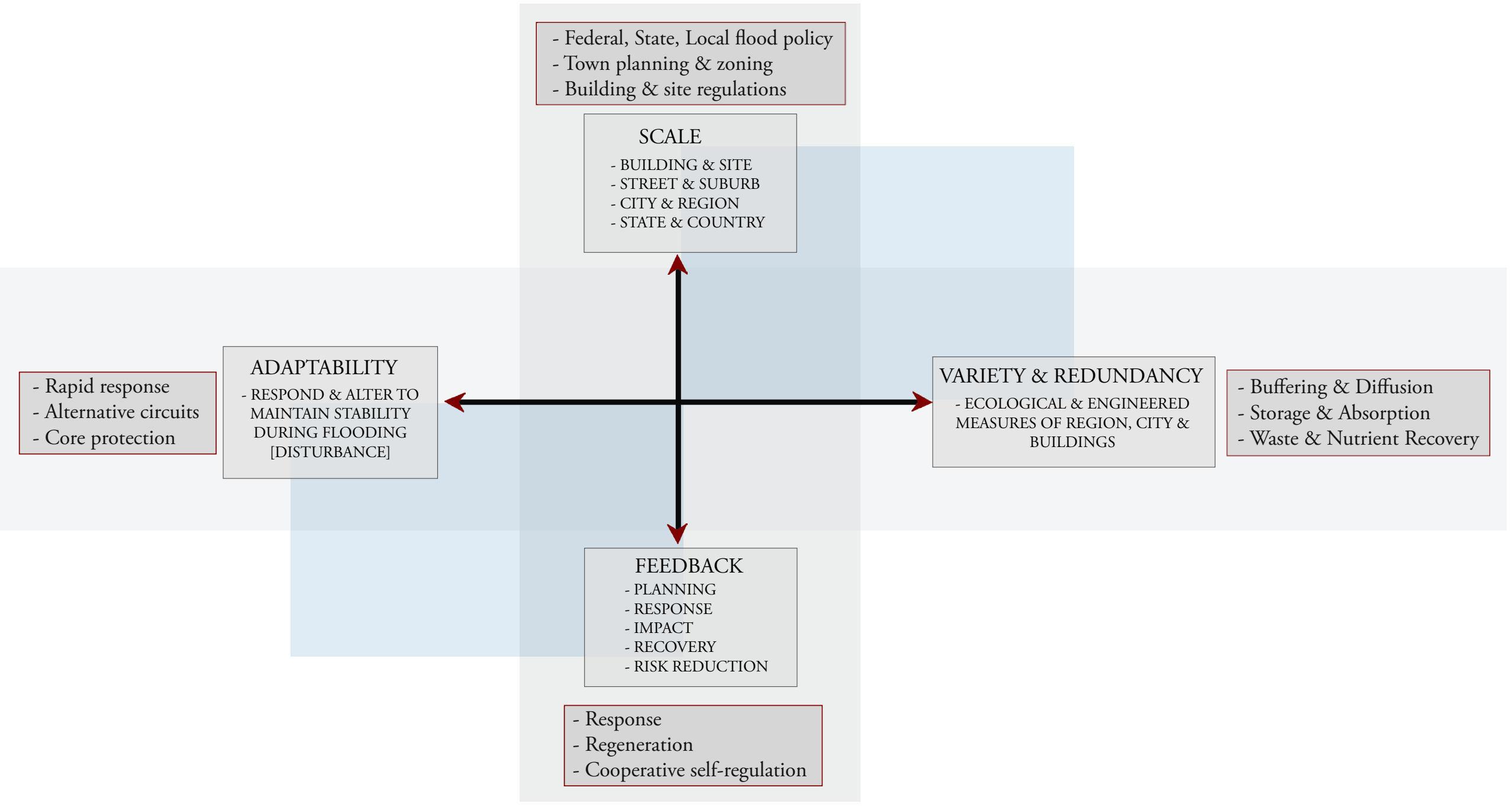

Figure 15: The interrelating flood resilience principles with flood management practices 
3.6 Conclusion: The need for a resilience flood management network

A socio-spatial resilience network may provide a more cohesive means to categorise flood management. An increased understanding of the interconnections between ecosystems and human societies can influence vulnerability and resilience levels to natural hazards, such as flooding (Adger, Hughes, Folke, Carpenter, \& Rockstrom, 2005). A multi-scale interlinked management process will promote robustness to flooding by ensuring there is redundancy within the system.

Scientists predict that climate change will cause an increase in the frequency and severity of flooding. It hence becomes increasingly important that uncertainty is integrated into planning. Ecological flood resilience principles indicate the need for increased flexibility and robustness at a series of socio-spatial levels. Climate change and population growth provide an opportunity to re-think traditional relationships between infrastructure, ecology and society within the urban environment (Nordenson, Seavitt, \& Yarinsk, 2010, p. 14). Whilst recognising that a single solution for all cultural, socio-economic, political and site specific characteristics a singular resilience solution does not exist, there is an opportunity to develop a variety of resilience measures which promote redundancy and flexibility and thus a city's ability to adapt and respond to floods that can be adapted to suit specific situations.
There is currently a lack of practical explorations and applications of resilience theory. Much of the resilience literature remains theoretical, due to a lack of site specific resilience testing. A multi-scale socio-spatial resilience approach provides opportunities for new flood interventions to reduce the adverse impacts of flooding. A variety of interventions at multiple scales will potentially increase the flexibility and robustness of a city, a community and an individual living in a flood prone region.

Four key resilience principles arose from analysis of ecosystem resilience to flooding; scale, adaptability, variety and redundancy, and feedback. The significance of multiscale interactions was also highlighted. Applying these flood resilience principles to existing flood management measures will provide a means to re-categorise existing practices and create a new socio-spatial resilience flood management matrix. 
Resilience is the ability of a system, community or society exposed to hazards to resist, absorb, accommodate to and recover from the effects of a hazard in a timely and efficient manner, including through the preservation and restoration of its essential basic structures and functions... The resilience of a community in respect to potential hazard events is determined by the degree to which the community has the necessary resources and is capable of organizing itself prior to and during times of need. 


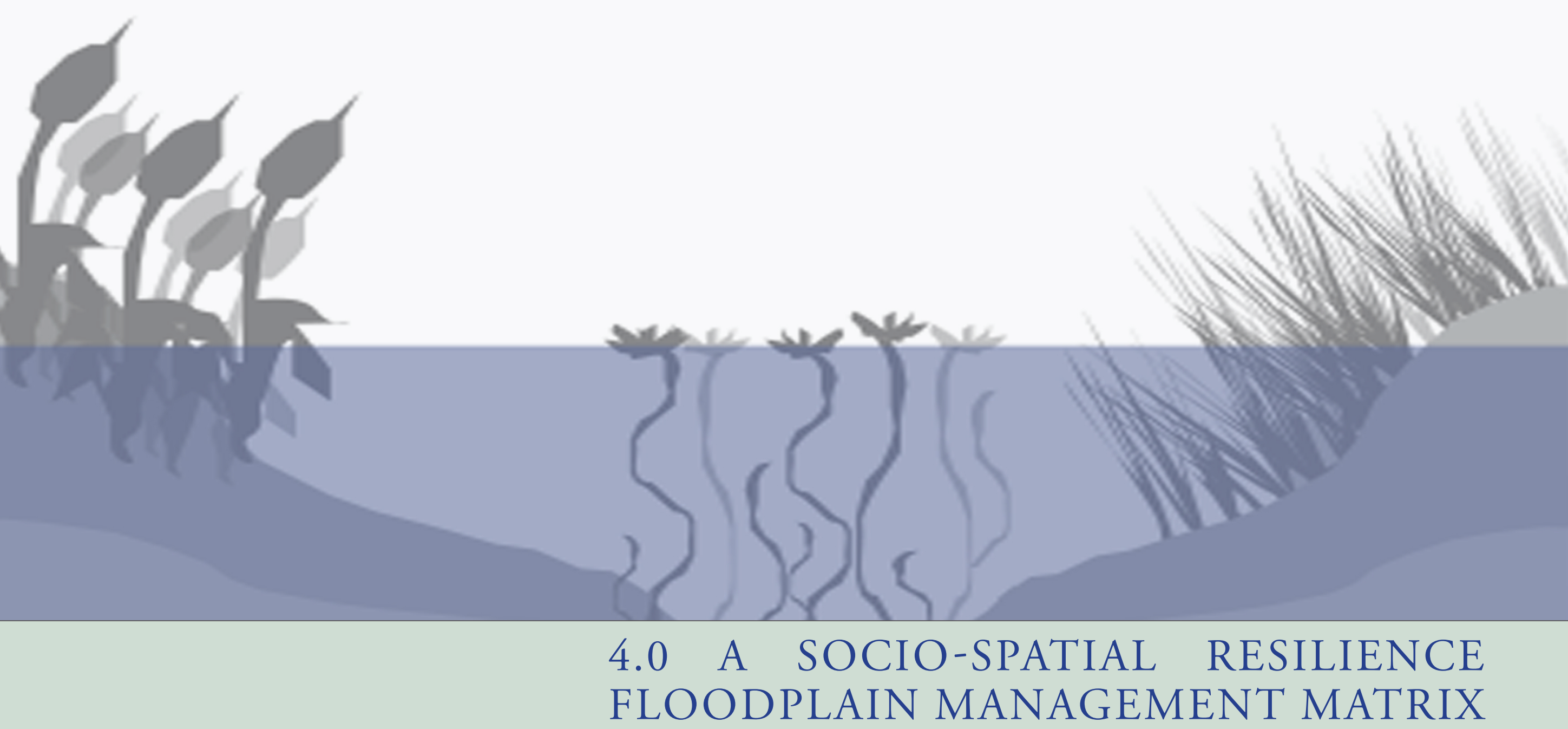

In this section, the flood resilience principles previously identified, as scales, adaptability, variety and redundancy and feedback will be further detailed. Diagrams translate literature theory into visually refined flood resilience strategies. A matrix system is then used as an analytical tool to re-categorise existing ecological resilience processes and flood management strategies. The benefits and limitations of a resilience flood management matrix are outlined and the importance of a balanced top down and bottom up approach highlighted. 
Key flood resilience principles previously identified in ecosystem resilience to flooding as scales, adaptability, variety and redundancy and feedback need to be further defined with regards to flood management. Resilience philosophies enable buildings, communities and city services to anticipate and respond to flooding (Watson \& Adams, 2011, p. 257). The interacting scales of a city allow interventions to have knock-on effects upon other spatial scales. Pre-planning for flooding in the built, natural and social environments enhances the resilience capabilities of a floodplain city to maintain its basic structure and function during flood disturbances. Sociospatial flood resilience is measured by the "magnitude of disturbance that can be absorbed before the system is restructured with different controlling variables and processes" (Gunderson, Holling, \& Pritchard, Resilience of large-scale resource systems, 2002, p. 4).

\section{Flood characteristic indicators}

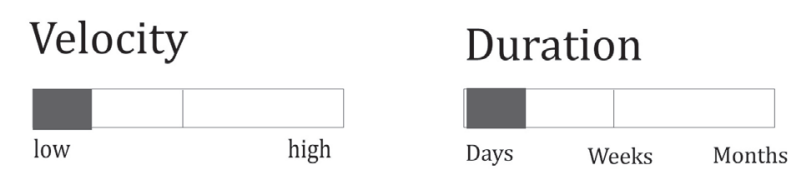




\section{Depth}

$<1 \mathrm{~m}$

\section{Frequency}

Annual Decade Century

Figure 16: Flood characteristics diagrams

\subsection{Socio-spatial scales}

Strategies aimed at strengthening the flexibility and robustness of a system need to be employed at a series of spatial scales. The flood resilience principle of scales includes interacting spatial and social domains; region, city, suburb, street and the lot, alongside society, community and the individual. Flood characteristics and their spatial impact, including velocity, duration, depth and frequency, are also seen to vary across socio-spatial scales.

The flood velocity, otherwise known as flow rate, impacts hazard levels and is a determining factor when choosing appropriate flood strategies. Flood duration and period of inundation have social, ecological, health and economic impacts. The flood depths determine the suitability of particular flood proofing measures. The flood frequency also referred to as flood occurrence rates, influence the preparedness, response and recovery of floodplain cities and its inhabitants. 
Resilience is the ability of a system, community or society exposed to hazards to resist, absorb, accommodate to and recover from the effects of a hazard in a timely and efficient manner, including through the preservation and restoration of its essential basic structures and functions... The resilience of a community in respect to potential hazard events is determined by the degree to which the community has the necessary resources and is capable of organizing itself both prior to and during times of need.

(UNISDR, 2009, p. 24)

Social vulnerabilities:

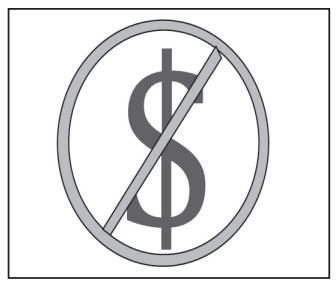

Low socio-economic status

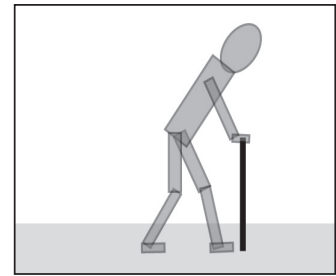

Elderly

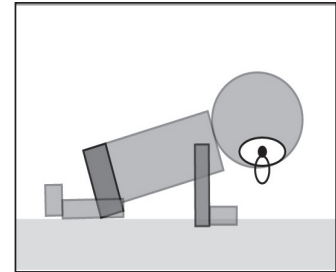

Young

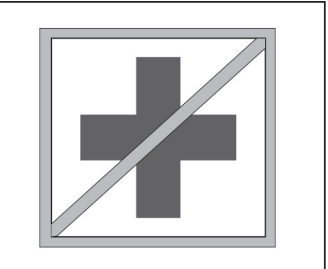

Poor health and sickness
Everyday key services:

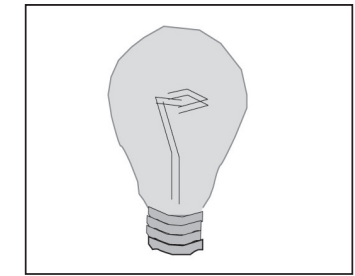

Power/electricity 
Social exposure to the spatially acting flood characteristics, described above, vary greatly. Buckle (2006) suggests flood vulnerability levels of individuals and communities can be assessed in their ability to access key resources such as food, water, shelter, health care, education and cultural activity. This sentiment is to be further highlighted through precedent analysis (Chapter 5).

To achieve social resilience the specific contextual political, economic, social, historical and cultural influences need to be understood. These greatly impact the societal capacity to plan, respond and adapt to flooding (Wisner, 2004). From literature analysis a number of key social characteristics were seen to significantly influence vulnerability levels. However, there is a need to site specifically analyse the social vulnerabilities of floodplain cities in order to determine whether there is a singular social aspect which if improved can improve overall social resilience to flooding. (See Chapter 5 Precedent Analysis for further detail)

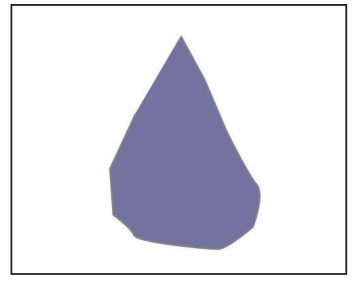

Fresh drinkable water

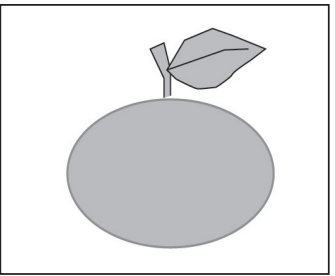

Availability of food

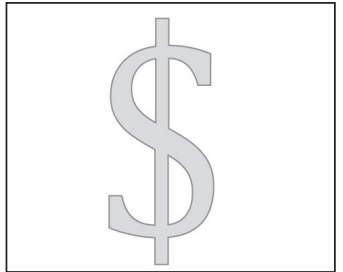

Access to work and income

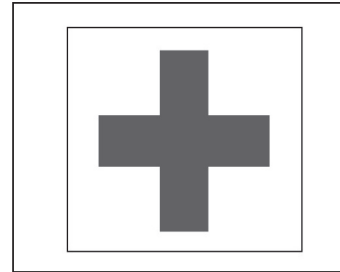

Access to medical services

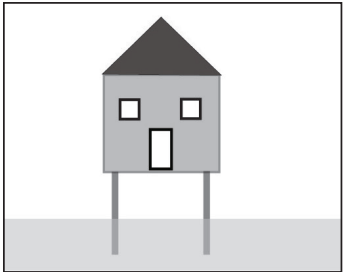

Provision of shelter

Figure 17: Social vulnerability and everyday key service diagrams 


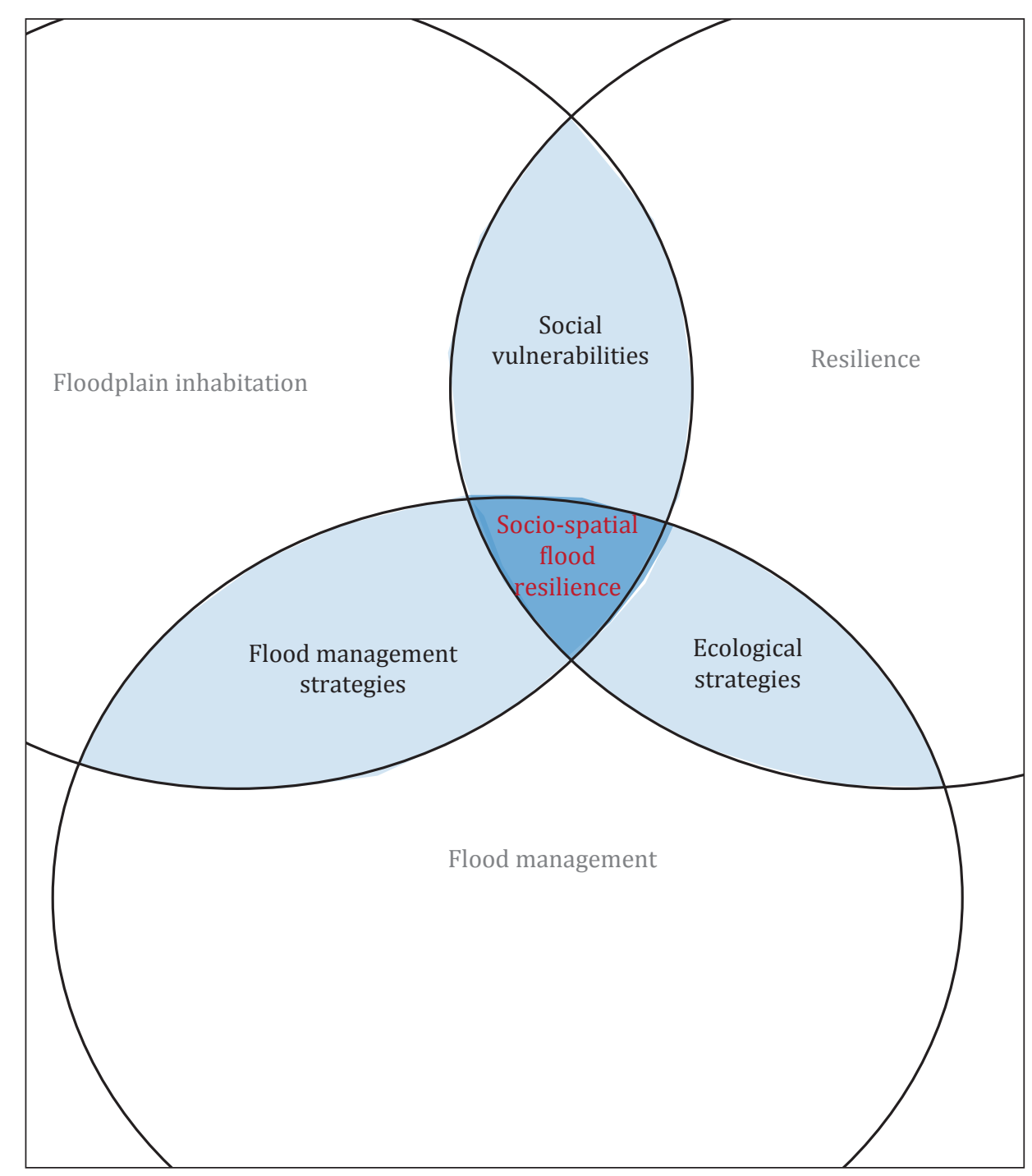

Figure 18: Refining the primary, secondary and tertiary themes of this thesis 


\subsection{Adaptability}

When dealing with flood hazards social resilience is greatly impacted upon by spatial resilience both within the natural and urban environment. For example, spatial factors such as urban density not only influence surface run off, stormwater volumes, and subsequently flood heights, but are also intrinsically linked to the ability of residents to adapt and cope with the resultant flood hazards. This is readily evidenced in urban environments where volume and peak flows of storm water runoff are significantly altered, and the flash flooding causes widespread social and spatial implications (Toubier \& White, 2007, p. 14).

Zvenbergen et al (2008) indicate that there is a lack of studies attempting to "formulate systematic principles of and proxies for flood resilience and apply them to flood-risk management systems" (Zvenbergen, Veerbeek, Gersonius, \& van Herk, 2008, p. 83). This chapter establishes a set of guiding ecological flood resilience principles to re-categorise flood management practices with an aim to enhance resilience, rather than focus on flood prevention. The flood resilience principles of scales, adaptability, variety and redundancy, and feedback are further broken down into functional ecological and spatial processes. A definition of the resilience principle and an explanation of the ecological and spatial examples will be provided. It should be noted that the resilience principle of scales have been applied through the range of strategy options provided for each principle.
Adaptability is defined in ecology as "the capacity of actors in a system to influence resilience", such as species in an ecosystem (Walker, Holling, Carpenter, \& Kinzig, 2004). It is the ability of system components to adjust their structure and response to changing external environmental drivers, such as flooding. This enables the system to remain within a stable functional domain. Adaptability in relation to flood resilience is made up of several key resilience principles; rapid response, alternate circuits, and core protection. Adaptability strengthens the flexibility of a system. In a flood prone city adaptability is the adaptive capacity of infrastructure and services. If infrastructure is designed to respond and cope with a variety of flood conditions flood damage will be reduced, functionality maintained and societal degradation reduced. 
Rapid response

In relation to flood resilience rapid response involves the components of the system being autonomous, so they are able to intrinsically respond and function in the altered environmental conditions without human intervention. Infrastructural solutions can be as simple as elevating and structurally reinforcing roads up to a design flood height. The aim of rapid response system components is that their everyday function remains the same in the altered environmental conditions imposed by flooding. The flood-adapted function is a continuation of its everyday use to ensure social awareness and understanding. An effective response of floodplain inhabitants to floods is reliant on flood resilience and resistance strategies being an intrinsic component of their everyday lives. Rapid response interventions involve strategies implemented prior to floods.
Ecological Strategy

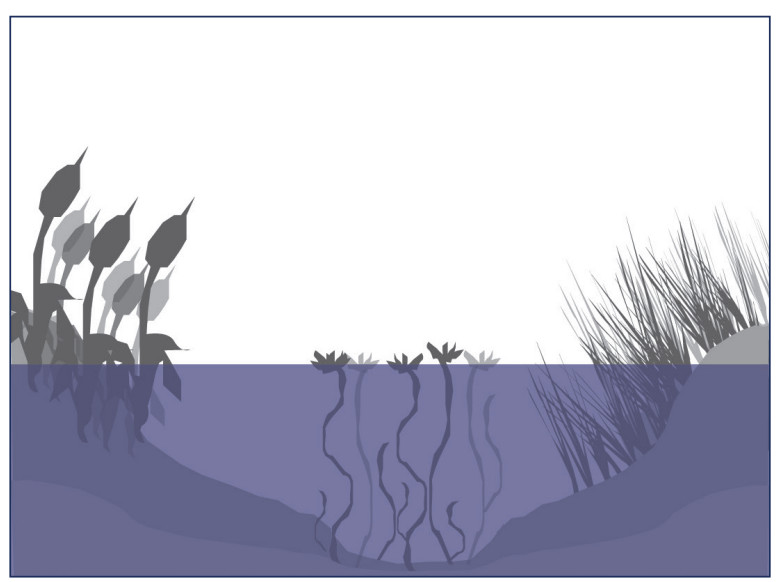

Figure 19: Flood adapted vegetation 
Spatial Strategies

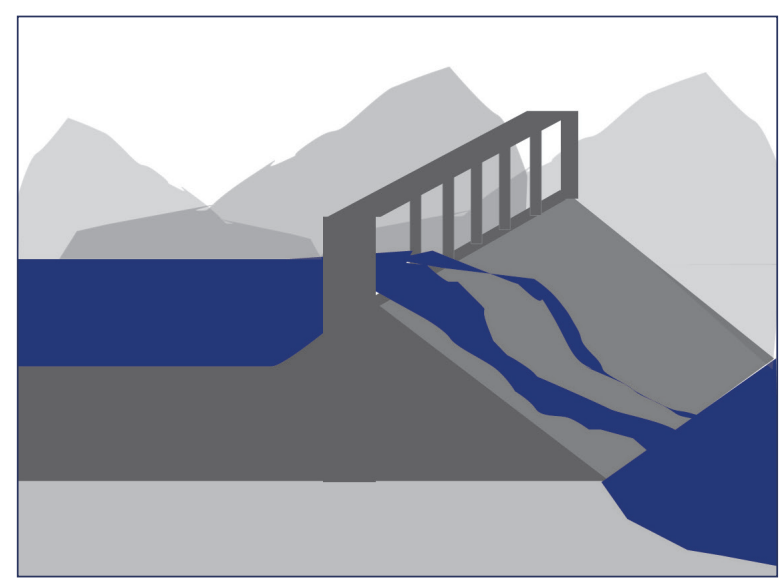

Ecological rapid response involves flood adapted vegetation which thrives in a variety of hydrological conditions, due to their water adaptive structures, functional processes, nutrient capturing, and water borne location.

Engineered flood management strategies such as dams and levees respond rapidly to rising floodwaters by storing and diverting water.

Figure 20: Engineered Flood Works 
Spatial Strategies

These strategies work with adaptive infrastructure such as raised roads, floating promenades and terraced landscapes to reduce the impact of floodwaters. Dry flood proofing measures prevent floodwaters from penetrating buildings up to a design flood level (DFL). This can involve elevating the structure on columns, walls, or earth. Permanent or temporary shields can be used to prevent water penetration up to and equal to 0.9 metres (SCARM, Report 73, 2000). This is the point at which the hydrostatic pressure differences between the interior and exterior may lead to structural failure.

Wet flood proofing measures involve the implementation of measures to allow for water penetration. Interior drainage, waterproof material use, and pumps and sumps in basements, all ensure that water minimally impacts upon interiors (and structural integrity), although it must be acknowledged that issues with debris and contaminated muddy floodwaters can hamper recovery processes post immersion.

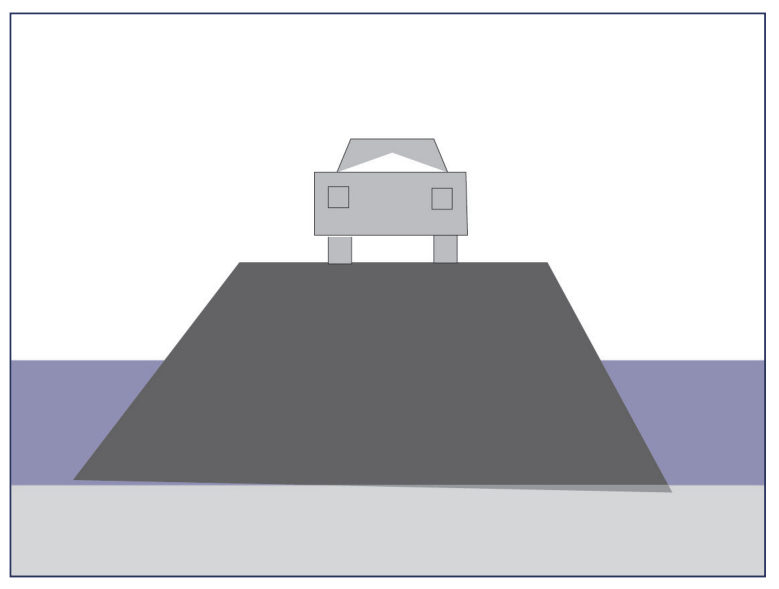

Figure 21: Adaptive infrastructure 


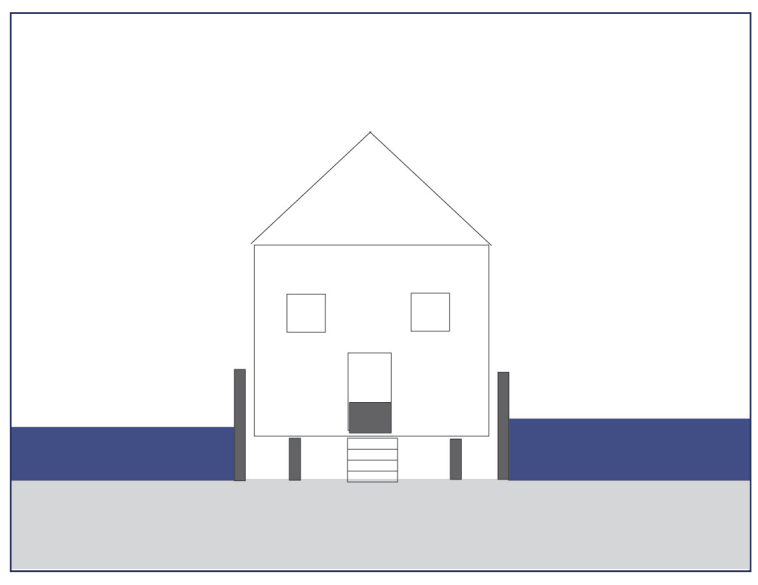

Figure 22: Dry flood proofing (permanent/temporary)

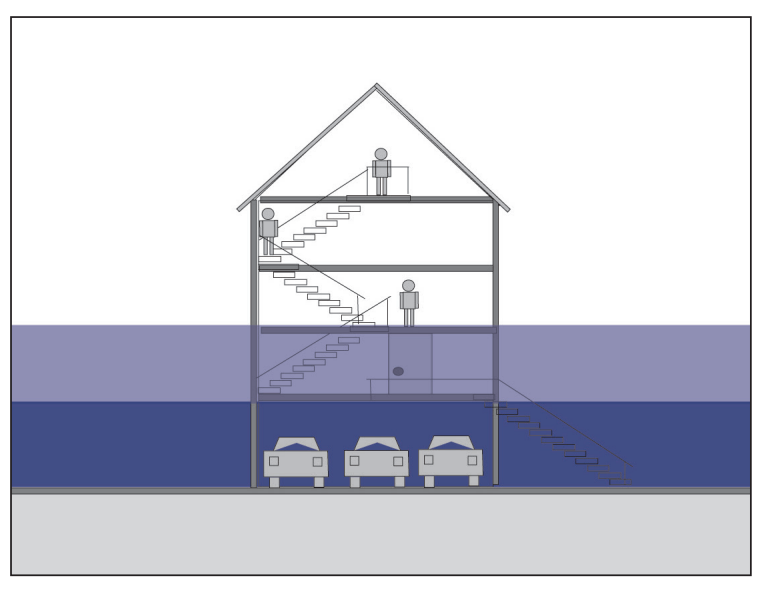

Figure 23: Wet flood proofing (permanent) 
Core protection means the protection of a 'core' with sacrificial elements. Core protection ensures the primary structure and function of a system remains within a stable domain when disturbed by external hydrological extremes. It requires a multiscale approach, encompassing both systems and the individual components making up a system. Core protection establishes a complex web of interconnected and interdependent multi-scale relationships to increase the robustness of the system to external disturbances. A variety of functional sub-systems establishes flexibility and increases the ability of the system to adapt and reduce the impact of disturbances such as flooding. In flood management it relies upon a balance between ecological soft measures and wet and dry flood proofing strategies working together to reduce the adverse impacts of flooding at a city, urban and residential scale.

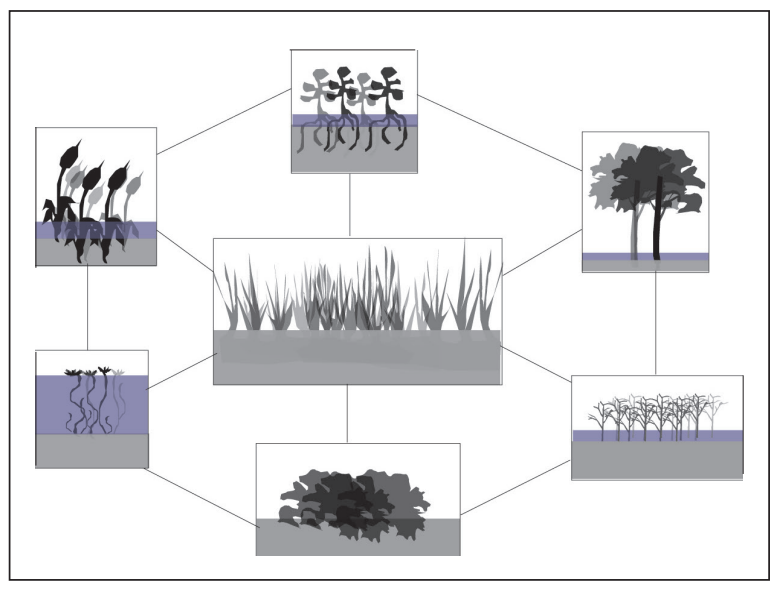

Figure 24: Keystone species 


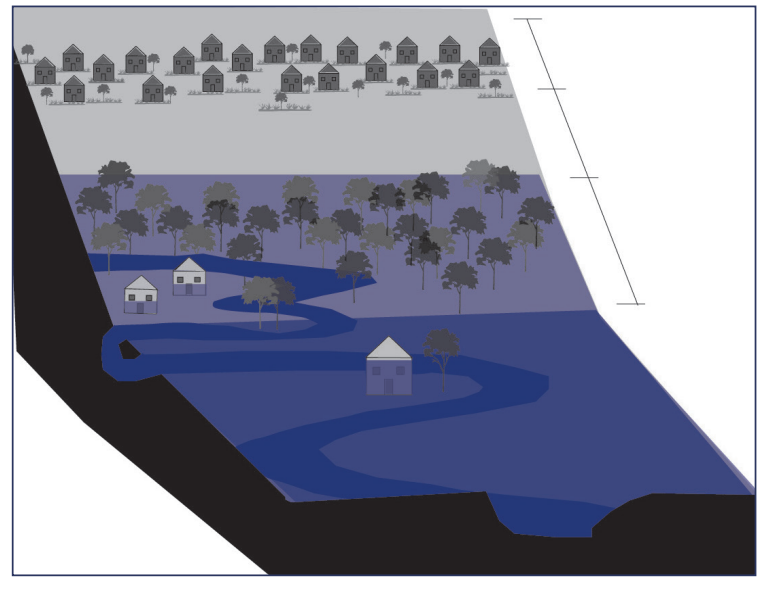

Figure 25: Land zoning
Core protection is achieved in ecosystems through keystone species, which function as the system drivers. They provide key functional services to the guilds of species making up an ecosystem. Guilds, also referred to as functional groups, perform specific services or functions within the ecosystem and are responsible for resource sharing. Keystone species control the number of guilds and thus species diversity within the ecosystem. A diversity of guilds enable the ecosystem to adapt to changed environmental conditions and maintain functional and structural stability. Thus all species of the ecosystem are dependent on the abundance of keystone species.

In flood management these same core protection principles are evidenced by land zoning practices that ensure green spaces benefit from regular inundation, whilst inhabitants are set back and protected from regular inundation by floodwaters. 
Core protection is improved through the use of water-resistant material with the structural integrity of materials not being adversely impacted upon by water. These materials include concrete, marine plywood, solid brickwork, galvanized metal, aluminum, stainless steel and closed or foam cell insulation. Flood adaptive service connections allow key services to continue to function during and post floods. It involves flood adapted mechanisms, adaptive connections and additional storage facilities, such as using non-return valves in drainage and sewerage pipes to increase a building's core protection from flooding. Self-reliant subsystems provide key services without relying on a city's water mains and electricity grid. It includes alternate energy sources such as solar energy and wind energy, grey water systems, and rainwater collection tanks. Independent connection mechanisms and storage facilities allow key services to continue to function during and post floods.

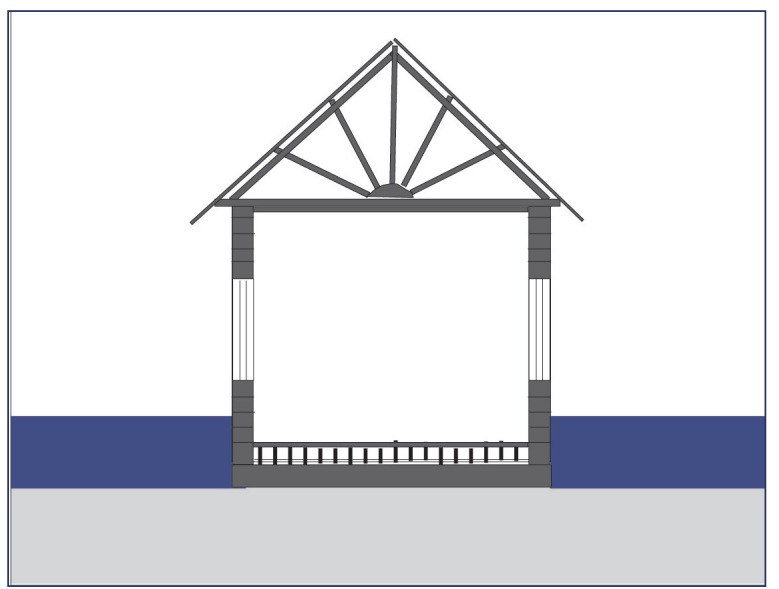

Figure 26: Water-resistant material 


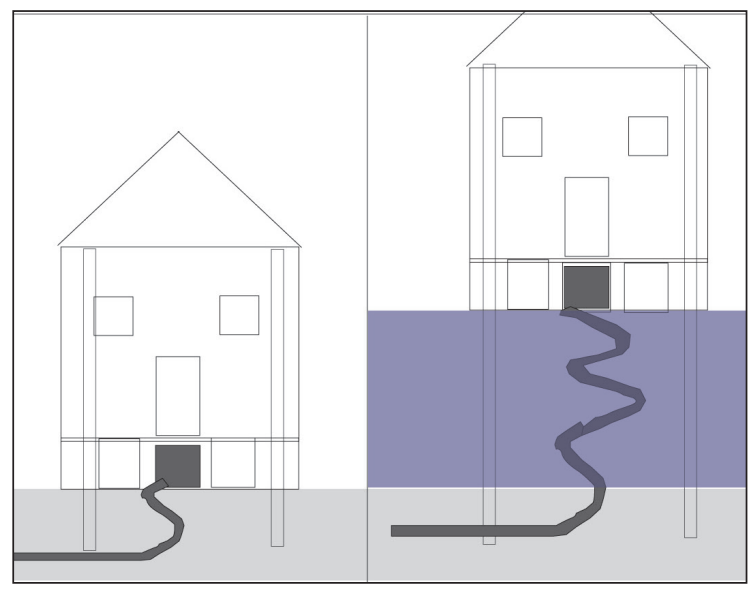

Figure 27: Adaptive service connections

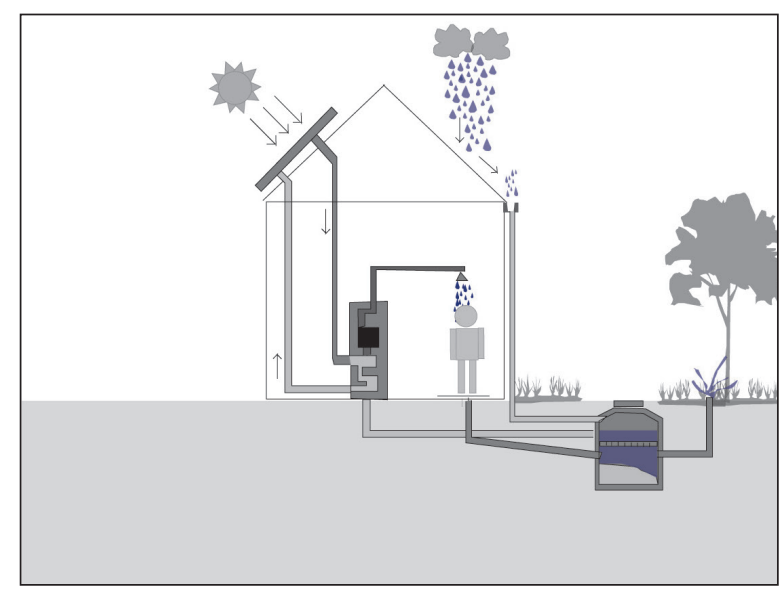

Figure 28: Self-reliant subsystems 


\section{Alternate circuits}

Alternate circuits refer to the availability of multiple paths for floodwaters and inhabitants. The aim is to reduce flood impact through the distribution of floodwaters, whilst providing a variety of circulation pathways for floodplain inhabitants. A variety of transport options and multiple circulation pathways reduce the chances of individuals, communities or a city becoming isolated during floods. Alternate circuits also contribute to redundancy in the system, a vital concept in improving flood resilience.
Ecological Strategies

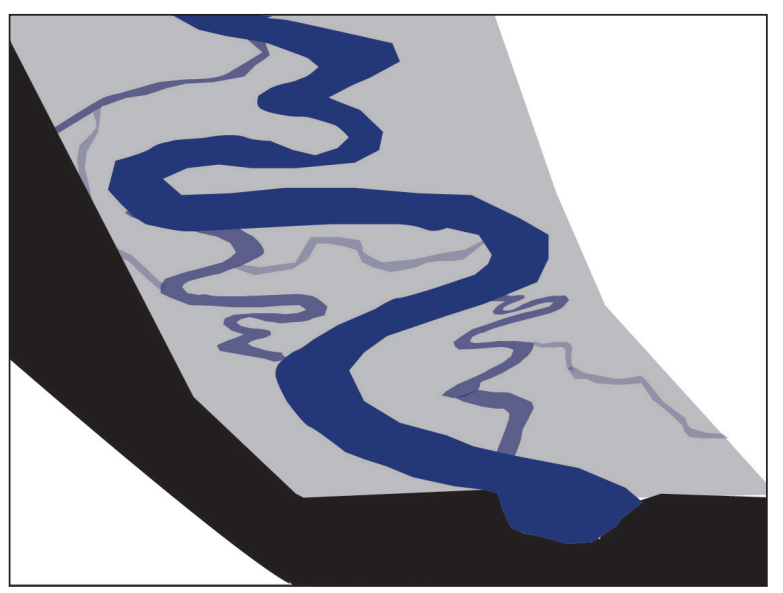

Figure 29: Natural flow paths

Ecological resilience processes involve the natural diversion of floodwaters into a variety of natural flow paths, such as rivers, streams, and creeks. Floodwaters are released into established wetlands, green spaces, lakes, or oceans. 
Ecological Strategies

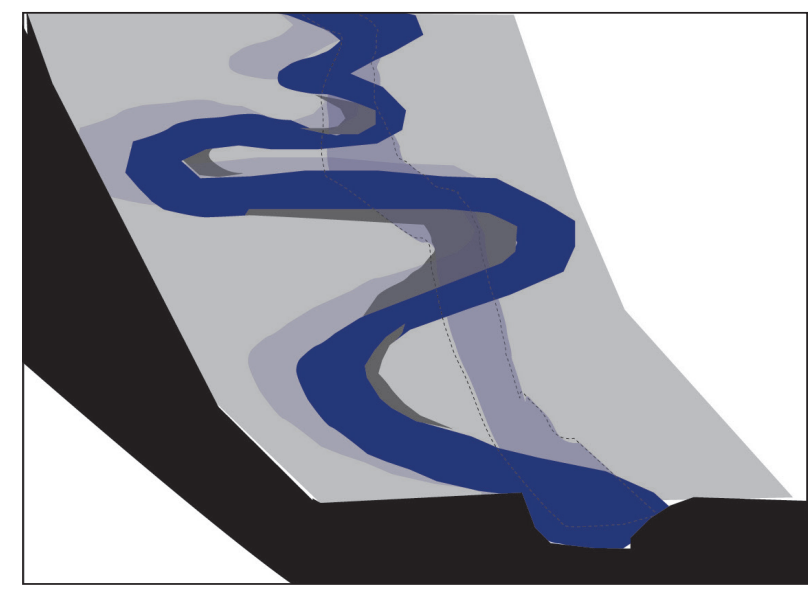

Figure 30: Meandering rivers

Meandering rivers also establish alternate paths during flood events, further diverting floodwaters, as accumulated sedimentation in a river channel reduces the river's depth and width, leading to water overtopping of banks and new pathways for river passage.
Spatial Strategies

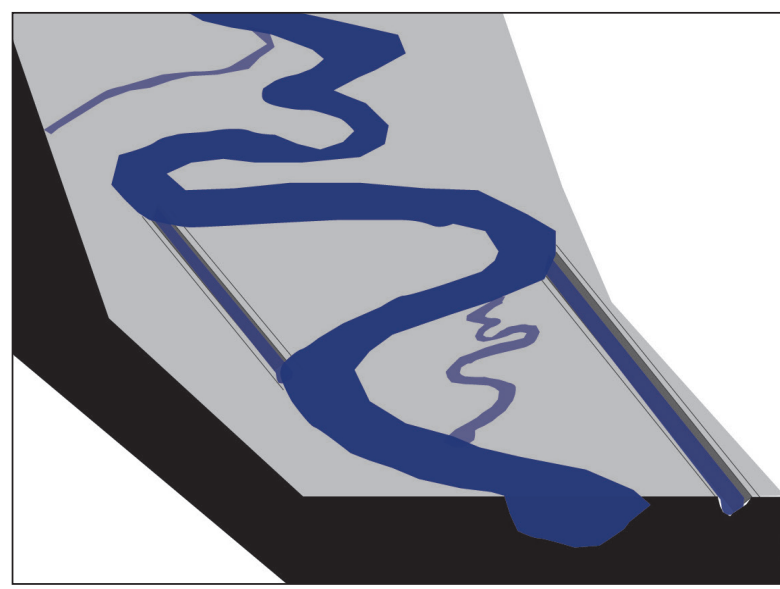

Figure 31: Flood control channels

In flood management flood control channels establish artificial paths for diversion of excess floodwaters away from the high flood zone, and vital urban infrastructure. 
The decentralisation of infrastructures and services in a city further improves its resilience to flooding by reducing the chances of inundation of all service providers. Such flood diversion is also important for access and escape for the inhabitants. The aim is fundamentally to improve mobility options to reduce safety concerns associated with urban circulation of flood waters.

A variety of roads and transport access (train, bus, vehicular, water vessels, and helicopter options) facilitate evacuation processes. A connected street network establishes a variety of options for vehicular and pedestrian movement. This reduces the chances of inhabitants becoming isolated when roads are inundated by floodwaters.

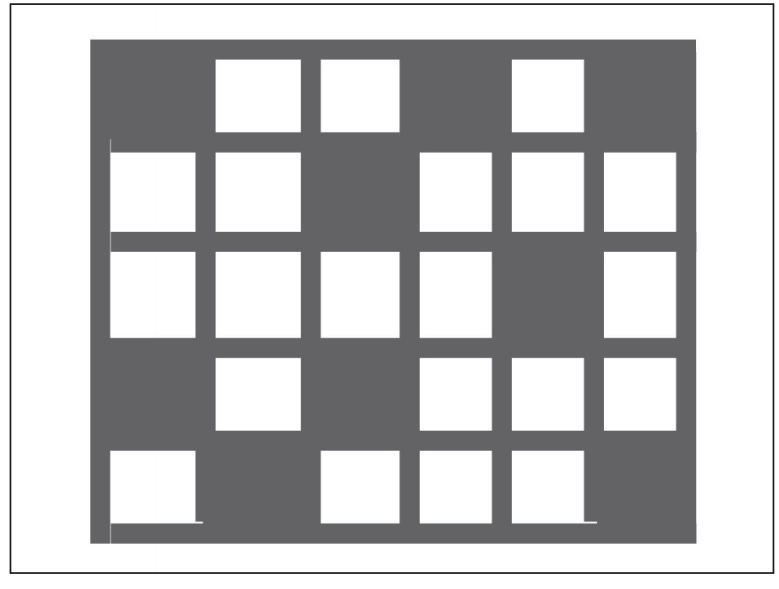

Figure 32: Decentralisation 


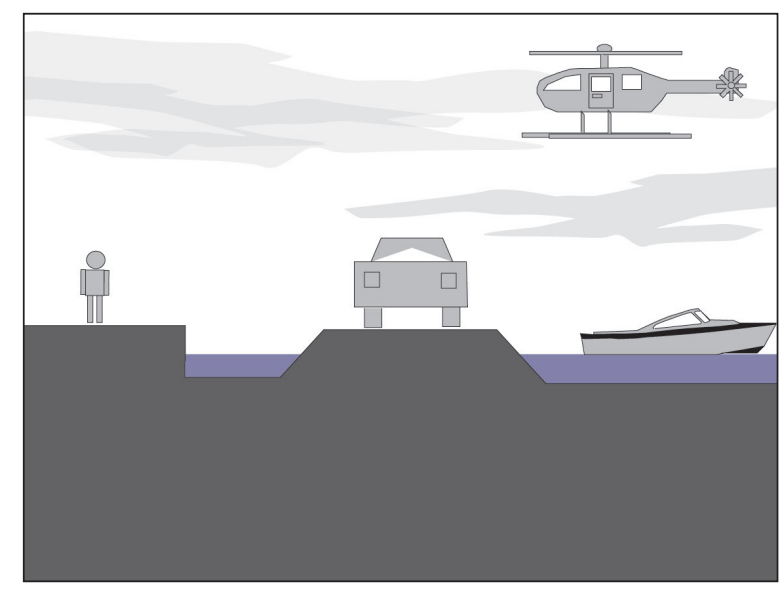

Figure 33: Access and escape

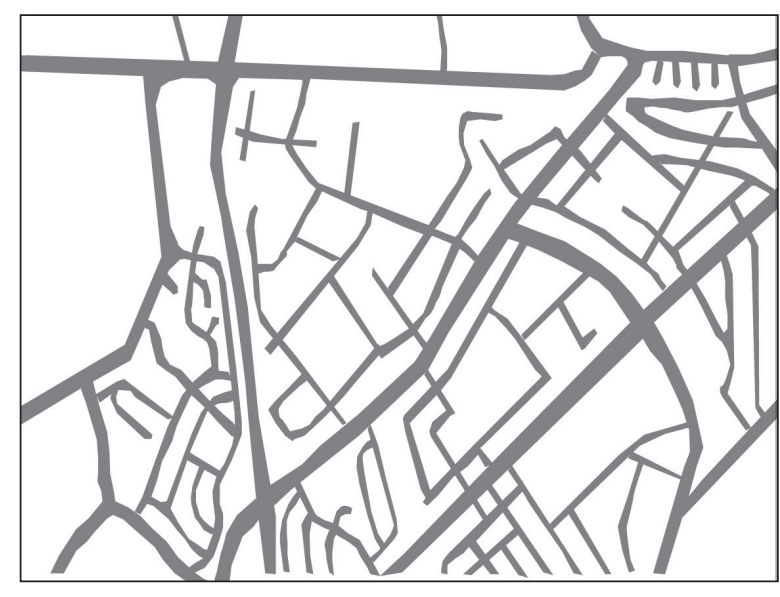

Figure 34: Connected street network 


\subsection{Variety and Redundancy}

Establishing diversity and thus redundancy in a city strengthens the robustness of the system, its ability to continue to function whilst inundated by floods. Implementing a variety of flood management measures improves the socio-spatial response at the city, urban and residential scales to flooding. Buffering, diffusion, storage, absorption, nutrient and waste recovery are the key flood resilience principles that ensure redundancy is adequately integrated across the socio-spatial scales.

\section{Buffering}

Buffering strategies aim to reduce the velocity of floodwaters and facilitate absorption. Both ecological and flood management measures can successfully utilise buffering principles to soften the impact of floodwaters. A variety of vegetation establishes a succession of spatially separated ecological barriers that function to reduce water velocity.
Ecological Strategy

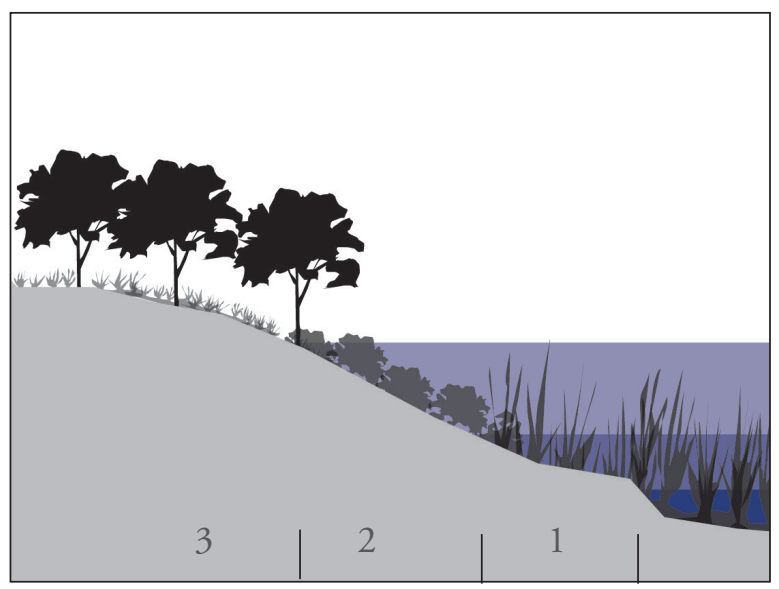

Figure 35: Riparian buffers

Riparian buffers are dense native vegetative areas which run adjacent to the river and are comprised of three zones. Zone one comprises large tree species and reeds whose role is to stabilise and shade the riverbank and reduce erosion. Zone two contains native shrubs that reduce rainwater runoff velocity, improving absorption rates and the filtering of contaminants. Zone three's native grasses further reduce surface water runoff volumes and encourage the absorption of the final water contaminants. 
Spatial Strategies

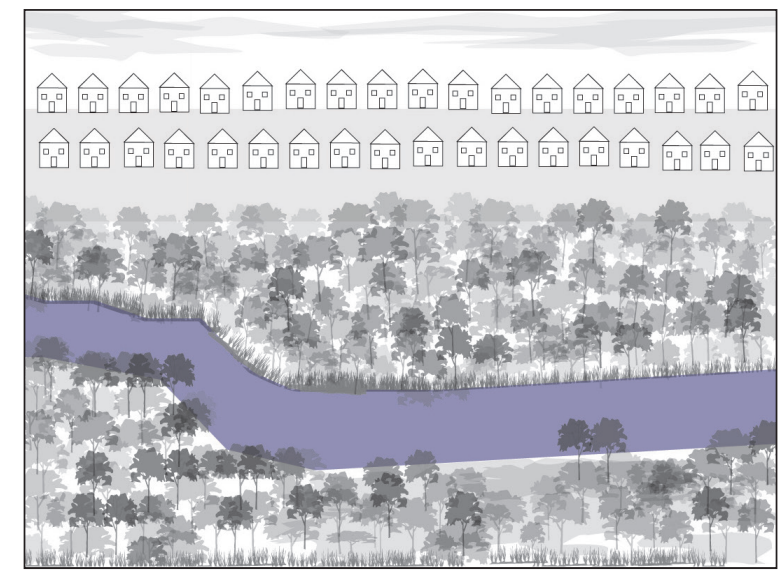

Figure 36: Green corridors

Green corridors are vegetative zones that act to slow the velocity of urban surface runoff of storm water or floodwaters. Green infrastructure involves core vegetative zones positioned within green hubs. These hubs are natural ecological open spaces such as wetlands, grasslands and forests.

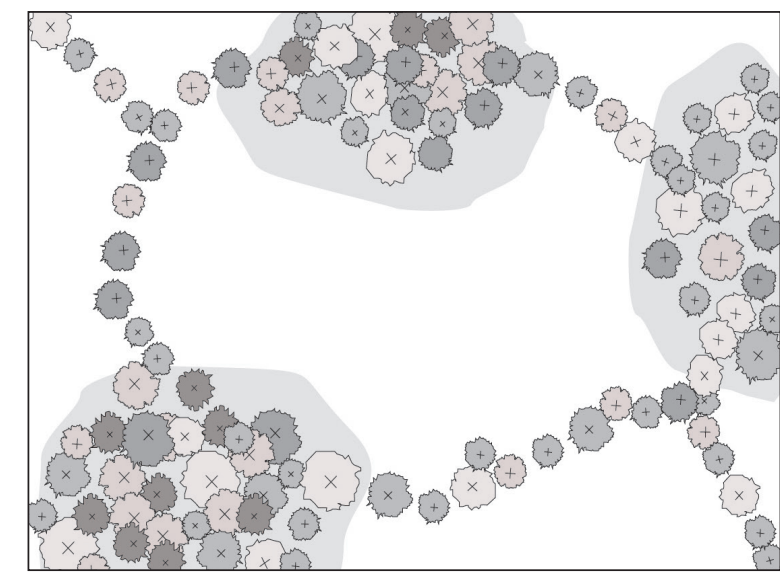

Figure 37: Green infrastructure (vegetative components)

These ecological hubs are positioned within urban zones and are connected via green corridors and smaller vegetative links to establish vegetative buffer zones to facilitate the storage and absorption of stormwater and floodwater. 


\section{Diffusion}

Diffusion strategies aim to dissipate water volume through ecological or artificial flood management means. Distributing the water across a larger spatial area mitigates the effects of local flood water depths, improves the ability of stormwater systems and ecological absorption processes to function effectively.
Ecological Strategy

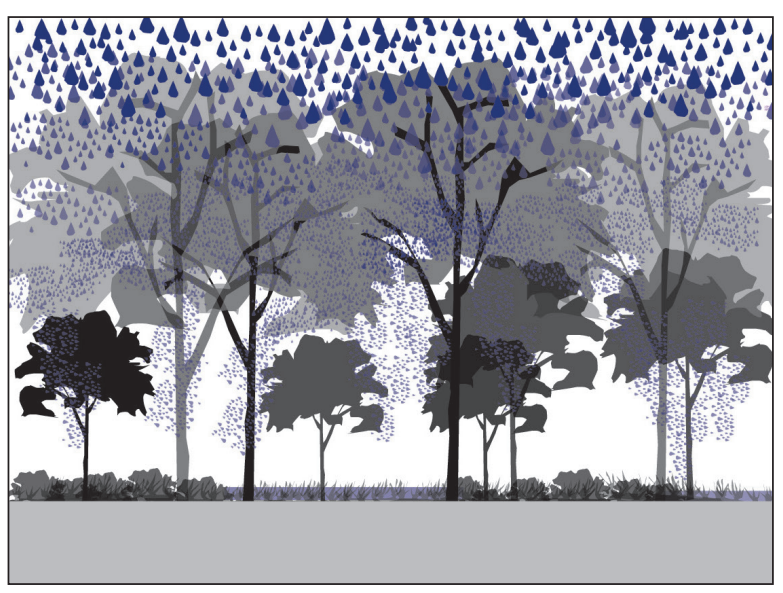

Figure 38: Dense canopy layer

Dense canopy layer of forests dissipates rain, reducing its velocity and spreading it over a larger surface area to improve absorption rates. The thick high foliage prevents sunlight and large amounts of precipitation from penetrating. This leads to the ground area between the large tree trunks being sparsely vegetated, providing greater surface area for floodwaters. The nutrient filled soil, with large tree roots and shallow vegetative roots promotes absorption. 
Spatial Strategies

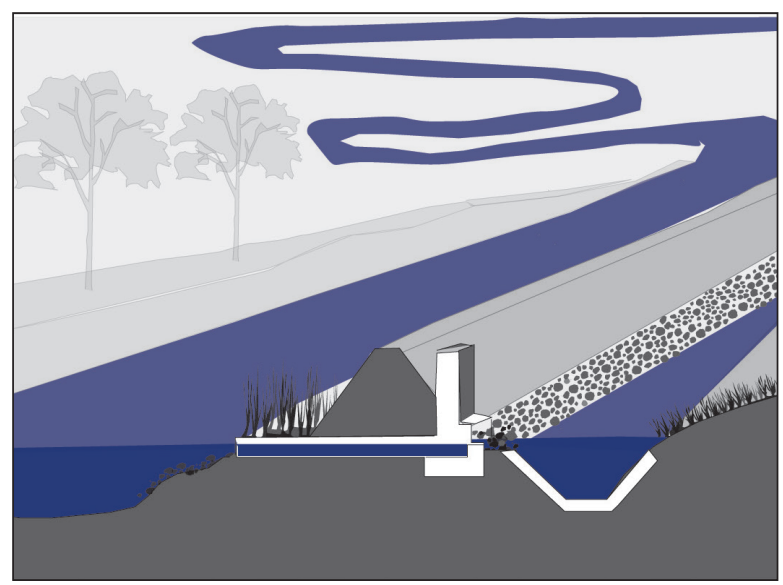

Figure 39: Diversion channels

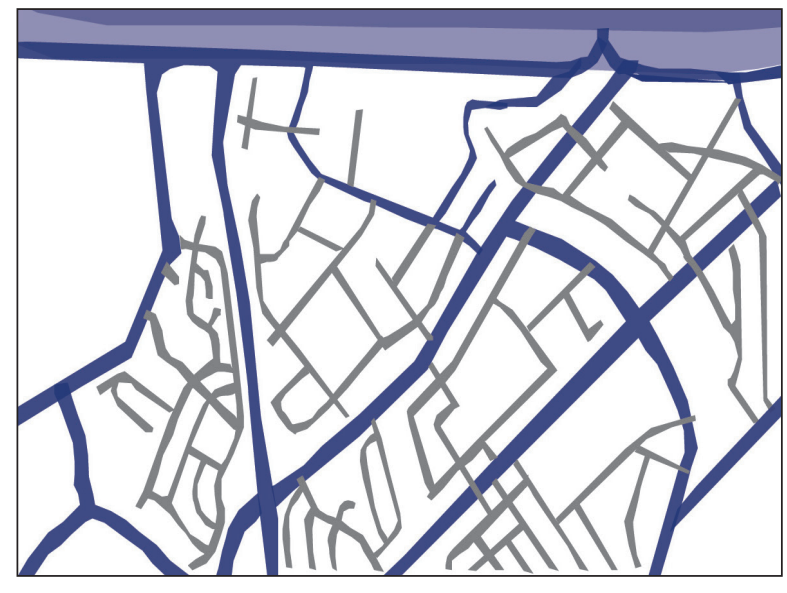

Figure 40: Circulation paths
Urban flood management uses diversion channels to diffuse floodwaters from the main channel into secondary water channels, to reduce the volume of water in the main channel.
Circulation pathways such as streets can be positioned to respond and re-direct urban surface rainwater into stormwater drains, and in larger flood events can be used to channel floodwaters into a series of secondary green space storage zones, away from urban built up areas. Australian roads are designed up to a one in one hundred annual exceedance probability (AEP) standard. This means that roads become water channels in flood events. 
Storage

Storage measures encompass naturally occurring and artificial soft or hard edged solutions with a functional role to collect and store rain and flood waters. Soft ecological storage strategies enable water to be naturally absorbed back into the soil as an intrinsic part of the hydrological system. Hard edged strategies promote water collection and reuse within dam or water storage areas.
Ecological Strategy

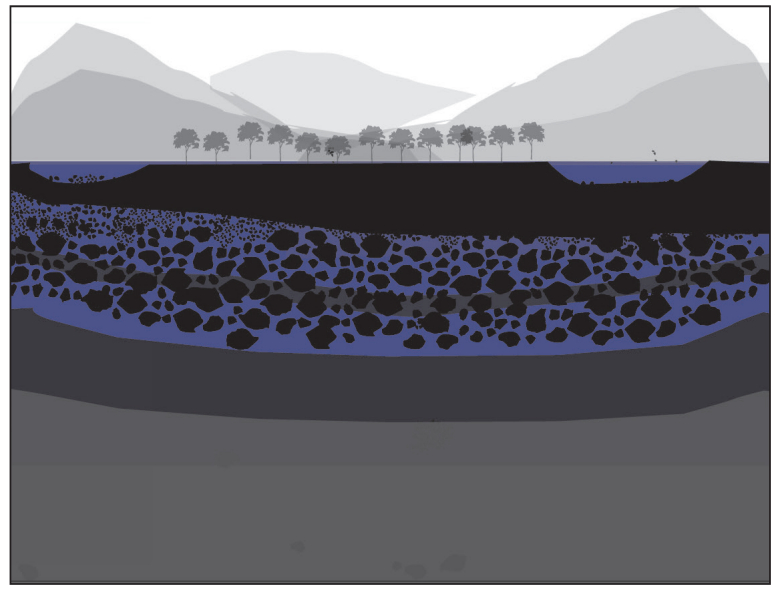

Figure 41: Aquifers

Ecological resilience strategies involve above and below ground water storage areas. Wetlands, marshlands and swamps are above ground areas seasonally or permanently inundated by water. Aquifers are a wet underground water-bearing layer of permeable rock, sand and gravel. 
Spatial Strategies

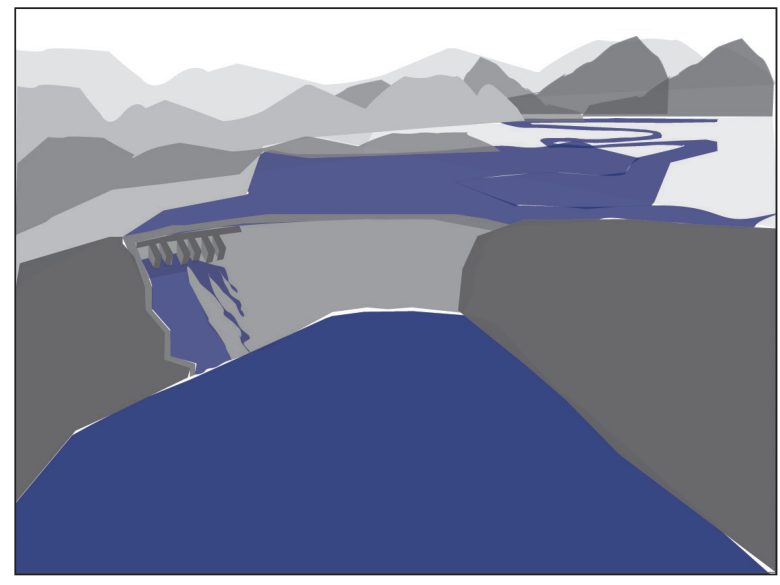

Figure 42: Dams and wet retention basin

Flood management practices utilise dams and wet retention basins as artificial water storage devices to control and lower downstream water volumes and reduce flood risks up to a design flood standard (water carrying capacity). Wet retention basins are permanent artificial lakes and ponds designed to capture and store stormwater runoff and floodwaters.

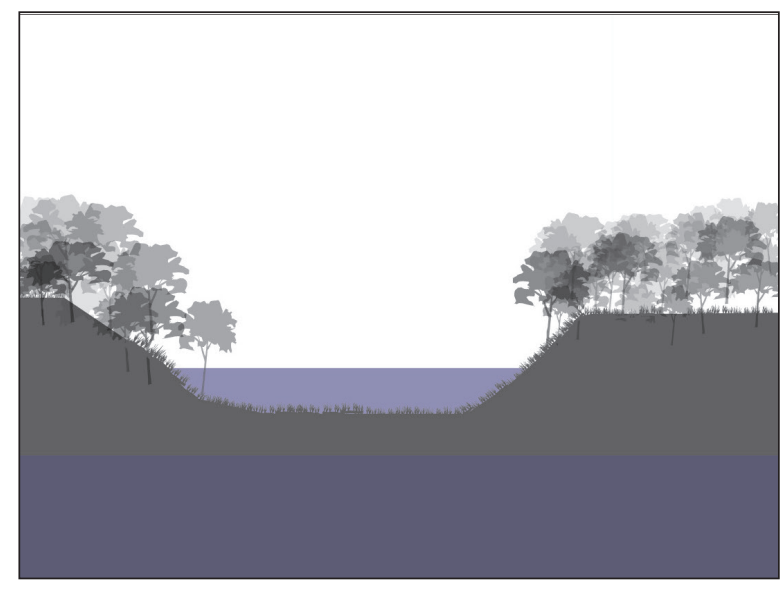

Figure 43: Dry retention basins

Dry retention basins are dry green spaces with gradient depressions to direct, capture, store and absorb seasonal precipitation, stormwater and floodwaters 
Absorption principles encompass both artificial and natural processes. Natural water absorption in soils involves the uptake and retention of water, with efficiency largely determined by the structural makeup and components of the soil. Artificial absorption refers to absorption levels of construction materials in the built environment, where the structural impact of water absorption determines the appropriateness of material use in a given environment.

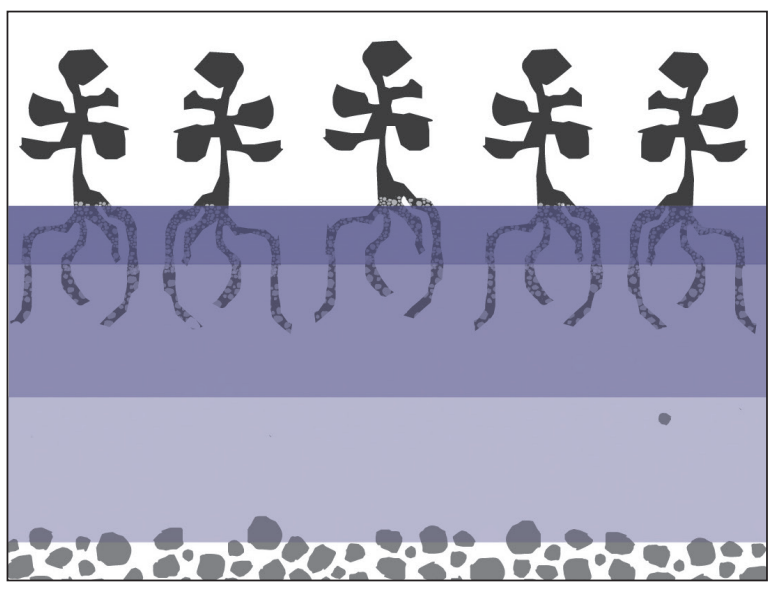

Figure 44: Root structure and soil composition

Ecological absorption processes are reliant upon root structure and soil composition. The shallow or deep root structures of plants influence the depth at which water penetrates. Soil particle density involving the sand, gravel and rock content and its compaction also influence the transmission and storage of water. Soil moisture content investigates the capacity of the soil to absorb water before a maximum saturation point is reached.. 
Spatial Strategies

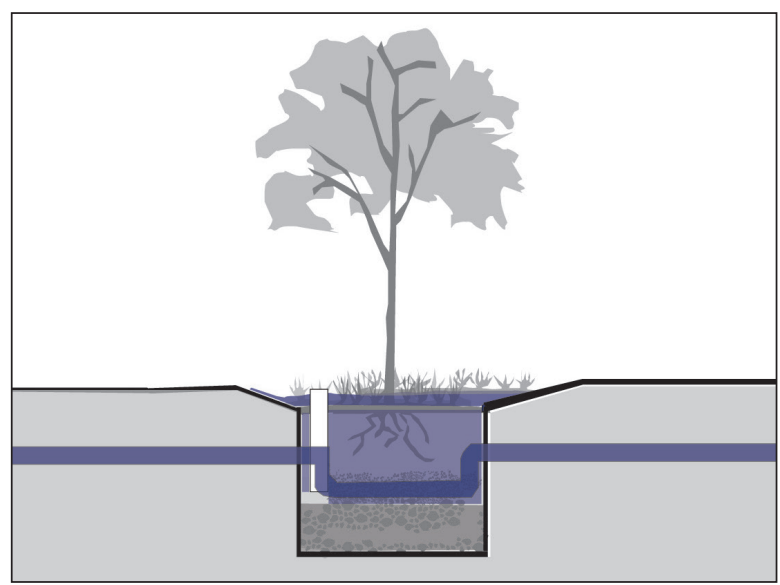

Figure 45: Rain garden

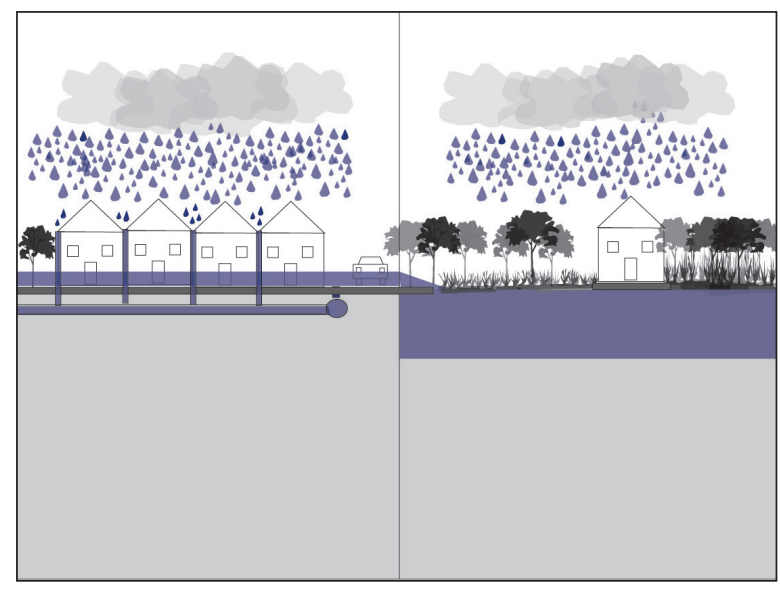

Figure 46: Urban density
In the built environment, rain gardens are artificially constructed gardens, sidewalks and medium strips. Dense vegetation rests within a gravel and sandy soil substrate to facilitate rapid absorption of surface water runoff to reduce storm water runoff volumes. Rain gardens help to absorb, redistribute and slow the flow rate of floodwaters in urban zones.
Increasing urban density results in reduced water penetrable surfaces, lowered absorption rates and increased water runoff leading to a high level of reliance in stormwater drainage. 
Nutrient and waste recovery

Nutrient and waste recovery involves the recapturing of water and waste products to recycle and reduce their adverse impacts on the urban and ecological environment. There are once again both ecological and artificial strategies available to achieve this.

\section{Ecological Strategy}

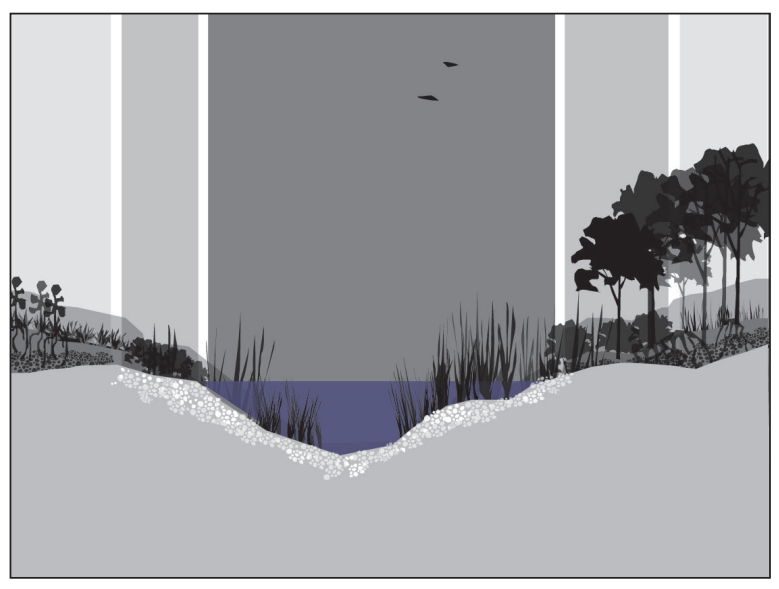

Figure 47: Wetlands

Ecological strategies utilise wetlands, including marshes, swamps and bogs, to capture, store, filter and release clean water back into the ecosystem. Specific plant and wildlife species and saturated soil conditions create a productive nutrient filled boundary between the terrestrial-aquatic environments. Their function is to facilitate waste recovery and nutrient absorption. 
Spatial Strategies

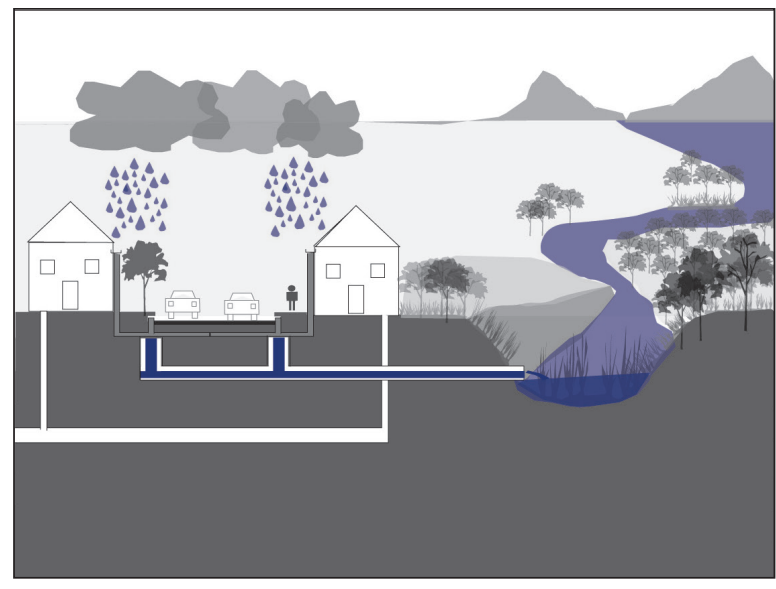

Figure 48: Stormwater management

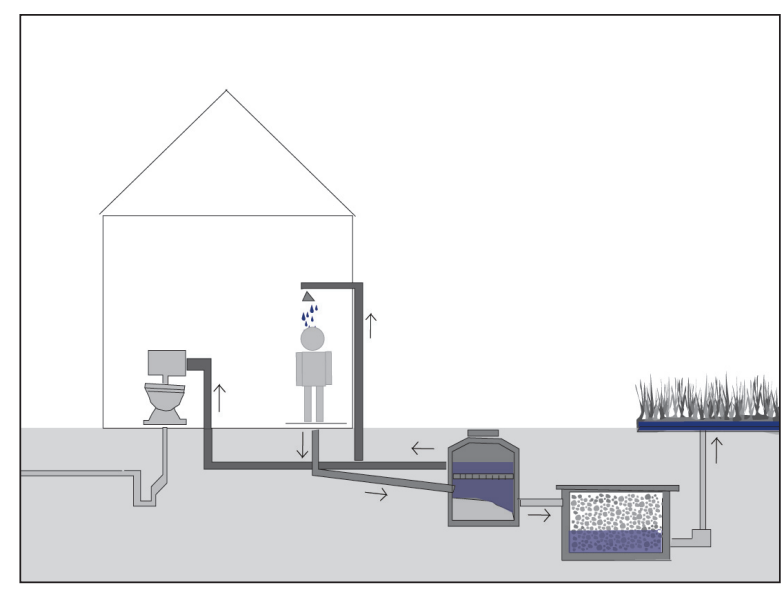

Figure 49: Grey water systems
Stormwater management controls the quality and quantity of stormwater. Urban surface runoff originating from domestic, commercial and industrial activities, precipitation and floodwaters, is collected and redirected by above ground (e.g. bioretention swales) and below ground (e.g. sand and gravel chamber filters) stormwater drainage systems. The water is then collected, filtered of wastes and the contaminants removed to improve water quality before it is either reused or released back into the surface water hydrological system.
Grey water systems involve the recapturing of wastewater generated from domestic activities in the laundry and kitchen along with bathing. The water can be stored and reused for on site irrigation or can be filtered, purified and re-used as nonpotable household water. Soft biological processes and hard mechanical processes are involved. These include sand filtration, ultra violet radiation, membrane filtration systems, or constructed wetlands and treatment ponds. 


\subsection{Feedback}

Feedback involves a multi-scale loop process of analysing an input in order to deliver an output. With regards to flooding feedback of a system and its components takes place prior, during and post flood disturbances in an effort to positively influence resilience to the flooding in the short and long term. Feedback involves response, regeneration, and self-regulation processes. There are ecological services and flood management practices that ensure feedback takes place pre, during and post flooding to enhance resilience and reduce short and long term flood damage. In cities, ensuring effective feedback mechanisms involves designing the spatial urban environment to allow communities to quickly and effectively respond to floods. Feedback processes foster flood awareness and fast response rates, whilst promoting ongoing analysis and post flood improvements to resilience strategies.

\section{Response}

Response relates to ecological and flood management systems that immediately react and initiate processes in reaction to floods. Response strategies have proven more successful when implemented prior to floods, thus an element of predicting and pre-planning for flooding is required. 
Ecological Strategy

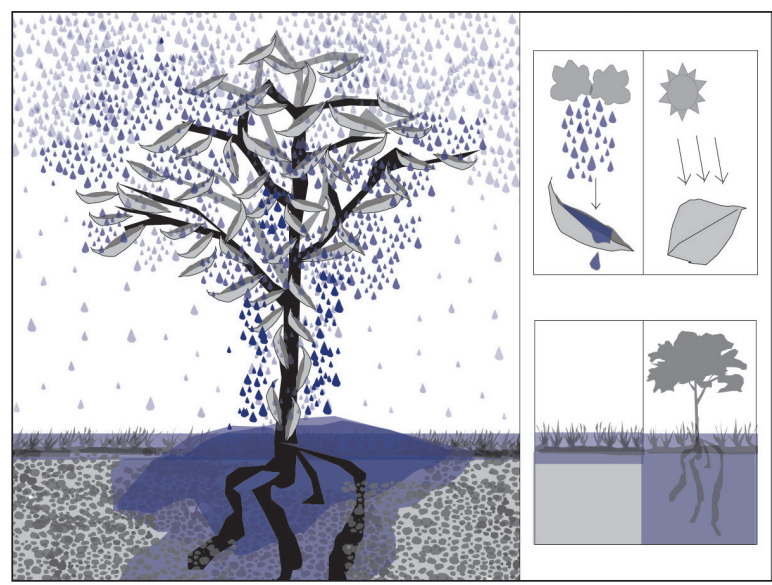

Figure 50: Predictive and reactive homeostasi

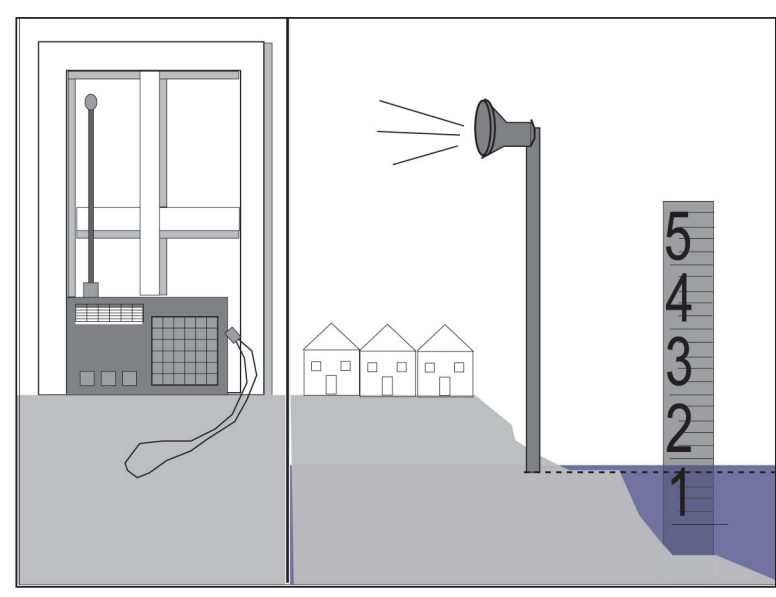

Figure 51: Flood warning systems

Ecology utilises homeostasis to allow species of an ecosystem to react to flooding. Predictive homeostasis involves the development of functions in anticipation of known seasonal variables, such as flooding. It allows species to respond immediately to flooding, such as the root systems of plants enhancing water penetration. However as predictions may be incorrect there is a need for reactive homeostasis. Reactive homeostasis involves species functionally mediating their response to physiological variables, such as flood events which can threaten the stability of the ecosystem. As a simple example leaves react to environmental stimuli altering their shape to capture sunlight or to direct rainwater towards its roots.

Planned flood management utilises a variety of passive and active flood warning systems to visually or acoustically inform residents of flood risks. Flood warning systems are both predictive and reactive in function. Warning systems such as flood gauges and flood sensor alarms are implemented prior to flooding at the city, urban and residential scale to facilitate community response. Whilst reactive flood warning systems are initiated in response to a flood as it takes place, including door knocking, and emergency flood broadcasts on the radio, the internet and television. 


\section{Regeneration}

Environmental regeneration relates to the restoration, renewal and new growth of lost species in an ecosystem. In ecosystems, regeneration is a naturally reproducing process which allows the ecosystem to re-establish stability after a disturbance, such as flooding. In urban flood management it refers to the reconstruction of damaged infrastructure and the development of new improved built components better suited to flood prone conditions.
Ecological Strategy

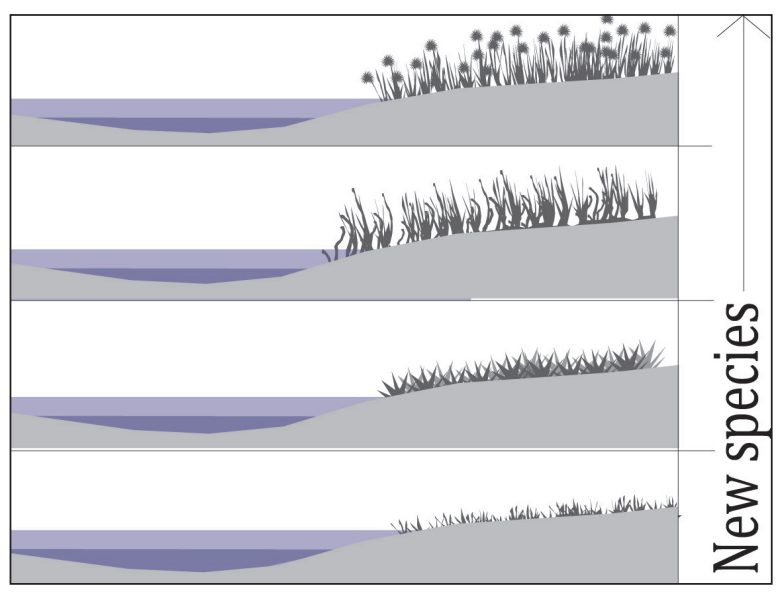

Figure 52: Speciation

Ecological succession and speciation are the environmental processes responsible for allowing an ecosystem to transform and become progressively suited to the environmental flood prone conditions. Speciation is a lengthy evolutionary process in which new biological species arise through adaptive regeneration. 


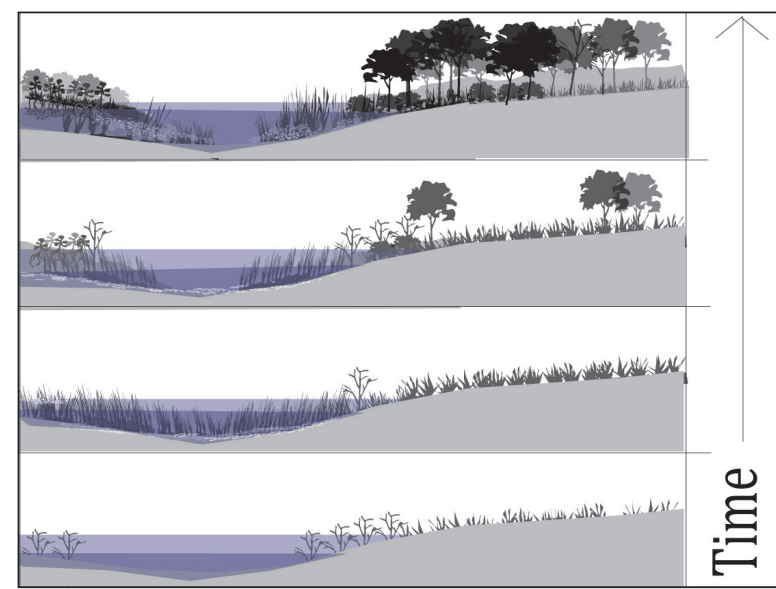

Figure 53: Ecological succession

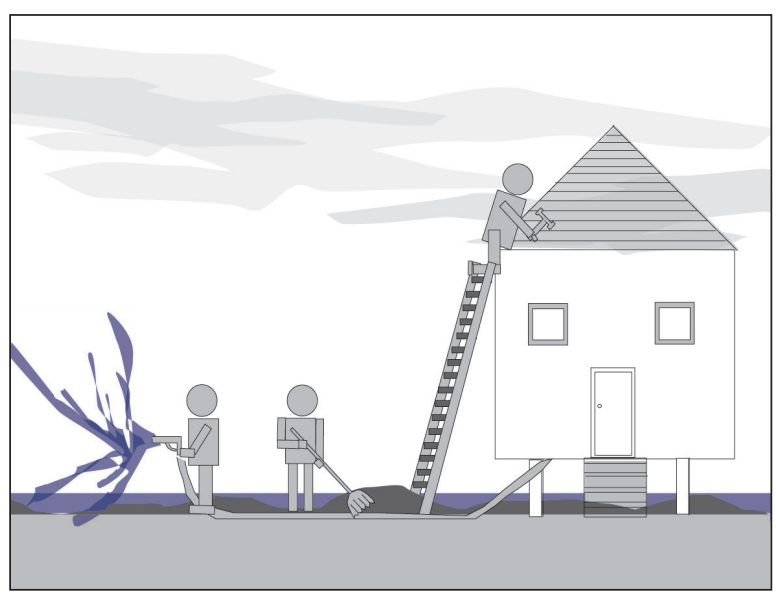

Figure 54: Restoration and renewal

Ecological succession involves the ecological community altering and reproducing species post flood disturbance to establish an ecosystem better suited to the local climatic conditions. Ecological succession has predictable regeneration stages following a disturbance, with fast growing species initially dispersing and dominating. Slower more adapted species follow and continue to compete and develop reliance upon other species to create a complex and diverse interacting system.

In urban flood management restoration and renewal after flooding, there are opportunities for the individual to alter and improve their residences to reduce future flood damage. The process strengthens the community who collectively clean up and restore local built infrastructure to ensure the area and its inhabitants are better prepared for future flooding. 
Cooperative self-regulation

Cooperative self-regulation can enable both ecosystems and built city systems to respond to environmental disturbances. The process relies upon action by individuals at each hierarchical level and the self-processing of information by local communities. The self-regulation process must be cooperative to ensure the effect is distributed and reduced across the hierarchical multi-scale system.

Ecology achieves self-regulation through its interacting and interdependent hierarchical organisation of species (individual) and ecosystems (community). In ecology a hierarchical organisation allows ecosystems to be divided into a collection of subsystems: physical, chemical, and biological processes, which interact with the individual and collective species of ecosystems to maintain system functioning and reduce the impact of environmental disturbances.
Ecological Strategy

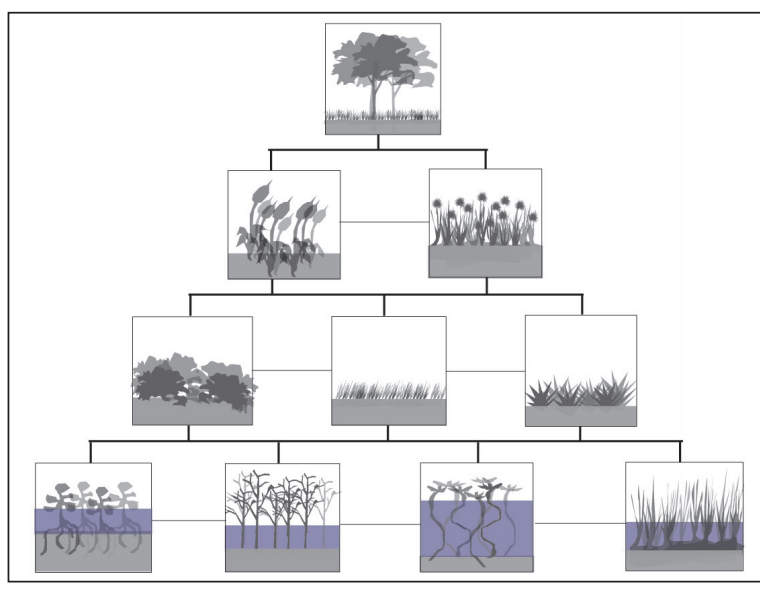

Figure 55: Hierarchical organisation 
Spatial Strategies

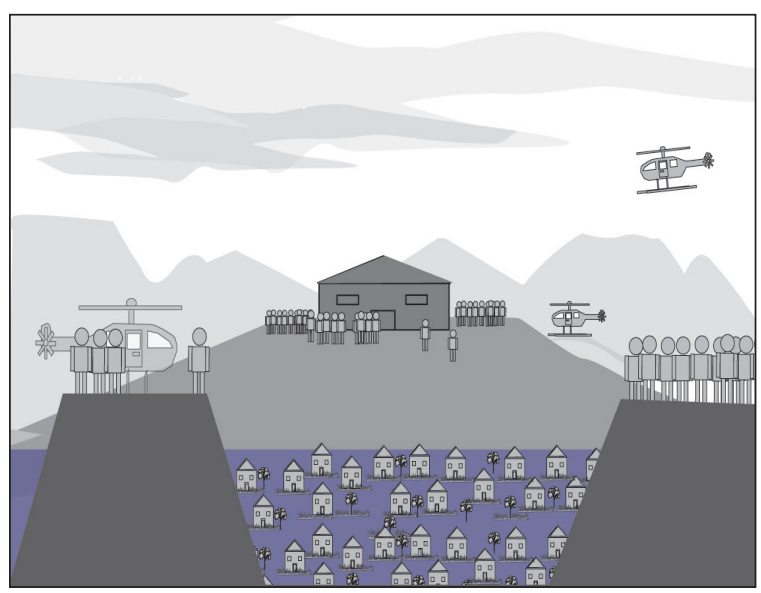

Figure 56: Emergency management
In community emergency management these sub-systems are mitigation, response, preparedness and recovery. These interact with the local flood affected inhabitants and collective governing bodies (federal, state and local government) to reduce the local physical, financial and emotional flood impact. Self-regulation allows a method to monitor and alter the city in an effort to reduce flood impact and thus facilitates a cooperative hierarchical response to flood events. In order to successfully achieve emergency management and prevent disastrous flood repercussions it is necessary to have a cooperative self-regulating system. 


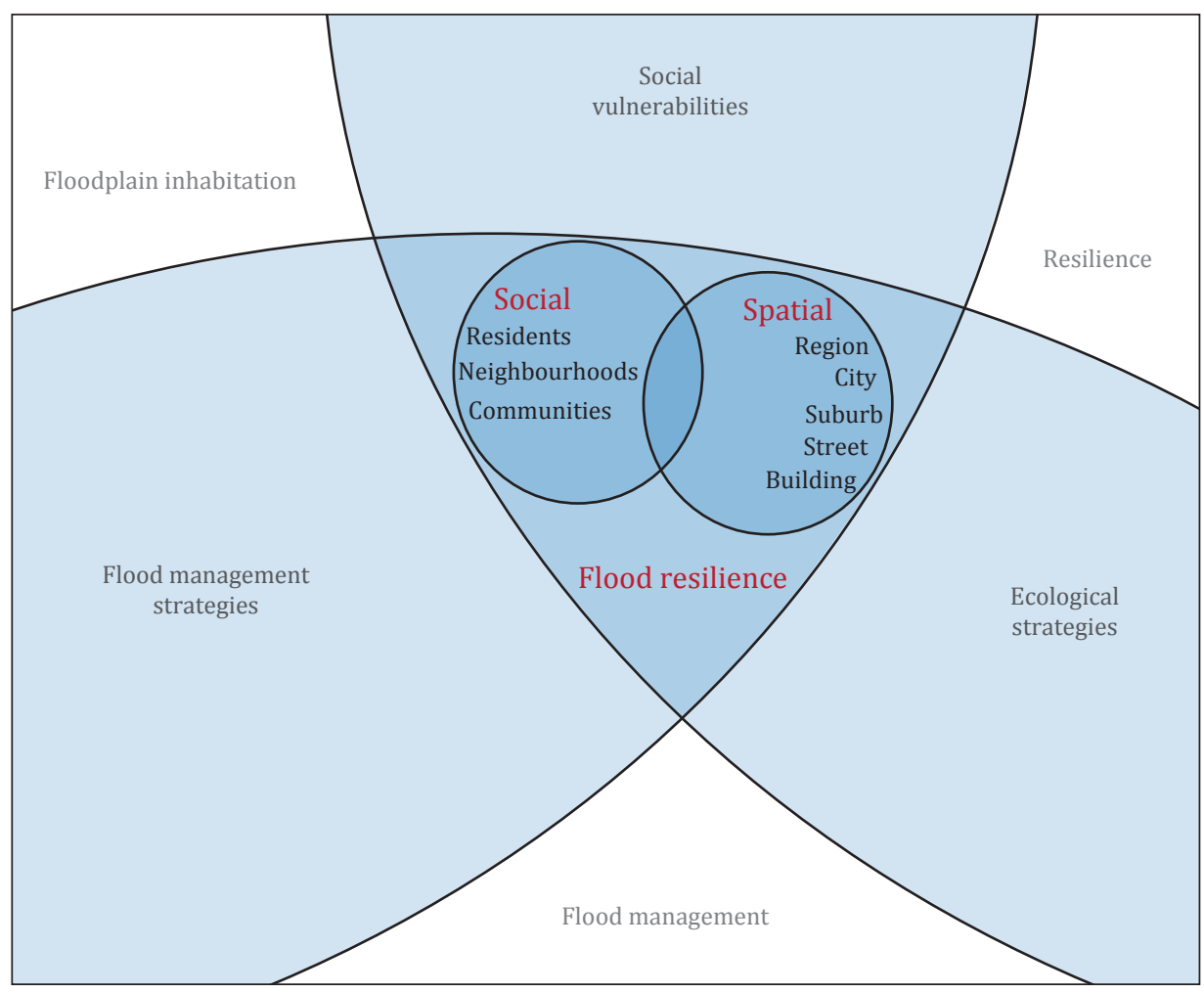

Figure 57: Socio-spatial flood resilience themes of this thesis 
4.6 Summary of flood resilience strategies for floodplain cities

The flood resilience strategies discussed in the previous section demonstrate how certain ecological principles and urban flood management practices improve the resilience of floodplain cities. The adaptive capacity of the city is dependent on the natural environment and the built infrastructure that enable it to continue to function when inundated by floods. Educated flood awareness, pre-planning and adaptive infrastructure reduce flood impact and enable a city to maintain basic structure and function during and after flood events.

Flood preparedness involves implementing a variety of time-based and cross-scale initiatives in anticipation of floods. These pre-flood initiatives could include warning systems, evacuation procedures and emergency supplies (as detailed previously). Implementing more resilience principles would provide opportunity for innovative future developments to become part of the solution to flood events, reducing the personal, local and more general societal impacts of flooding.

The ability of a society to return to its previous state of functioning post disaster is limited by the physical, social and psychological effects of the disaster. If the review of the disaster were utilized to implement a variety of new prevention strategies guided by resilience principles, there would be an opportunity for ALL those affected by the disaster to contribute to the solutions (societal, community and individual dynamics are an intimate part of a resilience strategy). It would enable inhabitants to understand the risks inherent in floodplain living, and facilitate individual ownership of components of the floodplain management plan (e.g. land use restrictions, urban planning and design). It is within "this context that disaster can be conceptualised as a catalyst for change” (Paton \& Johnston, 2006, p8).

There is an opportunity for resilience principles to foster innovative design, construction and planning policy to increase community preparedness, awareness and adaptive capacity to flooding. A balance between resilient design principles and mitigation practices will enhance opportunities for community investment and renewal (Ryan, 2010, pp. 259-257). Flood resilience principles provide an opportunity to re-categorise ecological strategies and flood management practices into a new multi-scaled resilience flood management approach with improved community and individual understanding, acceptance and implementation. 
The Resilience Flood Management Matrix

\begin{tabular}{|c|c|c|c|c|c|c|}
\hline & Scales: & Region & City & Suburb & Street & Building \& Site \\
\hline & Adaptability: & & & & & \\
\hline Flood impact & Alternative circuits & \begin{tabular}{|l|} 
Engineered flood \\
works
\end{tabular} & $\begin{array}{l}\text { Flood defences } \\
\text { eg town levees }\end{array}$ & \begin{tabular}{|l|l|} 
Adaptive \\
infrastructure
\end{tabular} & \begin{tabular}{|l|}
$\begin{array}{l}\text { Connected } \\
\text { grid }\end{array}$ \\
\end{tabular} & \begin{tabular}{|l|} 
Flood proo@ing \\
permanent/temporary
\end{tabular} \\
\hline \multirow{3}{*}{$\begin{array}{l}\text { building/site } \\
\text { circulation }\end{array}$} & Rapid Response & Flow paths & $\begin{array}{l}\text { Access and } \\
\text { escape }\end{array}$ & \begin{tabular}{|l|} 
Green \\
infrastructure
\end{tabular} & \begin{tabular}{|l|}
$\begin{array}{l}\text { Circulation } \\
\text { pathways }\end{array}$ \\
\end{tabular} & \begin{tabular}{|l|} 
Adaptive service \\
connections
\end{tabular} \\
\hline & Core Protection & Land zoning & $\begin{array}{l}\begin{array}{l}\text { Decentralisation } \\
\text { (town planning) }\end{array} \\
\end{array}$ & Network & \begin{tabular}{|l|} 
Self reliant \\
subsystems
\end{tabular} & $\begin{array}{l}\text { Material \& construction } \\
\text { strength }\end{array}$ \\
\hline & Variety \& Redundancy: & & & & & \\
\hline Flooding issues & Buffering & Land use planning & \begin{tabular}{|l|}
$\begin{array}{l}\text { Wildlife } \\
\text { corridors }\end{array}$ \\
\end{tabular} & Riparian buffer & Rain gardens & Building orientation \\
\hline \multirow{2}{*}{$\begin{array}{l}\text { debris } \\
\text { water velocity }\end{array}$} & Diffusion & $\begin{array}{l}\text { Meandering river \& } \\
\text { Diversion channels }\end{array}$ & \begin{tabular}{|l} 
Town planning \\
(meandering)
\end{tabular} & Landscaping & \begin{tabular}{|l|}
$\begin{array}{l}\text { Water } \\
\text { channels }\end{array}$ \\
\end{tabular} & Building form \\
\hline & Storage & Dams \& wetlands & $\begin{array}{l}\text { Reservoirs/dry } \\
\text { retention basins }\end{array}$ & Aquifers & $\begin{array}{l}\text { Storm water } \\
\text { collection \& } \\
\text { swales }\end{array}$ & $\begin{array}{l}\text { Rainwater storage } \\
\text { tanks }\end{array}$ \\
\hline water volume & Absorption & Watershed planning & Urban density & \begin{tabular}{|l|}
$\begin{array}{l}\text { Retention } \\
\text { ponds }\end{array}$ \\
\end{tabular} & \begin{tabular}{|l|} 
Surface \\
permeability
\end{tabular} & \begin{tabular}{|l|}
$\begin{array}{l}\text { Green roof \& building } \\
\text { footprint }\end{array}$ \\
\end{tabular} \\
\hline \multirow[t]{2}{*}{ water quality } & \begin{tabular}{|l|} 
Nutrient \& Waste \\
Recovery
\end{tabular} & Wetlands & Storm water & Waste systems & $\begin{array}{l}\text { Rainwater } \\
\text { harvesting }\end{array}$ & Grey water use \\
\hline & Feedback: & & & & & \\
\hline Weather event & Response & \begin{tabular}{|l}
$\begin{array}{l}\text { Official weather } \\
\text { information }\end{array}$ \\
\end{tabular} & \begin{tabular}{|l|} 
Local radio \& \\
water gauges
\end{tabular} & \begin{tabular}{|l}
$\begin{array}{l}\text { Neighbourhood } \\
\text { watch }\end{array}$ \\
\end{tabular} & \begin{tabular}{|l}
$\begin{array}{l}\text { Flood height } \\
\text { indicators }\end{array}$ \\
\end{tabular} & Flood Sensor alarms \\
\hline \multirow[t]{2}{*}{ precipitation } & Regeneration & Industry functioning & $\begin{array}{l}\text { Town } \\
\text { infrastructure }\end{array}$ & \begin{tabular}{|l|}
$\begin{array}{l}\text { Ecological } \\
\text { recovery }\end{array}$ \\
\end{tabular} & \begin{tabular}{|l} 
Debris \& \\
cleaning
\end{tabular} & \begin{tabular}{|l} 
Household repair \\
debris \& cleaning \\
\end{tabular} \\
\hline & $\begin{array}{l}\text { Cooperative } \\
\text { Self-Regulation }\end{array}$ & $\begin{array}{l}\text { Emergency } \\
\text { volunteers (eg SES) }\end{array}$ & \begin{tabular}{|l} 
Local disaster \\
coordinator \& \\
plans
\end{tabular} & $\begin{array}{l}\text { Emergency } \\
\text { shelters }\end{array}$ & \begin{tabular}{|l} 
Elevated Safe \\
zones
\end{tabular} & $\begin{array}{l}\text { Educated flood } \\
\text { awareness }\end{array}$ \\
\hline
\end{tabular}




\subsection{Establishing a resilience flood management matrix}

This new resilience flood management matrix allows ecological and hard engineered solutions to be re-categorised and potentially enhanced by further strategies resulting from the flood resilience principles. A matrix system allows the "linkages, dependences and networks of resilience and vulnerability" to be systematically analysed and categorised (Buckle, 2006, p89). A resilience flood management matrix will promote the restoration of ecological systems and the retrofitting of the urban environment to cope with a variety of flood events. Ecological theories and resilience principles remain aspirational and poorly defined with regards to specific strategies of planning, construction and flood management (Ashley, Garvin, Pasche, Vassilopoulos, \& Zevenbergen, 2007). The resilience flood management matrix will work towards practically defining flood resilience.

\subsubsection{The resilience flood management matrix}

The resilience principles previously derived from ecosystem analysis, including scales, adaptability, variety and redundancy, and feedback provide a guiding rationale for re-categorising existing ecological measures and flood management strategies and suggest where new strategies need to be incorporated. The resilience flood management matrix set out below has been constructed using insight gained from previous resilience frameworks (Fleischhauer, 2008); (Watson \& Adams, 2011); (Foster, The Ozymandias Principles, 1997) and existing flood management practices (SCARM Report 73, 2000); (Rigby, Floodplain Development Manual (NSW) A document in need of review, February 2007); (Keys, Towards better practice: the evolution of flood management in NSW, 1991).

The new resilience flood management matrix provides a means to analyse the existing resilience and vulnerability of floodplain cities. The multi-scale approach allows flood resilience to be analysed at a national, regional, town, suburb and the residential scale. The resilience matrix enables resilience flood management to be accessible and comprehensible not only to Federal, State and Local Governments but also to community groups and individuals. There are opportunities for flood exposure to be reduced once flood resilience principles and their relationship to flood hazards are defined.

The flood characteristics listed vertically are linked to the flood resilience principles used to mitigate flood impact. The flood management strategies listed in the matrix enable the functional and spatial requirements of resilience principles to be readily identified. The strategies are not to be viewed as the only solution, but merely an example of a suitable strategy. Any plan would need to be fashioned to reflect the specific characteristics and risks inherent in a given situation.

A resilience matrix must recognise the significant, but differing roles a top down and bottom up flood management approach fulfils. Bottom up approaches empower individuals to take control and put in place the necessary measures to improve their resilience at a local level, whilst top down measures ensure there are suitable policies, institutions, services, and infrastructure in place for a region and city. This matrix establishes top down approaches at the regional and town scale, whilst bottom up approaches are utilised on the building and site scale. The urban scale of the suburb and street encompasses a combination of top down and bottom up approaches to foster a collaborative involvement between stakeholders. Individual and community measures need to be readily available and accessible to property owners. Bottom up approaches empower individuals to adapt, strengthen and improve measures based on direct post flood performance feedback. Top down approaches will have more far reaching and permanent impacts, but are characteristically costly and lengthy in their implementation. 
It must be recognised that a matrix system fails to indicate the interactions taking place between different sub-systems (Buckle, 2006). This is higlighted in the new resilience flood management matrix which details measures that can be used to improve spatial resilience, but does not address social resilience and vulnerability. Anderson and Wood (1998) acknowledge that social vulnerability is more complex where factors to be considered include gender, demographics and socio-economic issues. These factors therefore need to be analysed alongside the matrix. Social flood vulnerabilities also remain poorly understood and less advanced, when compared to the physical processes involved in flood mitigation (Merz, Hall, Disse, \& Schumann, 2010). However evidence suggests that a range of community focused bottom up strategies can actually improve social capital and social resilience. Social vulnerabilities, such as low socio-economic conditions, provide guiding doctrines on the selection of appropriate spatial flood resilience strategies.

Established ecological, hydrological, engineering and geological methods can be used to quantify resilience flood management measures and thus determine their relative effectiveness in seasonal fluctuations of drought and flood. This thesis recognises the limitations in measuring the success of the matrix due to an inability to directly apply and test the matrix on a city. Ideally, establishing a resilience flood management matrix would have involved a range of stakeholders including ecologists, hydrologists, architects, representatives of government, business, and community groups, and individuals. However, through precedent analysis, this thesis seeks to propose a category of innovative solutions at a series of socio-spatial scales within a site specific context. Despite the limitations, a resilience flood management matrix provides a new categorised approach to analysing existing floodplain contexts. A matrix allows existing vulnerabilities to be located alongside those which foster and strengthen flood resilience.
Finally, whilst this thesis specifically investigates socio-spatial resilience with regard to its potential for reducing flood hazards, it must be acknowledged that particular population groups experience greater vulnerability. Flood hazards cannot be viewed uniformly across a floodplain. Particular demographic groups, including the young, elderly, poor, and disabled experience increased vulnerability to flood risks (Bolin \& Standford, 1998; Buckle, 2006). This is as a result of reduced personal mobility, education and awareness. Peoples of lower socio-economic status also experience greater susceptibility to flood hazards as a result of inequalities, including often a higher flood risk housing location and reduced capacity for the flood proofing of houses. 


\subsection{Conclusion}

Despite the difficulties in pre-defining recovery processes due to the unpredictable nature of socio-spatial vulnerabilities and flood characteristics, it is possible to define the underlying factors responsible for the successful everyday functioning of the city, and thus what constitutes the system's resilience (Paton \& Johnston, 2006, p. 7). In the context of floodplain cities, these include individual and community values and the provision of vital infrastructure and services to the city. The continued functioning of these crucial elements during and post flooding is vital in the city's capacity to adapt and continue to function when inundated. Preplanning for flooding in the built, natural and social environments enhances the resilience capabilities of a city to maintain basic structure and function during flood disturbances.

The development of a resilience floodplain matrix strengthens social and community engagement and commitment to the management of floods. Resilience philosophies enable buildings, communities and city services to anticipate and react to flooding (Watson \& Adams, 2011, p. 257). The identification of interacting spatial scales allows for a successful implementation of interventions that have knock-on effects at other scales. Zvenbergen et al (2008) indicate that there is a lack of studies attempting to "formulate systematic principles of and proxies for flood resilience and apply them to flood-risk management systems" (Zvenbergen, Veerbeek, Gersonius, $\&$ van Herk, 2008, p. 83).

A matrix system provides an analytical tool to site specifically understand the flexibility and the robustness of existing floodplain cities at multiple scales. Comparative analysis of floodplain cities will provide insight into universal vulnerabilities experienced by floodplain cities. The insight gained will provide a focus for new strategies aimed at improving the socio-spatial resilience of floodplain cities to flooding. 


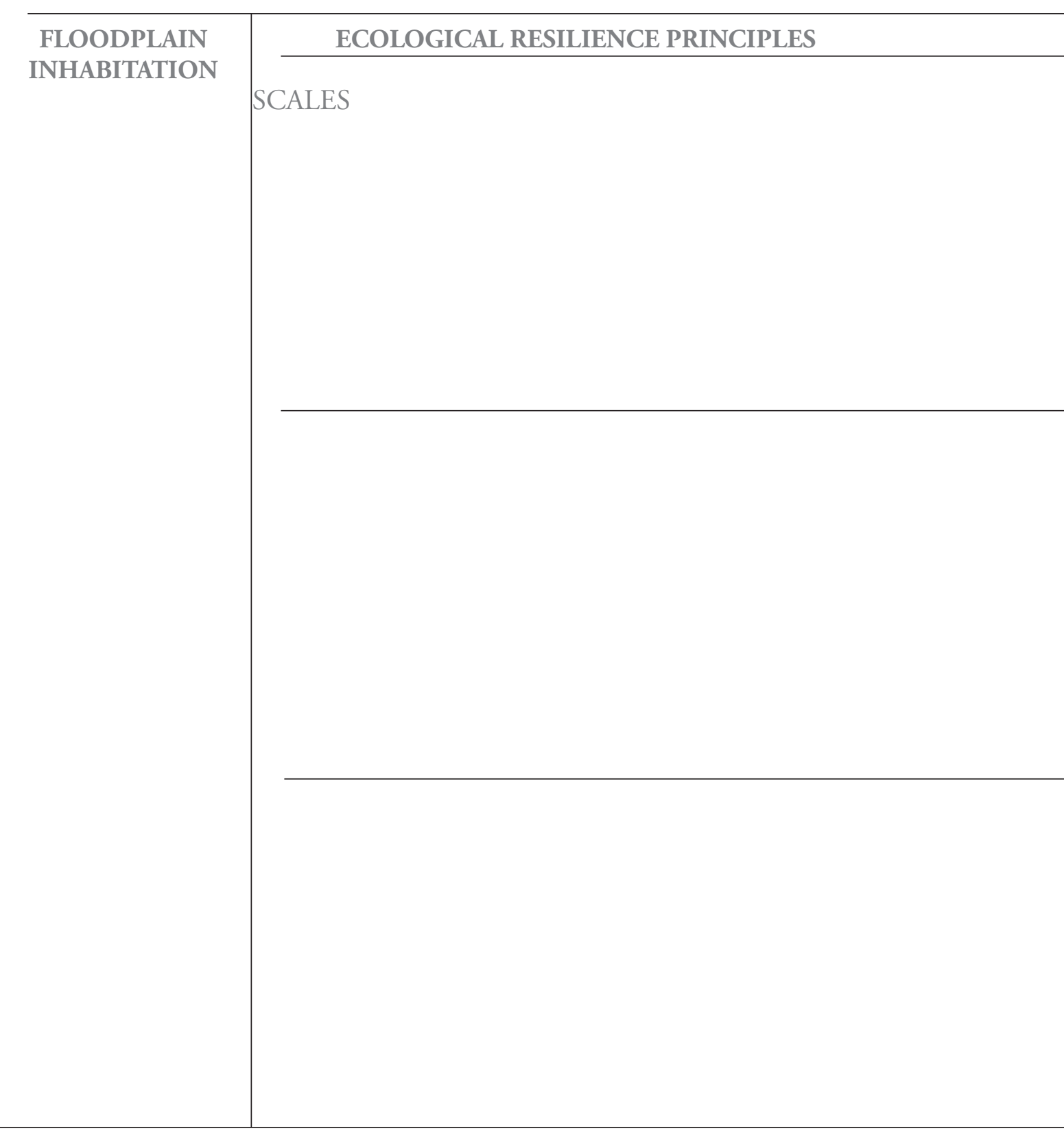


FLOOD RESILIENCE PRINCIPLES
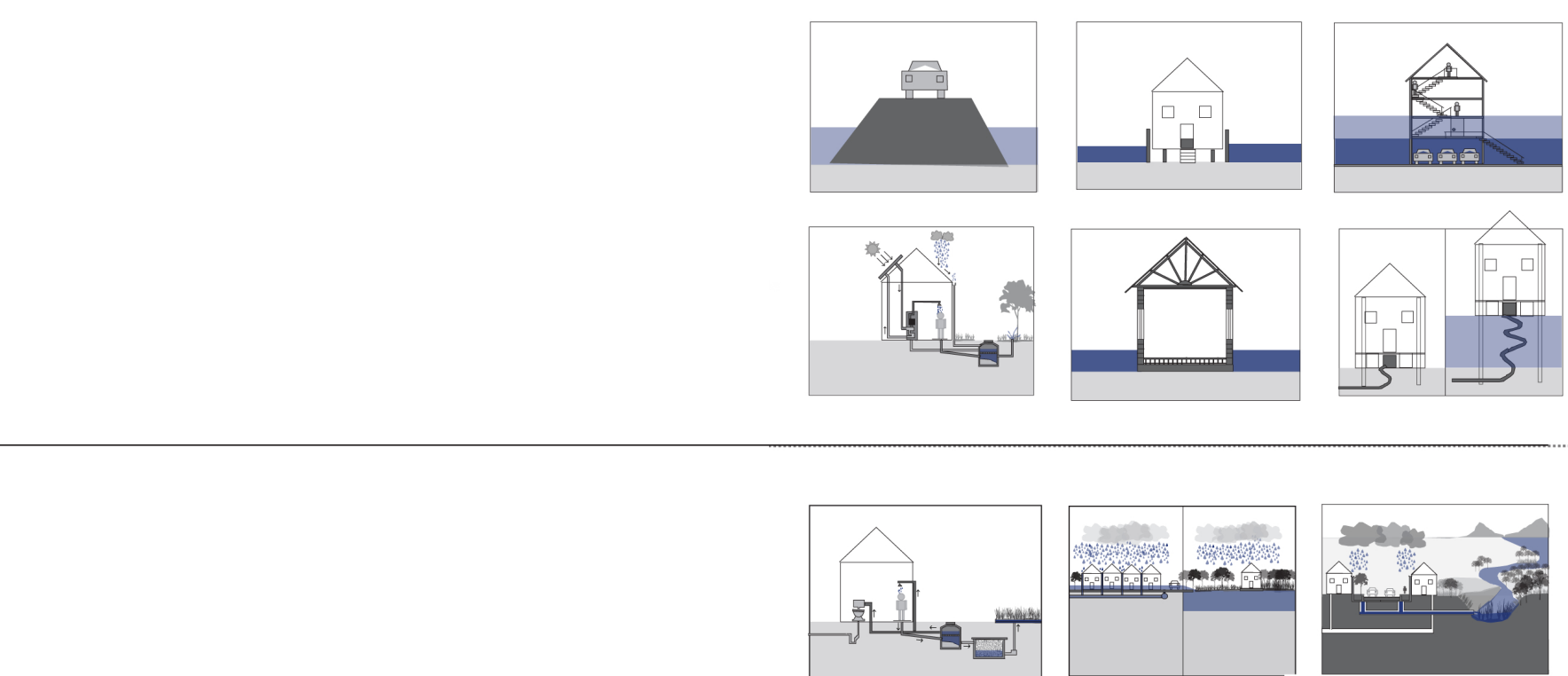

$$
\text { जी_a }
$$
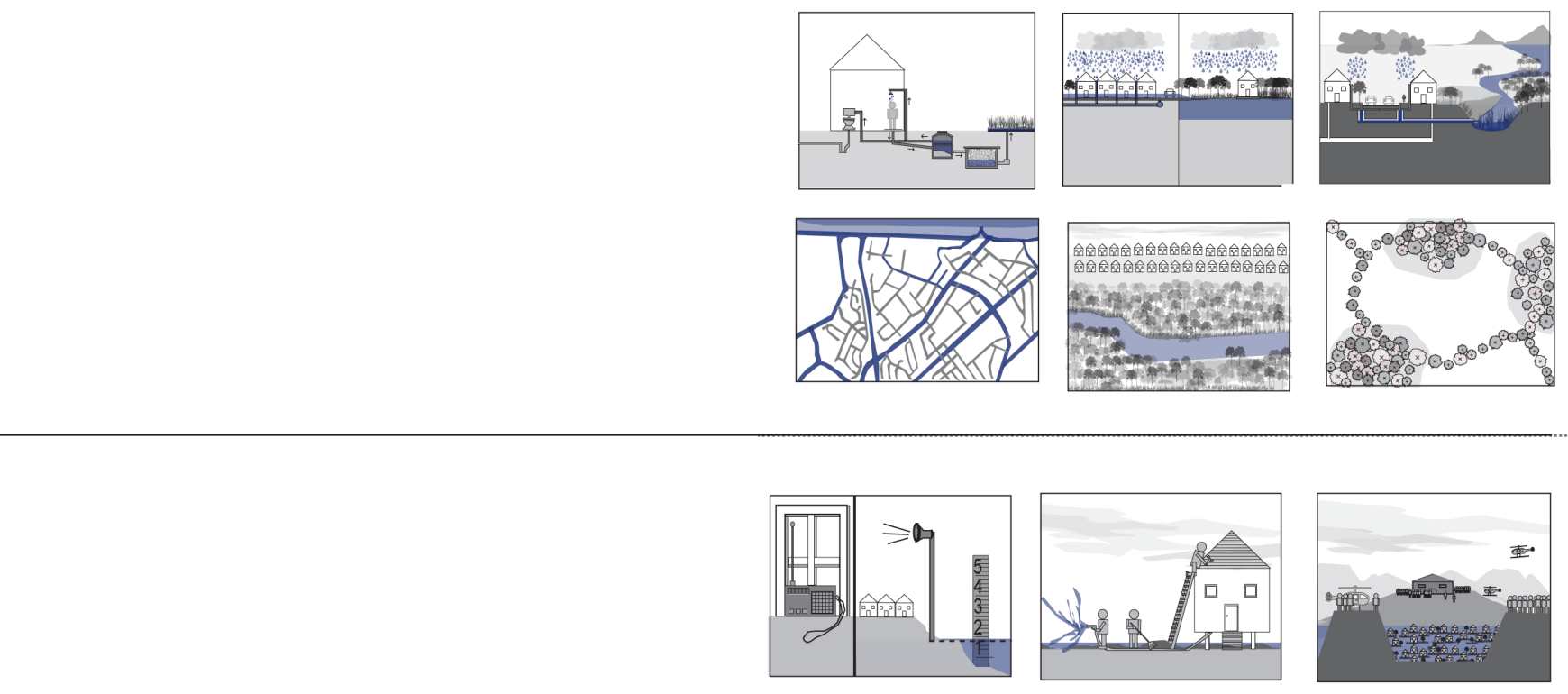
An open minded approach results in ground breaking solutions that are responsive to local and wider-scale needs promoting diversity, education, creativity, tolerance and allowing for the exchange of ideas... introducing new ways of living, working and playing on water. 



\subsection{Resilience strategies}

Resilience strategies, although lacking in Australian floodplain management, have recently been integrated into the Netherlands philosophical approach. Extensive international research has focused on shifting former flood preventative philosophies to concepts of resilience. This has arisen from societal and academic spheres recognising the innovative potentials a resilience approach provides (De Bruijn \& Klijn, 2010); (Godschalk D. , 1999); (Fleischhauer, 2008). New amphibious concepts are being explored in New Orleans following the devastation of cyclone Katrina. Amphibious approaches have transformed existing vernacular floating methods, such as those used in Cambodia, which evolved over time to meet the specific needs of flood prone inhabitants. The distinct physical (infrastructure), social and environmental risks associated with floods in Cambodia, New Orleans and the Netherlands highlight opportunities for new flood resilience strategies to be implemented.

\subsection{A criteria to analyse precedents}

\section{- SOCIO-SPATIAL SCALES:}

\section{(flood characteristics, social vulnerabilities and access to services)}

Socio-spatial scales investigate the flood characteristics, social vulnerabilities and the local ability to access key services during and post inundation from floods. The flood characteristics of velocity, duration, depth and frequency vary greatly across local and regional scales. A vulnerability profile involves analysis of the social characteristics of each location and whether vital services can be accessed during and post flooding. Social and spatial components are shown to limit the ability of local inhabitants to achieve resilience to flooding.

\section{- ADAPTABILITY: \\ (strengthens the flexibility of the city)}

Flood adapted residences and infrastructure components increase the ability of a floodplain city to respond to and reduce the impact of local floods. Throughout history a variety of residential flood proofing and adaptive strategies have developed in response to local culture, material availability and flood experiences. The adaptive capacity of a city's infrastructure and inhabitants relies upon community coordinated responses to living with water. 


\section{- VARIETY AND REDUNDANCY: (strengthens the robustness of the city)}

A variety of flood proofing measures, flood works and ecological solutions increase the robustness of a floodplain city system. A balance between resistance and resilience measures is achieved when the specific social vulnerabilities are understood. Inclusion of multiple solutions ensures that if one structure, service, or infrastructure solution fails there are other strategies in place that will continue to function when a city is inundated by floodwaters. This systematic redundancy will reduce the impact of flooding on the natural and built environment.

\section{- FEEDBACK:}

\section{(preparation, evacuation and recovery processes)}

Feedback involves the planning and review of immediate and long term responses to flooding. Flood warning systems that facilitate response; reconstruction and renewal processes implemented in the wake of floods; the system of cooperative self-regulation in which governments provide funding and support for local communities; all are shown to be vital to an effective response to flooding. A resilient system empowers those directly impacted by floods to take control of flood preparation and recovery processes.

\section{- RESILIENCE FLOOD MANAGEMENT MATRIX: (macro/micro analysis identifies strengths \& weaknesses)}

The resilience flood management matrix allows a means to summarise and categorise each component of the flood management process. Common shortcomings of current flood management approaches become evident when the matrix is applied to different locations. Where current flood management measures fail to enhance flood resilience, there are opportunities to explore new innovative resilience solutions. 
Socio-spatial scales: flood characteristics, social vulnerabilities and access to key services

More than sixty million people inhabit the Lower Mekong river basin of Cambodia (International Federation of Red Cross and Red Crescent Societies (IFRC), 2009, p. 40). Floods from the Mekong River and its tributaries occur annually from monsoonal rains between August and November from tropical storms originating from typhoons in the Chinese Sea. Flooding is characteristically slow in velocity, lengthy in duration and has large fluctuations in depth (from one to ten metres) across the Lower Mekong river basin. The basin's Tonle Sap Lake swells during monsoonal months and then releases water back into the Mekong River in low precipitation months, providing fresh water downstream. The livelihoods of the regions inhabitants are highly dependent on this hydrological process in its provision of fish, rice cultivation, forest products, transport, drinking water and sanitation practices. Flooding subsequently has major adverse social and economic impacts and results in local and regional food shortages. This has national implications with the region being the country's major rice and fish producing area.

\section{Flood characteristics of the Tonle Sap}

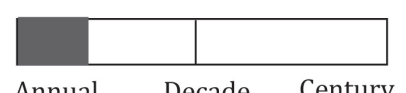

Frequency

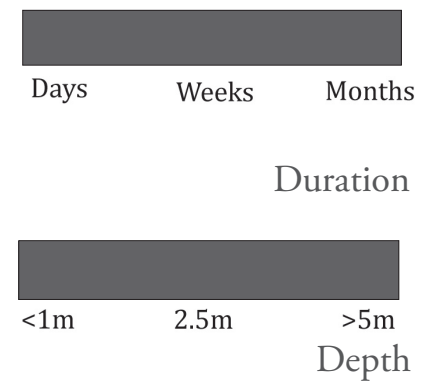

Despite local anticipation of the annual monsoonal rains the provinces of the Lower Mekong River Basin remain severely affected by flooding (International Federation of Red Cross and Red Crescent Societies, 30 September 2011). Climate change and environmental degradation increase the unpredictably of flood frequency and severity (Nuorteva, Keskinen, \& Varis, 2010). Socio-economic inequity, a lack of flood adapted infrastructure and a lack of flood safe zones and emergency centres further intensifies the region's vulnerability. Locals are without food, fresh water, safe shelter or access to markets to sell produce during floods for extended periods. Seasonal migration between provinces and population growth have further "exacerbated the communities' level of vulnerability, rendering traditional coping strategies inadequate" (IFRC, 2000, p.40).

\section{Key services lacking in villages of the Tonle Sap}

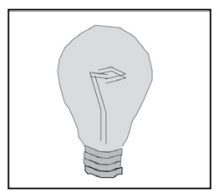

- Villages are without power, and thus a means to communicate externally.

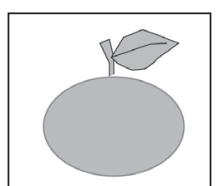

- Locals are unable to access markets to buy and sell fishing and agricultural produce. Food shortages result in villages.

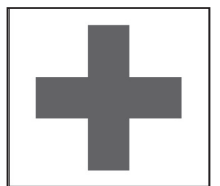

- There are a lack of local medical facilities when people are injured or become sick from water bourne illnesses. 


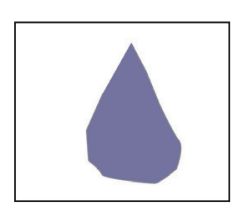

- A lack of fresh water supply causes health concerns.

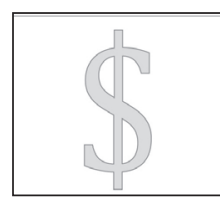

- As a result of being unable to access markets and the short term adverse impact floods have on agriculture, inhabitants suffer economically.

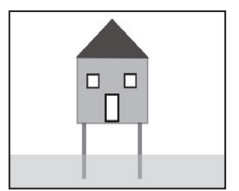

- There is a lack of evacuation centres, or flood safe shelters once statically elevated houses are breached and floating houses become unsafe, due to wave heights.

\section{Social vulnerabilities experienced}
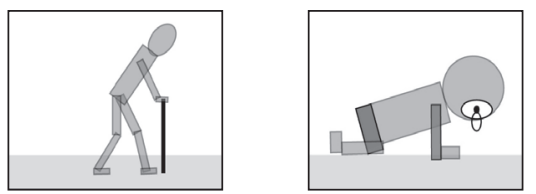

- The elderly and young experience greater safety concerns as a result of reduced mobility and a reliance on support/aid.

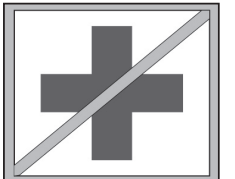

- Health concerns arise from a lack of fresh water, food and sanitation standards.

- The low socio-economic status of inhabitants results in lower flood proofing, construction standards and lengthy recovery periods. Floods adversely impact on agriculture and fishing, which results in long term financial hardship.

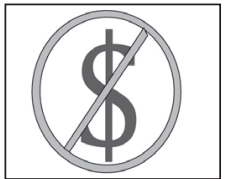

Adaptability, Variety and Redundancy: strengthens the flexibility and robustness of the city

The agriculturally and fishing based lifestyles of the Lower Mekong River Basin are intrinsically connected to natural flood frequencies. The inhabitants live with floods, rather than trying to prevent them. Flood prone villages have developed a range of bottom-up autonomous flood adaptive initiatives such as water adapted circulation using water borne transport and floating timber and reed pontoons. These infrastructure adaptations are integrated into the everyday village function to reduce the adverse impacts of periodically residing on water. The floating and elevated structures cater to the fluctuations in the Mekong River depth (from one to ten metres) coincident with flood pulses of the Tonle Sap Lake and flood events (MRCS/WUP-FIN, 2007).

Of major concern is a lack of flood adaptive measures applied to vital infrastructure and services such as fresh water, sewerage and food sources. A lack of urban adaptive strategies result in mobility and transport issues when main roads are cut and villages become isolated. There are safety risks, health concerns, and economic ramifications when locals are unable to access surrounding villages, access vital health infrastructure or reach markets to sell produce.

Floating and statically elevated houses are constructed from locally sourced, renewable timber and reeds suited to the wet conditions. The low cost local construction traditions empower communities to self-organise and adapt to seasonal variations (Marschke \& Berkes, 2006). Inhabitants of floating villages are also able to relocate from high risk climatic zones. Statically elevated houses on stilts and floating homes are vulnerable to severe floods due to risks of inundation and debris from increased waves and water velocities. Both housing types become accessible only via boats during floods. 
The Food and Agriculture Organization of the United Nations Mekong River Commission Secretariat, March, (1999) report calls for the implementation of flood control works to protect floodplain inhabitants and to reduce flood impact on agricultural production. A lack of flood adapted infrastructure and services in the Mekong River Basin is due to the low top down, institutional and governmental investment in the region. The low socio-economic status of villagers means they are without the means to implement these large-scale developments, upgrades and maintenance. Despite the rich tapestry of traditional bottom up solutions to seasonal flooding, a lack of coordinated implementation hinders a collective effectiveness. "Local autonomous and largely spontaneous adaptation strategies... need to be complemented with more macro-level, long-term policy responses" (Nuorteva, Keskinen, \& Varis, 2010, p. 98). Hien highlights the need for a balance between structural (roads and levees) and non-structural (mangroves, forests and diversification of crops of the delta and its inhabitants) measures to reduce current vulnerabilities (Mekong River Commission, 29-30 July, 2010, p. 26). There are opportunities for new innovative resilience strategies that utilise existing local knowledge and traditional technologies to be locally developed and constructed, encouraging an integrated and coordinated top down and bottom up approach.

\section{Flood prone adaptive strategies being used}

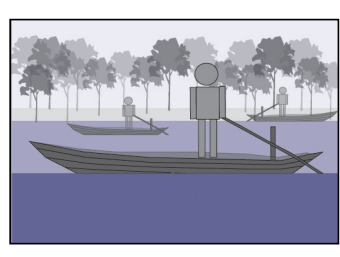

- Water transport

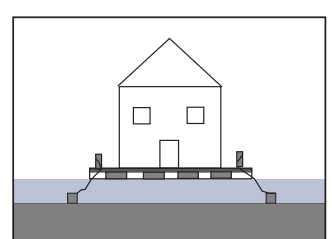

- Floating and stilt house construction are the main housing types

\section{Shortcomings of existing flood management strategies}

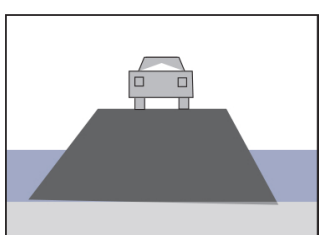

- Flood adapted infrastructure

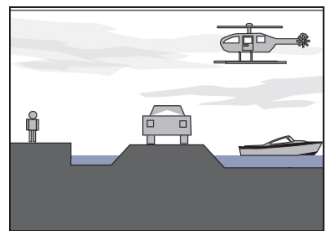

- Isolation from a lack of inland transport

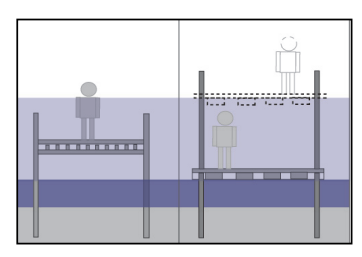

- Static and floating paths
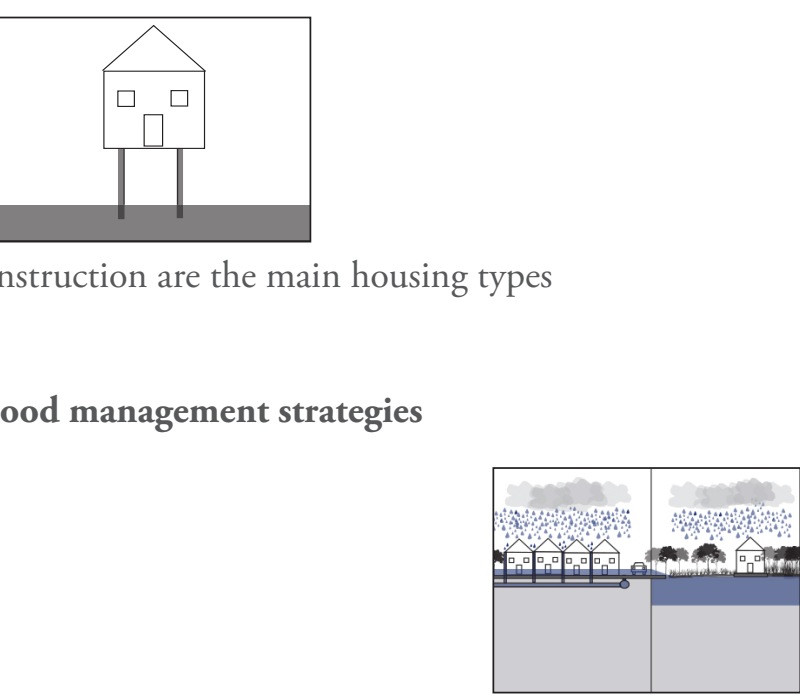

- A stormwater management system

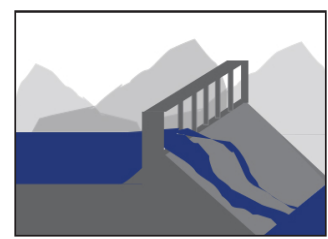

- Regional/urban flood infrastructure 
From post flood analysis it is clear that fishing villages of the Mekong River basin experience the greatest flood impact, as the livelihoods of the villagers is dependent on the hydrological environment (Nuorteva, Keskinen, \& Varis, 2010, p. 95). Poor fish numbers resulting from floods, exposure to water related risks (wave heights, water velocity, debris, and water quality), a lack of access to potable fresh water (sewage contamination and contaminated agricultural run off) and a lack of access to markets (roads unusable) were all significant factors influencing vulnerability. As a result, villagers of the Lower Mekong River basin are highly reliant on external aid funding and support after flood events. Such aid is typically focused on immediate relief of suffering and often fails to enhance the long-term adaptive capacity of the region. A lack of food, sanitation, water and emergency shelter along with a loss of urban circulation are major factors that reduce socio-spatial resilience levels.

A lack of flood warning systems hinders the ability of locals to rapidly respond to flood risks and take appropriate action. Flood warning systems, from flood markers to local radio warnings, are needed to reduce flood exposure and improve local knowledge and adaptive capacity to flood hazards (International Federation of Red Cross and Red Crescent Societies (IFRC), 2009, p. 42). Effective communication of flood forecasts requires the integrated involvement of local communities, governments and authorities to deliver effective and diverse flood warning systems.
Improved flood warning systems, forecasting and flood mapping will have knockon benefits to all nations affected by the Mekong River. Improving collaboration between governments, institutions, local village communities and individual inhabitants will ensure flood measures are socio-culturally suited and locally understood. Whilst collaborative efforts are improving, continued flood devastation highlights a need for further strengthening of coordinated flood management, evacuation and recovery process. Local understanding of the structure and function of measures will ensure effective flood responses, enable continued maintenance of the integrity of measures and promote site specific adaptations based on feedback.

\section{Issues inhibiting preparation, evacuation and recovery:}

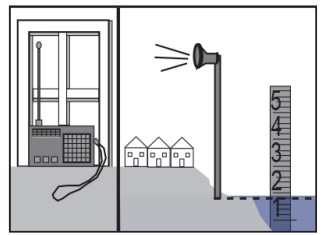

- A lack of local, urban and regional flood warning systems hinders the flood response of local inhabitants.

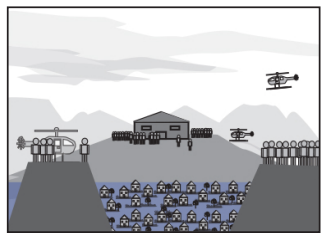

- There is a need to strengthen emergency management through a collaborative planned approach involving governments and local community members.

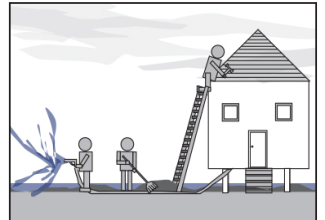

- Local material use and construction aids recovery processes on a residential scale. But there is a lack of investment in renewal of damaged public infrastructure. 
Socio-spatial scales: flood characteristics, social vulnerabilities and access to key services

New Orleans, Louisiana is located on the Mississippi Plain of the Lower Mississippi River, approximately 160 kilometers from the Gulf of Mexico. Prior to the development of The Mississippi River Levee Flood Protection System, flooding periodically took place between April and August, whilst hurricane risk occurred between June and October (Independent Levee Investigation Team, 31 July, 2006). Flooding of the Mississippi impedes its functional roles, which include transport, foreign trade, recreation, tourism and as a key hub for oil and petroleum production. Flooding in New Orleans nowadays occurs from levee failures, the Mississippi River overtopping, Gulf of Mexico storm surges, or inundation from surrounding lakes following high precipitation (Steinberg $\&$ Shields, 2008). Flood characteristics vary greatly depending on the source of flooding.

Hurricane Katrina in 2005 caused a devastating storm surge of the Mississippi River which breached poorly maintained and inappropriately designed levees. The Lower Ninth Ward, bounded by the Mississippi River, the Industrial Canal, the St Bernard Parish line and the Florida Avenue levee, experienced high velocity, high volume and prolonged flooding. Slow velocity waters and long periods of inundation were experienced in dense low lying urban zones as storm water pumping was unable to cope with the high precipitation. Powered pumping services were the only available means to drain the waters.

\section{Flood characteristics of the Lower Ninth Ward:}

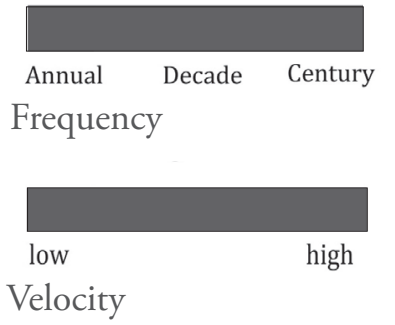

High density commercial development of the 1970's, the implementation of large scale flood works on the Mississippi River and a cyclical period of few hurricane events encouraged social complacency and increased population numbers in the New Orleans region (Steinberg \& Shields, 2008). The flooding caused by Hurricane Katrina in 2005 revealed the significant socio-economic inequality and racial difference in flood exposure and vulnerability within the city (Ryan, 2010). Low socio-economic groups experienced greater flood exposure as a result of the lower land values in high risk zones and poorly maintained residential flood proofing standards. The diverse ethnicity of the region triggered accusations of political injustice, with a long term failure of governments to invest in areas such as the Lower Ninth Ward. Social vulnerabilities were further compounded by poor infrastructure standards and limited access to key services including police, hospitals and fire services before, during and after the flooding in the Lower Ninth Ward.

\section{Social vulnerabilities experienced:}

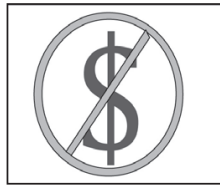

- Socio-economic and racial inequalities increase flood exposure due to a lack of government funding for racial minorities and lower land values in high risk flood zones.

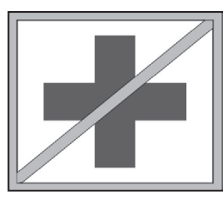

- Health concerns arise from poor living standards, due to a lack of sanitation, fresh water and power sources post flooding.
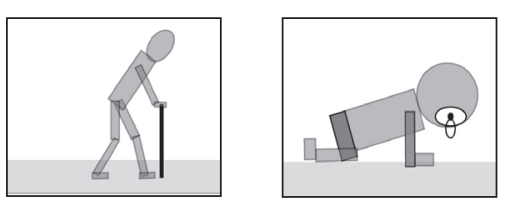

- The elderly and young experience greater flood exposure due to dependence and mobility issues. 


\section{Key services lacking in the Lower Ninth Ward:}
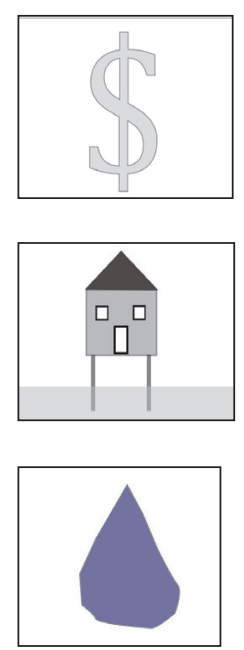

- Flooding damages freshwater infrastructure supply services.
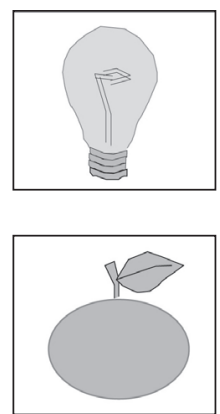

- A lack of public infrastructure post flooding means inhabitant are unable to return post flooding. The city and its inhabitants suffer financially as a result of this.

- The evacuation centres are poorly planned and inadequate standards of sanitation result.

- Flooding results in a lack of power supply. Communication systems, heating/cooling, cooking and lighting are hence lost.

- Food sources in the are become limited as a result of shop closes and access issues for suppliers.
Adaptability, variety and redundancy: strengthens the flexibility and robustness of the city

Existing housing types of the Lower Ninth Ward, including shotgun houses, creole cottages and modern mansions (Upton, 2006) proved inadequate in providing flood protection. Following the devastation the area became a testing ground for flood prone architecture, including statically elevated and amphibious construction. Although new and retrofit amphibious construction techniques using polystyrene blocks, metal frame, timber guide posts and light weight timber were tested, current FEMA standards and insurance policies hindered their implementation.

\section{Flood prone adaptive strategies being used:}
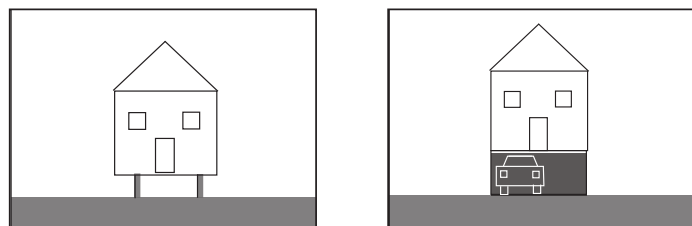

- Existing creole cottages and modern mansions
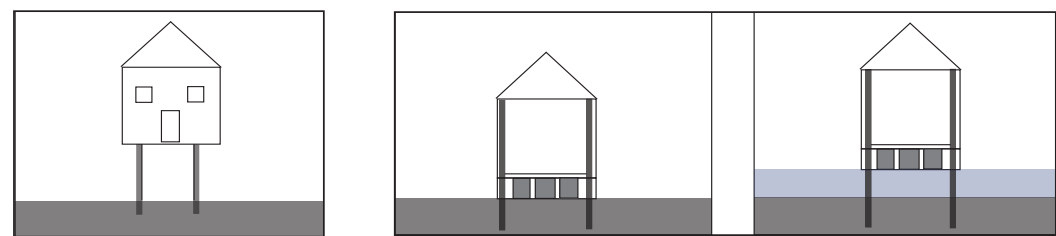

- New houses post Katrina flooding are being raised several metres and amphibious new and retrofit housing solutions are being tested, approved by the US Federal Emergency Management Agency (FEMA) and implemented. 
The Make it Right Foundation, founded by actor Brad Pitt and the Buoyant Foundation Project, founded by Dr Elizabeth English, Associate Professor of the School of Architecture at University of Waterloo, are two organisations involved in post Katrina residential reconstruction effort. Although both organisations are working to improve the residential flood resilience, they fail to address community resilience within the urban surrounds. A lack of flood adapted roads continues to hinder the ability of residents to evacuate from low lying areas, including the Lower Ninth Ward. A failure to provide a variety of public transport options further hinders circulation, as many residents have no vehicular access.

Land reclamation for urban expansion has resulted in large parts of New Orleans resting below sea level. As a consequence, inhabitants have become reliant on flood prevention structures, drainage systems and the active pumping of local groundwater to prevent regular inundation (Upton, 2006). Following Hurricane Katrina the American Army Corps initially began discussing ways to further control the Mississippi River system by strengthening existing flood control structures and implementing further preventative strategies. However, it became clear that a more comprehensive approach as needed, as it became evident that flood control works on the Mississippi River had resulted in a loss of wetland ecology, reduced ecosystem diversity and ecological degradation. The resultant high salinity levels, increased soil erosion rates and increased sedimentation had led to a narrowing of the river channel and an increase in flood risks. Existing urban and residential flood control measures have become inadequate to their planned task to hold back floods. Mumford believes there is a need for a "more delicately poised equilibrium between the landscape and the modes of human occupation" (Mumford, The culture of cities, 1938, p. 335).

\section{Shortcomings of existing flood management strategies:}

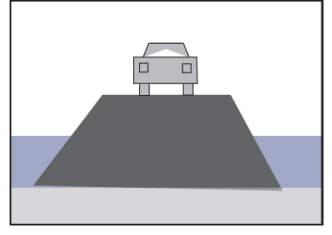

- Flood adapted infrastructure

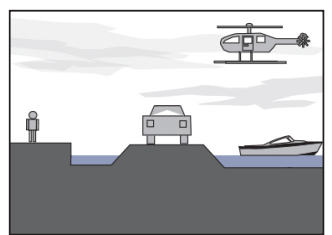

- Insufficient public transport for emergency evacuation.

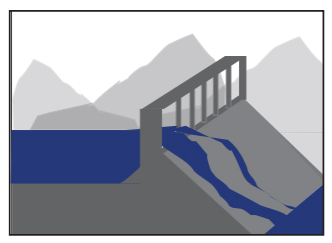

- Poorly maintained and inadequate engineered flood works at the regional, city and urban scales have caused significant ecological degradation and increased risks to inhabitants who become reliant on its 'protection' from inundation. 


\section{Issues inhibiting preparation, evacuation and recovery:}
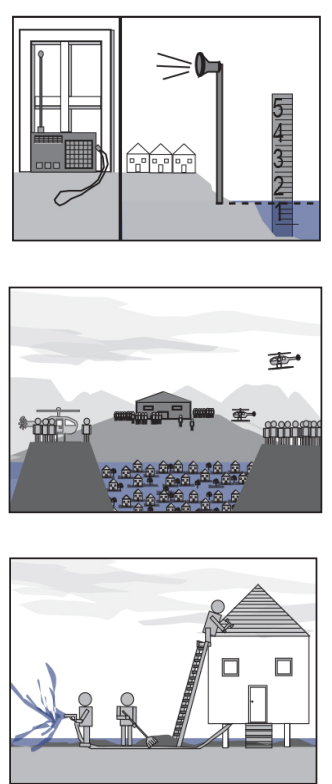

- A lack of local, urban and regional flood warning systems hinders the flood response of local inhabitants.

- Emergency management needs to be strengthened; following the poorly implemented evacuation processes and inadequately equipped emergency shelters.

- Local recovery processes are hindered by a lack of basic public infrastructure and services. Lengthy recovery due to low insurance levels and socio-economic standards.

\section{Feedback : preparation, evacuation and recovery processes}

Post analysis of the 2005 Hurricane Katrina event revealed the shortcomings of America’s Flood Emergency Management Agency (FEMA) (Steinberg \& Shields, 2008, p. 22). Emergency evacuation processes were badly planned, implemented and managed, with poor transport options for people to reach evacuation centres. Access to the Superdome and Convention Centre, which became make-shift evacuation shelters, were poorly planned and inadequately equipped with electricity, food, fresh water and sanitary supplies. Alternate energy sources such as back up generators and renewable energy sources would have greatly improved initial evacuation and recovery efforts. Post analysis also revealed a need to strengthen pedestrian and vehicular mobility through a diversification of everyday flood adapted circulation pathways, to facilitate effective evacuation and recovery processes.

The recovery process post Katrina was hindered by a lack of potable water, electricity and gas services, a dysfunctional sewer system and a lack of shelter options for returning residents. Recovery processes were further hampered by the low socioeconomic resources of the area, with reconstruction being costly and insurance levels low. A lack of investment in public infrastructure and services from all levels of government resulted in further flood exposure and lengthier recovery processes (Steinberg \& Shields, 2008, p. 22). 
Socio-spatial scales: flood characteristics, social vulnerabilities and access to key services

The landmass of the Netherlands is formed from the estuary of the three major European rivers the Rhine, the Meuse and the Schelde Rivers and is bounded by Germany, Belgium and the North Sea. Flooding results from the overtopping of rivers, storm surges of the North Sea, and urban storm water runoff. "Without the protection of dikes, dunes and hydraulic structures approximately $60 \%$ of the country would be flooded regularly" (Jonkman, Kok, \& Vrijling, 2008, p. 1358).

A Dutch national flood experience has enabled a high level of investment in flood-adapted infrastructure. The flood management plans attempt to ensure that key services will be available during flood events. The strength and height of flood structures is calibrated to the economic, industrial, commercial and human consequences of a flood event (Jonkman, Kok, \& Vrijling, 2008). Socio-demographic groups including the poor, elderly, young and sick continue to experience greater flood exposure. Land valuations have historically resulted in low socio-economic groups being located in higher flood risk zones. However niche markets of floating and amphibious villages, such as Maasbommel, located on the river are challenging these historical socio-economic trends.

The disastrous flood event of 1953, caused by a storm surge from the North Sea, resulted in widespread flooding across the south west of the Netherlands. Over 1,800 people drowned from the floods (Jonkman, Kok, \& Vrijling, 2008, p. 1358). In reaction to such a devastating flood, the Netherlands flood management standards were increased and flood control structures diversified and strengthened to provide flood protection from a 1250 year to a one in 4,000 year flood reoccurrence flood rating (De Bruijn \& Klijn, 2010, p. 1). These flood standards greatly differ from the one in one hundred year standard of New Orleans and the annual anticipated flood height standard in Cambodia.

In the Netherlands, large scale government, community and individual investment in flood infrastructure and flood proofing standards has greatly reduced flood exposure. However, the continued threats posed by flooding and the environmental degradation caused by flood works has bred societal resistance (Oosthoek, 2006). A societal shift has taken place in which the safety benefits of making space for varying levels of water in the built environment, recognising the functional benefits natural ecological systems in reducing flood impacts, has been recognised (De Bruijn K., 2005, p.1).

\section{Flood characteristics of Maasbommel:}

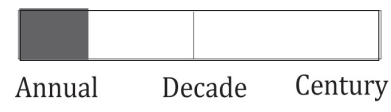

Frequency

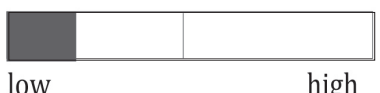

Velocity
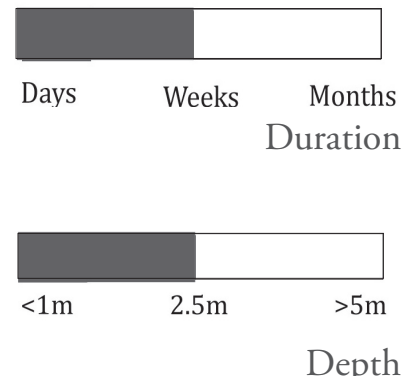

\section{Social vulnerabilities experienced:}

- Low socio-economic demographics experience greater flood exposure as a result of low land values in high risk flood zones.
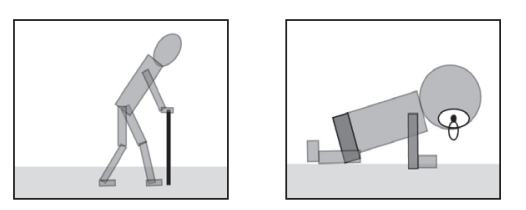

- The elderly and young experience greater flood exposure due to dependence and mobility issues. But amphibious and floating solutions decrease adverse flood impact. 


\section{Key services lacking in Maasbommel:}

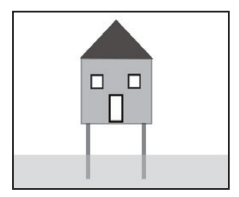

- Due to the scale of large number of possible evacuees, evacuation centres need to be well planned and established prior to flooding.

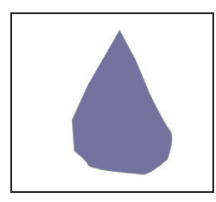

- Flooding damages freshwater infrastructure supply services.

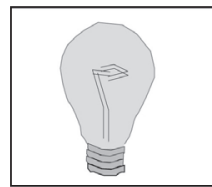

- Flooding often results in a lack of power supply.; communication systems, heating/cooling, cooking and lighting are hence lost.

Adaptability, Variety and Redundancy : strengthens the flexibility and robustness of the city

The Netherlands has a rich history of building dykes and other flood engineering structures in an effort to prevent flooding. Inhabitants of the Netherlands have historically used mounds, plinths and poles to elevate structures above anticipated flood levels. Floating homes in the Netherlands initially began post World War Two with the adaptation of river barges into residential dwellings (Nillesen \& Singelenberg, 2011, p. 12). This housing type has historically been perceived as an alternate hippie lifestyle, rather than as a social norm.
The 1993 and 1995 dramatic Netherlands floods prompted architects, designers, engineers and construction companies to begin investigating new solutions to living in such a flood prone land (Herman Hertzberger, Koen Olthuis of Waterstudio, Michael Louman and Chris Zvenbergen of Dura Vermeer). In 2005 construction of amphibious and floating residences began at the Maasbommel district on the Maas River (Dura Vermeer and Koen Olthuis) as part of the Government's Experiments in Adaptive Housing (EMAB) program. Amphibious approaches are currently a niche high socio-economic market and have thus far focused on "technical solutions to matters like stability and connections to on shore facilities" such as sewerage pipes, water and electricity sources (Nillesen \& Singelenberg, 2011, p. 9). Although new amphibious solutions are increasing individual resilience to floods they fail to increase resilience to high velocity floods, there continues to be "catastrophic potential of large-scale flooding of low-lying areas in the Dutch delta" (Jonkman, Kok, \& Vrijling, 2008, p. 1358).

As a result of historic economic prosperity in the Netherlands and its geography there has always been a high level of investment in flood infrastructure. An extensive flood control system known as the Delta Works consists of over 15,000 kilometers of structures including dikes, flood barriers, floodgates, polders, dams and mechanical pumping and draining systems. The flood structures and mechanical hydraulic systems control groundwater levels and fluctuations in river heights to such an extent that the natural waterways have become artificial channels. However in the face of rising sea levels, increased precipitation rates and urban development these structures are increasingly inadequate to the task. 
Flood prone adaptive strategies being used:
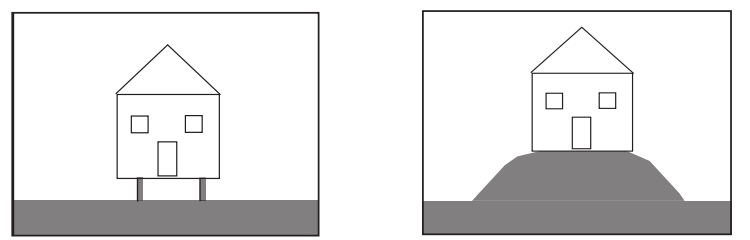

- Nearby farm houses are constructed using traditional flood proofing techniques.
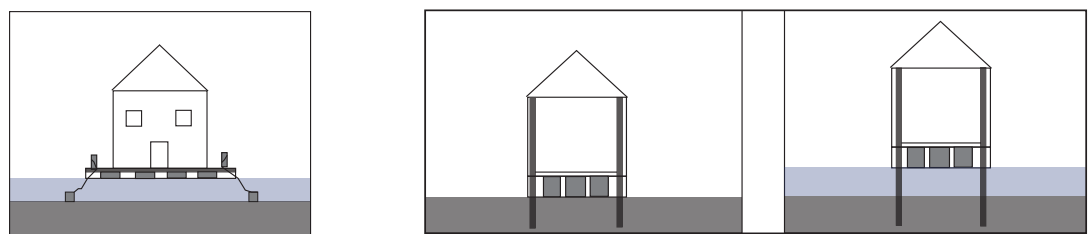

- New floating and amphibious housing
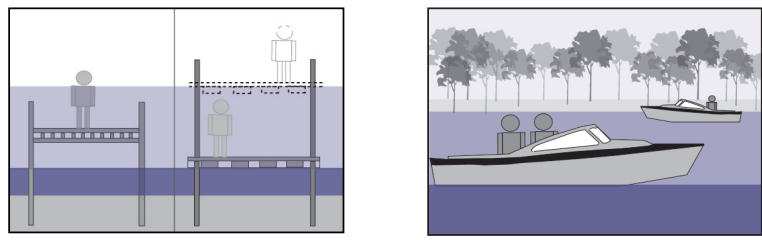

- Static and floating paths and water transport

Recent resilience strategies have begun to investigate the notions of controlled flooding. In response to altered social values the Dutch Government has started to implement a fifty to one hundred year planned retreat from coastal and riverine areas, entitled 'Making room for water' (Watson \& Adams, 2011). Flood management plans have also been transformed to advocate more resilience practices in mitigating and "living with floods" (De Bruijn \& Klijn, 2010, pp. 4-5). The tops of levees have been adapted to benefit their nearby urban structures, with new elevated roads on the top of the structures working to establish greater connection for inhabitants. An increased diversity of circulation paths reduces the risks of all roads becoming impassable during flood events.

\section{Shortcomings of existing flood management strategies:}
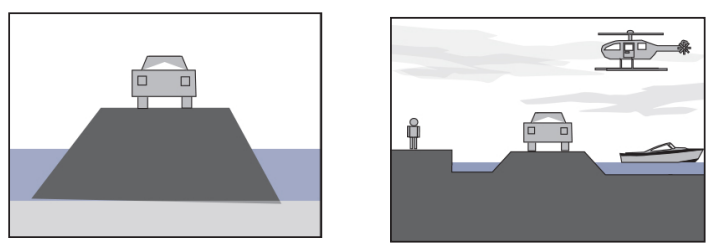

- Inhabitants lacking floating pathways or access to water transport become isolated during floods.

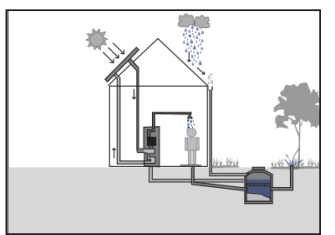

- Alternate energy and water collection and filtration systems are implemented on a residential, but not urban or city scale.
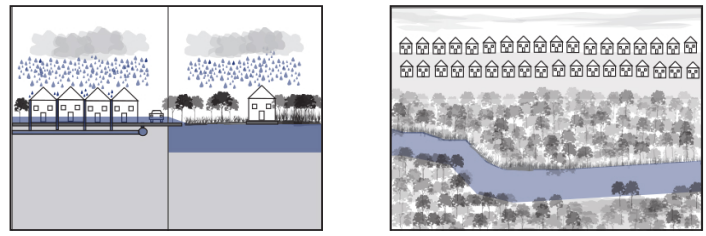

- Residential development reduces the amount of water penetrable surfaces and a loss of green corridors. Urban runoff and flood risks will resolutely be increased.
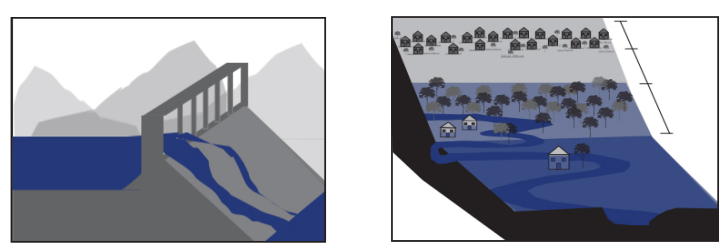

- Flood engineering structures are vital in protecting thousands of inhabitants, due to the low lying land of the Netherlands. 
A history of regulating and coping with floods has ensured large-scale evacuation plans and back up flood adaptive infrastructure are in place prior to flood events. Jonkman, Kok, \& Vrijling (2008) highlight the large and densely populated areas of South Holland continue to expose many thousands of people to extreme flood hazards and evacuation will be difficult due to the low lying land (Jonkman, Kok, \& Vrijling, 2008, p. 1357). Past flood events have been characterised both by widespread devastation and by the effective and successful evacuation of thousands of people. The two extremes demonstrate that resilience to flooding may vary greatly between regions and time periods due to the vastly differing flood characteristics and exposure levels.

Economic prosperity and effective flood policy have resulted in successful top down evacuation processes and recovery investments, compared to the New Orleans and Cambodian examples. In Maasbommel the area becomes isolated from surrounding cities and major infrastructure during floods and boats become the only means of transport during the lengthy inundation period. Recovery processes in the Netherlands vary on the scale of devastation, but a history of flood impact ensures the process is continually refined and strengthened through feedback and review. High flood insurance levels further strengthen recovery processes. Despite the Netherlands flood experience there are still opportunities for feedback resilience strategies to further reduce flood impact and provide innovative solutions to living with floods. The Netherlands recognises the benefits and is moving down the path of resilience planning.

\section{Issues inhibiting preparation, evacuation and recovery:}

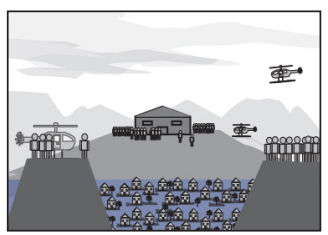

- Emergency management is well practiced, however the scale of evacuation due to the low lying land complicates the process of relocating and sheltering the sometimes thousands of evacuees.

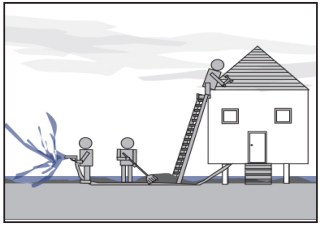

- The scale of flood damage will influence the time length of recovery processes and the ability of the government to provide financial assistance to those affected.

\subsection{Resilience Flood Management Matrix: Macro/Micro analysis}

\section{(Identifies strengths \& weaknesses)}

Applying the resilience flood management matrix to each precedent location allows the strength and weaknesses of existing flood management practices to be analysed at the multiple interacting scales. The matrix encourages a multi-disciplinary approach in which top down and bottom up initiatives are integrated. 


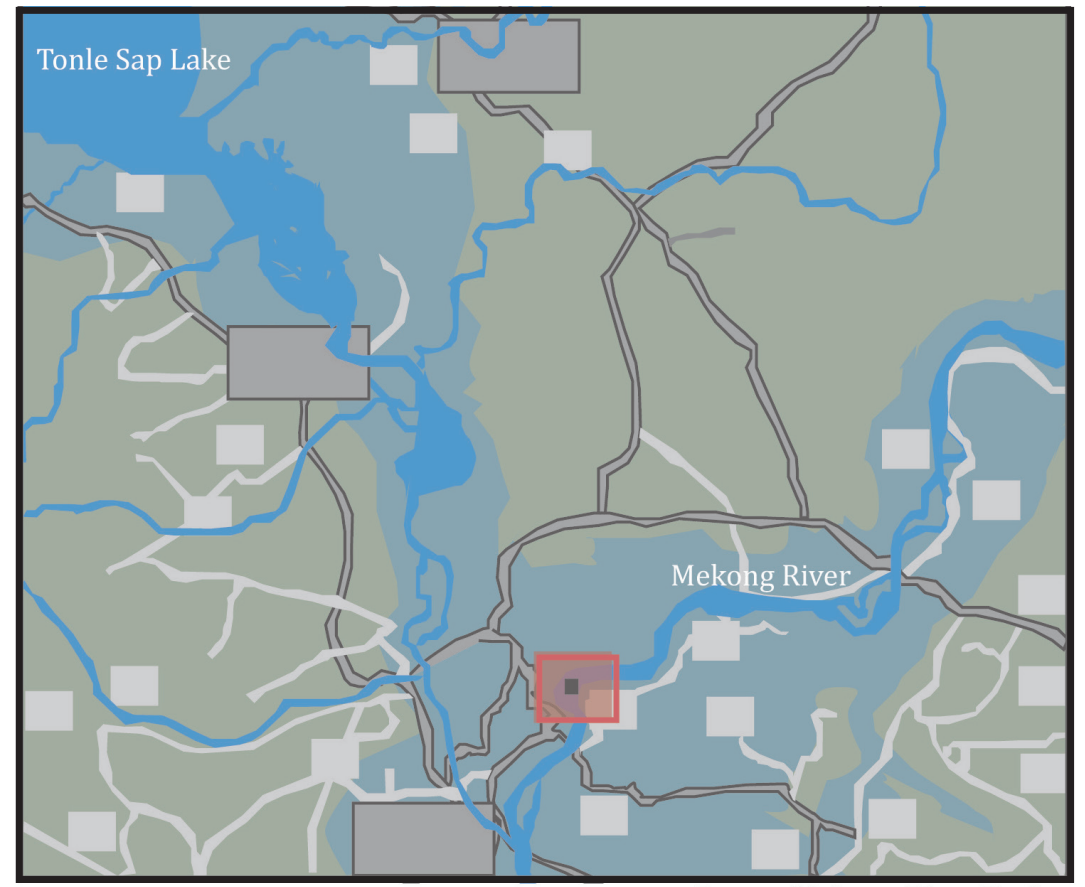

Lower Mekong River Basin

Green/Agricultural land

Large Villages and major roads

Small villages and secondary roads

$1: 1000,000$

Seasonal flood zone

Floating/elevated buildings of study area

$\square$ Study area

Figure 60: Lower Mekong River Basin, Cambodia

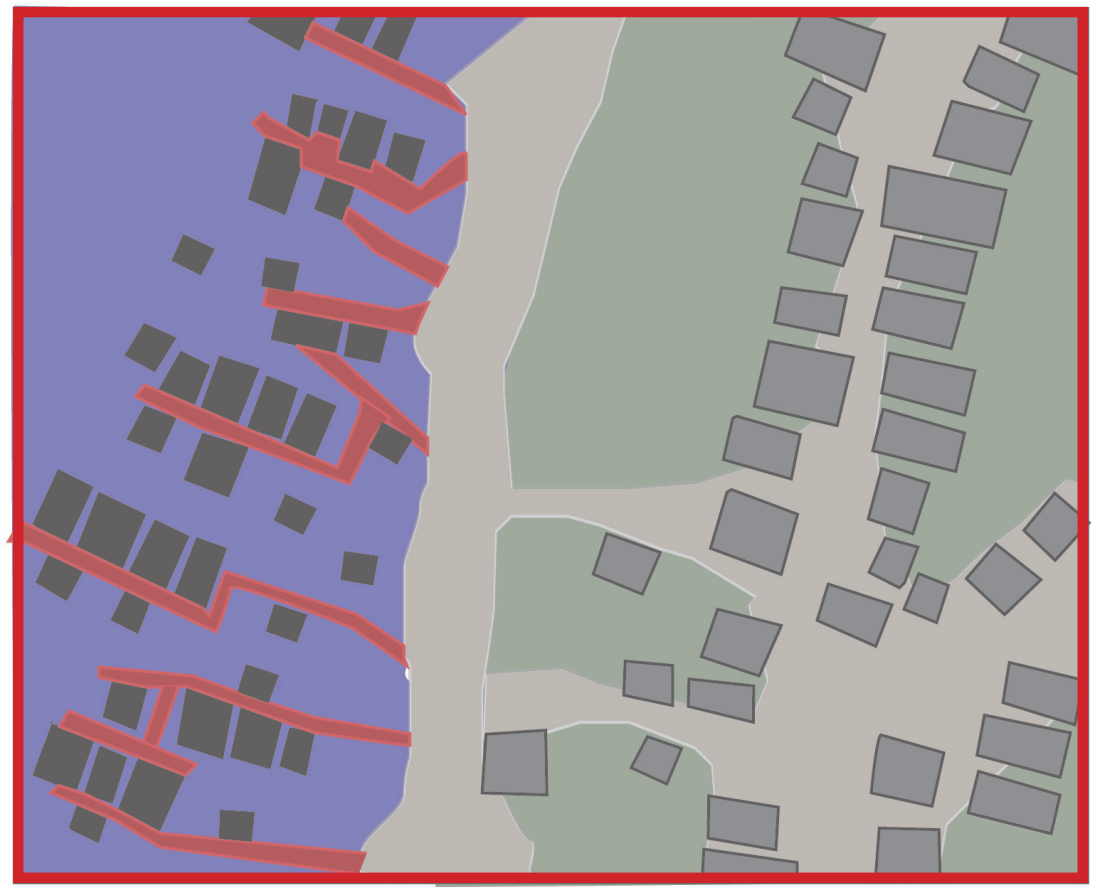

Vegetative/Agricultural land

Dirt roads

Phumi Prek Dambang,

Lower Mekong River

Temporary pontoons

1:5000

Floating houses

$\square$ Elevated houses

Figure 61: Phumi Prek Pambana, Tonle Sap, Lower Mekong River Basin, Cambodia 
Resilience flood management matrix analysis of the Lower Mekong River, Cambodia

\begin{tabular}{|c|c|c|}
\hline \multirow{3}{*}{ RAPID RESPONSE: } & Region: & Flood works up stream of the Mekong River and the Tonle Sap acts as a water storage basin. \\
\hline & Suburb: & Natural banks of the river/lake, but no flood infrastructure in place. \\
\hline & Building/site: & $\begin{array}{l}\text { Timber houses are elevated houses above anticipated water levels, other houses float. No flood defences. } \\
\text { Construction methods can't withstand high velocity floods. Timber weathers and weakens from water emersion. }\end{array}$ \\
\hline \multirow{3}{*}{ ALTERNATIVE CIRCUITS: } & Region: & The Mekong River and it tributaries direct flow paths into floodplains and storage zones, eg Tonle Sap lake \\
\hline & Suburb: & Poor quality roads result in in-land access being cut during floods. Water transport option in low velocity floods. \\
\hline & Building/site: & Pontoon structures and canoes connect individuals to community. \\
\hline \multirow{3}{*}{ CORE PROTECTION: } & Region: & The area is mainly used for agriculture and fishing with large green spaces for water catchment. \\
\hline & Suburb: & A lack of flood adapted infrastructure, such as roads, power, water or sewerage services. \\
\hline & Building/site: & Floating and elevation provides protection for low velocity and low-high volume floods. \\
\hline \multicolumn{3}{|l|}{ DIVERSITY \& REDUNDANCY } \\
\hline \multirow{3}{*}{ BUFFERING: } & Region: & Areas cleared for agricultural land use, whilst in other areas wetlands, marshland, forrests remain. \\
\hline & Suburb: & Natural foilage such as mangroves and reeds act to buffer, diffuse and store water. \\
\hline & Building/site: & $\begin{array}{l}\text { Timber stilt and floating timber construction will buffer water, alongwith plastic/metal drums. } \\
\text { Water already in the river and lake acts to buffer the velocity of flood waters entering the area. }\end{array}$ \\
\hline \multirow{3}{*}{ DIFFUSSION: } & Region: & Both natural meandering Mekong River and its tributaries along with artificial channelling for agriculture. \\
\hline & Suburb: & No water channels in place to tranport flood waters. Agricultural practices alter land gradients and flow paths. \\
\hline & Building/site: & The building form will not impact on water diffussion. \\
\hline \multirow{3}{*}{ STORAGE: } & Region: & Hydraulic dams upstream provide water storage, along with floodplains of the watershed catchment. \\
\hline & Suburb: & Agricultural low lying land acrts as a dry retention basin. The Tonle Sap provides additional space. \\
\hline & Building/site: & No on site rainwater storage devices. \\
\hline \multirow{3}{*}{ ABSORPTION: } & Region: & Watershed soil conditions (dry/damp/wet) \& vegetative cover (volume/type) affect absorption efficiency. \\
\hline & Suburb: & Low urban density, a lack of bitumen and other impermeable covered surfaces improve absorption rates. \\
\hline & Building/site: & Small or no building footprints increase area for absorption. \\
\hline \multirow{3}{*}{$\begin{array}{l}\text { NUTRIENT \& } \\
\text { WASTE RECOVERY: }\end{array}$} & Region: & Natural wetland and reed bed ecosystems filter the water. \\
\hline & Suburb: & No local urban sewerage devices or underground water supplies (results in river having poor water quality). \\
\hline & Building/site: & River/Lake water used for cooking, sanitation and cleaning practices (poor water quality) \\
\hline \multicolumn{3}{|r|}{ 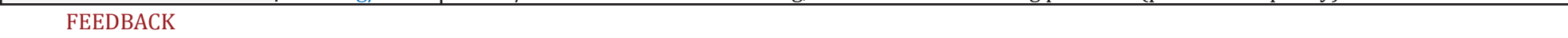 } \\
\hline \multirow{3}{*}{ RESPONSE: } & Region: & National Committee for Disaster Management(NCDM), flood management in Cambodia lacks flood mapping/data collection. \\
\hline & Suburb: & Lack of early warning systems. Radio flood warning begining to be issued. \\
\hline & Building/site: & Local flood knowledge and anticipation of floods facilitates action and preparation. \\
\hline \multirow{3}{*}{ REGENERATION: } & Region: & Reliance on government/external aid for infrastructure repair. Agricultural/fishing industry economy impacted. \\
\hline & Suburb: & Reliance on external aid for vital services: food, water, sanitation. \\
\hline & Building/site: & Floating and elevated houses reduce flood impact. Local construction techniques empower locals to rebuild. \\
\hline \multirow{3}{*}{$\begin{array}{l}\text { COOPERATIVE } \\
\text { SELF-REGULATION: }\end{array}$} & Region: & Reliance on external aid for evacuation due to a lack of mobility options. \\
\hline & Suburb: & $\begin{array}{l}\text { Floating building withstand floods of low wave heights and can relocate. Elevated housing become emergency } \\
\text { shelters for communities during more severe floods. }\end{array}$ \\
\hline & Building/site: & Flood knowledge improves response, but is limited by lack of food, water, shelter, poor sanitation and health. \\
\hline
\end{tabular}

Figure 62: Resilience flood management matrix of the Lower Mekong River Basin, Cambodia 


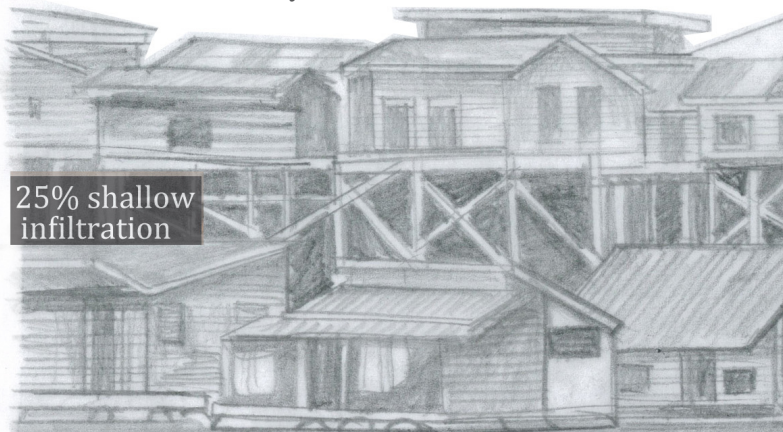

\section{$0 \%$ impervious surfaces}

$$
\text { aring }
$$

Key features impacting on water absorption, runoff and water retention (water volume, velocity and flood duration)

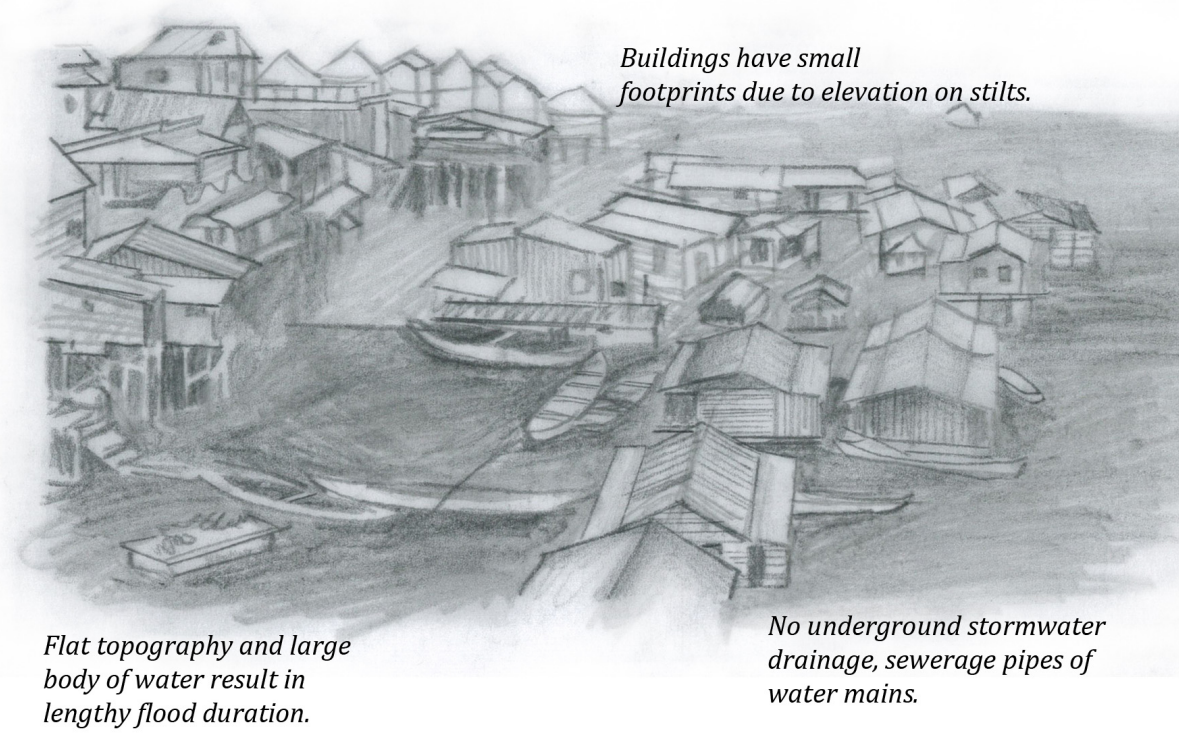

Locally available materials implemented; economic and sustainable construction.

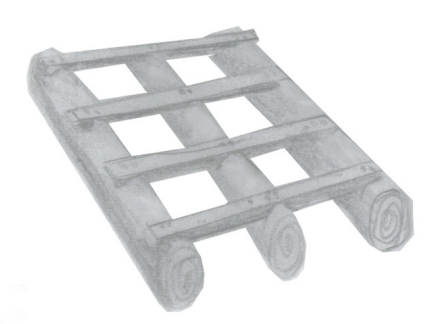
lengthy flood duration.

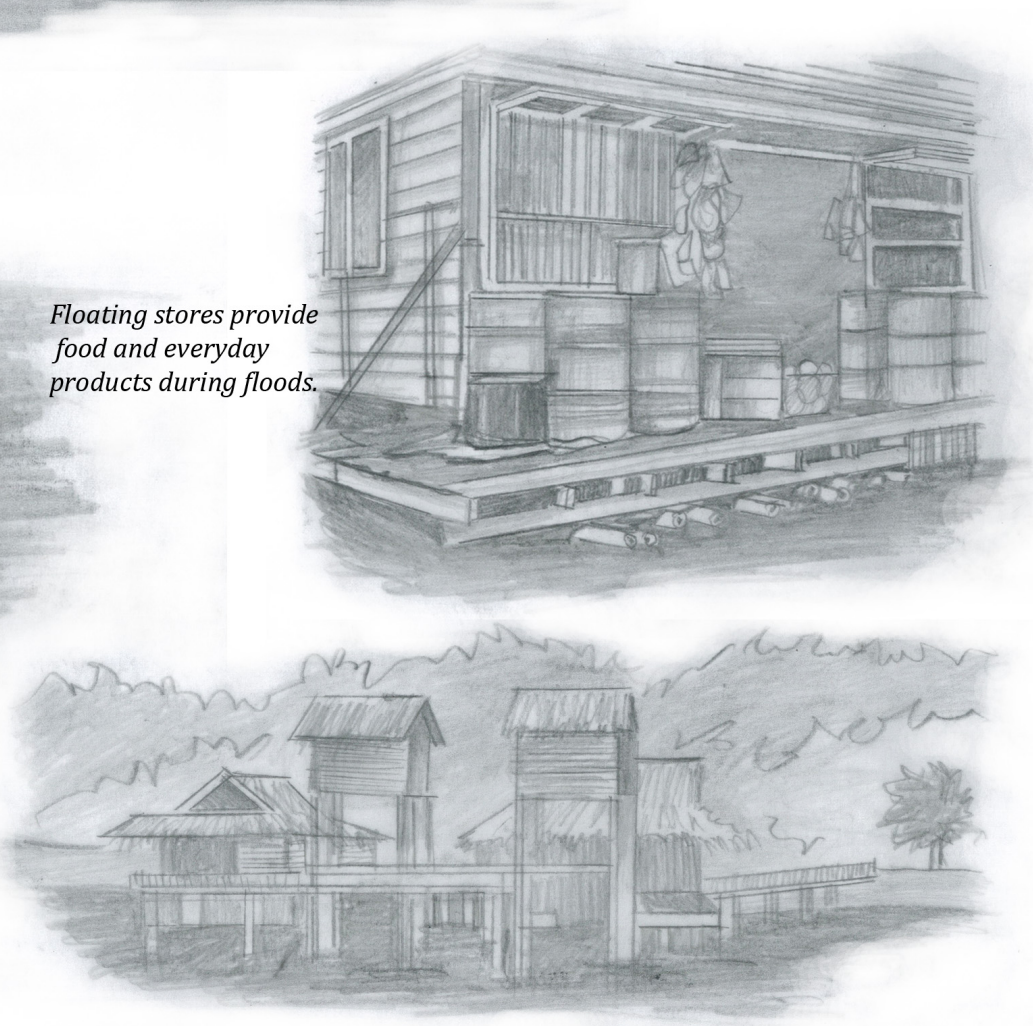




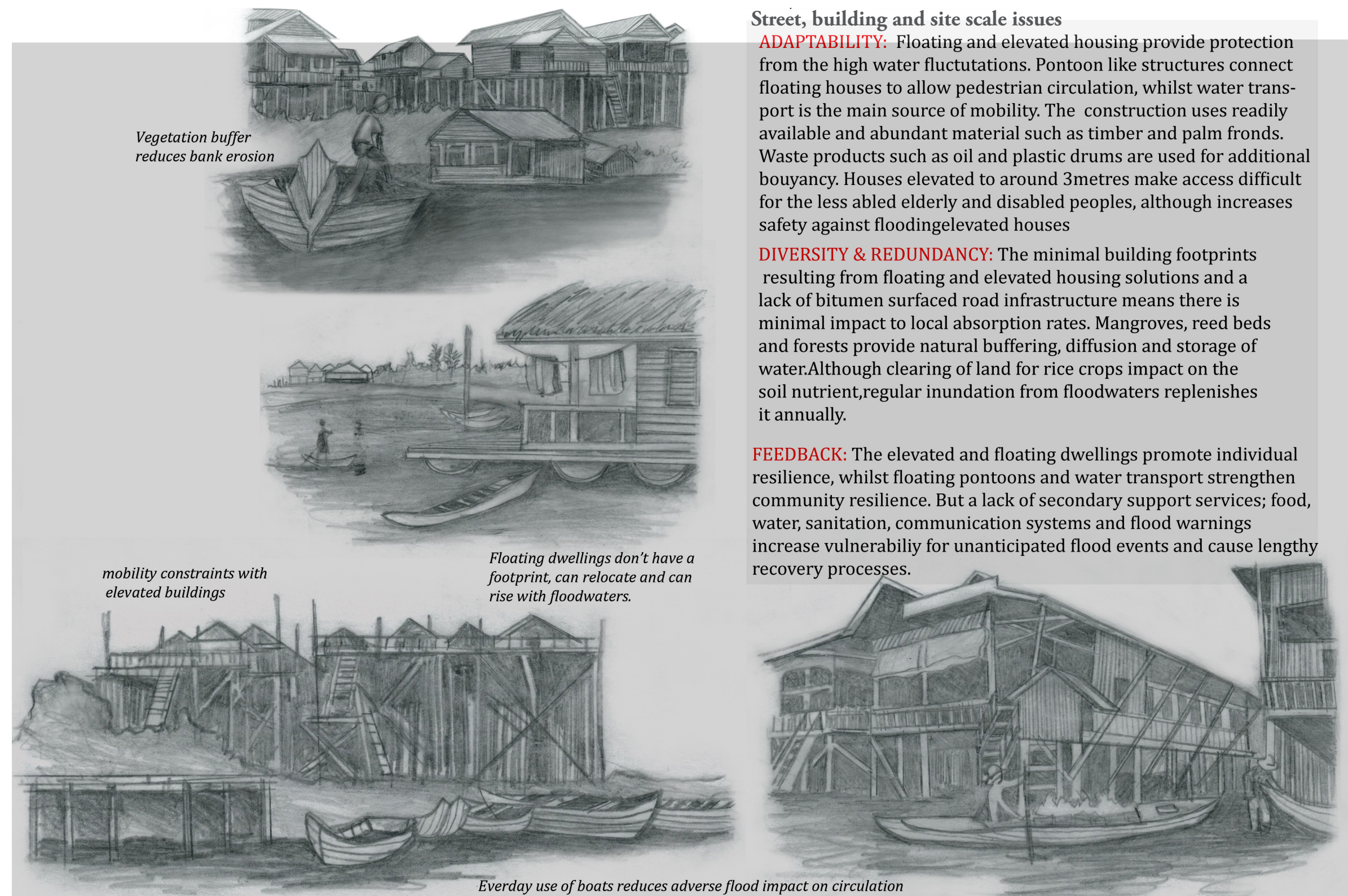

Figure 64: Lower Mekong River Basin Street, building and site scale analysis 


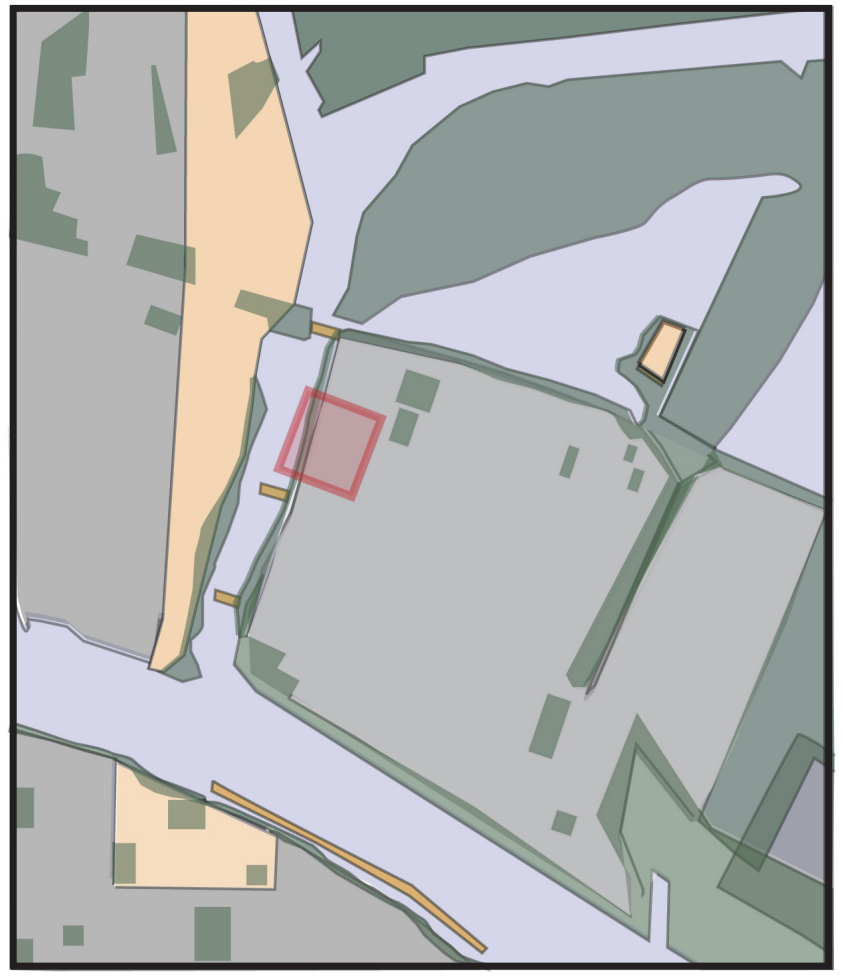

New Orleans

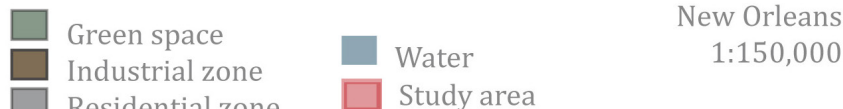

$\square$ Residential zone $\quad \square$ Study area

Figure 65: Lower Ninth Ward, New Orleans, USA

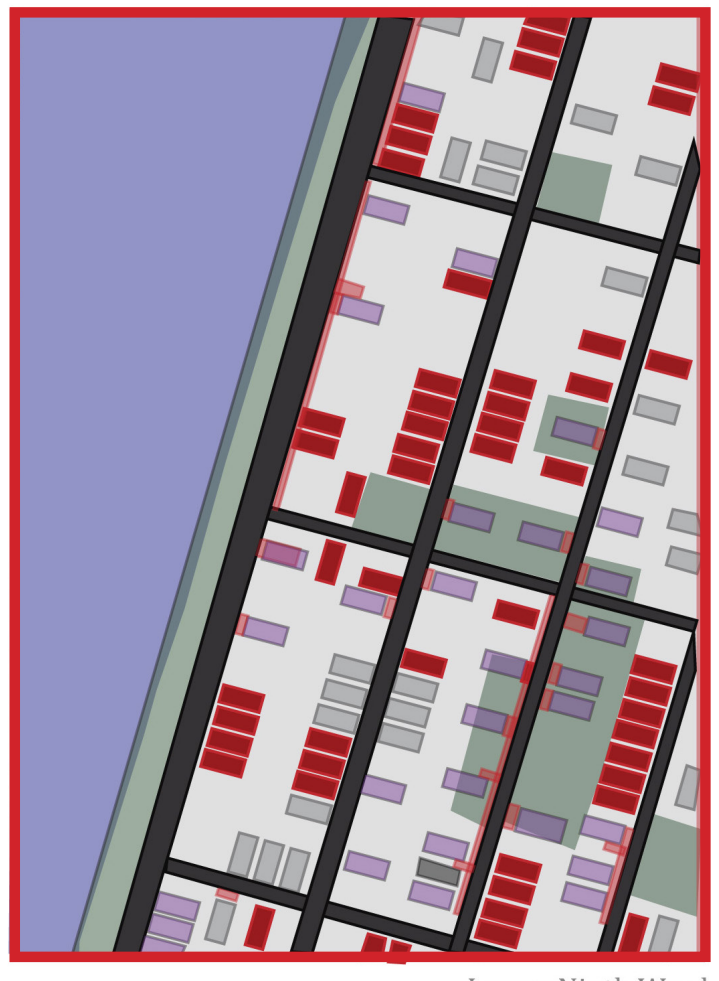

Lower Ninth Ward

$\square$ Roads

Driveways \& paths

Grassed, bushes, trees Water

Earth, Mud and debris

Exposed foundations

Existing damaged houses $\square$ New Flood proof houses $\square$ Amphibious house

Figure 66: Lower Ninth Ward, New Orleans, USA 


\begin{tabular}{|c|c|c|}
\hline \multirow[b]{3}{*}{ RAPID RESPONSE: } & Region: & A system of flood works proects the city. But poorly maintained infrastructure is unable to cope with flood volumes. \\
\hline & Suburb: & There is an exisiting town levee protection, but this failed at several points. There was no back up systems in place. \\
\hline & Building/site: & $\begin{array}{l}\text { Poor flood proofing standards lead to greater flood damage and hindered local response. } \\
\text { New residential development is elevating structures and increasing flood proofing standards. }\end{array}$ \\
\hline \multirow{3}{*}{ ALTERNATIVE CIRCUITS: } & Region: & Artificial flood channels in place to transfer floodwaters. Natural river system and tributaries carry floodwaters. \\
\hline & Suburb: & Low lying land lacks raised accessible roads during floods. A lack of alternate transport/circulation options. \\
\hline & Building/site: & The walkawys, driveways and roads are low lying, not raised from roads. No circulation during floods. \\
\hline \multirow{3}{*}{ CORE PROTECTION: } & Region: & The land was not zoned according to flood risks, with green space but densely zoned into blocks and streets. \\
\hline & Suburb: & No flood adapted network exists between the diverse housing types (varying elevation types \& construction). \\
\hline & Building/site: & New developments are including solar power \& some septic systems to reduce waste water \& reduce flood risks. \\
\hline \multicolumn{3}{|l|}{ DIVERSITY \& REDUNDANCY } \\
\hline \multirow{3}{*}{ BUFFERING: } & Region: & High urban density with minimal green spaces and a lack of dense vegetative covering \\
\hline & Suburb: & The grass levee acts as a barrier, but there is no riparian buffer or green zone. \\
\hline & Building/site: & $\begin{array}{l}\text { Minimal site vegetation (grass/small shrubs). Elevated buildings will reduce area of structure buffering floodwaters. } \\
\text { Floating foundations will buffer the water, but horizontal movement of the pylons reduces the impact. }\end{array}$ \\
\hline \multirow{3}{*}{ DIFFUSSION: } & Region: & The natural and managed topography establishes diversion channels \& distribute flow of floodwaters. \\
\hline & Suburb: & The low lying land acts as dry retention basins, but this is where the Lower Ninth Ward area is located. \\
\hline & Building/site: & The building forms \& site orientation is not relective of flood paths, creating barriers rather than directing water. \\
\hline \multirow{3}{*}{ STORAGE: } & Region: & Extensive flood defence infrastructure on the river system, eg dams. But loss of ecological storage eg wetlands. \\
\hline & Suburb: & There is a nearby swamp zone for water. The low lying residential area acting as storage. \\
\hline & Building/site: & No on site rainwater storage devices are implemented on a large scale. \\
\hline \multirow{3}{*}{ ABSORPTION: } & Region: & Watershed planning; quality of soil \& vegetative cover determines efficiency of water transfer above \& below ground. \\
\hline & Suburb: & The low urban density of the area reduces storm water issues, but with more development this will be reduced. \\
\hline & Building/site: & Demolished and new building footprints have increased impermeable land. Grassed area beside concrete areas. \\
\hline \multirow{3}{*}{$\begin{array}{l}\text { NUTRIENT \& } \\
\text { WASTE RECOVERY: }\end{array}$} & Region: & Ecological degredation and loss of wetlands has reduced natural waste filtration and nutrient producing processes. \\
\hline & Suburb: & Munincipal wastewater is collected and treated, but poor stormwater infrastructure and sewerage systems failed. \\
\hline & Building/site: & Grey water reuse systems or water collection systems were not locally adopted or promoted as options. \\
\hline \multicolumn{3}{|l|}{ FEEDBACK } \\
\hline \multirow{3}{*}{ RESPONSE: } & Region: & National Weather Service issues weather warnings. The Federal Emergency Management also issued warnings and evacuation calls. \\
\hline & Suburb: & Local radio warnings. Lack of local flood warning systems and services enabling locals to respond effectively. \\
\hline & Building/site: & Lack of local flood warning systems, lack of flood education and awareness of flood risks. \\
\hline \multirow{3}{*}{ REGENERATION: } & Region: & Lack of funding from all levels of government, poor infrastructure and local services hinder recovery processes. \\
\hline & Suburb: & Debris removal, fixing road infrastructure, levee failure points. Damaged sewerage, freshwater and electricity mains. \\
\hline & Building/site: & Low insurance levels in the area and lengthy insurance payouts for those with correct flood insurance types. \\
\hline \multirow{3}{*}{$\begin{array}{l}\text { COOPERATIVE } \\
\text { SELF-REGULATION: }\end{array}$} & Region: & Lack of local flood management proceedures. FEMA poorly implemented and managed emergency response. \\
\hline & Suburb: & Recent flooding improves local flood awareness, preparedness and flood risk assessment. \\
\hline & Building/site: & $\begin{array}{l}\text { Recent flooding improves residential uptake rates of flood proofing measures. But recent developments are } \\
\text { elevatating houses at great heights, but create disabled access issues and islation issues during floods. }\end{array}$ \\
\hline
\end{tabular}

$$
\text { Figure 67: Resilience flood management matrix analysis of the Lower Ninth Ward, New Orleanss }
$$


Urban and residential scale analysis
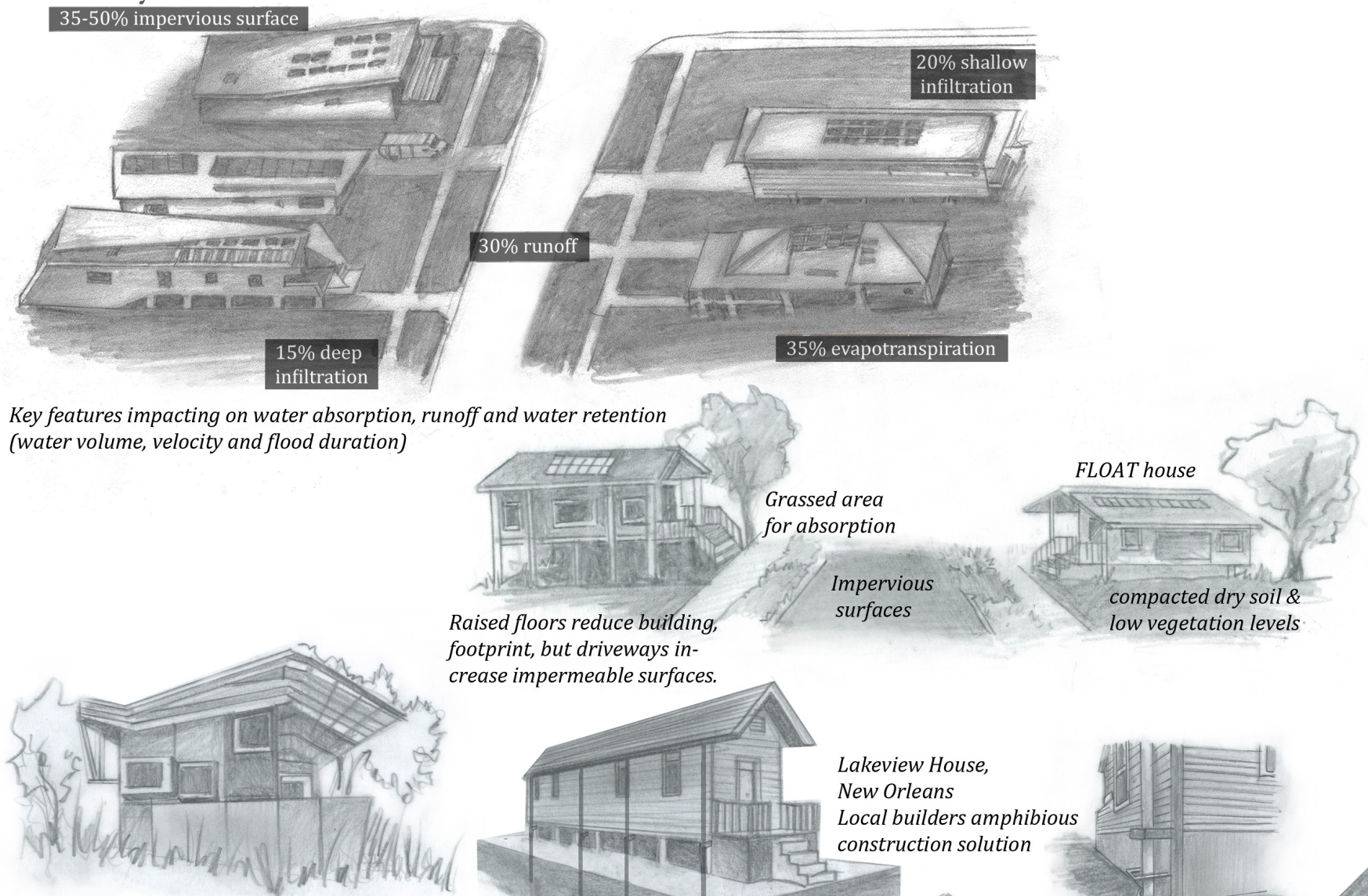

footprint, but driveways in-

crease impermeable surfaces.

Morphosis Architects, The FLOAT House

The first new amphibious building in New Orleans.
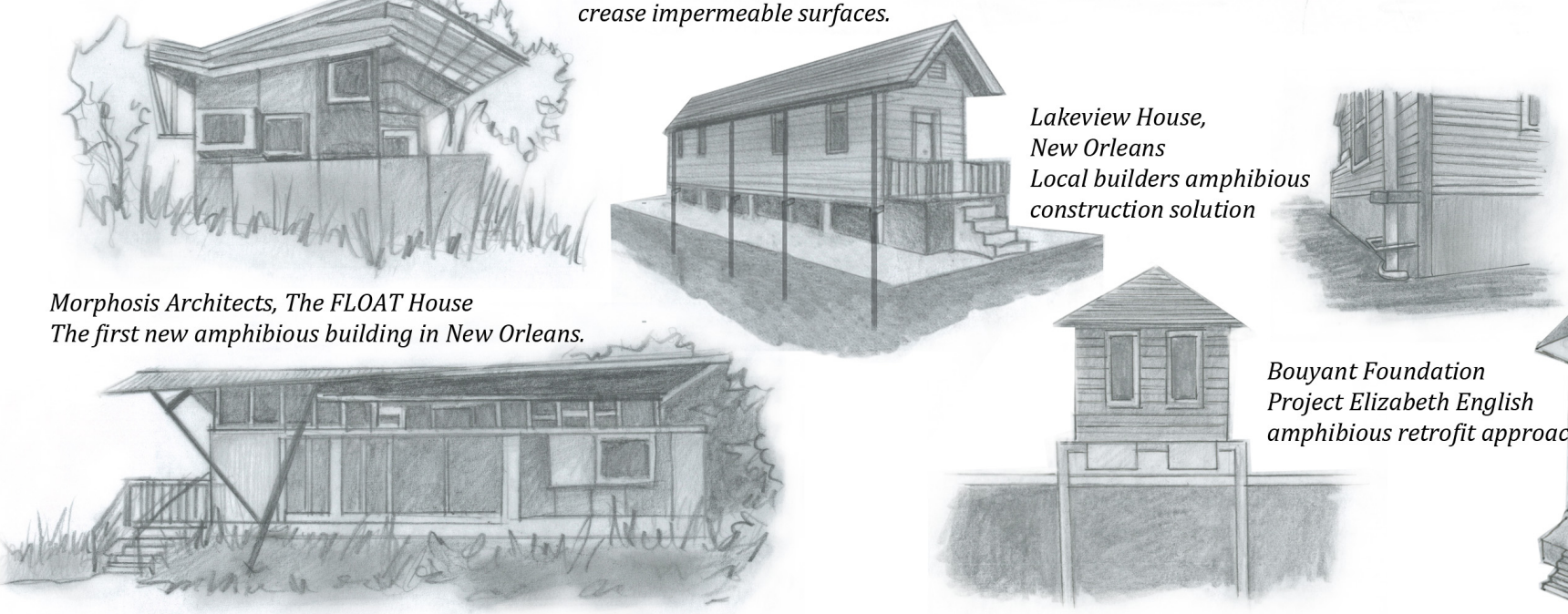

Figure 68: Urban and residential scale analysis of the Lower Ninth Ward, New Orleans 


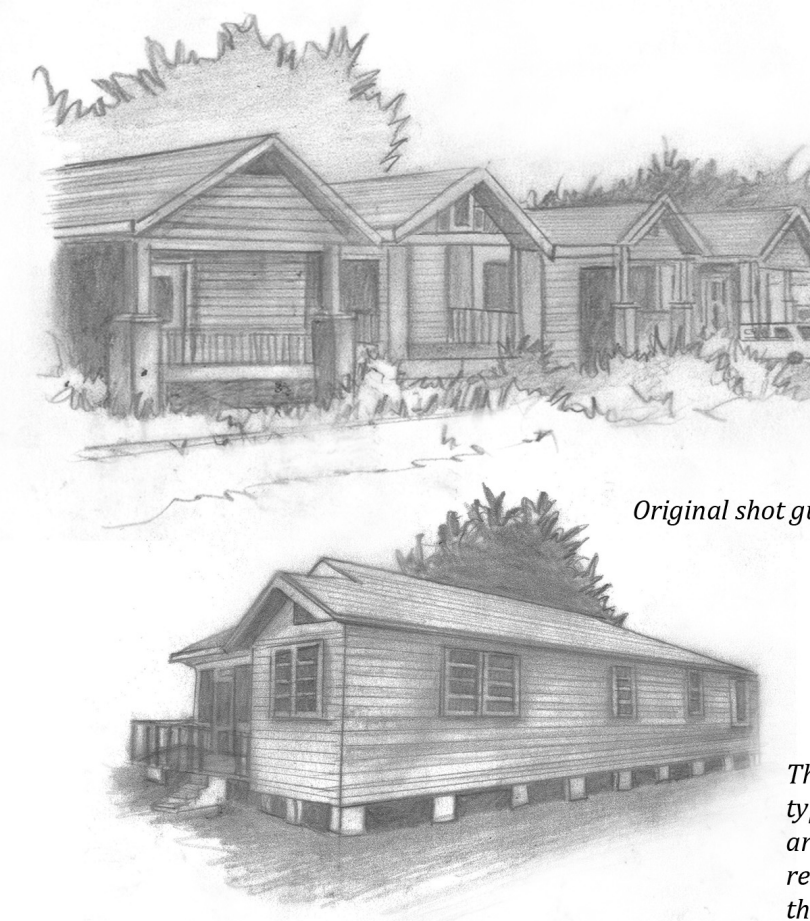

Street, building and site scale issues

ADAPTABILITY: FEMA raised floor elevation standards following the disaster

This has caused disbabled access concerns. The Lower Ninth Ward has

become a testing ground for flood \& hurricane proof housing. The traditiona

shot gun house style is being lost in the modern architectural solutions.

There is no flood adapted circulation network between houses,; new or

existing. One amphibious house has been approved and constructed, whil

the remainder are traditionally elecated, although a t varying heights.

DIVERSITY \& REDUNDANCY: Individual resilience is being improved through the new flood/hurricane proof housing solutions. But, ground permeability is
reduced by bitumen and compacted soils resulting from the addition of

driveways, sidewalks, the construction process, \& lack of buffering vegetation

Grassed areas resulting in drier soils. Cars will add to debris due to lack of

garages. There is a lack of site \& town landscaping to diffuse flows, detention

areas \& establish water channels.

FEEDBACK: The amphibious FLOAT house and the work of the BFP promotes a new solution for the region. The flood/hurricane proof housing improves

former standards of protection, but fails to address site orientation

(lot placement), raised circulation pathways \& housing density.

The shot gun housing types were the dominant housing type in the region. Now modern architectural solutions are being implemented post Katrina. Whilst the flood resilience of residential buildings is being improved there are concerns for the cities heritage and cultural

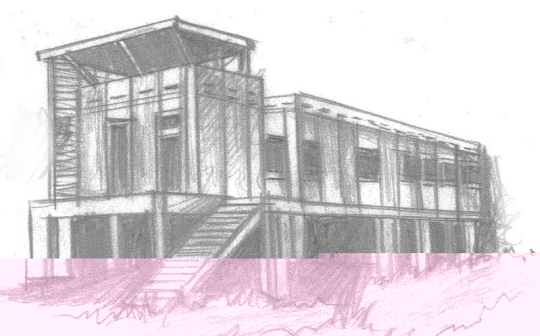

character being lost.
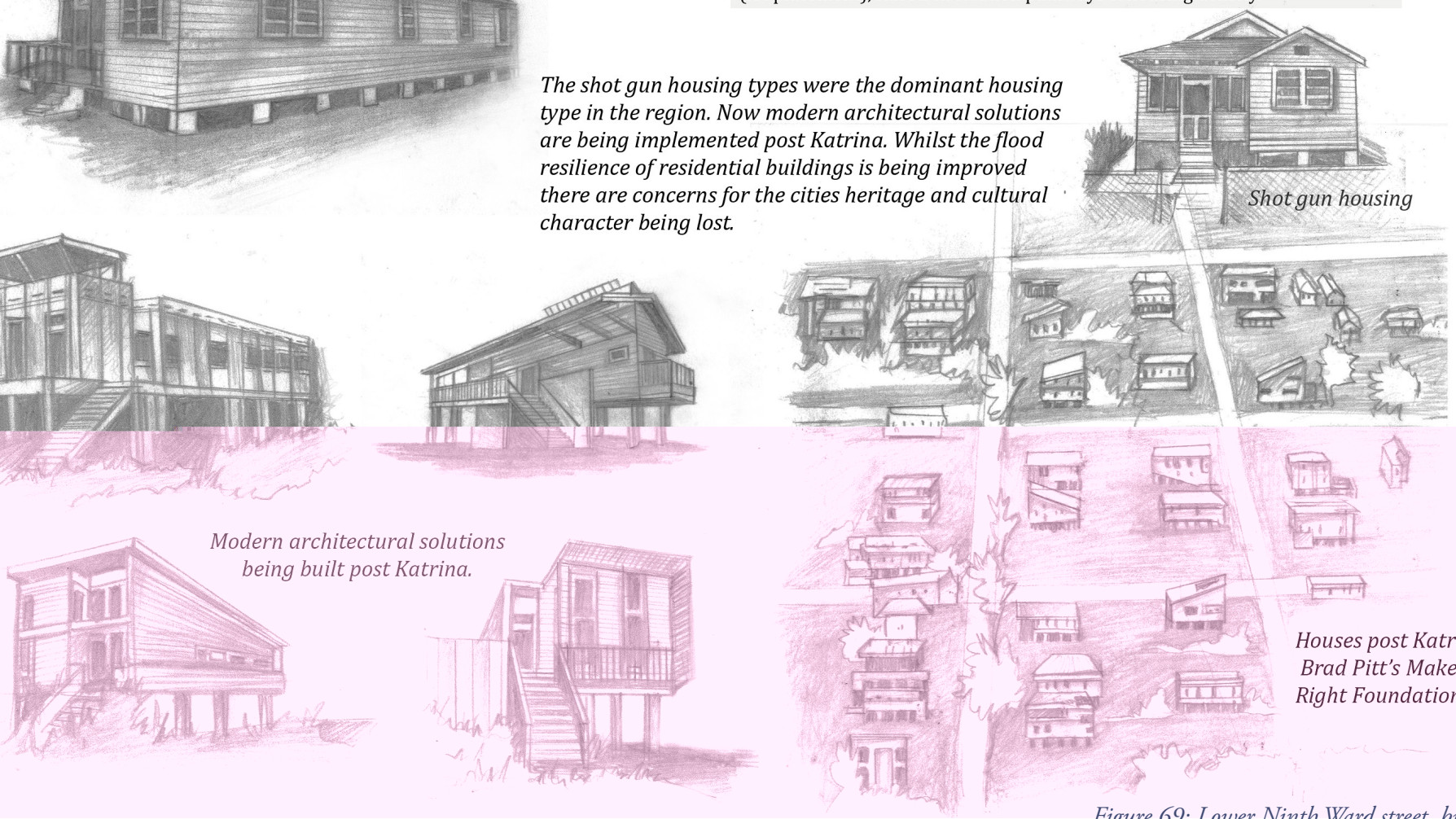

Figure 69: Lower Ninth Ward street, building and site scale analysis 
Maasbommel, West Maas en Waal, The Netherlands

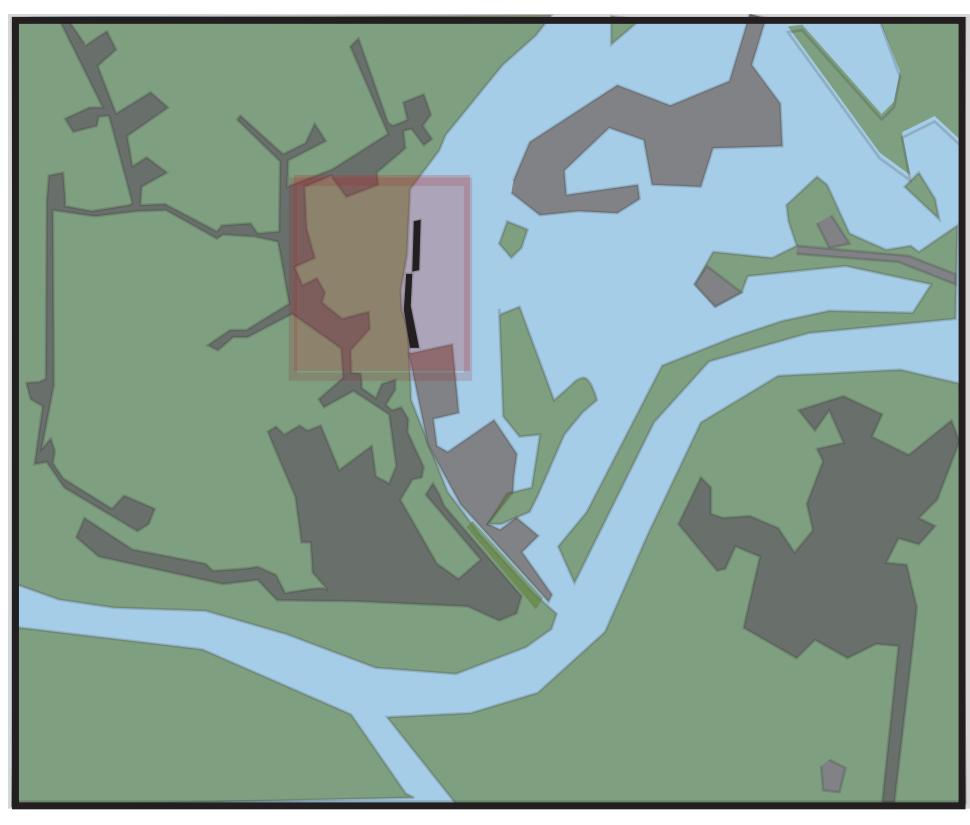

West Maas en Waal

Agricultural lan

$1: 20,000$

Amhibious \& Floating buildings

Study area

Figure 70: West Maas en Waal, The Netherlands

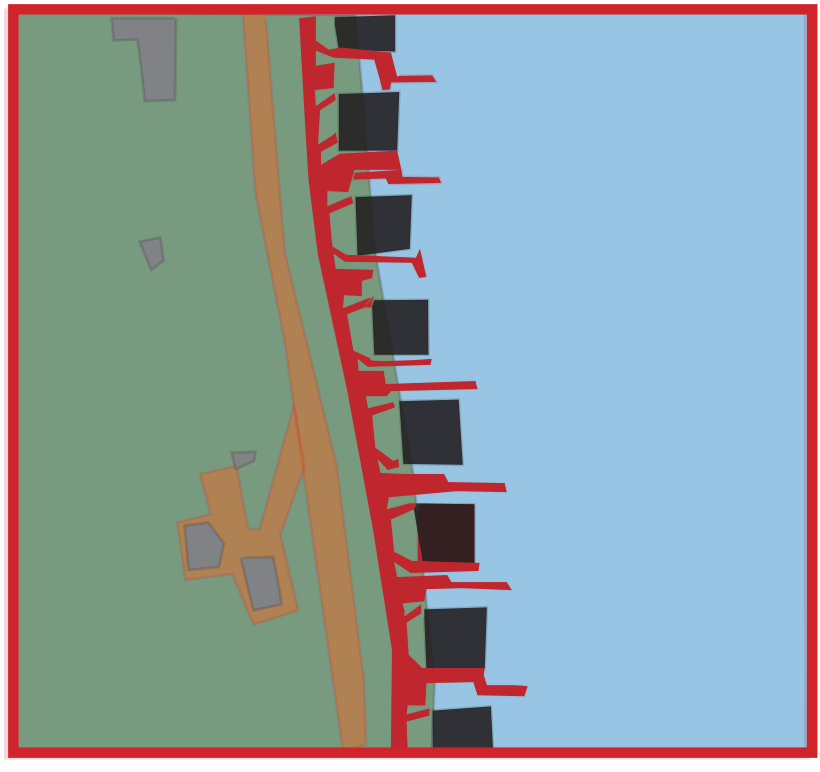

Agricultural land

Existing roads and driveways

Additional road and pathways

Amphibious buildings

Traditional farm houses

Figure 71: Maasbommel, West Maas en Waal, The Netherlands 
Maasbommel, West Maas en Waal. The Netherlands

ADAPTABILITY

\begin{tabular}{|c|c|c|}
\hline \multirow{3}{*}{ RAPID RESPONSE: } & Region: & There is a system of flood works proecting nearby town. The agricultural land acts as a dry retention basin. \\
\hline & Suburb: & There is exisiting levee protection.Upon which the amphibious houses rest in front of. \\
\hline & Building/site: & The houses have flood proof materials, but no on site flood defence, other than amphibious means. \\
\hline \multirow{3}{*}{ ALTERNATIVE CIRCUITS: } & Region: & Artificial flood channels direct floodwaters into green space and wet retention basins.. Natural channels carry water. \\
\hline & Suburb: & The major road is placed on the levee, raised above the low lying land to function in low flood inundation. \\
\hline & Building/site: & Sidewalks and roads connecting amphibious houses are flood liable, but boats and floating pontoons are available. \\
\hline \multirow{3}{*}{ CORE PROTECTION: } & Region: & Land is mostly zoned as agricultural due to flood risks. Large government investment in infrastructure reduces risks. \\
\hline & Suburb: & No flood adapted network exists between amphibious houses, but exists between floating houses there are pontoons. \\
\hline & Building/site: & $\begin{array}{l}\text { Building/site: Amphibious houses have self-reliant systems and flexible pipes, providing services during floods. } \\
\text { The concrete pylons are embedded in the rockbed to increase strength, but they cannot withstand high velocity floods. }\end{array}$ \\
\hline \multicolumn{3}{|l|}{ DIVERSITY \& REDUNDANCY } \\
\hline \multirow{3}{*}{ BUFFERING: } & Region: & Agricultural land, minimal vegetative covering. Amphibious housing is further reduce riverine vegetative buffer zones. \\
\hline & Suburb: & The grass levee acts as barrier, but there is no riparian buffer. \\
\hline & Building/site: & $\begin{array}{l}\text { Minimal site vegetation (grass/small shrubs). Floating foundations buffer surface wave heights. } \\
\text { Horizontal movement of the pylons reduces the velocity and water pressure on the structure. }\end{array}$ \\
\hline \multirow{3}{*}{ DIFFUSSION: } & Region: & Meandering rivers and gradiented agricultural land, artificial landscaping and diversion channels transport floodwaters. \\
\hline & Suburb: & Raised road running adjacent to the river will not contribute to water tranfer. \\
\hline & Building/site: & The building form will not impact on water diffussion. The position of driveways and vegetation will also not contribute. \\
\hline \multirow{3}{*}{ STORAGE: } & Region: & Flood defences and green infrastructrure provide water storage zones. But urban sprawl and densification reduce area. \\
\hline & Suburb: & A network of polders, canals, pumps and dykes regulate the flow of water. Agricultural land acts as dry retention basins. \\
\hline & Building/site: & On rainwater storage devices would be suitable, however there are few explamples of these currently in the area. \\
\hline \multirow{3}{*}{ ABSORPTION: } & Region: & Watershed planning; quality of soil \& vegetative cover determines the efficiency of water transfer above \& below ground \\
\hline & Suburb: & Low urban density reduces stormwater volumnes and provides greater green space for water absorption. \\
\hline & Building/site: & Building footprint in low floods, but will float during high floods. But concrete driveway reduces lot absorptio \\
\hline \multirow{3}{*}{$\begin{array}{l}\text { NUTRIENT \& } \\
\text { WASTE RECOVERY: }\end{array}$} & Region: & Munincipal wastewater is conveyed, collected \& treated. Ground water is collected, treated \& reused. \\
\hline & Suburb: & Waste water is transferred via flexible pipes into the main underground system. \\
\hline & Building/site: & Grey water reuse systems are not currently in widespread use,but would be suitable in the flood prone environment. \\
\hline \multicolumn{3}{|l|}{ FEEDBACK } \\
\hline \multirow{3}{*}{ RESPONSE: } & Region: & $\begin{array}{l}\text { The Royal Netherlands Meteorological Institute (KNMI) delivers weather forecasts and warnings. Dutch Government's } \\
\text { Flood Information Warning System (FIWS) is operational at the national, regional and local levels. }\end{array}$ \\
\hline & Suburb: & Flood height indicators, a complex system monitoring fluctuations in waterlevels, and local radio broadcasts are used. \\
\hline & Building/site: & Floor sensor alarms implemented in high risk zones. Flood proofing standards, flood education and awareness are high. \\
\hline \multirow{3}{*}{ REGENERATION: } & Region: & Agricultural industry impacted short term, but benefits long term. Adaptive infrastructure to floods reduces damage. \\
\hline & Suburb: & Debris removal, fixing road infrastructure, damaged services and maintenane of flood proofing structures in recovery. \\
\hline & Building/site: & urance by local farmhouses aid recovery. \\
\hline \multirow{3}{*}{$\begin{array}{l}\text { COOPERATIVE } \\
\text { SELF-REGULATION: }\end{array}$} & Region: & Netherlands police, fire brigades and ambulances establish a network to facilitate streamline emergency response. \\
\hline & Suburb: & Local organisations ensure evacuation plans are in place and community is flood aware. \\
\hline & Building/site: & The floating buildings become emergency shelters for nearby residents. \\
\hline
\end{tabular}

Figure 72: Resilience flood management matrix analysis of Maasbommel, The Netherlands 
Urban and residential scale analysis

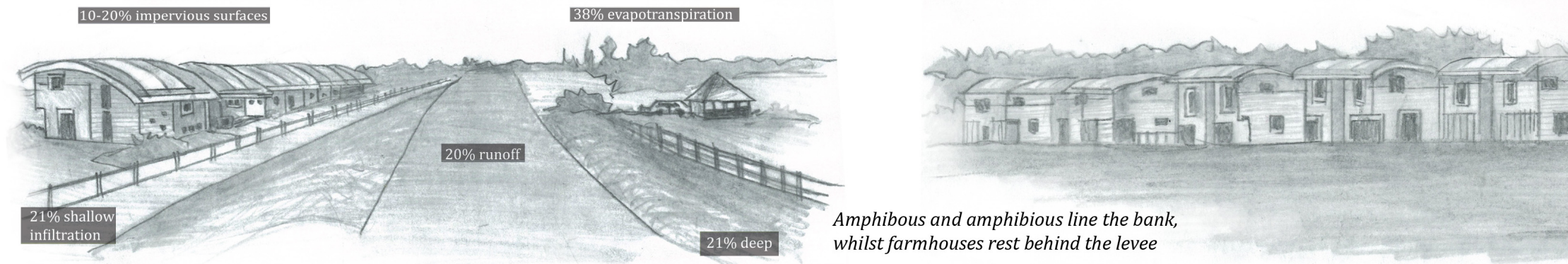

Key features impacting on water absorption, runoff and water retention

(water volume, velocity and flood duration)

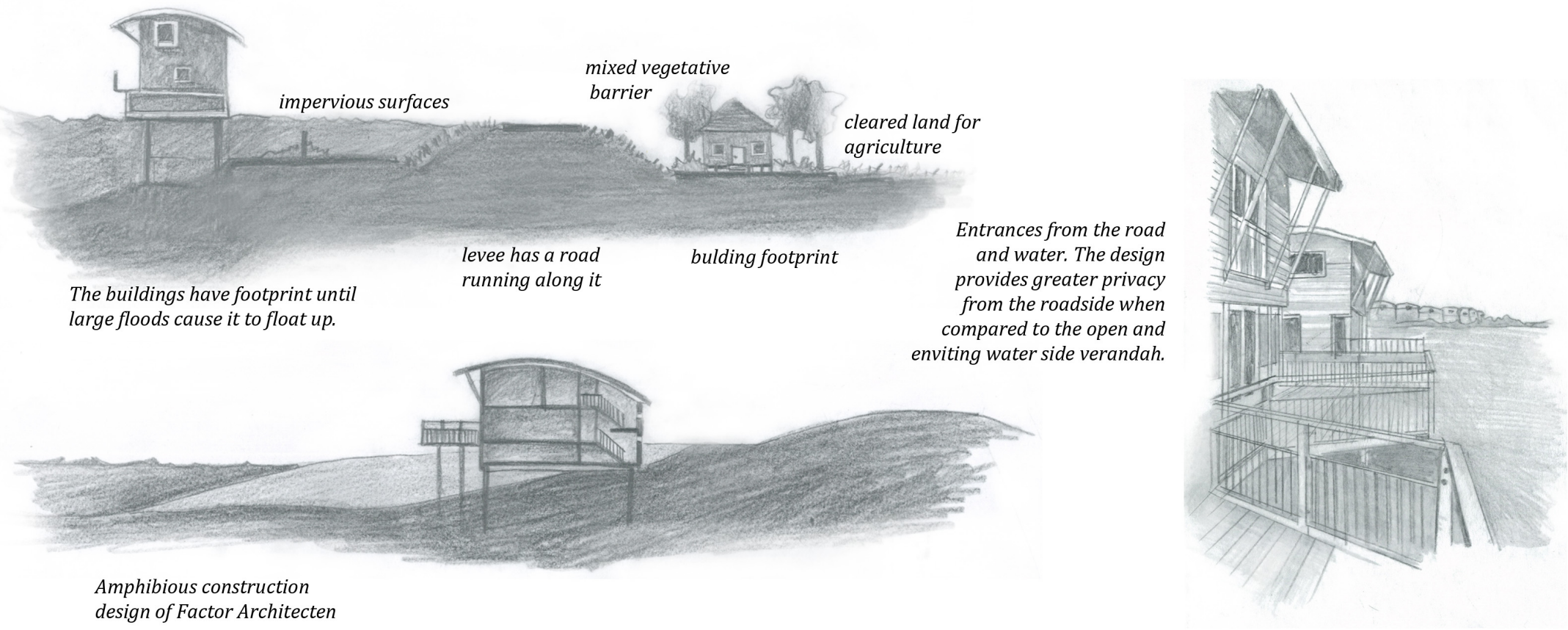

Figure 73: Urban and street scale analysis of the Maasbommel, The Netherlands 
Street, building and site scale analysis

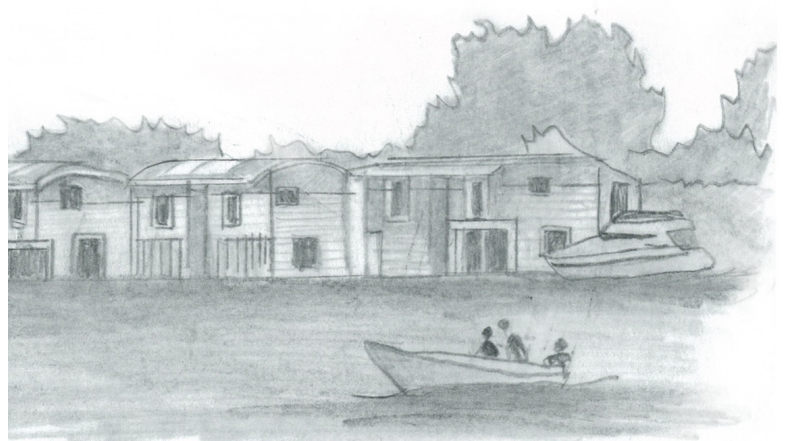

Alternate transport during floods; boats.

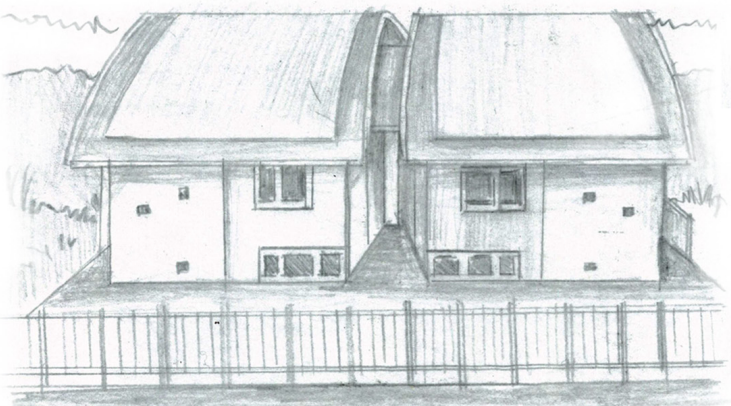

Elevation from road entrance

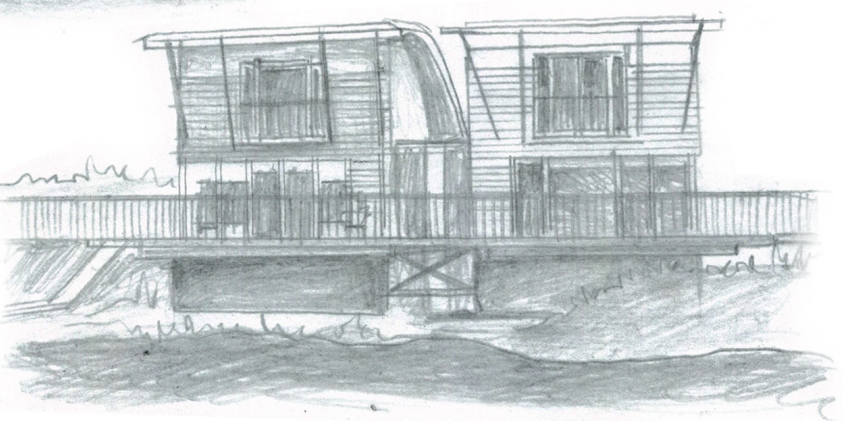

Elevation from water

Factor Architecten designed

amphibious and floating housing

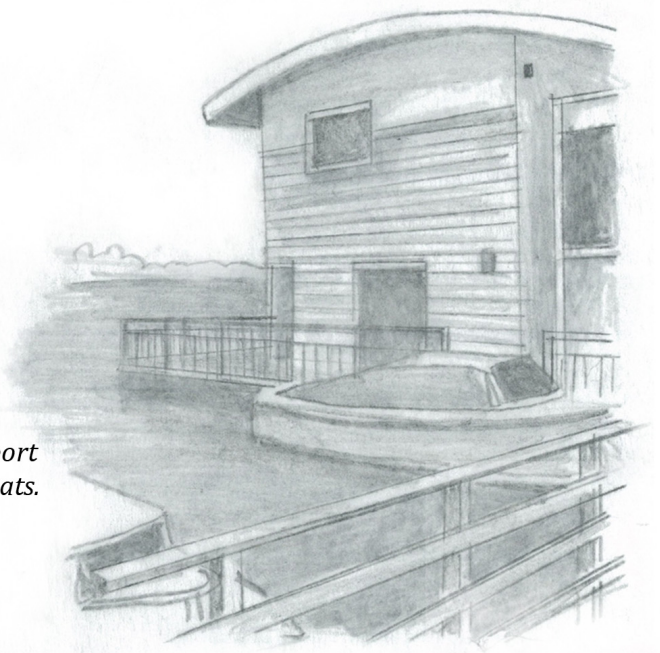

ADAPTABILITY: The design is not reminiscent of nearby

agricultural farm houses. The floating foundationsare attached to lexible pipes for wastes and services. Due to the adaptive floating building flood risks are not increased for suburb or region. There is no flood adapted circulation network between houses; new or existing. The pylon construction is not suitable for high velocity

DERITY \& REDUNDANCY: The offsite construction of the buildings results in minimal on site impact, such as ground

compaction (absorption inpacts). But ground permeability is

reduced by the addition of roads and driveways. There is minimal

subs

to the lack of garages. There are now water on site storage devices.

FEEDBACK: The amphibious buildings promote individual esilience. Building position behind the levee, doesn't impact on xisting farmhouses. Recovery tates are high due to floating

y escape is by boat.

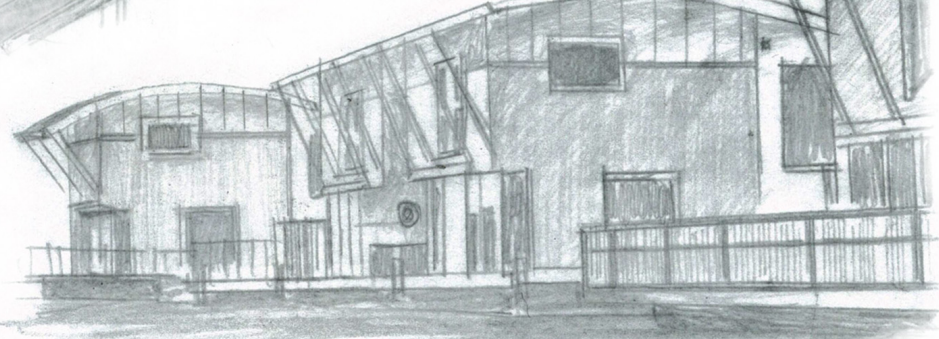

Floating housing solution

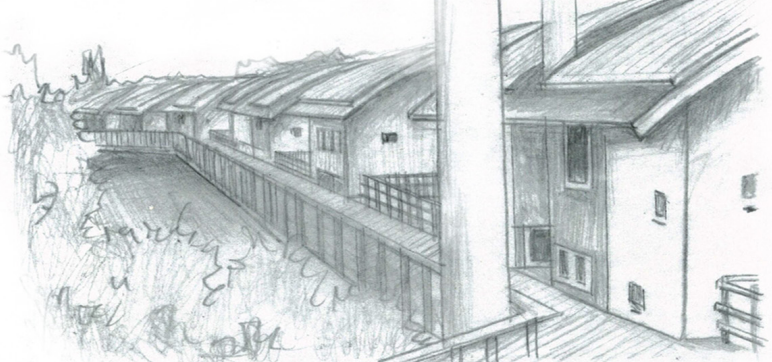

Pontoon paths connect residents of the floating houses

Figure 74: Maasmbommel street, building and site scale analysis 


\subsection{Key findings from precedent analysis}

Precedent analysis revealed the benefits of viewing floods as natural hydrological processes, rather than as disastrous events requiring prevention. The uniform focus of improving individual resilience through flood proofing and amphibious construction of residential housing was shown to be limited in enhancing sociospatial resilience. Whilst the benefits of making space for water proved beneficial, a common weakness in the approach was a lack of consideration of the need to make room for people whilst the floodplain is inundated by floodwaters. There is an opportunity to explore interventions that allow floodplain communities to remain safely connected to one another and vital services during flood events. The Netherlands has accepted the challenge to foster new innovative solutions that "can contribute to the development of meaningful new urban structures" (Nillesen \& Singelenberg, 2011, p. 9). However, despite the advancements in amphibious construction and the increasing use of floating residential options, resilience at the "lowest spatial level as part of a top-down and bottom-up approach" needs to be enhanced (Nillesen \& Singelenberg, 2011, p.9). Improvements in top down and bottom up approaches will enhance the integration of residential environments, infrastructure services and ecological systems to improve the flood resilience of a city.
Feedback has regularly demonstrated that social resilience impacts significantly on the successful uptake and implementation of flood resistance and resilience strategies. Social resilience to flood hazards was shown to be largely determined by demographic groups, health status, economic status, political policies, educated awareness and cultural values (Smit, et al., 2001). It is imperative that the social and spatial vulnerabilities of a community are understood in order for local communities to accept, plan, respond and adapt to flooding (Wisner, 2004).

The basic function of the city system needs to be maintained during and post flood inundation. These functions include the provision of basic services including water, food, shelter and energy. Secondary city function including access to health services and means of mobility, whether on foot or by other means of transport (boat, helicopter, 4WD) are also key, so individuals are not isolated without an ability to escape. Vulnerability levels were shown to increase from a loss of access to mains electricity and subsequent heating, cooling, cooking facilities, sanitary drainage and telecommunication services. The duration of the flood event and the spatial distribution of inundation also greatly affect the number of people impacted by flooding and thus the basic services required. Flood proofing of residential, infrastructure and urban landscapes needs to enhance the adaptive capacity of both individual environments and city infrastructure to respond to flooding. 
The relocation of towns in high risk flood zones is regularly raised. Such a solution was resisted in New Orleans where post construction is taking place on the previous housing footprint. Perseverance in Cambodia has resulted in traditional construction techniques being adapted to take advantage of modern material. Corrugated iron is replacing palm fronds to improve longevity, weather proofing and structural integrity (Oliver, 2003). In the Netherlands amphibious and floating architectural solutions have arisen from a history of flood experience. "Katrina can teach us about the ways in which cities as urban forms mediate between nature, community, and commerce" (Steinberg \& Shields, 2008, p. 22). These examples also serve to highlight the importance of remembering: "the resilience of an ecological system depends on the actions of the interlinked social systems, leading to a strong and dynamic interdependence between the two" (Nuorteva, Keskinen, \& Varis, 2010, p. 91).

Societal confusion was demonstrated to arise in all three examples when singular standards of flood occurrence rates and anticipated flood levels were established but not understood by the community. Concerns were raised in the feedback period over a lack of planning or implementation of resistance or resilience measures for more extreme events. This situation could be improved by designing for low, medium and high flood events, which are representative of different functional stages of the city system to manage flood impact; low floods being anticipated and manageable floods with minimal disruption; medium floods being recognised as potentially hazardous and requiring improvements to infrastructure for resilience without evacuation; and high floods where the focus should be on rescue and survival with planning for the rapid return of the city to a functional state after the flood event.
Such flood planning would have to involve both top down and bottom up approaches, implemented by an informed and empowered community so the plan could be understood, accepted, implemented and sustained. The natural and artificial components of systems need to be able to anticipate, prepare, cope and adapt to flooding to enhance socio-spatial resilience capabilities. The difference in socially accepted flood risk standards in the Netherlands, America and Cambodia was seen to be greatly influencedby their historic flood experience, flood policy, economic value and socio-cultural accepted levels of risk.

In the three case studies culture impacted significantly on the nature of engineered solutions to flooding, the communities' understandings of flood risk and the societal consequences of the flood event. The flood history of places was also seen to greatly impact on the ability of inhabitants to adapt to the changed circumstances. Adaptive-capacity was demonstrated to influence flood damage and recovery rates. It is at this point that floods become a catalyst for positive renewal and change. Those with lifestyles and professions directly connected to the river system, such as farmers and fisherman, were seen to be more accommodating and prepared for floods, whilst cities that turned their back on the rivers behind levee systems experienced greater vulnerability. 


\section{Common issues for floodplain inhabitants:}

- Amphibious and floating houses proved more successful than static elevation techniques.

- Preventative flood measures (flood engineering structures) resulted in greater long term risk and exposure to severe floods from failure.

- Flood defences such as levees were shown to disconnect communities from the river, and result in the city becoming complacent about its vulnerability, and its inhabitants becoming ignorant of the risk.

- Evacuation response was hindered by a lack of flood warning, a lack of flood safe circulation pathways, and the publication of inappropriate time frames for action.

- Residents became isolated, and recovery periods lengthy despite flood adapted housing methods (elevated/floating/amphibious) due to a lack of urban circulation options whilst inundated.

- Public infrastructure damage, resulting in a lack of energy, water and sewerage services, meant external aid was necessary. Centralised infrastructure was at greater risk of failure.

- Absent, poorly planned or inadequately prepared evacuation shelters increased evacuation risks, social vulnerability and recovery periods. Inadequate power, food and sanitation supplies and services were critical factors.

- Evacuating communities to remote locations was seen to cause issues with community structure and to adversely impact on local support structures.

- Evacuation processes are commonly hindered by a lack of vehicular and pedestrian mobility options.

\section{City trends identified to increase flood risks:}

- Urban expansion and increasing urban density promote previous land zoning to be altered. Former high risk flood zones are drained, developed and 'flood protected' to cater for urban growth.

- Urban areas regularly inundated by flooding are reluctant to relocate after settlement. Adaptive and resilient measures need to be implemented during post feedback analysis to reduce flood vulnerability and increase adaptive capacity in the long term.

- Technological advances are often resulting in centralised infrastructure systems (e.g. electricity, water mains and storm water management).

This means infrastructure has greater flood exposure to a total system failure. The technical complexity of the infrastructure often results in longer recovery time, Decentralising and diversifying infrastructure services often results in longer recovery time. Decentralising and diversifying infrastructure services may help to reduce flood vulnerability. 
Precedent analysis highlighted that social values, lifestyle, environment and economic availability influence a variety of urban, housing and flood management solutions. The differing housing types of Cambodia, New Orleans and the Netherlands highlighted that "no dwelling tradition is isolated from its surroundings; each is a constituent part of its environment" (Oliver, 2003, p. 167). Innovative solutions responsive to the local and regional scale were seen to foster resilience and strengthen educated awareness of flood risks. A lack of key infrastructure services such as electricity, water, food and emergency shelter was shown to increase flood impact and inhibit recovery.

The relationships between a city's architecture, its infrastructure, environment and hydrological patterns need to be investigated to establish suitable solutions which reduce vulnerability and improve the adaptive capacity, while implementing a variety of resilience solutions and feedback processes. Such an approach will promote a reenvisioning of cities and foster new ways to inhabit flood prone land. Socio-spatial resilience measures will enable a balanced approach to making safe spaces for water and people, whilst minimally impacting on flood hydraulics.
The Food and Agriculture Organization of the United Nations Mekong River Commission Secretariat, March 1999 Report highlighted the need to view the spatial distribution of floods, human settlements, infrastructure and the urban environment in order to strengthen the adaptive capacity of the region.

The relationships between a city's architecture, its infrastructure, environment and hydrological patterns need to be investigated in order to establish suitable solutions which reduce vulnerability and improve the adaptive capacity, variety of resilience solutions and feedback processes. This will promote opportunities for reenvisioning cities and foster new ways to inhabit flood prone land. Socio-spatial resilience measures will enable a balanced approach in making safe spaces for water and people, whilst minimally impacting on flood hydraulics. 
The revenge of nature seems to be part and parcel of the essence of Australia - a land of extreme landscapes that has dictated a particular response with regards to buman occupation of its territory. 

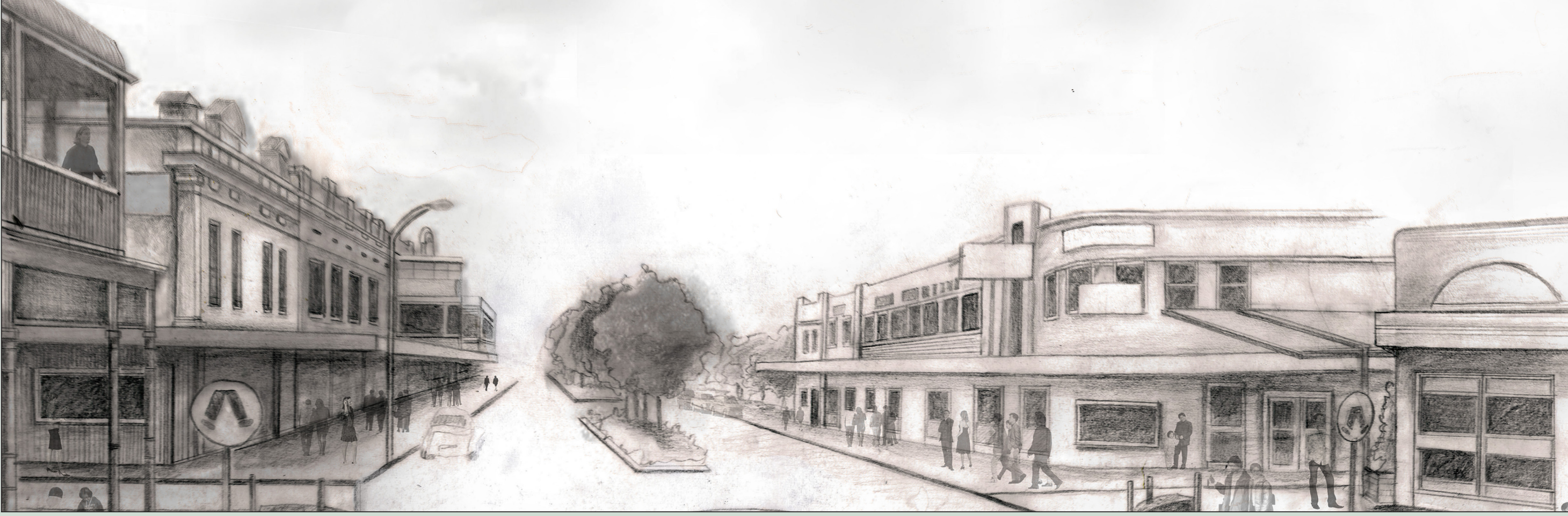

\subsection{MAITLAND CASE STUDY: CONTEXT \& SOCIO-SPATIAL RESILIENCE ANALYSIS}

This chapter establishes the context of Maitland, a floodplain city in New South Wales, Australia where large-scale development is proposed. It discusses the impact the flood history and the implementation of extensive flood works has had on the city. The structure and function of the city, urban and residential scales is analysed. The flood resilience principles (scales, adaptation, variety and redundancy, feedbacks) and key findings from precedent analysis are applied to Maitland. This provides insight as to where socio-spatial resilience for floodplain inhabitants can be strengthened. 
Maitland is located 32 kilometers from the coastal centre of Newcastle (Bergsteiner, McInnes \& Rigby Pty Ltd, 1977) where the Hunter River meets the ocean. The Hunter River catchment and its tributaries make up an area of approximately 20,000 square kilometers, making it one of the largest coastal basins in New South Wales (Hunter Catchment Management Trust, 1998, p. 10). Maitland consists of four major suburbs: Lorn, Central Maitland, East Maitland and Rutherford. Central Maitland, rests on the low-lying floodplain of the Hunter River (six to eight metres above water level) and is separated from its neighbouring townships via agricultural land and the river. The entire area is flood liable, however particular areas experience greater exposure to more frequent flooding. The surrounding cleared agricultural land differs greatly from the diverse temperate rainforests, open woodlands and grasslands that originally covered the Maitland area (Walsh \& Archer, 2007).

Before European settlement indigenous Australian tribes hunted and gathered across the Hunter River catchment. The indigenous Australians were spiritually connected to the land and had an intrinsic understanding of its extreme drought and flooding forces (Walsh \& Archer, 2007). In 1818 the colonial Government selected high land to the east of the floodplain, now known as East Maitland, as the official site to erect the town due to the evident flood risks. However, the first Europeans defied settlement plans and progressively built on the agricultural rich floodplain known then as Wallis Plains (Walsh \& Archer, 2007).

Maitland owes much of its historical significance to the Hunter River as a water resource, a provider of fertile land for agriculture, forests for timber and as the main source of transport, until the railway line and highway were developed. Its history of flooding has also shaped the community, urban landscape and rural surrounds in fundamental ways (Keys, 2008, p. 1). Despite five floods inundating the area between 1830 and 1834 , settlement growth continued undeterred until the disastrous flood of 1955 (Hunter \& Maitland City Heritage Group, 2000). Rapid population growth during the 1830's and 40's caused widespread land subdivision, changes in ownership, and an irregular lot pattern that persists in present day Maitland (Bergsteiner, McInnes and Rigby and Maitland Council, 1977).
Flooding continued to be an intermittent problem, until in 1949 after a particularly disastrous flood, the NSW Government established the Hunter Valley Conservation Act 1950 in an effort to coordinate the Local, State and Commonwealth Government's flood mitigation practices (Walsh \& Archer, 2007, p. 71). The Hunter Valley Flood Mitigation Scheme, now known as the Hunter-Central Rivers Catchment Management Authority was formed and the trust invested \$250 million over forty years to establish:

" $160 \mathrm{k}$ of levees, $4 \mathrm{~km}$ of spillways, $120 \mathrm{~km}$ of flood drains, 245 flood gates, and $270 \mathrm{~km}$ of protected stream banks along the Hunter River and its tributaries in the Maitland area" (Wlash \& Archer, 2007, p.71.)

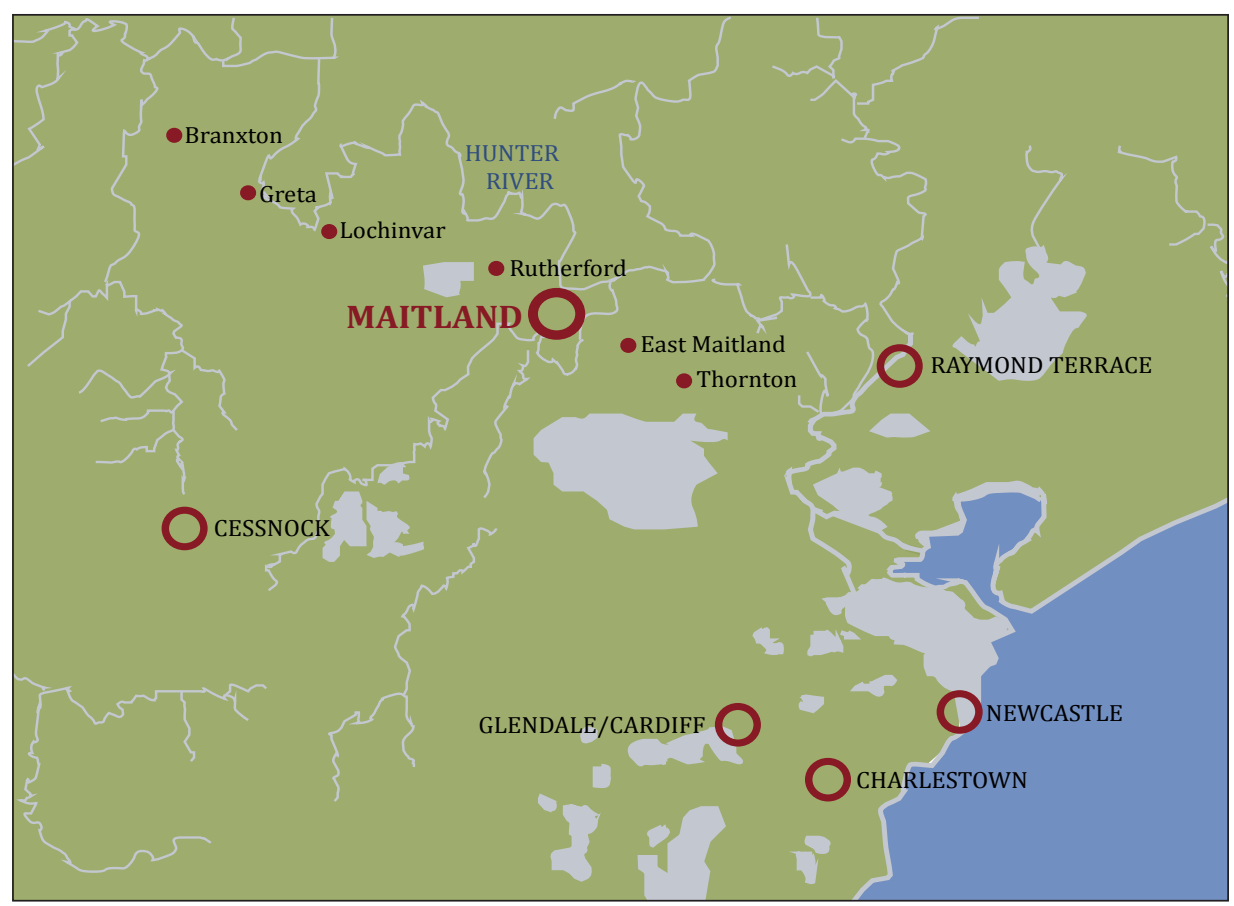

Figure 75: Maitland and the major town centres of the Lower Hunter Valley Region at 1:500,000 
Throughout history Maitland has flooded, an occurrence which will continue into the future.

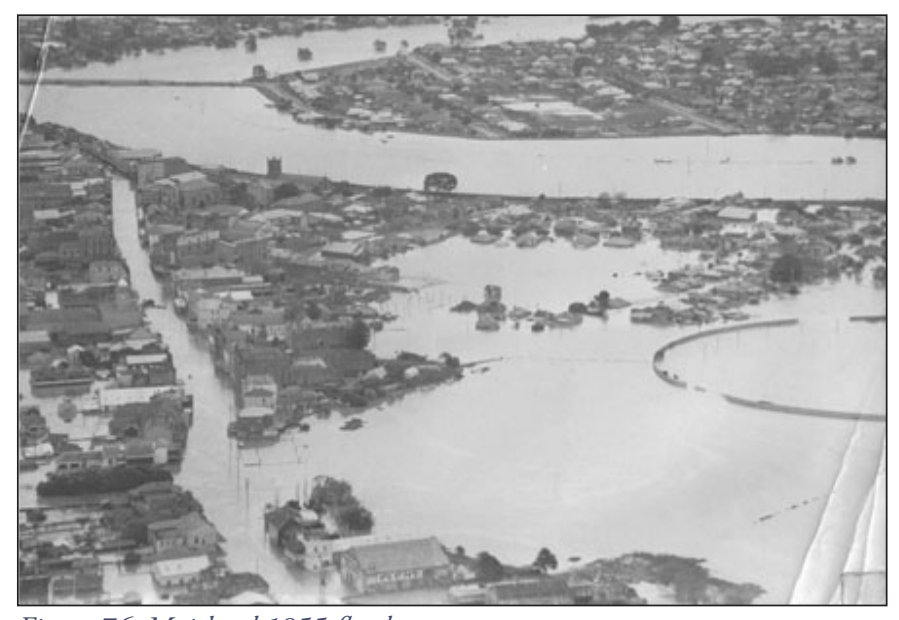

Figure 76: Maitland 1955 floods

[Photographer unknown] Source: Fiarleigh \& Doherty, (2008) p.23.

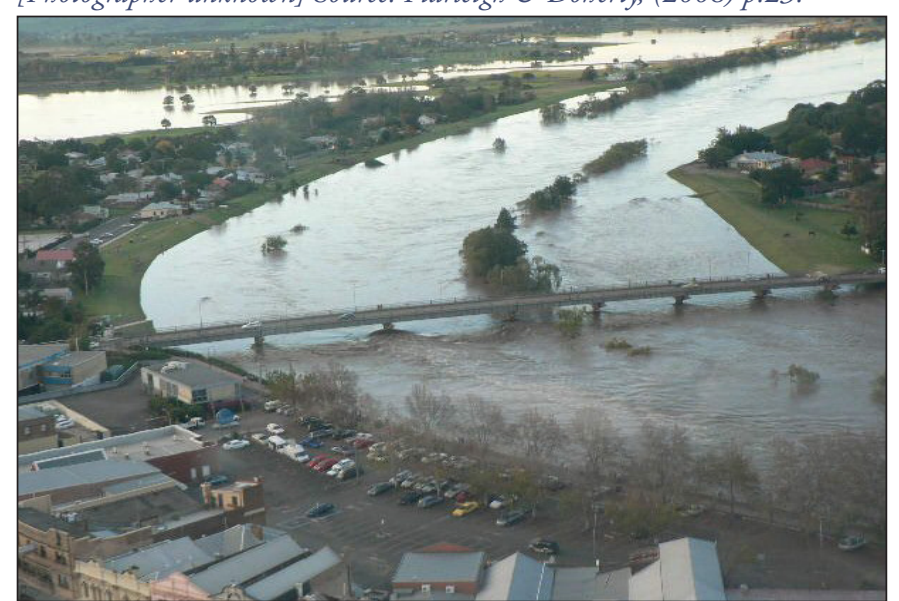

Figure 77: Maitland 2007 floods

[Photographer unknown] Source: NSW State Emergency Service (2007).

(www.bom.gov.au.nsw/sevwx/facts/events/june-07-3cllel-8-9jun.shtml.)
In February 1955 the flood scheme failed to hold back devastating floods. Eleven people died, thousands of homes were inundated and much of the city was destroyed (Keys, Learning to tame deadly floods, 2008). More flood works were engineered, existing structures were strengthened, and work has continued in an effort to prevent populated zones being inundated. The extensive flood mitigation scheme has reduced the frequency and severity of flooding on the Maitland floodplain. Nevertheless flooding continues to take place. The flood prone land continues to be rezoned and additional flood works implemented through the accumulation of flood data. Traditional approaches to floodplain development have focussed on preventing any further development. This has led to the degradation of the existing housing stock, the deterioration of services and infrastructure and a decreased population density in the township.

Rezoning previous flood liable land so it can be developed was "initially considered socially unacceptable and contrary to the [Maitland] council's aim of reduced residential development in flood prone areas" (City Plan Urban Design, November 2009). However, following the release of the Draft Lower Hunter Region Strategy, this is being reviewed as a possible long-term solution for the strategic planning of floodplain cities. The redevelopment of floodplains with higher density development, such as shop-top living and residential apartments, is being seen as a possible solution. These solutions enable residents to be elevated above anticipated flood levels and ensure development projects have a reduced footprint when compared to traditional single detached houses (Bewsher \& Grech, 1999). Councils across Australia are exploring a development driven solution in order to rejuvenate towns and cater for a growing population. 


\subsubsection{Maitland City Council development proposals}

The Floodplain Management Committee of Maitland (1994) believe regional flood works and levees operating around Maitland allow for further development to proceed in the area. This is due to the belief that there would not be a significant increase in flood hazard until the levees are overtopped (City of Maitland Floodplain Management Committee, August, 1994). However, the continued threat of floods must be recognised.

The flood prone nature of Maitland was most recently evidenced in the severe weather of June 2007 which saw extensive flooding of the Hunter River take place, with the highest peaks in thirty six years. Back flooding of Maitland took place and the levees held, but townships further downstream of Maitland, such as Newcastle, were badly flood ravaged. If greater storage space for floodwaters had been available on floodplains upstream, such as at Maitland, flood heights and thus flood hazards, would have been significantly decreased for these downstream townships.

Despite these recent flood threats development driven sentiment continues today. The Central Maitland Structure Plan (2010) indicates a key strategy is to "solve the problems that have made the flood liable area unbuildable" in order to reverse trends and promote population growth within the city (Maitland City Council, 2010, p. 3). Floodplain councils across Australia are facing similar challenges as a result of the adverse impacts flood prone land has for the long term sustainability of floodplain cities.

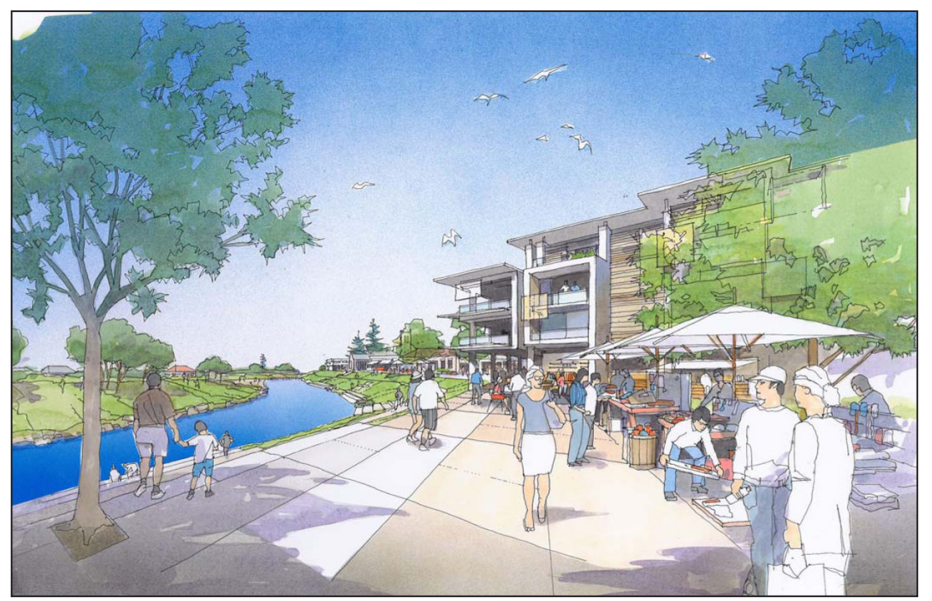

Figure 78: Maitland City Council's riverside vision for Central Maitland Source: City Plan Urban Design \& Maitland City Council (2009). p. 45

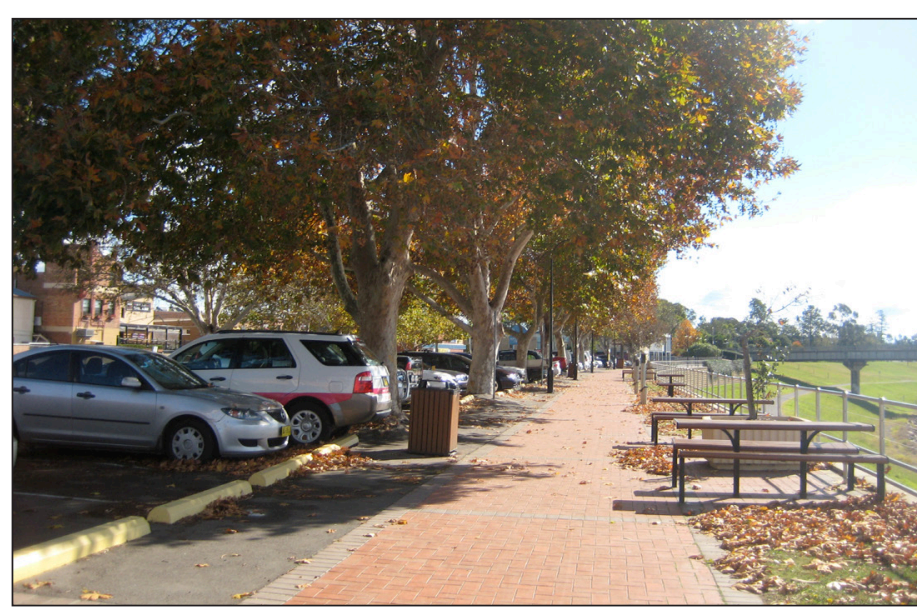

Figure 79: The current car park site 


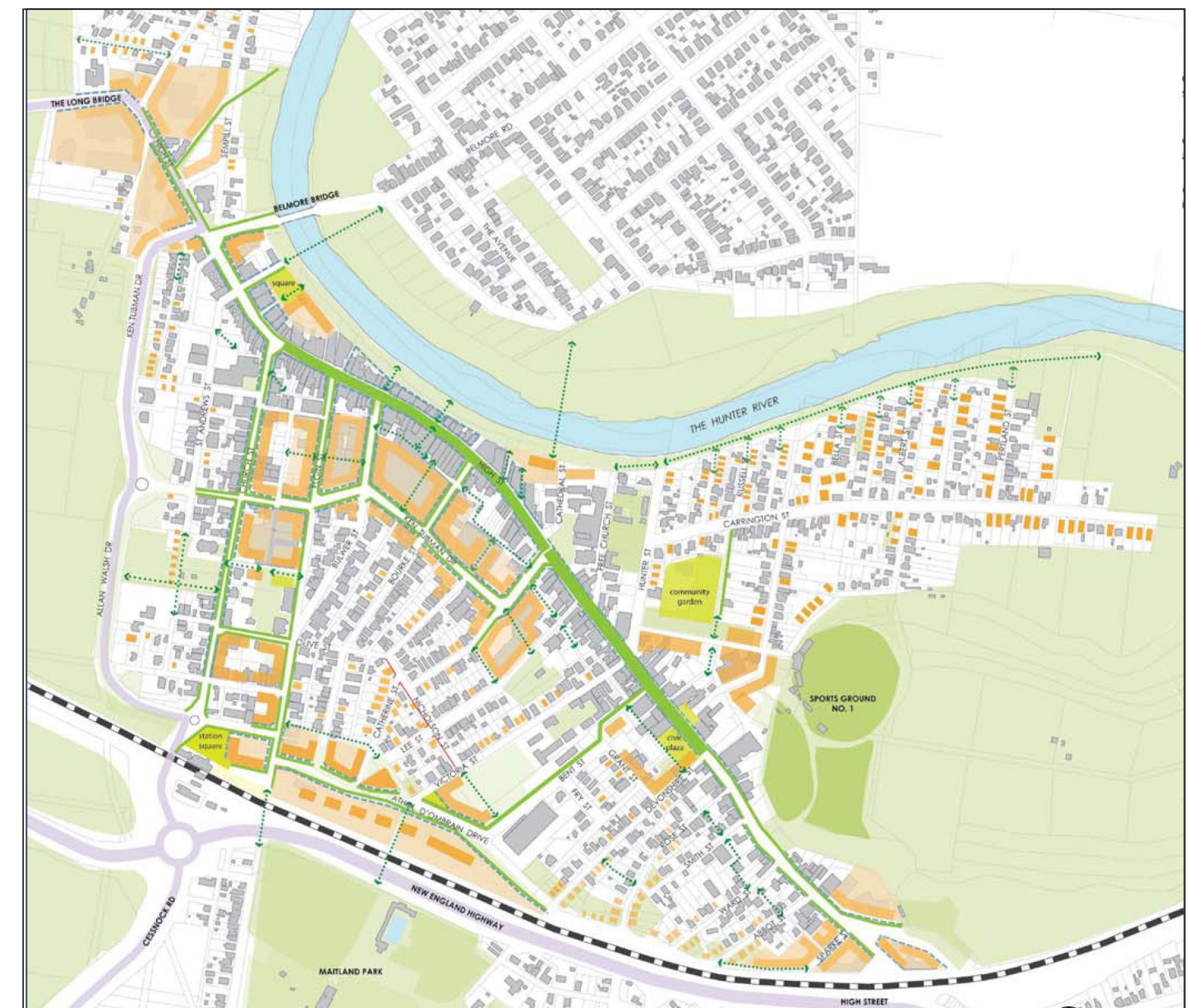

Pedestrian priority retail strip (mmi) Desired link

- Pedestrian domain upgrade

Indicative building mass

Indicative residential infil Existing buildings

Area for taller buildings

\section{Active ground floor}

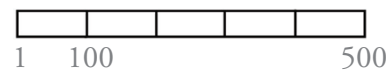

New public open space

Figure 80: The Central Maitland Structure Plan Source: City Plan Urban Design \& Maitland City Council, (2009) p. 46.
Whilst the Australian Federal and State Government's play a role in establishing overall guiding standards local government agencies, such as regional councils, are primarily responsible for implementing specific flood policies and measures to reduce their local flood risks. But with around 900 local government agencies in Australia, approximately half of which are flood prone to varying degrees, there is estimated to be several hundred different versions of floodplain management in Australia (Smith, 1998, p. 58). Consequently grave concerns accompany urban expansion and densification with escalating numbers of people exposed to the risks of flooding, without a uniform approach aimed at increasing resilience. Vulnerability is also being further increased from the effects of climate change.

Urban densification and sprawl continues to take place across Australia and is to persist into the future to cater for a growing population. With much of the inhabitable regions of Australia being flood prone, development is a widespread concern, but necessary and inevitable. It is therefore imperative that development is undertaken with a resilience approach that ensures the future inhabitation of floodplains does not increase flood severity and exposure levels. The flood characteristics, current flood mitigation strategies, social vulnerabilities and inhabitation traditions of Maitland need to be analysed. Applying the flood resilience principles and precedent analysis findings to the Maitland contextual findings provide further insight into how a socio-spatial flood resilience network can be established. It is time to explore the opportunities a socio-spatial flood resilience network provide for future floodplain inhabitants 


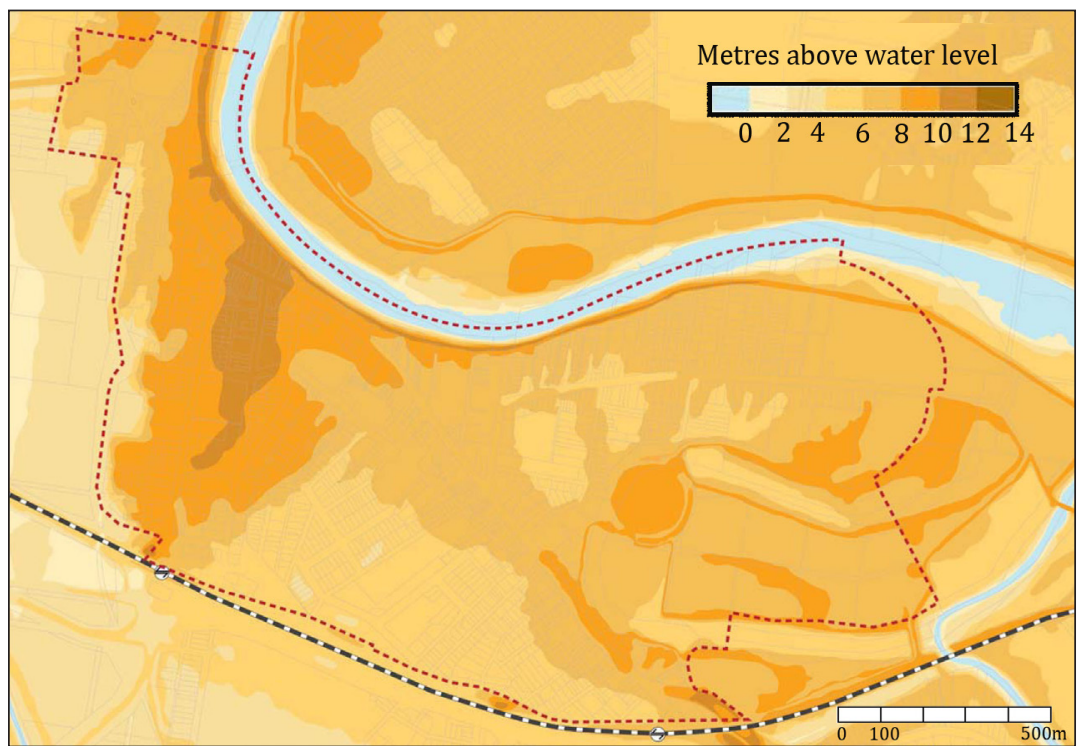

Figure 81: Topographic ground of Maitland

Source: City Plan Urban Design \& Maitland City Council, (2009) p. 31.

The majority of the land upon which Maitland resides is low lying. Maitland's major commercial zone resides on the highest topographical point of the land. The residential zones of South Maitland are the most low lying and thus prone to flooding. Industrial and agricultural buildings also reside on the lowest lying land of the area.

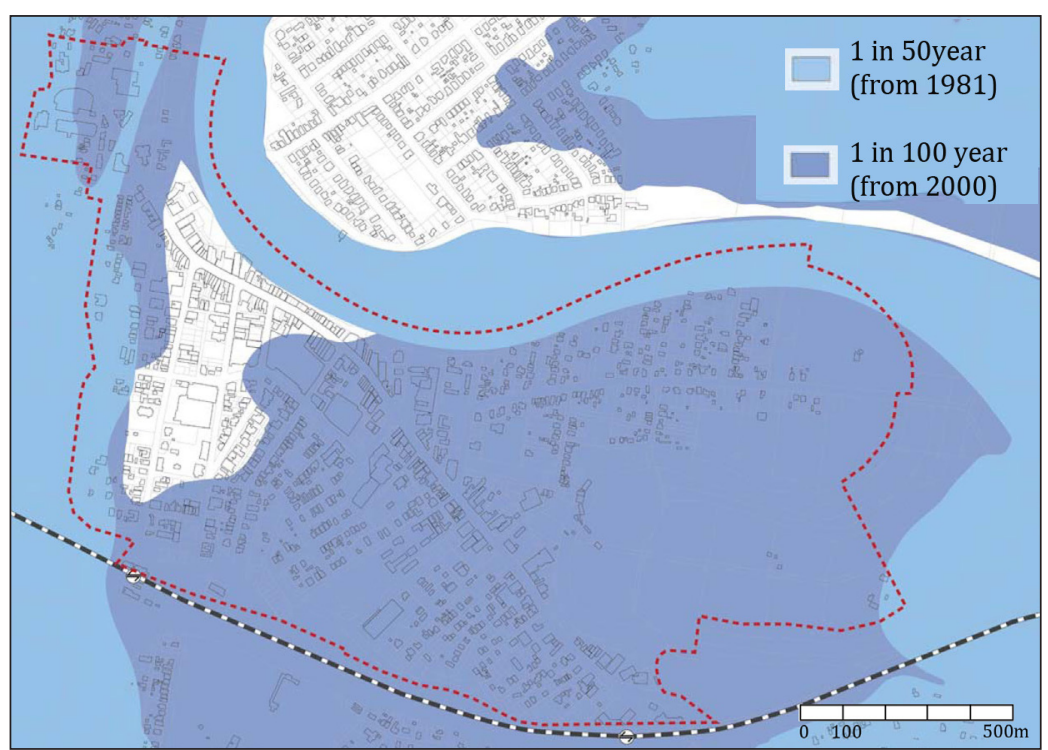

Figure 82: Impact of one in fifty and one in one hundred year floods on Maitland

Source: City Plan Urban Design \& Maitland City Council, (2009). p. 30.

A large portion of Maitland is flooded in a one in one hundred year annual exceedance probability event. Whilst all except for the highest topographical point of Maitland is subject to flooding in a one in fifty year event. The flood heights vary greatly within Maitland. These are determined by their topographic heights and land gradients. 


\begin{tabular}{|c|c|c|}
\hline \multirow{3}{*}{ RAPID RESPONSE: } & Region: & The Lower Hunter Valley Flood Mitigation Scheme. Agricultural land and floodplain acts as a water storage basin. \\
\hline & Suburb: & Ring levees, dry retention basins and flood gates are in place. \\
\hline & Building/site: & $\begin{array}{l}\text { Varying levels of static elevation, floodproofing and quality of masonry and timber construction. Masonry absorbs water } \\
\text { but maintains structural integrity up to a design flood standard, whilst timber varies in performance depending on type. }\end{array}$ \\
\hline \multirow{3}{*}{ ALTERNATIVE CIRCUITS: } & Region: & Hunter River and its tributaries transport water, but flood works attempt to control where it is collected and stored. \\
\hline & Suburb: & Back flooding results in main roads being cut and access in and out of Maitland becomes cut due to floodwaters. \\
\hline & Building/site: & Maitland roads carry and redirect floodwaters, but many footpaths and lots are insufficiently raised. \\
\hline \multirow{3}{*}{ CORE PROTECTION: } & Region: & The area is predominately agricultural cleared land providing water storage, although towns/cities are often isolated. \\
\hline & Suburb: & A lack of flood adapted infrastructure (roads, electricity, water or sewerage services hinders the cities function in floods \\
\hline & Building/site: & Elevated and flood proofing of houses reduces the impact of low velocity and low-medium volume floods. \\
\hline \multicolumn{3}{|l|}{ DIVERSITY \& REDUNDANCY } \\
\hline \multirow{3}{*}{ BUFFERING: } & Region: & Areas cleared for agricultural land use, whilst in other areas wetlands, marshland, forrests remain. \\
\hline & Suburb: & Natural foilage such as mangroves and reeds act to buffer, diffuse and store water. \\
\hline & Building/site: & $\begin{array}{l}\text { Timber pylons and floating timber construction will buffer water, with plastic/metal drums providing additional } \\
\text { buffering. Water already in the river and lake acts to buffer the velocity of flood waters entering the area. }\end{array}$ \\
\hline \multirow{3}{*}{ DIFFUSSION: } & Region: & Both natural meandering Mekong River and its tributaries along with artificial channelling for agriculture. \\
\hline & Suburb: & No water channels in place to tranport flood waters, but diversion channels re-direct floodwaters. \\
\hline & Building/site: & There is minimal landscaping on Maitland lots. Buildings will also minimally impact on flowpaths. \\
\hline \multirow{3}{*}{ STORAGE: } & Region: & Dams upstream of Maitland store water along with wet and dry retention basins including wetlands and floodplains. \\
\hline & Suburb: & Agricultural low lying land acts as dry retention basins. \\
\hline & Building/site: & Few rainwater tanks. NSW Govenrment's BASIX legislation encourages the use of rainwater tanks for non potable uses. \\
\hline \multirow{3}{*}{ ABSORPTION: } & Region: & Watershed soil conditions (dry/damp/wet) \& vegetative cover (volume/type) determine efficiency of water transfer. \\
\hline & Suburb: & Vacant lots and vegetative zones improve absorption rates. Climate eg drought and heavy rain influence absorption. \\
\hline & Building/site: & Small or no building footprints increase area for absorption. \\
\hline \multirow{3}{*}{$\begin{array}{l}\text { NUTRIENT \& } \\
\text { WASTE RECOVERY: }\end{array}$} & Region: & Natural wetland and reed bed ecosystems filter the water. \\
\hline & Suburb: & Stormwater management and sewerage systems are in place in Maitland. However are unable to cope in high floods. \\
\hline & Building/site: & No artificial grey water systems in place, however local gardens and vegetation filter wastes and absorb nutrients. \\
\hline \multicolumn{3}{|l|}{ FEEDBACK } \\
\hline \multirow{3}{*}{ RESPONSE: } & Region: & $\begin{array}{l}\text { Hunter Valley Flood Mitigation, Maitland City Council Hunter River Floodplain Management, warnings and alerts issued } \\
\text { by the Bureau of Meterology are broadcast on the Australian Broadcasting Corporation radio station. }\end{array}$ \\
\hline & Suburb: & Flood guages, water markers, flood warning systems e.g. alarms \\
\hline & Building/site: & Local flood educated awareness and preparedness e.g. flood proofing measures and household flood emergency kits. \\
\hline \multirow{3}{*}{ REGENERATION: } & Region: & Reliance on government financial aid to repair damaged infrastructure. SES volunteers aid. Agriculture is impacted. \\
\hline & Suburb: & Reliance on external aid for vital services: food, water and power in during floods and in recovery periods. \\
\hline & Building/site: & Flood insurance reduces financial impact. Government disaster emergency grants provide further recovery funding. \\
\hline \multirow{3}{*}{$\begin{array}{l}\text { COOPERATIVE } \\
\text { SELF-REGULATION: }\end{array}$} & Region: & Reliance on external aid for evacuation post inundation due to lack of mobility options. eg NSW SES volunteers \\
\hline & Suburb: & Maitland City Council flood evacuation and emergency plans. \\
\hline & Building/site: & $\begin{array}{l}\text { Local flood experience improves response actions. But low mobility and flood proofing hinders response. } \\
\text { Emergency shelters set up at Maitland High School and East Maitland provide first aid, power food and water. }\end{array}$ \\
\hline
\end{tabular}

Figure 83: Resilience flood management matrix analysis of Maitland, Australia 


\subsubsection{Maitland flood characteristics and flood mitigation strategies}

Floods in Maitland are characteristically low in velocity and vary substantially in depth, depending on the topography and proximity of flood preventative structures (City Plan Urban Design, November 2009, p. 30). A series of control banks in the Hunter River act to reduce floodwater velocities, however high velocity catastrophic flood events can still be caused by the structural failure of dams positioned upstream from Maitland. Inundation periods of the low lying agricultural land can be up to several weeks, with the floodplain becoming a dry retention basin. Wallis Creek and Fishery Creek swamps similarly become temporary storage basins for floodwaters, before they re-renter the Hunter River. Flood peak heights can also be dramatically affected from the tidal nature of the Hunter River up to Maitland's Belmore Bridge.

As a result of the Lower Hunter Valley Flood Mitigation Scheme (LHVFMS) controlled backwater flooding occurs at South Maitland and Lorn, the suburbs geographically furthest away from the river. South Maitland is protected by a ring levee up to approximately a 5\% AEP event, whilst Lorn is protected by three levees. Floodgates on the creeks reduce the occurrence of backflow of floodwaters. A 2\% AEP event overtops levees inundating Central Maitland and Horseshoe Bend (City of Maitland Floodplain Management Committee, August, 1994). Thus it must be noted that the severity of a $1 \%$ AEP or $0.5 \%$ AEP are not reduced by the LHVFMS (City of Maitland flood risk management plan 2007).

Levees stretch along a majority of the Hunter River from Oakhampton to Morpeth and continue to protect urban areas further downstream. These levees aim to confine floodwaters to the main channel up to a $10 \%$ AEP event and prevent flood inundation to nearby urban and rural areas. For flood events larger than a 10\% AEP floodwaters will overtop upstream levees, releasing floodwaters into spillways No.1 and No.2 at Oakhampton on the west bank and into the zig-zag Bolwarra spillway on the east. These spillways act to direct water into floodways or flow paths.

Levee construction, river diversion channels and cut-offs have dramatically altered the Hunter River's path and length. The length of the river has been shortened from 27 to 9 kilometres between Maitland and Morpeth between 1860 to 1965 (Hunter Catchment Management Trust, 1998). This has significantly reduced the rivers water carrying capacity. Although the LHVFMS has reduced the frequency of flood inundation in Maitland, the hard edge structural solutions have had farreaching and devastating impacts on the environment. Land degradation, water salinity, soil erosion, reduced wildlife biodiversity and the loss of significant riverine ecosystems, such as wetlands, increase long-term flood and drought risks in the region. Mileti (1999) believes "natural and related technological catastrophes of the next millennium are likely to be larger than any ever before experienced" (Mileti, 1999 , p. 35) as a result of climate change, ecological degradation, increased flood mitigation measures and the urban expansion of increasing populations. Predicted changes in climate, such as increased precipitation rates, will also alter previously set flood prediction levels and cause further vulnerability unless unpredictability begins to become an intrinsic component of planning for the future.

\section{Flood characteristics for medium to high flood events}

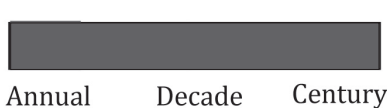

Frequency

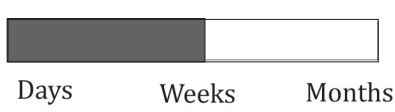

Duration

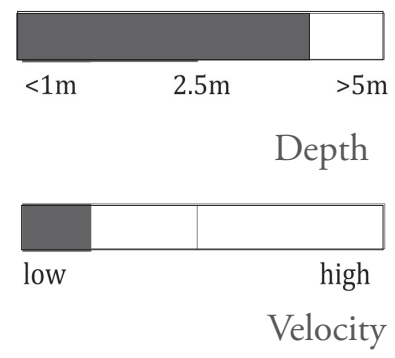




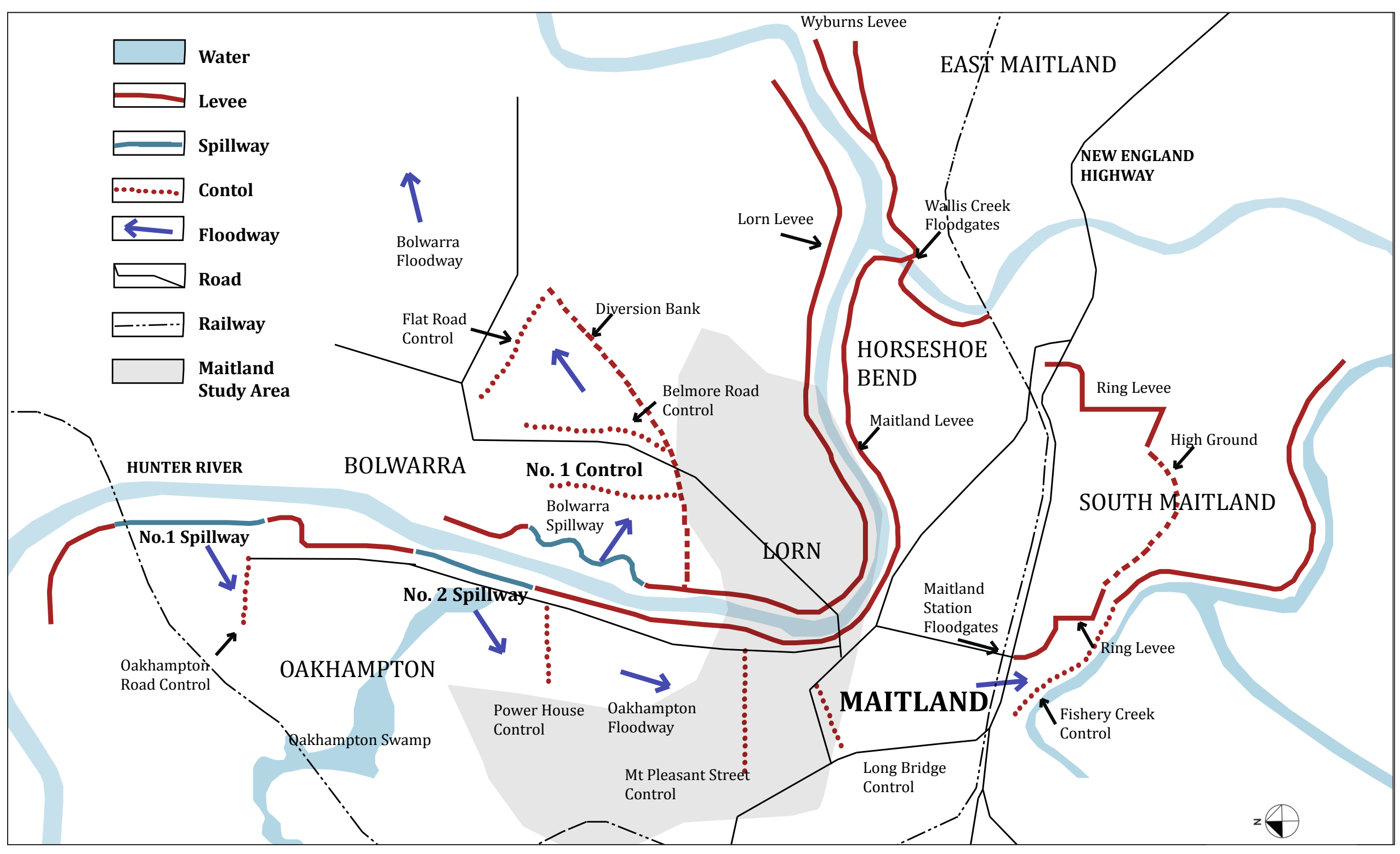

Figure 84: The Maitland Floodways Scheme Source: Authors image adapted from a sign on the Maitland levee produced by the Hunter-Central Rivers Catchment Management Authority (CMA). 
The most recent Census (2006) recorded 61,880 for Maitland's population, which was an increase of 8,497 from 2001 data. This inevitably means there are greater numbers of people now exposed to flooding. Although flood mitigation measures have greatly reduced the prevalence of flooding in Maitland, flood vulnerabilities have increased as a direct consequence of a socially perceived flood safety. According to Chas Keys, "it is not universally understood or acknowledged locally, but the city of Maitland occupies one of the most flood prone areas in Australia" (Keys, 2008, p. 5). Maitland's flood history has caused "massive dislocation to human activity, destroyed much that was of value and brought desperation to many individuals" (Keys, 2008, p. 5). However as Keys highlights communities that occupy floodplains, such as Maitland, cannot afford to perceive floods as negative, with much of its agricultural and industrial sectors being highly reliant on periodic flooding. The flooding ensures local ecosystems continue to provide the services and functions upon which they rely, for example rich soils, timber products and fishing.

Cities such as Maitland are progressively deteriorating due to a lack of development opportunities on the flood prone land. In Maitland, this has lead to a decline in the housing stock standard and eventually a socio-economic stigmatisation (Bewsher \& Grech, 1999). The socio-demographic context of Maitland's inhabitants is summarised (City Plan Urban Design and Maitland City Council, November 2009):

- The 2006 Census recorded 61,880 for Maitland's population, 8,497 more than 2001 data.

- 20-40\% low income households, compared to 4-9\% high income households

- Average household income is below the NSW state average

- One parent families are higher in ratio that national averages

- An average 2.35 people per household, compared to a 2.6 NSW state average.

- Average age in Maitland is 41 years

- Low house prices and high flood insurance (due to flood prone nature of the land)

- Low car ownership levels (20\% of households are without vehicles)

- The majority of residents travel to work outside the city (via car, bus or train)
The annual exceedance probability of floods greatly impacts social vulnerability and flood damage to infrastructure and housing stock. The majority of buildings in Maitland are single storey, with some two storey building examples and a few three storey commercial buildings. Maitland City Council has not promoted initiatives such as house raising and flood proofing, as these would prove insufficient in many locations due to the large depths experienced in numerous locations. Although it must be noted that there are a few examples of statically elevated houses in Horseshoe Bend and South Maitland, which have been successful in surviving previous floods. The council has at different historical points established land buy back schemes for those houses in high risk flood zones. The most notable came following a succession of floods in the eighties. Calls were made to relocate Maitland up onto the high topographical land of East Maitland. But these proposals were inevitably strongly opposed and rejected.

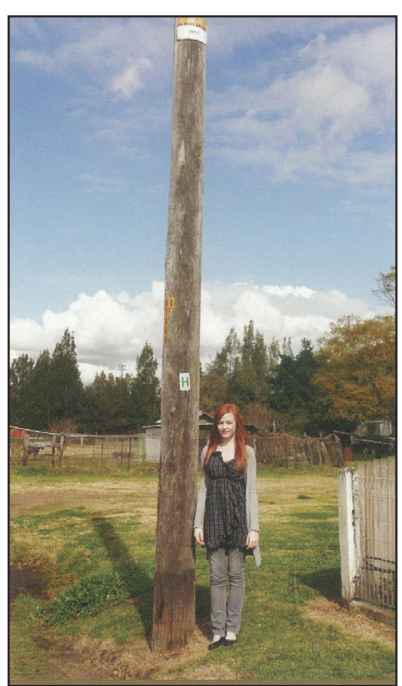

Figure 85: 1955 Flood height marker 4.2m in Central Maitland [Photographer unknown] Source: Keys, (2008), p.20. 


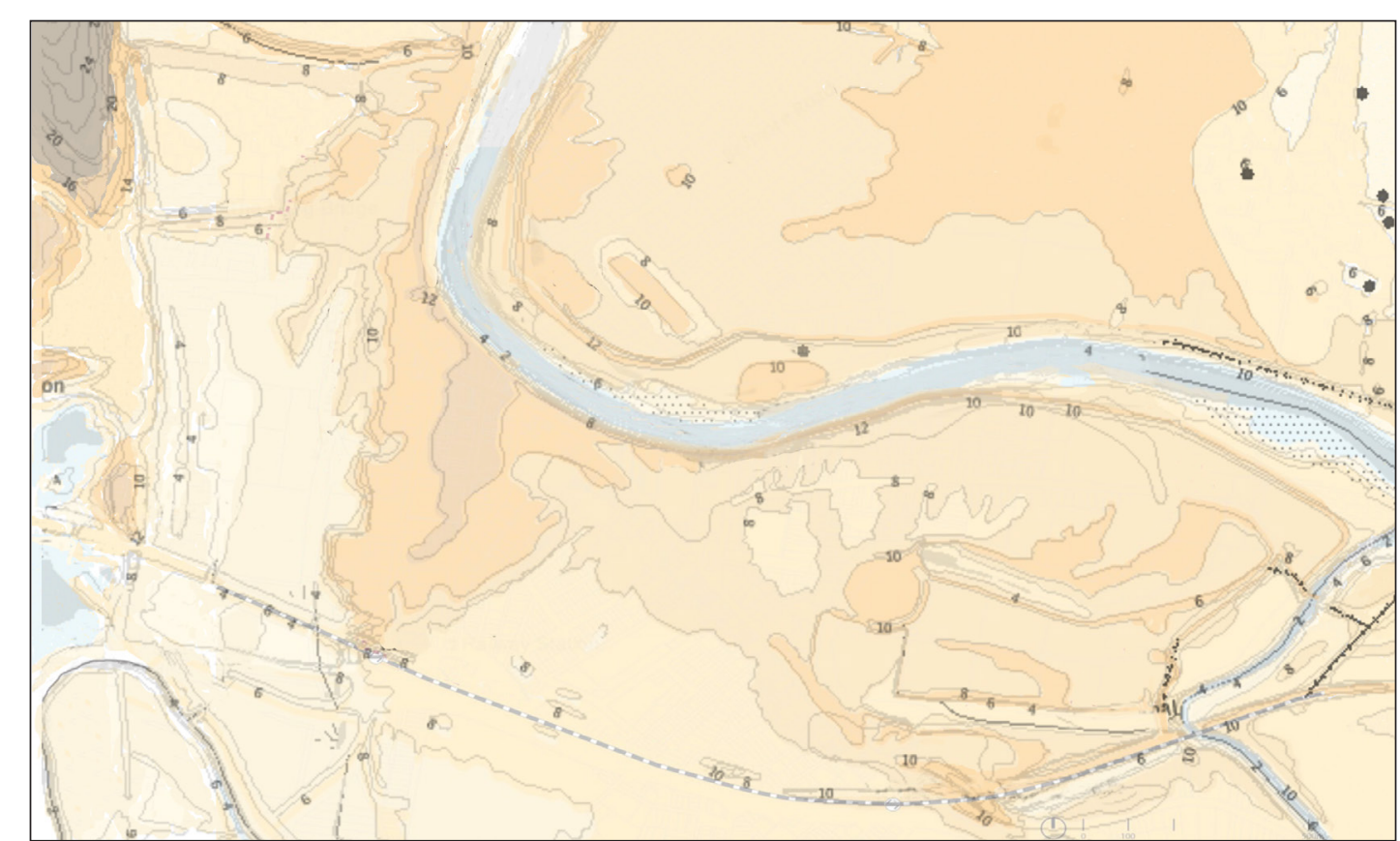

Figure 86: Maitland topographic map Source: Authors image constructed using information from City Plan Urban Design \& Maitland City Council, (2009) p. 31.
Due to back flooding in Maitland taking place prior to the levees being breached residents need to be evacuated several hours prior to the flood peak, with Maitland road and train access routes being cut by floodwaters. Time versus height graphs from hydraulic models indicate the timing of inundation across the floodplain for anticipated flood events. Whilst these aid in evacuation processes it must be recognised these are only estimations. They do not take into account local site conditions, but are based on regional topographic land characteristics.

The effect floods can have on Maitland was most recently evidenced in 2007, when it was feared floods would breach levees. Around 4,000 people were evacuated from Maitland in anticipation of peak flood heights. Electricity and water mains were cut, sewerage lines damaged and many stormwater drains were not functional during floods. Access to food supermarkets and the hospital, became limited or non-existent during floods. Community flood education and awareness directly influenced the uptake of flood preventative measures and ownership of flood survival kits. Evacuation centres were established on the higher topographic land of East Maitland following evacuation calls by State Emergency Service volunteers on the $\mathrm{ABC}$ radio and door knocking.

The Australian Broadcast Corporation radio station is the Australian Government's official broadcaster of weather warnings. The Australian Bureau of Meteorology is a non-government organisation which is responsible for advising the Australian Government, citizens and involved parties, such as SES volunteers of flood risks and warnings. SES volunteers are responsible for instigating evacuation procedures and post flood recovery processes. Local evacuation procedures are also put in place by the Maitland City Council Floodplain Management Committee. These nationally established organisations and government bodies have greatly reduced safety concerns, improved evacuation processes and reduced post flood recovery time. However, improving the ability of individuals to remain connected to neighbouring residents and maintain access to local services and city infrastructure, would further reduce the adverse impact of a range of floods in Maitland. 


\subsubsection{City scale analysis}

Central Maitland focus area

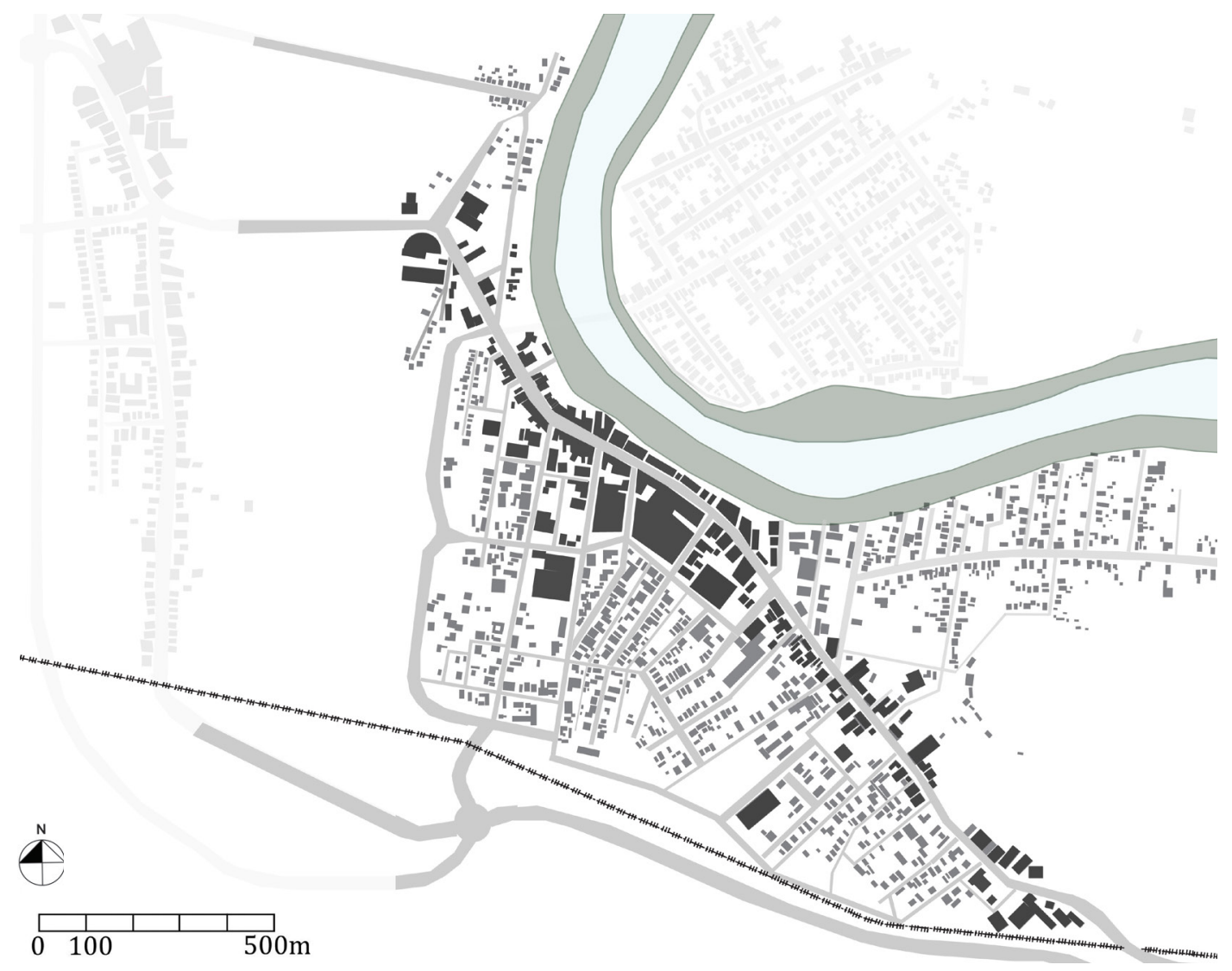

Figure 87: The focus area of Maitland with the adjacent townships of Lorn and Morpeth

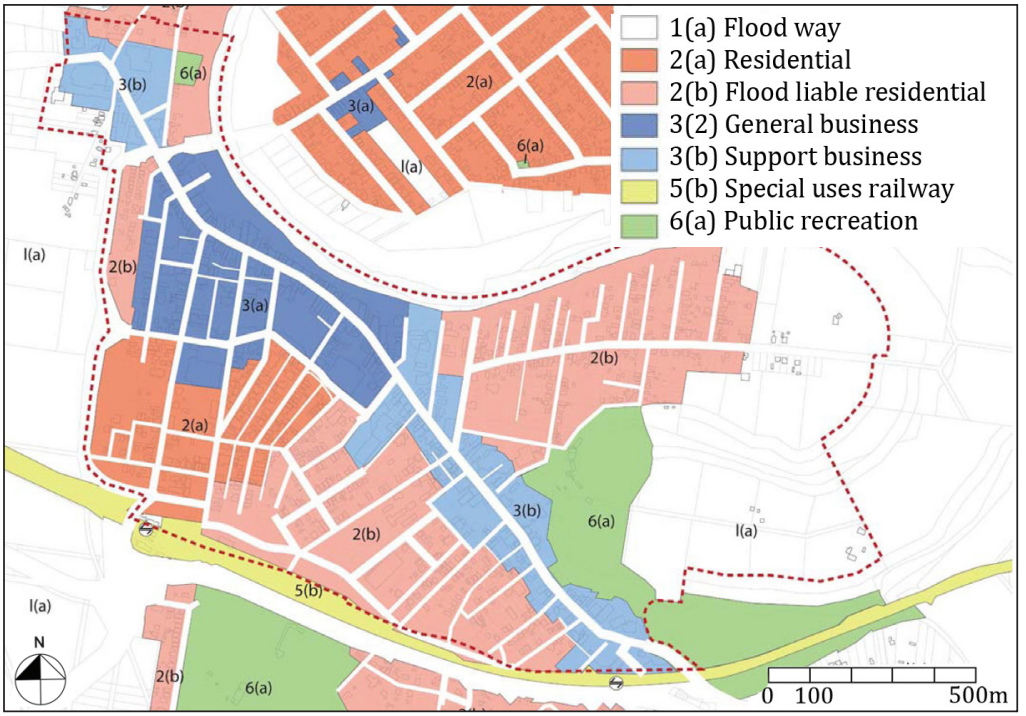

Figure 88: Existing land use zoning: a large portion of the residential area is flood liable Source: City Plan Urban Design \& Maitland City Council, (2009). p. 35.

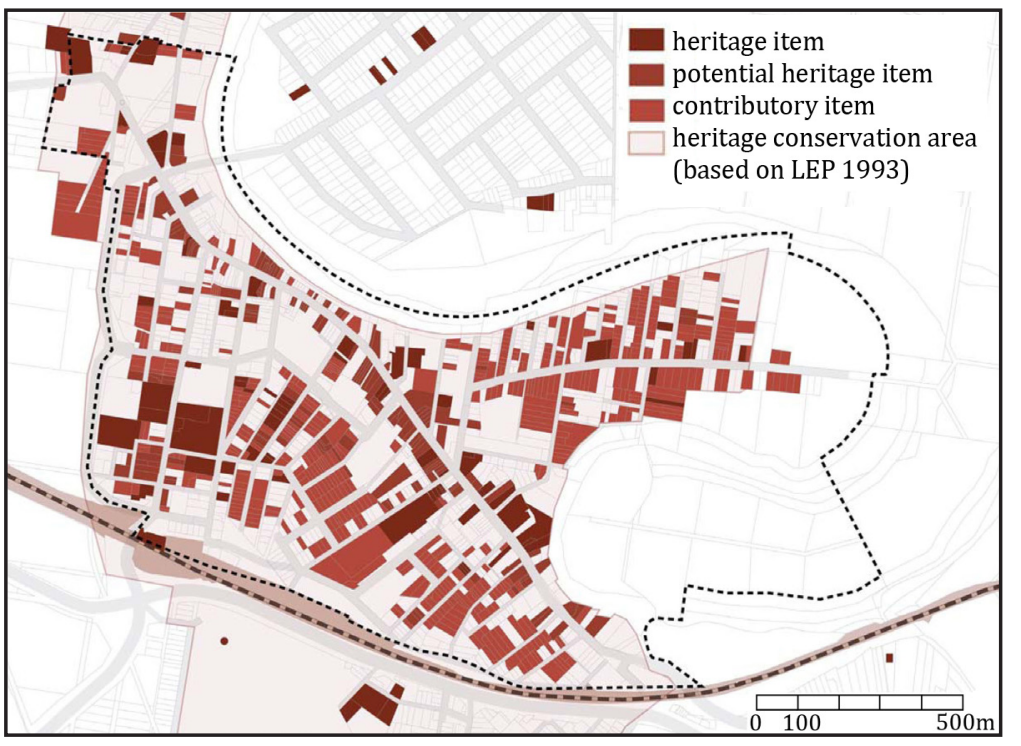

Figure 89: Assessment of heritage value: flood proofing standards were implemented in 1970 Source: City Plan Urban Design \& Maitland City Council, (2009) p. $24 .$. 


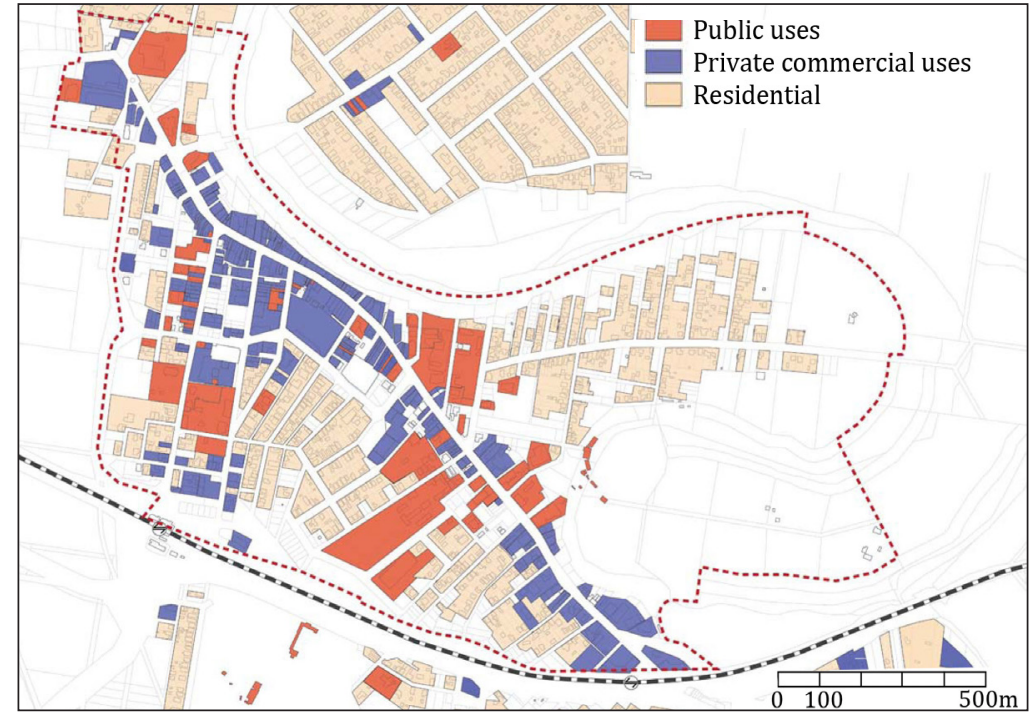

Figure 90: Existing land use: building function and economic value influence flood proofing standards Source: City Plan Urban Design \& Maitland City Council, (2009), p. 34.

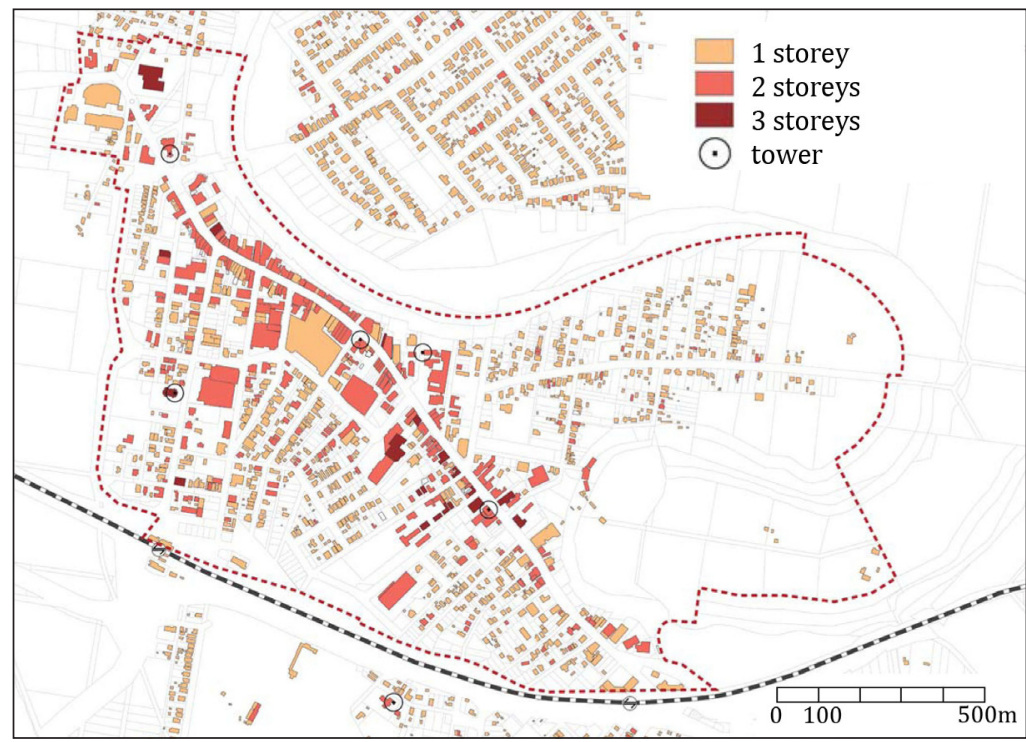

Figure 91: Existing building heights: locating living spaces above ground level reduces risk. Source: City Plan Urban Design \& Maitland City Council, (2009). p. 33.

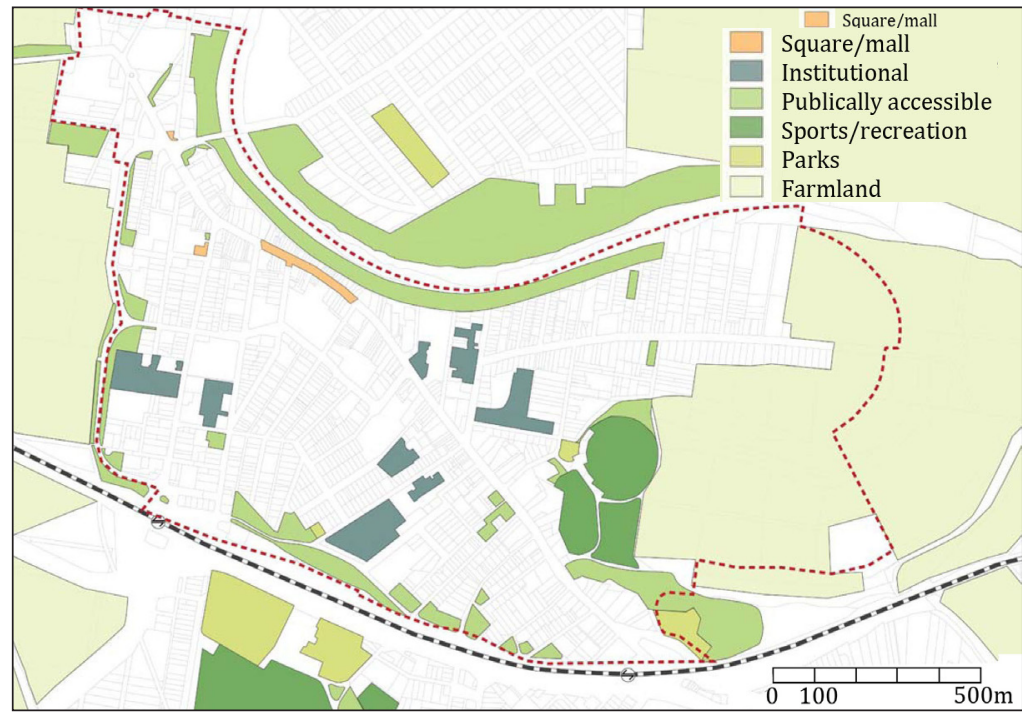

Figure 92: Existing open space typologies: green zones function as dry retention basins. Source: City Plan Urban Design \& Maitland City Council, (2009). p. 36

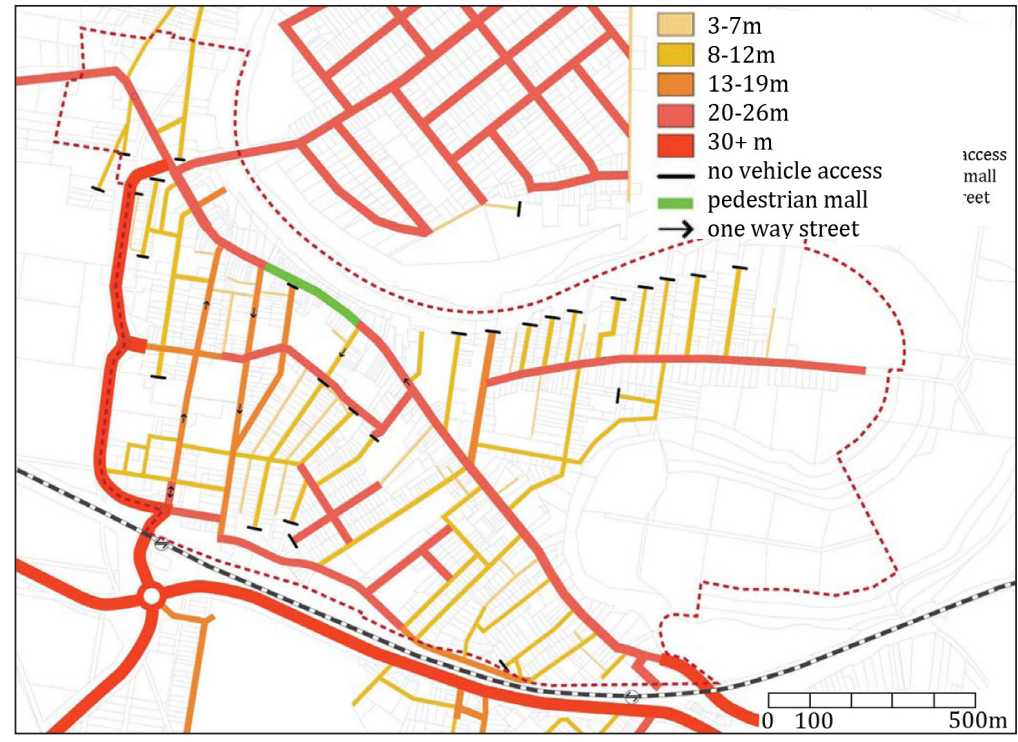

Figure 93: Access and circulation: road widths determine the carrying capacity of flood waters. Source: City Plan Urban Design \& Maitland City Council, (2009). p. $38 .$. 
Block and street scale opportunities within the Maitland suburb of Horseshoe Bend.

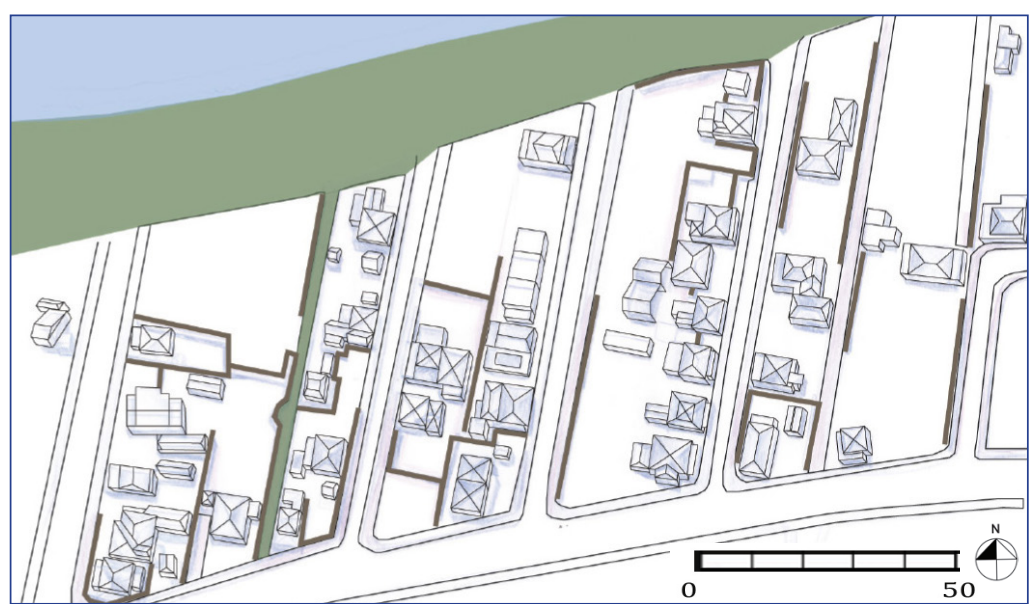

Figure 94: Residential block densities

$$
0
$$

Existing houses

Levee

Hunter River

Dxisting fences

Response: Relationship between houses and circulation paths

Diffusion: Irregular buildings positioning directs water, whilst roads transfer it.

Buffering: Fences and foliage reduce the velocity of floodwaters.

Regeneration: Levee creates barrier between residents and river; no visual axis.

Absorption: Vacant lots increase the amount of water penetrable land.

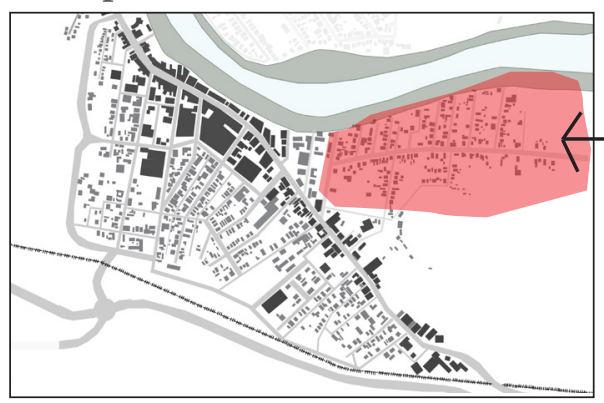

Horseshoe Bend, Maitland

urban and residential study area

Figure 95: Horseshoe Bend, Maitland

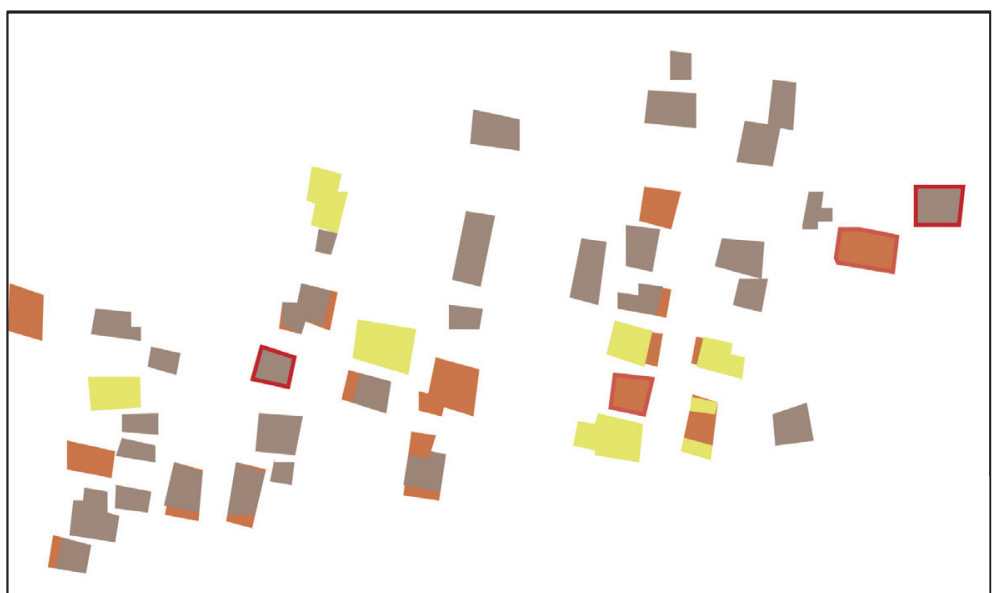

Figure 96: Existing building construction types

Masonry construction

Timber construction

Masonry verandah with timber construction

Elevated timber building on masonry plinth

Elevated timber building, masonry verandah

$\square$ Elevated timber building on columns

Elevated masonry building on columns

Rapid response: Permanent flood proofing measures of elevated timber floors and masonry construction reduce vulnerabilities.

Core protection: Elevated services, flood resistant materials, self reliant systems and building strength (at a variety of flood heights). 


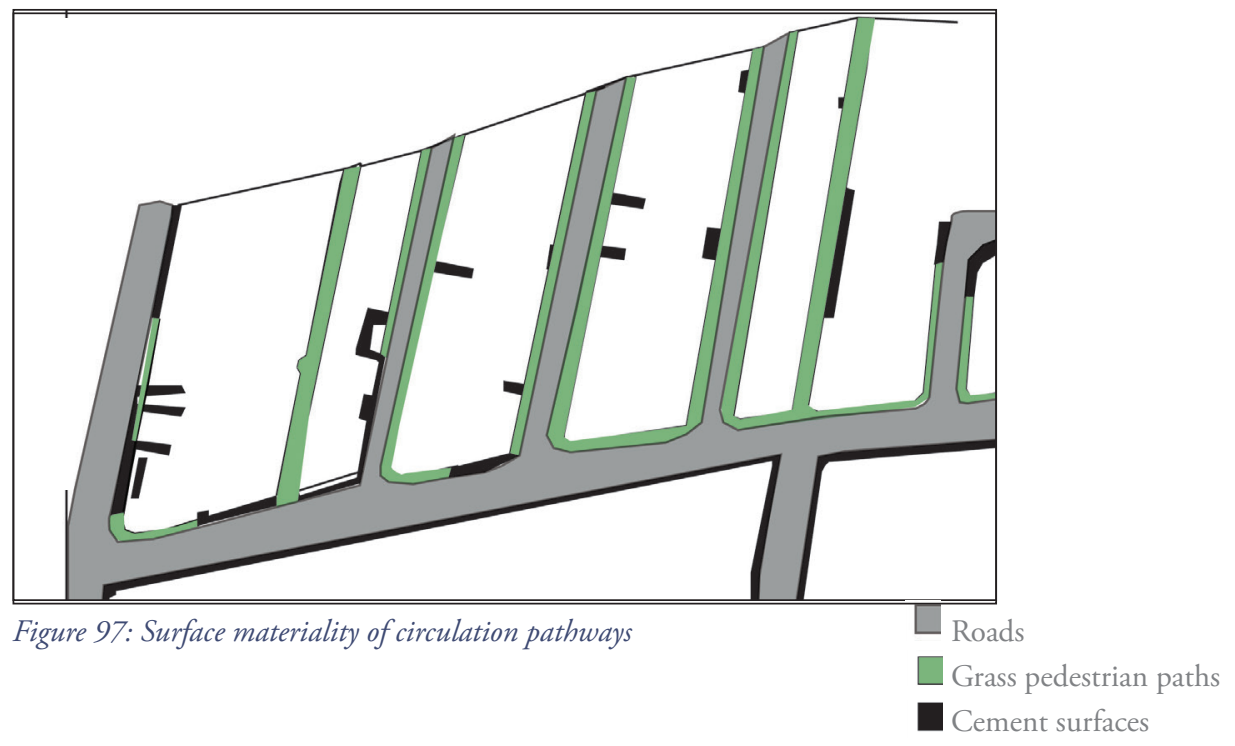

Alternate circuits: Back paths provide additional paths to roadways, however there is a lack of raised circulation options in Horseshoe Bend.

Absorption: Surface permeability of circulation pathways is increased by grass rather than concrete pedestrian sidewalks in the area. Vacant lots also increase the amount of water penetrable land.

Diffusion: Roads act to linearly direct water flow if the levee was to breach. An underground stormwater drainage system is not operational on all roads in the area. Storage capacity: Nearby agricultural land provides dry retention basins for floodwaters. Vegetation covered vacant lots increase the area available for water.

Rapid response: There is a lack of infrastructure solutions in the area to facilitate rapid response to floods. Prior to the levee breaching the city begins to be flooded as a result of a river diversion channel. This results in the city becoming isolated as a result of the main access roads being inundated.

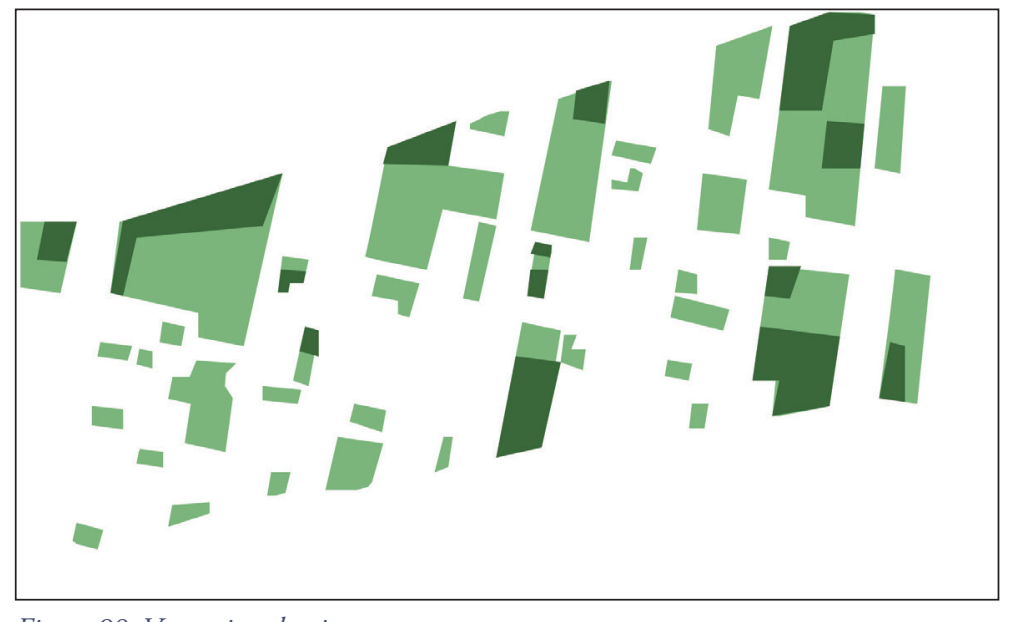

Figure 98: Vegetation density

Absorption: Surface permeability, housing density and water retention areas. Foliage aids soil in absorption capabilities. Seasonal climatic environments impact on soil absorption efficiencies (saturated or dry).

Buffering: Foliage separates residences from river, but steep grass levee increases velocity of floodwaters, reducing the efficiency of vegetative buffers.

Storage: The low lying land acts as a dry retention basin, both in the urban and nearby agricultural land. 
The three main residential typologies of Horseshoe Bend, Maitland.

Weatherboard timber construction;

Depending on the grade, water resistant type and dimensions timber varies in its flood performance. Immersion in water can cause the material to warp, however its innate elasticity ensures structural stability is minimally impacted upon. The timber must be thoroughly dried following floods to prevent rotting and mould issues.

Masonry construction is an example of a wet proofing solution within Maitland. Mortar types influence erosion rates and the uptake of water. Cracks may result is hydrostatic increases past a critical threshold, at which point structural performance may be affected.

Timber construction elevated on columns endeavours to raise living spaces above hazardous flood levels. The timber or concrete column dimensions, depth of submersions, and cross bracing support influence the structural stability of the structure when impacted upon by moving floodwaters.

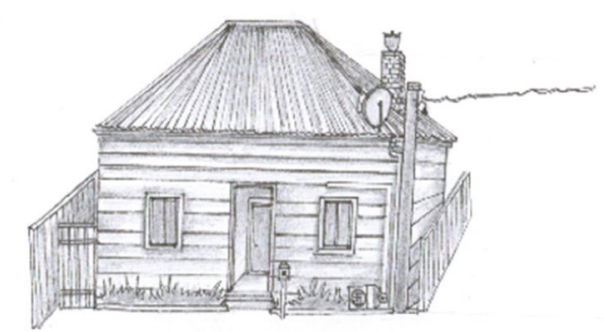

Weatherboard timber construction

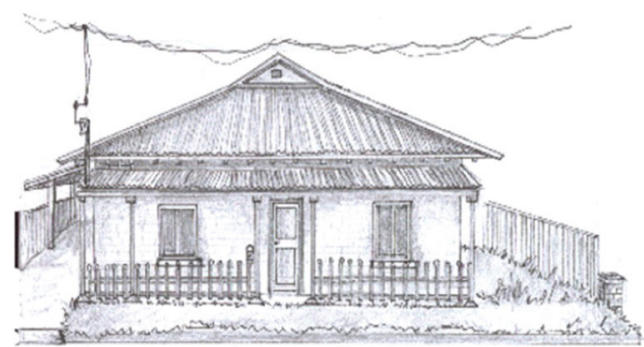

Masonry construction

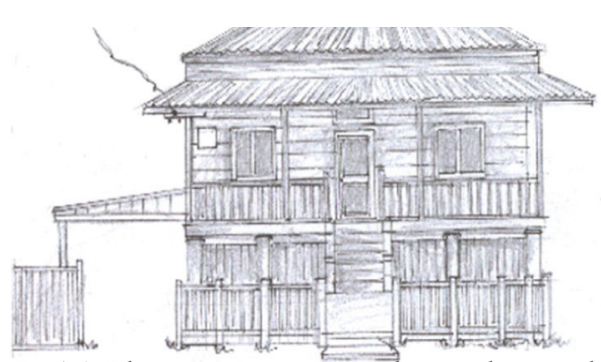

Timber construction elevated on columns
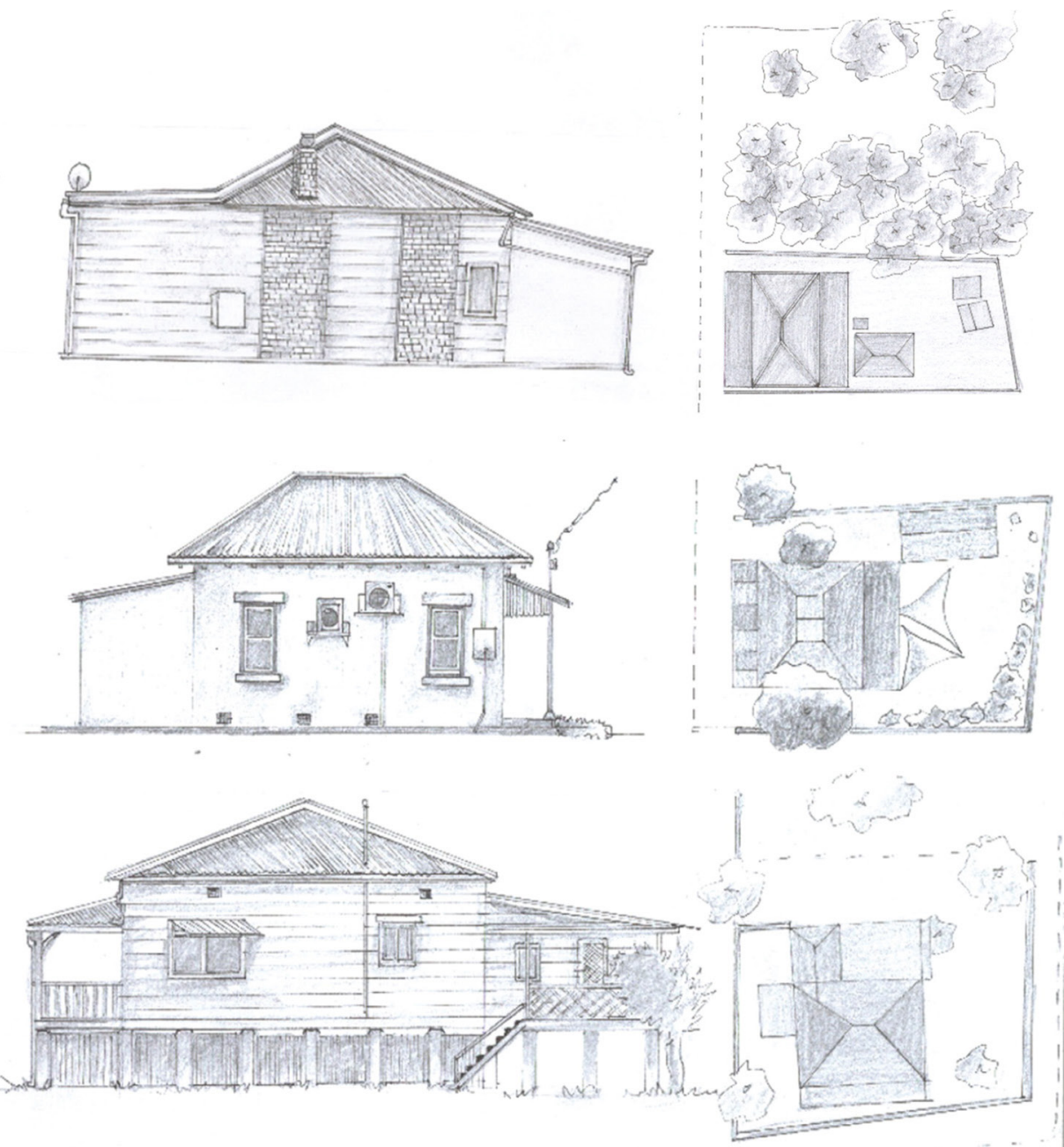

Figure 99: Horseshoe Bend residential typologies 


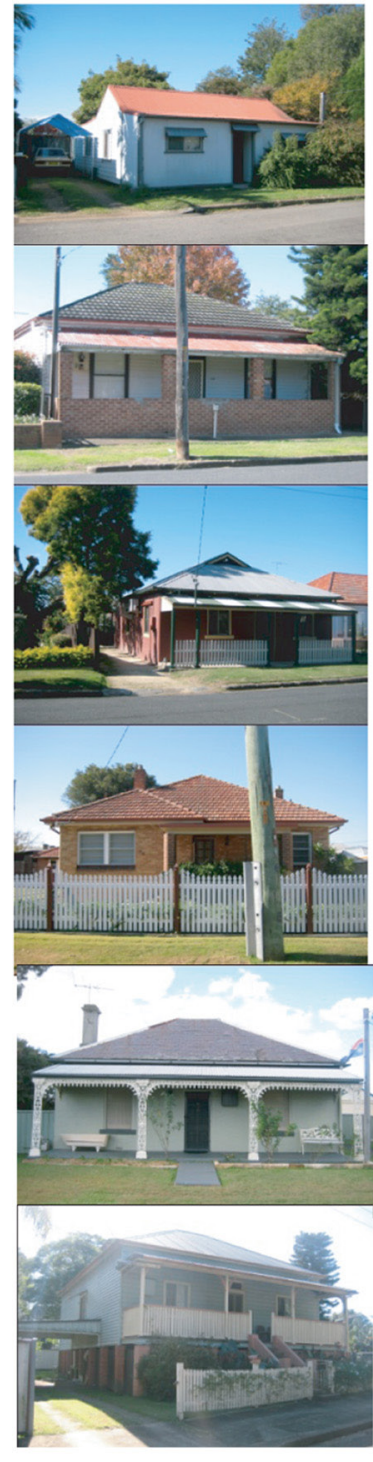

Figure 100: Houses within Horseshoe Bend, Maitland

The six main residential construction types represented in Maitland. The styles are both representative of Australian architectural history, but also the influence flood events have had on building types and construction.

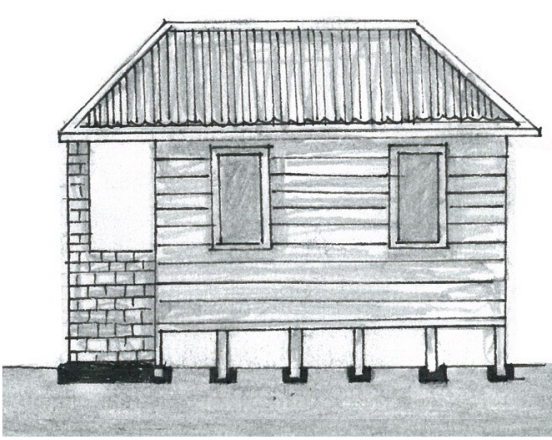

Timber frame on timber posts with a masonry verandah covered entrance.

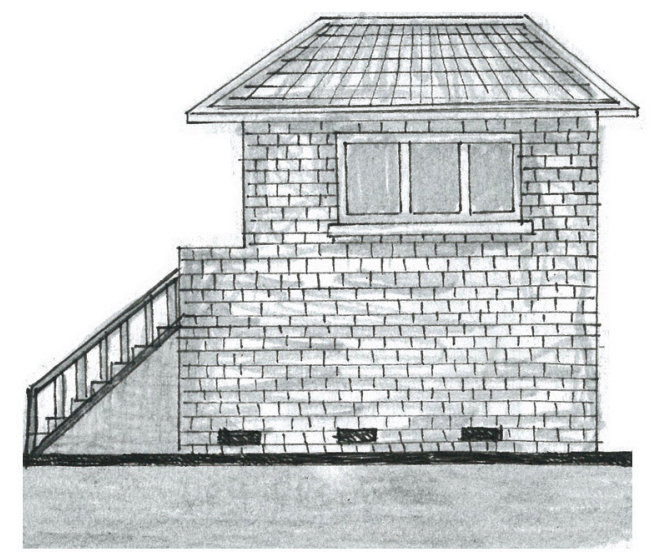

Masonry construction on a concrete foundation, and concrete stairway

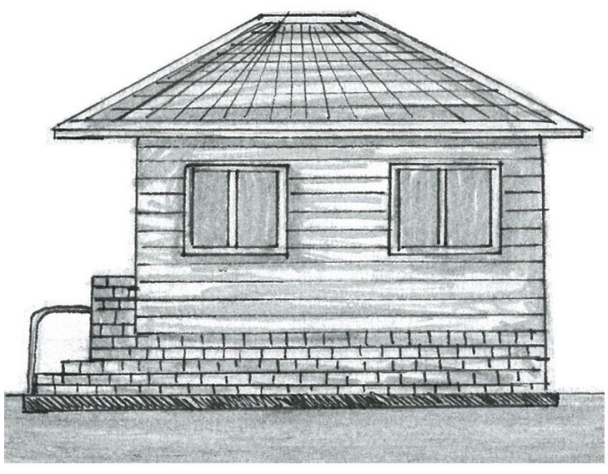

Masonry perimeter wall, timber posts and timber construction resting on top.

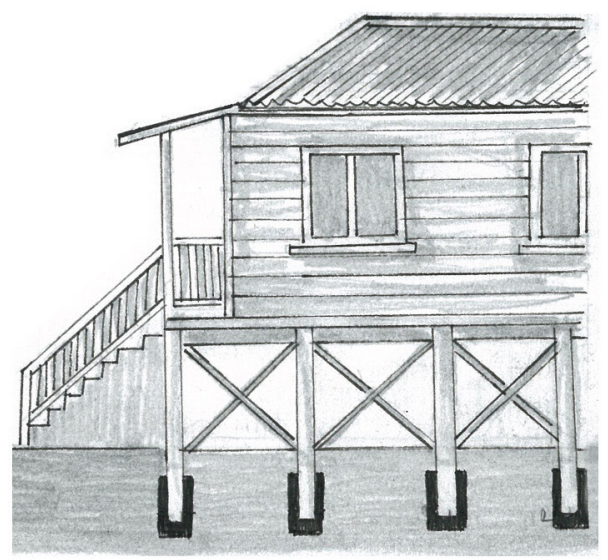

Concrete or timber pylons, with steel or timber cross bracing supporting timber frame construction.

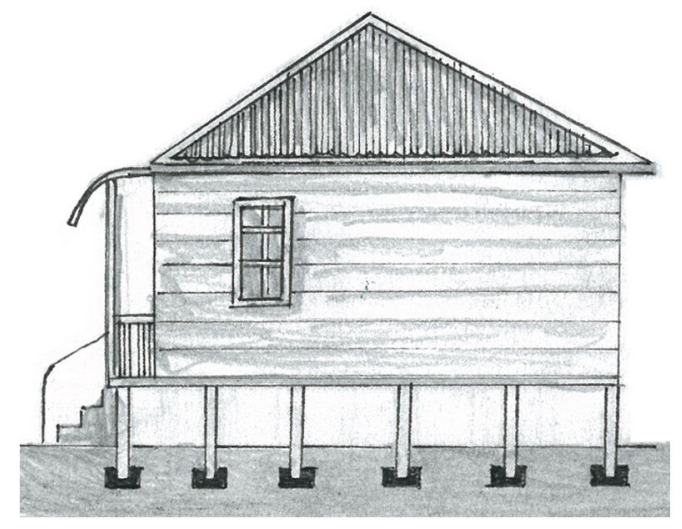

Masonry columns supporting timber construction with timber open stairs.

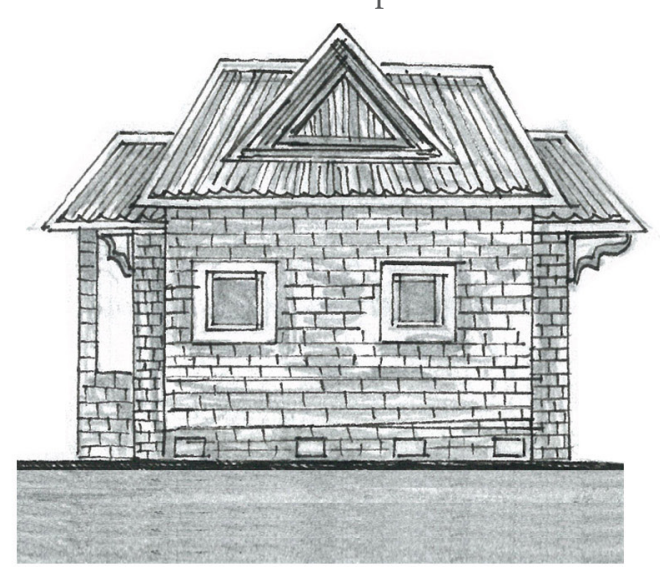

Masonry construction with raised timber flooring.

Figure 101: The main residential construction types of Maitland 

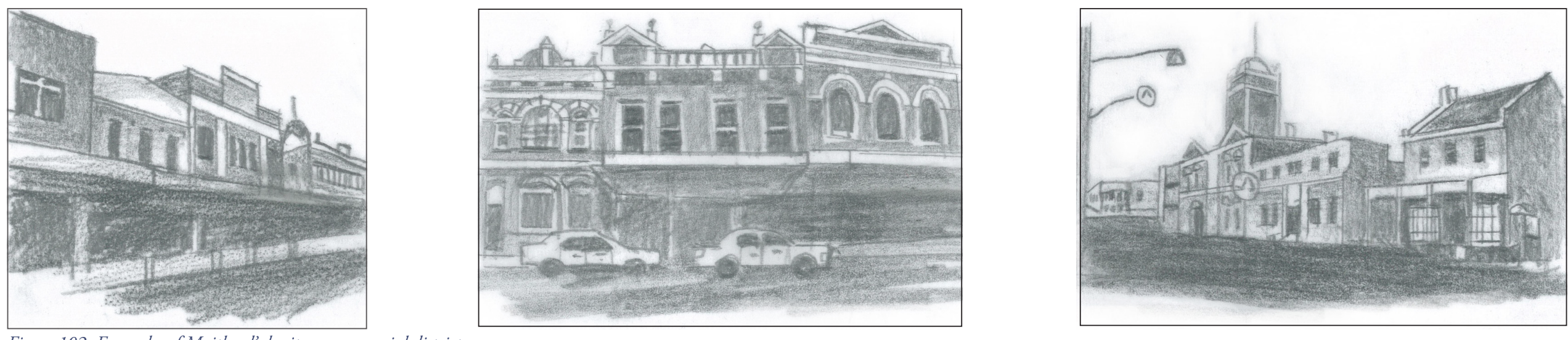

Figure 102: Examples of Maitland's heritage commercial district 


\subsection{Key Findings}

National improvements to flood proofing measures and international progress in amphibious construction continue to strengthen individual resilience. But there continues to be a lack of interventions being tested within the urban landscape with aims to strengthen community and city resilience. Current Australian stormwater management standards address street gradients for floods up to a one in one hundred event, however many of the old streets in Maitland are without adequate above or below ground stormwater infrastructure. This increases flood risks and results in greater flood heights.

Back flooding of Maitland as a result of the LHVFMS reduces flood velocities and inundation rates of the CBD and residential zones, but causes parts of South and Central Maitland to flood prematurely. Back flooding also adversely effects circulation, with the main access roads into Maitland being cut from floods well before levees are at risk of breaching. The city hence becomes isolated from surrounding townships, and is without a means to safely evacuate if floods continue to rise. Sidewalks are also inadequate in providing circulation paths for pedestrians from low inundation. This means that whilst the city is isolated its inhabitants become increasingly detached from neighbours and are unable to safely gain access to the cities services and infrastructure.

Socio-spatial resilience within the characteristic slow velocity floodwaters and prelonged periods of inundation may be enhanced through interventions that aim to establish flood adapted pedestrian pathways. These pathways would enable inhabitants to remain connected to community and provide access key city services. This will help the city in remaining functional during low anticipated periods of flood inundation. Building urban systems that have an intrinsic ability to respond to flood disturbances will enable flood resilience to evolve within cities.

Socio-spatial flood resilience strategies have the potential to strengthen the adaptive capacity of Maitland city and its inhabitants to inundation. Watson \& Adams (2011) believe resilient design can be expressed as; "multiple scales of impact, collaborative design, and innovation in design, technology and policy". A socio-spatial network interlinking key social and spatial aspects at multiple scales of the city may prove the most effective way to achieve this. The concept of a spatial resilient network is supported in landscape architecture by Ware, Raxworthy, Weller, Russell-Clarke, \& Meyer, (2011) who believe it can "create new systems of interconnected or interwoven networks that complement existing structures" (Ware, Raxworthy, Weller, Russell-Clarke, \& Meyer, 2011, p. 10).

A range of interventions at multiple scales will work towards improving the robustness and flexibility of the city system in its ability to anticipate, respond and function in low (anticipated), medium (potentially hazardous) and high flood (survival) events. The categorising of flood events based on the functional capabilities of the city responding to different flood levels provide a new less misleading categorisation of flood risks when compared to current annual exceedance probability classifications. Establishing a socio-spatial flood resilience network recognises the importance of maintaining community connection, access to key services and functional infrastructure within the urban and city scales during flood disturbances. This has been highlighted as a common issue in floodplain inhabitation through analysis of existing circumstances on the Mekong River in Cambodia, the Lower Ninth Ward of New Orleans, Maasbommel in the Netherlands and Maitland, Australia. 
The history of Maitland's engagement with floods has been one in which the community sought...to do battle with the 'menace' of flooding and defeat it. In the battle there were some victories, but flooding usually had the last word and over the past half century it has been increasingly well recognised that a different approach [is] necessary. 


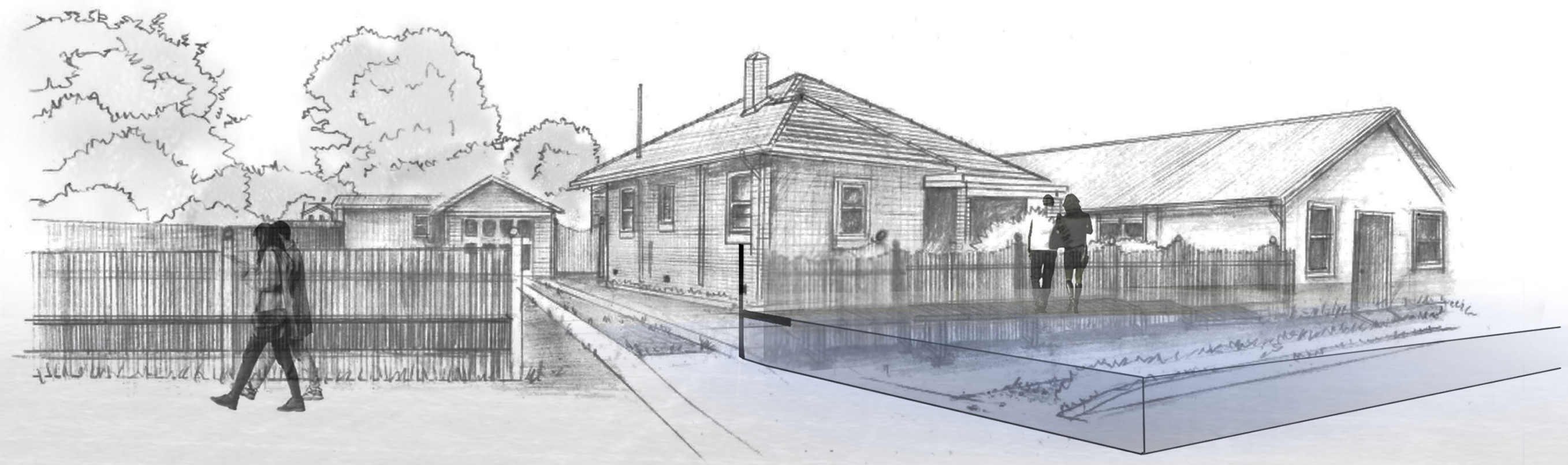

\subsection{MAITLAND CASE STUDY : A SOCIO-SPATIAL FLOOD RESILIENCE NETWORK}

This chapter analyses the potential social, structural and functional response of Maitland to low, medium and high flood category events. This chapter reveals the opportunities and challenges that arise from establishing a multi-scale socio-spatial resilience network. Interventions aim to strengthen the adaptive capacity of the city system to floods. The case study establishes a category of possible solutions, with each exploring different vulnerable aspects of Maitland's socio-spatial resilience network. 
A multi-scale socio-spatial resilience network will aim to improve the flexibility and robustness of a floodplain city system to flooding and reduce the functional impact floods have on the city system. A reduction in the short and long term social and physical flood damages, will aim to improve the post flood recovery time frames of residents, communities and the city's infrastructure. As previously discussed there is a need for bottom up approaches to be concurrent with top down planning and development. Bottom up approaches on an individual and community scale will aim to encourage ownership of the risks. Fleischhauer (2008) acknowledges the importance spatial planning plays in mitigating flood hazards through urban structures in order to strengthen urban resilience prior to a natural disturbance. Precedent analysis reveals the need for spatial measures to be interconnected in a network system rather than implemented in isolation.

A balance of natural and artificial solutions is needed to avoid the catastrophic failures that often result from an emphasis on prevention. The natural and artificial components of systems need to anticipate, prepare, cope and adapt to flooding to enhance the city's socio-spatial resilience capabilities. A socio-spatial resilience network will aim to connect a variety of spatial scales to provide flexibility and robustness to flooding as a series of interrelating systems within the city. The community centre, levee, flood adapted pedestrian paths, urban shelters and flood safe house design components create a network of strategies aiming to strengthen the socio-spatial resilience of Maitland. The interventions will explore possible solutions to common issues encountered inhabiting flood prone land (refer to Chapter 5 for further detail).
The network utilizes the new low, medium and high flood categorizing model of this thesis, instead of the current flood probability model. Low, medium and high 'flood category events', are representative of specific structural and functional shifts within the city. The low flood category event refers to anticipated inundation, the medium as potentially hazardous and high as survival. In the low category events, anticipated flooding minimally impacts on houses and resident lifestyles.. In medium category events floodplain residents are reliant on flood adapted city infrastructure providing shelter, energy, food, and water. Whilst in the high category events the focus is to be on the evacuation of residents and ensuring key services and facilities of the city are functional post flooding.

The three major structural and functional states a city transitions between when impacted upon by flooding are determined by the social and spatial components of the city; the capacity of inhabitants to access key services and the ability of infrastructure to continue to function and provide services. Anticipated, potentially hazardous and survival become the structural and functional states of the sociospatial resilience network. The network aims to reduce the adverse impact each stage will have on community, safety of inhabitants and the damage to public infrastructure. 
City social and spatial function

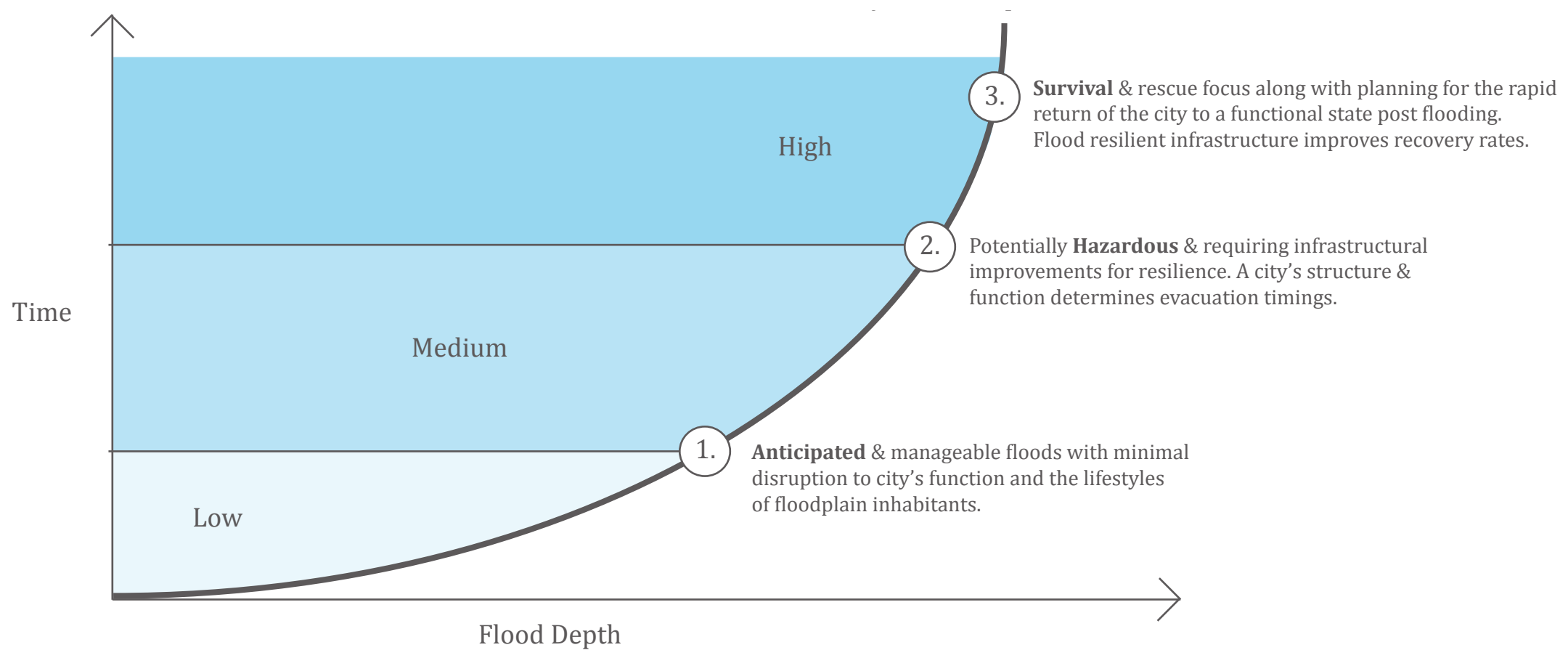

Figure 103: The low, medium, high flood categorisation approach 
Integrating new strategies into Maitland's resilience flood management matrix

\begin{tabular}{|c|c|c|c|c|c|c|}
\hline & Scales: & Region & City & Suburb & Street & Building \& Site \\
\hline & Adaptability: & & & & & \\
\hline Flood impact & Alternative circuits & \begin{tabular}{|l|} 
Lower Hunter Valley \\
Flood Mitigation Scheme
\end{tabular} & \begin{tabular}{|l|} 
Flood defences \\
(eg main ring levee)
\end{tabular} & $\begin{array}{l}\text { Adaptive } \\
\text { infrastructure }\end{array}$ & Connected grid & $\begin{array}{l}\text { Permanent \& temporary } \\
\text { wet \& dry flood proofing }\end{array}$ \\
\hline \multirow{3}{*}{$\begin{array}{l}\text { building/site } \\
\text { circulation }\end{array}$} & Rapid Response & $\begin{array}{l}\text { Rivers, streams } \\
\text { and creeks }\end{array}$ & $\begin{array}{l}\text { Flood adapted } \\
\text { mobility options }\end{array}$ & \begin{tabular}{|l} 
Green \\
infrastructure
\end{tabular} & $\begin{array}{l}\text { Self-reliant } \\
\text { subsystems }\end{array}$ & $\begin{array}{l}\text { Adaptive \& flexible } \\
\text { service connections }\end{array}$ \\
\hline & Core Protection & $\begin{array}{l}\text { Maitland LEP } 2011 \\
\text { Land zoning }\end{array}$ & \begin{tabular}{|l|}
$\begin{array}{l}\text { Decentralisation of } \\
\text { city infrastructure }\end{array}$ \\
\end{tabular} & $\begin{array}{l}\text { flood adaptive } \\
\text { network }\end{array}$ & \begin{tabular}{|l|}
$\begin{array}{l}\text { Multiple } \\
\text { circulation paths }\end{array}$ \\
\end{tabular} & $\begin{array}{l}\text { Material construction } \\
\text { \& strength }\end{array}$ \\
\hline & Variety \& Redundancy: & & & & & \\
\hline Flooding issues & Buffering & $\begin{array}{l}\text { Maitland LEP } 2011 \\
\text { Land use planning }\end{array}$ & $\begin{array}{l}\text { Levee vegetative } \\
\text { recreation zone }\end{array}$ & Riparian buffers & $\begin{array}{l}\text { Vegetative } \\
\text { barriers }\end{array}$ & $\begin{array}{l}\text { Vegetative \& architecturally } \\
\text { built buffers }\end{array}$ \\
\hline debris & Diffusion & $\begin{array}{l}\text { LHVFMS diversion } \\
\text { channels and levees }\end{array}$ & \begin{tabular}{|l|} 
Street pattern, \\
CBD on high land
\end{tabular} & \begin{tabular}{|l|} 
Terraced \\
landscaping
\end{tabular} & $\begin{array}{l}\text { artificial water } \\
\text { channels }\end{array}$ & $\begin{array}{l}\text { Site and building floor } \\
\text { plans channel floodwaters }\end{array}$ \\
\hline water velocity & Storage & $\begin{array}{l}\text { Glenbawn, Chichester } \\
\text { and Lostock dams }\end{array}$ & $\begin{array}{l}\text { Maitland Floodplain, } \\
\text { dry \& wet retention } \\
\text { basins }\end{array}$ & $\begin{array}{l}\text { Stormwater } \\
\text { managment } \\
\text { system }\end{array}$ & \begin{tabular}{|l|} 
Stormwater \\
collection (rain \\
gardens \& swales)
\end{tabular} & Rainwater storage tanks \\
\hline water volume & Absorption & $\begin{array}{l}\text { Lower Hunter Valley } \\
\text { Watershed planning }\end{array}$ & $\begin{array}{l}\text { Urban density and } \\
\text { green space }\end{array}$ & $\begin{array}{l}\text { Dry retention } \\
\text { basins }\end{array}$ & Rain gardens & $\begin{array}{l}\text { Low building footprint (stilt } \\
\text { \& amphibious construction) }\end{array}$ \\
\hline water quality & $\begin{array}{l}\text { Nutrient \& Waste } \\
\text { Recovery }\end{array}$ & $\begin{array}{l}\text { Hunter Estuary } \\
\text { Wetlands }\end{array}$ & \begin{tabular}{|l|} 
Maitland Stormwater \\
/Lakes Management \\
Plan 2006
\end{tabular} & \begin{tabular}{|l|} 
Maitland on-site \\
sewage \\
management \\
\end{tabular} & $\begin{array}{l}\text { Rainwater } \\
\text { harvesting }\end{array}$ & Grey water filtration system \\
\hline & Feedback: & & & & & \\
\hline Weather event & Response & \begin{tabular}{|l} 
The Australian \\
Bureau of Meteorology
\end{tabular} & $\begin{array}{l}\text { ABC flood warnings } \\
\text { local flood gauges }\end{array}$ & \begin{tabular}{|l|} 
Local flood watch \\
\& evacuation plans
\end{tabular} & $\begin{array}{l}\text { Flood height } \\
\text { indicators }\end{array}$ & $\begin{array}{l}\text { Flood sensor alarms } \\
\text { visual and acoustic }\end{array}$ \\
\hline precipitation & Regeneration & $\begin{array}{l}\text { Lower Hunter Region } \\
\text { industry functioning }\end{array}$ & $\begin{array}{l}\text { Elevated roads \& } \\
\text { renewable energy }\end{array}$ & $\begin{array}{l}\text { > ecological } \\
\text { recovery }\end{array}$ & \begin{tabular}{|l|}
$<$ flood damage \\
to infrastructure
\end{tabular} & $<$ residential flood damage \\
\hline Flood duration & $\begin{array}{l}\text { Cooperative } \\
\text { Self-Regulation }\end{array}$ & $\begin{array}{l}\text { NSW State } \\
\text { Emergency Service } \\
\text { volunteers }\end{array}$ & \begin{tabular}{|l|} 
State Emergency \\
Service Maitland \\
City Local Flood Plan
\end{tabular} & $\begin{array}{l}\text { Evacuation Centres } \\
\text { (community } \\
\text { centre) }\end{array}$ & \begin{tabular}{|l|} 
Urban flood \\
shelters (equipt \\
with key services)
\end{tabular} & $\begin{array}{l}\text { Flood safe houses (flood } \\
\text { kits, evacuation plans, } \\
\text { residential floodproofing) }\end{array}$ \\
\hline \multicolumn{5}{|c|}{ Figure 104: New strategies for Maitland's resilience flood management matrix } & \multicolumn{2}{|c|}{$\begin{array}{l}\text { Stategies became primary focus in designing } \\
\text { the network and its components } \\
\text { Strategies integrated into design components }\end{array}$} \\
\hline
\end{tabular}


Applying the resilience flood management matrix to Maitland enables the strengths and weaknesses of existing flood management solutions in place to be identified (refer to 6.3 Resilience flood management matrix: Maitland). These existing strategies have been analyzed at multiple scales to ensure that floods are mitigated using a variety of techniques. Greater insight is gained when the findings are compared to the previous precedent resilience matrix analysis (refer to 5.6 Resilience flood management matrix: macro/micro analysis). Several core sociospatial vulnerabilities are once again identified. Key strategies at the city, urban, suburb and residential scale provide guiding principles for designing the Maitland socio-spatial resilience network and its components. These have been identified in the new Maitland resilience flood management matrix.

\section{Existing vulnerabilities and short comings of Maitland's flood resilience;}
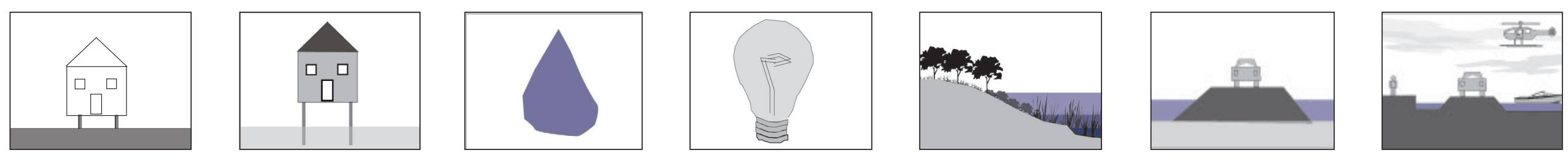
Access roads and railway line cut from back flooding

Residential houses on low lying land with largest flood heights

Community services, facilities and gathering points

Paved footpaths elevated from roads
7.4 Key issues of the Maitland city system

- Traditional methods of flood proofing are insufficient in areas prone to high flood depths.

- Major access roads to Maitland are cut during flooding, isolating the town or limiting circulation and evacuation options.

- Pedestrian circulation is limited during flood inundation, resulting in further isolation of individuals from neighbours and vital services.

- Reliance on external aid for evacuation processes, once the major highway is cut by flooding.

-A loss of community gathering points where services, facilities and alternate energy sources are provided pre, during and post flooding.

- Poor functional and structural flood adaptability by urban and city infrastructure and services (major services such as electricity, freshwater supply and sewerage processes are commonly not operational during floods).

Figure 105: The key existing issues influencing Maitland's flood vulnerability 
7.5 Understanding the socio-spatial system

(city, urban and residential scale)

The impact low, medium and high flood category events have on Maitland

A socio-spatial system essentially involves the everyday structure and functions of a city. Understanding how the city system functionally responds to a variety of floods (low, medium, and high flood category events) is vital when strengthening the resilience of a socio-spatial system to flooding. The impact of flood categories on community connection and inhabitant safety must also be analysed.

\section{Central Maitland, Lower Hunter Valley Region, NSW, Australia}

The nearby townships of Lorn and Morpeth are depicted in low opacity.

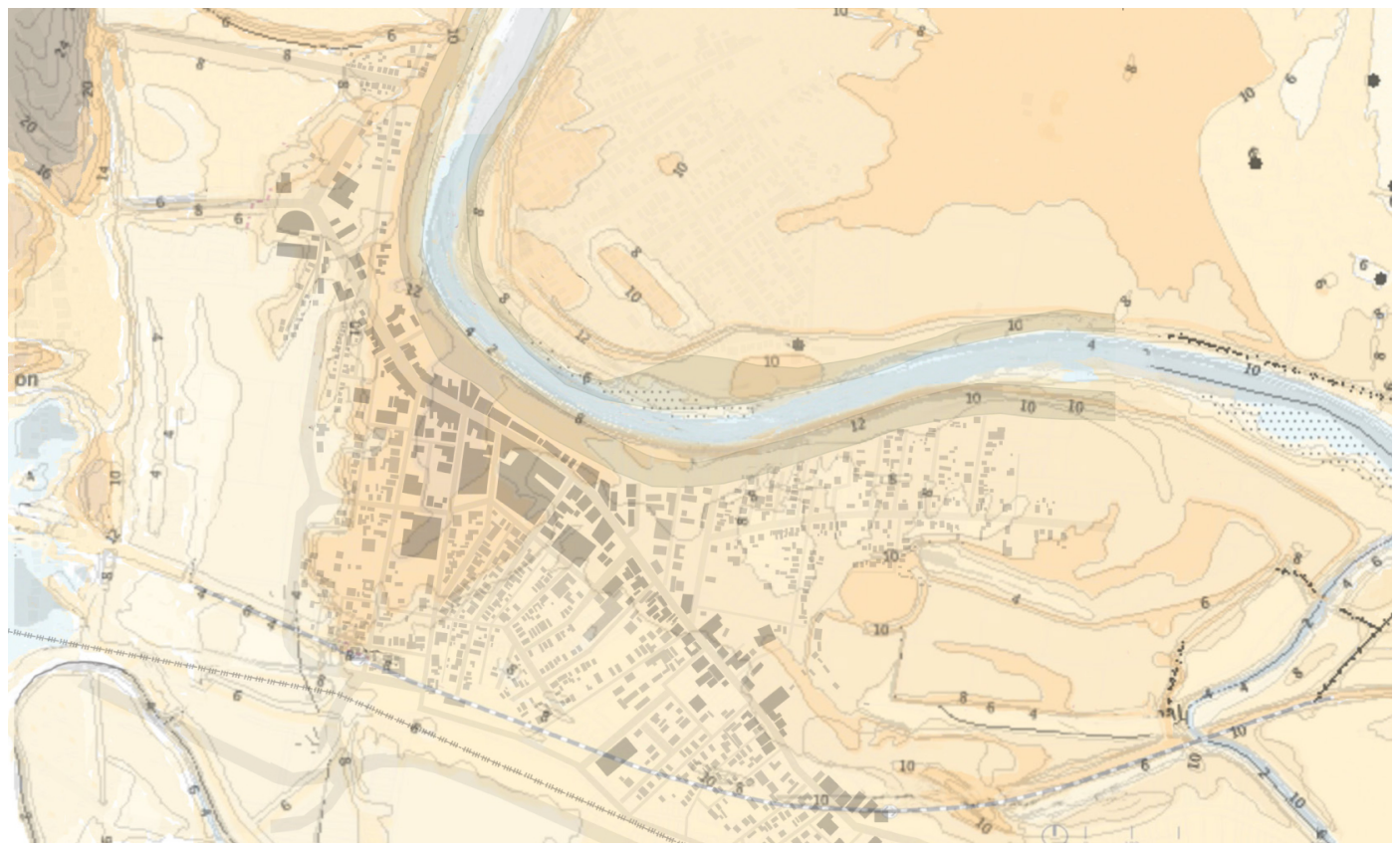

Figure 87: The focus area of Maitland with the adjacent townships of Lorn and Morpeth
1. Low flood ( $<5 \%$ AEP)

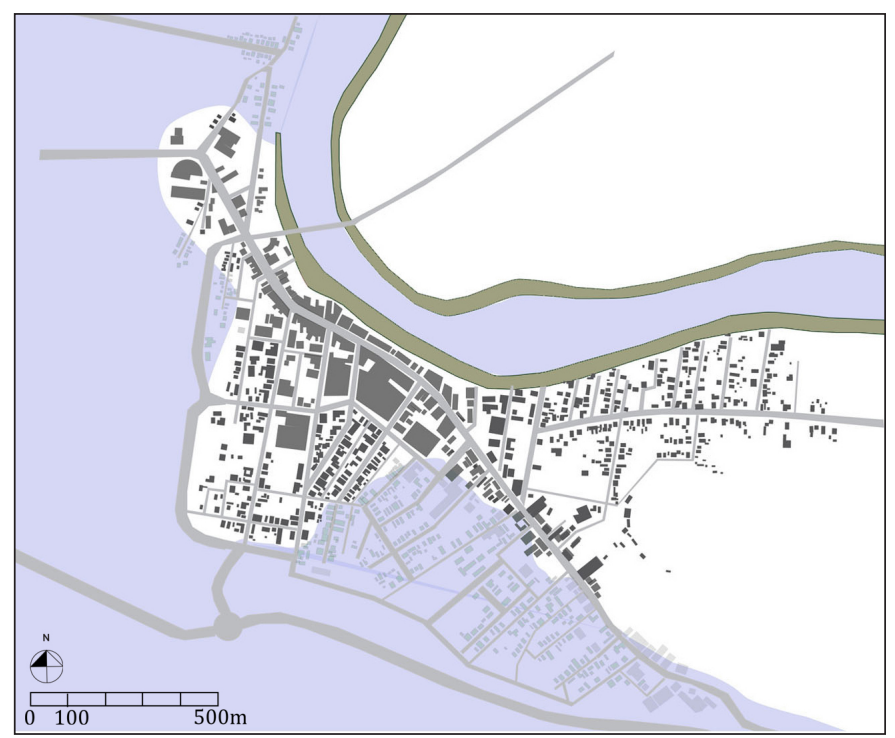

Figure 106: Maitland's low flood category event

\section{AHD flood heights}

Maitland Business District $\quad 8.0 \mathrm{~m}$

Horseshoe Bend

$7 \mathrm{~m}$

Central Maitland

$7.5 \mathrm{~m}$

Total number of residential buildings

Maitland Business District $\quad 578$

Horseshoe Bend 192

Central Maitland $\quad 510$ 


\section{Medium flood (>2\% AEP)}

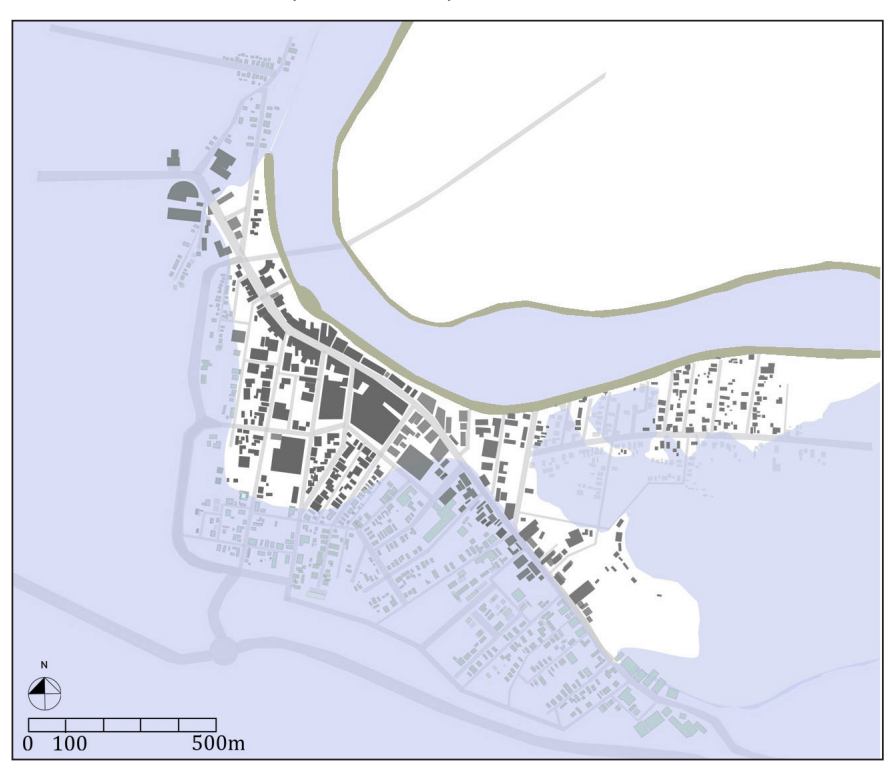

Figure 107: Maitland's medium flood category event

\section{AHD flood heights}

Maitland Business District $\quad 9.3 \mathrm{~m}$

Horseshoe Bend

$9.3 \mathrm{~m}$

Central Maitland

$9.3 \mathrm{~m}$

\section{Average ground level (AHD)}

Maitland Business District

$9.8 \mathrm{~m}$

Horseshoe Bend

$8.2 \mathrm{~m}$

Central Maitland

$7.1 \mathrm{~m}$

\section{High flood (= or $>1 \%$ AEP)}

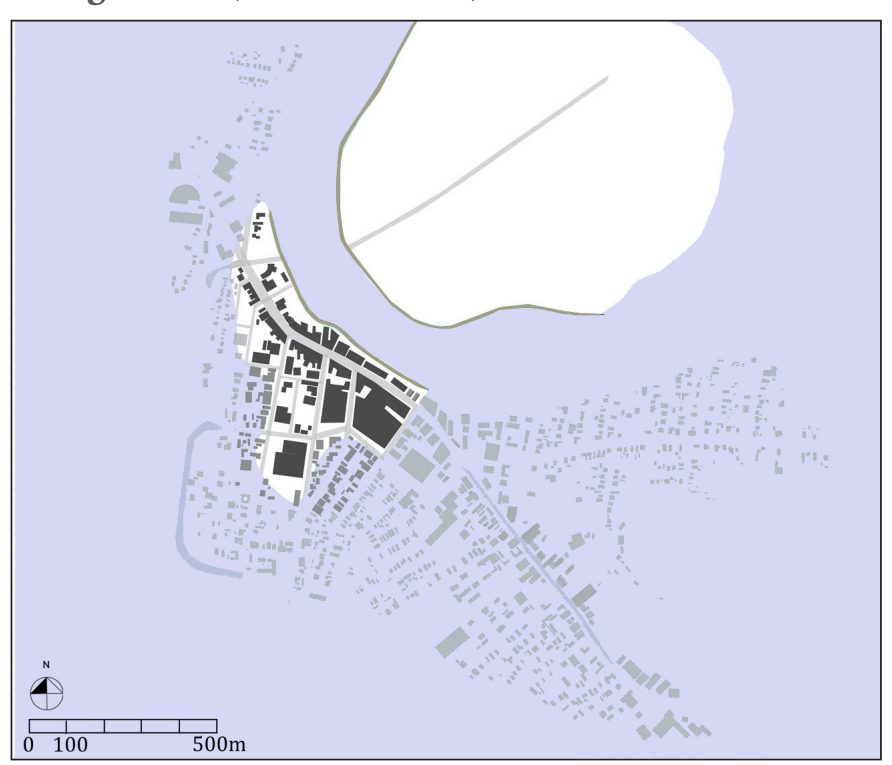

Figure 108: Maitland's high flood category event

\section{AHD flood heights}

Maitland Business District 10.4-14.2m

Horseshoe Bend 12.9m

Central Maitland $\quad 13.4 \mathrm{~m}$

\section{Average flood level (AHD)}

Maitland Business District

Horseshoe Bend

Central Maitland

$10.1 \mathrm{~m}$

$8.7 \mathrm{~m}$

$7.6 \mathrm{~m}$

Flood statistics collated from Maitland City Council \& WMA Water (September, 2010); Maitland City Council LEP \& DCP 2011; Central Maitland Structure Plan (November, 2009) 
Analysis of the location of public services, public infrastructure and existing circulation pathways, provides insight into where the system is vulnerable. The impact flood category events have on the ability of services to be accessed must also be analysed. A series of everyday residential, urban and city strategies enable a city's infrastructure to continue to function and its inhabitants remain connected to one another and able to access city services during a variety of flood events.

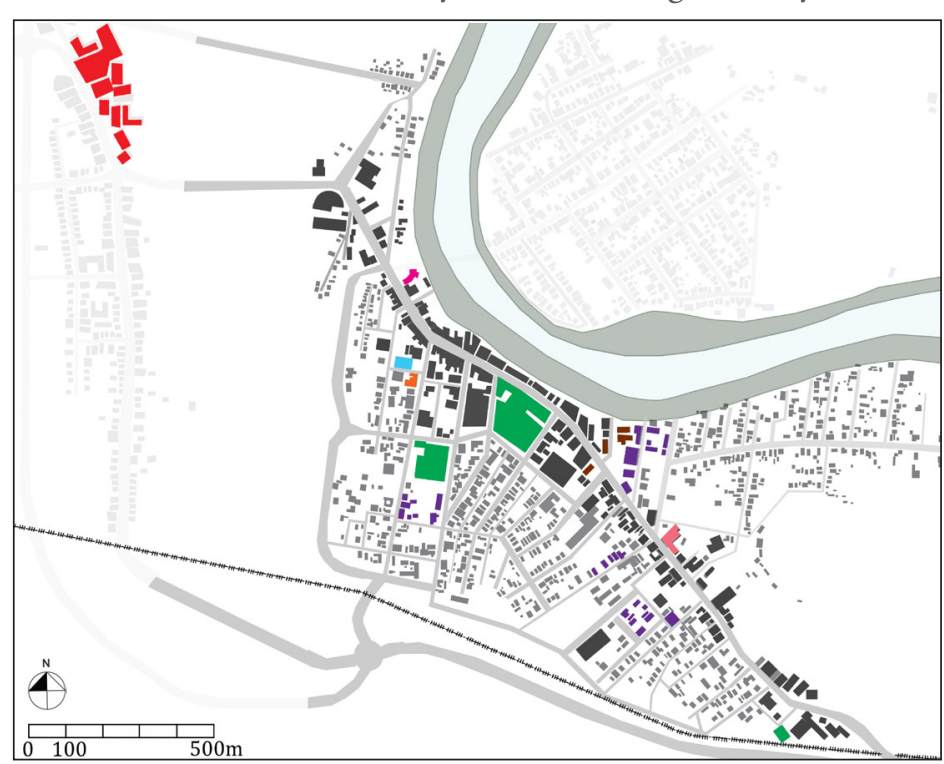

Figure 109: Existing Maitland services and infrastructure

\begin{tabular}{|c|c|c|c|c|c|c|}
\hline$\nabla$ & $\begin{array}{l}\text { Supermarkets \& } \\
\text { Grocery Stores }\end{array}$ & & 0 & & & \\
\hline$\square$ & Maitland Hospital & & $\mathbf{A}$ & C & 4 & 金年 \\
\hline - & $\begin{array}{l}\text { Fire Brigades NSW } \\
\text { Maitland Police Station }\end{array}$ & & & 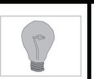 & 4 & \\
\hline 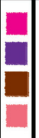 & $\begin{array}{l}\text { Maitland City Library } \\
\text { School } \\
\text { Church } \\
\text { Maitland Regional Art Ga }\end{array}$ & & & e & & $\widehat{0_{0}^{0}}$ \\
\hline
\end{tabular}

The impact floods have on commercial buildings and community services

1. Low flood category event

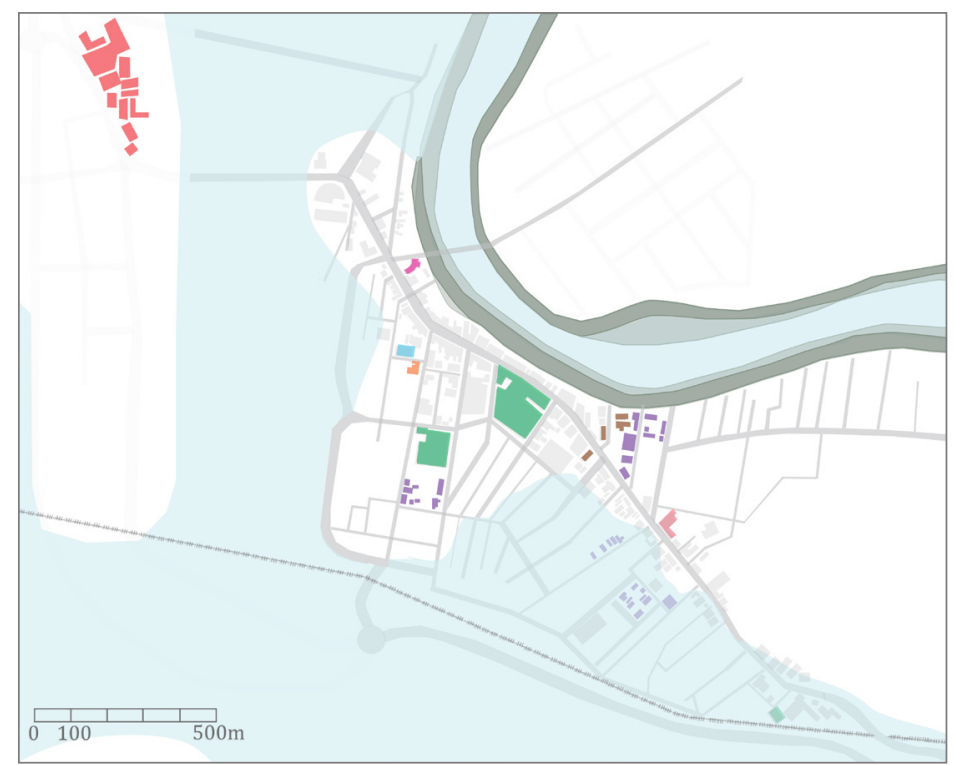

Figure 110: Low flood impact on Maitland services and infrastructure

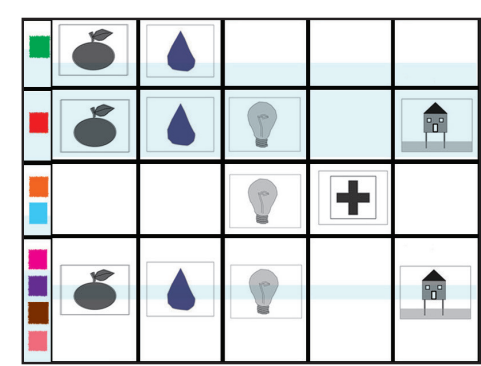


2. Medium flood category event

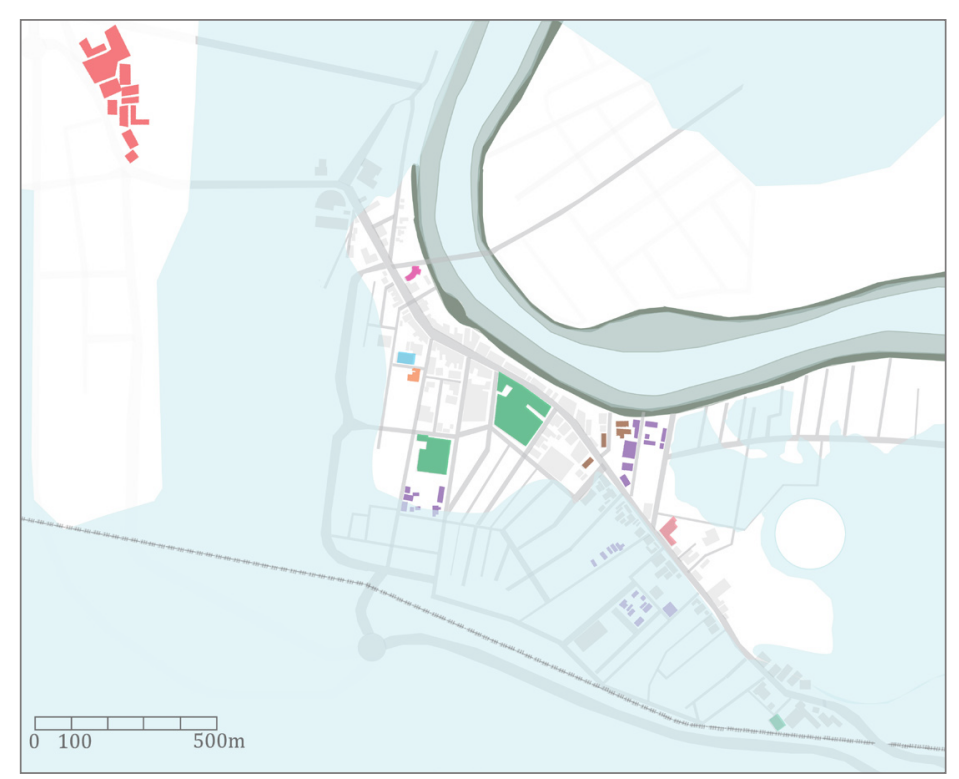

Figure 111: Medium flood impact on Maitland services and infrastructure

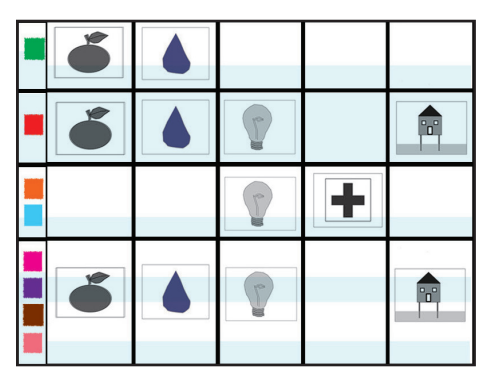

3. High flood category event

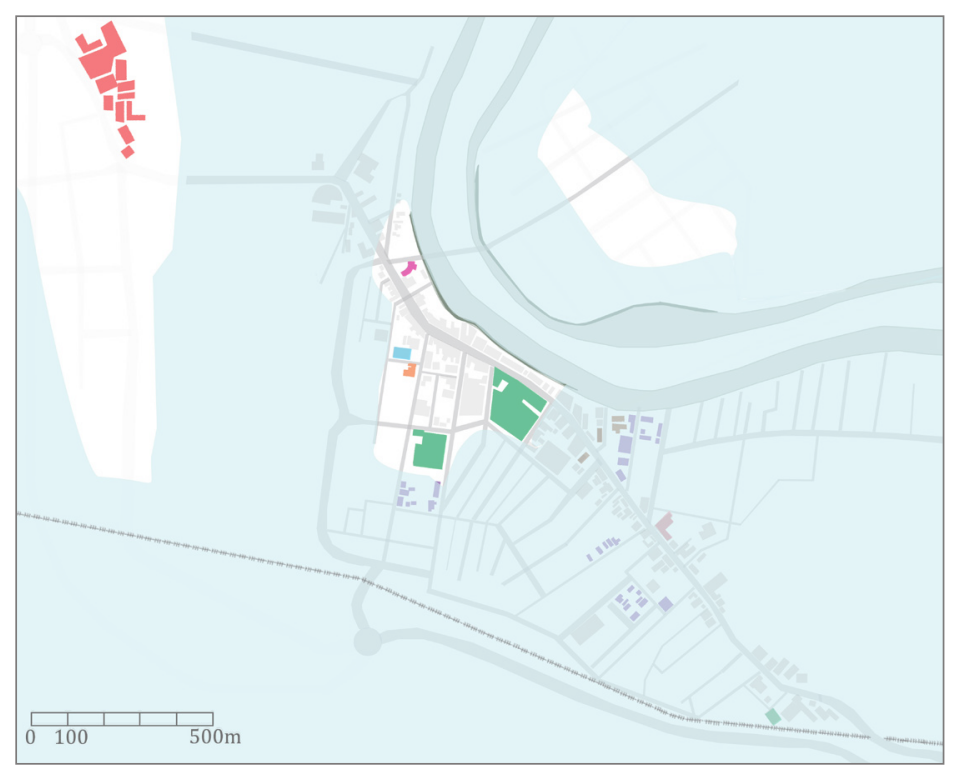

Figure 112: High flood impact on Maitland services and infrastructure

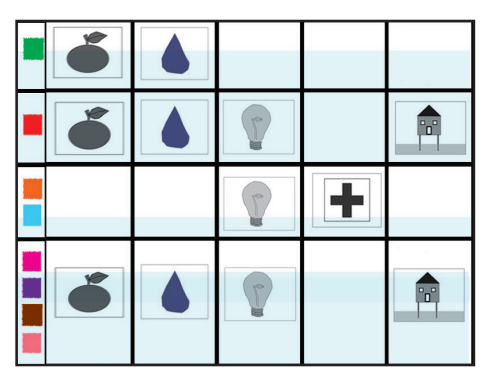


A socio-spatial resilience network is made up components at the multiple scales of the city

\section{- Maitland : city services, urban $\&$ residential scales $\bullet$}

\section{A socio-spatial resilience network connects residents with communities and infrastructure using a}

- Low flood category event network

- Medium flood category event network

- High flood category event network 
7.6 Maitland's socio-spatial resilience network

(Linking the residential, urban and city scales)

A series of everyday residential, urban and city strategies enable a city's infrastructure to continue to function and its inhabitants remain connected to one another and able to access city services during a variety of flood events.

The multi-scale socio-spatial resilience network involve a series of strategies:

- Residential flood safe houses : wet/dry flood proofing and amphibious construction - Alternate pedestrian circulation: statically elevated and amphibious urban features - Urban shelters with power/water/services : bus stop, barbeque and picnic shelters

- Community centre and civic square: provision of shelter, power, food and water.

The everyday city network system linking residents, communities and city services to one another via a system of roads

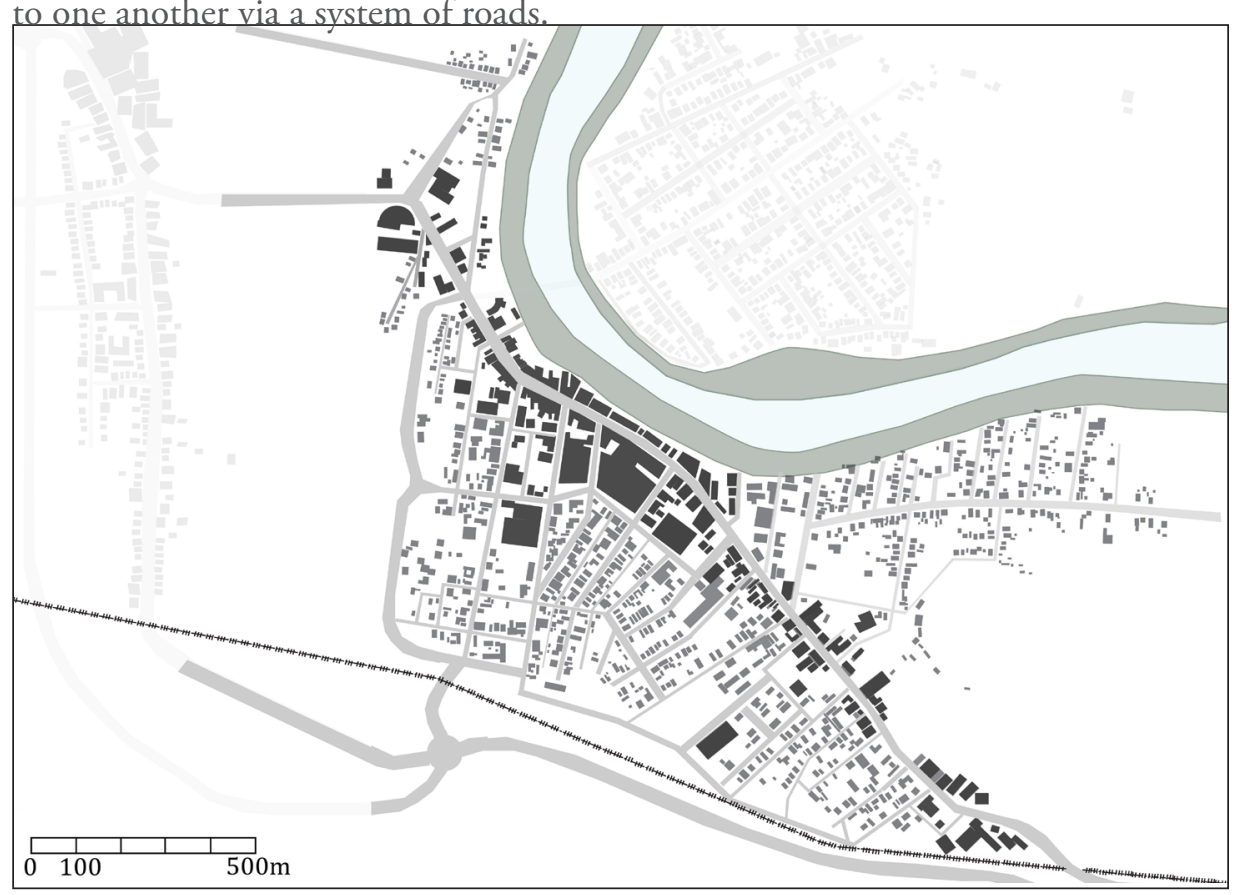

Figure 87: The focus area of Maitland with the adjacent townships of Lorn and Morpeth

\section{Low flood category event}

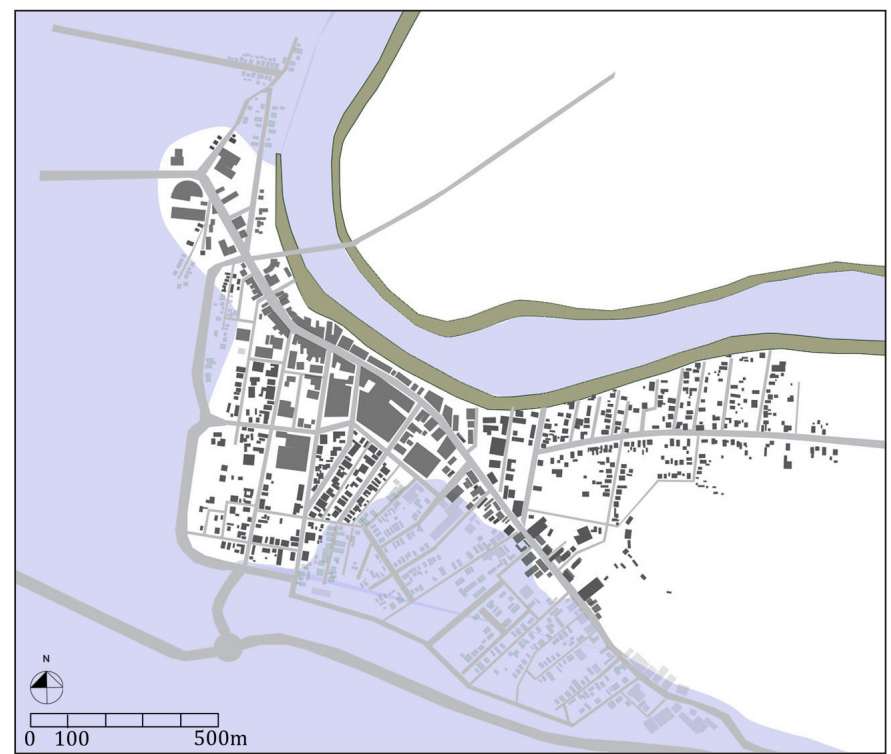

Figure 106: Maitland's low flood category event 


\section{The buildings at risk of being inundated in a low flood category event}

Traditional wet and dry flood proofing measures should be sufficient for flood heights associated with a first category low flood event.

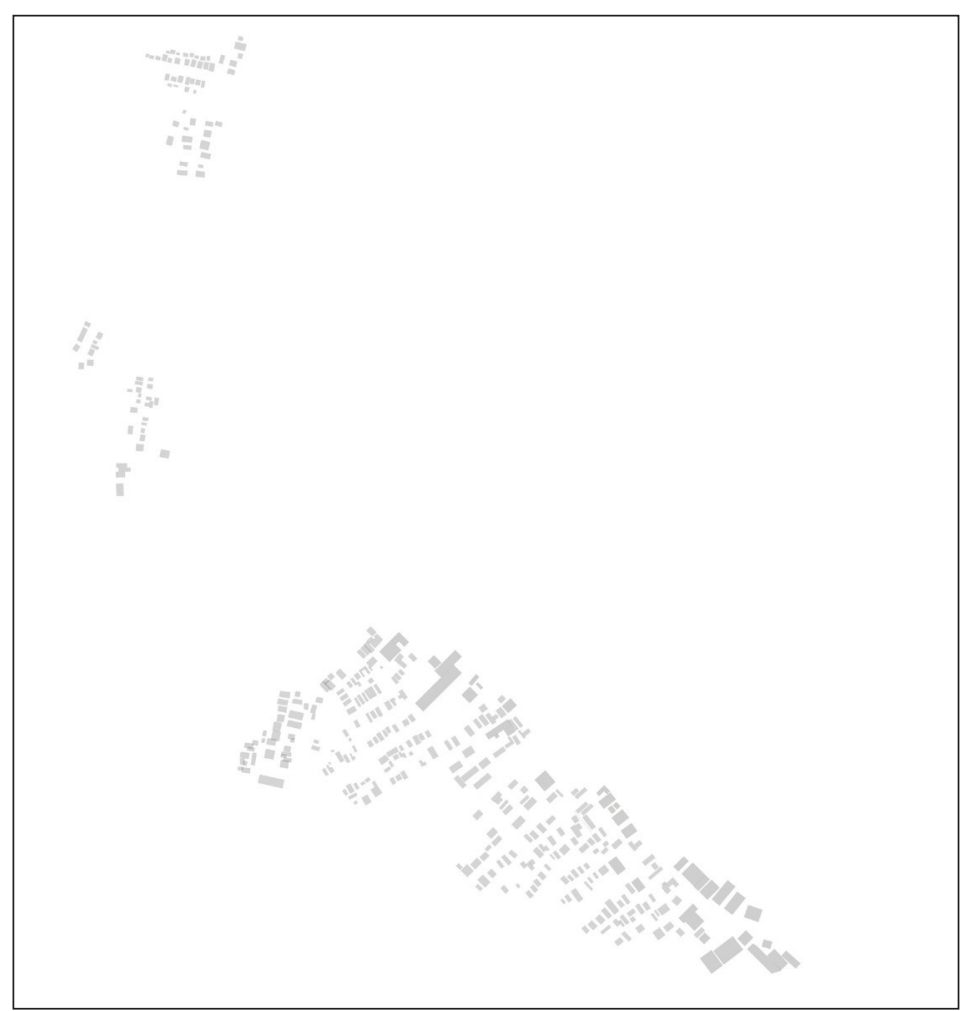

Figure 113: The buildings at risk of being inundated in a low flood

\section{A socio-spatial resilience network in low floods}

A low socio-spatial resilience network ensures those within the inundated area remain connected to one another, the community and city at large. Allowing them to continue to access services and be minimally impacted upon by floods.

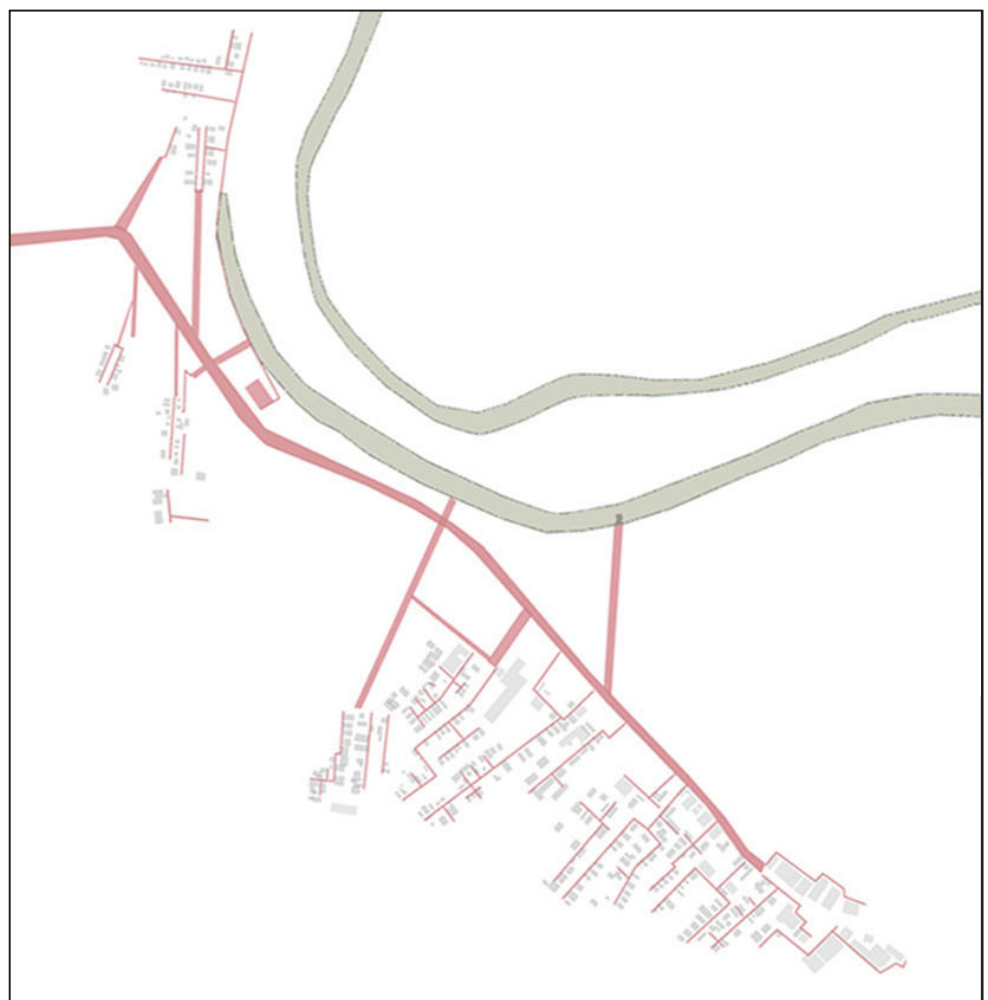

Figure 114: Maitland's socio-spatial resilience network in low floods

A low flood network connects inundated residents to the higher ground of the CBD. This thesis proposes the development of a community centre and shelters located within local neighborhoods to ensure shelter, emergency supplies and services remain available to those residents of South Maitland inundated by back flooding. 


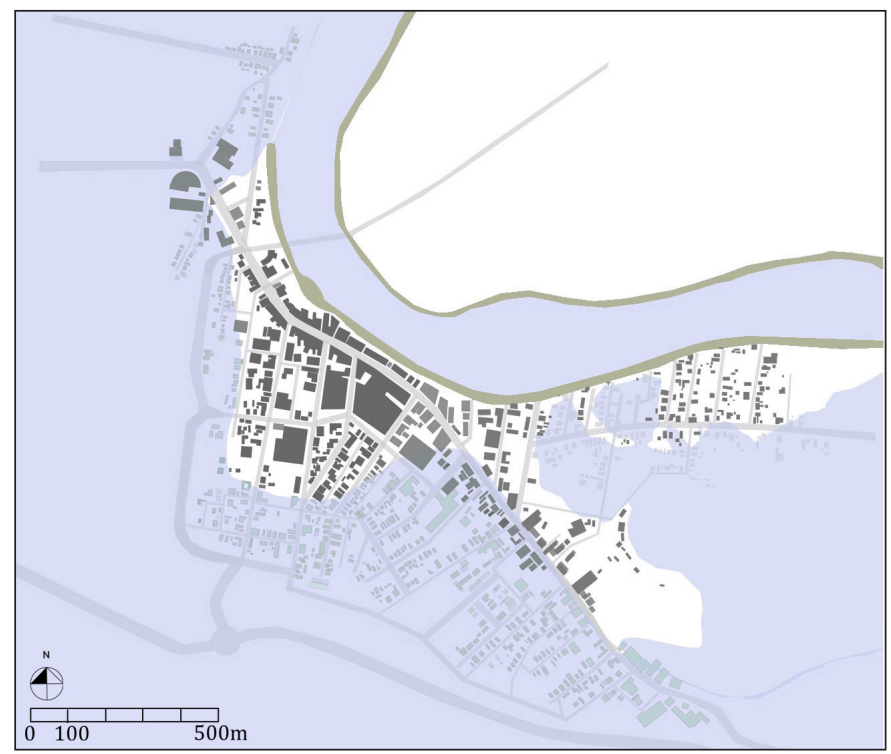

Figure 107: Maitland's medium flood category event 


\section{The buildings at risk of being inundated in a medium flood category event}

Traditional flood proofing measures will most likely be adequate for buildings inundated for the first time. But for those previously inundated from low events traditional measures begin to fail as flood heights increase. Amphibious construction becomes a more suitable solution for these buildings.

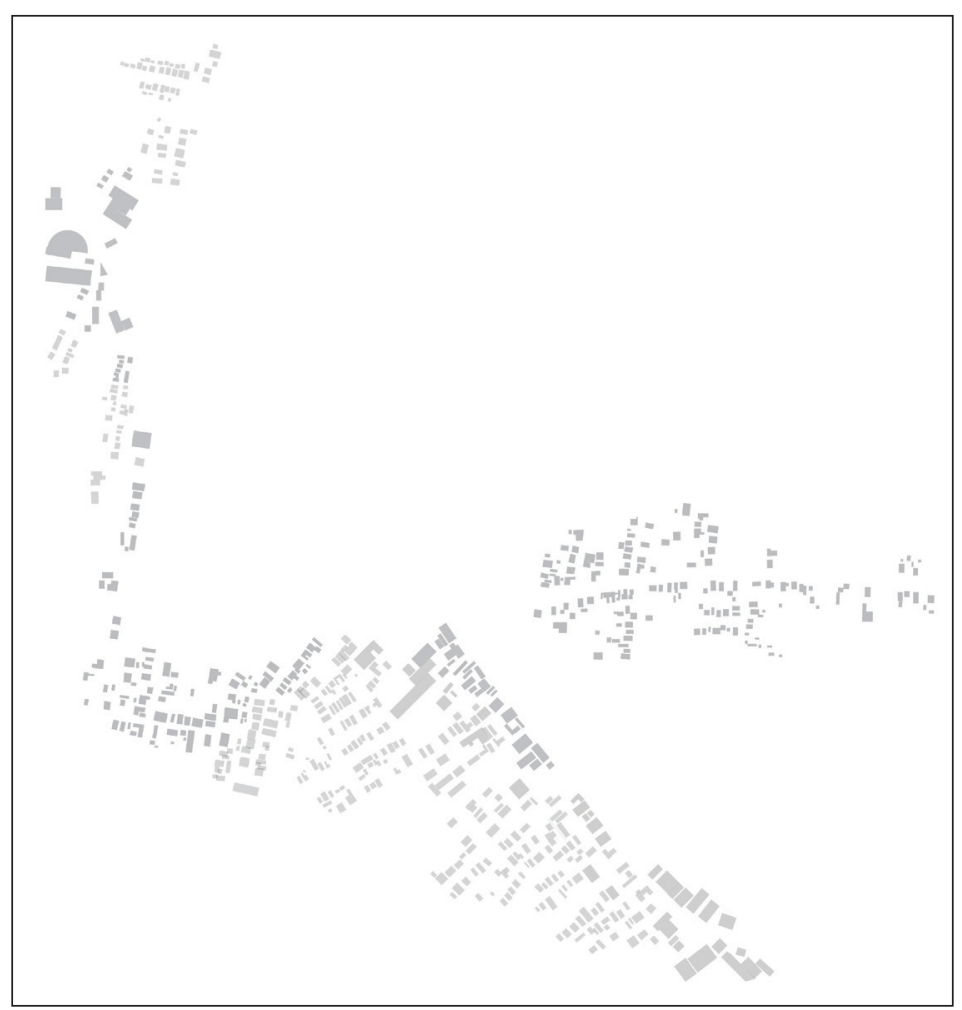

Figure 115: The buildings at risk of being inundated in a medium flood

\section{A socio-spatial resilience network in medium floods}

A socio-spatial resilience network allows those affected by flooding to remain connected to one another, the community and city at large. Inhabitants are able to access services and begin to evacuate to alternate flood safe shelters as the flood situation becomes hazardous.

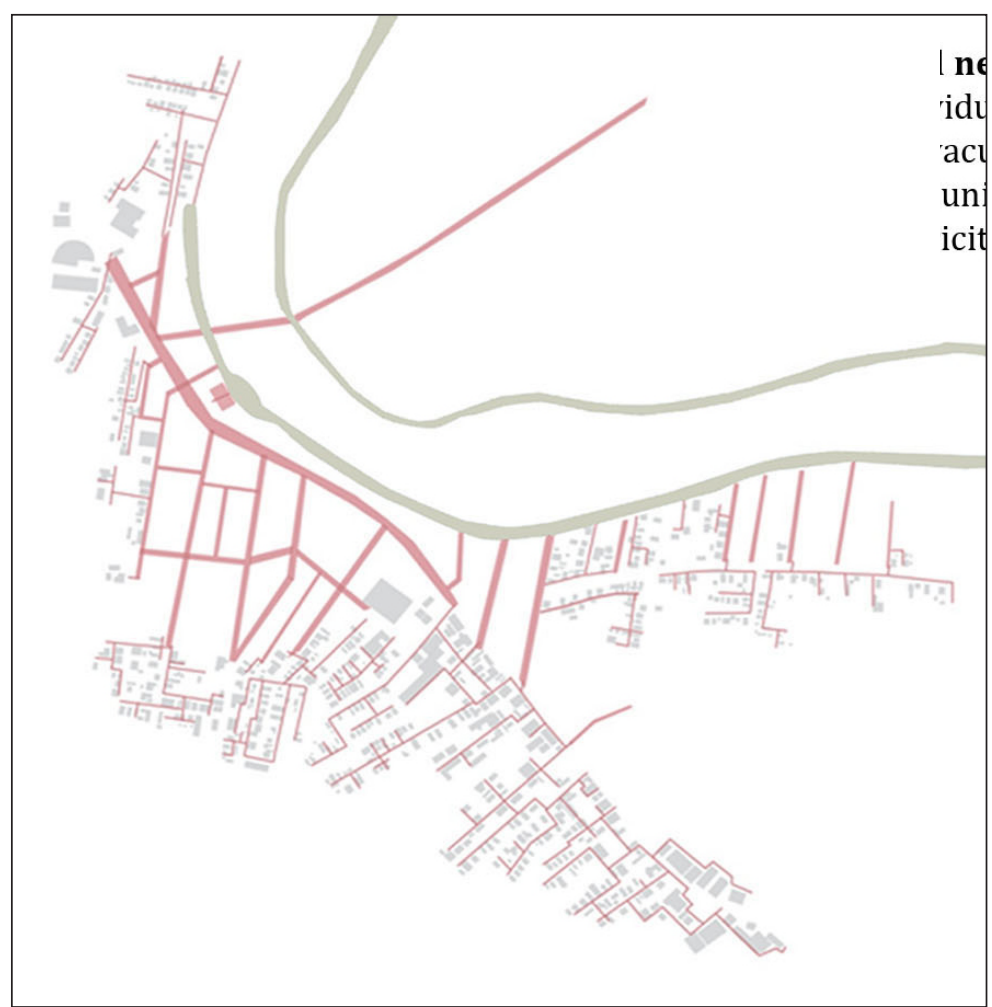

Figure 116: Maitland's socio-spatial resilience network in medium floods

A medium flood network links individuals to surrounding communities where temporary urban shelters provide water, electricity and cooking facilities. This not only improves local social support but also strengthens evacuation paths from Horseshoe Bend and South Maitland to the levee, and the high topographical Maitland Business District. It is here that a community centre is located, functioning as an evacuation gathering point where key services and facilities are provided. 


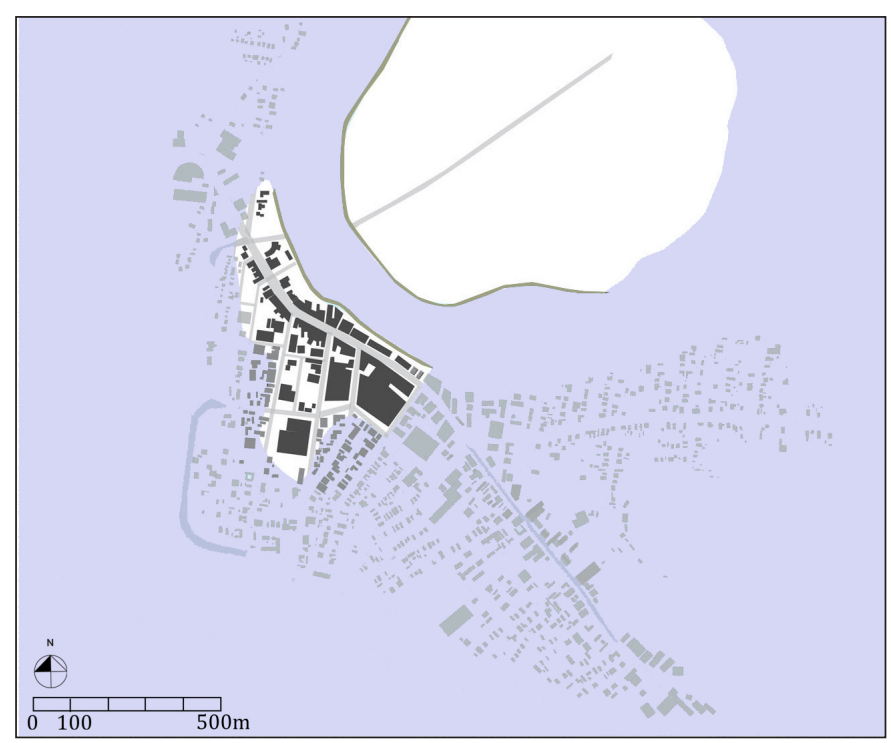

Figure 108: Maitland's high flood category event 


\section{The buildings at risk of being inundated in a high flood category event}

Traditional wet and dry flood proofing measures will be insufficient for buildings previously inundated by low and medium flood events as a result of the high flood heights. Amphibious construction techniques enable evacuated buildings to be minimally impacted upon by floods. However, floating debris and possible high velocity floods could cause damage.

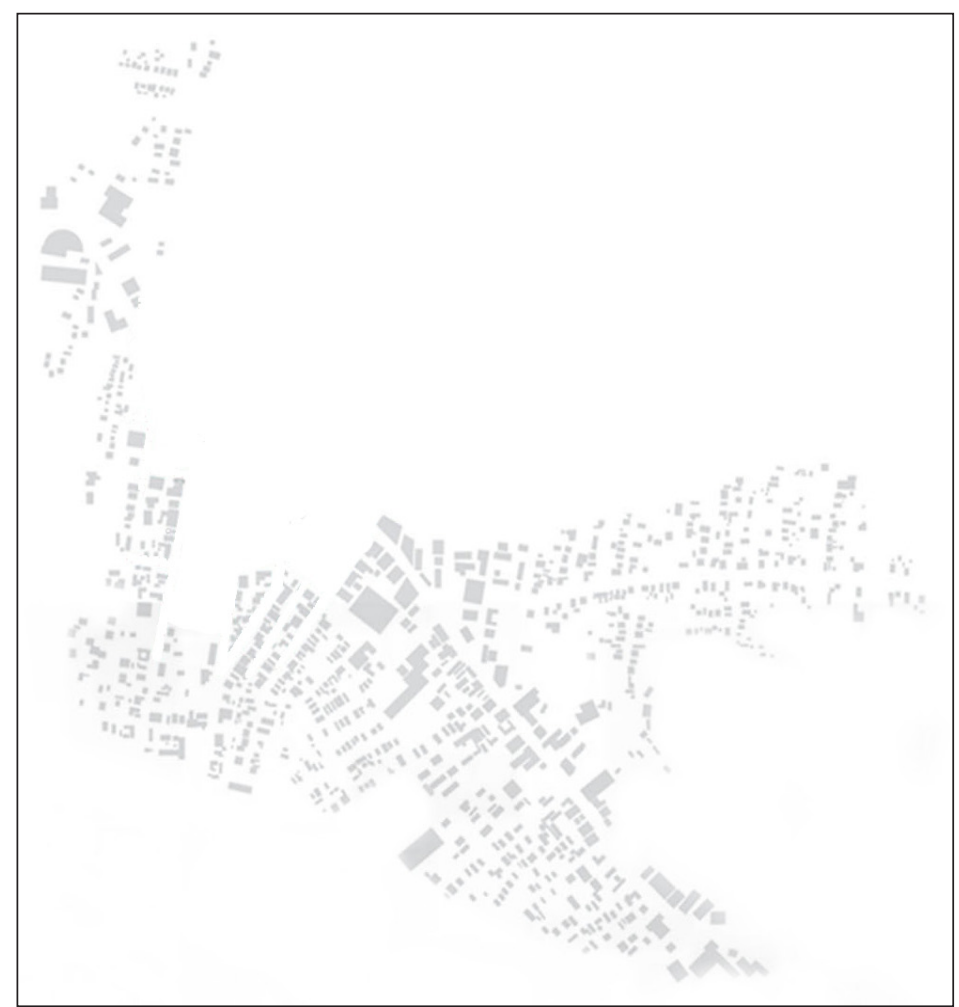

Figure 117: The buildings at risk of being inundated in a high flood

\section{A socio-spatial resilience network in high floods}

A high socio-spatial resilience network focuses on survival. It is made up of the city components located on the high topographical land. The city's residents at this point will have been evacuated, but any remaining people can shelter within the community centre, which will not be structurally or functionally impacted upon by the floods.

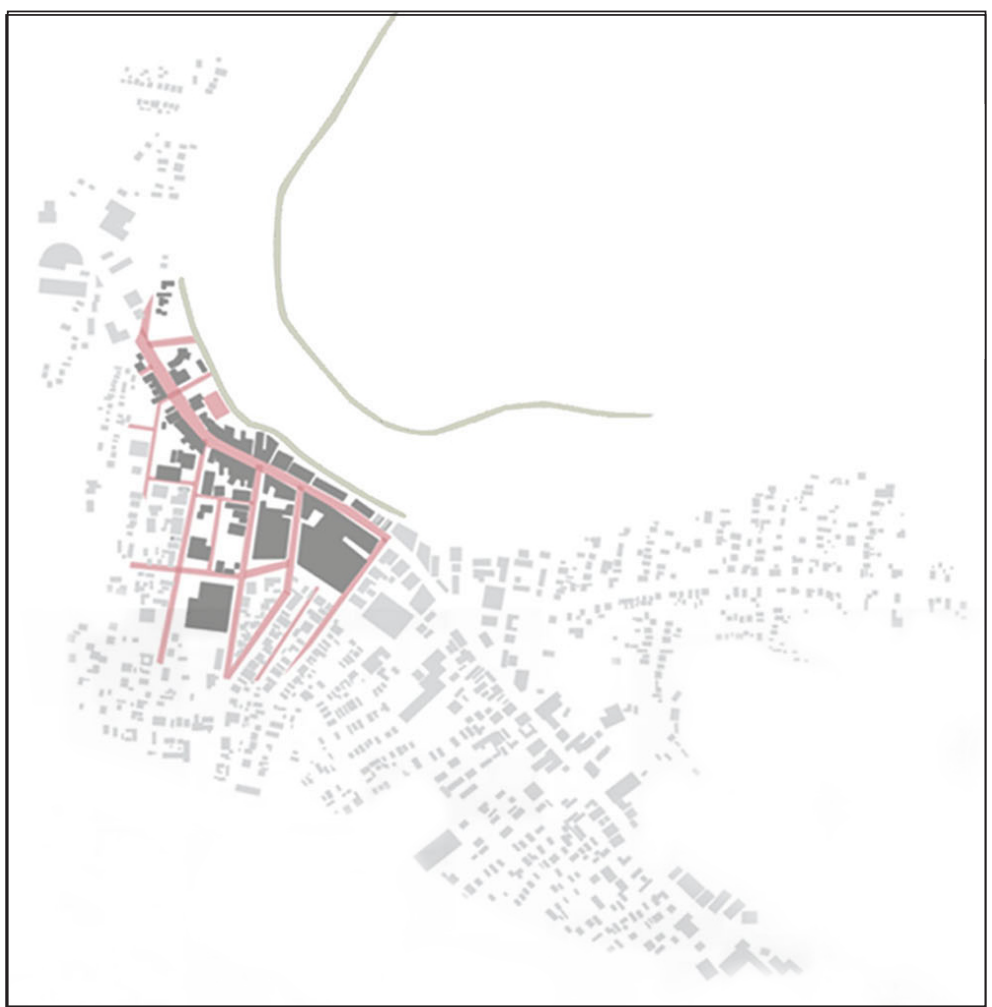

Figure 118: Maitland's socio-spatial resilience network in high floods

Roads not impacted upon by flooding form important roles in post flood recovery processes, along with the community centre, and any functional commercial businesses. Functional city facilities and services enable residents to more readily return post flooding. The high flood network is strengthened by increasing the amount of city infrastructure which remains structural and functional post flooding. 
7.7 Current socio-spatial vulnerabilities and opportunities

The key issues of the Maitland City system arise from several existing socio-spatial vulnerabilities. These adverse system components or lack of sufficient strategies provide opportunities where socio-spatial flood resilience can be strengthened. A series of innovative components (detailed later in the chapter) are designed to provide a category of possibilities rather than a singular solution. This recognizes the multi-scale interrelating complexity of a resilience flood management approach. Strengthening the socio-spatial resilience network of Maitland involves ensuring key infrastructure services remain functional pre, during and post flooding and accessible by communities, who remain connected and minimally impacted upon by low to medium flood category events. As high flood category events approach the network ensures there is adequate warning, circulation, and transport options for the city's inhabitants to evacuate whilst key infrastructure are designed to survive and remain functional post flooding. 
A socio-spatial resilience network is made up components at the multiple scales of the city

\section{- residents : building \& lot scale}

New and retrofit amphibious construction

- vulnerabilities

- opportunities

- strategies 
7.7.1 Residents : building and lot scale

\section{(flood proofing, elevation \& amphibious construction)}

The Urban Development Institute of Australia's NSW Lower Hunter State of the Land Report 2010 highlighted the increasingly problematic under supply of housing in the Hunter Region. The report raised concerns about the unaffordability of housing in the region after the NSW and Local Governments failure to "provide an attractive and stable environment for investment" (UDIA, 2010). Maitland's flood liable land has inhibited development opportunities and lowered land values, whilst heritage values in Maitland have impacted on the implementation of flood proofing strategies. The UDIA report recommends revolutionising how the NSW Government and Local Councils view housing in the region. As Smith (1998) notes, "the majority of the potential failures are to single storey, detached, [and] weatherboard dwellings that are a relatively common house type in older urban areas in Australia”, such as Maitland (Smith, 1998, p. 58).

"It was distressing in flood time to see families having to leave their homes and return to them desolated and damp enough to kill them".

West Maitland Council's Chairman Owen Evans (c. 1866) in Hunter \& Maitland City Heritage Group, 2000, p. 18)

\section{Suitability of existing housing types to a range of flood types:}
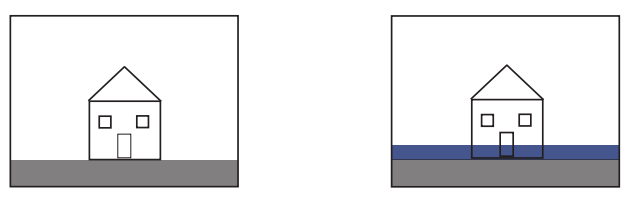

Slab on grade construction

- Flood prone construction

- Suitability improved through water resistant material use e.g. concrete.

- Not suitable for floods
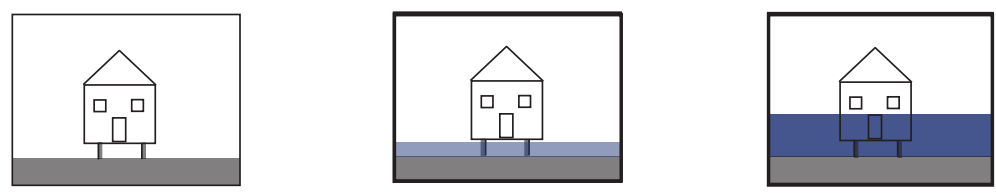

Pile or post construction

- Minimal flood proofing measure

- Low flood suitability improved through the use of marine grade timber.

- Not suitable for medium flood velocities and depths.
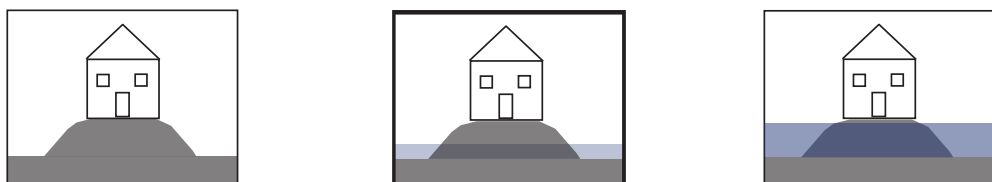

Houses raised on earth mounds

- Medium flood proofing measure

- Flood suitability improved with greater topographical height.

- Not suitable for unanticipated large flood heights.

Figure 119: Suitability of existing housing types to a range of flood heights 

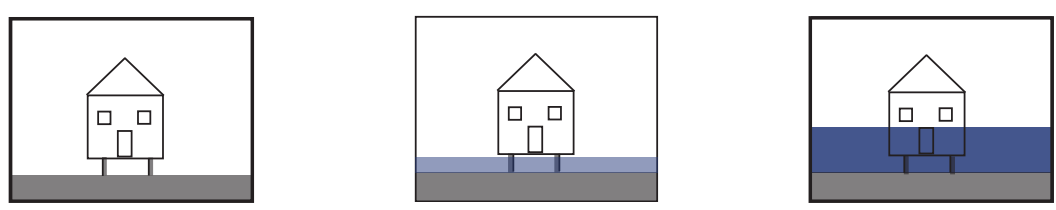

Stilt house construction

- Medium flood proofing measure

- High flood suitability improved by water resistant material use and stilts extending deep below ground.

- Not suitable for high velocity floods.
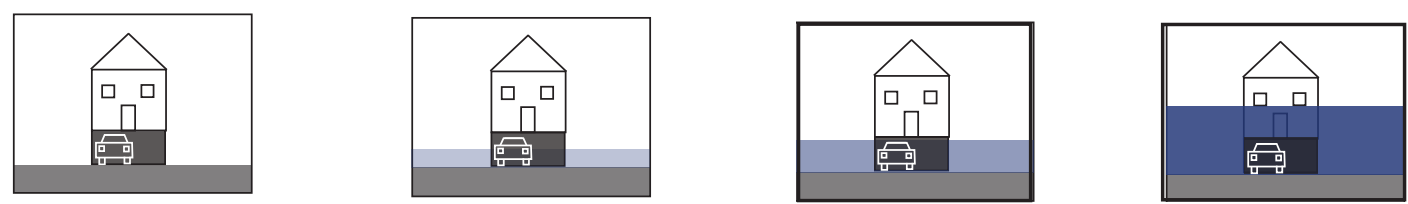

Living spaces raised above garages

- Medium flood proofing measure

- High suitability if water resistant materials are used and valuables kept above garages.

- Not suitable for unanticipated large flood heights.

\section{Suitability of amphibious construction to a range of flood types:}
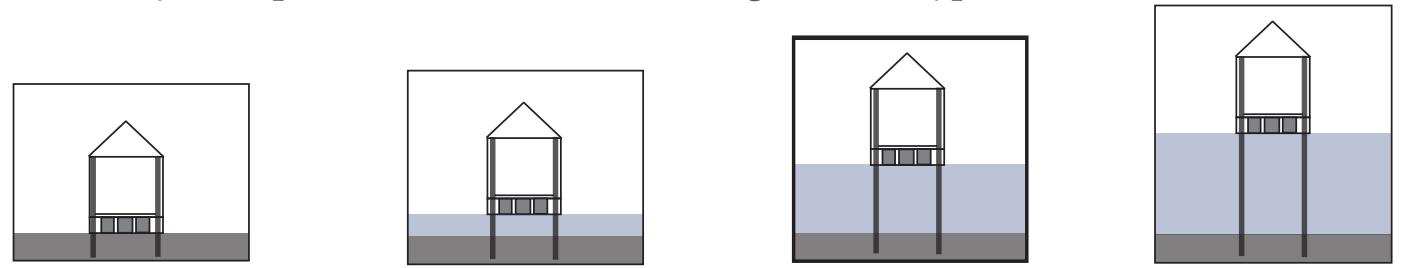

Amphibious construction

- High flood proofing measure

- Suitable in a variety of flood heights if water resistant materials are used e.g. steel, timber, lightweight concrete.

- Not suitable for high velocity floods and large wave heights.

Figure 120: Suitability of amphibious construction to a range of flood types
Elevating houses on stilts greatly reduces the building footprint, but can be inappropriate for disabled access or impractical in areas of Maitland which experience high flood heights. Due to the heritage character of many Maitland residences and high flood heights, traditional measures such as elevation have failed to be implemented, leaving many residents extremely vulnerable to the flood risks.

Amphibious construction methods, being trialed and implemented internationally have proven highly successful in slow rising, high depth, low velocity, long duration floods, which are characteristic to Maitland. Amphibious construction varies in economic cost, but can be likened to the cost of raising weatherboard houses on masonry blocks or timber pylons. Thus amphibious construction is a suitable low-economic flood proofing option for Maitland residents.

There are opportunities to further enhance socio-spatial resilience through the provision of alternate energy sources and services to allow the occupant to continue to practice everyday lifestyles during inundation periods. This is particularly useful for floodplain inhabitants, with floods typically being long in duration. 
Resilience design strategies which may improve the socio-spatial resilience of flood safe housing include:

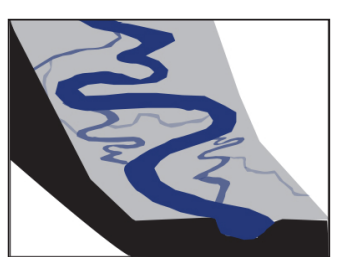

Understanding local flood hydraulics will ensure buildings are appropriately sited and structurally suited to the flood characteristics.

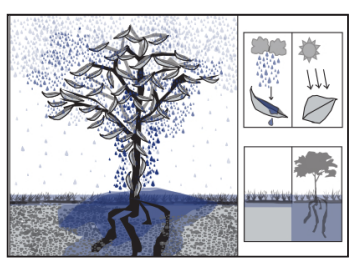

Structures designed with an ability to anticipate and react to flooding, such as amphibious construction, will reduce flood damage.

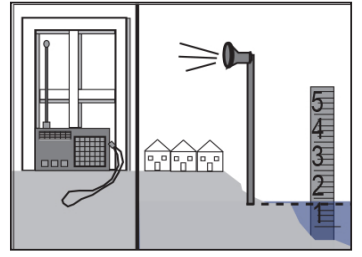

Acoustic and visual flood warning strategies will enable residents to be informed of the fluctuating water levels.

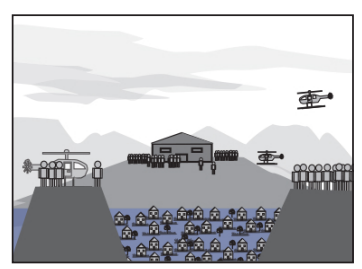

Residents being prepared for flooding include preparing flood kits prior to flood events and having evacuation plans in place.
Having self-reliant systems such as alternate energy means and water collection and filtration systems, ensures residents are not without power, water and sanitation facilities when electricity and water mains are shut down.
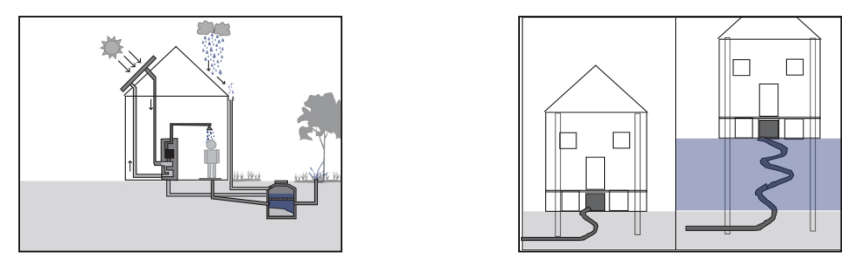

The use of water resistant material and site specifically selecting dry and wet flood proofing measures based on local flood characteristics will ensure a balance between preventing and embracing floods is achieved.
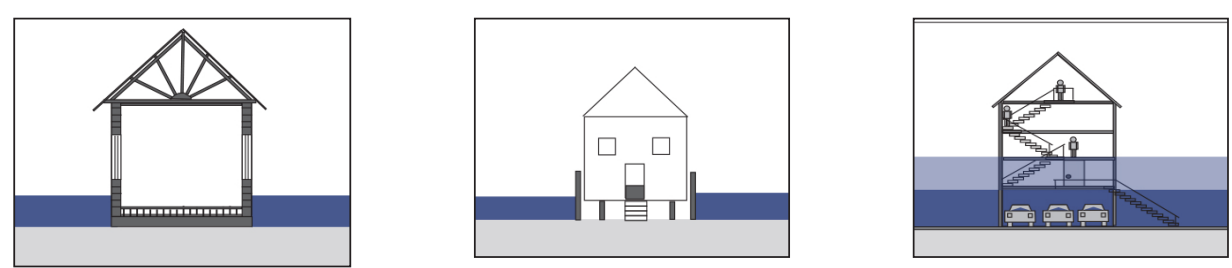


\section{New and retrofit amphibious construction}

Maitland's heritage weatherboard housing will need to be amphibiously retrofitted to ensure the construction does not affect the town's heritage significance. The amphibious construction provides a new economically feasible solution for Maitland, supplementing current wet and dry flood proofing initiatives and elevated construction techniques. Residential flood proofing measures and amphibious retrofit construction will greatly reduce the long term financial, physical and emotional costs of flood damage. However, these residential scale benefits must be viewed within the multifaceted concept of resilience; acknowledging the impact the surrounding site features, urban landscape and regional structures will have on flood characteristics.

In acknowledging that residential flood resilience differs greatly within communities, there is an opportunity to develop a new community support program; 'flood safe houses' which would work towards further strengthening a socio-spatial flood resilience network. A 'flood safe houses' program could be modeled off the existing Child Safety Australia's 'Safety House program', which involves collaboration between community and police in which a network of houses and businesses, checked by police, provide a safe place to those feeling unsafe within the community. Flood safe houses could similarly involve collaboration between a range of stakeholders including, hydrologists and residential owners within the community. This would enable residents to remain locally sheltered, supported and safe. This will work at strengthening local support networks, whilst reducing the emotional stress associated with people being unable to check and improve the sandbagging of residents, along with the stress involved in losing contact with those evacuated from the city.
There are also opportunities for Maitland City Council to consider implementing amphibious developments as part of their 2021 Maitland vision for the creation of a 'vibrant river city'. New and retrofit amphibious residences will significantly improve residential flood resilience (refer to Chapter 5 Precedent analysis: floodplain inhabitation for further detail) without affecting the existing character of residential suburbs. Maitland has an opportunity to become more than just a river city tourist attraction, but the first Australian city to implement amphibious housing solutions in Australia and join its international flood prone counterparts in working towards a flood resilience future.

\section{Strategy intentions:}

- Reducing flood damage and thus speeding up flood recovery

- Establishing flood safe shelters within the local residential community

- Ensuring alternate and renewable energy sources, water collection and sanitation facilities are available.

Architectural resilience adaptability strategies: regeneration

Cleaning and improving flood proofing solutions by applying knowledge of the recent flood event will improve the ability of the structure to cope with future floods.

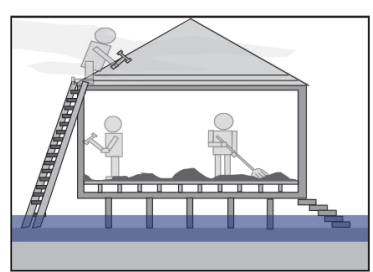

Figure 121: An architectural regeneration strategy 
Examples of existing amphibious construction techniques

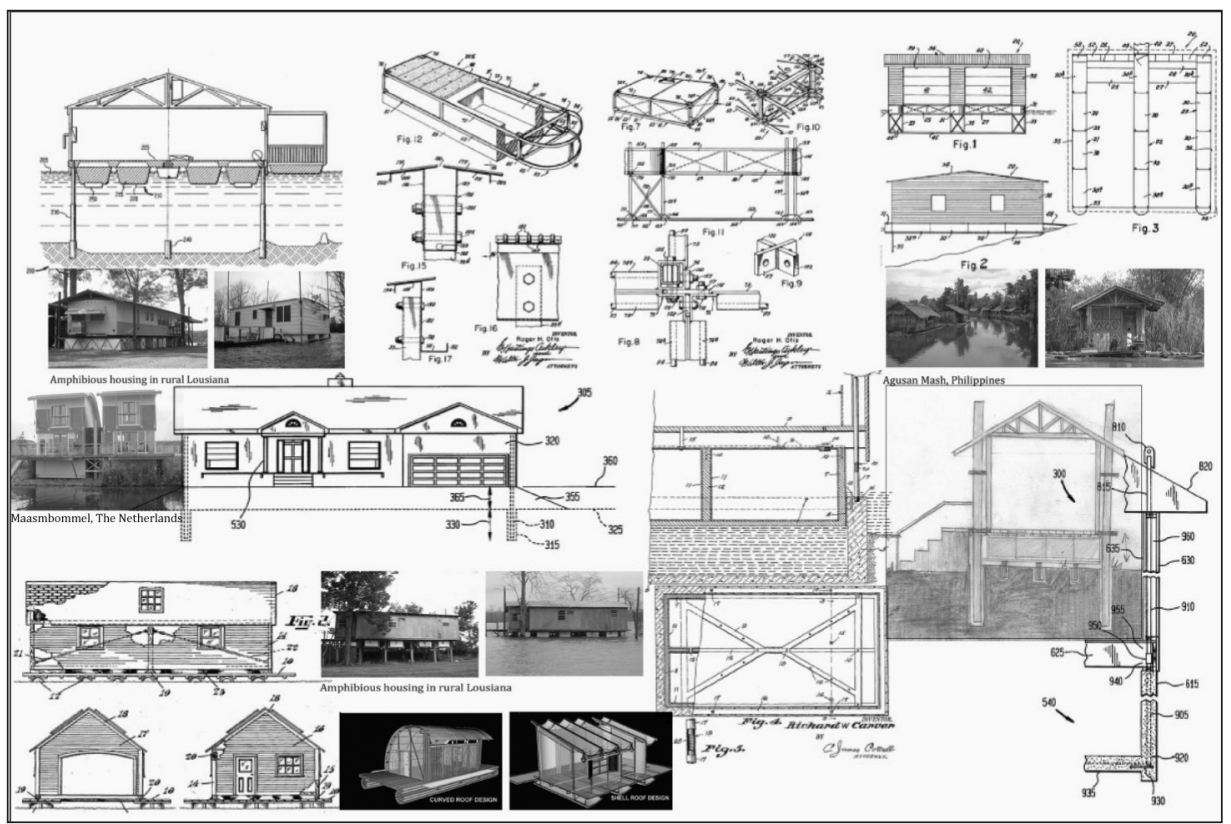

Figure 122: Examples of existing amphibious construction techniques
There are a diversity of existing amphibious construction methods, many of which are based around the theory of long steel, timber or concrete pylons supporting a buoyant foundation structure made from light weight concrete or polystyrene blocks within a metal frame. Furthering exploration into amphibious residential construction is beyond the scope of this thesis. This thesis highlights the problems arising from current emphasis on the residential scale and the need to address issues within the urban and city scales (refer to $5.7 \mathrm{Key}$ findings from precedent analysis).

Architectural resilience adaptability strategies:

rapid response
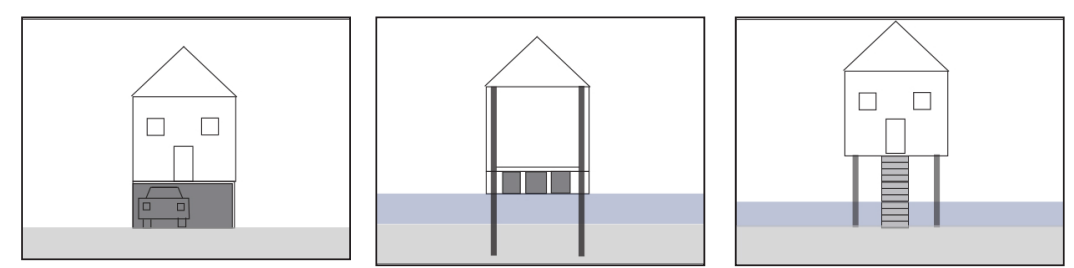

Permanent flood adaptation measures such as static elevation of living spaces on poles or above garages and amphibious construction reduce the adverse impacts of floods on buildings.

Figure 123: An architectural rapid response strategy 
An example of how amphibious retrofit of existing Maitland residences could take place

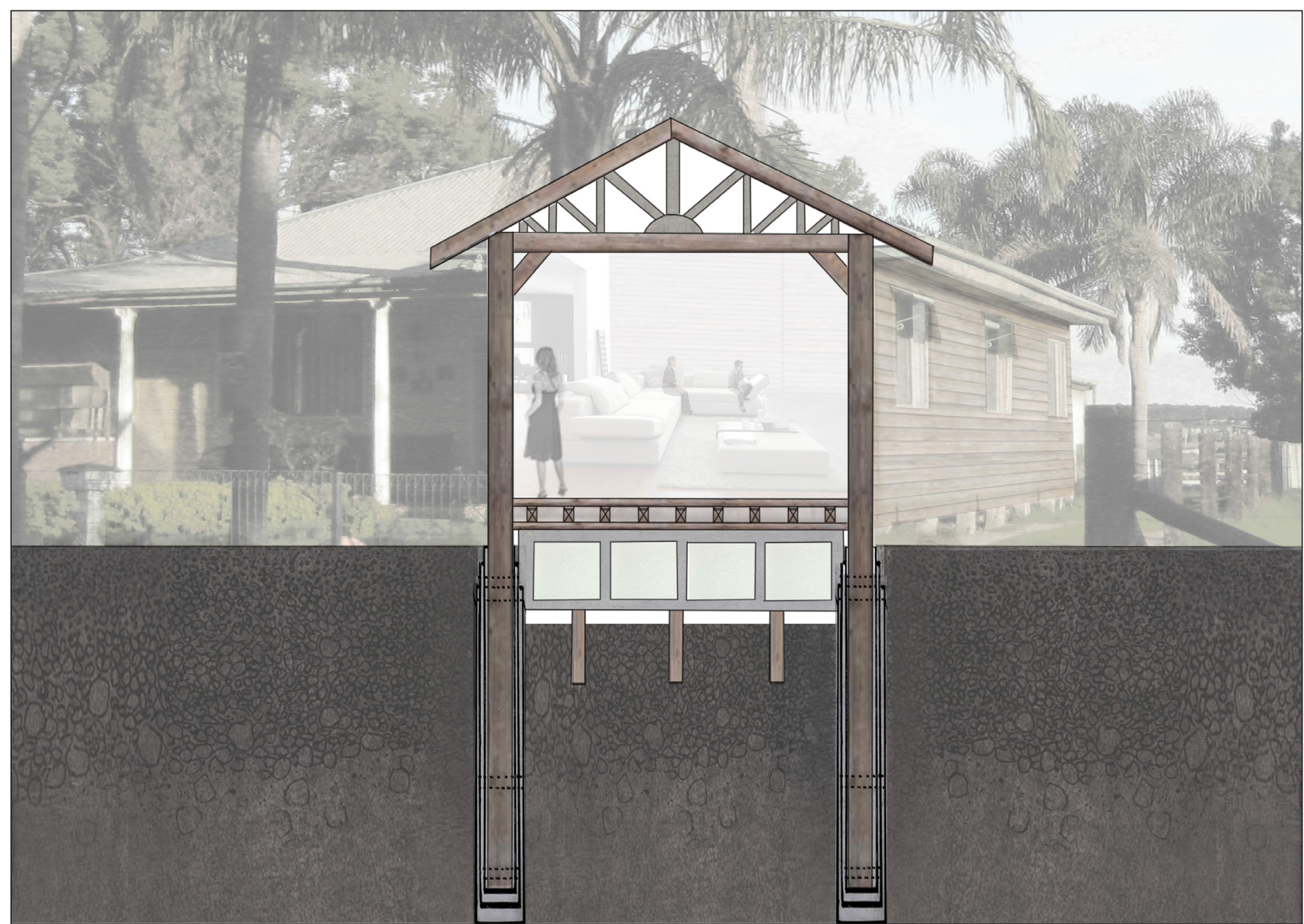

Figure 124: An amphibious retrofit of an existing Maitland residence 
A socio-spatial resilience network is made up components at the multiple scales of the city

\section{- neighbourhoods : urban street scale}

Flood adapted pedestrian circulation

- vulnerabilities

opportunities

- strategies 


\section{(Pedestrian mobility concerns)}

Mobility options in Maitland have diversified over time to include water, rail, and road transport options. Historically the Hunter River fulfilled a significant recreational and industrial functional role; "the Hunter River provided a livelihood for many people and was always busy with the passage to and fro of small boats carrying goods and people" (Hunter \& Maitland City Heritage Group, 2000, p. 6). But the development of a railway line in the 1850 's provided a new more effective means of transport for industry and people. The Hunter Railway line continues to provide transport south to Newcastle and north to Dungog, although this is hindered by flooding at Maitland's two flood liable railway stations: High St and Maitland. The most recent development of the New England Highway provided additional means to transport people and industrial goods between the north and south, however it too remains flood liable. The railway is cut and the highway impassable when back flooding of Maitland takes place. It is at this point that Maitland City becomes isolated from surrounding townships.

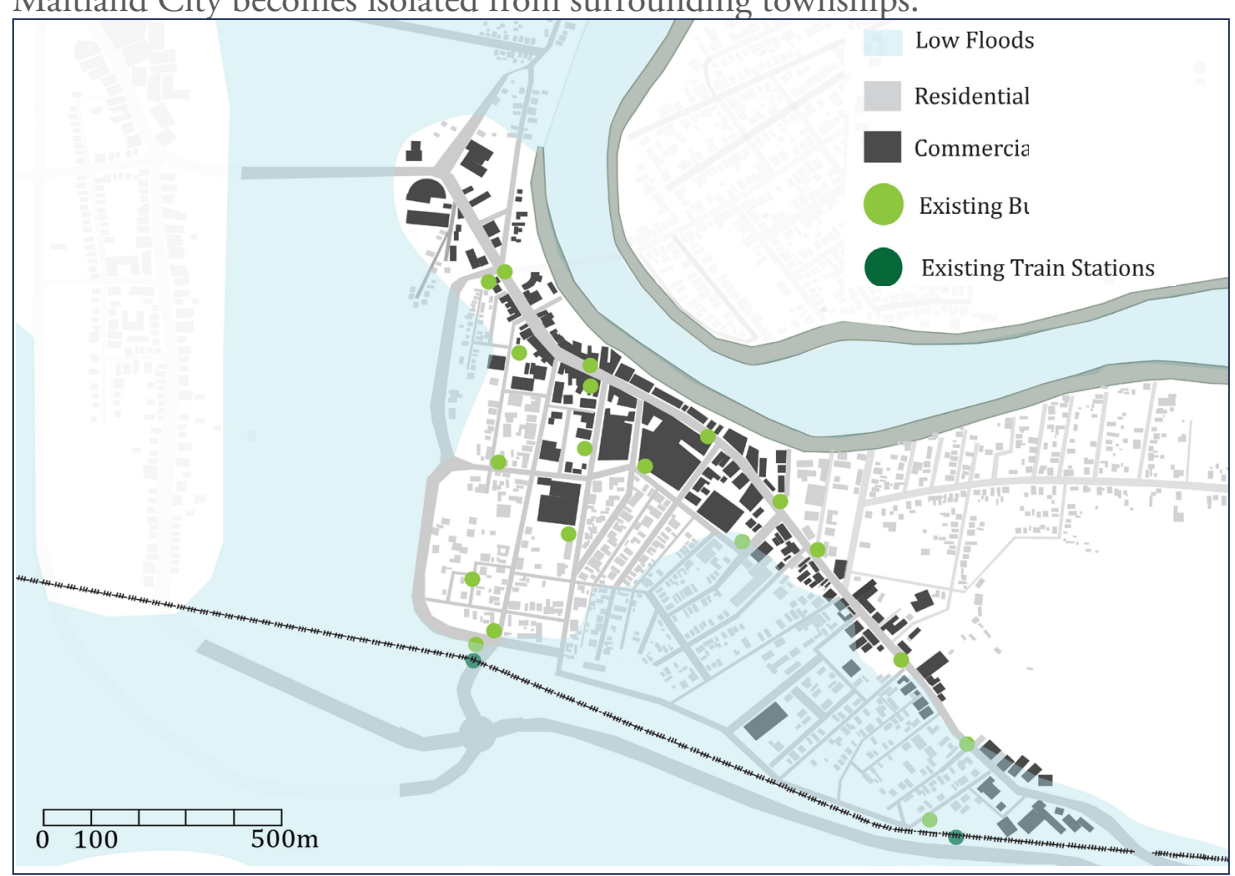

Within Maitland there are also mobility concerns resulting from floods. With the Central Maitland land having initially been subdivided for agricultural farming rather than for a township the primary street pattern, such as High Street, was historically designed to respond to the river's meandering path (City Plan Urban Design, November 2009). Maitland City Council has recognized the issues surrounding access and circulation in Maitland for many years. In 1998 the Maitland Council released the Central Maitland Area Improvement Plans (CMAIP): Landscape Concept Plan which recognized the need for improved road entry points and pedestrian mobility within the city. Strategic proposals such as improving access to the riverbank and enhancing the circulation of the riverbank for public use however failed to be implemented.

Most recently the Maitland City Council's Central Maitland Structure Plan 2009 and Maitland 2021 Community Strategic Plan have identified pedestrian and transport weaknesses within Maitland (City Plan Urban Design, November 2009);(Maitland City Council, February 2011). One such issue identified was the limited number of connections between the CBD and residential areas in South Maitland, which are the first to be inundated by back flooding.

Residents become isolated from their neighbours, communities, and city services as a result of public infrastructure not being designed to adapt to seasonal flood conditions. There is a need to explore flood adapted pedestrian circulation options which do not rely upon existing road infrastructure. This will enable residents to continue everyday lifestyles in low flood category events whilst strengthening access to flood safe shelters in medium flood events and evacuation procedures ahead of high flood category events. Thereby reducing the impact of floods on pedestrian mobility and strengthening everyday community networks and connections.

Figure 125: Impact of low floods on residential and commercial buildings, roads, bus stops \& train stations 
Resilience design strategies that could to improve the socio-spatial resilience of pedestrian mobility include:

Understanding both the natural and artificial flow paths of floodwaters is crucial in establishing flood safe circulation pathways.
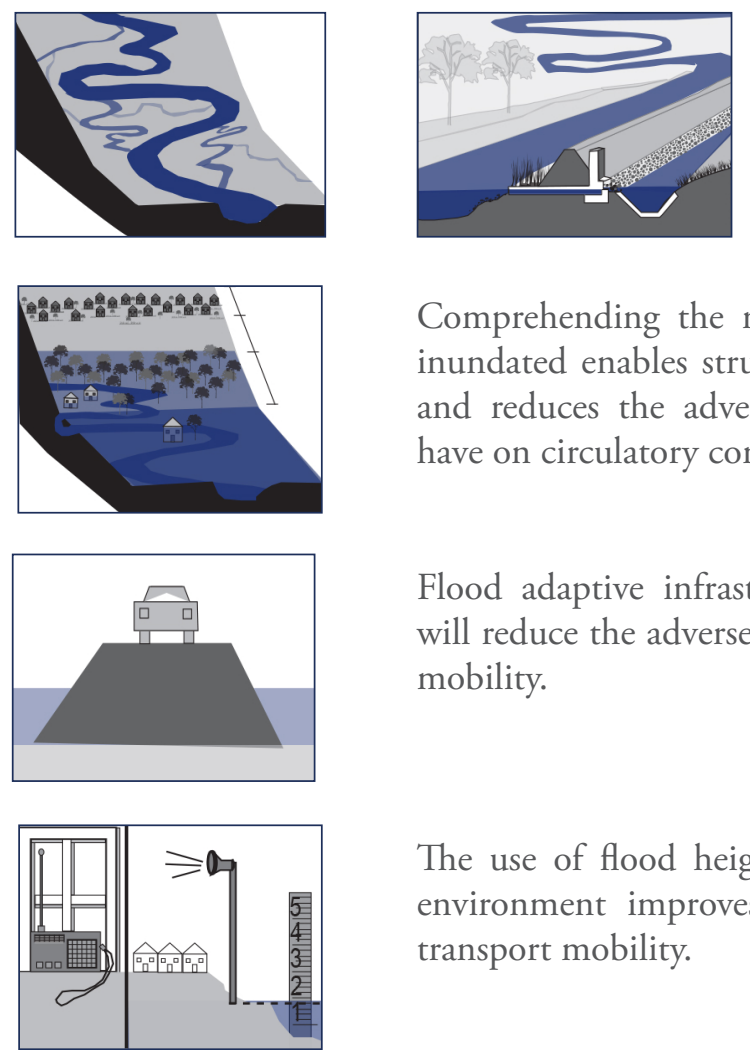

Comprehending the risk levels of urban areas being inundated enables structures to be appropriately sited and reduces the adverse impact flood characteristics have on circulatory connections.

Flood adaptive infrastructure such as elevated roads will reduce the adverse impact of inundation on urban mobility.

The use of flood height indicators within the urban environment improves the safety of pedestrian and transport mobility.
The use of water resistant material, along with dry and wet flood proofing measures, will enhance the longevity and structural integrity of the flood prone circulation structures.
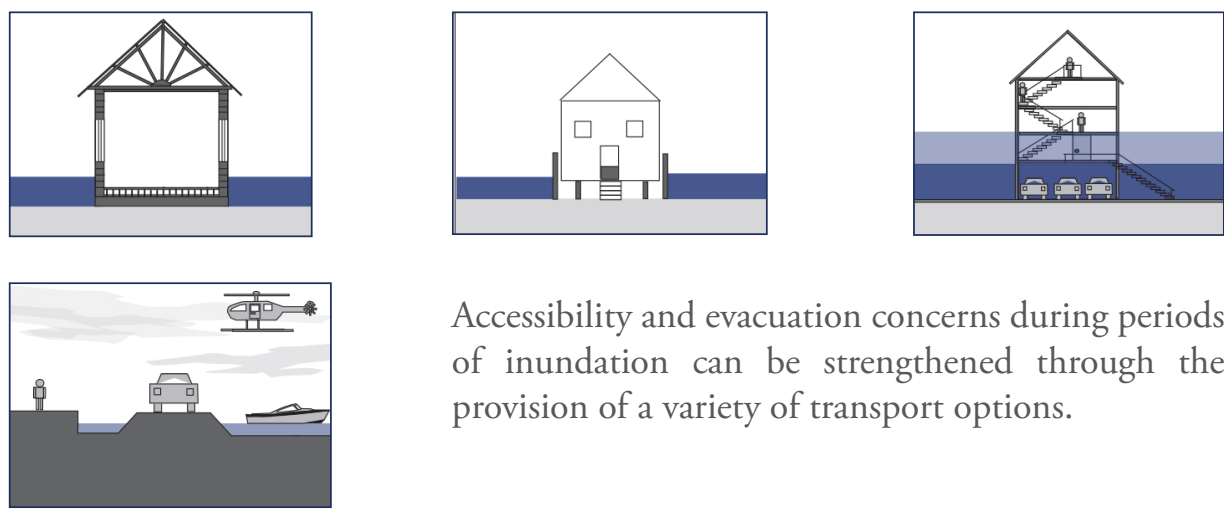

Accessibility and evacuation concerns during periods of inundation can be strengthened through the provision of a variety of transport options.

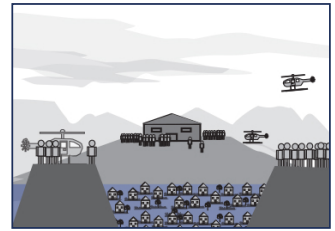

The ability of inhabitants to escape flood risk zones and access evacuation centres greatly impacts upon the efficiency of Emergency Management.

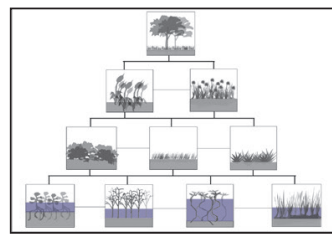

Structures designed to anticipate and react to changing flood conditions will reduce the adverse impact of floods.

Natural and artificial strategies will strengthen the ability of the urban environment to mitigate flooding (reducing velocity and volume).
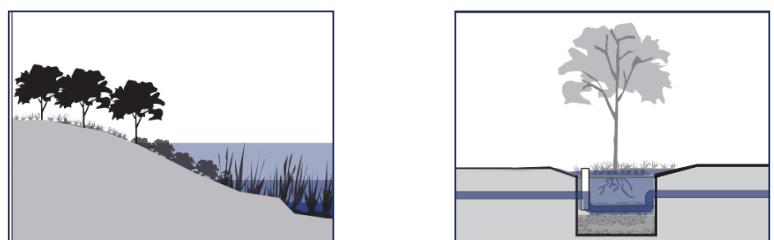

- Case study design 


\section{Flood adapted pedestrian circulation}

(At the urban scale: connecting residential and city scales)

There are opportunities for existing urban infrastructure and services to be retrofitted or implemented to provide local communities with power, water and shelter pre, during and post flooding through renewable self-sufficient sources.

A combination of bottom up and top down approaches facilitate an ongoing collaborative process between residents and governments working to improve pedestrian circulation during low and medium flood inundation. The bottom up construction methods need to be economically suited to residential development, when compared to top down measures which have higher investment and construction costs.

Floods currently result in individual residents becoming isolated in their houses. The cutting of electricity, water supplies and limited access to food outlets and other city services means the ability of individuals to achieve resilience is limited. The provision of an alternate flood pedestrian circulation path which is functional in a variety of flood events will improve community support during periods of inundation as residents are able to remain physically and socially connected to one another. Flood hazard levels need to be understood with regards to depth, velocity and rate of rise.

There are a series of specific statically elevated and amphibious circulation strategies appropriate in low and medium floods, whilst in the hazardous environment of the city high flood category events become reliant on boat and helicopter transport. Connecting a variety of strategies to one another improves the ability of residents to access key services and facilities during and post floods. Thus the adverse impact of floods is reduced, evacuation procedures are improved and recovery processes enhanced

\section{Strategy intentions:}

- Intention to connect individuals to communities and key services (e.g. bus shelters, barbeque and picnic shelters, community centre and the commercial district).

- Speed up recovery rates by allowing inhabitants to access city services and their place of employment.

- Reduce the impact of floods by improving the ability of the inhabitants to live with floods and circulation infrastructure to function during periods of inundation.

- Utilise existing urban features to establish an autonomous solution which can be easily implemented (e.g. fences, sidewalks, bridges, pedestrian crossings and park shelters).

There are opportunities for inhabitants to establish and benefit from a symbiotic relationship with water in the urban landscape.

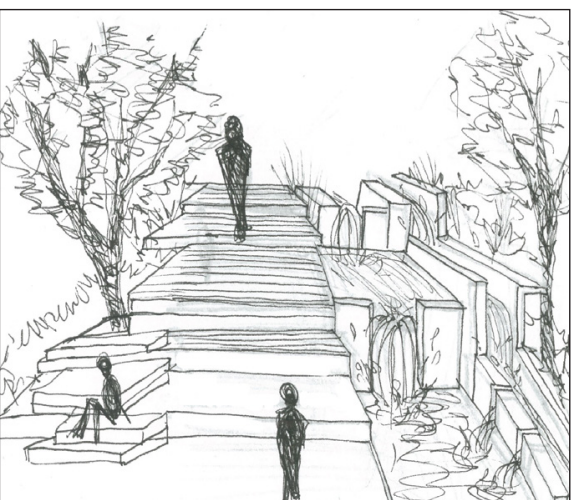

Figure 126: Waterscapes design idea 
Pedestrian pathways are primarily sited in relation to residences. They fulfill a secondary role in establishing pathways to key services and infrastructure, and achieve a tertiary relationship with existing road infrastructure. With roads becoming water channels during floods, pedestrian pathways, where possible, have been sited at a distance from roads to areas where floodwaters are more characteristically stationary and debris and water velocity risks are reduced.

An example of low flood category event circulation paths: $\quad$ Alternate pedestrian paths Maitland levee - Functional roads Inundated roads

- Existing roads and flood - Buildings at risk of adapted pedestrian paths. being inundated.

- Flood adapted pedestrian circulation paths.
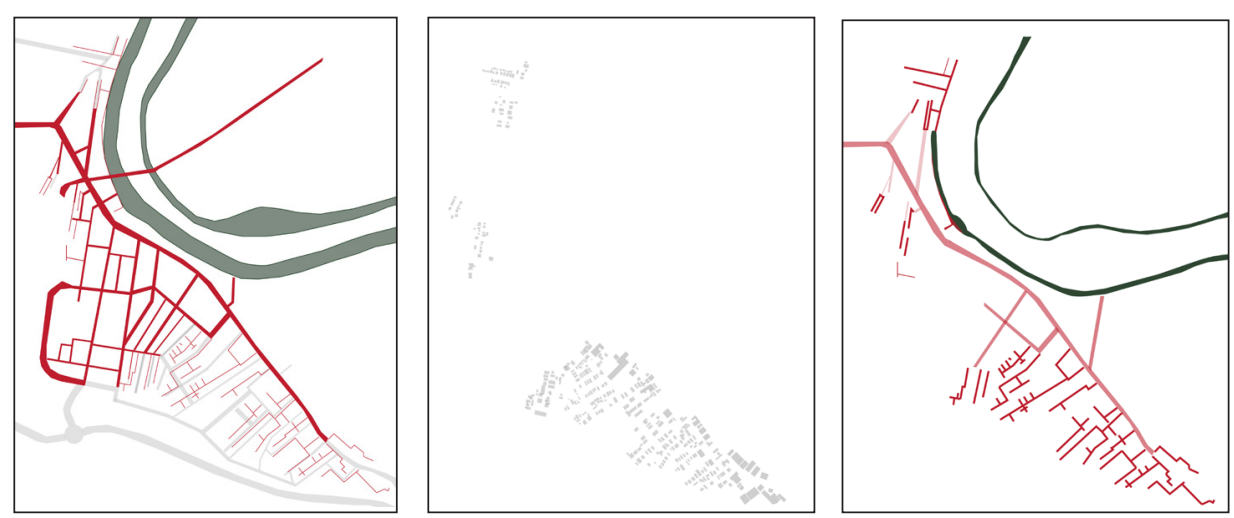

Figure 127: Circulation paths for a low flood category event
The urban environment of Maitland must begin to seek improved balances between flood preventative dry flood proofing measures and water embracing wet proofing, ecological vegetation and water channels to reduce the adverse impacts of inevitable flooding.
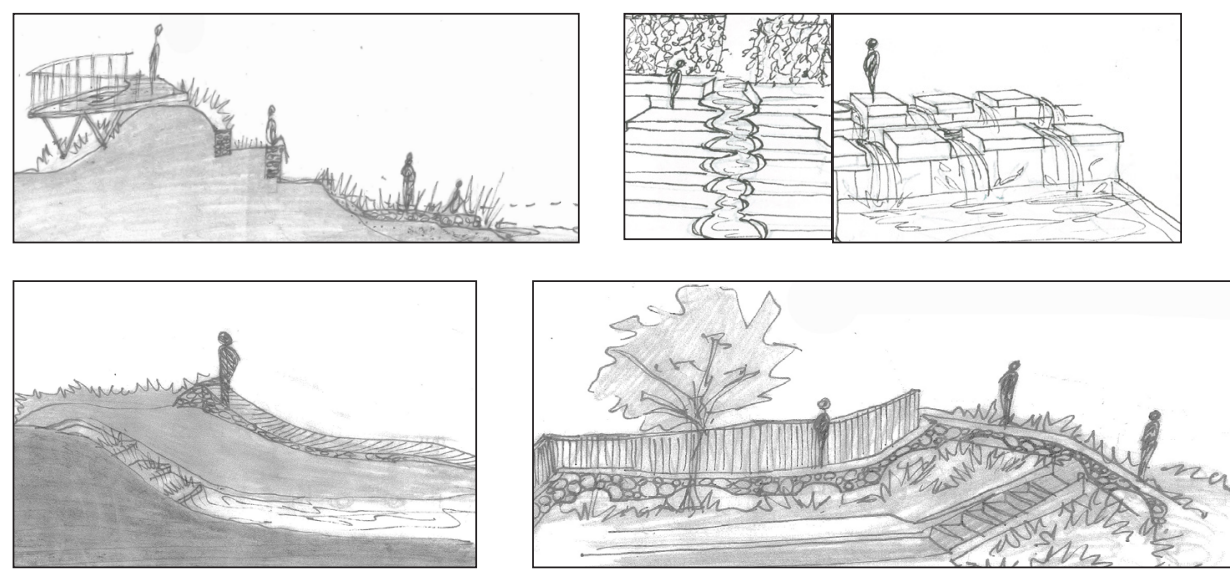

Figure 128: Sketches exploring relationships between pedestrians, water, ecology and urban can be formed

There are opportunities for the urban landscape features to interact with and celebrate rainwater, whilst preemptively planning how floodwaters will be guided through the urban landscape in order to reduce its adverse socio-spatial impacts; mitigating risks for inhabitants and built infrastructure, whilst increasing the ecological benefits of flooding. 


\section{Traditional pedestrian circulation infrastructure investigated}

Pedestrian bridges can provide elevated circulation paths across roads, which become water channels during floods. However, within the heritage low lying, predominately single residential storey and double storey commercial districts, there are aesthetic street character concerns. Due to heritage concerns, despite the practical appropriateness of this traditional measure, it is unable to be implemented as a widespread solution within Maitland.

Site-specific hydraulic studies need to explore velocity, duration, flood heights and debris concerns to ensure adequate structural strength and water resistant material is incorporated into the bridge design and construction.

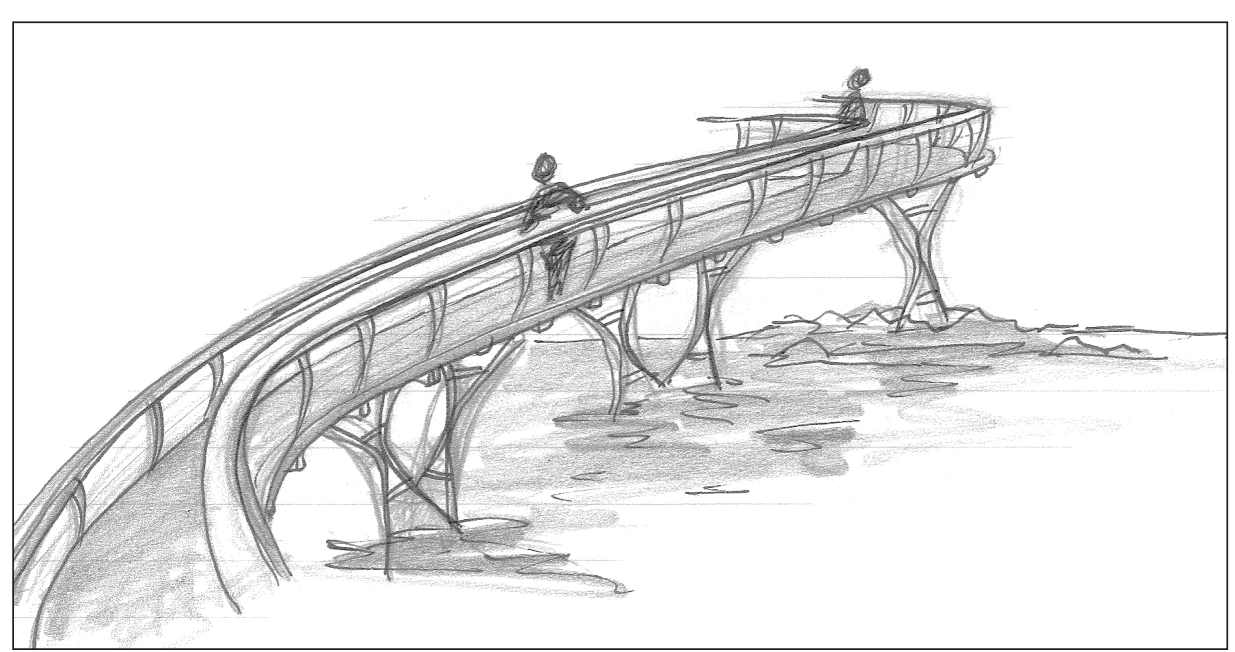

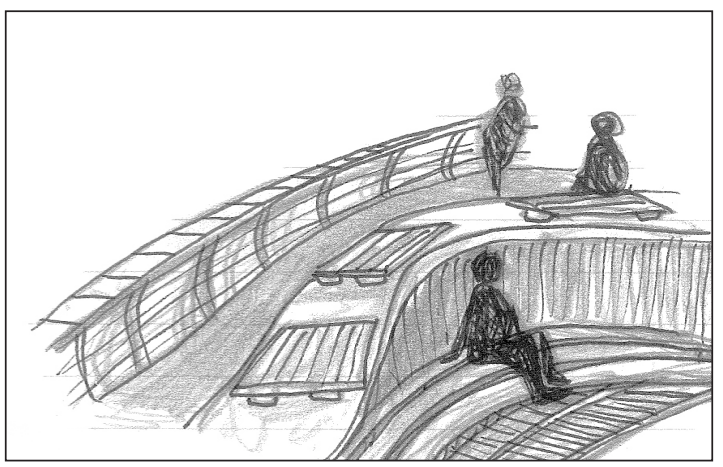

The pedestrian bridges establish direct elevated circulation paths whilst providing opportunities for passive resting spaces..
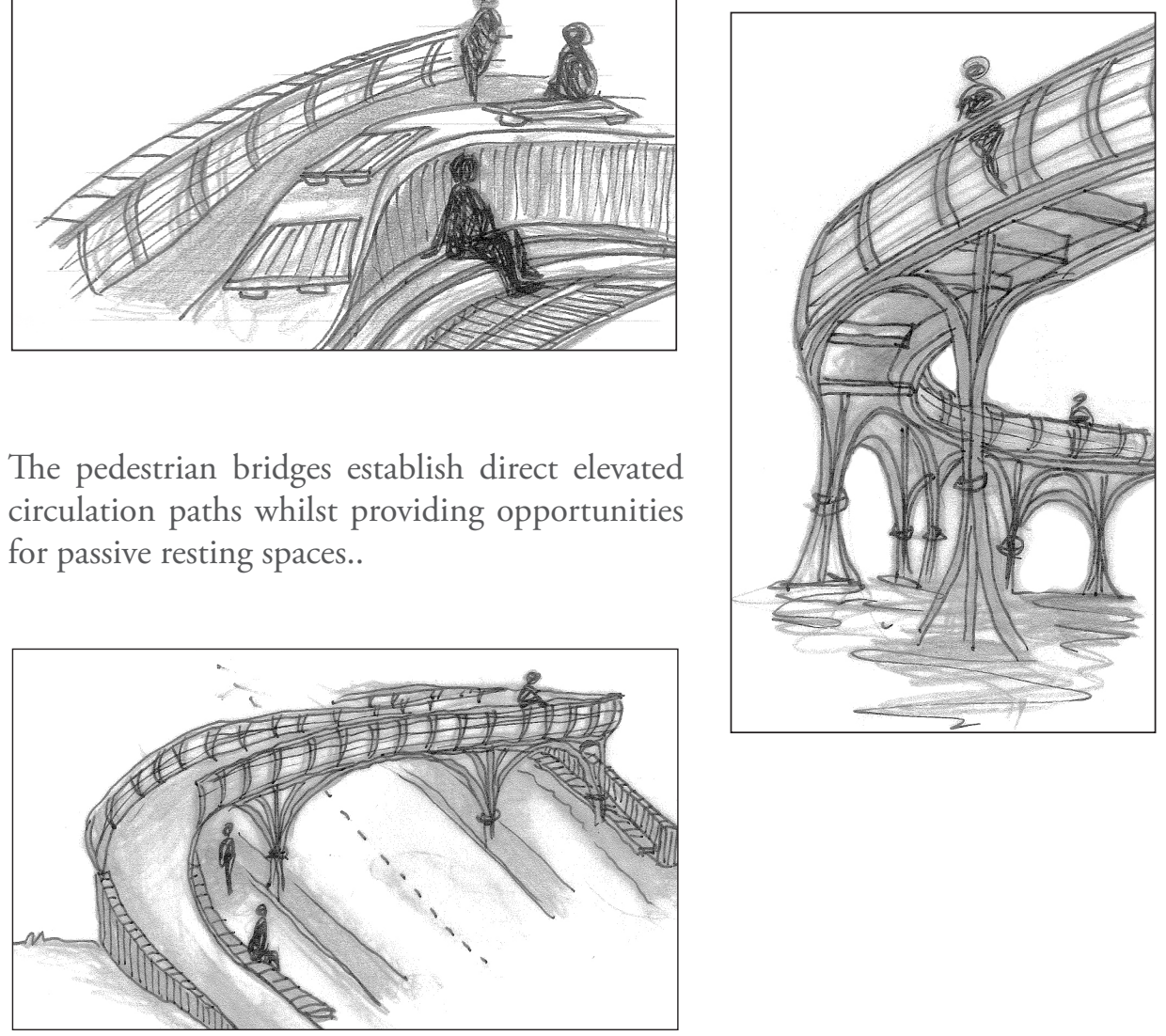

Figure 129: Sketches exploring the possibilities of pedestrian bridges 


\section{The issue of crossing roads during floods}

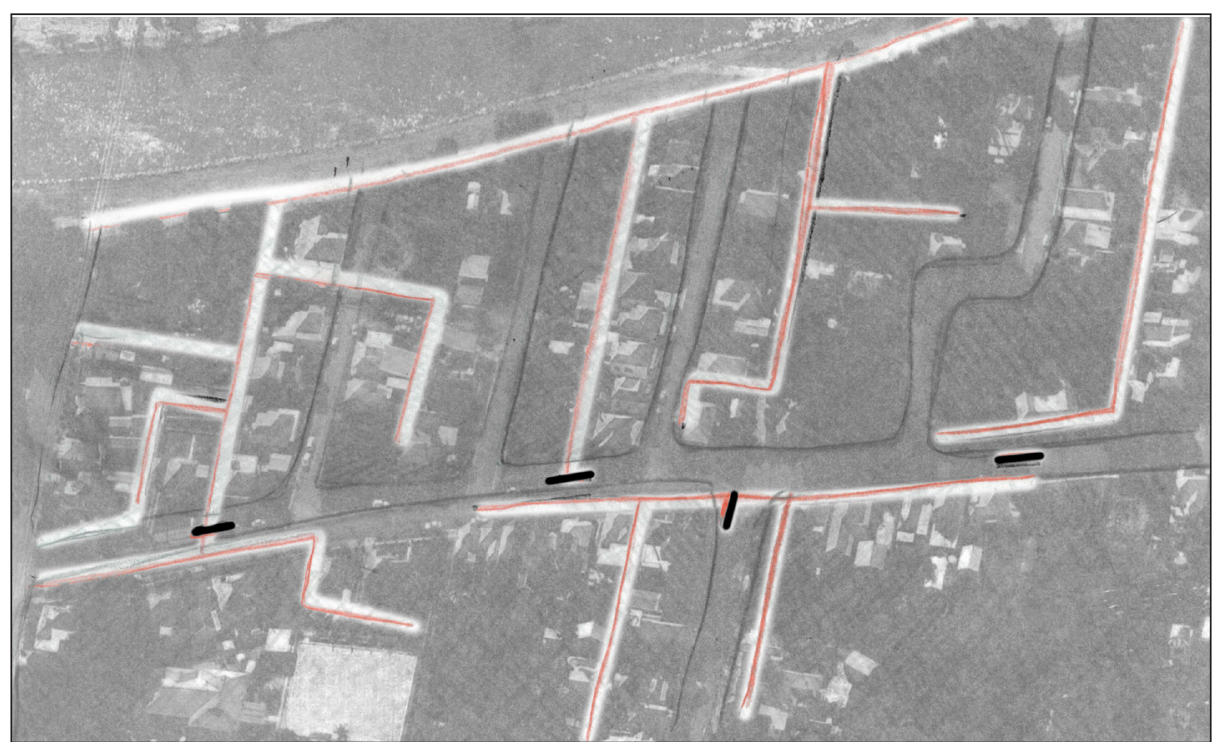

Figure 130: Analysing the existing fence layout of Horseshoe Bend

(Refer to 6.3.4 Urban scale analysis for further information on the Maitland suburb of Horseshoe Bend.).
Analysis of the existing fences in Horseshoe Bend, Maitland reveals the challenge of crossing inundated roads. Because pedestrian bridges are inappropriate as a widespread solution within the heritage town, an alternative solution needs to be investigated.

Inhabitants encounter difficulties when attempting to cross roads when urban areas are inundated by floodwaters. Unless residents have access to alternate water borne forms of transport they quickly become isolated from neighbours, public infrastructure and services. Applying socio-spatial resilience concepts to the problem, the importance of functional autonomy, such as the need for rapid response of both infrastructure and inhabitants is recognized.

Architectural resilience adaptability strategies: alternate circuits

Static or amphibious circulation pathways increase safety and provide pedestrian paths during and post flood inundation periods.

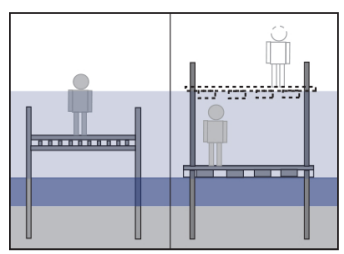

Figure 131: An architectural alternate circuit strategy 


\section{Design explorations into possible solutions of crossing flood inundated roads}

A series of possible options such as floating road sections and amphibious median strips were tested before pedestrian crossings arose as the most appropriate functional autonomous solution.

The social meaning behind pedestrian crossings, seen as the safe place to cross roads, is hence able to be maintained pre, during and post flooding. This allows existing urban infrastructure features to serve additional flood adapted functions, which are an extension of their everyday roles. This ensures there is social understanding of the urban features, improving inhabitant use and response, whilst allowing the alternate functions not to inhibit or disrupt the spatial aspects of the structures.

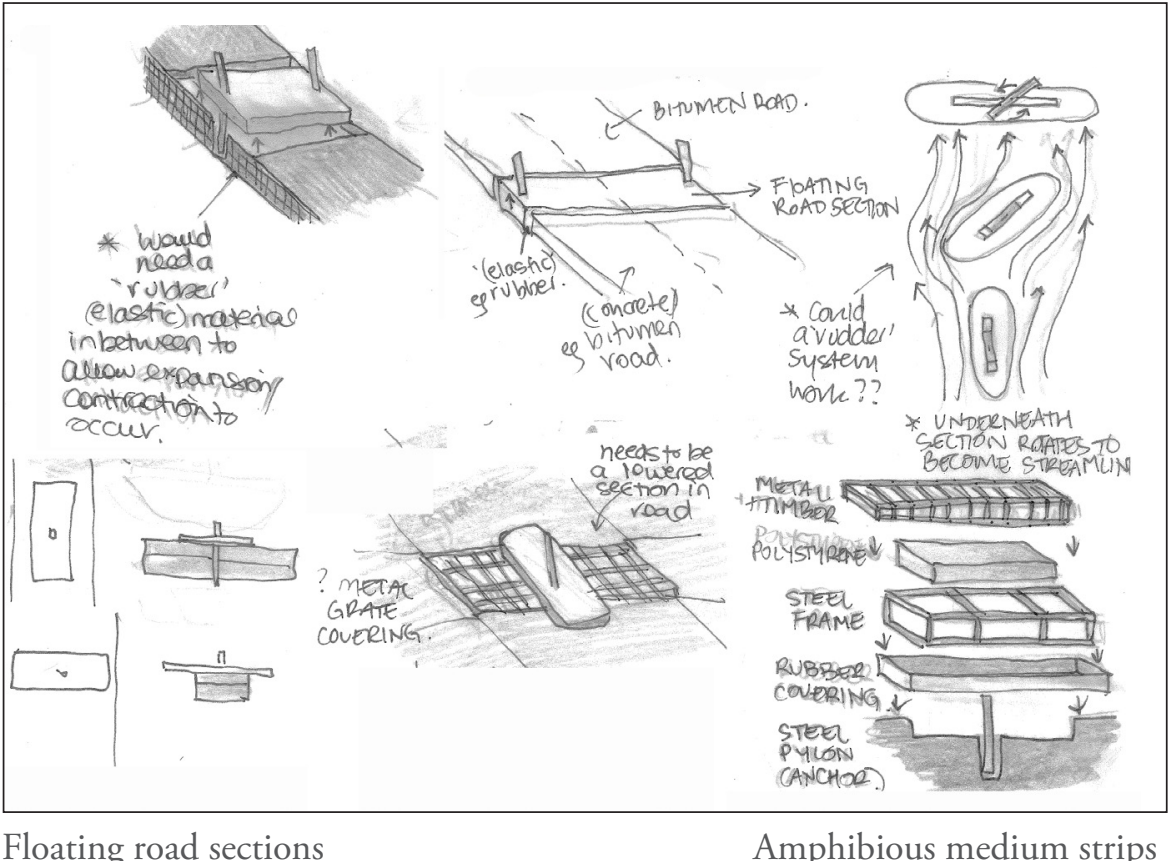

Figure 132: Exploring possible amphibious and floating solutions to crossing flood inundated roads 
This construction section of an amphibious pedestrian crossing, highlights how the construction is hidden beneath the road. This ensures the design is respectful and does not detract from the heritage character of Maitland's main street, High Street.

The construction method used in this amphibious solution maintains the key concepts behind amphibious construction, but differs greatly to the economical residential amphibious fence methods (detailed; ater in the chapter). This is a direct result of the pedestrian crossing being a top down initiative, requiring a long durable lifespan. The function of the road as a load bearing structure for cars also influences the material selection and the choice of construction methods.

Light weight concrete encases polystyrene blocks allow the concrete to absorb and reflect under loads, rather than be rigid in response to the cars passing over. Telescopic large steel pylons reduce the depth of the supporting structure, to provide greater stability and support. Stainless steel welded and bolted brackets connect the concrete to the metal pylons.

A steel reinforced ribbed slab allows water to collect beneath the hollow structure. As the water collects an upwards pressure mounts. The stainless steel telescopic pylons have a series of weeping holes which allow water to pass through and the pressure mount, establishing a hydraulic mechanism. This method has been trialed and previously implemented internationally in amphibious residential buildings. 


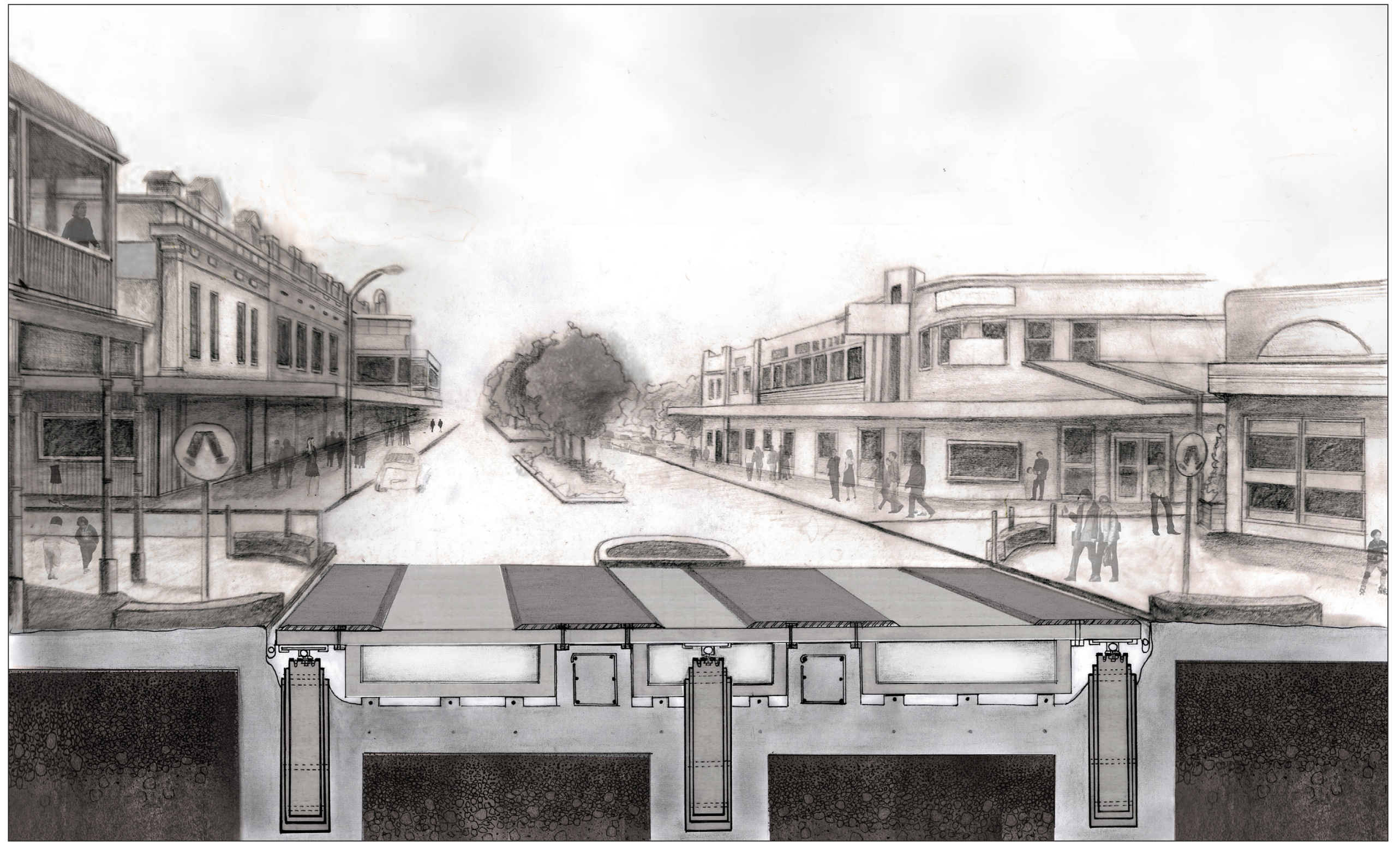

Figure 134: A 1:100 section of the amphibious pedestrian crossing design for High Street, Maitland

- Case study design - neighbourhoods : urban street scale $\quad$ strategies

- 181 
Analysis of existing urban patterns reveals the significance of fences (refer to Figure 192: Analysing the existing fence layout of Horseshoe Bend and section 6.3.4 Urban scale analysis). Fences in the everyday functioning of the city act as barriers, defining the privacy and ownership of lots between neighbours. But spatially they can be viewed as connecting veins within the urban landscape. There are opportunities for fences to perform an alternate connecting function during floods, such as establishing alternate circulation paths for pedestrians. This would allow residents to continue to be connected to their neighbours and have access to city services.
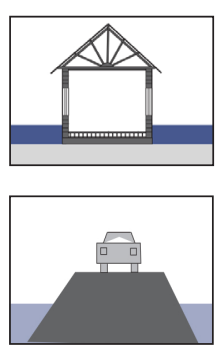

Amphibiously retrofitting Maitland's existing heritage fence types requires understanding of the flood hydraulics and influence debris has on construction. For example mesh fences will quickly become solid as vegetative and waste debris becomes trapped when floodwaters pass through. However, allowing water to flow beneath fences will reduce the hydrostatic pressure on the structure. The connections between fences need to be flexible and ductile to fluctuations in the water levels. The Maitland City Council's specific heritage fence type guidelines improve retrofit opportunities due to the minimal construction variations in existence within the city.

Strict heritage guidelines restrict the fence types options within Maitland. The Maitland City Council provides examples of suitable fence types for the heritage town. The simple design, material use and construction techniques of many of the fences lend themselves to being retrofitted. The fence types provide options for elevated circulation paths around residential boundaries, with intersecting points to neighbours, and the urban streets beyond.

Where large flood heights are experienced there are also opportunities to implement amphibious fence types. This design solution will work at strengthening community connection, as fences will need to be connected to one another to ensure structural stability during inundation periods. This solution also challenges preconceived notions of city and urban design; challenging what is and what can be possible when innovative socio-spatial resilient design is incorporated into the strengthening of flood adapted pedestrian networks within a flood prone city.

\section{Maitland City Council's heritage fence types and static retrofit examples}

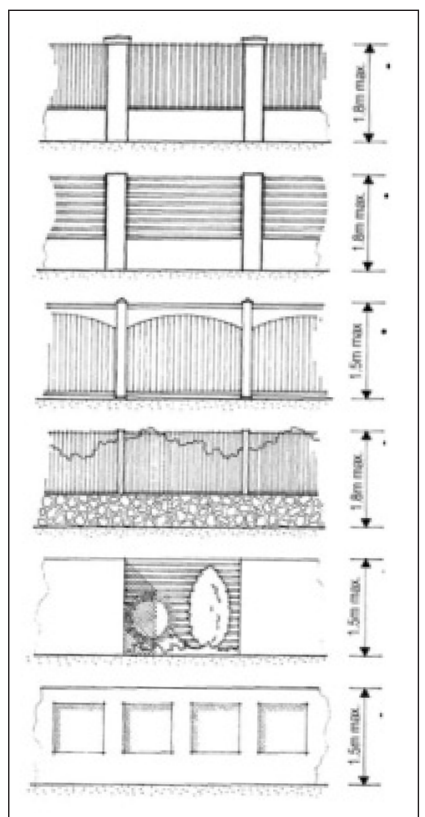

Figure 135: Maitland City Council heritage fence type examples Source: Maitland City Council (2011), p. 200

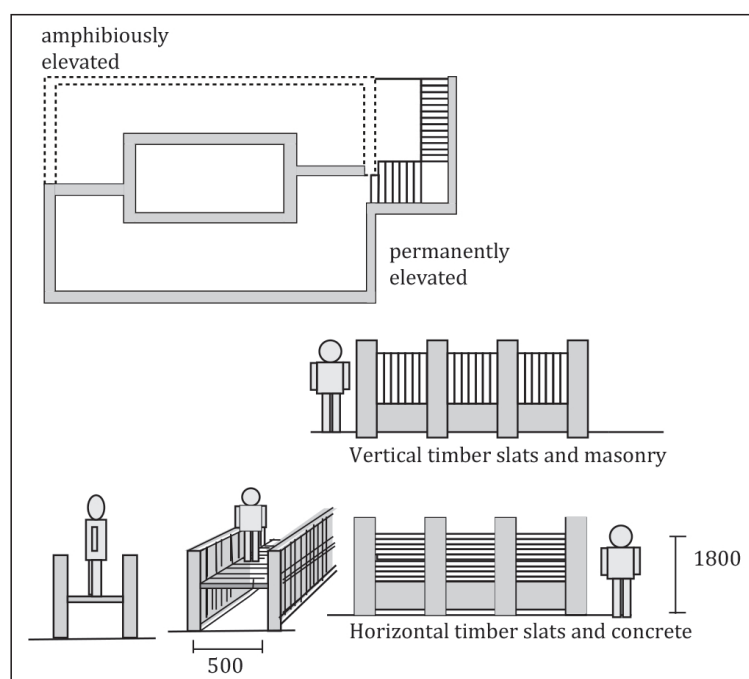

Fences between bulings need fifty percent pracy and fifty percent vision.

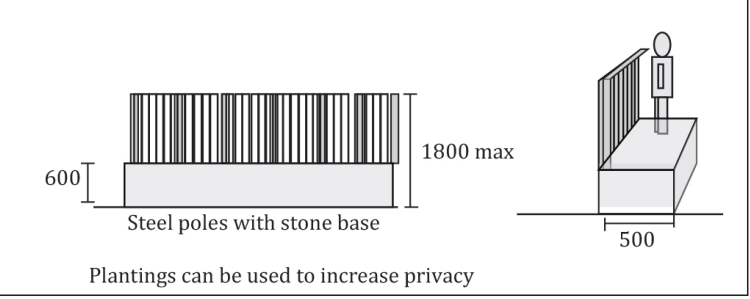

Figure 136: Retroftting existing fence types to provide static elevated paths 
The static elevated modular design serves to educate the community on the possibilities fences provide for elevated circulation during floods. The urban seating feature breaks down the boundaries between public and private zones and encourages social interaction within the community, whilst reinvigorating neighbourhoods.

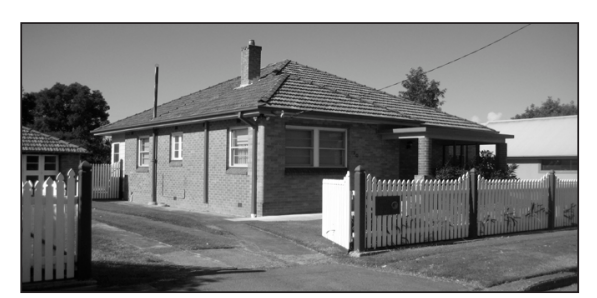

Figure 137: Photo of the existing Horseshoe Bend site

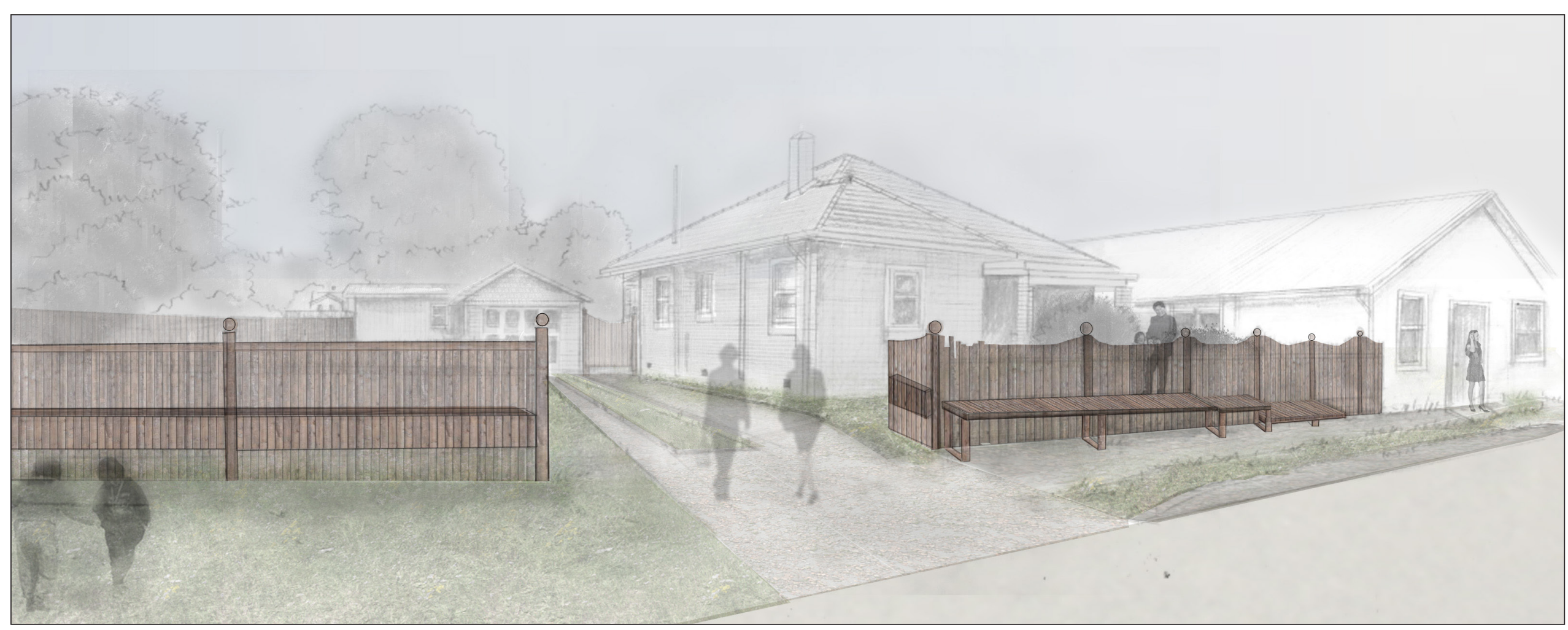

Figure 138: Horseshoe Bend retrofit example of a statically elevated pedestrian pathway solution 
Sculptural fence features

Sculptural fence features can further rejuvenate previously neglected spaces, enriching the everyday urban landscape, whilst providing alternate elevated pedestrian paths through Maitland. These work to improve current issues associated with circulation shortages, as a result of increasing urban connectivity. The sculptural yet practical urban features also allow Maitland's heritage back pathways to become more vital circulation links within the city.

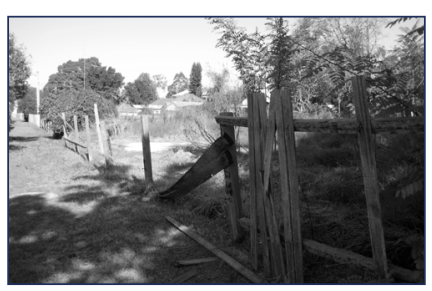

Figure 139: An existing back pathway in Horseshoe Bend

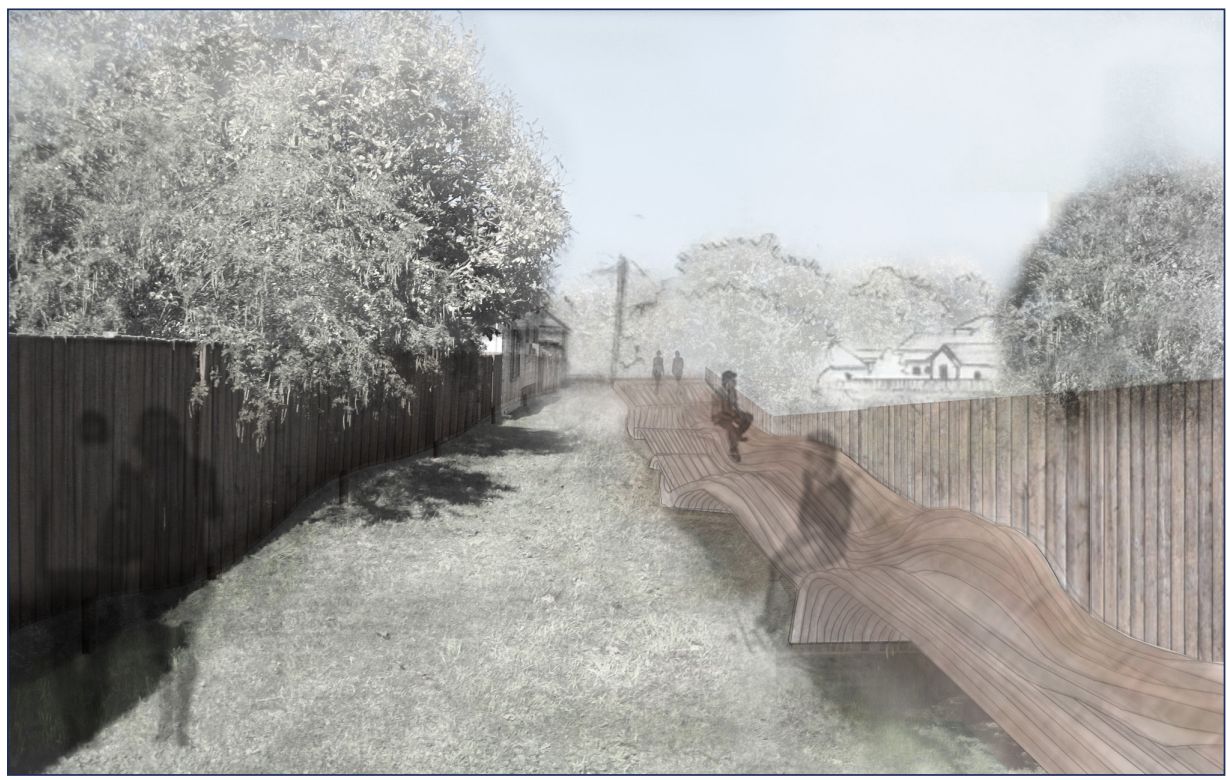

Figure 140: A design example of a sculptural statically elevated fence within Horseshoe Bend
Elevated fence connection zone

Where elevated fence pathways come together there are opportunities for previously neglected vegetative zones to become revitalized public recreational spaces. The space will act as a dry retention basin and rainwater collection points will allow residents to be informed of the areas flood storage purposes, whilst celebrating the occurrence of rain. Vegetation will serve to buffer and reduce the risks associated with fast moving flood waters whilst the grassed area will improve the absorption rates of the urban environment.

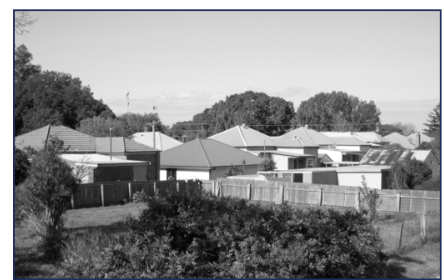

Figure 141: An existing neglected vegetative space within Horseshoe Bend

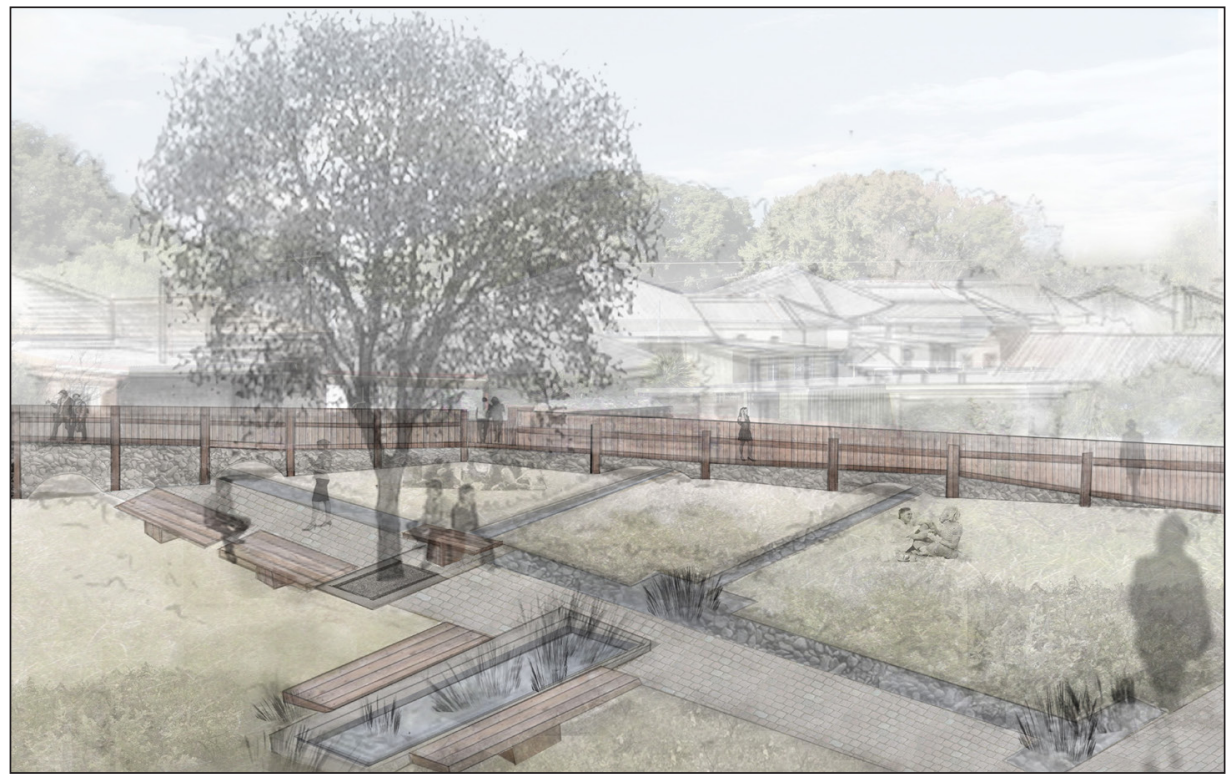

Figure 142: An example of a currently neglected vegetative zone within the urban landscape 
A residential amphibious fence example

The timber pylons support a stainless steel metal cylinder which encases a series of polystyrene blocks. There are also options for light weight concrete to encase the polystyrene blocks. This construction method would increase the longevity of the strategy, however is also a more expensive solution to implement.

Neighbouring fences lock into one another to create an urban network, whilst strengthening individual fence lines. The amphibious fences allow residents to remain connected to one another and urban and city services during low and medium flood inundation periods.

Ideally amphibious fence types will connect into amphibious residential construction types. Enabling the structures to respond and react together. But alternatively access to residents can be gained when the walkways attached to the fences link into existing stairs (a typical feature of elevated housing entrances.

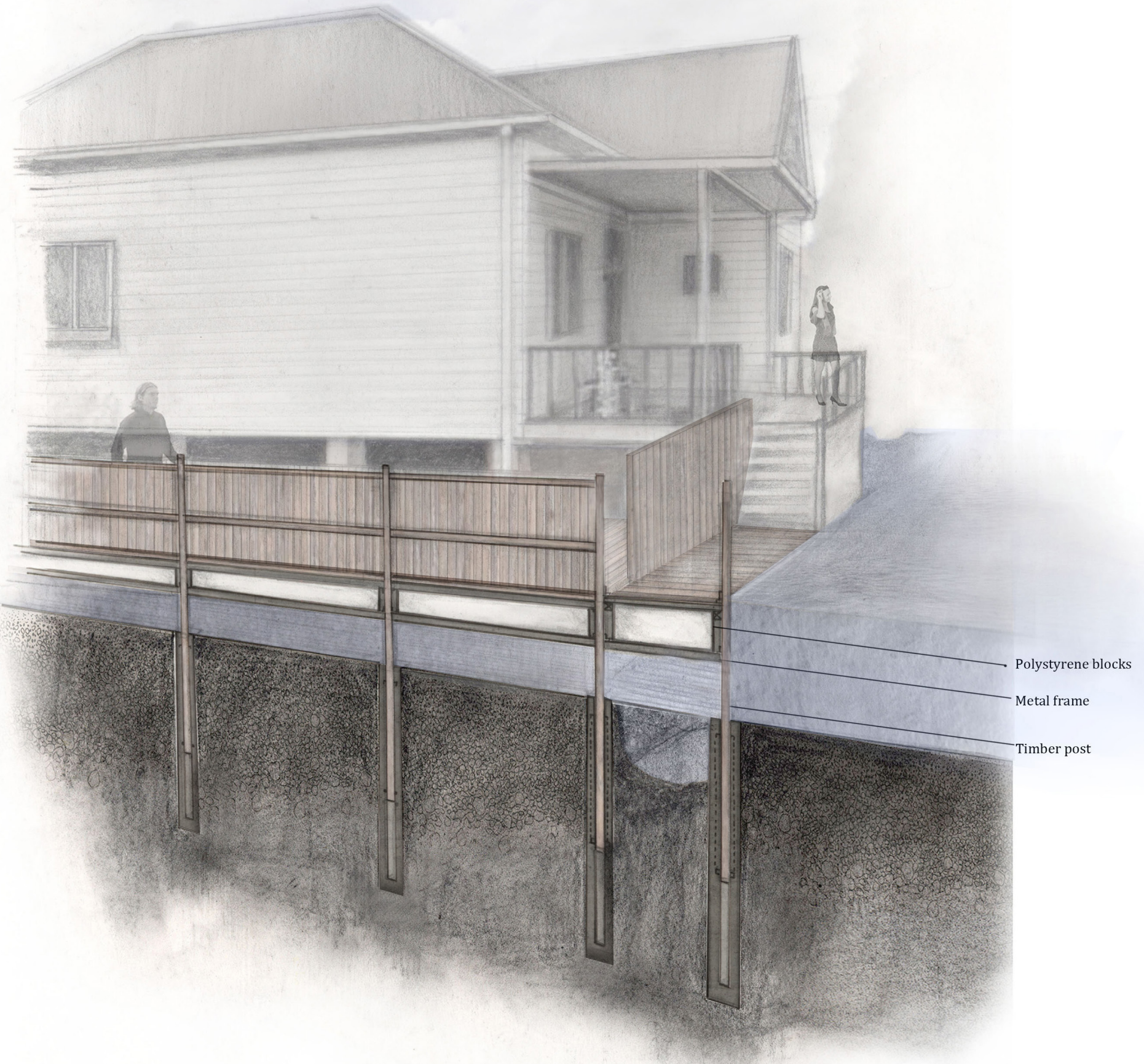

Figure 143: A sectional perspective of a bottom up amphibious fence design example 
New relationships form between amphibious circulation and urban furniture within the everyday and flood function of the city

The adverse impacts of flooding on the social and spatial functions of the urban environment can be reduced and the location enhanced through applying the flood resilience principles to existing vulnerabilities. Material selection, wet and dry flood proofing measures, are just a few of the diverse options which need to be site specifically considered with respect to the vulnerabilities and socio-spatial resilience strengthening opportunities. Urban furniture, circulation paths, shelters and fences need to be explored outside preconceptions of their existing functions and relationships to one another and the urban landscape at large.

The urban feature functionally transforms during floods, becoming a rain shelter and a platform to board water borne transport, whilst maintaining its function as a alternate pedestrian pathway.

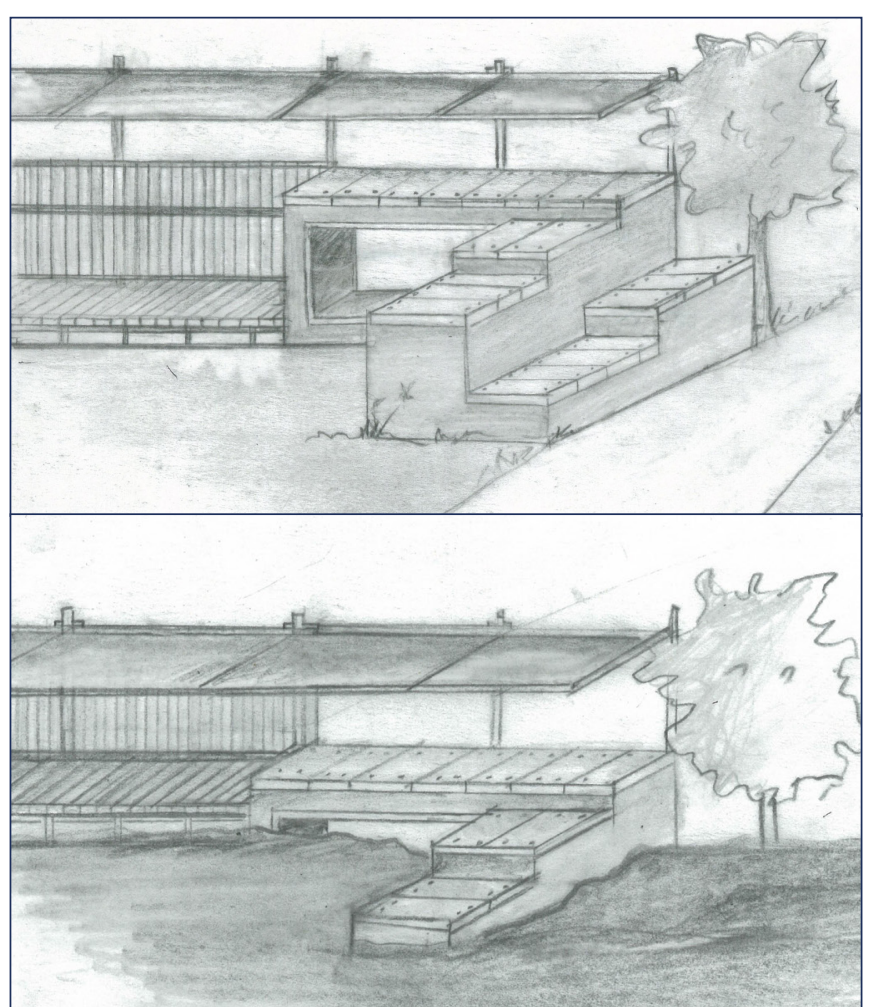

Figure 144: The urban furniture that provides alternate pedestrian paths 
New relationships form between amphibious circulation, ecological environments and stormwater infrastructure

The spaces resting below these structures are able to supplement existing stormwater infrastructure, essentially functioning as artificial flood channels; aiding the transfer of water from urban zones, where non-permeable surfaces dominate, to ecological greens zones, where floodwaters are absorbed, stored, nutrients are transferred and waste products filtered. Bushy vegetation aids in catching debris and serves to buffer and reduce the velocity of floodwaters. Trees help transport floodwaters deep into the soils, below non-permeable surfaces.

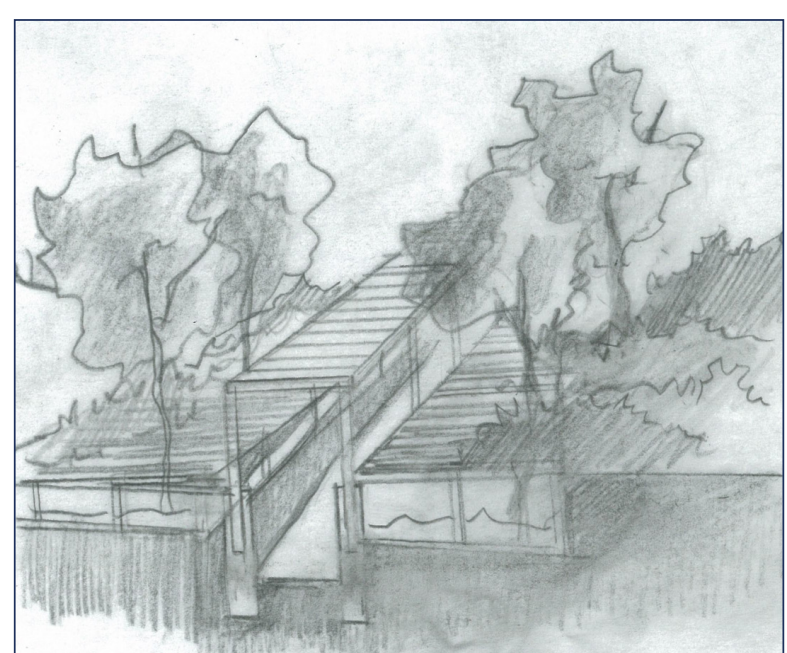

Figure 145: Exploring new urban amphibious pedestrian solutions 
A socio-spatial resilience network is made up components at the multiple scales of the city

\section{- neighbourhoods : urban levee scale}

The levee : a socio-ecological recreational space

- vulnerabilities

- opportunities

- strategies 
(From an infrastructure barrier to a recreational space connecting residential communities to the city and river.)

The area adjoining the levee was historically covered in gigantic cedar trees, figs and gum trees, swamp oak trees, tall rushes and paper bark scrub (Walsh \& Archer, 2007 , p. 8); (Hunter \& Maitland City Heritage Group, 2000, p. 10). But all of this changed when the town developed, residents moved in and agriculture prospered. The once harmless soil nourishing floods quickly became viewed as hazardous events. Historical accounts indicate that since 1857 private landholders were constructing a number of dams and embankments in an attempt to hold back the floods, however these non-collaborative approaches continually proved unsuccessful. After years of flooding the Maitland City Council erected the Horseshoe Bend embankment, which over time has been strengthened, increased in height and transformed to become the current Maitland main ring levee.

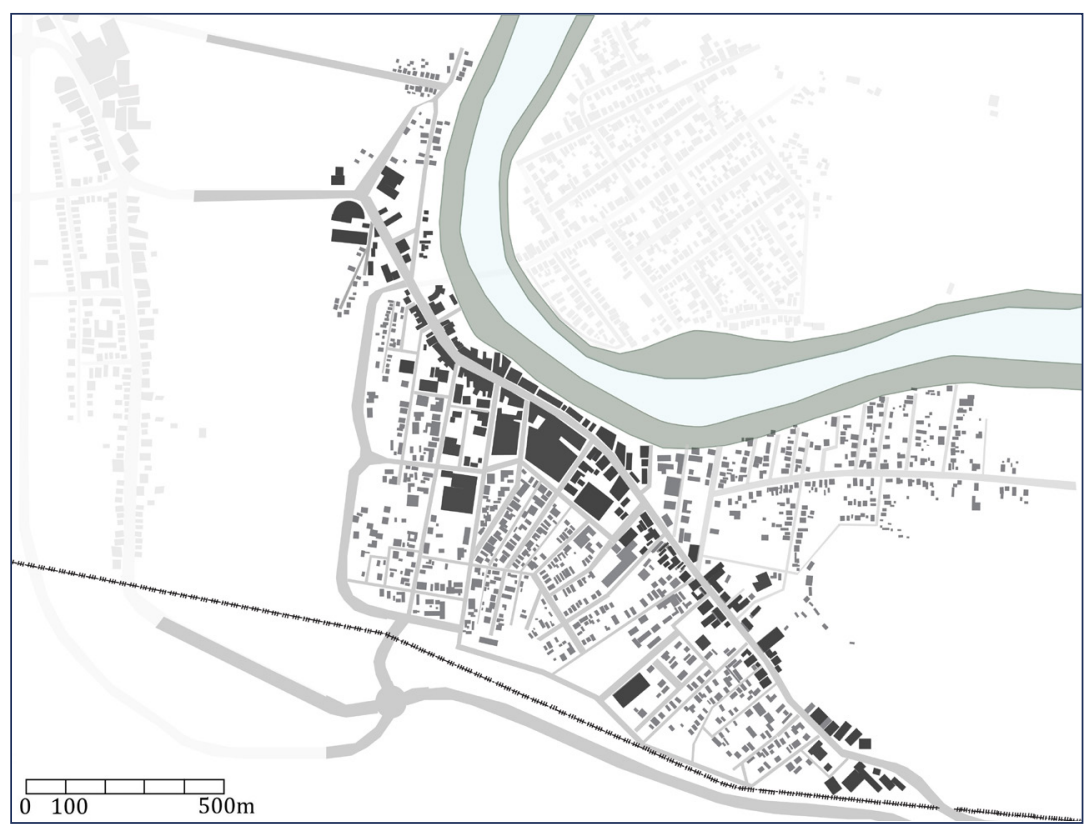

Figure 87: The focus area of Maitland with the adjacent townships of Lorn and Morpeth
Upon initial analysis, the flood mitigating benefits of the levee running alongside Central Maitland and Horseshoe Bend can be readily identified in its role of reducing the inundation frequency of urban zones adjoining the Hunter River. However, socio-spatial analysis reveals several adverse effects. Firstly, the levee confines floodwaters to the river channel carrying them further downstream and inhibiting the natural process of floodplain inundation. Subsequently higher water volumes and velocities are experienced downstream of the city where residents and townships are lacking in flood mitigation infrastructure. Secondly, the levee acts as a physical barrier between the city and the river. The city has subsequently turned its back on the levee and the river it adjoins. The buildings positioned on the levee effectively inhibit visual and physical connections from the streets to the river. The backs of buildings also adversely impact on the character and atmosphere of the levee as a public recreational green space.

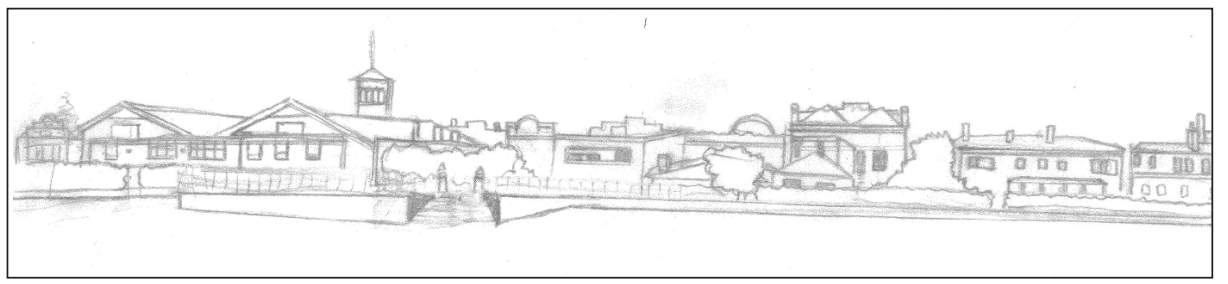

Figure 146: A riverside view of the levee and buildings that align it

The buildings along the levee block visual and physical access to the river from Maitland's main street, High Street and the pedestrian Heritage Mall. 
Resilience design strategies that are likely to improve socio-spatial flood resilience of the Maitland ring levee include:

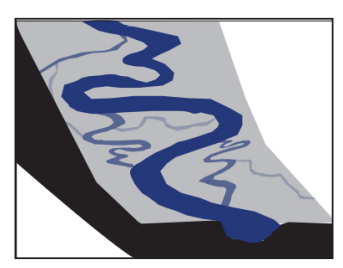

Daily and seasonal fluctuations in the Hunter River need to be understood and anticipated. Recognition of the river system provides insight into the hydraulic conditions the ring levee will be subjected to.

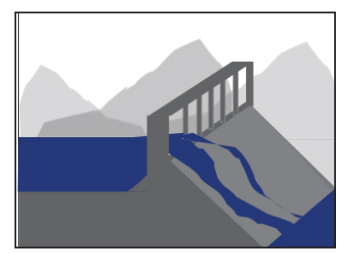

Understanding how existing engineered flood works, such as dams, diversion channels and levees, affect local flood characteristics and flood occurrence rates ensures the levee is able to efficiently mitigate flood risks.

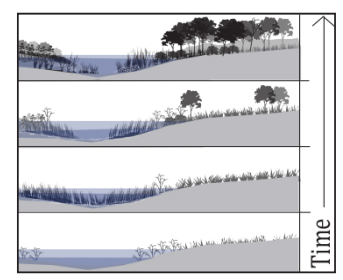

Ecological succession recognizes the transience of the levee's riverine ecosystem, which will continue to modify itself overtime as it responds and reacts to changing climatic and environmental conditions.

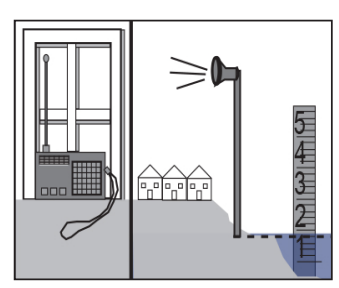

There is an opportunity for the levee design to further inform floodplain inhabitants of the river's fluctuating river levels.

Establishing a riparian buffer on the levee will enable it to effectively interact with changing water levels and
The use of water resistant material and other permanent wet flood proofing measures will ensure the water environment will not have adverse effects on the longevity of the levee structures.
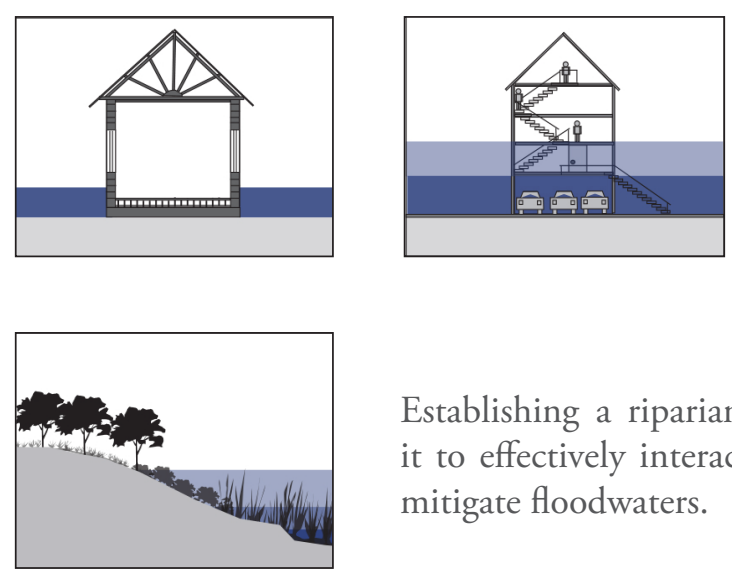

Establishing a riparian buffer on the levee will enable it to effectively interact with changing water levels and mitigate floodwaters.

The use of appropriate water borne vegetation, nutrient soils and deep root structures will aid the levee in mitigating floods (filtering contaminants, stabilizing river beds and removing sediments and debris to improve water quality).
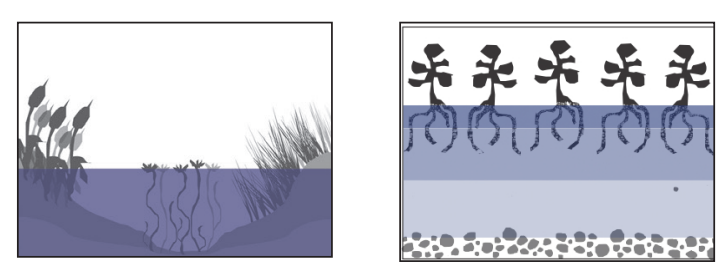


\section{The levee : A socio-ecological recreational space}

There is an opportunity for the levee to become more actively engaged with connecting the Maitland community to the Hunter River. As a consequence of back flooding, the levee becomes a vital circulation path connecting residential communities to the commercial area in low and medium flood category events. In 1998 the Maitland Council released the Central Maitland Area Improvement Plans (CMAIP): Landscape Concept Plan which recognized the need for improved road entry points and pedestrian mobility within the city. However, strategic proposals such as improving access to the riverbank and enhancing the circulation of the riverbank for public use failed to be implemented.

Levees traditionally consist of grass, which is mowed and maintained in an effort to ensure vegetation does not reduce the velocity of floodwaters and thus promote a larger volume of water to collect. The city has become reliant on the levees performance to protect it from direct inundation from the Hunter River. (The levee is overtopped by high flood category events with a current rating of a 5\% AEP event).

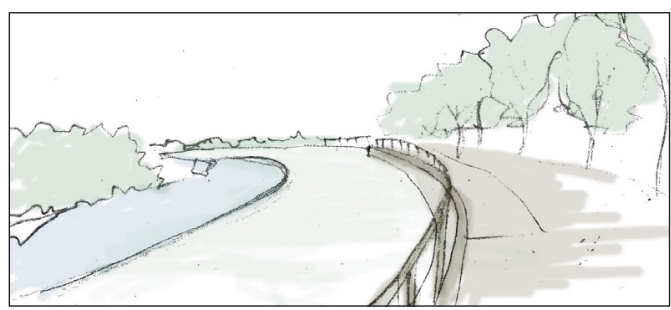

Figure 147: A grass hill and main walkway along the top with trees distributed along it

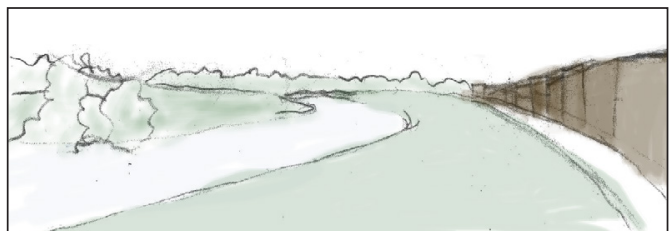

Figure 148: The single and double storey heritage buildings along the top of the levee
Whilst retaining the flood mitigating benefits of the levee, there are opportunities to promote Maitland as a river city and re-establish socio-spatial connections between the city and the river. Maitland City Council has also recognized the aesthetic and touristic benefits this would provide for the city in its Central Maitland Structure Plan (City Plan Urban Design, November 2009). Ware, Raxworthy, Weller, RussellClarke \& Meyer (2011) purport that landscape architecture has "the capacity to articulate the restorative and resistive social and cultural formation of territories... and realize a constructive interplay between ecological and urban strategies" (p.23).Viewing the levee as a recreational green space will promote restoration and rejuvenation of the riverine ecosystem to create a more attractive space for community gathering.

\section{Strategy intentions:}

- Create a public recreational space, that re-connects floodplain inhabitants to the river

- Provide a vital circulation spine alongside the river, connecting residents to the commercial precinct and community centre.

- Rejuvenate riverine ecosystems and improve local ecological functioning.

- Design a site which informs and celebrates the Hunter River's daily and seasonal fluctuations. 


\section{Levee design thoughts:}

There is an opportunity for the levee to functionally respond and inform inhabitants of rising water levels with respect to the three flood category levels. Allowing the water to infiltrate the levee at specific points and be channeled through the urban streets to encourage a transformation of the urban design of the city, in which water, whether from the river, floods, or rainwater are integrated, celebrated and adverse impacts reduced within the urban landscape of Maitland. Thus pushing Maitland Council visions of a river city further than just river-side development.

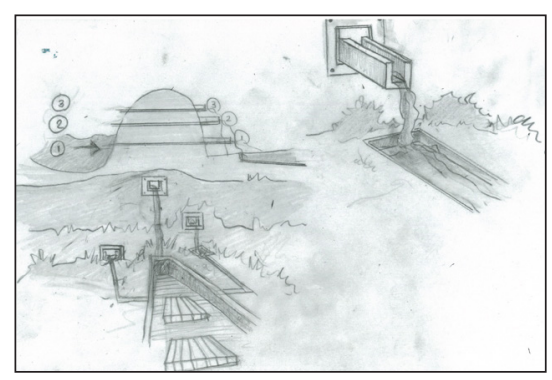

Figure 149: Water infiltrating the levee as informative urban water features

The levee design needs to encourage and allow for interaction with the waters edge. Gabion structures allow an edge to be defined and greater appreciation of the river's daily tidal fluctuations, along with reinforcing the structural stability of the levee.

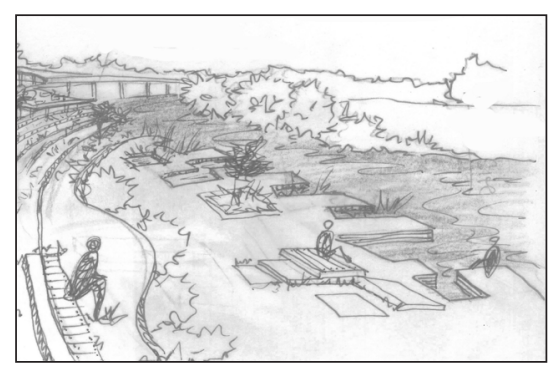

Figure 150: Levee design exploration into social and structural function of the rivers edge
Sculptural elements are able to inform users of the waters levels. The levee is broken down into different zones for occupation and a series of paths encourage use of the entire levee. These paths then connect to the $\mathrm{CBD}$ and direct users to the civic square and community centre.

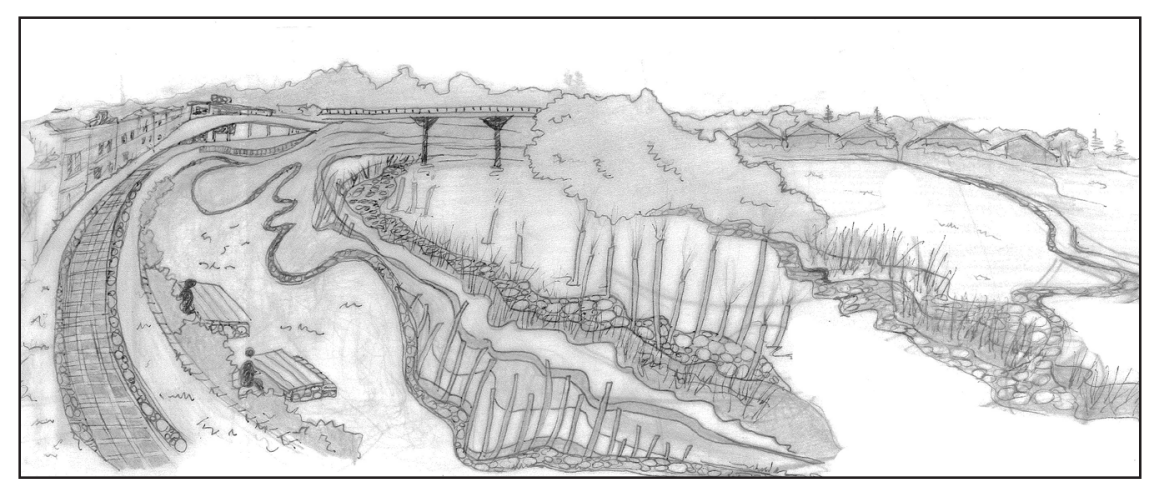

Figure 151: Levee design exploration into informative sculptural elements

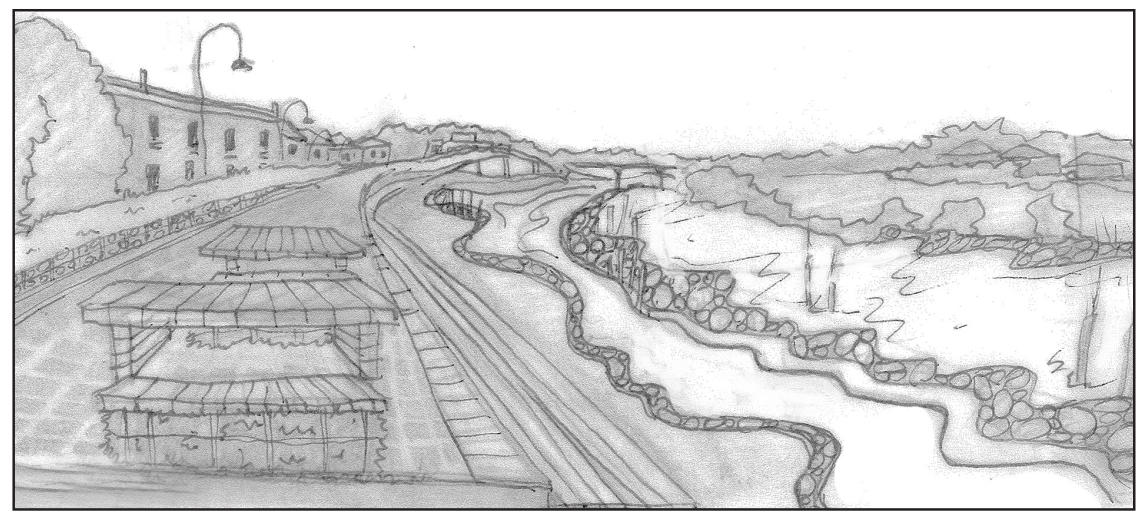

Figure 152: Levee design exploration into level changes and the social functions

- Case study design

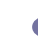

- neighbourhoods : urban levee scale

opportunities 
The macro scale plan of the levee

At a macro scale the levee has been designed to resemble the natural topographical and hydrological riverine conditions. The organic design allows the levee to transform and become more natural in its configuration whilst establishing platforms and park zones to encourage the use of its spaces for recreation and ecological regeneration of the riverine ecosystem. A series of habitable zones enhance the spatial qualities of the levee and encourage everyday community use. The design combines artificial and ecological attributes allowing it to respond to the low, medium and high flood categories. The design aims to break down the current barrier and encourage the city to begin to respond and interact more with the river's modulating edge.

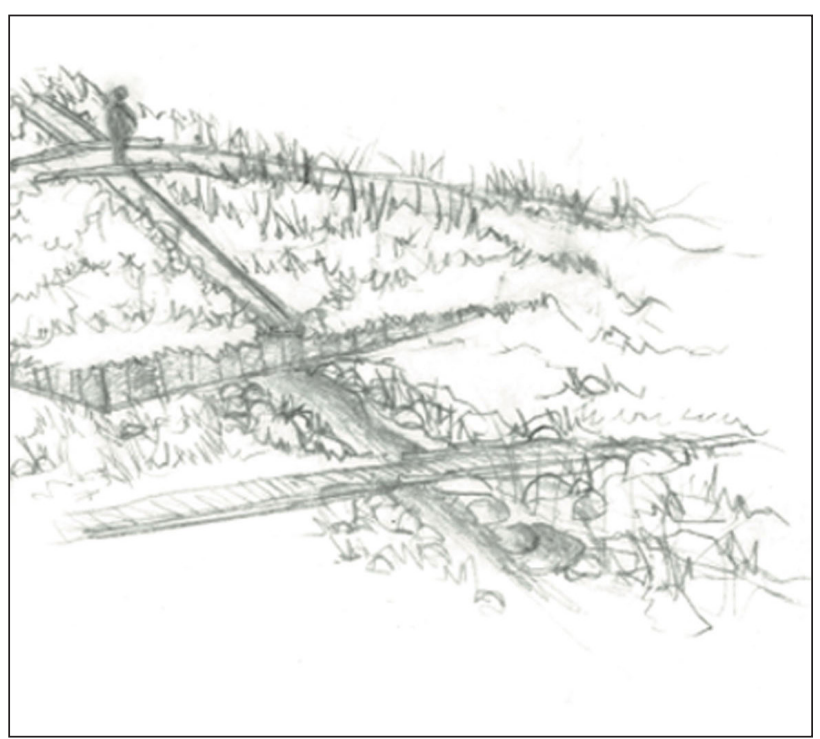

Figure 153: Rejuvenated ecological zones
A balance between natural ecological zones and structured recreational spaces enable the levee to perform a series of functions. Rejuvenated native vegetation ensures the levee will continue to evolve and be enriched over time by the natural drought and flood climatic extremes of the site.

A series of paths interweave through different ecological and socially activated spaces to allow appreciation of the levee in its varying ecological, flood management and social functions.

Natural and artificial design features establish passive and active systems that inform and warn users of the Hunter River's water levels. These promote community early response and strengthen understanding and appreciation of the rivers natural daily and seasonal fluctuations.

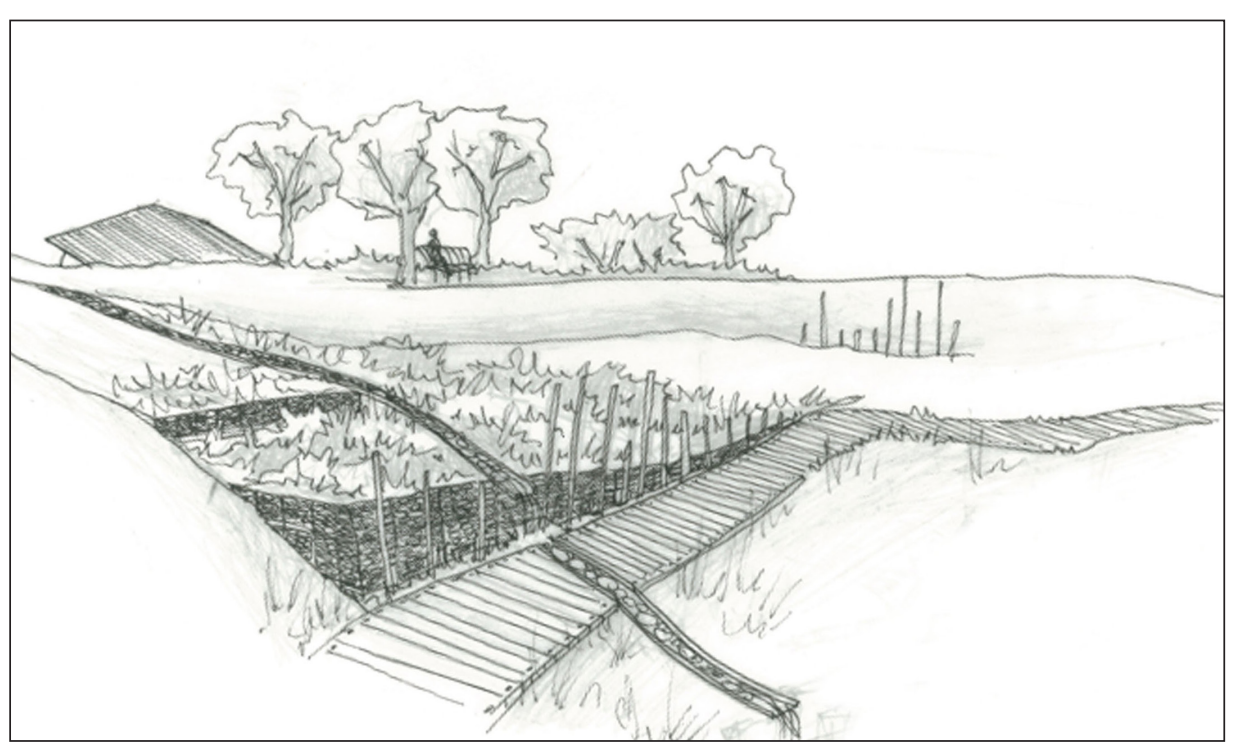

Figure 154: Artificially terraced spaces define pathways for pedestrians and water 


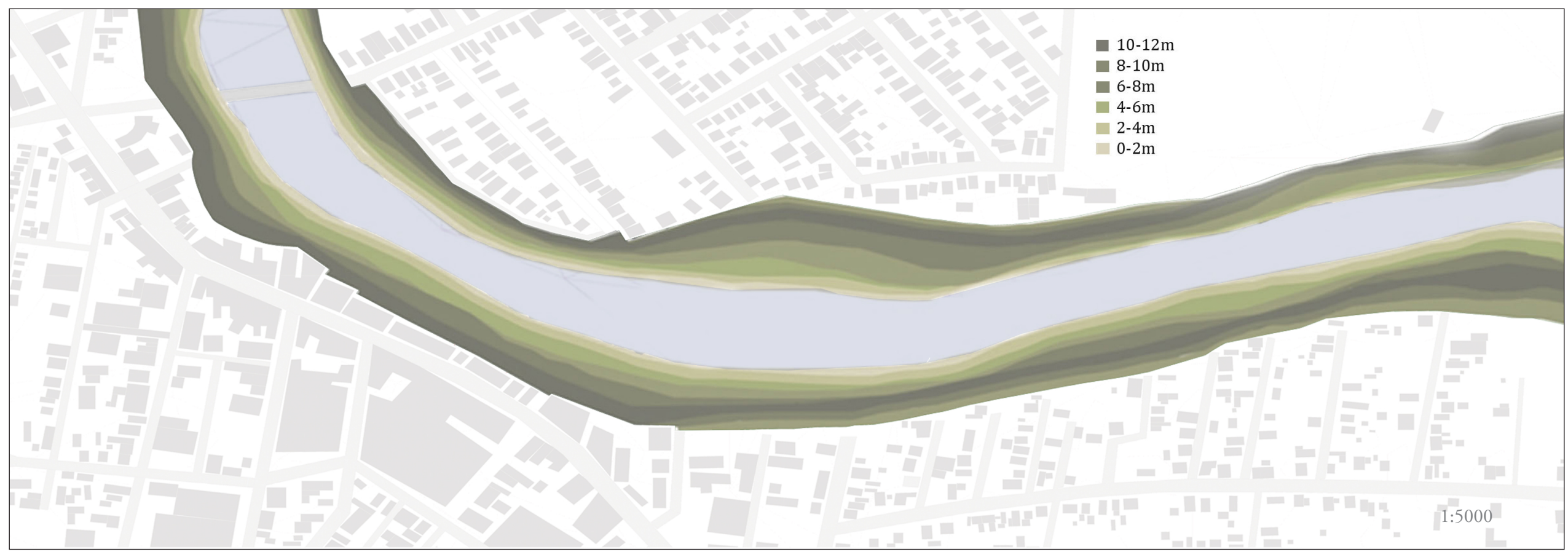

Figure 155: A macro scale 1:5,000 plan of the proposed Maitland levee design 
Vegetation shifts are interwoven into the three riparian zones of the levee, with native riparian species being reintroduced. The three zones of the riverine ecosystems functionally and spatially reinforce the low, medium and high flood category events, with the inundation of each zone reflecting the inundation of the Maitland suburbs furthest away from the levee (as a result of back flooding). Improved ecological functioning will lead to the return of native wildlife and further improve the ability of the levee to maintain its structure and stability during the two climatic extremes of droughts and floods. This in turn will improve the flood mitigating role of the levee. The once abundant wildlife and healthy ecosystems have over time been adversely affected by the Lower Hunter Regions agricultural production and flood work infrastructure.

From the river's edge, zone one is the narrowest area and consists of reeds and large native trees which function to stabilize and shade the bank. The tidal nature of the water means the submersion of the zone fluctuates. Its adjacent area, zone two, consists of a variety of native shrubs. These work to reduce rainwater runoff, promote absorption and improve the river's water quality through the filtering of contaminants and buffering of debris. Zone three is the largest zone and consists of native grasses. It serves as a dry retention zone, slowing water runoff and absorbing contaminants. Native oaks and red cedars are also to be planted. The deep root structures of the trees aid in water transfer and soil stabilization, reducing the chances of land-slips and erosion rates.
The vegetation shifts and terracing passively inform the community of the rivers fluctuating water levels. Sculptural elements further highlight the heights at which people need to begin responding and acting in response to rising floods. Gradients within the levee not only function as circulation pathways, but also serve to direct rainwater to collection points where sculptural and ecological features respond to its presence. The rainwater features celebrate the climatic occurrence. Boardwalks bridging over water channels strengthen interaction and site participation. The meandering paths encourage exploration of the site, whilst recreational features such as a children's playground, picnic tables and tiered seating establish a variety of passive and active recreational spaces

Architectural resilience adaptability strategies: diffusion

Artifcial diffusion channels can be achieved by positioning and gradienting exterior circulation pathways and interior corridors to re-direct flood waters across the site and through the building.

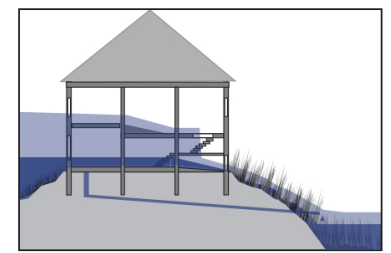

Figure 156: An architectural diffusion strategy 


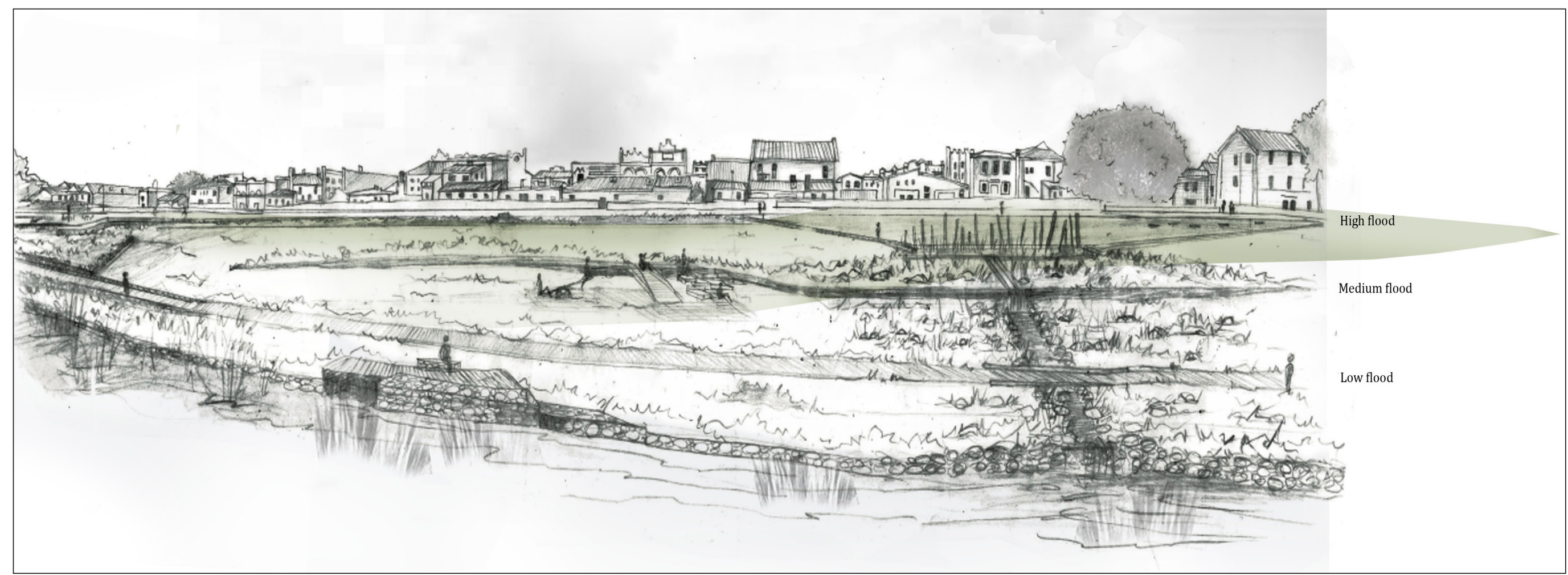

Figure 157: A view of the Maitland levee design from the Lorn side of the Hunter River. 
Architectural resilience adaptability strategies: absorption

Permeable surface membrances reduce runoff volumes in urban areas by allowing water to penetrate below ground via natural filtration.

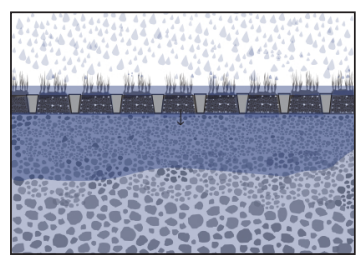

Figure 158: An architectural absorption strategy

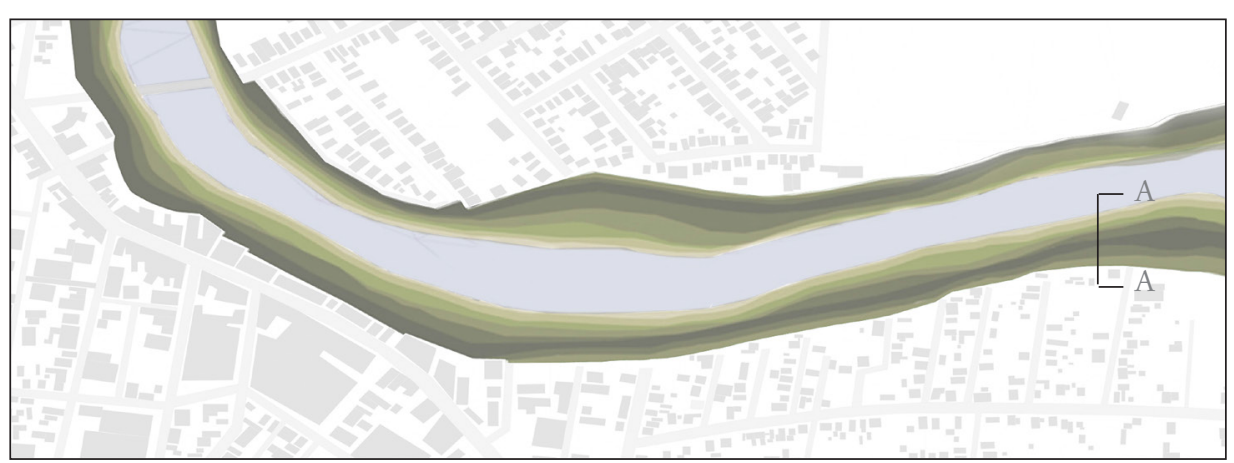

Figure 159: The Maitland levee design 1:10,000 plan highlighting Section A 


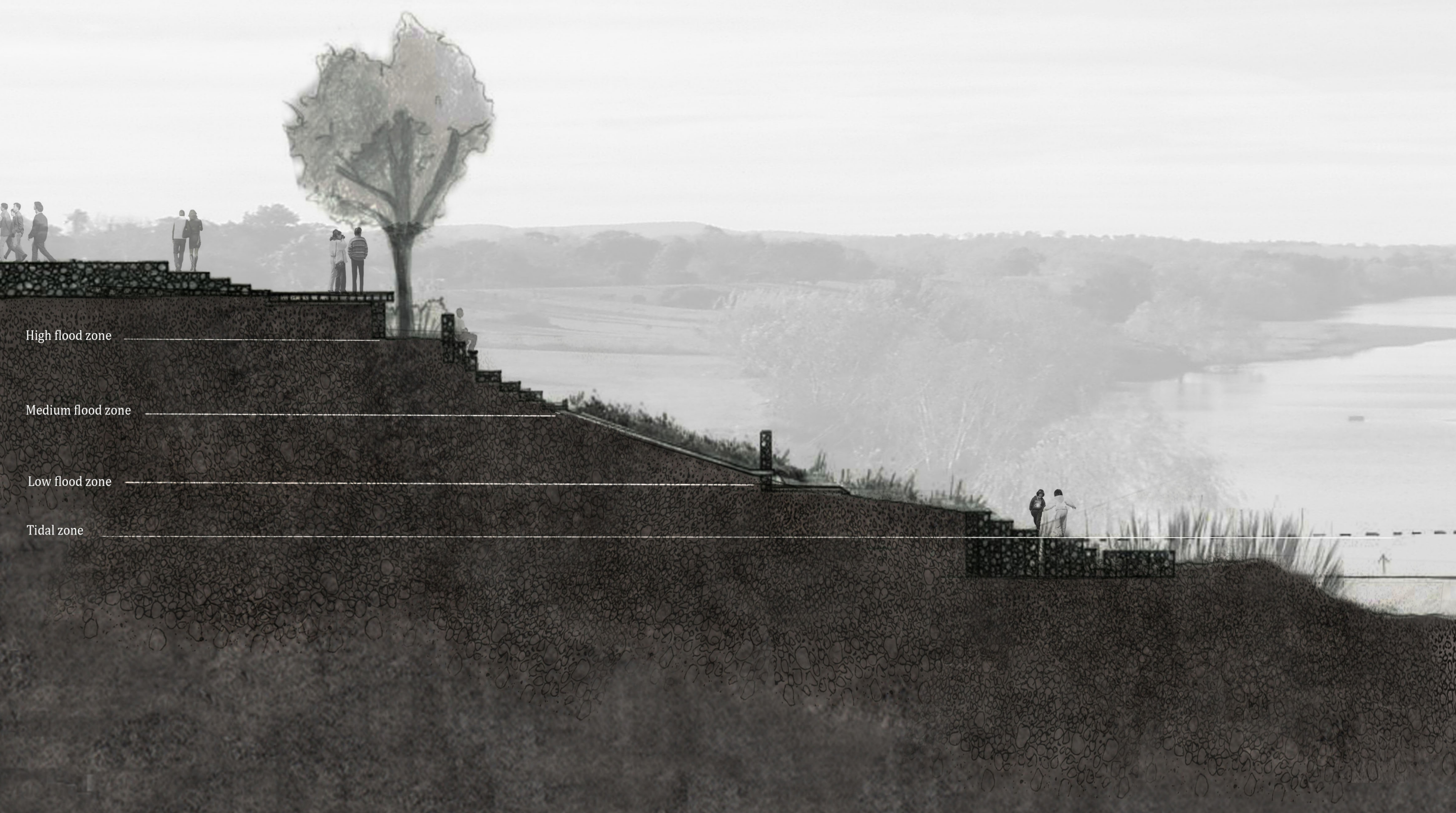

Figure 160: 1:200 Section A of the Maitland levee design 


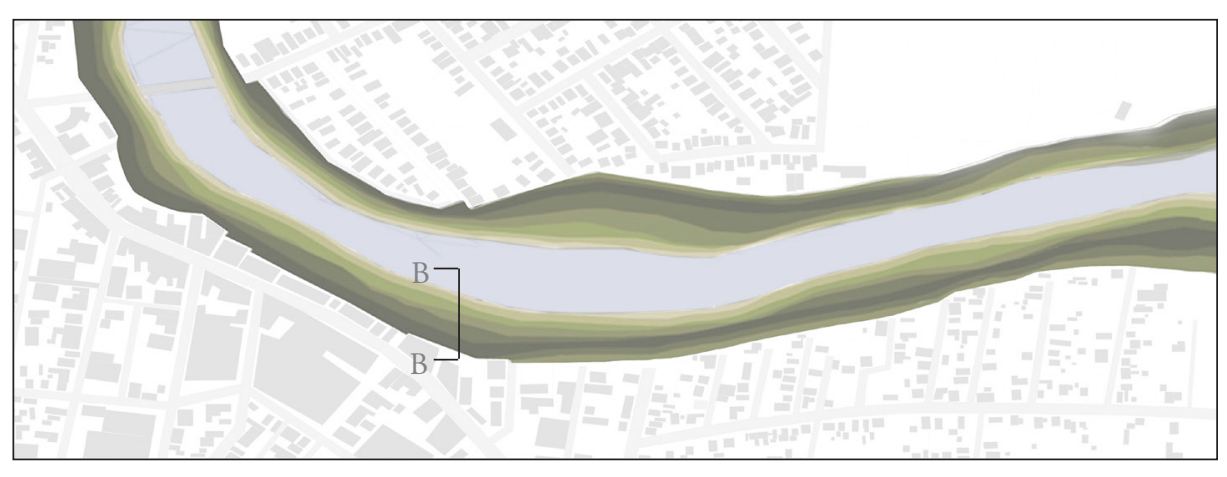

Figure 161: The Maitland levee design 1:10,000 plan highlighting Section B 


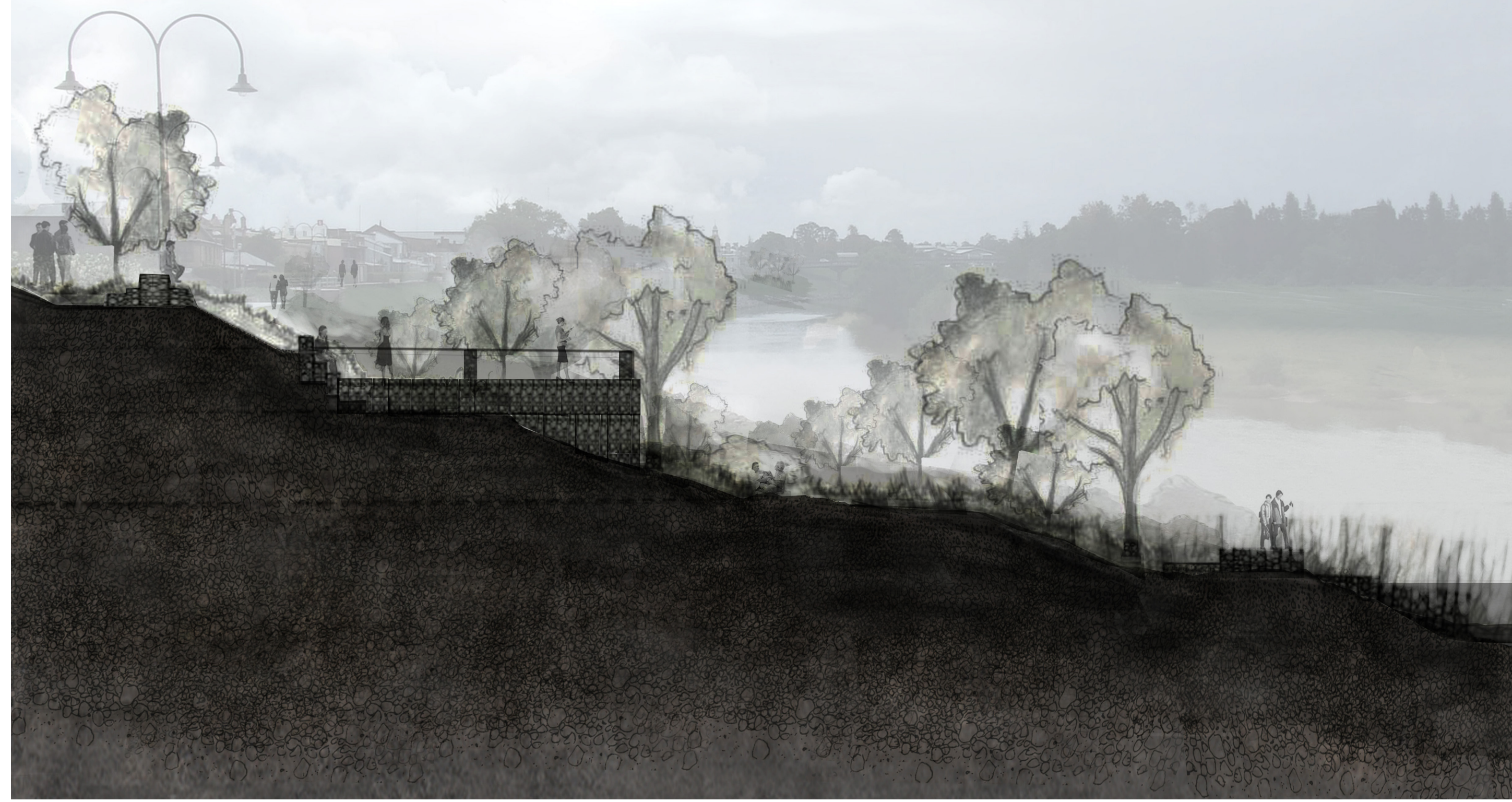

Figure 162: 1:200 Section B of the Maitland levee design 


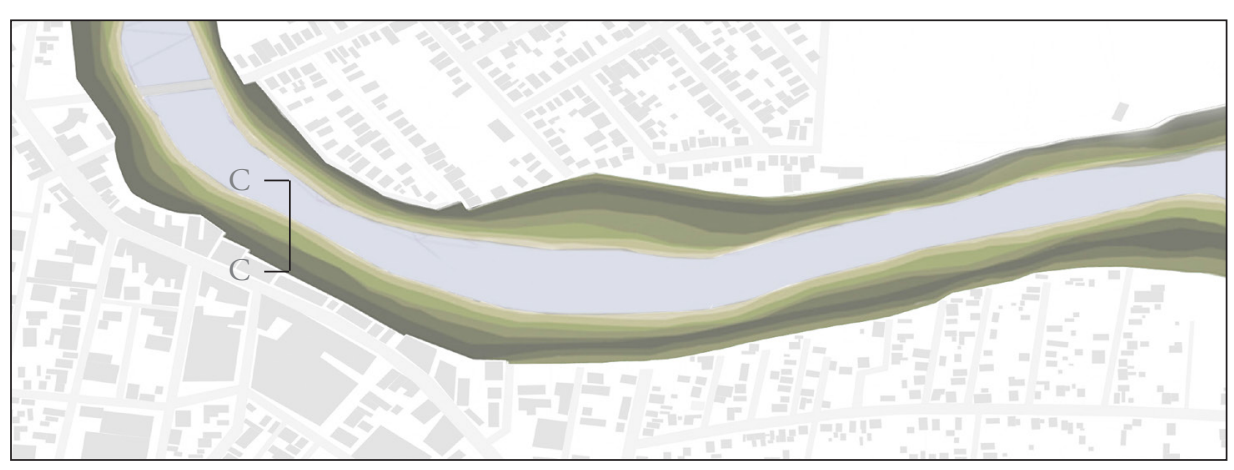

Figure 163: The Maitland levee design 1:10,000 plan highlighting Section C 


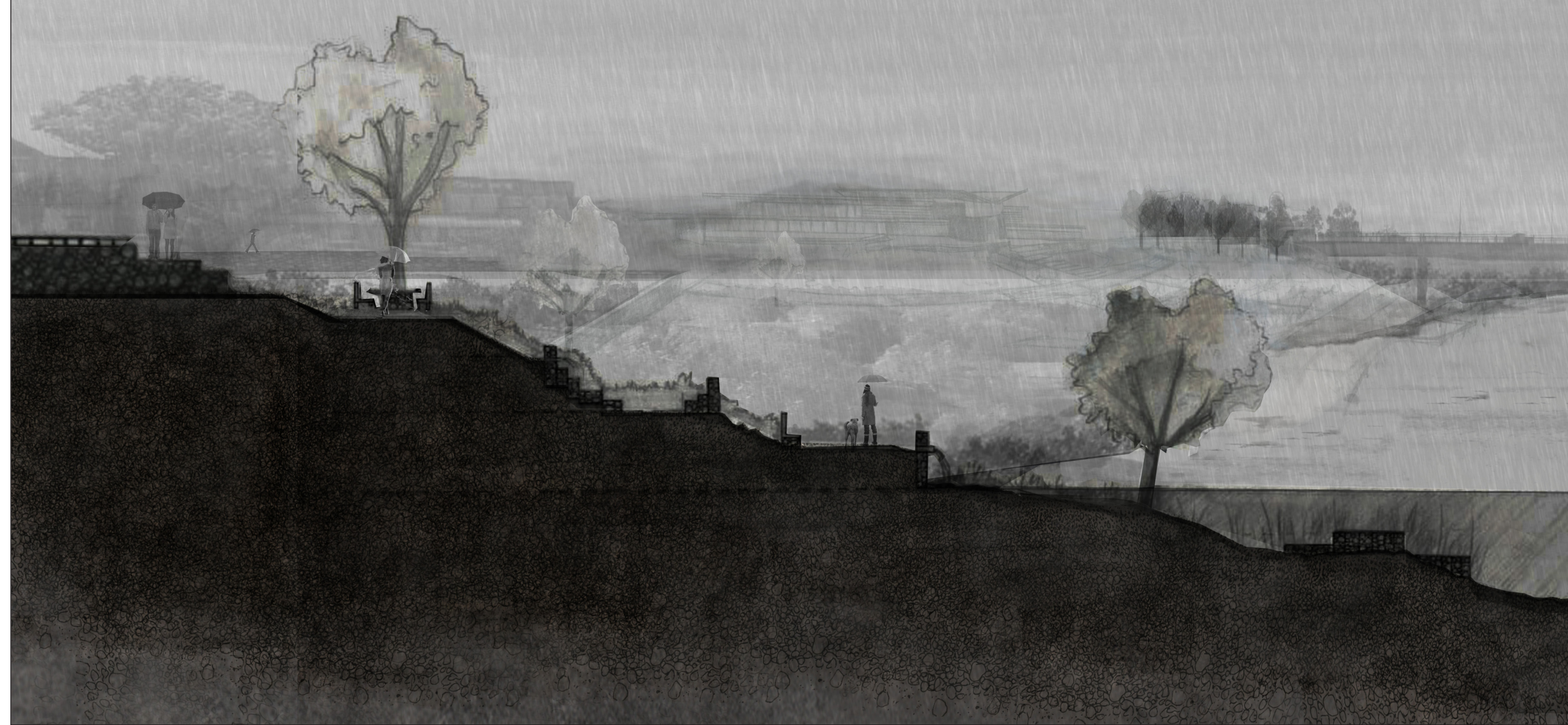

Figure 164 1:200 Section C of the Maitland levee design 
A socio-spatial resilience network is made up components at the multiple scales of the city

\section{- communities : city services scale}

Flood safe shelters providing vital services $\&$ facilities

- vulnerabilities

- opportunities

- strategies 
Flood evacuation centres in Australia are in many cases established once a flood hazard is imminent. This is different from fire and cyclone evacuation centres, which are determined prior to the event to ensure they are structurally sound for the climatic conditions to be experienced. SES crews work with the Bureau of Meteorology to determine flood risks and evacuation timings. Shelters, such as school and community halls in East Maitland and Morpeth are then hastily established, with information communicated to flood affected residents via radio, television and door knocking.

Developing a flood evacuation centre within Maitland, with an everyday function as a community centre, would strengthen existing social relationships and allow residents to recover within their community. This would reduce the emotional and financial impact of floods, and subsequently the flood impact on city functioning. There is a need to address the issues surrounding the provision of vital services and shelter options for Maitland at the different functional stages of city inundation. A lack of flood adapted infrastructure and mobility options in Maitland inhibit evacuation procedures. Flood evacuation gathering points and evacuation shelters therefore perform a significant safety function. Analysis of existing community centres in and around Maitland provides insight into the services already available and the cultural, recreational and community functions currently lacking.
Existing community buildings in the Lower Hunter Region include:

- Bruce Street Community Hall, East Maitland: Community Health Centre

- Maitland Senior Citizens Hall, Maitland: community hall for functions/meetings but no catering facilities

-Visitor's Information Centre, Maitland: information centre and café

With an aim to compliment rather than duplicate existing community services, it must be noted that Maitland currently lacks community exhibition spaces, meeting rooms, outdoor performance spaces and halls equipped with cooking and showering facilities. Maitland is also without a main civic space. At present the largest public gathering space is at the sports oval situated at the edge of town, which although elevated remains exposed to the climatic elements and limited in shelter options. There are existing community services which are distributed throughout the city. However none of these are appropriately sited and constructed to act as flood evacuation shelters. Establishing a flood safe community centre and urban community structures would ensure key services (temporary shelter, power, water, food, health and communication) are provided prior, during and post flooding at multiple socio-spatial scales. The site would become autonomous in the everyday city functioning as a community gathering point, where connections are strengthened and support gained. 
Resilience design strategies that are likely to improve the socio-spatial resilience of flood safe community shelters include:
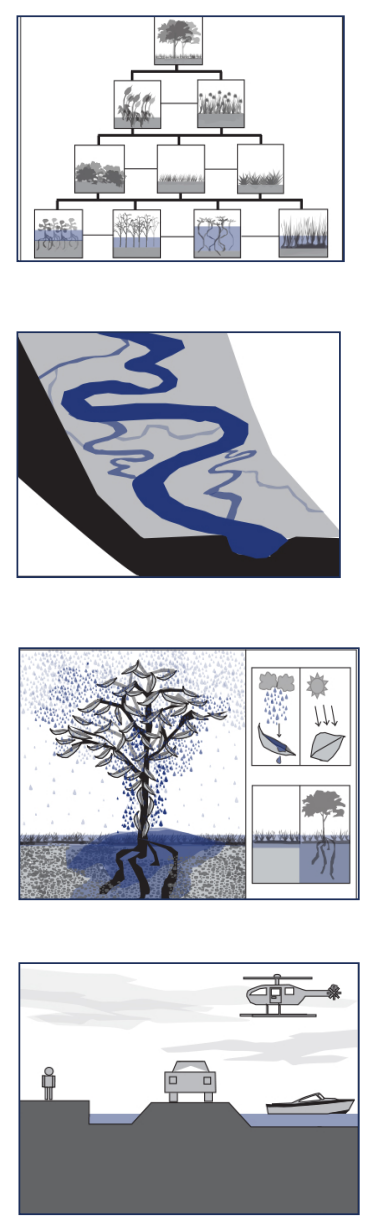

Flood paths need to be integrated into planning to ensure appropriate placement of community flood safe structures.

Flood safe shelters and their services need to be designed to preempt flood conditions, whilst being able to react

to new flood conditions.

A balanced top down and bottom up approach is vital in the implementation of a community centre.

Community members must be able to access services at multiple the socio-spatial scales of the city (city, urban, residential).

Flood adaptive infrastructure must not only be achieved structurally but must integrate alternate energy sources to ensure services remain available during periods of inundation. Adaptive service connections, such as flexible plumbing, will increase the flexibility of services and reduce the adverse impact of flooding.
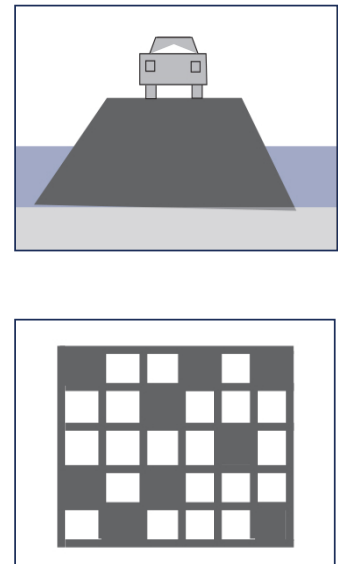

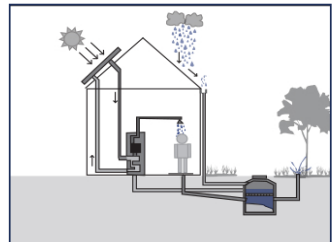

Distributing community flood safe shelters providing key services across multiple socio-spatial scales will increase the robustness of the city system in its ability to deliver during periods of inundation.

Temporary and permanent wet and dry flood proofing measures and water resistant material selection strengthen the robustness of structures and reduce the likelihood of flood damage.
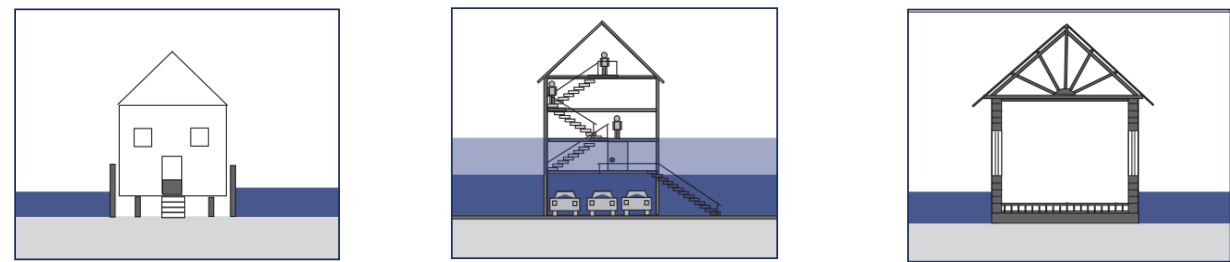


\section{Bus shelters, barbeque and picnic shelters (urban scale)}

On an urban scale there is a need to establish 'modules' where services can be obtained and temporary shelter provided. These points within the urban landscape could also become established evacuation points within Maitland. In keeping with autonomous principles, the retrofitting of bus shelters arose as a suitable solution. This ensures that the everyday functioning of the bus shelter is maintained; a temporary meeting point where people are transported to other locations.

There are also opportunities for urban picnic shelters sited within recreational parks to provide barbeque cooking services and alternate energy sources for inhabitants within the urban landscape. Alternate energy sources and the provision of drinkable water will not only provide benefit during floods, but will enhance the urban environment and also improve available public services and amenities within Maitland. The socio-spatial resilience of Maitland will be strengthened by the provision of key services, whilst flood adapted pedestrian paths of the socio-spatial network ensure residents are able to gain access to the urban services and shelters during a variety of flood events.

\section{Strategy intentions}

- Establish a series of autonomous urban modules, which remain functional and accessible during floods.

- Urban shelters can become transitional points and flood safe evacuation shelters from which the SES, police and other relevant parties can retrieve people.

- Provide key services; water, power, cooking amenities and shelter pre, during and post a variety of inundation flood heights.
Initial design thoughts

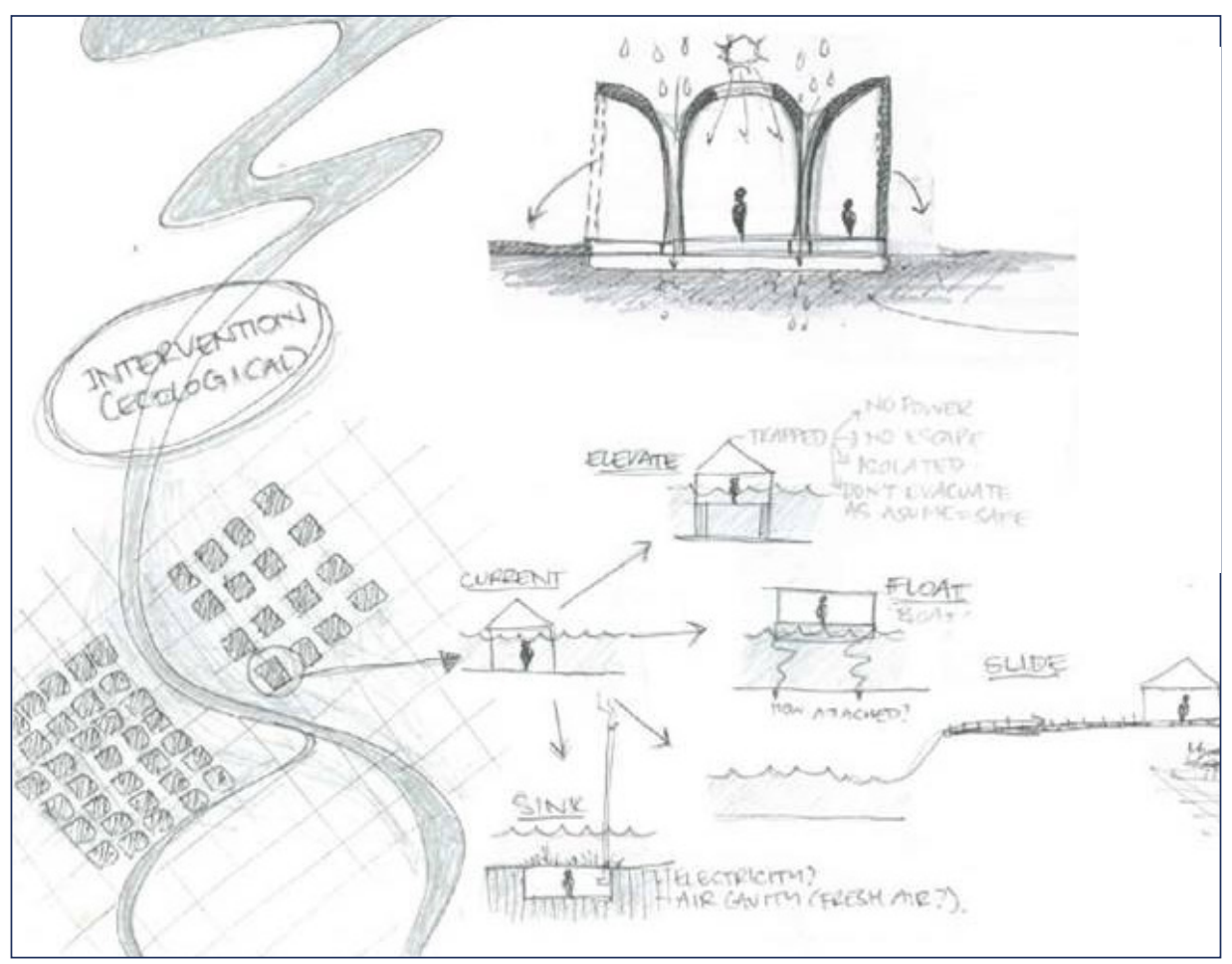

Figure 165: Initial urban shelter design thoughts

Architectural resilience adaptability strategies: core protection

Permanent dry and wet flood proofing measures and alternate energy and rainwater collection improve the adaptive capacity of the building to flooding and ensure vital services are available during floods.

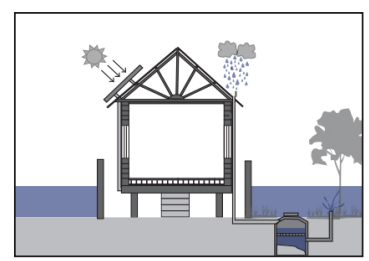

Figure 166: An architectural core protection strategy 


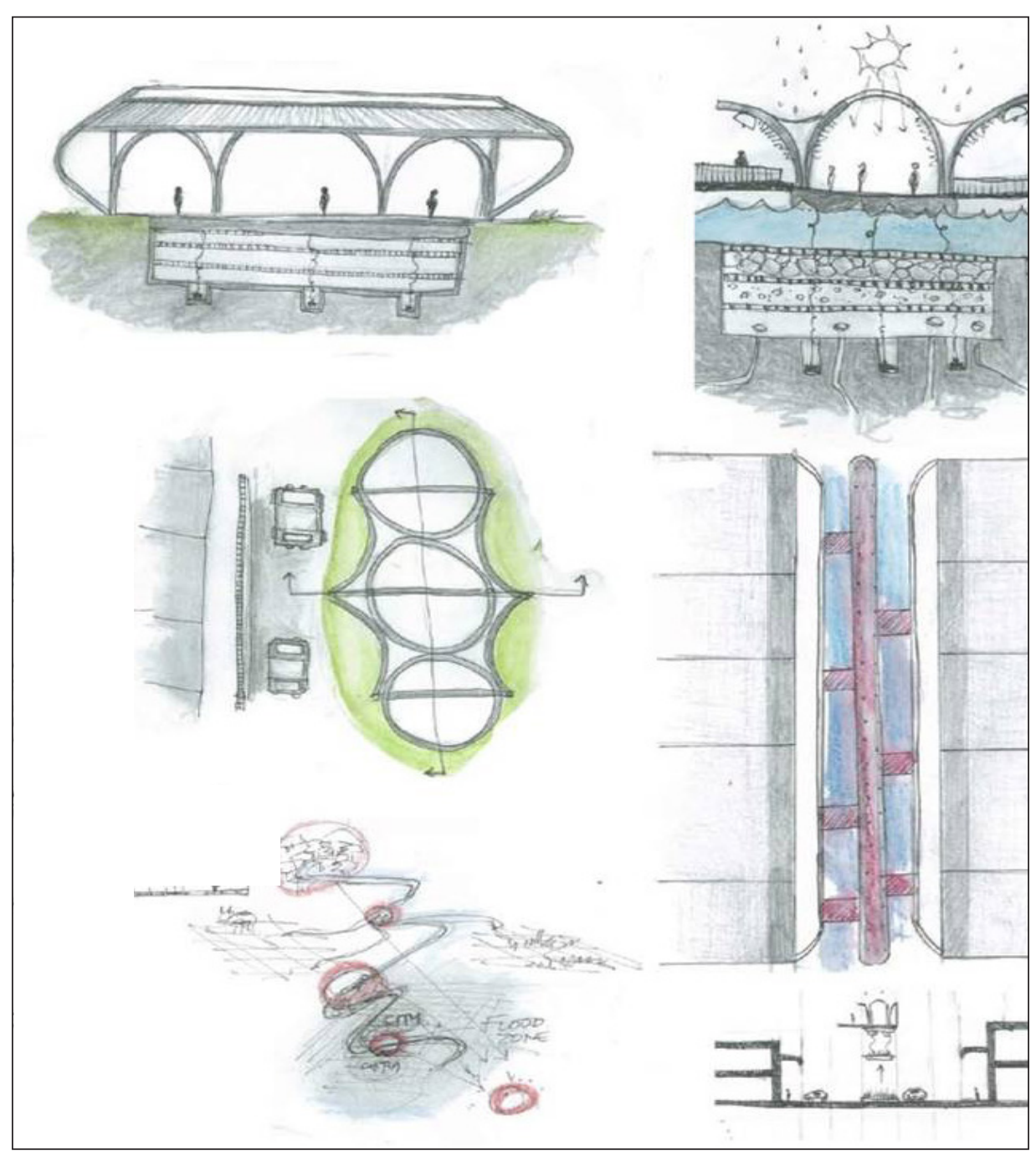

Figure 167: Exploring retrofit opportunities of existing urban structures
There are opportunities for the urban structures to enhance the lifestyles of Maitland's inhabitants during the varying climatic environments; temporal, drought stricken and flood prone climatic periods. The collection, storage and filtration of rainwater allow drinking water to be provided in drought stricken times when water restrictions are imposed and shortages become a local issue. The distribution of water storages throughout the city also ensures there is redundancy within the city system, so that when the city's water mains are not functional during or post flooding water remains available.

Solar and hydraulic alternate energy methods are suitable to Maitland as a power creating source which is not reliant on the city's energy mains. Solar energy takes advantage of the large amounts of sun available annually, whilst hydraulic energy methods provide a backup system, and allow structures to benefit from moving floodwaters and large amounts of rainfall. These methods ensure power is available to charge technological devices such as mobile phones, which become vital forms of communication during flooding. The power can also allow the urban shelters to be lit at night, aiding in their emergency shelter role and allowing them to become beacons of light within the urban realm.

The urban shelters strengthen and challenge existing city design objectives, whilst invigorating existing urban public structures to encourage community interaction and enrich the lives of nearby inhabitants. These urban features are at present either inhibited in their accessibility during floods, are inappropriately sited with respect to floodways or do not remain functional once urban streets are inundated.

Architectural resilience adaptability strategies: response

Construction techniques, such as amphibious methods, enable a building to effectively respond and react to flooding, reducing the adverse impacts of floods

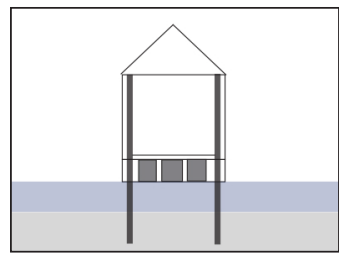
have on a building's structural integrity.

Figure 168: An architectural response strategy 
Amphibious bus shelters

Previously trialed and tested residential amphibious construction solutions are transferable to the urban shelter designs. New structures will improve the quality and aesthetics of urban spaces. Designs must however respect the heritage character of the town. But there are also options for existing urban shelters, such as bus stops and picnic shelters to be retrofitted with renewable energy and amphibious construction methods, to provide a more economically efficient and more immediate solution. Bus stops function as everyday transitional points within the city. During floods they can serve as flood safe shelters where residents can be evacuated using water borne transport. These shelters benefit local suburban environments and improve existing shelters, which have dated poorly.

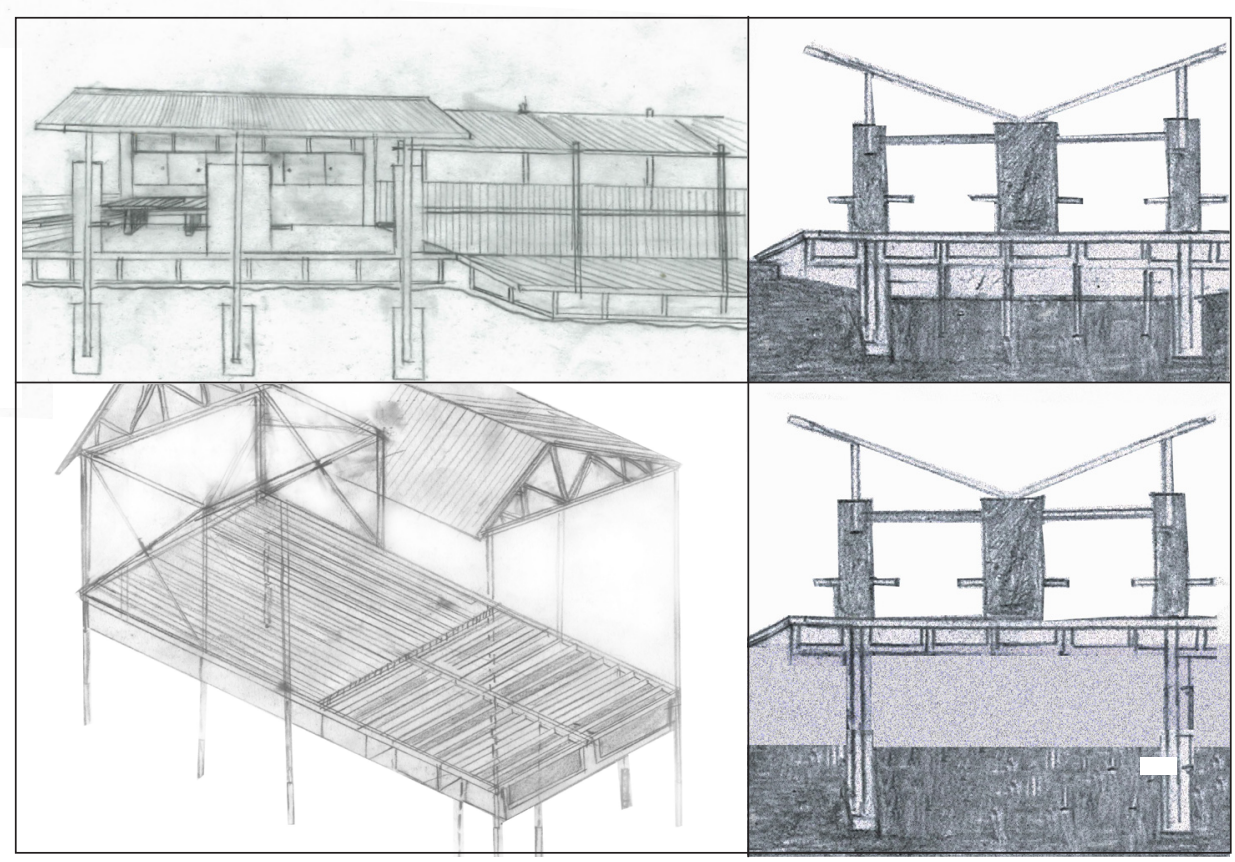

Figure 167: Bus shelter amphibious construction concepts

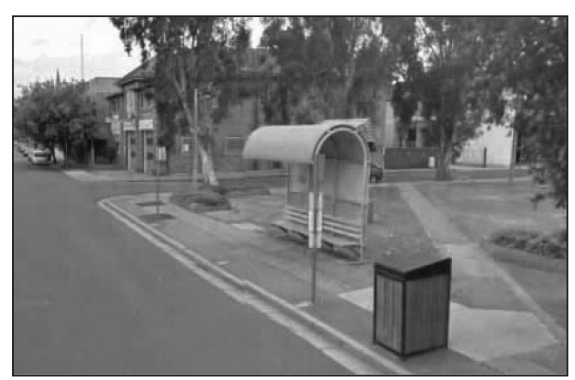

Figure 168: An existing Maitland bus shelter
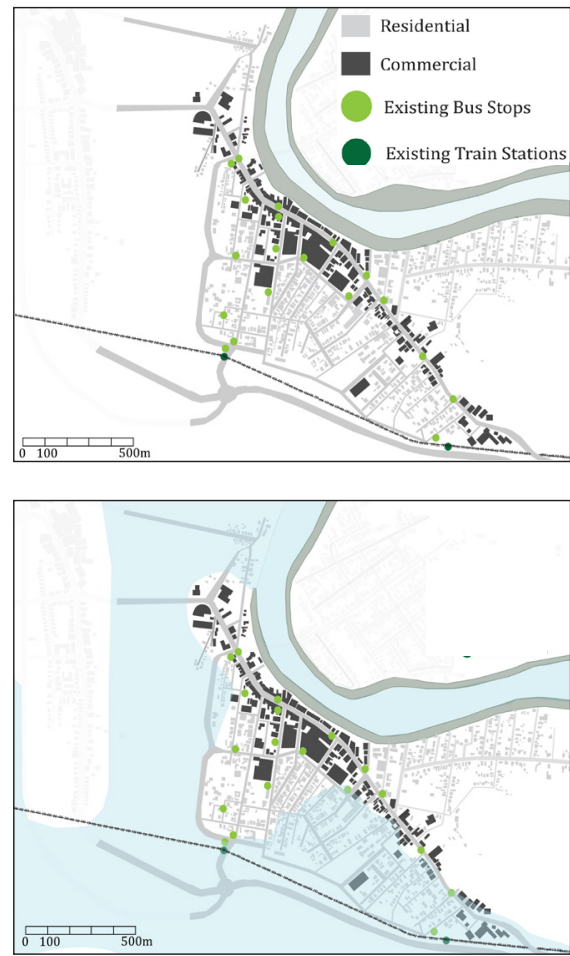

Figure 169: The impact of low floods on existing bus stops and train stations in Maitland 


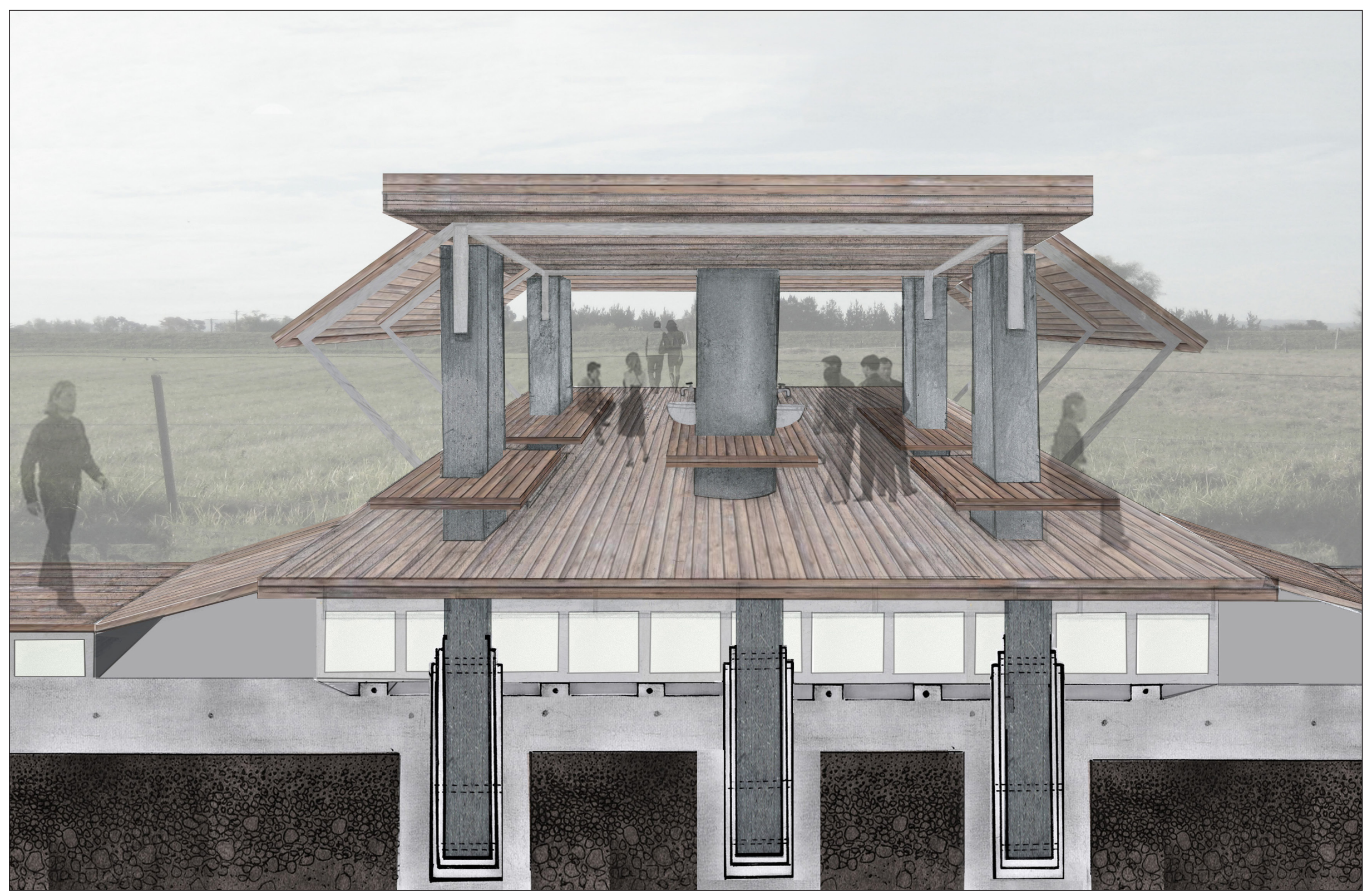

Figure 170: A sectional perspective of an amphibious bus shelter design for Maitland 
Amphibious shelter, water, sanitation and cooking facilities

A lack of functional sanitation services during and post flooding dramatically impacts on the health of a city's inhabitants. Services are lost when electricity and water mains of a city are cut due to flood damage or safety concerns. Sanitation facilities distributed within the urban landscape will improve the everyday availability of these key public services, whilst ensuring they remain available when traditional methods fail. The sanitation facilities will need to utilize rainwater storage and alternate energy systems. The services will also provide temporary shelter options during periods of inundation.

Consideration of the flood resilience principles reveals greater insight of how the structure can improve the social, ecological and infrastructure aspects of Maitland in the everyday city system, along with the functioning of a flood affected system.

The sanitation facilities may also establish functional relationships with picnic barbeque shelters. There are also options for existing facilities, such as at sports and recreational grounds, to be amphibiously retrofitted or flood proofing measures improved and alternate energy measures, water storage and filtration devices implemented.

Architectural resilience adaptability strategies: storage

Above or below ground rainwater tanks made from concrete or steel collect and store water for occupant use.

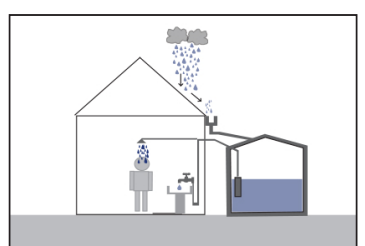

Figure 171: An architectural storage strategy 


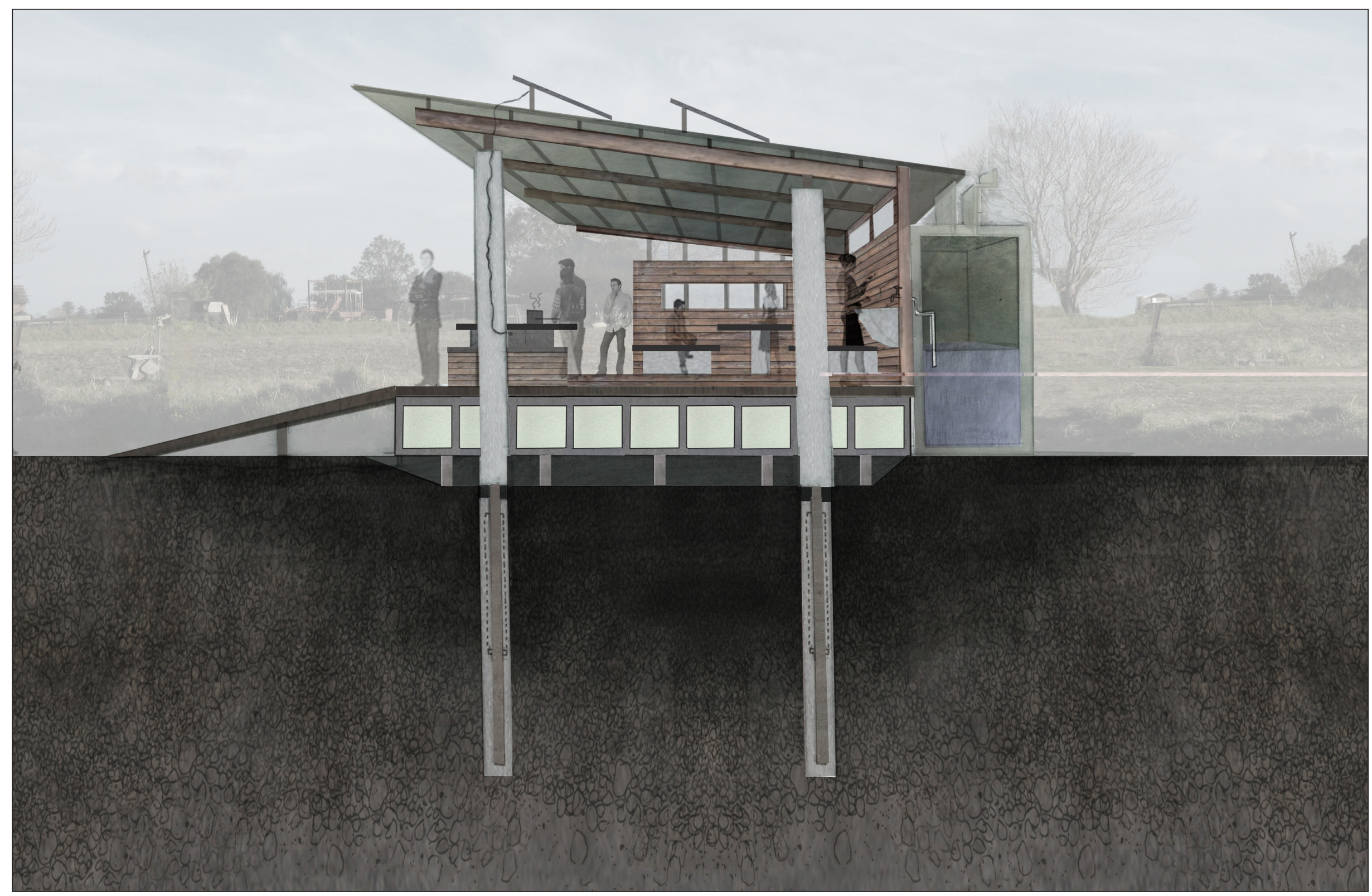

Figure 172: A sectional perspective of a Maitland barbeque shelter design 
The community centre is to form an integral part of the community through the provision of everyday services and facilities. The site of the community centre is located within Maitland's commercial district on the highest topographical point, the least flood liable land of the city. The space surrounding the community centre is to become a public civic space, physically connecting the levee to the city. The building floats above and nestles within the levee adjoining the Hunter River. The community centre is to provide a café, performance space, toilets, change rooms, an exhibition space, meeting and picnic spaces, hall, storage facilities, a pick up and drop off zone, car parking and an emergency helipad. The site is to perform a community strengthening role and enrich Maitland. The civic square and community centre become an everyday gathering point within the city, a practice that will continue when the site functions as a flood evacuation point. The community centre will provide alternative energy, water, sanitation, food and storage facilities during floods, when services are limited or unavailable.

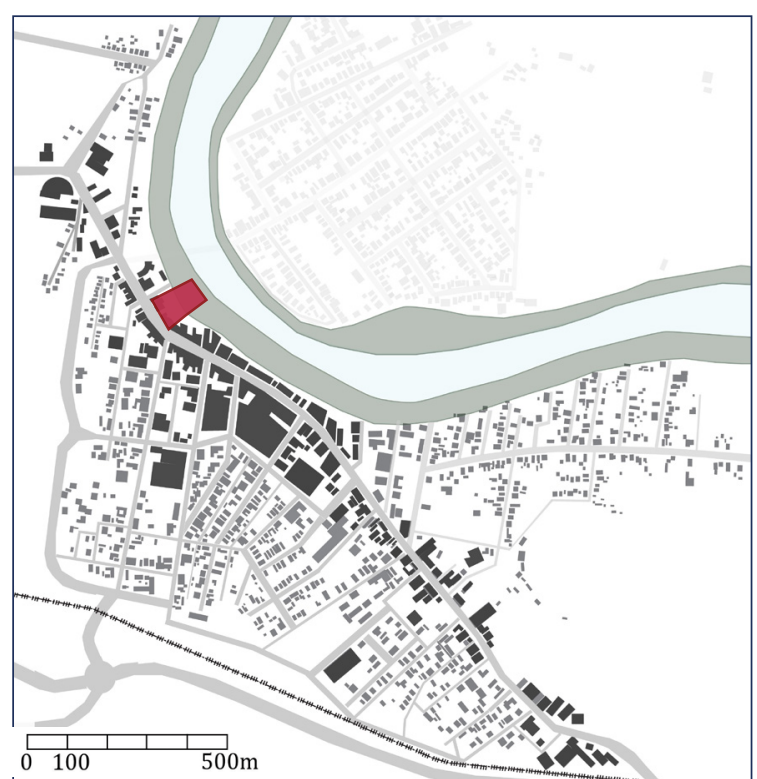

Figure 173: Community centre location within Maitland

\section{Strategy intentions:}

- Provide vital alternate and renewable energy sources, water collection, filtration and re-use, flood safe shelter, sanitation facilities, food and medical services pre, during and post flooding.

- Establish a civic space, community hall and an events space for public gathering.

- Allow the community centre to further inform occupants of the three category flood heights.

- Establish a symbiotic relationship between the building and the surrounding riverine ecosystem, rather than the development further perpetuating flood hazards.

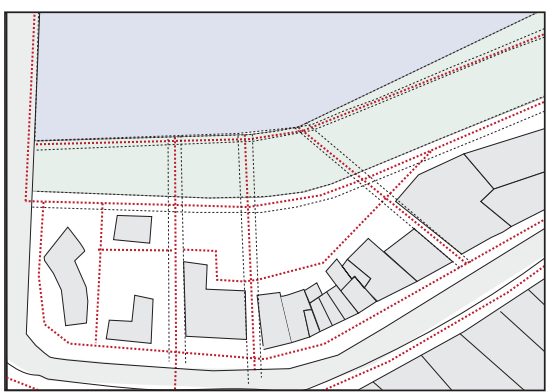

Figure 174: Existing pedestrian circulation on and around the site of the community centre

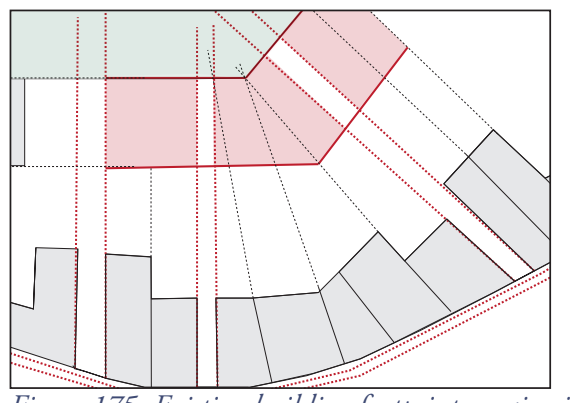

Figure 175: Existing building footprints, main circulation paths and view shafts from the street to car park 


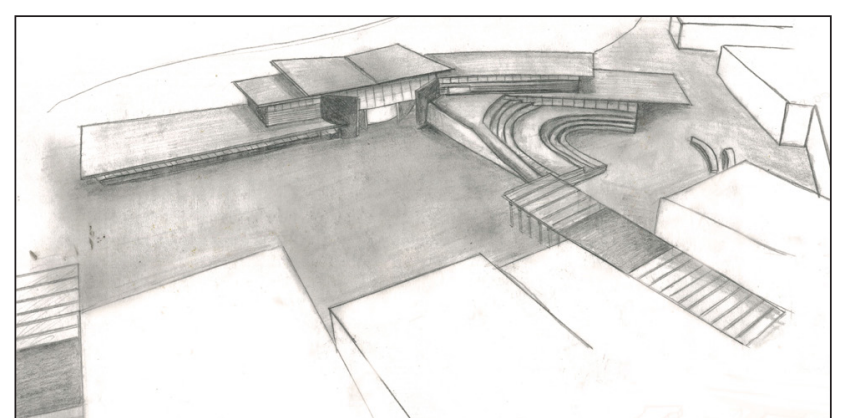

Figure 176: A view of the community centre from above the existing Maitland rectilinear buildings

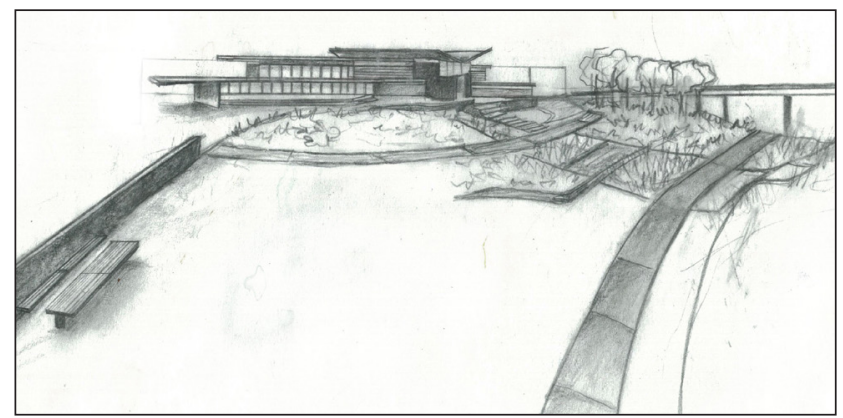

Figure 177: A view of the community centre from along the more natural ecological end of the levee

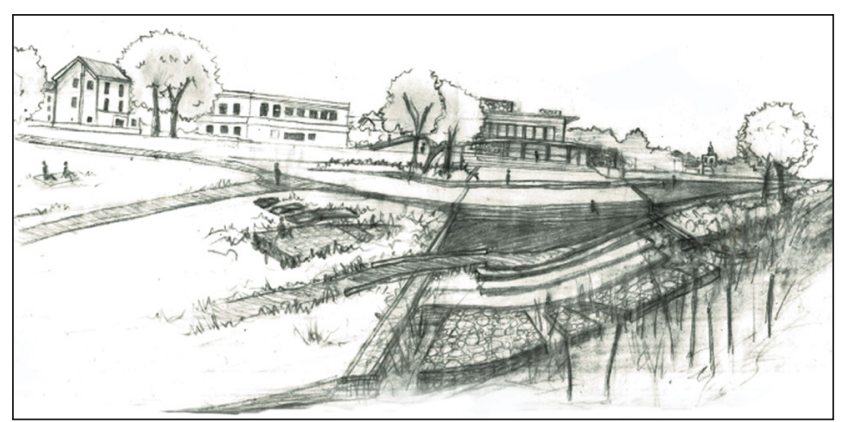

Figure 178: A riverside view of the levee leading up into the community centre
The meandering circulation paths of the levee converge onto the civic square and community centre site. A series of concentric terraces radiate out from the community centre's central circulation and water storage/ filtration core across the levee. Visually and functionally linking back to the core water based function of the community centre. The three flood category events are integrated into the terracing of the levee and the levels of the community centre. The building steps up from the urban environment to elevated cafe and hall spaces, where views extend southwards down the levee. At the northern end the market, exhibition and picnic meeting spaces step down into the levee and interact with the high flood zone.

The community centre establishes a transitional zone between the passive ecological levee and the active urban city. The building form arose from site analysis of surrounding buildings and existing circulation paths of the levee and urban surrounds. The nearby Maitland City Library is also reflected in the form of the community centre. The curved section of the building cradles and provides shelter to the outdoor amphitheater. The enclosed concrete surfaced space contrasts with the large open semi-porous paved space that adjoins it. Two canopy sheltered entrances lead into this large market space. The market space lends itself to the gathering of large numbers of people. This ensures the civic square will adequately function as a flood evacuation point for the city of Maitland.

Architectural resilience adaptability strategies: cooperative self-regulation

Moving furniture, appliances and important posessions above anticipated flood heights. Living spaces on the second level are equipped with flood kits and a flood evacuation plan.

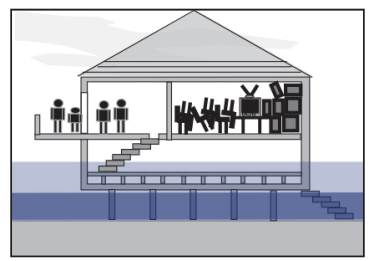

Figure 179: An architectural cooperative self-regulation strategy 
The community centre plans
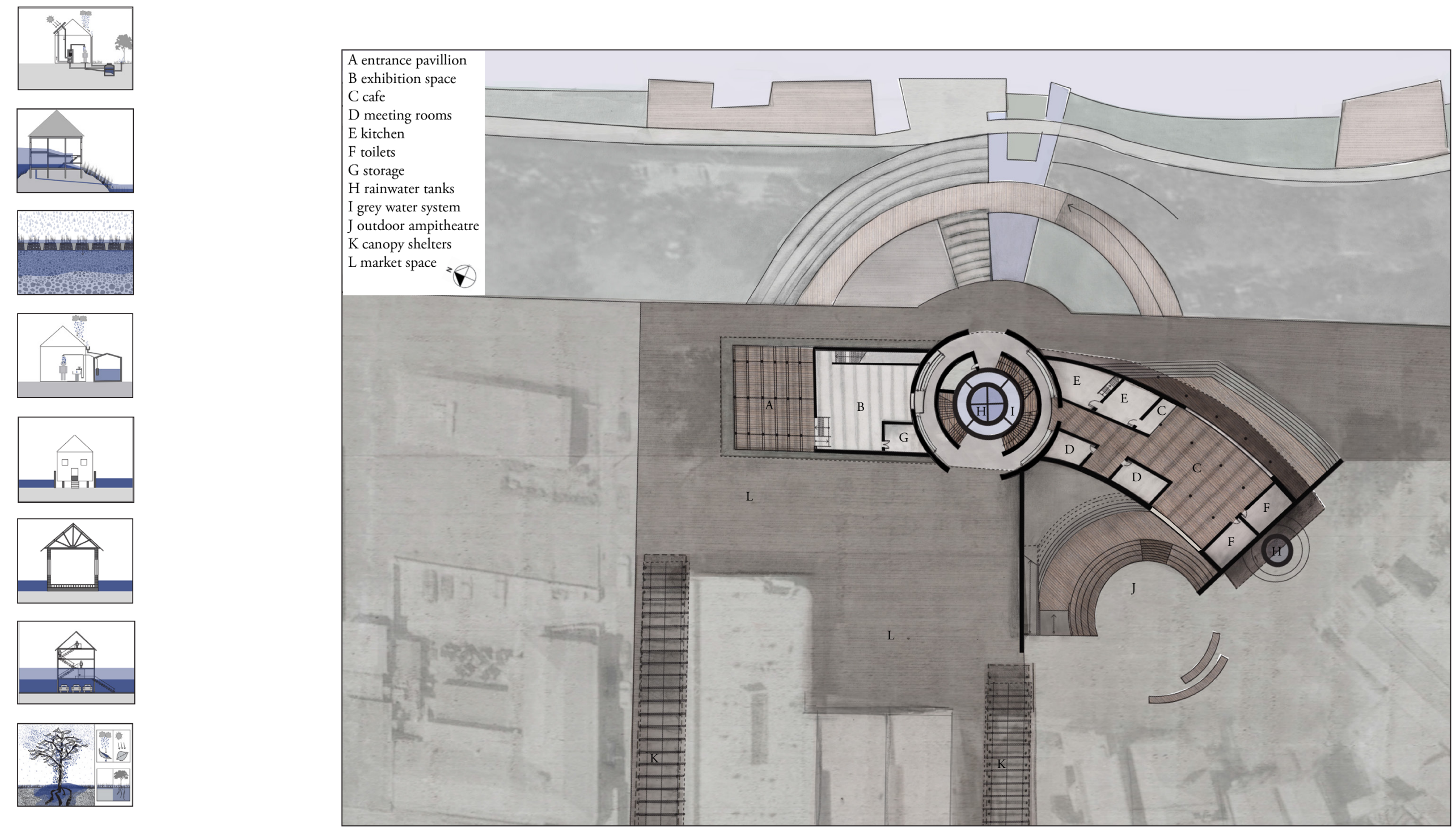

Figure 180: 1:100 Community centre level two plan 


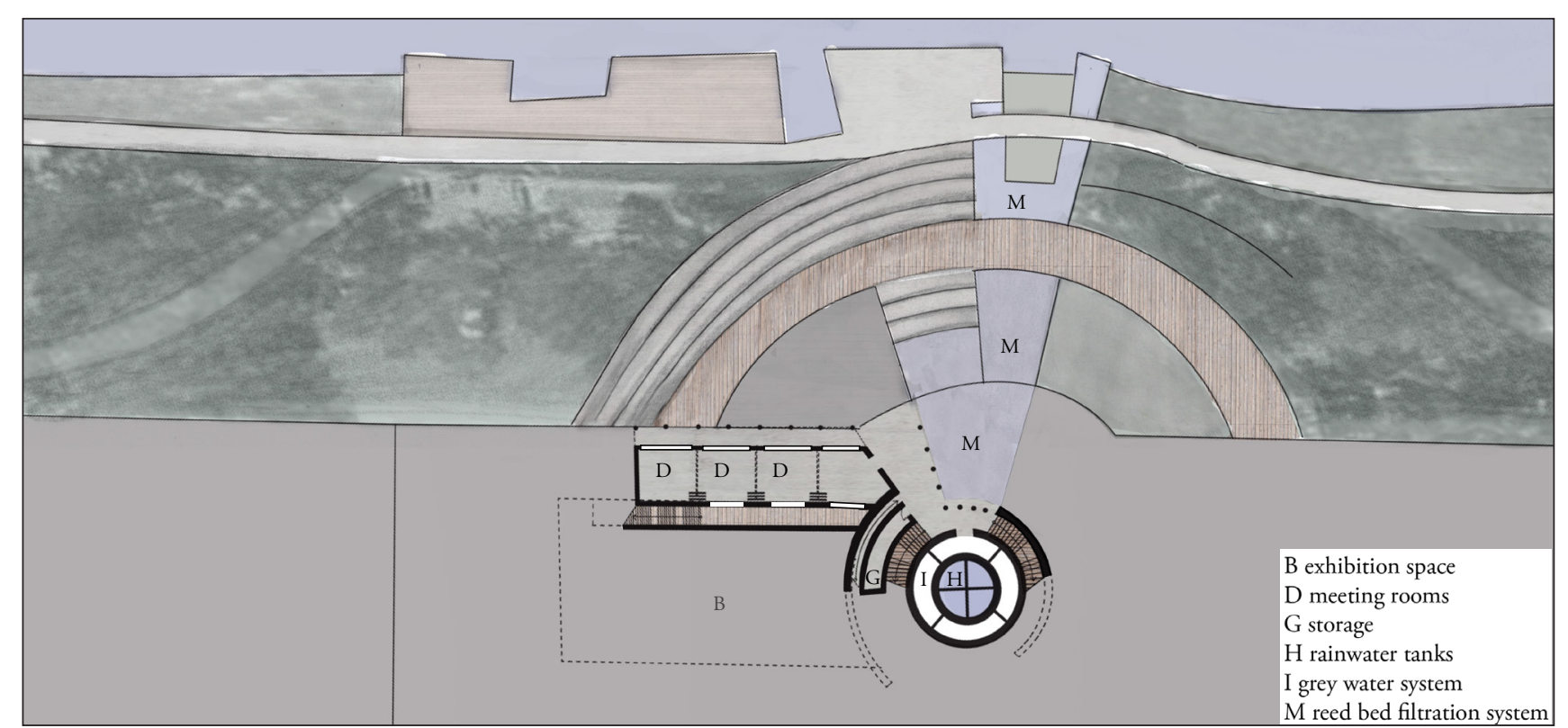

Figure 181: 1:100 Community centre level one plan

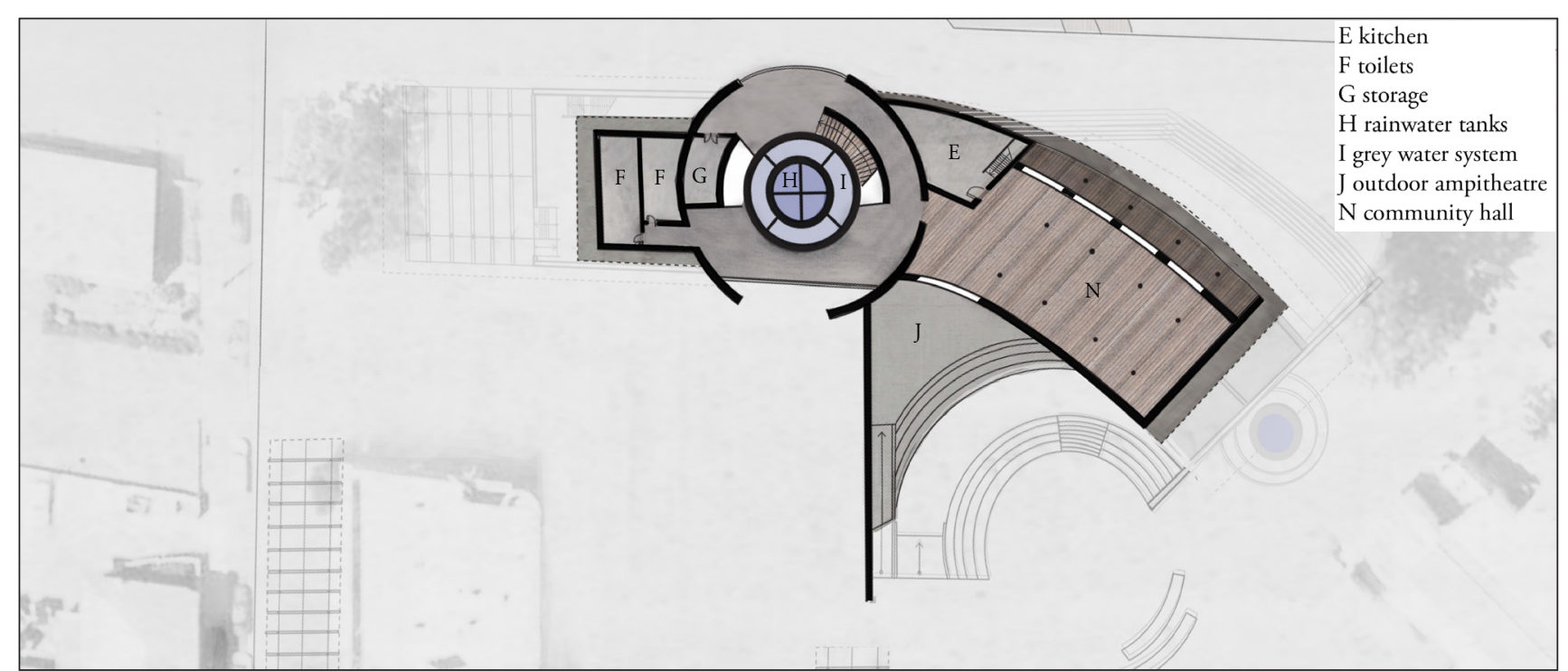

Figure 182: 1:100 Community centre level three plan
The flood resilience principle of scales, adaptability, variety and redundancy and feedback, are integrated socially and spatially into the community centre. Ecological, flood management and architectural flood resilience measures are integrated into the civic square, levee and community centre designs.

Functional redundancy is incorporated into the community centre. The large multi-purpose and flexible exhibition, hall and cafe spaces ensure they can be spatially altered to respond to the changing needs of society over time, as well as being changeable to suit their alternate flood emergency shelter roles. Redundancy exists in the double storey kitchen, which has the capacity to service the cafe and hall space.

A variety of stair and ramp circulation options respond to the multiple entrances and series of elevated and sunken platforms upon which the building's spaces are positioned. The multiple ramps and stairways traversing the building ensure those mobility disabled, such as the elderly, pregnant women, sick and young persons are not disadvantaged in their use of the community centre. The central circulation core also serves an alternate function as a rainwater storage and filtration system.

The central core is broken down into four rainwater filtration tanks. There are also additional rainwater collection tanks that service the cafe toilets at the southern end of the building on the elevated ground floor. The modular building design allows it to be implemented and developed in stages in response to community consultation and use. This also plans for future population increases. There are also future options for Maitland Council's proposed riverside residential developments to attach onto its northern entrance and extend along the levee. 
The community centre becomes intrinsically connected to the levee's riverine ecosystem; the building and levee establish a symbiotic relationship with respect to their social, ecological and structural flood management functions. This is in recognition of the benefits the building will have for the levee during drought periods, and the protection the building will gain from an ecologically healthy and efficient riverine levee system.

The building has a central core containing a series of rain water tanks and a grey water treatment system enabling the building's water to be reused. What is unable to be reused is then released out across the reed bed natural filtration system of the levee; benefiting the local surrounds in drought stricken times. The building also integrates backup septic and solar energy systems. Buffering is reinterpreted architecturally through a double skin structure; sacrificial spaces (basement/garage etc.) utilize non load bearing columns which act to reduce water velocity, whilst the exterior wall is closed allowing the space to function from the interior.

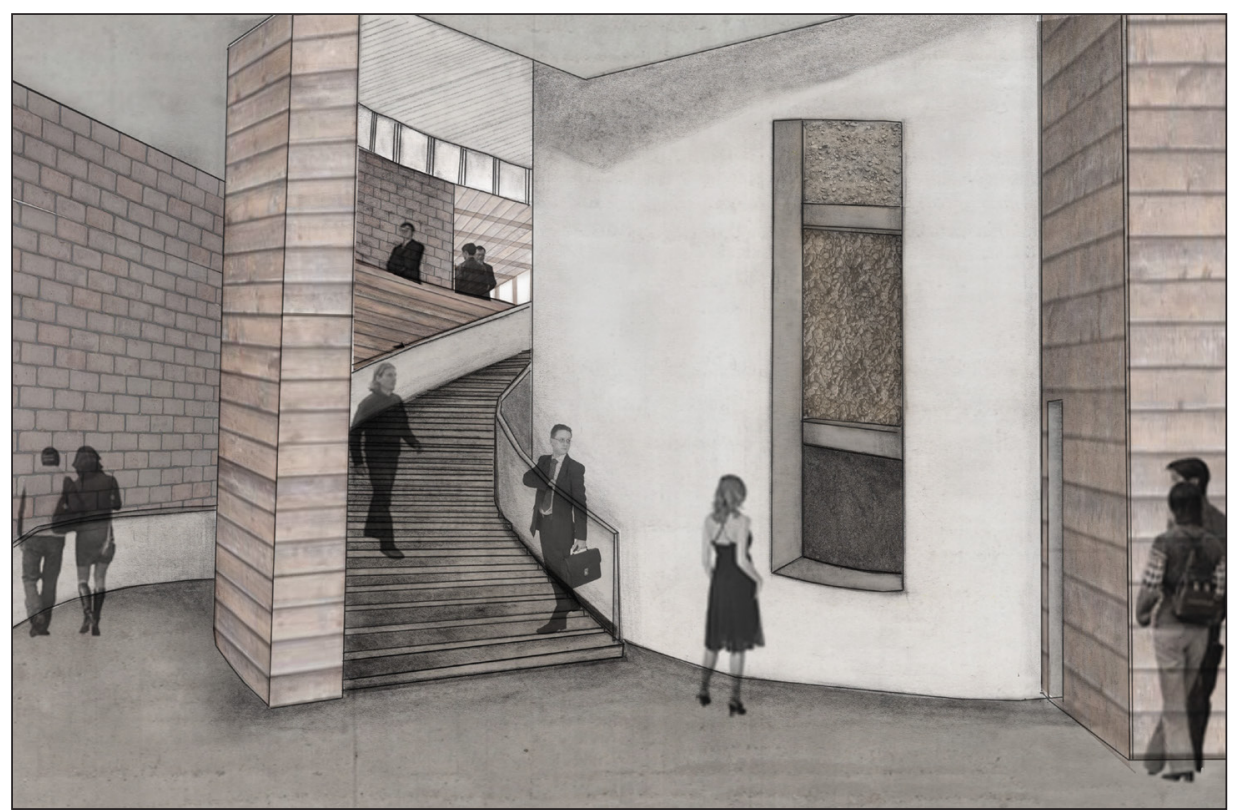

Figure 183: The central circulation core with a grey water system viewing window
The thermal and structural benefits of concrete and brick aids in the building passively heating and cooling itself through annual seasonal changes. A central spiral stair case runs along the circumference of the central core. As people move up and down the spiral staircase they are able to interact with the core's rainwater collection tank and grey water filtration system. The building's grey water is reused for nonpotable uses and black water is treated before being released across the natural reed bed filtration system of the levee or into a below ground septic tank system.

The building interacts structurally, spatially and functionally with the high flood category levels. Elevated spaces ensure the flood safe emergency shelter is available for extreme flooding.

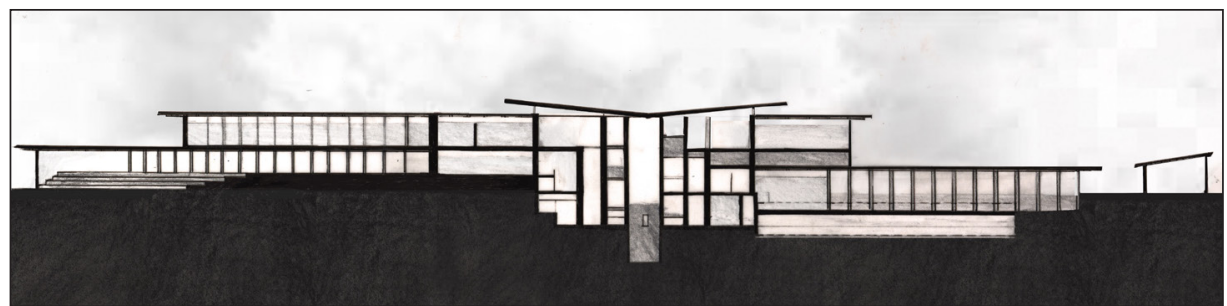

Figure 184: A 1:500 longitudinal section of the rainwater collection \& grey water filtration system 
The community centre's grey water system and the levee's reed bed filtration system

Grey water is initially filtered in the central core and reused before being released across the levee's ecological filtration system.

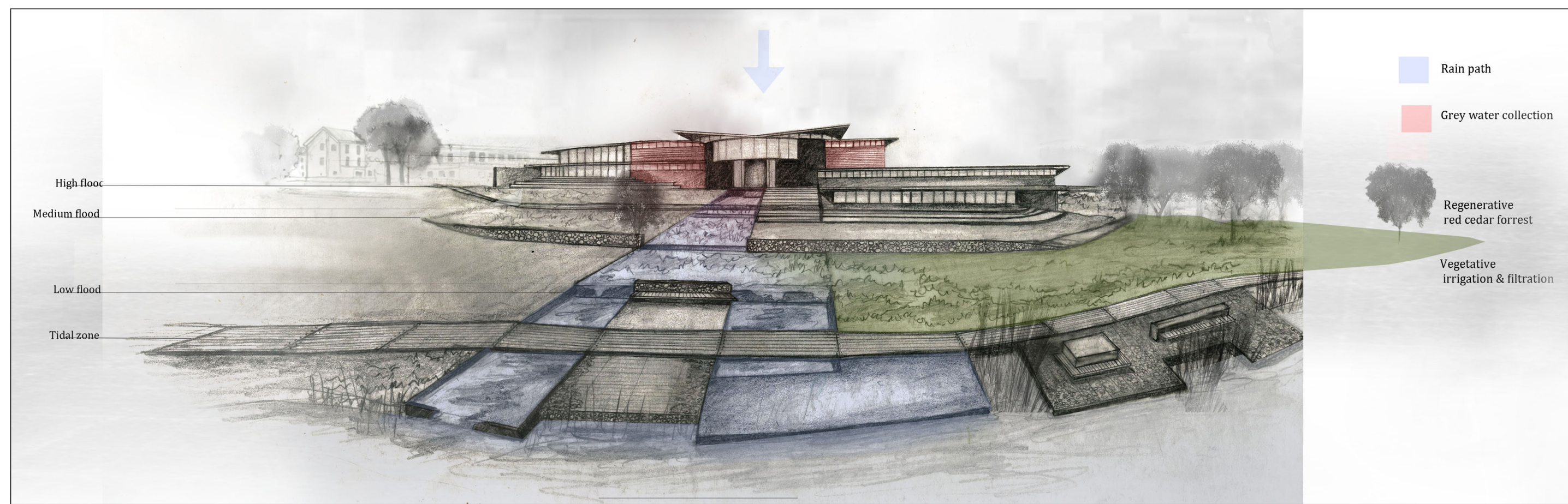

Figure 185: The community centre's grey water system and the levee's reed bed filtration system

Architectural resilience adaptability strategies: nutrient and waste recovery

Grey water systems and rain water collection tanks allow water to be captured, stored, filtered and reused before being released back into the natural vegetative surroundings.

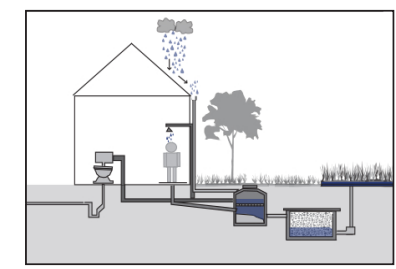

Figure 186: An architectural nutrient and waste recovery strategy 
A canopy structure extends down the length of the two High Street entrances. This allows the community centre and civic square, which are predominately hidden from the main street, to have a street presence. The simple canopy structure rests in harmony with existing heritage shop front canopy structures. There are materiality shifts within the canopy roof structure, ensuring natural light is able to permeate the spaces and enhance a processional entrance onto the site. The covered spaces provide protection against rain and sun and benefit the markets to be held within the space.

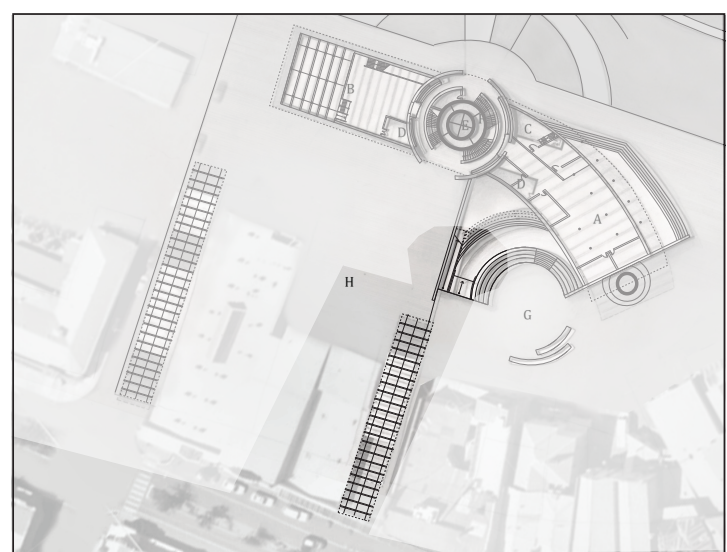

Figure 187: A plan highlighting one of the two canopy entrances
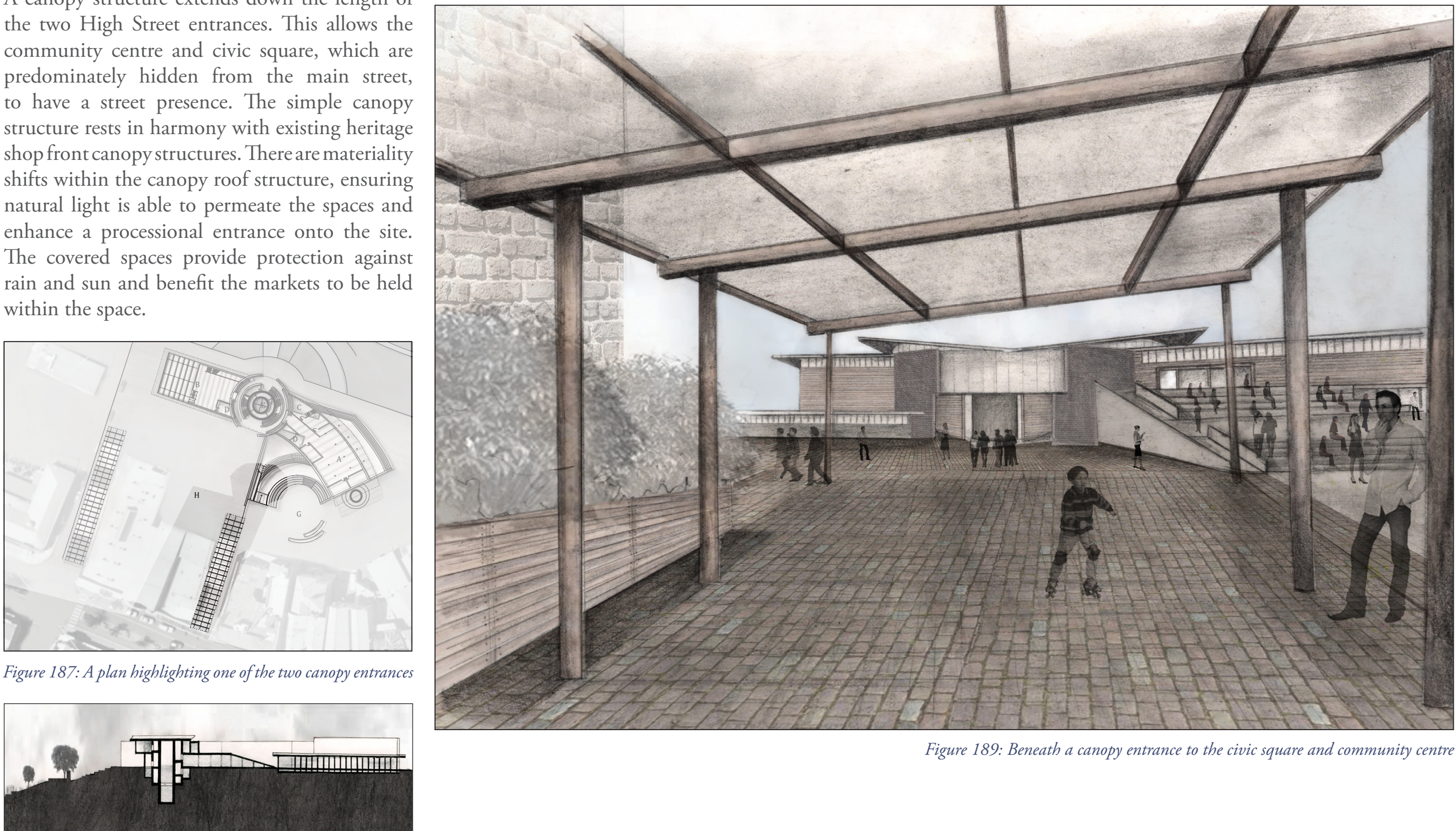

Figure 189: Beneath a canopy entrance to the civic square and community centre

Figure 188: A transverse 1:1000 section of the community centre's canopy entrance 


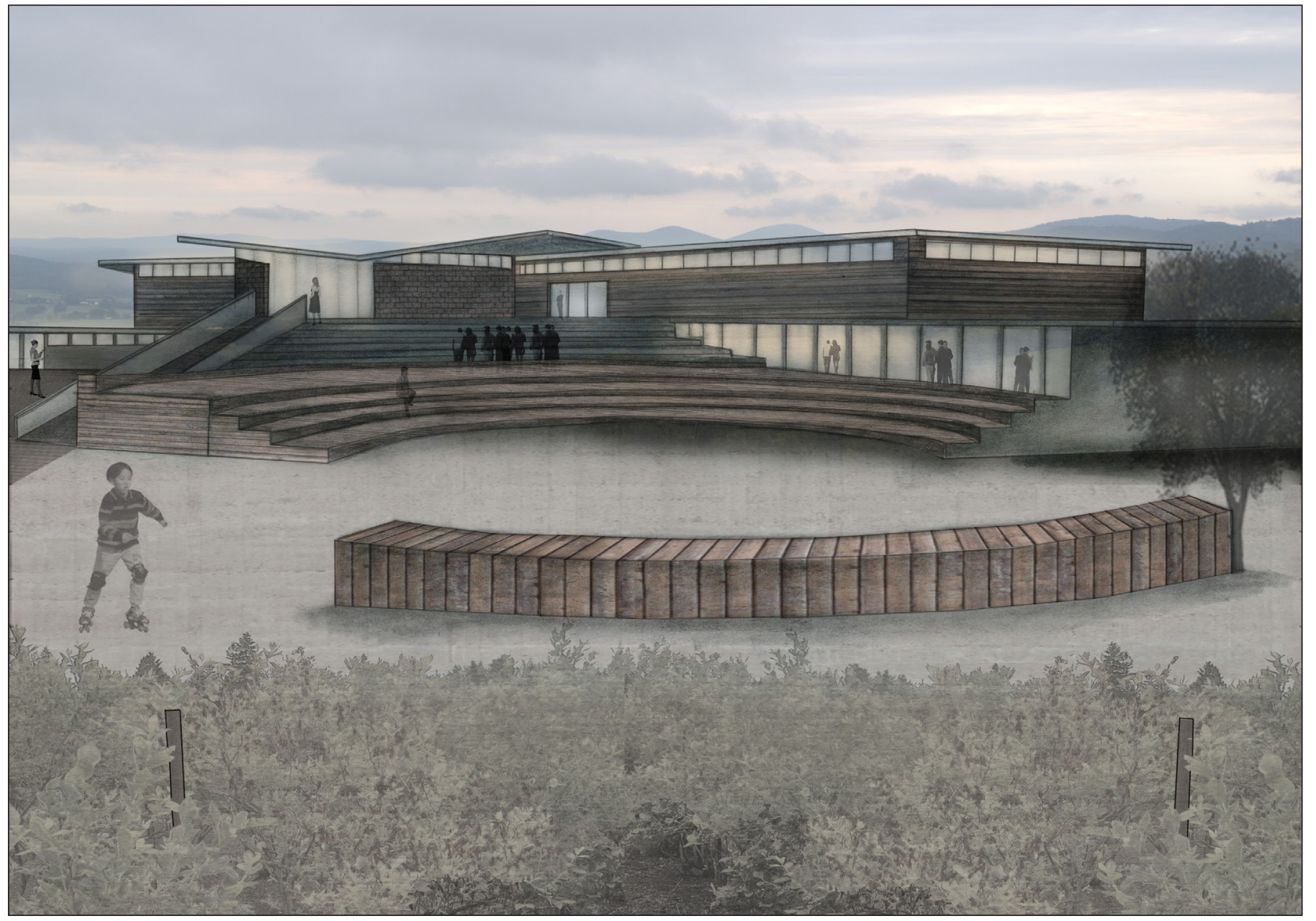

Figure 190: An exterior view of the community garden, outdoor amphitheatre area, community hall \& café at night
The heritage buildings and community centre shelter the outdoor amphitheater from wind and direct sun from the east and west during summer. The building wraps around to create a protected and intimate space, improving the spatial qualities of the gathering space. The more enclosed and defined space differs from the large open courtyard space that lies beyond. This allows the enclosed and open spaces to be appreciated and adapted in use to suit their alternate flood related functions. A community garden and vegetative spaces begin to activate the backs of existing buildings; encouraging existing building occupants to utilize back entrances to strengthen circulation from High Street into the civic space.

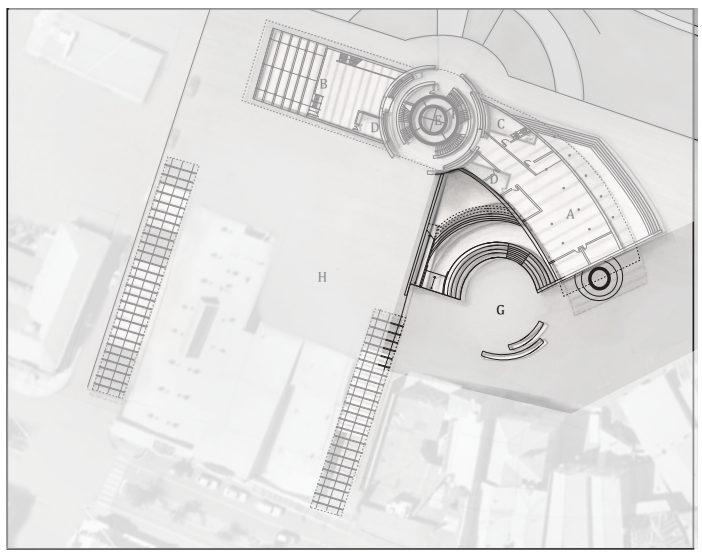

Figure 191: A plan highlighting the outdoor amphitheatre 


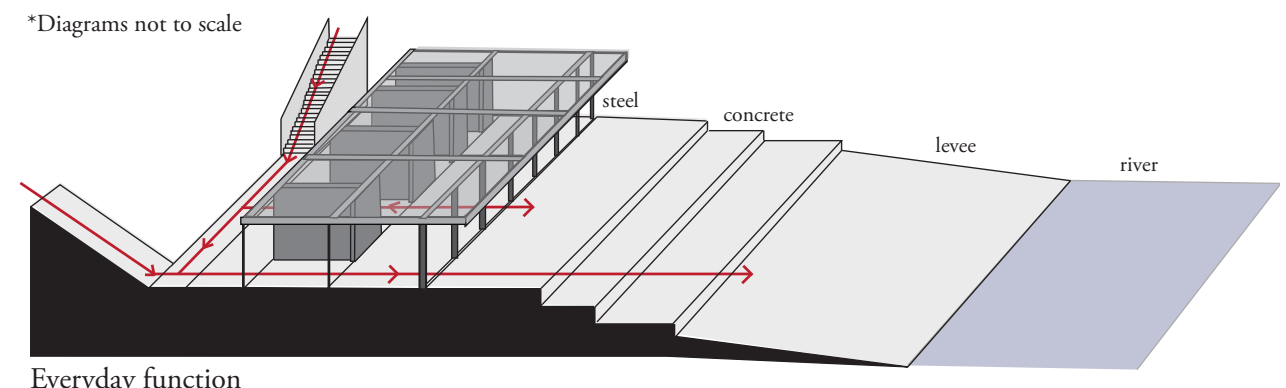

eryday function

Spaces are accessible via an internal ramp or stairway as well as through an entry from the levee.

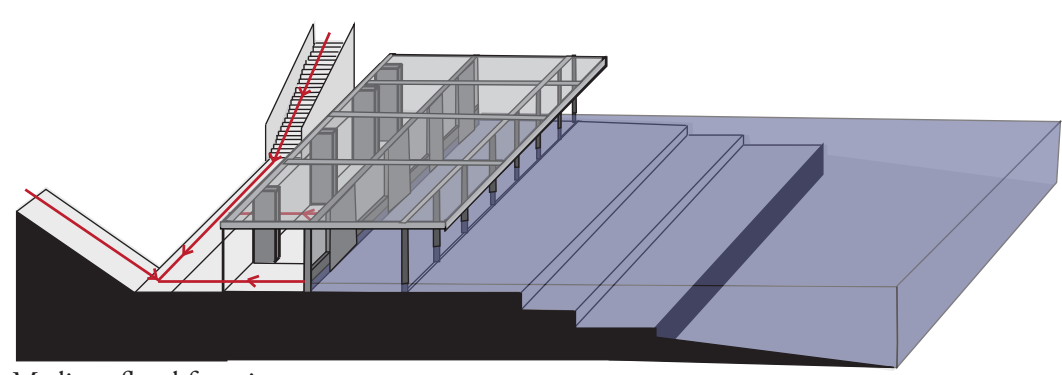

Medium flood function

Walls on the levee side are closed, and the spaces operate from the interior only

Up to a $0.9 \mathrm{~m}$ height flood proofing barriers function to keep water out of the spaces.

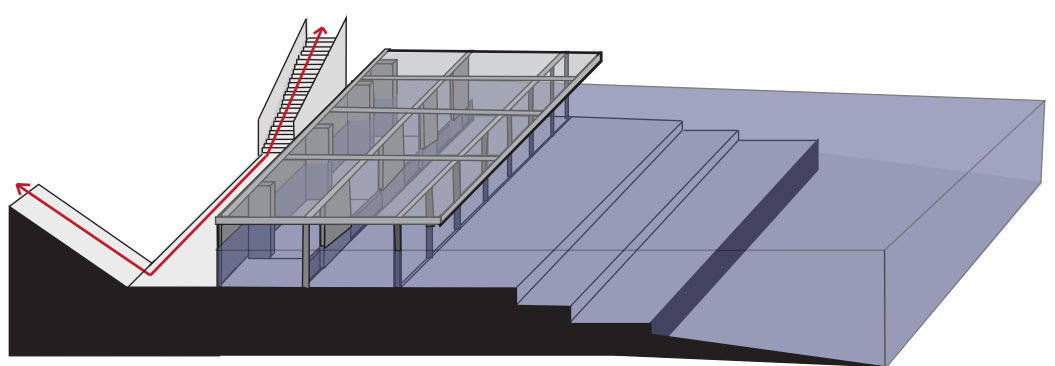

Not functional during high floods

Spaces are inundated by floodwaters and not during extreme flood events. Back walls prevent it from inundating the rest of the building. Spaces are minimally impacted due to water resistant material use.

Figure 192: The meeting and picnic area of the community centre functional interaction with high floods

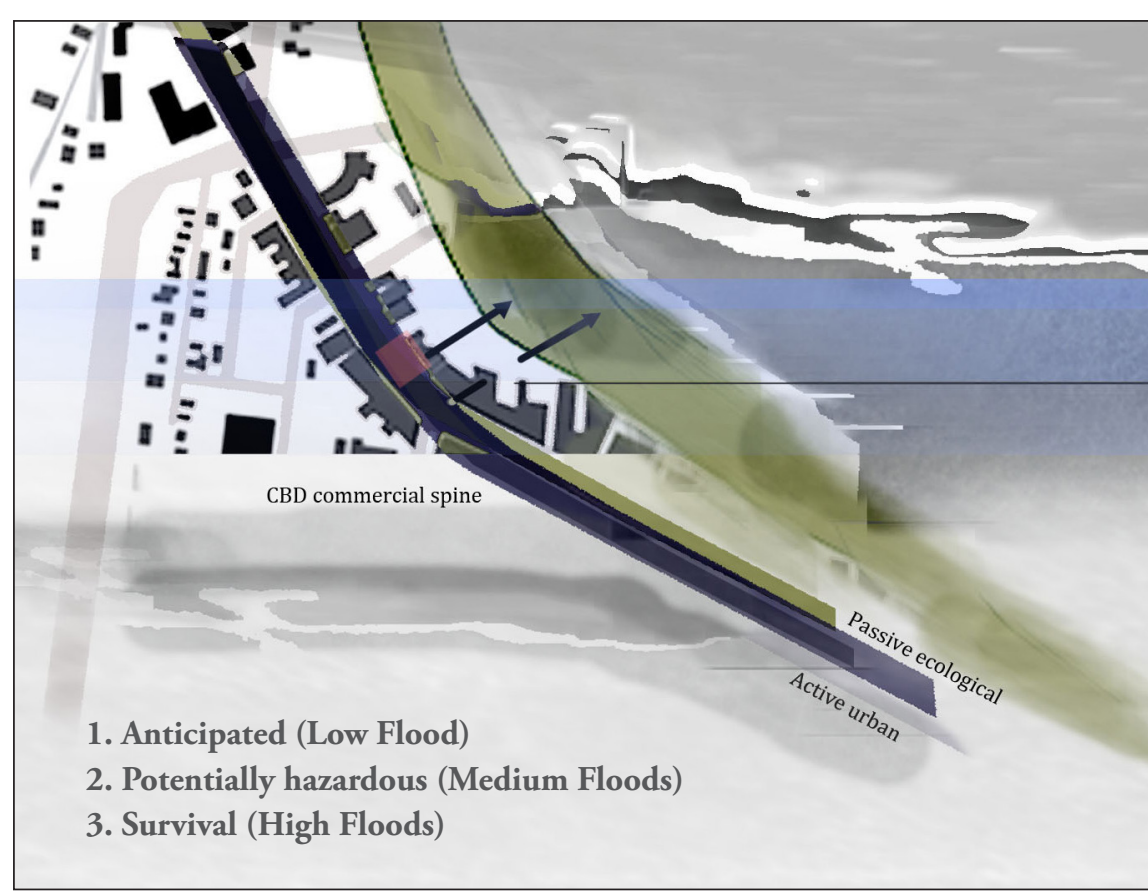

The longitudinal section above demonstrates the impact low, medium and high flood category events have on the building function and structure. The three stages are incorporated into the structural and spatial function of the building. The community centre caters for the increasing numbers who become reliant on different services. The building both steps down to embrace flooding and steps up to form an elevated safe zone. It is in this way that the building aims to establish a balance between embracing and preventing floods.

The meeting and picnic spaces are designed to react to flooding and spatially respond to the changing conditions. The normally open pavilion like spaces, become enclosed and shut off from the levee side. The area then becomes accessible via an internal stairway leading up into the exhibition space, or via the central ramp that leads into the core circulation zone. 


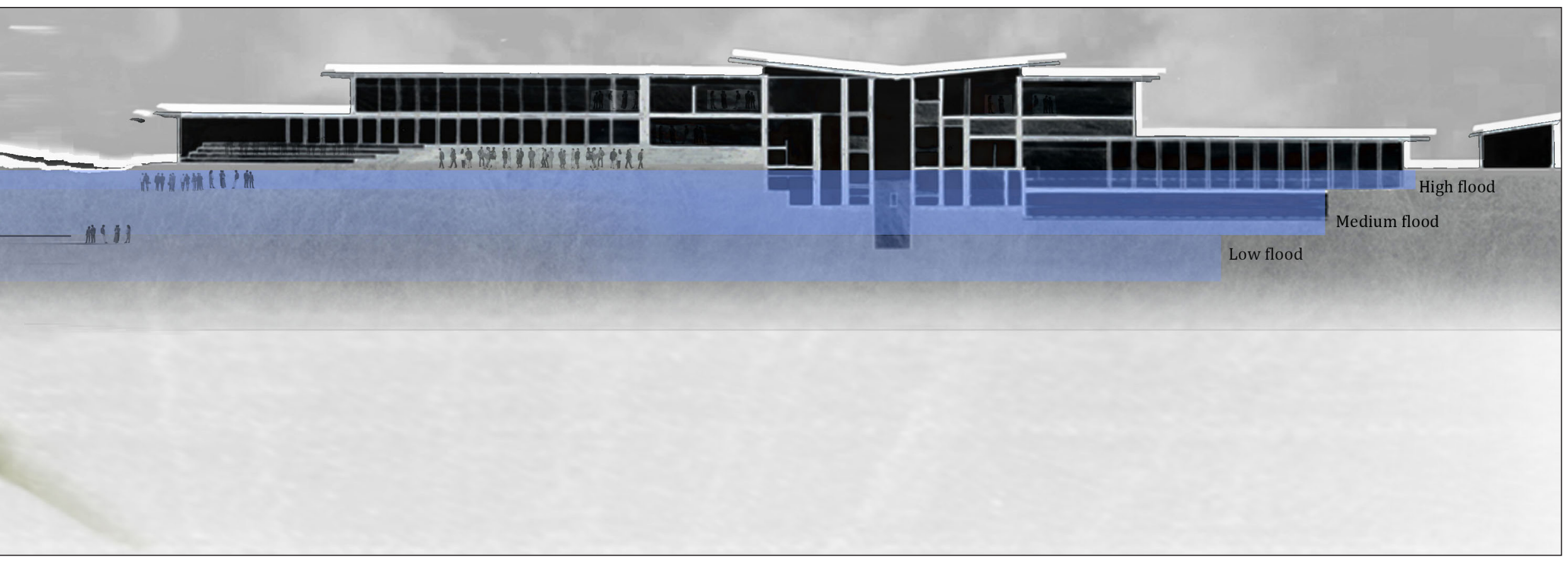

Figure 193: A longitudinal section of the community centre highlighting the impact of floods on the building

Water resistant materials, such as concrete and brick along with wet proofing measures, have been incorporated into the meeting picnic rooms. The spaces begin to be inundated at a medium category flood event. This represents the beginning of inundation for residents within South Maitland (located furthest away from the Hunter River) . Feedback will enhance flood awareness, response and preparedness of Maitland residents not yet effected; a warning to begin to take action.

Dry flood proofing measures initially protect the space from floodwaters up to 0.9 metres. It is at this point that due to hydrostatic pressure, water begins to be buffered into the spaces. A series of ramps integrated into each level change allow the building to release flood waters back across the levee and into the river once the levels begin to fall. This also aids in reducing post flood recovery and clean up time frames.
Architectural resilience adaptability strategies: buffering

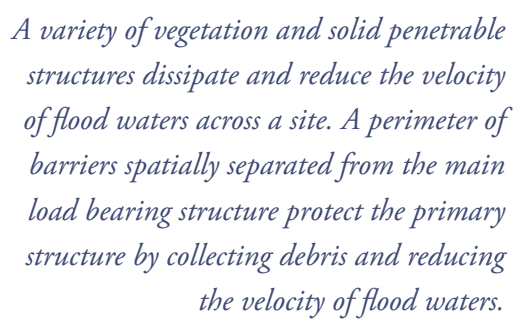

A variety of vegetation and solid penetrable structures dissipate and reduce the velocity of flood waters across a site. A perimeter of barriers spatially separated from the main load bearing structure protect the primary structure by collecting debris and reducing the velocity of flood waters.

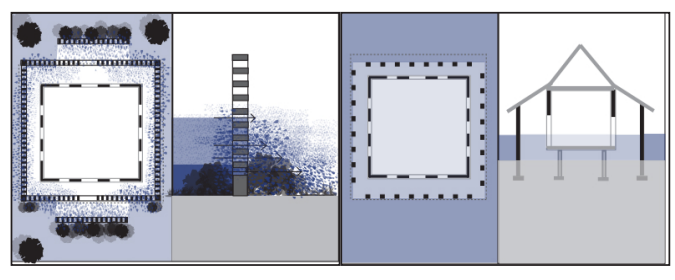

Figure 194: An architectural buffering strategy 
The front of the community centre opens out and onto the levee. A series of platforms radiate out from the central core, with reed beds and vegetative zones acting to naturally filter the centre's waste water. The centre promotes interaction with the levee and provides a place to rest, recuperate, be entertained or satisfy thirst or hunger.

It is a building which due to its modular design is able to transform and alter to suit the changing needs of the Maitland community it serves. The community centre terraces up from within the levee, welcoming people into and out of the building at each level. At night and especially during floods the building becomes a beacon on the landscape. The community centre in this instance becomes a flood safe emergency shelter within which water, power, sanitation and communication services, food, and cooking facilities, are provided.

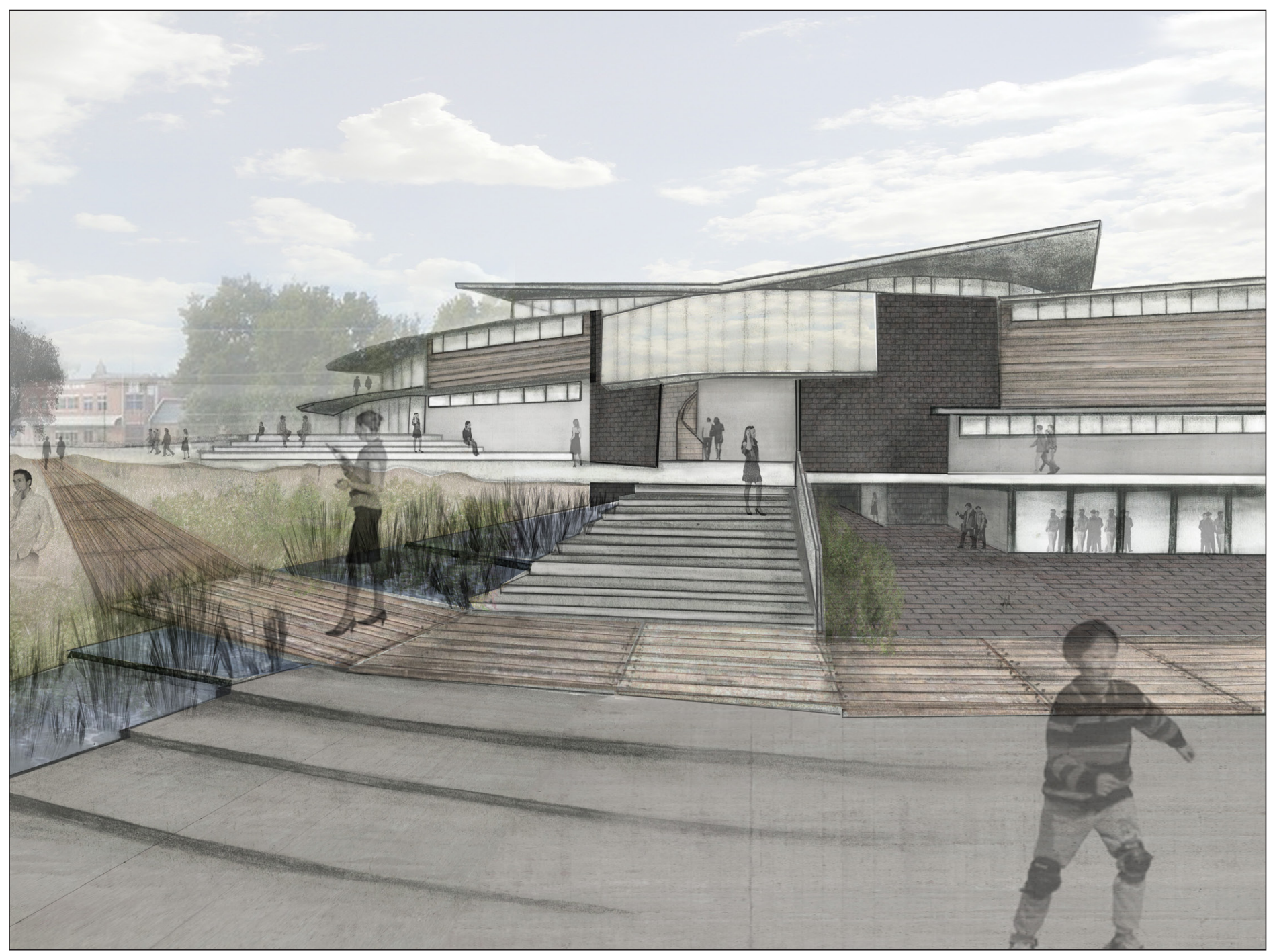

Figure 195: A view of the community centre from the levee's terracing spaces and reed bed filtration system 


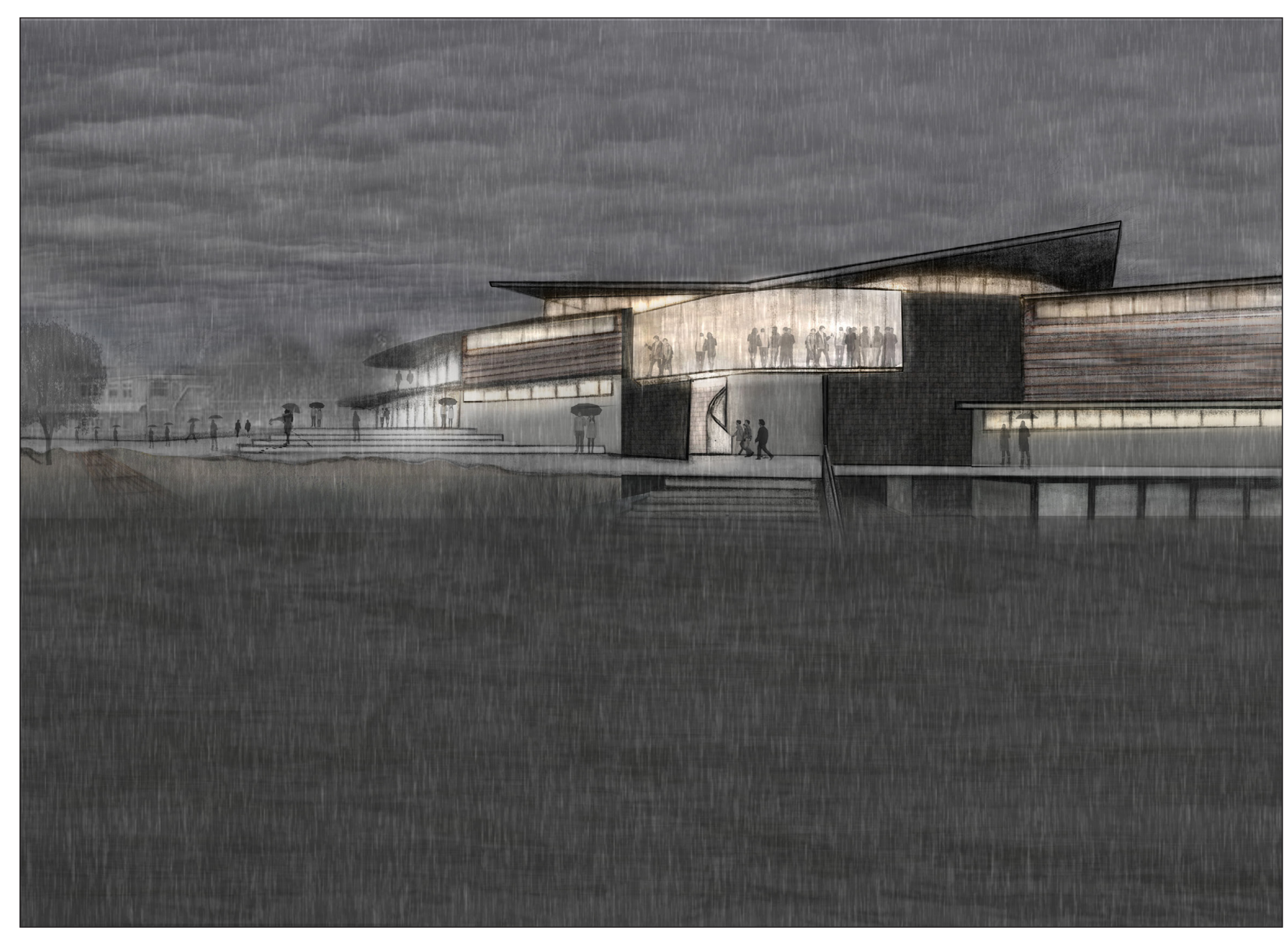

Figure 196: A view of the community centre at night from the river during high floods 
7.8 Architectural flood resilience principles

Flood resilience principles (scales, adaptability, variety and redundancy and feedback) were architecturally reinterpreted through the designing process to establish guiding resilience strengthening strategies. A diverse range of possibilities arise when these architectural resilience strategies are combined with previously detailed ecological and flood management resilience. The Architectural flood resilience principles establish guiding practical solutions on a building construction and site scale. The ecological, flood management and architectural resilience strategies allow a floodplain city's socio-spatial flood resilience to be strengthened. 


\section{Architectural flood resilience principles}

ADAPTABILITY

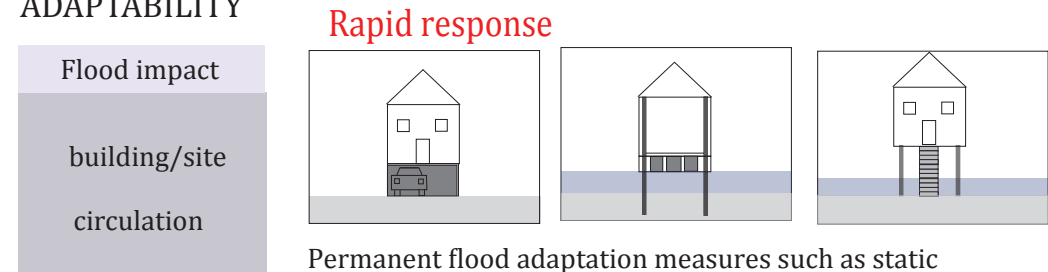

Permanent flood adaptation measures such as static elevation of living spaces on poles or above garages or amphibious construction reduce the adverse impacts of floods.

\section{Alternate circuits}

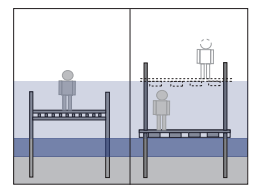

Static or amphibious cirulation pathways increase safety and enable greater pedestrian movement during and pos flood events.

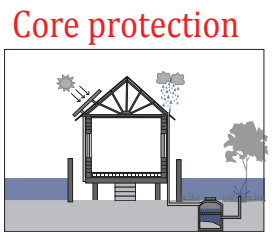

Permanent dry and wet flood proofing measures and alternate energy and back up services improves the adaptive capacity of the building to flooding and ensures vital needs services are available.

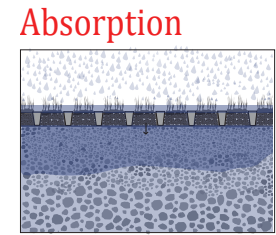

Artificial difussion channels can be achieved by

A variety of vegetation and solid

penetrabl structures dissipate and reduce the velocity of flood waters across a site. A perimetre of barriers spatially separated from the main load bearing structure protect the primary structure by collecting debris and reducing the velocity of flood waters.

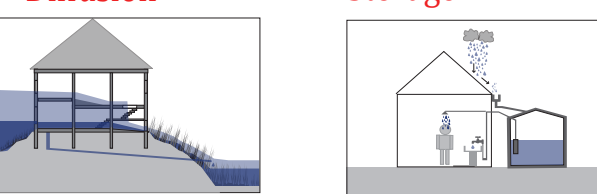

Above or below groun rainwater tanks made from concrete or steel positioning and gradienting exterior circulation pathways collect and store water and interior corridors to

re-direct flood waters across the site and through the building.
Permaeable surface membranes reduces run off volumes in urban areas by allowing water to penetrate below ground through natural filtration.
Nutrient \& waste recovery

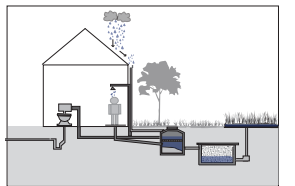

Grey water systems and rain water collection tanks allow water to be captured, stored, filtered and reused before being released back into the natural vegetative surroundings.

\section{FEEDBACK}

Weather event
precipitation
Flood duration

Response

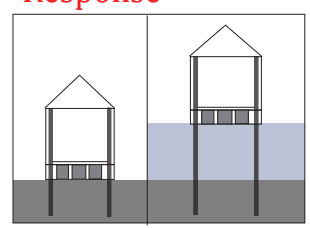

Construction technique and material selection ensure a structure is not adversly impacted upon by flooding. Construction methods, such as amphibious techniques, enable a structure to effectively respond and react to flooding.
Regeneration

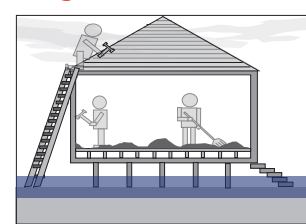

Cleaning and improving flood proofing solutions by applying knowledge of the recent flood event will improve the ability of the structure to cope with future floods.
Cooperative self regulation

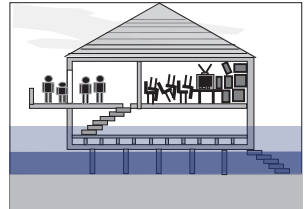

Moving furniture, appliances and important posessions above anticipated lood heights. Livable spaces on the second storey, equipt with flood kits and a flood evacuation plan.

Figure 197: The architectural flood resilience principles 
Analysis of the social and spatial impact that low, medium and high floods have on the structure and function of Maitland highlight the strengths and weaknesses of the city system. Key issues are able to be identified through the use of the resilience flood management matrix, with a social vulnerability profile of Maitland's residents providing greater insight. Strategies address Maitland's key issues of a building's suitability to flood conditions, disconnect between river and city, community connectivity and access to functional services and infrastructure. Strategies endeavor to strengthen the flood resilience of Maitland. A socio-spatial resilience network ensures strategies acting at the multiple scales of the city system remain functional, connected and accessible.

A variety of architectural strategies arise when the flood resilience principles of scale, adaptability, variety and redundancy and feedback are applied in a site specific floodplain context. Each intervention is focused at a specific scale but remains connected through the interlinking social and spatial realms.

At a resident : building and lot scale amphibious construction methods provide opportunities to enhance current wet and dry flood proofing measures, and improve resilience to large flood heights in which traditional methods are insufficient. Flexible service connections, alternate and renewable energy sources, and on site rainwater collection and filtration systems are measures which would further enhance socio-spatial flood resilience. Although it must be recognised this is an area that is currently receiving a large amount of attention within the architectural discipline, despite a lack of flood codes in the Building Code of Australia.
At a neighbourhood : urban street scale issues of residential connectivity and access to community services during flood inundation can be reduced through the provision of flood adapted pedestrian circulation. A range of static and amphibious strategies provide opportunities to strengthen and diversify urban relationships, whilst improving the everyday walkability of the urban realm. An example of this is with the flood adapted fence types, in which everyday barriers dividing private and public zones become essential artillery links connecting residents. The planning, implementation and user processes strengthen community relations and improve flood awareness and preparedness. A range of initiatives also increase redundancy within the system, reducing the likelihood of residents becoming isolated during flood events. A flood adapted pedestrian network improves community connectivity, allows for continued access to vital services, improves current inhibited evacuation processes and allows for a more hasty return to the city as floods begin to recede.

At a neighbourhood : urban levee scale transforming the levee into a recreational and informative community strengthening site provides a means to reconnect the city to the Hunter River. This in turn will increase awareness of the dynamic nature of the river and allow it to once again become an intrinsic part of Maitland's everyday functioning. Reintroducing native vegetative species to the site and rejuvenating the existing riverine ecosystem will not only improve the flood mitigating capabilities of the levee, but will also result in a return of once native wildlife to the area and further enhance the recreational green space for users. 
At a community : city services scale there is a need to decentralise and improve the adaptive capacity of infrastructure to flooding. Floods commonly result in the malfunction of sewerage systems, water and electricity mains, occasioning in residents being without vital services during and post flood events. Renewable and alternate energy sources need to be integrated into the public realm to reduce the adverse effects of infrastructure damage. There are opportunities for strategies to be autonomous with their everyday function. An example of this is seen in the amphibious bus shelters; flood safe temporary shelters, which provide rainwater collection tanks and alternate energy sources. Bus shelters continue to function as designated pick up points within a city enabling people to be transported to alternate locations during floods.

A community centre with relationships to a city's civic square enables it to not only become a central part of everyday community gathering, but ensure the site is also capable and practiced at hosting large numbers of people. This allows the community centre to serve additional community strengthening and supportive roles as a recognized flood emergency shelter. A flood emergency shelter must have the flood resilience principles integrated into the designing and construction process to ensure the building is sufficiently strengthened, correctly sited and appropriate water resistant materials used. This will ensure shelter, electricity, water, toilets, showers, food and cooking facilities are readily available for flood affected residents pre, during and post flooding. If functional redundancy is integrated into the functional, structural and service components of the building the probability that services will remain functional during and post flood events will be improved.
The decentralisation of services and infrastructure, elevating structures on high topographical land, fixed columns or utilising amphibious construction methods, will further improve the probability that key services remain functional and available to communities during periods of inundation. Interventions will further increase the everyday service availability of a city, whilst reducing the adverse effects of flooding. The interrelating ecological and flood management realms provide greater opportunities to diversify the architectural solutions employed, allowing a building to become more intrinsically connected to its environmental surrounds and thus achieve greater flood resilience.

Architecture fulfills a significant role in the strengthening of socio-spatial flood resilience at a city, urban, and residential scale. When the flood resilience principles guide the designing process innovative solutions that are unrestrained by conventional approaches begin to take shape. When the architectural resilience strategies are combined with resilience flood management and ecological strategies a rich tapestry of solutions begin to develop. The involvement of a range of disciplines in the analytical, decision making and implementation stages will further contribute to the strategy options and instigate pioneering thought as to how the resilience of floodplain cities and their occupants can be strengthened. Future risks and vulnerabilities of inhabiting flood prone land will be subsequently lessoned as cities and inhabitants continue to evolve to form symbiotic relationships between the social and spatial realms. 
"Innovation does not come about simply by evolution or by revolution. Creative and innovative design came about by resolution, that is by the human resolve to bring creativity and insight to improve on the standard ways of doing things"

(Ryan, 2010, pp. 269-270)

Figure1: Wagga Wagga, in NSW, Australia inundated by floods in 2012

[Photograph: Johnathan Samuels, Australia Correspondent]

Source: Sky News (2012). (http:I/news.sky.com/home/world-news/article/16182786) 


\subsection{CONCLUSION}

This chapter reflects on the motivation for this thesis, current shortcomings in the literature and the need for further investigation into how resilience can improve future floodplain inhabitation. This thesis proposes a series of new approaches to identify existing vulnerabilities and provides practical solutions to improve flood resilience; a resilience flood management matrix, a sociospatial resilience network and a new flood categorising approach. Architectural principles played a significant role in strengthening resilience at multiple scales when applied to the case study city of Maitland, NSW, Australia. 
This thesis arose from a concern for the future sustainability of floodplain inhabitation. Climate change, population growth and an aging population are intensifying flood exposure levels and the risks associated with residing on flood prone land. Yet, humans continue to enjoy residing near bodies of water. A new flood categorising approach based on specific structural and functional shifts that take place within a city system is needed. A low flood category is representative of anticipated flooding, which minimally impacts on the city function and social connectedness. A medium flood category corresponds to potentially hazardous flooding, in which the city must functionally adapt to being inundated and socially respond to escalating risk levels. A high flood category focuses on survival. The aims of a high category event are for city infrastructure to be minimally flood damaged, whilst inhabitants are evacuated to safe zones.

In this approach specific flood heights are not established, but rather changing community and city functional circumstances are addressed and adverse impacts reduced. This new flood categorising approach attempts to improve current confusions surrounding specific heights being proposed for annual probability flood occurrence rates. The new approach acknowledges what the probability method fails to address; the interrelating social, structural, functional and ecological components upon which floods differ in their adverse impact. A socio-spatial resilience network provides a means to incorporate this new flood categorising method into practice.

The network provides a means to connect the multiple scales that act simultaneously within a city, through reducing issues around urban connectivity and access to functional infrastructure during periods of inundation. The network nurtures new ways of viewing relationships between the structural, functional, ecological, and social components of floodplain cities. It promotes evolutionary philosophies, identifying the periodic need to reside on water. This in turn will decrease the need for evacuation, reduce flood damage and lessen the time frame of post flood social and structural recovery. A socio-spatial resilience network works to reduce the emotional, financial and physical damages sustained from floods.
Recentadvances in amphibious construction provide a means to strengthen traditional flood proofing measures and improve the flood resilience of individual buildings. But if architects focus exclusively at the residential scale, flood management practices will continue to concentrate on flood hydraulics at the regional and catchment scale. Such a process is likely to lead to a lack of investment in community resilience within the urban environment of floodplain cities. A new approach encompassing the multiple interacting social and spatial scales will enable a more holistic and resilient approach. Analysis of the strategies employed by ecosystems, which enable them to respond, adapt and continue to function when inundated by flooding provide guiding flood resilience principles: scales, adaptability, variety, redundancy and feedback. However, when viewing the resilience of cities the interrelationship between the ecological, the built and the social realms needs to be recognized to improve flood resilience strategies.

Resilience is a concept which continues to be refined. This thesis re-categorises existing flood management strategies based on flood resilience principles to establish a new resilience flood management matrix approach. The resilience flood management matrix can be used as an analytical method or as an holistic action-oriented tool. The matrix provides a means to site specifically investigate the strengths and weaknesses of existing flood management practices. Applying the resilience flood management matrix to the floodplains of Tonle Sap in the Lower Mekong River Basin, Cambodia, the Lower Ninth Ward, in New Orleans and Maasbommel, in the Netherlands several universal vulnerabilities were highlighted. A lack of community connection, emergency flood safe shelters and access to functional services inhibited the ability of floodplain inhabitants to achieve resilience when inundated by flood waters. Current flood probability categorization methods proved misleading and ineffective in guiding local plans to reduce flood exposure to the likely range of flood events, continuing to rely on the singular arbitrary one in one hundred anticipated annual exceedance probability event standard. 
Applying the resilience flood management matrix and socio-spatial resilience network approach to the Maitland case study identified significant existing vulnerabilities. Current Australian stormwater management standards address street gradients for floods up to a one in one hundred event, but many of the old streets in Maitland are without adequate above or below ground stormwater infrastructure. Back flooding of Maitland as a result of the current management plan reduces flood velocities and inundation rates of the CBD and residential zones, but causes parts of South and Central Maitland to flood prematurely. Back flooding also adversely effects circulation, as the main access roads into Maitland are cut by floodwaters well before levees are at risk of breaching. The city becomes isolated from surrounding townships, and is without a means to safely evacuate if floods continue to rise. Sidewalks are also inadequate in providing circulation paths for pedestrians from even low level inundation. This means that whilst the city is isolated its inhabitants become detached from neighbours and are unable to safely access the city's infrastructure.

The resilience flood management matrix and socio-spatial resilience network approach not only identifies weaknesses in current approaches to flood plain inhabitation, it suggests innovative solutions to improve our capacity to "live with floods". In Maitland city adapted pedestrian connections would enhance sociospatial resilience by allowing inhabitants to connect to surrounding communities and access key services during inundation. Elevated civic zones would enable residents to access a portion of the city that could remain functional during periods of flood inundation. Addressing vehicle access would be a clear priority for both evacuation and for support of resilient civic zones. The models provides insight as to where interventions are needed to strengthen flood resilience at a residential, an urban, and a city scale. A multi-scale socio-spatial network allows a means to incorporate ecological, flood management and architectural principles into a resilience strengthening cohesive approach.
The discipline of architecture is shown to play a significant role in strengthening the socio-spatial flood resilience at multiple scales of Maitland. A range of architectural resilience principles arise when new designed components are integrated within the city structure to strengthen a socio-spatial resilience network. Architecture provides a means to enhance the aesthetic qualities of flood management strategies to enrich everyday city function. The discipline of architecture plays an important role in addressing the complex relationships between structure, function, social values and inhabitation. A lack of technical expertise means this thesis is unable to test and quantify the effectiveness of design interventions in a variety of flood circumstances. Rather it aims to instigate further exploration and collaborative testing by experts in fields such as flood management, resilience, ecology, and architecture. Preplanning and implementation will facilitate the capacity of a city to maintain its basic structure and function during and post flooding. Post flood analysis enables inhabitants to continue to evolve and improve their ability to cope with floods, despite the inherent unpredictability of specific flood heights.

A resilience framework will allow flood management to be accessible to a range of disciplines, governments and flood prone inhabitants. It recognises the importance of a balanced bottom up and top down approach; top down strategies are implemented at a regional and city scale including, infrastructure, planning and policy initiatives; bottom up strategies arise at the residential and neighbourhood scale; whilst the urban scale fosters collaboration between governments and communities. It is through recognising the inter-scale relationships between social and spatial domains that a truly resilient management strategy can be conceived, designed and successfully implemented in a culturally appropriate and architecturally sound process. 


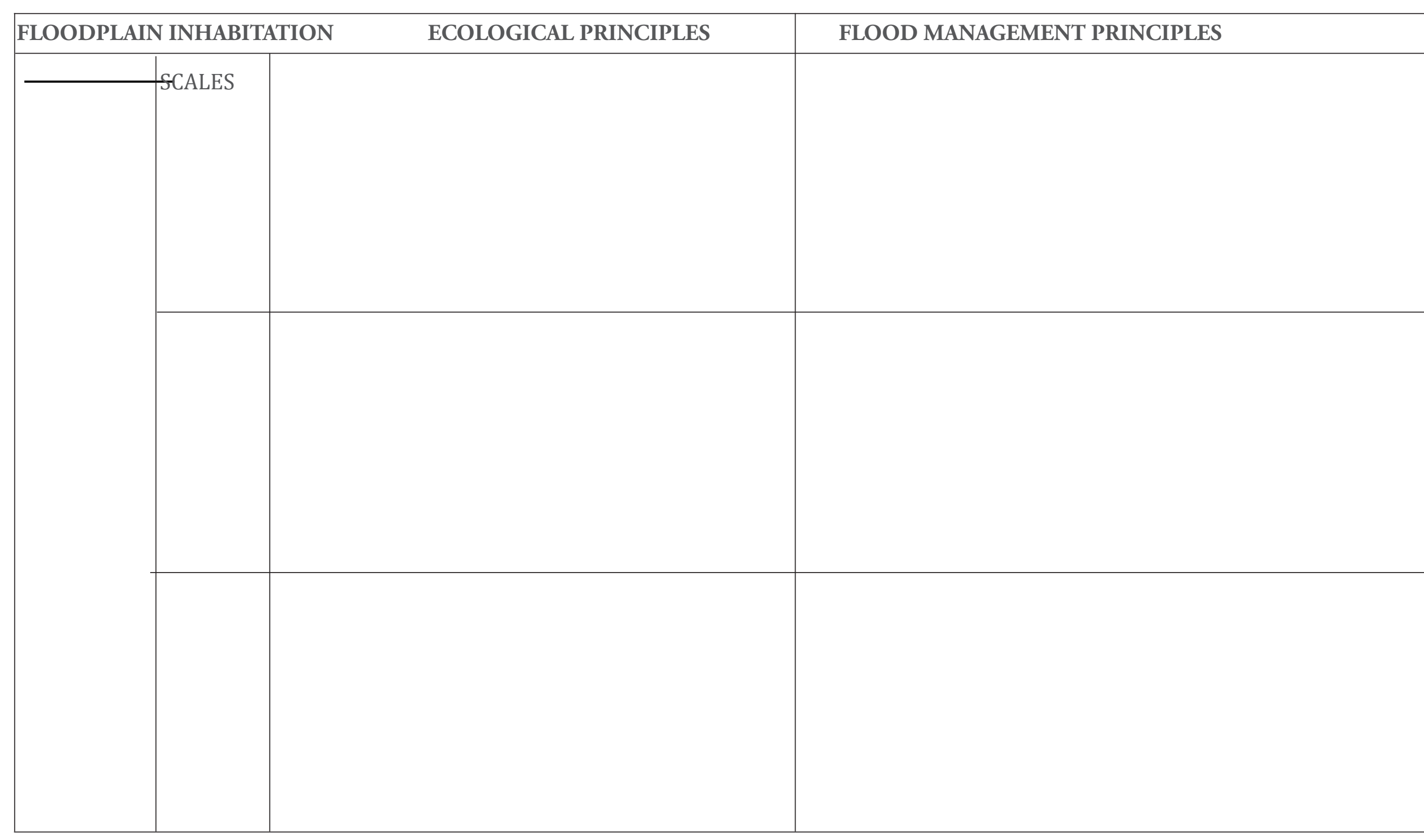




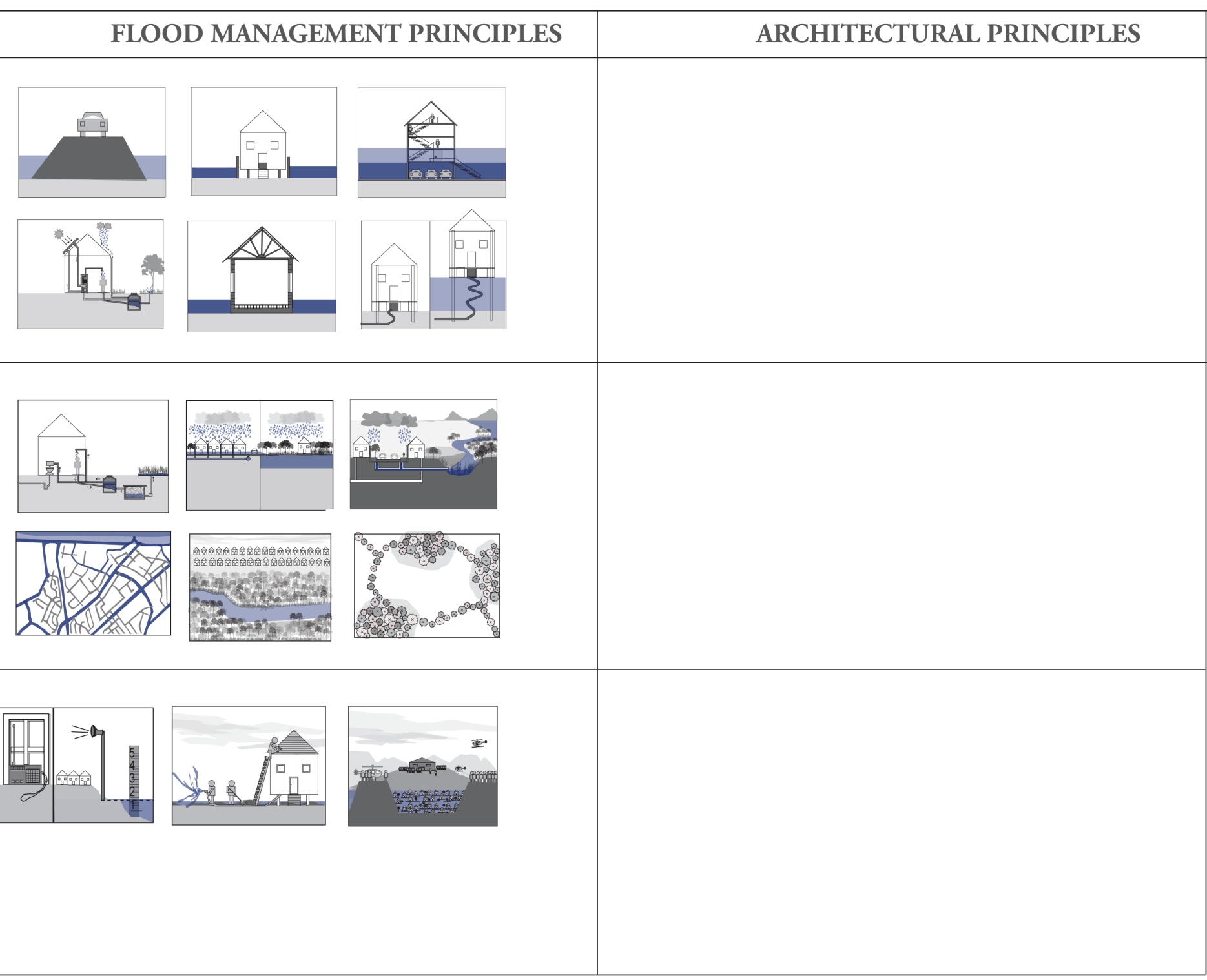

Figure 198: The floodplain, ecological, flood management and architectural flood resilience principles 
LIST OF FIGURES 
Figure 1: Wagga Wagga, in NSW, Australia inundated by floods in 2012

[Photographer: Jonathan Samuels, Australia Correspondent]

Samuels, J. (2012) Thousands Evacuated In Deadly Oz Floods. Sky News.

World News. Retrieved: March 5, 2012, from Sky News: http://news.sky. com/home/world-news/article/1618786

Figure2: The intersection of primary and the subsequent secondary themes of this thesis (p.19) [Author's image] (2011)

Figure 3: Floodplain, floodway and flood fringe (p. 22) [Author's image] (2011)

Figure 4: History of floodplain inhabitation (p.23)[Author's image] (2011)

Figure 5: Pile, stilt and raft housing construction (p.24)[Author's image] (2011)

Figure 6: Flood engineering structures, levees and rejuvenated ecosystems (p.26) [Author's image] (2011)

Figure 7: Land use policies and emergency management (p.27) [Author's image] (2011)

Figure 8: Exploring ecological impacts of flood management and land use (pp.28-29) [Author's image] (2011)

Figure 9: Exploring new resilience ways to inhabit floodplains (pp.30-31) [Author's image] (2011)

Figure 10: Ecosystem strategies that provide insight for resilience design and construction (p. 34) [Author's own table] (2011)

Adapted from Watson, D., \& Adams, M. (2011). Design for flooding. Architecture, landscape and urban design for resilience

to climate change. (p.258). New Jersey: John Wiley and Sons Inc.

Figure 11: Ecological flood resilience principles (p.35) [Author's image] (2011)
Figure 12: Maitland's Belmore Bridge flood gauge recordings for the June 2007 floods (p.36) [Table : Australian Bureau of Meteorology] Australian Government Bureau of Meteorology. (2012). The Queen's Birthday East Coast Low: 8-9 June 2007. Retrieved March 5, 2011, from Bureau of Meteorology: http://www.bom.gov.au/nsw/sevwx/facts/events/ june-07-ecl/el-8-9jun.shtml.desc.

Figure 13: The interacting scales of a catchment system (p.37) [Author's image] (2011)

Figure 14: Highlighting the socio-spatial resilience themes and existing methods of living on floodplains (p.39) [Author's image] (2011)

Figure 15: The interrelating flood resilience principles with flood management practices (p.44) [Author's image] (2011)

Figure 16: Flood characteristics diagrams (pp.48-49) [Author's image] (2011)

Figure 17: Social vulnerability and everyday key service diagrams (pp.50-51) [Author's image] (2011)

Figure 18: Refining the primary, secondary and tertiary themes of this thesis (p.52) [Author's image] (2011)

Figure 19: Flood adapted vegetation (p.54) [Author's image] (2011)

Figure 20: Engineered Flood Works (p.55) [Author's image] (2011)

Figure 21: Adaptive infrastructure (p.56) [Author's image] (2011)

Figure 22: Dry flood proofing (permanent/temporary) (p.57) [Author's image] (2011)

Figure 23: Wet flood proofing (permanent) (p.57) [Author's image] (2011)

Figure 24: Keystone species (p.58) [Author's image] (2011) 
Figure 25: Land zoning (p.59) [Author's image] (2011)

Figure 26: Water-resistant material (p.60) [Author's image] (2011)

Figure 27: Adaptive service connections (p.61) [Author's image] (2011)

Figure 28: Self-reliant subsystems (p.61)[Author's image] (2011)

Figure 29: Natural flow paths (p.62)[Author's image] (2011)

Figure 30: Meandering rivers (p.63)[Author's image] (2011)

Figure 31: Flood control channels (p.63)[Author's image] (2011)

Figure 32: Decentralisation (p.64)[Author's image] (2011)

Figure 33: Access and escape (p.65)[Author's image] (2011)

Figure 34: Connected street network (p.65)[Author's image] (2011)

Figure 35: Riparian buffers (p.66)[Author's image] (2011)

Figure 36: Green corridors (p.67)[Author's image] (2011)

Figure 37: Green infrastructure (vegetative components) (p.67)[Author's image] (2011)

Figure 38: Dense canopy layer (p.68) [Author's image] (2011)

Figure 39: Diversion channels (p.69) [Author's image] (2011)

Figure 40: Circulation paths (p.69) [Author's image] (2011)

Figure 41: Aquifers (p.70) [Author's image] (2011)
Figure 42: Dams and wet retention basins (p.71) [Author's image] (2011)

Figure 43: Dry retention basins (p.71) [Author's image] (2011)

Figure 44: Root structure and soil composition (p.72) [Author's image] (2011)

Figure 45: Rain garden (p.73) [Author's image] (2011)

Figure 46: Urban density (p.73)[Author's image] (2011)

Figure 47: Wetlands (p.74)[Author's image] (2011)

Figure 48: Stormwater management (p.75)[Author's image] (2011)

Figure 49: Grey water systems (p.75)[Author's image] (2011)

Figure 50: Predictive and reactive homeostasis (p.77)[Author's image] (2011)

Figure 51: Flood warning systems (p.77)[Author's image] (2011)

Figure 52: Speciation (p.78)[Author's image] (2011)

Figure 53: Ecological succession (p.79)[Author's image] (2011)

Figure 54: Restoration and renewal (p.79)[Author's image] (2011)

Figure 55: Hierarchical organization (p.80)[Author's image] (2011)

Figure 56: Emergency management (p.81)[Author's image] (2011)

Figure 57: Socio-spatial flood resilience themes of this thesis (p.82)[Author's image] (2011)

Figure 58: The resilience flood management matrix (p.84)[Author's image] (2011) 
Figure 59: Ecological and flood management resilience strategies (p.85)

[Author's image] (2011)

Figure 60: Lower Mekong River Basin, Cambodia (p.106) [Author's image] (2011)

Figure 61: Phumi Prek Pambana, Tonle Sap, Lower Mekong River Basin, Cambodia (p.106) [Author's image] (2011)

Figure 62: Resilience flood management matrix analysis of the Lower Mekong River Basin, Cambodia (p.107) [Author's image] (2011)

Figure 63: Urban and residential scale analysis of the Lower Mekong River Basin, Cambodia (p.108) [Author's image] (2011)

Figure 64: Lower Mekong River Basin street, building and site scale analysis (p.109) [Author's image] (2011)

Figure 65: Lower Ninth Ward, New Orleans, USA (p.110) [Author's image] (2011)

Figure 66: Lower Ninth Ward, New Orleans, USA (p.110) [Author's image] (2011)

Figure 67: Resilience flood management matrix analysis of the Lower Ninth Ward, New Orleans (p.111) [Author's image] (2011)

Figure 68: Urban and residential scale analysis of the Lower Ninth Ward, New Orleans (p.112) [Author's image] (2011)

Figure 69: Lower Ninth Ward street, building and site scale analysis (p.113) [Author's image] (2011)

Figure 70: West Maas en Waal, The Netherlands (p.114) [Author's image] (2011)

Figure 71: Maasbommel, West Maas en Waal, The Netherlands (p.114)

[Author's image] (2011)
Figure 72: Resilience flood management matrix analysis of Maasbommel, The Netherlands (p.115) [Author's image] (2011)

Figure 73: Urban and street scale analysis of the Maasbommel, The Netherlands (p.116) [Author's image] (2011)s

Figure 74: Maasmbommel street, building and site scale analysis (p.117) [Author's image] (2011)

Figure75: Maitland and the major town centres of the Lower Hunter Valley Region at 1:500,000 (p.124) [Author's image] (2011)

Figure 76: Maitland 1955 floods (p.125) [Photographer unknown]

Fairleigh, M \& Doherty, P. (2008). The Louth Park mob: memories come flooding back. (p.23). Maitland City Council.

Figure 77: Maitland 2007 floods (p.125) [Photograph:NSW SES]

The Australian Government Bureau of Meteorology. (2012). The Queen's Birthday East Coast Low: 8 - 9 June 2007. Retrieved March 5 , 2011, from Bureau of Meterology: http://www.bom.gov.au/nsw/sevwx/ f acts/events/june-07-ecl/e1-8-9jun.shtml\#desc.

Figure 78: Maitland City Council's riverside vision for Central Maitland (p.126) [Map:Maitland City Council]

City Plan Urban Design \& Maitland City Council.

(November 2009). Central Maitland Structure Plan. (p.45). Maitland: Maitland City Council.

Figure 79: The current car park site (p.126) [Author's photograph] (2011)

Figure 80: The Central Maitland Structure Plan (p.127)

[Map:Maitland City Council]

City Plan Urban Design \& Maitland City Council.

(November 2009). Central Maitland Structure Plan. (p.46).

Maitland: Maitland City Council. 
Figure 81: Topographic ground of Maitland (p.128) [Map:Maitland City Council] City Plan Urban Design \& Maitland City Council. (November 2009). Central Maitland Structure Plan. (p.31).

Maitland: Maitland City Council.

Figure 82: Impact of one in fifty and one in one hundred year floods on Maitland (p.128) [Map:Maitland City Council]

City Plan Urban Design \& Maitland City Council. (November 2009). Central Maitland Structure Plan. (p.30). Maitland: Maitland City Council.

Figure 83: Resilience flood management matrix analysis of Maitland, Australia (p.129) [Author's table] (2011)

Figure 84: The Maitland Floodways Scheme (p.131) [Author's image Adapted from a sign on the Maitland levee produced by the Hunter Central Rivers Catchment Management Authority (CMA) in partnership with the Department of Environment, Climate Change and Water and Maitland City Council. Viewed on site by the author and retrieved online March 7, 2011, from the CMA: http://www.hcr.cma.nsw.gov.au/articles/ news.asp?news.id $=208$.

Figure 85: 1955 Flood height marker 4.2m in Central Maitland (p.132) [Photograph: Chas Keys]

Keys, C. (2008). Maitland City on the Hunter. Fighting floods or living with them? Paterson, NSW, (p.20). Australia: Hunter-Central Rivers Catchment Management Authority.

Figure 86: Maitland topographic map (p.133)

[Author's image] (2011) ]

Constructed using information from the City Plan Urban Design \& Maitland City Council. (November 2009). Central Maitland Structure Plan. (p.31). Maitland: Maitland City Council.
Figure 87: The focus area of Maitland with the adjacent townships of Lorn and Morpeth (p.134, 150, 156, 172) [Author's image] (2011)

Figure 88: Existing land use zoning: a large portion of residential land is flood liable land. (p.134) [Map:Maitland City Council]

City Plan Urban Design \& Maitland City Council. (November 2009). Central Maitland Structure Plan. (p. 35). Maitland: Maitland City Council.

Figure 89: Assessment of heritage value: flood proofing standards were implemented in 1970 (p.134) [Map:Maitland City Council]

City Plan Urban Design \& Maitland City Council. (November 2009). Central Maitland Structure Plan. (p. 24). Maitland: Maitland City Council.

Figure 90: Existing land use: ownership and functional use influences flood proofing uptake. (p.135) [Map:Maitland City Council]

City Plan Urban Design \& Maitland City Council. (November 2009). Central Maitland Structure Plan. (p. 34). Maitland: Maitland City Council.

Figure 91: Existing building heights: locating living spaces above ground level reduces risk. (p.135) [Map:Maitland City Council]

City Plan Urban Design \& Maitland City Council. (November 2009). Central Maitland Structure Plan. (p. 33). Maitland: Maitland City Council.

Figure 92: Existing open space typologies: green zones function as dry retention basins. (p.135) [Map:Maitland City Council]

City Plan Urban Design \& Maitland City Council. (November 2009).

Central Maitland Structure Plan. (p. 36). Maitland: Maitland City Council.

Figure 93: Access and circulation: road widths determine the carrying capacity of flood waters. (p.135) [Map:Maitland City Council]

City Plan Urban Design \& Maitland City Council. (November 2009). Central Maitland Structure Plan. (p. 38). Maitland: Maitland City Council. 
Figure 94: Residential block densities (p.136) [Author's image] (2011)

Figure 95: Horseshoe Bend, Maitland (p.136) [Author's image] (2011)

Figure 96: Existing building construction types (p.136) [Author's image] (2011)

Figure 97: Surface materiality of circulation pathways (p.137) [Author's image] (2011)

Figure 98: Vegetation density (p.137) [Author's image] (2011)

Figure 99: Horseshoe Bend residential typologies (p.138) [Author's sketches] (2011)

Figure 100: Photos of houses within Horseshoe Bend, Maitland (p.139) [Author's photographs] (2011)

Figure 101: The main residential construction types of Maitland (p.139) [Author's sketches] (2011)

Figure 102: Examples of Maitland's heritage commercial district [Author's sketches] (2011)

Figure 103: The low, medium, high flood categorisation approach (p.145) [Author's diagram] (2011)

Figure 104: New strategies for Maitland's resilience flood management matrix (p.146) [Author's diagram] (2011)

Figure 105: The key issues influencing Maitland's flood vulnerability (pp.148-49) [Author's image] (2011)

Figure 106: Maitland's low flood category event (p.150, 156) [Author's image] (2011)

Figure 107: Maitland's medium flood category event (p.151, 158) [Author's image] (2011)
Figure 108: Maitland's high flood category event (p.151, 160) [Author's image] (2011)

Figure 109: Existing Maitland services and infrastructure (p.152) [Author's image] (2011)

Figure 110: Low flood impact on Maitland services and infrastructure (p.152) [Author's image] (2011)

Figure 111: Medium flood impact on Maitland services and infrastructure (p.153) [Author's image] (2011)

Figure 112: High flood impact on Maitland services and infrastructure (p.153) [Author's image] (2011)

Figure 113: The buildings at risk of being inundated in a low flood (p.157) [ Author's image] (2011)

Figure 114: Maitland's socio-spatial resilience network in low floods (p.157) [Author's image] (2011)

Figure 115: The buildings at risk of being inundated in a medium flood (p.158) [Author's image] (2011)

Figure 116: Maitland's socio-spatial resilience network in medium floods (p.158) [Author's image] (2011)

Figure 117: The buildings at risk of being inundated in a high flood (p.161) [Author's image] (2011)

Figure 118: Maitland's socio-spatial resilience network in high floods (p.161) [Author's image] (2011) 
Figure 119: Suitability of existing housing types to a range of flood heights (p.164-165)

[Author's image] (2011)

Figure 120: Suitability of amphibious construction to a range of flood types (p.165) [Author's image] (2011)

Figure 121: An architectural regeneration strategy (p.167) [Author's image] (2011)

Figure 122: Examples of existing amphibious construction techniques (p.168) [Author's collage] (2011)

Figure 123: An architectural rapid response strategy (p.168) [Author's image] (2011)

Figure 124: An amphibious retrofit of an existing Maitland residence (p.169) [Author's image] (2011)

Figure 125: Impact of low floods on residential and commercial buildings, roads, bus stops \& train stations (p.172)[Author's sketch] (2011)

Figure 126: Waterscapes design idea (p.174) [Author's sketch] (2011)

Figure 127: Circulation paths for a low flood category event (p.175) [Author's sketch] (2011)

Figure 128: Sketches exploring relationships between pedestrians, water, ecology and urban can be formed (p.175) [Author's sketch] (2011)

Figure 129: Sketches exploring the possibilities of pedestrian bridges (p.176) [Author's sketch] (2011)

Figure 130: Analysing the existing fence layout of Horseshoe Bend (p.177) [Author's sketch] (2011)
Figure 131: An architectural alternate circuit strategy (p.177) [Author's sketch] (2011)

Figure 132: Exploring possible amphibious and floating solutions to crossing flood inundated roads (p.178) [Author's sketch] (2011)

Figure 133: Exploring the amphibious construction solutions for a pedestrian crossing (p.179) [Author's sketch] (2011)

Figure 134: A 1:100 section of the amphibious pedestrian crossing design for High Street, Maitland (p.181) [Author's image] (2011)

Figure 135: Maitland City Council heritage fence type examples (p.182) Source: Maitland City Council (2011), p. 200

Figure 136: Retrofitting existing fence types to provide static elevated paths (p.182) [Author's image] (2011)

Figure 137: Photo of the existing Horseshoe Bend site (p.183) [Author's image] (2011)

Figure 138: Horseshoe Bend retrofit example of a statically elevated pedestrian pathway solution (p.183) [Author's image] (2011)

Figure 139: An existing back pathway in Horseshoe Bend (p.184) [Author's image] (2011)

Figure 140: A design example of a sculptural statically elevated fence within Horseshoe Bend (p.184) [Author's image] (2011)

Figure 141: An existing neglected vegetative space within Horseshoe Bend (p.184) [Author's image] (2011)

Figure 142: An example of a currently neglected vegetative zone within the urban landscape (p.184) [Author's image] (2011) 
Figure 143: A sectional perspective of a bottom up amphibious fence design example

(p.185) [Author's image] (2011)

Figure 144: The urban furniture that provides alternate pedestrian paths (p.186) [Author's image] (2011)

Figure 145: Exploring new urban amphibious pedestrian solutions (p.187) [Author's sketch] (2011)

Figure 146: A riverside view of the levee and buildings that align it (p.190) [Author's image] (2011)

Figure 147: A grass hill and main walkway along the top with trees distributed along it (p.192) [Author's sketches] (2011)

Figure 148: The single and double storey heritage buildings along the top of the levee (p.192) [Author's sketch] (2011)

Figure 149: Water infltrating the levee as informative urban water features (p.193) [Author's sketch] (2011)

Figure 150: Levee design exploration into social and structural function of the rivers edge (p.193) [Author's image] (2011)

Figure 151: Levee design exploration into informative sculptural elements (p.194) [Author's sketches] (2011)

Figure 152: Levee design exploration into level changes and the social functions (p.194) [Author's sketches] (2011)

Figure 153: Rejuvenated ecological zones (p.194) [Author's image] (2011)
Figure 154: Artificially terraced spaces define pathways for pedestrians and water (p.194) [Author's diagrams] (2011)

Figure 155: A macro scale 1:5,000 plan of the proposed Maitland levee design (p.195) [Author's diagrams] (2011)

Figure 156: An architectural diffusion strategy (p.196) [Author's image] (2011)

Figure 157: A view of the Maitland levee design from the Lorn side of the Hunter River. (p.197) [Author's photograph] (2011)

Figure 158: An architectural absorption strategy (p.198) [Author's image] (2011)

Figure 159: The Maitland levee design 1:10,000 plan highlighting Section A (p.198) [Author's photograph] (2011)

Figure 160: 1:200 Section A of the Maitland levee design (p.199)

[Author's image] (2011)

Figure 161: The Maitland levee design 1:10,000 plan highlighting Section B (p.200) [Author's image] (2011)

Figure 162: 1:200 Section B of the Maitland levee design (p.201) [Author's sketch] (2011)

Figure 163: The Maitland levee design 1:10,000 plan highlighting Section C (p.202) [Author's sketch] (2011)

Figure 164 1:200 Section C of the Maitland levee design (p.203)

[Author's sketch] (2011)

Figure 165: Initial urban shelter design thoughts (p.208) [Author's image] (2011) 
Figure 166: An architectural core protection strategy (p.208)

[Author's sketches] (2011)

Figure 167: Exploring retrofit opportunities of existing urban structures (p.209)

[Author's image] (2011)

Figure 168: An architectural response strategy (p.209) [Author's sketches] (2011

Figure 167: Bus shelter amphibious construction concepts (p.210)

[Author's images] (2011)

Figure 168: An existing Maitland bus shelter (p.211)

Author's photograph] (2011)

Figure 169: The impact of low floods on existing bus stops and train stations in Maitland (p.211) Author's image] (2011)

Figure 170: A sectional perspective of an amphibious bus shelter design for Maitland (p.212) Author's image] (2011)

Figure 171: An architectural storage strategy (p.212) [Author's image] (2011)

Figure 172: A sectional perspective of a Maitland barbeque shelter design (p.213) [Author's image] (2011)

Figure 173: Community centre location within Maitland (p.214)

[Author's diagram] (2011)

Figure 174: Existing pedestrian circulation on and around the site of the community centre (p.214) [Author's diagram] (2011)

Figure 175: Existing building footprints, main circulation paths and view shafts from the street to car park (p.214) [Author's sketch] (2011)
Figure 176: A view of the community centre from above the existing Maitland rectilinear buildings (p.215) [Author's sketch] (2011)

Figure 177: A view of the community centre from along the more natural ecological end of the levee (p.215)[Author's sketch] (2011)

Figure 178: A riverside view of the levee leading up into the community centre (p.215) [Author's image] (2011)

Figure 179: An architectural cooperative self-regulation strategy (p.215) [ Author's image] (2011)

Figure 180: 1:100 Community centre level two plan (p.216)

[Author's image] (2011)

Figure 181: 1:100 Community centre level one plan (p.217) [Author's image] (2011)

Figure 182: 1:100 Community centre level three plan (p.217) [Author's image] (2011)

Figure 183: The central circulation core with a grey water system viewing window (p.218) [Author's image] (2011)

Figure 184: A 1:500 longitudinal section of the rainwater collection \& grey water filtration system (p.219) [Author's image] (2011)

Figure 185: The community centre's grey water system and the levee's reed bed filtration system (p.219) [Author's image] (2011)

Figure 186: An architectural nutrient and waste recovery strategy (p.219)

[Author's image] (2011)

Figure 187: A plan highlighting one of the two canopy entrances (p.220) [Author's image] (2011) 
Figure 188: A transverse 1:1000 section of the community centre's canopy entrance

(p.220) [Author's image] (2011)

Figure 189: Beneath a canopy entrance to the civic square and community centre (p.220) [Author's image] (2011)

Figure 190: An exterior view of the community garden, outdoor amphitheatre area, community hall \& café at night (p.221) [Author's image] (2011)

Figure 191: A plan highlighting the outdoor amphitheatre (p.221) [Author's image] (2011)

Figure 192: The meeting and picnic area of the community centre functional interaction with high floods (p.222) [Author's image] (2011)

Figure 193: A longitudinal section of the community centre highlighting the impact of floods on the building (p.223) [Author's image] (2011)

Figure 194: An architectural buffering strategy (p223) [Author's diagram] (2011)

Figure 195: A view of the community centre from the levee's terracing spaces and reed bed filtration system (p.224) [Author's diagram] (2011)

Figure 196: A view of the community centre at night from the river during high floods (p.225) [Author's diagram] (2011)

Figure 197: The architectural flood resilience principles (p.227)

[Author's diagram] (2011)

Figure 198: The floodplain, ecological, flood management and architectural flood resilience principles (p.228) [Author's diagram] (2011) 
BIBLIOGRAPHY 
Adger, W. (2000). Social and ecological resilience: are they related? Progress in Human Geology, 24(3), 347-364.

Adger, W. (2006). Vulnerability. Global Environmental Change, 16(3), 268-281.

Adger, W., Hughes, T., Folke, C., Carpenter, S., \& Rockstrom, J. (2005, August 12). Social-ecological resilience to coastal disasters. Science, 309, 1036-1039.

Alexander, D. (1993). Natural disasters. Dordrecht, Netherlands: Kluwer Academic Publishers.

Allen, T., \& Starr, T. (1982). Hierarchy: perspectives for ecological complexity. Chicago: University of Chicago Press.

Anderies, J., Janssen, M., \& Ostrom, E. (2004). A framework to analyze the robustness of social-ecological systems from an institutional perspective. Ecology and Society, 9(1).

Ashley, R., Blanksby, J., Cashman, A., \& Newman, R. (2007). An adaptable approach to flood risk management for local urban drainage. Defra Flood and Coastal Erosion Risk Management Conference. York: University of York.

Ashley, R., Blanksby, J., Chapman, J., \& Zhou, J. (2007). Towards integrated approaches to increase resilience and robustness for the prevention and mitigation of flood risk in urban areas. In R. Ashley, S. Garvin, E. Pasche, A. Vassilopoulous, \& C. Zevenbergen (Eds.), Advances in urban flood management. (pp. 415-432). London: Taylor and Francis.

Ashley, R., Garvin, S., Pasche, E., Vassilopoulos, A., \& Zevenbergen, C. (2007). Advances in urban flood management. London: Taylor and Francis Group.
Asian Development Bank. (2011, November 7). Cambodia: replacing lost crops and cash-for-work schemes key to rebuilding lives after floods. Retrieved November 23, 2011, from Asian Development Bank. Fighting poverty in Asia and the Pacific: http://beta.adb.org/features/cambodia-replacing-lost-crops-and-cash-workschemes-key-rebuilding-lives-after-floods

Australian Government Bureau of Meteorology. (2012). The Queen's Birthday East Coast Low: 8 - 9 June 2007. Retrieved March 5, 2011, from Bureau of Meteorology: http://www.bom.gov.au/nsw/sevwx/facts/events/june-07-ecl/e1-8-9jun.shtml\#desc

Australian Government Geoscience Australia. (2011, December 7). Hazards: What is a flood? Retrieved January 24, 2012, from Geoscience Australia: http:// www.ga.gov.au/hazards/flood/flood-basics/what.html

Baker, J. (2007, June 10). Floodwaters will breach levee. Retrieved February 20, 2012, from The Sydney Morning Herald: http://www.smh.com.au/news/national/ floodwaters-will-breach-levee/2007/06/10/1181414117385.html

Baran, E., Starr, P., \& Kura, Y. (Eds.). (2007). Influence of built structures on Tonle Sap fisheries. Phnom Penh, Cambodia: Cambodia National Mekong Committee and the WorldFish Center.

Baskin, Y. (1998). The work of nature: how the diversity of life sustains us. Washington, D.C., USA: Island Press.

Beatley, T. (2009). Planning for Coastal Resilience. Best practices for calamitous times. Washington, USA: Island Press.

Bergsteiner, McInnes \& Rigby \& Maitland Council. (1977). Central Maitland: a study of its historic buildings and townscape. Maitland: Ray Millington Drafting Pty Ltd.

Berkes, F., Colding, J., \& Folke, C. (2002). Navigating social-ecological systems: building resilience for complexity and change. Cambridge: Cambridge University Press. 
Bewsher, D., \& Grech, P. (1997, May 6-9). A new approach to the development on planning controls for floodplains. 37th Annual Floodplain Management Conference: reducing the impact, pp. 1-11.

Bewsher, D., \& Grech, P. (1999). Redevelopment of flood prone areas. 40th Annual Conference, NSW Floodplain Management Authorities. NSW, Australia.

Bodin, P., \& Wiman, L. (2004, October 26). Resilience and other stability concepts in ecology: notes on their origin, validity and usefulness. ESS Bulletin, 2(2), pp. 3343.

Bolin, R., \& Standford, L. (1998). The Northridge earthquake: vulnerability and disaster. New York: Routledge.

Bowker, P. (May 2007). Flood resistance and resilience solutions: an R\&D scoping study. London: Department for Environment, Food and Rural Affairs Flood Management Division.

Brenson-Lazan, G. (2003). Groups and Social Resilience Building. Retrieved March 4, 2012, from West Hartford, CT, USA: Amauta International, LLC: http:// www. communityatwork.com/resilience/RESILIENCIAENG.pdf

Buckle, P. (2006). Chapter 6 Assessing social resilience. In D. Paton, \& D. Johnston, Disaster resilience. An integrated approach (pp. 88-104). Illinois, USA: Charles C Thomas Publisher.

Burkett, V., Wilcox, D., Stottlemyer, R., Barrow, W., Fagre, D., Baron, J., et al. (2005, December). Nonlinear dynamics in ecosystem response to climate change: case studies and policy implications. Ecological Complexity, 2(4), 357-394.

Capon, T., Parsons, M., \& Thoms, M. (October 2009). Floodplain ecosystems: resilience, value of ecosystem services and principles for diverting water from floodplains. Waterlines Report Series, Australian Government National Water Commission. Canberra: National Water Commission.
Carpenter, S., Walker, B., Anderies, J., \& Abel, N. (2001, June 6). From metaphor to measurement: resilience of what to what? Ecosystems, 4(8), pp. 765-781.

Chapin, F., Zavaleta, E., Eviner, V., Naylor, R., Vitousek, P., Reynolds, H., et al. (2000, May 11). Consequences of changing biodiversity. Nature, 405, pp. 234-242.

City of Maitland Floodplain Management Committee. (August, 1994). Determination of the flood standard report. Maitland City Council. Sydney: Webb, Mckeown and Associates Pty Ltd.

City Plan Urban Design \& Maitland City Council. (November 2009). Central Maitland Structure Plan. Maitland: Maitland City Council .

Crichton, D. (1999). The risk triangle. In J. Ingleton (Ed.), Natural disaster management (pp. 102-103). London: Tudor Rose.

Danby, M. (1971). Ganvie, Dahomey. In P. Oliver, Shelter in Africa (pp. 36-46). London: Barrie and Jenkins.

Dayton-Johnson, J. (2004, August). Natural disasters and adaptive capacity. OECD Development Centre working paper No. 237. Retrieved March 2, 2012, from OECD Development Centre : http://www.oecd.org/dataoecd/30/63/33845215.pdf

De Bruijn, K. (2005). Resilience and flood risk management: a systems approach applied to lowland rivers. DUP Science: Delft University Press.

De Bruijn, K., \& Klijn, F. (2010, November 5). Resilient flood risk management strategies. Retrieved November 23, 2011, from International Association of HydroEnvironment Engineering and Research: http://www.iahr.org/e-library/beijing proceedings/Theme_C/RESILIENT\%20FLOOD\%20RISK.HTML

Defra. (2004, July 29). Making space for water. Taking forward a new Government Strategy for flood and coastal erosion risk management in England. Retrieved April 23, 2011, from Department of Environment Food and Rural Affairs (Defra) Web site: http://archive.defra.gov.uk/environment/flooding/.../strategy-response1.pdf 
Dewar, R., \& Hardwick-Jones, R. (23 September 2010). Hunter River Branxton to Green Rocks flood study. Maitland City Council. Sydney, Australia: WMA Water.

Economic and social commission of Asia and the Pacific. (2010). Policy options for effective implementation of the Hyogo framework for action in Asia and the Pacific. Developing innovative strategies for flood-resilient cities. New York: United Nations.

Fairleigh, M., \& Doherty, P. (2008). The Louth Park mob: memories come flooding back. Maitland: Maitland City Council.

Fleischhauer, M. (2008). The role of spatial planning in strengthening urban resilience. In H. Pasman, \& I. Kirillov (Eds.), Resilience of cities to terrorist and other threats: learning from 9/11 and further research issues (pp. 273-298). Dordrecht, The Netherlands: Springer [online: www.springer.com].

Flesche, F., \& Burchard, C. (2005). Water house. London: Prestel Publishing.

Folke, C. (2006). Resilience: The emergence of a perspective for social-ecological systems analyses. Global Environmental Change, 16, 253-267.

Folke, C., Holling, C., \& Perrings, C. (1996, November). Biological diversity, ecosystems and the human scale. Ecological Applications, 6(4), 1018-1024.

Food and Agriculture Organization of the United Nations Mekong River Commission Secretariat. (March, 1999). Flood management and mitigation in the Mekong River basin. Ministry of Agriculture and Forestry of LAO P.D.R., Department of irrigation. Bangkok: Regional office for Asia and the Pacific (RAP) Publication

Foster, H. (1997). The Ozymandias Principles: thirty one strategies for surviving change. Victoria, Canada: Southdowne Press.

Godschalk, D. (1999). Natural hazard mitigation: recasting disaster policy and planning. Washington D.C.: Island Press.
Godschalk, D. (2003, August). Urban hazard mitigation: creating resilient cities. Natural Hazards Review, 4(3), pp. 136-143.

Godschalk, D., Beatley, T., Berke, P., Brower, D., \& Kaiser, E. (1999). Natural hazard mitigation: recasting disaster policy and planning. Washington DC, USA: Island Press.

Gregersen, H., Ffolliott, P., \& Brooks, K. (2007). Integrated watershed management connecting people to their land and water. Cambridge: Cambridge University Press.

Gregg, C., \& Houghteon, B. (2006). Natural hazards. In D. Paton, \& D. Johnston, Disaster Resilience. An integrated approach (pp. 19-39). Illinois, USA: Charles C Thomas .

Greiving, S., \& Fleischhauer, M. (2006). Spatial planning response towards natural and technological hazards. (P. Schmidt-Thome, Ed.) Natural and Technological Hazards and Risks Affecting the Spatial Development of European Regions, pp. $109-123$.

Gunderson, L., \& Holling, C. (2002). Panarchy: understanding transformations in humans and natural systems. Washington, D.C., USA: Island Press.

Gunderson, L., Allen, C., \& Holling, C. (Eds.). (2010). Foundations of ecological resilience. Washington DC: Island Press.

Gunderson, L., Holling, C., \& Light, S. (Eds.). (1995). Barriers and bridges to the renewal of ecosystems and institutions. New York, NY, USA: Columbia University Press.

Gunderson, L., Holling, C., \& Pritchard, J. P. (2002). Resilience of large-scale resource systems. In L. Gunderson, \& J. Pritchard (Eds.), Resilience and the behaviour of large-large scale systems. Washington, D.C., USA: Island Press. 
Haggett, P. (1972). Geography: A modern synthesis. New York: Harper and Row.

Handmer, J., \& Dovers, S. (1996, December). A typology of resilience: rethinking institutions for sustainable development. Industrial and Environmental Crisis Quarterly, 9(4), 482-511.

Harrison, B., \& Salt, A. (2006). Resilience thinking: sustaining ecosystems and people in a changing world. Washington DC: Island Press.

Heltberg, R., Siegel, P., \& Jorgensen, S. (2009, February). Addressing human vulnerability to climate change: toward a 'no regrets' approach. Global Environmental Change, 19(1), 89-99.

Henstra, D., Kovacs, P., McBean, G., \& Sweeting, R. (2004). Background paper on disaster resilient cities. Canada: Institute for Catastrophic Loss Reduction.

Holling, C. (1973). Resilience and stability of ecological systems. Annual review of ecology and systematics(4), 1-23.

Holling, C. (1991). The role of forest insects in structuring the boreal landscape. In H. Shugart, R. Leemans, \& G. Bonan (Eds.), A systems analysis of the global boreal forest (pp. 170-191). Cambridge: Cambridge University Press.

Holling, C. (1996). Engineering resilience versus ecological resilience. In P. Schulze (Ed.), Engineering within ecological constraints (pp. 31-44). Washington DC: National Academy Press.

Holling, C., Gunderson, L., \& Peterson, G. (2002). Sustainability and panarchies. In C. Holling, \& L. Gunderson (Eds.), Panarchy: Understanding transformations in human and natural systems (pp. 63-102). Washington DC, USA: Island Press.

Hunter Catchment Management Trust. (1998). The lower Hunter River: new knowledge - new opportunities. Newcastle: Department of Land and Water Conservation.
Hunter, C., \& Maitland City Heritage Group, N. (2000). Horseshoe Bend Maitland, historical study. Maitland: Maitland City Council.

Huq, S., \& Klein, R. (2003, December 1). Adapting to climate change: why and how. Retrieved November 16, 2011, from Science and Development Network: http:// www.scidev.net/en/policy-briefs/adapting-to-climate-change-why-and-how.html

Hurricane Katrina External Review Panel. (2007). The New Orleans hurricane protection system: what went wrong and why. Virginia: American Society of Civil Engineers.

Independent Levee Investigation Team. (31 July, 2006). An investigation of the New Orleans flood protection systems in hurricane Katrina on August 29, 2005. University of California, Civil and Environmental Engineering. [online\}: http:// www.ce.berkeley.edu/ - new_orleans/report/ .

International Federation of Red Cross and Red Crescent Societies. ( 30 September 2011). Information bulletin Cambodia: floods 2011. IFRC [online: www. ifrc.org].

International Federation of Red Cross and Red Crescent Societies (IFRC). (2009). World Disasters Report 2009: focus on early warning, early action. Satigny/ Vernie, Switzerland: ATAR Roto Presse.

Jonkman, S., Kok, M., \& Vrijling, J. (2008). Flood risk assessment in the Netherlands: a case study for dike ring south Holland. Risk Analysis, 28(5), $1357-$ 1373.

Kaplan, H. (2005). Chapter three Understanding the concept of resilience. In S. Goldstein, \& R. Brooks (Eds.), Handbook of resilience in children (pp. 39-47). Springer US. 
Kelly, D., \& Garvin, S. (2007). European flood strategies in support of resilient buildings. In R. Ashley, S. Garvin, E. Pasche, A. Vassilpoulos, \& C. Zevenbergen, Advances in urban flood management (pp. 339-358). London: Taylor and Francis Group.

Keys, C. (1991). Towards better practice: the evolution of flood management in NSW. Australian Disaster Conference (pp. 111-116). Canberra: New South Wales State Emergency Services.

Keys, C. (2008, October 31). Learning to tame deadly floods. Retrieved February 27, 2012, from The Maitland Mercury: http://www.maitlandmercury.com.au/news/ local/news/general/learning-to-tame-deadly-floods/1348388.aspx

Keys, C. (2008). Maitland. City on the Hunter. Fighting floods or living with them? Paterson, NSW, Australia: Hunter-Cantral Rivers Catchment Management Authority.

Keys, C. (2008). Making communities safer in times of flood: the story of the Floodplain Management Authorities of New South Wales. Wollongong: Floodplain Management Authorities of NSW.

Klein, R. (2003). Adaptation to climate variability and change: what is optimal and appropriate? In C. Giupponi, \& M. Schechter (Eds.), Climate change in the Mediterranean: socio-economics of impacts, vulnerability and adaptation (pp. 32-50). Cheltenham, UK: Edward Elgar.

Klein, R., Nicholls, R. J., \& Thomalla, F. (2004, February 10). Resilience to natural hazards: How useful is this concept? Environmental Hazards, 5, pp. 35-45.

Levin, S. (1999). Fragile Domain, complexity and the commons. Cambridge : Perseus.

Ludwig, D., Jones, D., \& Holling, C. (1978, February). Qualitative analysis of insect outbreak systems: The spruce budworm and forest. Journal of Animal Ecology, 47(1), pp. 315-332.
Maitland City Council. (2011, December). Maitland Development Control Plan Part C Design Guidelines. Retrieved January 25, 2012, from Maitland City Council: http://www.maitland.nsw.gov.au/UserFiles/File/Part\%20C\%20Design\%20 Guidelines\%202011.pdf

Maitland City Council \& WMA Water (September, 2010) Hunter River Branxton to Green Rocks Flood Study. Retrieved July 20, 2011, from Maitland City Council: http://www.maitland.nsw.gov.au/UserFiles/File/FloodStudy2010/ TextBranxtontoGreenRocksFloodStudySept2010.pdf

Make It Right Campaign. (2009). Our work and progress. (MGXLAB) Retrieved November 20, 2011, from Make it right. Helping to rebuild New Orleans' Lower 9th Ward: http://www.makeitrightnola.org/index.php/work_progress/\#

Marschke, M., \& Berkes, F. (2006). Exploring strategies that built livelihood resilience: a case from Cambodia. Ecology and Society, 11(1), 42-58. [online] URL: http://www.ecologyandsociety.org/vol11/iss1/art42/]

Mekong River Commission. (29-30 July, 2010). Decoding the development scenarios and strategy for basin development: what does the future hold? 3rd Regional Stateholder Forum on the Mekong River Basin Development Plan (pp. 1-59). Vientiane, Lao PDR: Mekong River Commission.

Merz, B., Hall, J., Disse, M., \& Schumann, A. (2010, March 16). Fluvial flood risk management in a changing world. Natural Hazards and Earth Systems Sciences, 10, pp. 509-527.

Mileti, D. S. (1999). Disasters by design: a reassessment of natural hazards in the United States. Washington DC: Joseph Henry Press.

MRCS/WUP-FIN. (2007). Hydrological, environmental and socio-economic modelling tools for the lower Mekong basin impact statement. Mekong River Commission, Water Utilisation Programme and Finnish Environment Institute Consultancy Consortium. Vientiane: Lao PDR. 
Muller, M. (2007, April). Adapting to clmate change: water management for urban resilience. Environment and Urbanisation, 19, 99-113.

Mumford, L. (1938). The culture of cities. New York: Harbour, Brace and Company.

Nillesen, A., \& Singelenberg, J. (2011). Amphibious housing in the Netherlands: architecture and urbanism on the water. Rotterdam: NAi Publishers.

Nordenson, G., Seavitt, C., \& Yarinsk, A. (2010). On the water. Palisade Bay. Zeppelinstrasse, Germany: Hatje Cantz Verlag.

Nuorteva, P., Keskinen, M., \& Varis, O. (2010). Water livelihoods and climate adaptation in the Tonle Sap Lake area, Cambodia: learning from the past to understand the future. Journal of Water and Climate Change, 87-101.

Odum, E. (1969, April 18). The strategy of ecosystem development. Science, 164, pp. 262-270.

Oliver, P. (2003). Dwellings. The Vernacular house world wide. New York: Phaidon Press.

O'Neil, R., DeAngelis, D., Waide, J., \& Allen, T. (1986). A hierarchical concept of ecosystems. Princeton, N.J.: Princeton University Press.

Oosthoek, K. (2006). Dutch river defences in historical perspective. Retrieved 1124 , 2011, from Environmental History Resources: http://www.eh-resources.org/floods. html

Osaka, N. (2004). Urban diagnosis and integrated disaster risk management. Journal of Natural Disaster Science, 26(2), 49-54.

Paton, D. (2006). Chapter 1 Disaster resilience: building capacity to co-exist with natural hazards and their consequences. In P. Douglas, \& D. Johnston, Disaster resilience. An integrated approach (pp. 3-10). Illinois, USA: Charles C Thomas.
Paton, D., \& Johnston, D. (2006). Disaster Resilience. An Integrated Approach. Illinois, USA: Charles C Thomas Publisher.

Pelling, M. (2003). The vulnerability of cities: natural disasters and social resilience. London: Earthscan Publications.

Peterson, G., Alen, C., \& Holling, C. (1998). Ecological resilience, biodiversity and scale. Ecosystems, 1, 6-18.

Pimm, S. (1984). The complexity and stability of ecosystems. Nature(307), 321326.

Poff, L. (2002, November 10). Ecological response to and management of increased flooding caused by climate change. The Royal Society, 360, 1497-1510.

Proverbs, D., Brebbia, C., \& Penning-Roswell, E. (Eds.). (2008). Flood recovery, innovation and response. Southampton, UK: WIT Press publishers.

Rigby, T. (February 2007). Floodplain Development Manual (NSW) A document in need of review. Floodplain Management Authority NSW Conference (pp. 1-7). Gunnedah: FMA NSW.

Rinne, K., Bloom, E., Cambra, J., \& Goodnight, C. (2009). Architecture for a hybrid landscape: proposals for the California delta. San Francisco, USA: California College of the Arts.

Ryan, Z. (2010). Building with water: concepts, typology, design. Basel: Birkhauser.

Safety House Australia Inc. (n.d.). The safety house program. Retrieved May 26, 2011, from Childsafety Australia: http://www.childsafetyaustralia.com.au/ community/safetyhouses/safetyhouses.htm 
Samules, P., Klijn, F., \& Dijkman, J. (2006, May 8). An analysis of the current practice of policies on river flood risk management in different countries. Irrigation and Drainage(55), 141-150.

Sapirstein, G. (2006). Social resilience: the forgotten dimension in disaster risk reduction. Jamba Quarterly Bulletin of the African Center for Disaster Studies, 1(1), 54-63.

SCARM Report 73. (2000). Floodplain management in Australia: best practice principles and guidelines. Standing Committee on Agriculture and Resource Management, Agriculture and Resource Management Council of Australia and New Zealand. Collingwood, Victoria: CSIRO Publishing.

Schulze, E., \& Mooney, H. (1993). Biodiversity and ecosystem function. Berlin , Germany: Springer.

SCOPE 60. (2002). Resilience and the behavior of large-scale systems. (L. a. Gunderson, Ed.) Washington DC, USA: Island Press.

Shellshear, T. a. (1987). High Street study Maitland, N.S.W.: an architectural assessment of the commercial buildings of High Street. Maitland, N.S.W., Australia: Maitland City Council.

Smit, B., Pilifosova, O., Burton, I., Challenger, B., Huq, S., Klein, R., et al. (2001). Adaptation to climate change in the context of sustainable development and equity. (J. McCarthy, O. Canziani, \& N. Leary, Eds.) Third Assessment Report of the Intergovernmental Panel on Climate Change, pp. 877-912.

Smith, D. (1998, Winter). Urban flood damage under greenhouse conditions: what does it mean for policy. Australian Journal of Emergency Management, 13(2), 56-61.

Steinberg, P., \& Shields, R. (2008). What is a city?: rethinking the urban after hurricane Katrina. Athens, Georgia: University of Georgia Press.
Szollosi-Nagy, A., \& Zvenbergen, C. (2005). Urban flood management measures. London: Taylor and Francis Group.

The National Flood Risk Advisory Group. (2008, November). Flood risk management in Australia. The Australian Journal of Emergency Management, 23(4), $21-27$.

The Resilience Alliance. (2002, October 29). Resilience. Retrieved July 15, 2011, from Resilience Alliance : http://www.resalliance.org/index.php/resilience

Tilman, D. (1999, July). The ecological consequences of changes in biodiversity: a search for general principles. Ecology, 80, pp. 1455-1474.

Tilman, D., \& Downing, J. (1994). Biodiversity and stability in grasslands. Nature(367), 363-365.

Timmerman, P. (1981). Vulnerability, resilience and the collapse of society: A review of models and possible climate applications. Canada: Institute for Environmental Studies, University of Toronto.

Toubier, J., \& White, I. (2007). Sustainable measures for flood attenuation: sustainable drainage and conveyance systems SUDACS. In R. Ashley, S. Garvin, E. Pasche, A. Vassilopoulos, \& C. Zevenbergen, Advances in urban flood management (pp. 13-28). London: Taylor and Francis Group.

UNISDR. (2009, May). 2009 UNISDR Terminology on Disaster Risk Reduction. Retrieved March 13, 2012, from United Nations International Strategy for Disaster Reduction: http://www.unisdr.org/files/7817_UNISDRTerminologyEnglish.pdf

United Nations (2010). Policy options for effective implementation of the Hyogo framework for action in Asia and the Pacific. Developing innovative strategies for floodresilient cities. United Nations, New York, Economic and Social Commission for Asia and the Pacific. Thailand: United Nations Publication. 
Upton, D. (2006). Chapter 19 Understanding New Orlean's architectural ecology. In E. Birch, \& S. Wachter (Eds.), Rebuilding urban places after disaster (pp. 275287). Philadelphia, Pennsylvania, USA: University of Pennsylvania Press.

von Bertalanffy, L. (1968). General systems theory : Foundations, development, applications. New York: Braziller.

Walker, B. (1995, August). Conserving biological diversity through ecosystem resilience. Conservation Biology, 9(4), pp. 747-752.

Walker, B., Carpenter, S., Anderies, J., Abel, N., Cumming, G., Janssen, M., et al. (2002). Resilience management in social-ecological systems: A working hypothesis for a participatory approach. Conservation Ecology 6(1):14, [online] URL:www. consecol.org/vol6/iss1/art14.

Walker, B., Holling, C., Carpenter, S., \& Kinzig, A. (2004). Resilience, adaptability and transformability in social-ecological systems. Ecology and Society, 9(2)(5), [online] URL: http://www.ecologyandsociety.org/vol9/iss2/art5.

Walker, B., Ludwig, D., C.S, H., \& Peterman, R. (1986). Stability of semiarid savanna grazing systems. Journal of Ecology(69), 473-498.

Walsh, B., \& Archer, C. (2007). Maitland on the Hunter (2nd Edition ed.). Tocal, Paterson, Australia: CB Alexander Foundation .

Ware, S., Raxworthy, J., Weller, R., Russell-Clarke, J., \& Meyer, B. a. (2011). Sunburnt, Landscape Architecture in Australia. Amsterdam: SUN architecture Publishers.

Watson, D., \& Adams, M. (2011). Design for flooding. Architecture, landscape and urban design for resilience to climate change. New Jersey: John Wiley and Sons Inc.
Wisner, B. (2004). Assessment of capacity and vulnerability. In G. Bankoff, G. Frerks, \& D. Hilhorst (Eds.), Mapping vulnerability: Disasters, development and people (pp. 194-205). London: Earthscan.

Wolsink, A. (2006). River basin approach and integrated water management: governance pitfalls for the Dutch space-water adjustment management principle. Geoforum, 37(4), 473-487.

Zvenbergen, C., Veerbeek, W., Gersonius, B., \& van Herk, S. (2008). Challenges in urban flood management: travelling across spatial and temporal time scales. Flood Risk Management , 1, 81-88. 


\section{APPENDIX: SOCIO-SPATIAL FLOOD RESILIENCE PRINCIPLES}




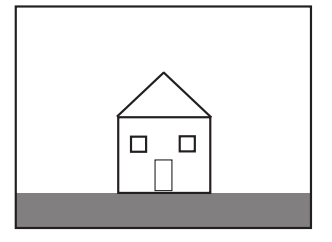

Slab on grade construction

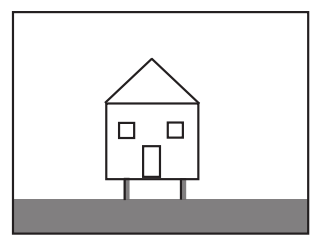

Pile or post construction

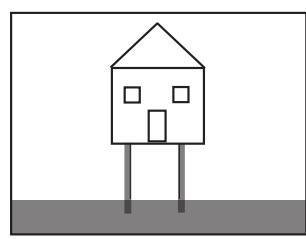

Stilt house construction

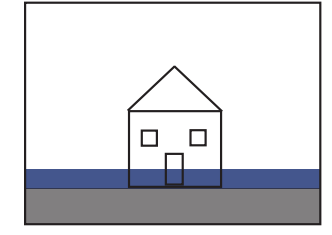

Flood prone construction

Suitability improved through water resistant material use e.g. concrete. Not suitable for floods.
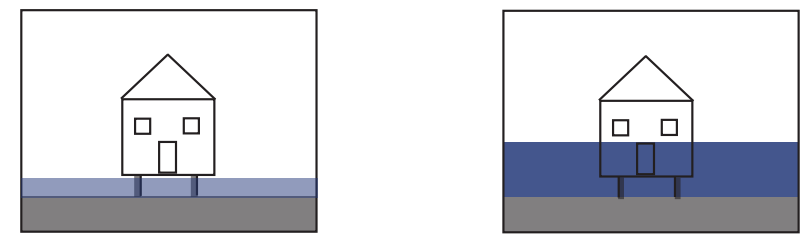

Minimal flood proofing measure

Low flood suitability improved through the use of marine grade timber Not suitable for medium flood velocities and depths.
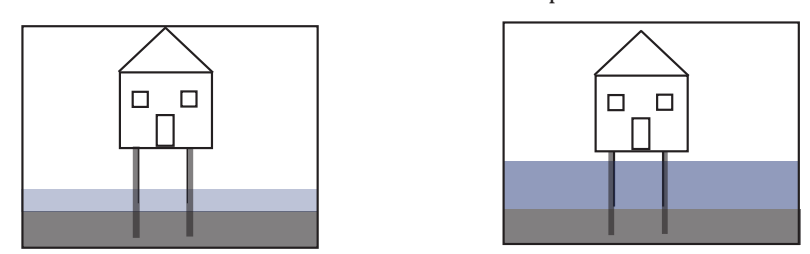

Medium flood proofing measure

High flood suitability improved through water resistant material use and stilts extending deep below ground. Not suitable for high velocity floods.

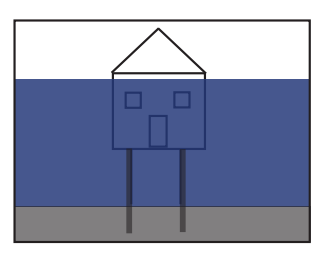




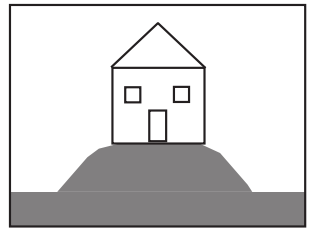

Houses raised on earth mounds

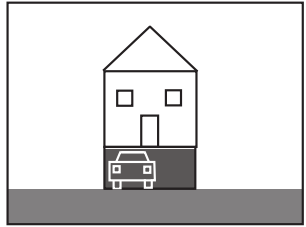

Living spaces raised above garages

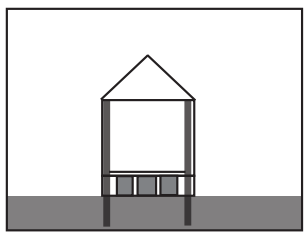

Amphibious construction

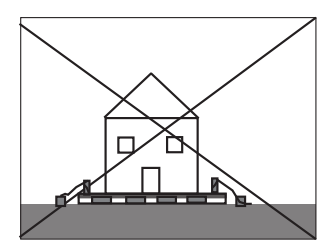

Floating construction
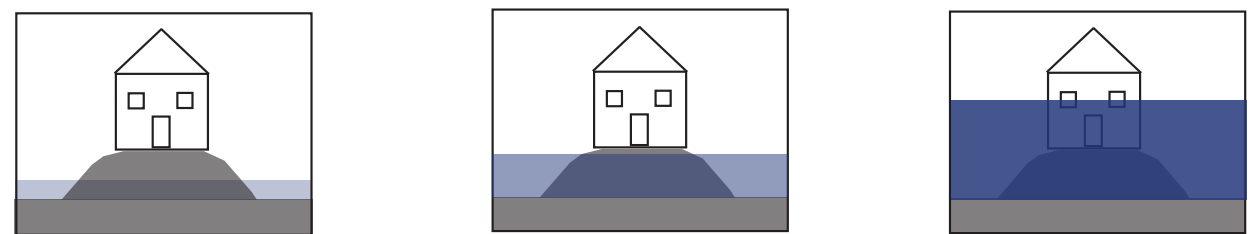

Medium flood proofing measure

High flood suitability improved with greater topographical height.

Not suitable for large flood heights.
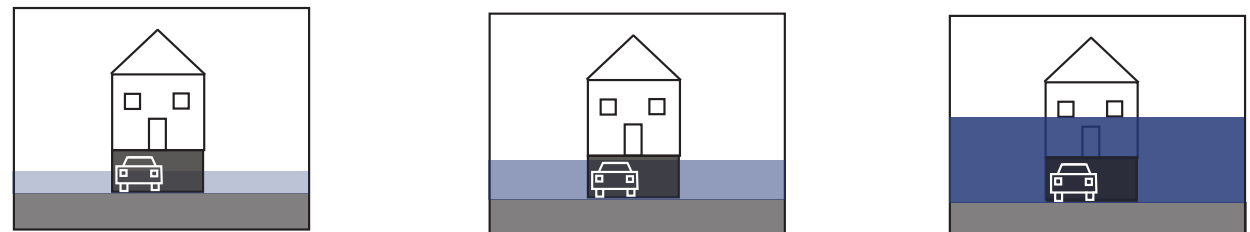

Medium flood proofing measure

High flood suitability if water resistant materials are used and valuables kept above garages.

Not suitable for large flood heights.
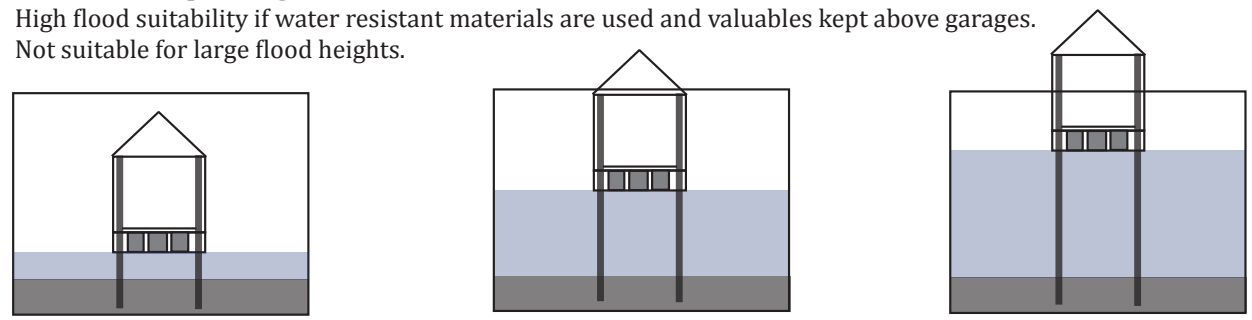

High flood proofing measure

Suitable for varying flood heights if water resistant materials are used e.g. steel, timber, light weight concrete Not suitable for high velocity floods and large wave heights.
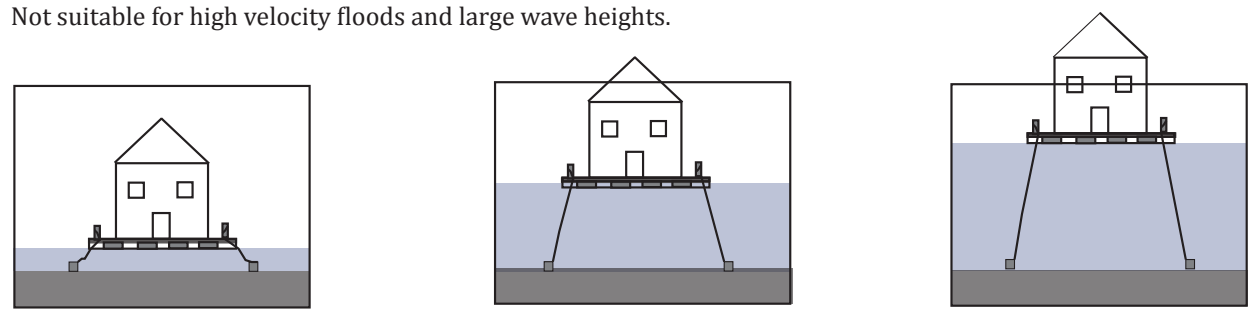

High flood proofing measure

environments improved with marine grade and treated timber use Not suitable for high velocity floods with large wave heights. 


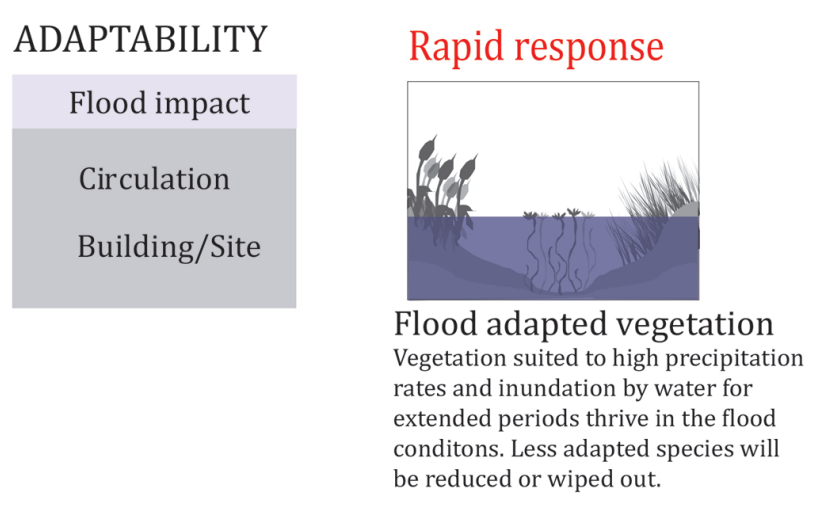

Alternate circuits

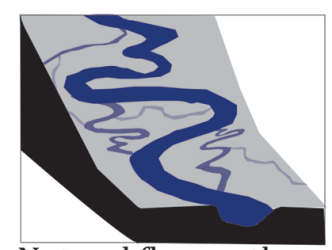

Natural flow paths Flood waters are naturally diverted into a variety of flow paths includin rivers, streams and creeks. These transport and release water into oceans.

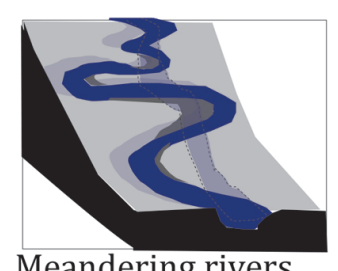

Meandering rivers

Accumulated sedimentation in the river reduces the channel depth and width leading to water overtopping the banks. Alternate water pathways are established during flood events.
VARIETY \& REDUNDANCY

Flooding issues

Debris

Water velocity

Water volume

Water quality

FEEDBACK

Weather even

Precipitation

Flood duration

\section{Buffering}

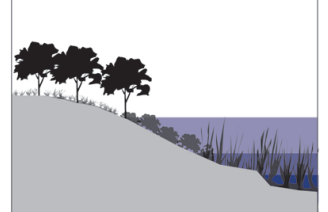

Riparian buffers Dense native vegetative areas which run adjacent to the river comprise of three zones. A variety of vegetation serves to reduce the velocity of floodwaters and its adverse impact.

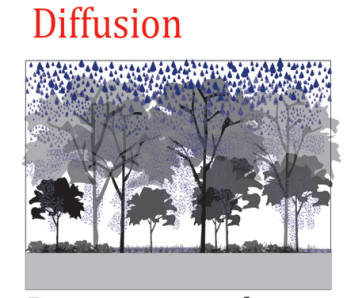

Dense canopy layer Dense foilage dissipates rainwater, reducing its velocity and spreading it over a larger surface area to improve absorption rates.
Storage

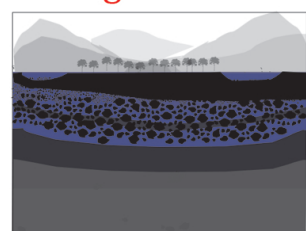

Aquifers

Naturally ocurring

underground water storage areas. Water bearing permeable rock, sand and gravel allow water to penetrat down to the bed rock.

\section{Absorption}

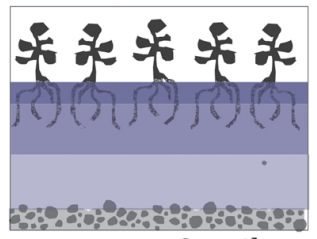
Root structure \& soil composit
Plant root structures influence the depth water penetrates. The soil particle density of sand, gravel and rock content influences the transmission of water. Soil moisture conten determines the amount of water that can be absorbed before saturation point is reached.
Core protection

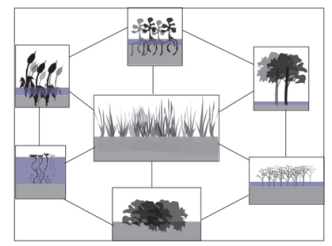

Keystone species

They are the system drivers who provide vital functional services. All species of an ecosystem are dependent on the
Response

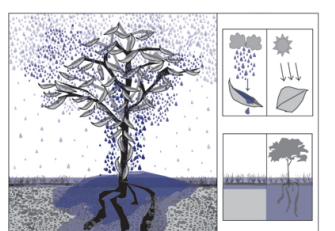

Predictive \& reactive homeostasis It allows species of an ecosystem to predict and react to flooding. It includes evolved functions that allow species to predict and benefit from seasonal variation coupled with physiological reactions that mediate a species response and adaption to variables.

\section{Regeneration}

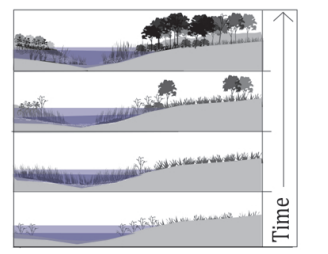

Ecological succession The process in which a community progressively transforms itself over time in an effort to achieve a stable ecosystem suited to the environmental conditions.

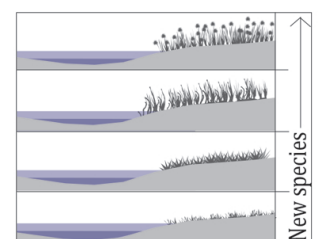

Speciation

An evolutionary process in which new biological species arise. These species are more suited to the unique local environmenta and ecosystem conditions.
Cooperative self regulation

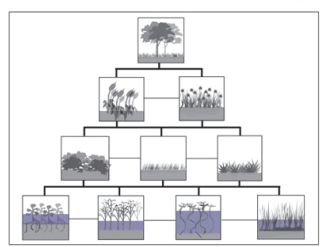

Hierarchical organisation Hierarchically divides ecosystems into subsystems including physical, chemical and biological processes. These interact with the individual and collective species to maintain ecosystem functioning during flooding.
Nutrient \& waste recovery

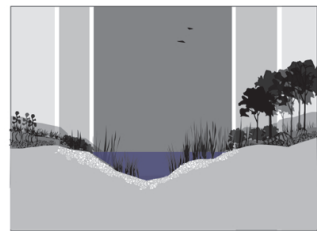

pecific plant and wildife species create productive nutrient fillled boundary between the terrestrial-aquatic environments. ters are stored, absorbed, filtered and leased back into the hydrological system. 


\section{ADAPTABILITY}

\section{Rapid response}

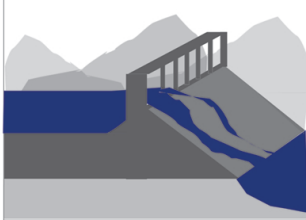

Engineered flood works A diversity of preventative structures hold back, store and divert floodwaters.

\section{Alternate circuits}

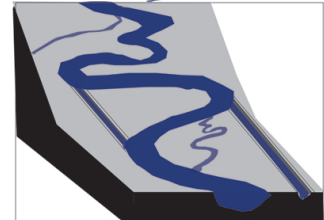

Flood control channels Artificial paths divert and transport floodwaters downstream from the high flood zone.

\section{Core protection}

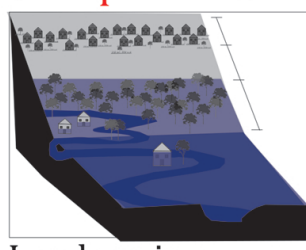

\section{Land zoning}

Determines, residential,

commercial, recreational and

vegetative zones. Limiting the adverse impact of floods on a cities inhabitants.

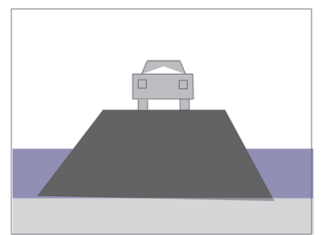

Adaptive infrastructure Infrastructure which is able to compensate for or adapt to rising flood waters.

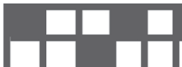 .}

Decentralisation Separating and diversifying infrastructural services reduces the likelihood of floods damaging all service providers.

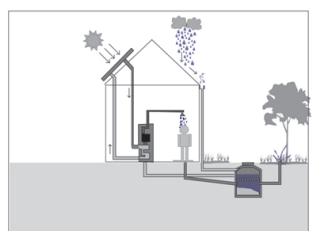

Self reliant subsystems Alternate energy, grey water systems and rainwater tanks provide services not reliant on the water mains and electricity grid.

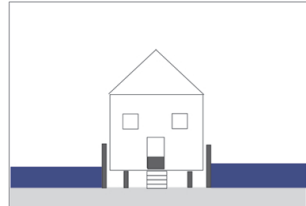

Dry flood proofing (permanent/temporary) Measures prent flosures prevent penetrating a building up to a design flood level (DFL).

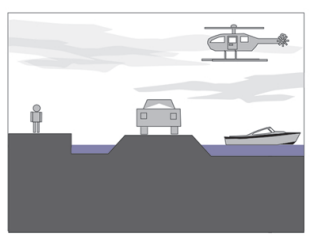

Access and escape A diversity of flood adapted roads and transport options facilitate evacuation processes.

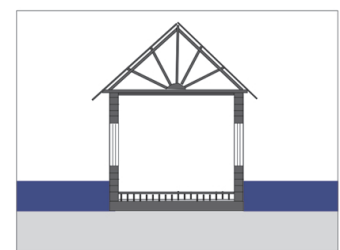

Water-resistant material Materials which maintain their structural integridy when inundated by water.

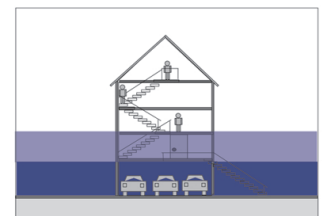

Wet flood proofing (permanent)

Measures ensure wate penetration minimally impacts upon the interio of buildings.

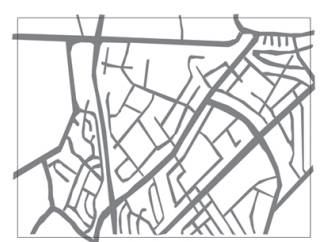

Connected street network A variety of options for

vehicular and pedestrian

movement allows inhabitants

to be connected during floods.

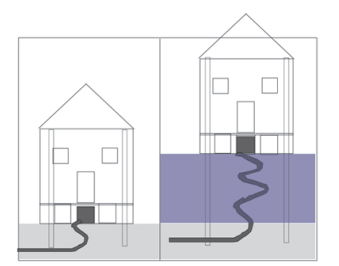

Adaptive service connections Alternate connections, mechanims and storage facilities allow key services to continue to function during and post floods. 
Buffering

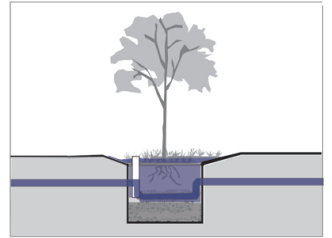

Rain garden

Vegetation in a pit filled with gravel and sandy soil substrate

to facilitate rapid absorption

storage of surface water runoff.

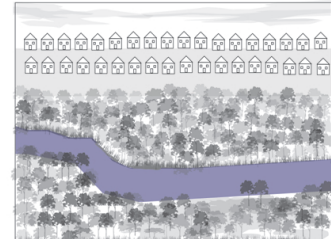

Green corridors

Vegetative zones that slow the velocity of floodwaters and are absorption zones for urban surface runoff.

Diffusion

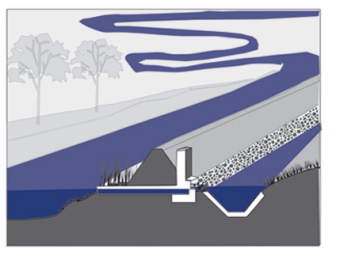

Diversion channels Divert excess water from the main river channel into secondary water channels. Reducing water volume in the main channnel

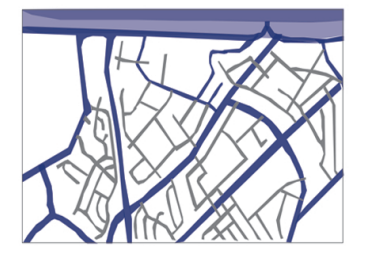

Circulation paths

Streets are positioned to become water channels during floods. Re-directing floodwaters into stormwater drains and secondary green space zones.

\section{Absorption}

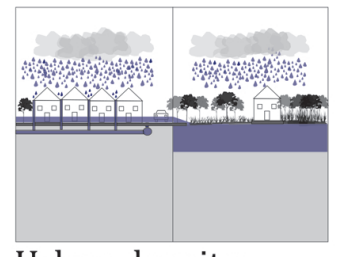

Urban density

Urban density influences the amount of water penetrable surfaces and absorption rates. Inceasing stormwater and surface runoff volumes.

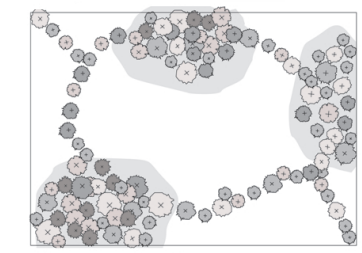

Green infrastructure

(vegetative components)

Core vegetative zones rest within green hubs. These ecological hubs are connected via green corridors and smaller vegetative links.

Storage

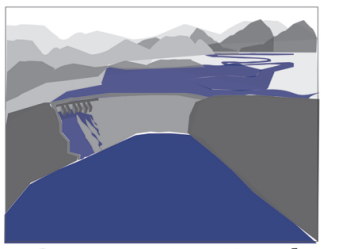

Dams \& wet retention basins Artificial permanent water storage devices that control and lowe downstream water volumes to reduc flood risks up to a design flood standard (water carrying capacity).

Nutrient \& waste recovery

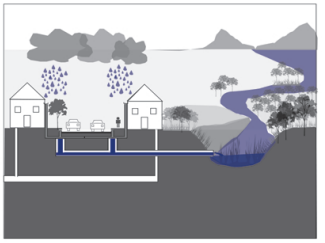

Stormwater management

The quality and quantity of stormwater is cotrolled by . Urban surface runoff, precipitation and floodwaters are

collected, redirected and contaminants filtered by above (eg. bioretention swales) and below ground (eg. sand and gravel chamber filters) stormwater drainage systems.

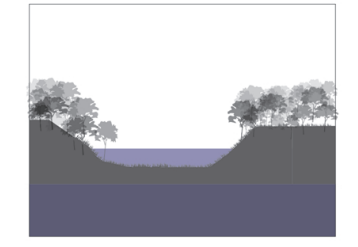

Dry retention basins Dry green spaces with gradient depressions direct, capture, stor and absorb seasonal precipitation, stormwater and floodwaters.

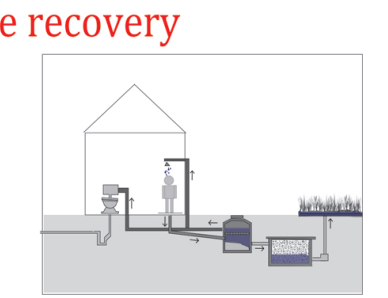

Grey water systems

Systems that recapture and store grey water to be re-used for irrigation. Soft biological processes and hard

mechanical processes can filter and purify the water further so it can be used as non-potable household water.
FEEDBACK

Response

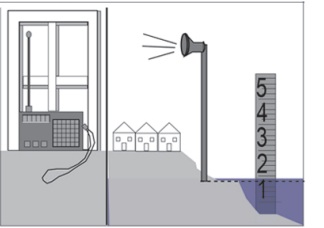

Flood warning systems Visually or acoustically inform residents of flood risks. They are implemented prior to, and activated in response to floods to facilitate community, loods

Regeneration

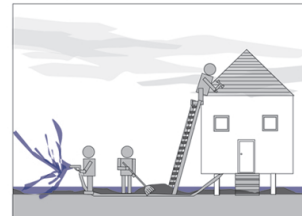

Restoration and renewal Flood damaged buildings and infrastructure are either restore with improved flood proofing or demolished to make way for new more flood adapted construction.

Cooperative self regulation

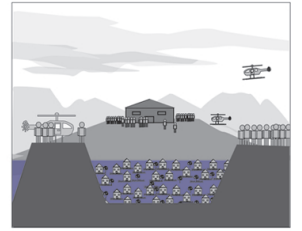

Emergency Management

ncludes mitigation, response, preparednes and recovery. It collaborates with the locas flood affected inhabitants and collective governing bodies to reduce the physical 


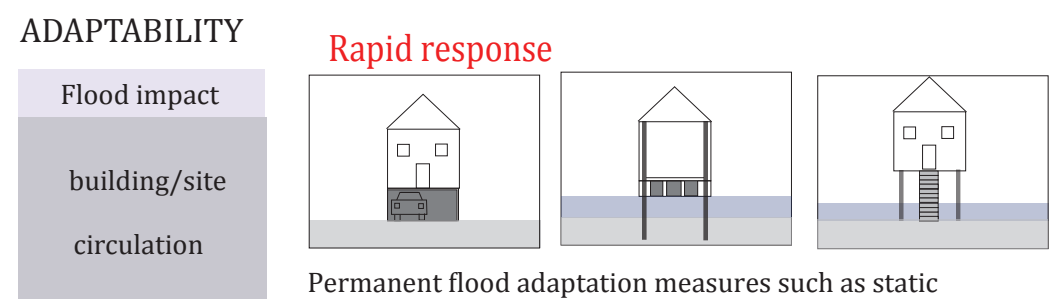

Permanent flood adaptation measures such as static elevation of living spaces on poles or above garages or amphibious construction reduce the adverse impacts of floods.

DIVERSITY \& REDUNDANCY

Flooding issues
debris
water velocity
water volume
water quality

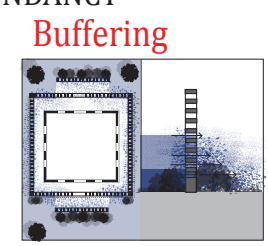

A variety of vegetation and solid penetrabl structures dissipate and

reduce the velocity of flood waters across a site. A perimetre of barriers spatially separated from the main load bearing structure protect the primary structure by collecting debris and reducing the velocity of flood waters.

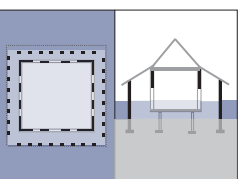

Alternate circuits

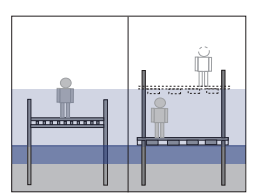

Static or amphibious cirulation pathways increase safety and enable greater pedestrian movement during and post flood events. from concrete or steel

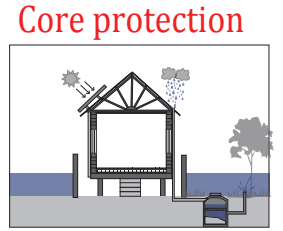

Permanent dry and wet flood proofing measures and alternate energy and back up services improves the adaptive capacity of the building to flooding and ensures vital needs services are available.

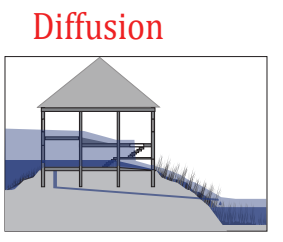

Artificial difussion channels can be achieved by

positioning and gradienting exterior circulation pathways and interior corridors to re-direct flood waters across

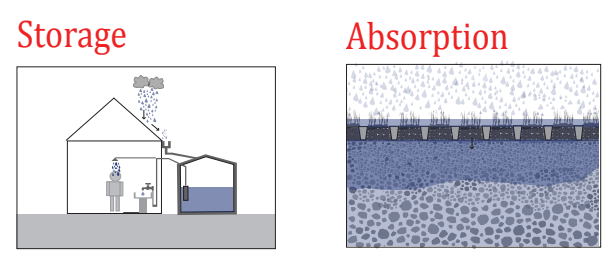

Above or below ground rainwater tanks made collect and store water for occupant use.

the site and through the

building.
FEEDBACK

Weather event
precipitation
Flood duration

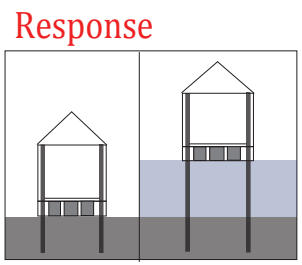

Construction technique and material selection ensure a structure is not adversly impacted upon by flooding Construction meth upon by flooding. Construction methods, such effectively respond and react to flooding.

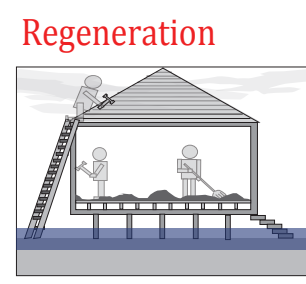

Cleaning and improving flood proofing solutions by applying knowledge of the recent flood event will improve the recent flood event will improve the
ability of the structure to cope with future floods.
Nutrient \& waste recovery

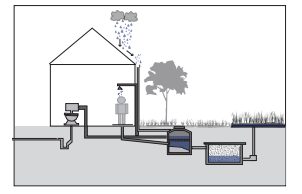

Grey water systems and rain water collection tanks allow water to be captured, stored, membranes reduces run off volumes in urban areas by allowing water to penetrate filtered and reused before below ground through being released back into the natural filtration. natural vegetative surroundings.

Cooperative self regulation

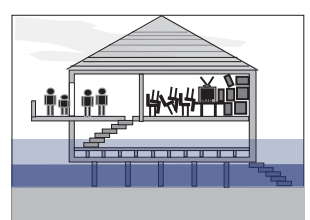

Moving furniture, appliances and important posessions above anticipated flood heights. Livable spaces on the second storey, equipt with flood kits and a flood evacuation plan. 